\title{
Untersuchung kulturbedingter Kategorisierungspräferenzen bei chinesischen und deutschen Probandinnen und Probanden
}

\author{
Dissertation \\ zur Erlangung des Doktorgrades \\ der Mathematisch-Naturwissenschaftlichen Fakultäten \\ der Georg-August-Universität zu Göttingen
}

vorgelegt von

Markus Reitt

aus Hildesheim

Göttingen 2012 
Referentin: Prof. Dr. Uta Lass

Korreferent: Prof. Dr. Dr. h.c. Gerd Lüer

Tag der mündlichen Prüfung: 22.01.2013 


\section{Danksagung}

Ich möchte an dieser Stelle die Gelegenheit nutzen, einer Reihe von Personen zu danken, die mich bei der Entstehung dieser Dissertation unterstützt haben:

Frau Prof. Dr. Uta Lass und Herrn Prof. Dr. Dr. h.c. Gerd Lüer, welche Referat und Korreferat übernommen haben;

Frau Prof. Dr. Song Yan, die mich an den Bereich der kulturvergleichenden Psychologie heranführte und mit deren Anregungen ich die zugrundeliegende Idee dieser Dissertation entwickeln konnte;

Frau Liu Xiaoyan, welche bei der Entwicklung und Kontrolle der chinesischen Stimuli wertvolle Impulse und versierte Sprachkenntnisse beisteuerte;

Herrn Prof. Chen für die herzliche Gastfreundschaft an der East China Normal University Shanghai und meinen großartigen chinesischen Helfern vor Ort in Shanghai, welche mir bei der Rekrutierung und Durchführung der Experimente in China behilflich waren: Jia Lili, Shen Nan, Li Bou, Xiao Ju und Bao Zhui;

Herrn Alexander Wiegmann und Herrn Simon Palmer, welche die Programmierung der Experimente maßgeblich vorantrieben;

Herrn Dr. Thorsten Albrecht für die Unterstützung bei der komplexen Auswertung des dritten Experimentes;

Frau Charlotte Lehmann, Frau Monika Budde und Herrn Dr. Michael Belz, die diese Arbeit Korrektur gelesen haben;

meinen Eltern, die mich während meines Studiums und meiner Promotion auf vielerlei Weise unterstützt haben und ohne deren wertvolle Unterstützung es diese Dissertation wahrscheinlich nicht gegeben hätte;

und zu guter Letzt ein großes Dankeschön an meine Probandinnen und Probanden, die an den Experimenten teilgenommen haben. 


\section{Inhaltsverzeichnis}

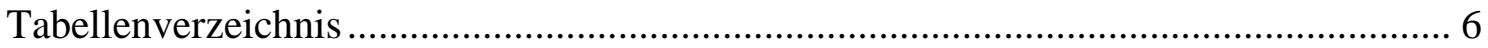

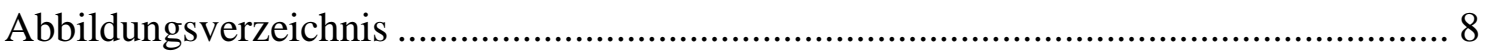

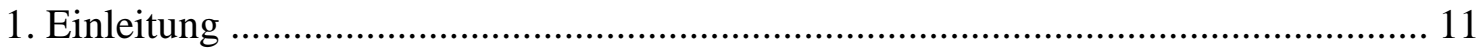

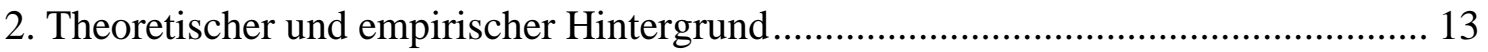

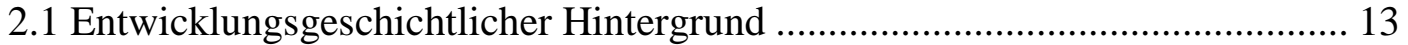

2.2 Denkstile in westlichen und ostasiatischen Kulturen ................................... 17

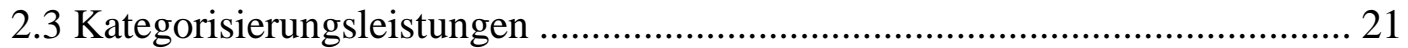

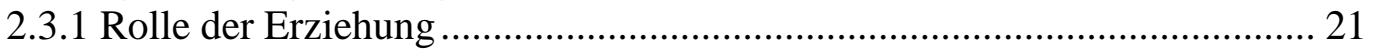

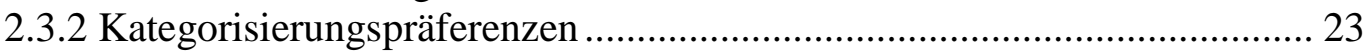

2.4 Interaktion von Sprache und Kognition - eine Frage des

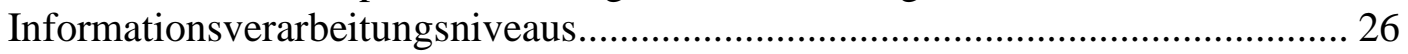

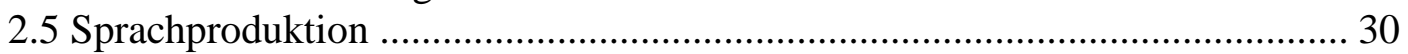

2.6 Zusammenfassungen der Hypothesen ....................................................... 34

3. Methode Experiment 1 „Kategorisierung über Bildpaare“ ........................................ 36

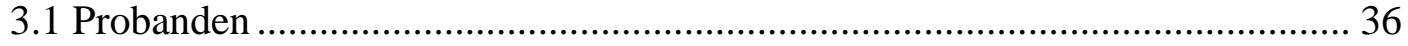

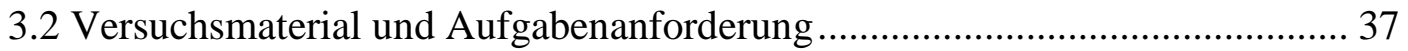

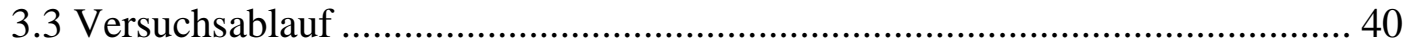

4. Ergebnisse Experiment 1 „Kategorisierung über Bildpaare“ ................................... 44

4.1 Overall-Analyse der Reaktionszeiten ....................................................... 44

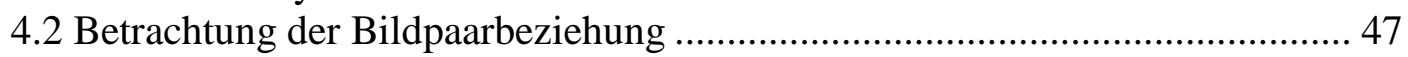

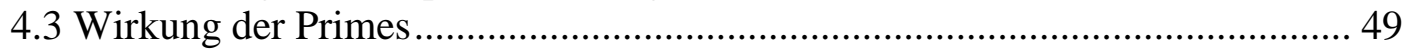

4.4 Auswertung der Fehlerprozente ............................................................... 52

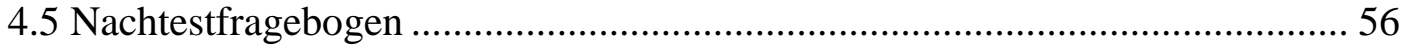

4.6 Fazit Experiment 1 „Kategorisierung über Bildpaare“.................................... 59

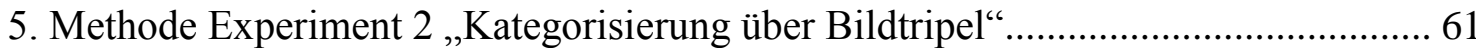

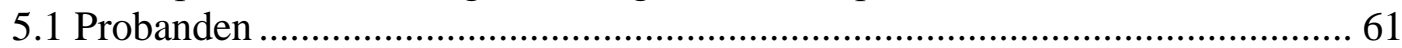

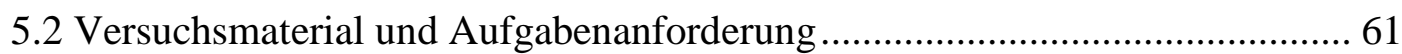

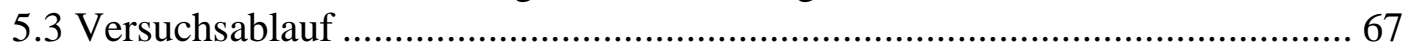

6. Ergebnisse Experiment 2 „Kategorisierung über Bildtripel“ ..................................... 70

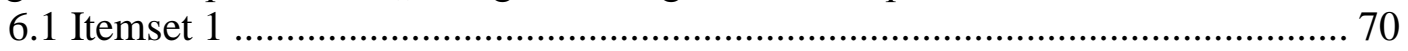

6.1.1 Analyse der Reaktionszeiten ......................................................... 70

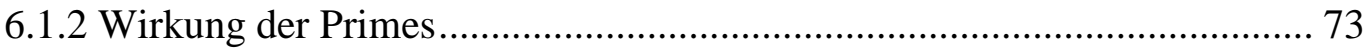

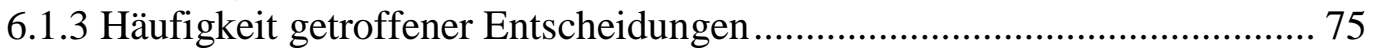

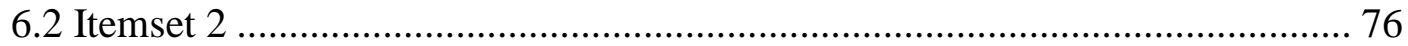

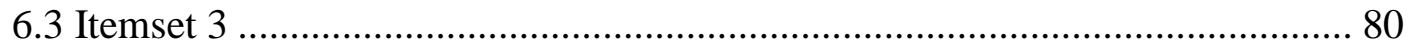

6.4 Fazit Experiment 2 „,Kategorisierung über Bildtripel“ ${ }^{\prime . . . . . . . . . . . . . . . . . . . . . . . . . . . . . . . . . . . . ~} 83$ 


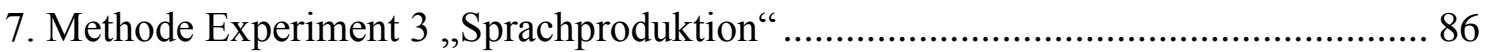

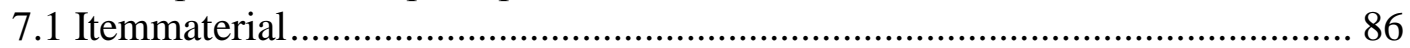

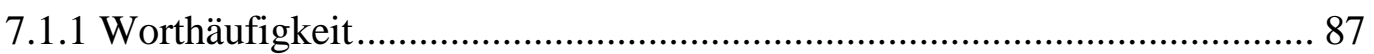

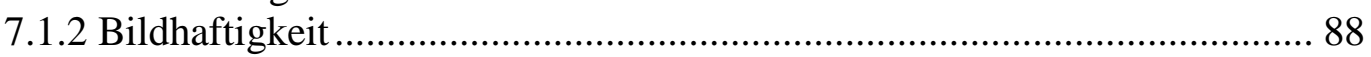

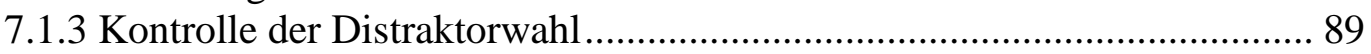

7.1.4 Zusammenfassende Bewertung des Itemmaterials .................................. 92

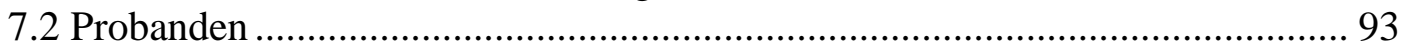

7.3 Versuchsanordnung und Aufgabenanforderung ......................................... 93

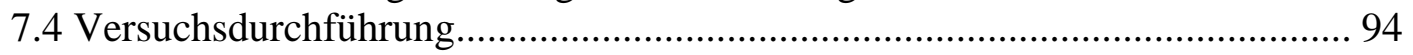

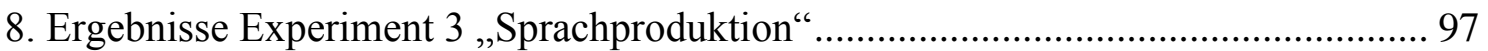

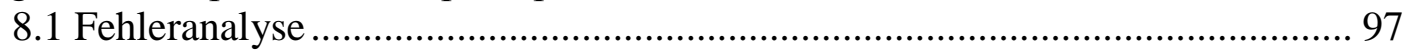

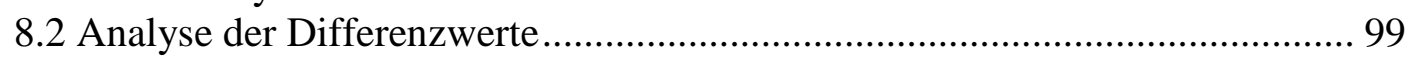

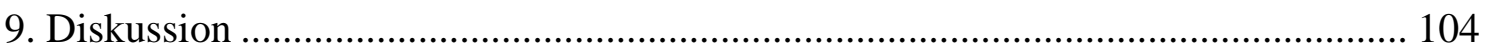

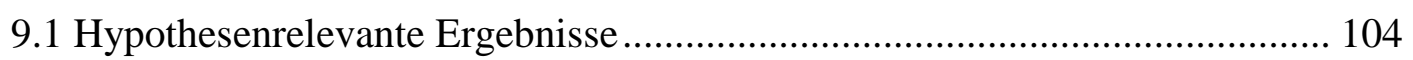

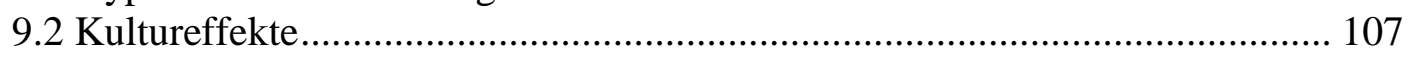

9.3 Kulturübergreifende Effekte.................................................................. 109

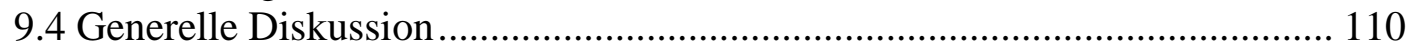

9.5 Kritik und Anregung für weitere Forschung ............................................ 112

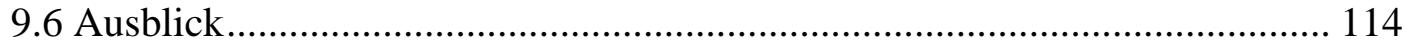

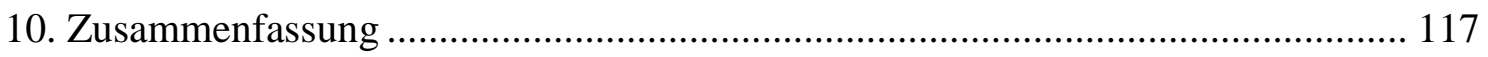

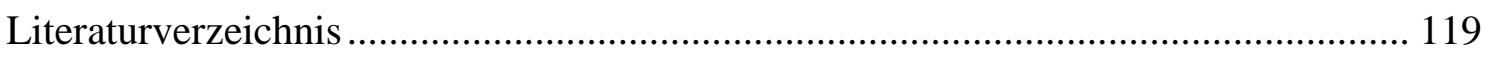

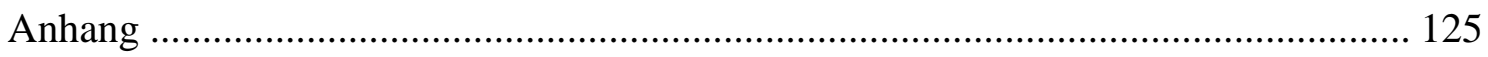

Anhang A: Itemmaterial Experiment 1 „Kategorisierung über Bildpaare“ ................. 125

Anhang B: Instruktion Experiment 1 „,Kategorisierung über Bildpaare““..................... 185

Anhang C: Nachtestfragebögen Experiment 1 „Kategorisierung über Bildpaare“ ...... 187

Anhang D: Itemmaterial Experiment 2 „Kategorisierung über Bildtripel“ “.................. 195

Anhang E: Instruktion Experiment 2 „,Kategorisierung über Bildtripel“ "...................... 269

Anhang F: Itemmaterial Experiment 3 ,Sprachproduktion“........................................ 273

Anhang G: Instruktion Experiment 3 ,Sprachproduktion“ ........................................ 279

Anhang H: Nachtestfragebögen Experiment 3 ,Sprachproduktion““.......................... 281 


\section{Tabellenverzeichnis}

Tabelle 1: Kombinationen kongruenter und inkongruenter Primes mit taxonomisch und relational verbundenen Bildpaaren

Tabelle 2: Mittlere Rektionszeiten in Abhängigkeit der Bildpaarbeziehung (taxonomisch versus relational) und des Primes (inkongruent, kongruent, neutral, kein) von $n=52$ chinesischen Probanden und $n=51$ deutschen Probanden. Mittelwerte sind in Millisekunden (ms) angegeben

Tabelle 3: Mittlere Fehlerprozente in Abhängigkeit der Bildpaarbeziehung (taxonomisch versus relational) und des Primes (inkongruent, kongruent, neutral, kein) von $n=52$ chinesischen Probanden und $n=51$ deutschen Probanden

Tabelle 4: Mittlere Fehlerprozente im Nachtestfragebogen in Abhängigkeit der Bildpaarbeziehung (taxonomisch versus relational) von $n=52$ chinesischen Probanden und $n=51$ deutschen Probanden

Tabelle 5: Kombinationen kongruenter und inkongruenter Primes mit taxonomisch und relational verbundenen Beziehungen aus einem Bildtripel

Tabelle 6: Kombinationen kongruenter und inkongruenter Primes mit taxonomisch und neutral verbundenen Beziehungen aus einem Bildtripel

Tabelle 7: Kombinationen kongruenter und inkongruenter Primes mit relationalen und neutral verbundenen Beziehungen aus einem Bildtripel

Tabelle 8: Mittlere Reaktionszeiten in Abhängigkeit der Entscheidung (taxonomisch versus relational) und des Primes (inkongruent, kongruent, neutral, kein) von $n=52$ chinesischen Probanden und $n=51$ deutschen Probanden. Mittelwerte sind in Millisekunden (ms) angegeben....

Tabelle 9: Mittlere Reaktionszeiten in Abhängigkeit des Primes (inkongruent, kongruent, neutral, kein) der Gesamtstichprobe $(N=103)$. Mittelwerte sind in Millisekunden (ms) angegeben.

Tabelle 10: Mittlere Reaktionszeiten in Abhängigkeit des Primes (inkongruent, kongruent, neutral, kein) von $n=52$ chinesischen Probanden und $n=51$ deutschen Probanden. Mittelwerte sind in Millisekunden (ms) angegeben

Tabelle 11: Mittlere Reaktionszeiten im Itemset 2 in Abhängigkeit des Primes (inkongruent, kongruent, neutral, kein) der Gesamtstichprobe $(N=103)$. Mittelwerte sind in Millisekunden (ms) angegeben 
Tabelle 12: Mittlere Reaktionszeiten in Abhängigkeit des Primes (inkongruent, kongruent, neutral, kein) von $n=52$ chinesischen Probanden und $n=51$ deutschen Probanden. Mittelwerte sind in Millisekunden (ms) angegeben

Tabelle 13: Mittlere Reaktionszeiten im Itemset 3 in Abhängigkeit des Primes (inkongruent, kongruent, neutral, kein) der Gesamtstichprobe $(N=103)$. Mittelwerte sind in Millisekunden (ms) angegeben ....

Tabelle 14: Mittlere Reaktionszeiten im Itemset 3 in Abhängigkeit des Primes (inkongruent, kongruent, neutral, kein) von $n=52$ chinesischen Probanden und $n=51$ deutschen Probanden. Mittelwerte sind in Millisekunden (ms) angegeben

Tabelle 15: Mittlere Differenzen in den Reaktionszeiten mit Angabe des Standardfehlers von Durchgängen mit taxonomischen minus neutralen Distraktoren sowie relationalen minus neutralen Distraktoren in den SOA-Bedingungen SOA -150 ms und SOA -300 ms ( $n=50$ chinesische Probanden, $n=51$ deutsche Probanden). 


\section{Abbildungsverzeichnis}

Abbildung 1: Beispiel eines zu bewertenden Bildpaares. In diesem Fall handelt es sich um eine taxonomische Beziehung zwischen den Bildern.

Abbildung 2: Zeitliche Sequenz der Ereignisse in einem Versuchsdurchgang in Experiment 1 „Kategorisierung über Bildpaare“ anhand eines Beispiels

Abbildung 3: Darstellung der mittleren Reaktionszeiten (ms) in Abhängigkeit von Bildpaarbeziehung (Angabe vor dem Schrägstrich) und Prime (Angabe hinter dem Schrägstrich). Die Werte für deutsche Probanden $(n=51)$ und chinesische Probanden $(n=52)$ sind einander gegenüber gestellt und der Standardfehler abgetragen.

Abbildung 4: Mittlere Reaktionszeiten in Sekunden bei taxonomischen Bildpaaren gegenüber mittleren Reaktionszeiten bei relationalen Bildpaaren innerhalb der gesamten Stichprobe mit Standardfehlern 48

Abbildung 5: Mittlere Reaktionszeiten (ms) bei taxonomischen Bildpaaren versus mittlere Reaktionszeiten bei relationalen Bildpaaren, getrennt für deutsche $(n=51)$ und chinesische Probanden $(n=52)$ mit Angabe des Standardfehlers des Mittelwertes.....

Abbildung 6: Darstellung der mittleren Reaktionszeiten (ms) in Abhängigkeit von der Primebeziehung in der chinesischen Stichprobe $(n=52)$ mit Angabe des Standardfehlers

Abbildung 7: Darstellung der mittleren Reaktionszeiten (ms) in Abhängigkeit von der Primebeziehung innerhalb der deutschen Stichprobe $(n=51)$ mit Angabe des Standardfehlers

Abbildung 8: Darstellung der Fehlerprozente in Abhängigkeit von der Primebeziehung über die gesamte Stichprobe $(N=103)$

Abbildung 9: Darstellung der Fehlerprozente in Abhängigkeit von der Primebeziehung innerhalb der chinesischen Stichprobe $(n=52)$.

Abbildung 10: Darstellung der Fehlerprozente in Abhängigkeit von der Primebeziehung innerhalb der deutschen Stichprobe $(n=51)$

Abbildung 11: Darstellung der Fehlerprozente im Nachtestfragebogen von $n=52$ chinesischen Probanden und $n=51$ deutschen Probanden

Abbildung 12: Darstellung der Fehlerprozente im Nachfragebogen und Computerexperiment von $n=52$ chinesischen Probanden und $n=51$ deutschen Probanden 
Abbildung 13: Stimulus aus Itemset 1 von Experiment 2. „A“, „B“ und „C“ markieren die Beziehung, die über eine Tastatur gewählt werden konnte. „B“ würde bedeuten, die Biene und der Bienenstock gehören zusammen; „C“ würde bedeuten, die Fliege und die Biene werden als zusammengehörig empfunden

Abbildung 14: Stimulus aus Itemset 2 von Experiment 2. „A“, „B“ und „C“ markieren die Beziehung, die über eine Tastatur gewählt werden konnte. „A“ würde bedeuten, die Zitrone und die Orange gehören zusammen; „C“ würde bedeuten, die Orange und der Bleistift werden als zusammengehörig empfunden

Abbildung 15: Stimulus aus Itemset 3 von „Experiment 2. „A“, „B“ und „C“ markieren die Beziehung, die über eine Tastatur gewählt werden konnte. „A“ würde bedeuten, der Schraubenzieher und die Banane gehören zusammen; „B“ würde bedeuten, die Banane und die Schraube gehören zusammen; „C“ würde bedeuten, der Schraubenzieher und die Schraube werden als zusammengehörig empfunden

Abbildung 16: Zeitliche Sequenz der Ereignisse in einem Versuchsdurchgang in Experiment 2 „, Kategorisierung über Bildtripel“" anhand eines Beispiel

Abbildung 17: Mittlere Reaktionszeiten in Abhängigkeit der Entscheidung (taxonomisch versus relational) und des Primes (inkongruent, kongruent, neutral, kein) von $n=52$ chinesischen Probanden und $n=51$ deutschen Probanden. Mittelwerte sind in Millisekunden (ms) angegeben und der Standardfehler ist abgetragen

Abbildung 18: Mittlere Reaktionszeiten in Abhängigkeit des Primes (inkongruent, kongruent, neutral, kein) der Gesamtstichprobe $(N=103)$. Mittelwerte sind in Millisekunden (ms) angegeben und der Standardfehler ist abgetragen.

Abbildung 19: Mittlere Reaktionszeiten in Abhängigkeit des Primes (inkongruent, kongruent, neutral, kein) von $n=52$ chinesischen Probanden und $n=51$ deutschen Probanden. Mittelwerte sind in Millisekunden (ms) angegeben und der Standardfehler ist abgetragen

Abbildung 20: Kontrast über die getroffenen Entscheidungen von $n=52$ chinesischen Probanden und $n=51$ deutschen Probanden: Summe der Anzahl taxonomischer Entscheidungen minus Summe der Anzahl relationaler Entscheidungen

Abbildung 21: Mittlere Reaktionszeiten im Itemset 2 in Abhängigkeit des Primes (inkongruent, kongruent, neutral, kein) der Gesamtstichprobe $(N=103)$. Mittelwerte sind in Millisekunden (ms) angegeben und der Standardfehler ist abgetragen

Abbildung 22: Mittlere Reaktionszeiten im Itemset 2 in Abhängigkeit des Primes (inkongruent, kongruent, neutral, kein) von $n=52$ chinesischen Probanden und $n=51$ deutschen Probanden. Mittelwerte sind in Millisekunden (ms) angegeben und der Standardfehler ist abgetragen 
Abbildung 23: Mittlere Reaktionszeiten im Itemset 3 in Abhängigkeit des Primes (inkongruent, kongruent, neutral, kein) der Gesamtstichprobe $(N=103)$. Mittelwerte sind in Millisekunden (ms) angegeben und der Standardfehler ist abgetragen

Abbildung 24: Mittlere Reaktionszeiten im Itemset 3 in Abhängigkeit des Primes (inkongruent, kongruent, neutral, kein) von $n=52$ chinesischen Probanden und $n=51$ deutschen Probanden. Mittelwerte sind in Millisekunden (ms) angegeben und der Standardfehler ist abgetragen

Abbildung 25: Mittlere Ratingscores mit Angabe des Standardfehlers für deutschsprachige Items (blau) und chinesischsprachige Items (rot) hinsichtlich der Frage, wie leicht sich die durch ein Distraktorwort und ein Targetwort bezeichneten beiden Objekte einer gemeinsamen Kategorie zuordnen lassen $(1=$ leicht, $6=$ schwer). $n=41$ deutsche Probanden und $n=40$ chinesische Probanden

Abbildung 26: Mittlere Ratingscores mit Angabe des Standardfehlers für deutschsprachige Items (blau) und chinesischsprachige Items (rot) hinsichtlich der Frage, wie leicht sich für die durch ein Distraktorwort und ein Targetwort bezeichneten Objekte eine logische, funktionale Beziehung herstellen lässt ( $1=$ leicht, $6=$ schwer). $n=41$ deutsche Probanden und $n=40$ chinesische Probanden

Abbildung 27: Darstellung eines Targets mitsamt standardisierter Bezeichnung in der Lernphase.

Abbildung 28: Zeitliche Sequenz der Ereignisse in einem Versuchsdurchgang mit taxonomischem Distraktor im Experiment 3 „Sprachproduktion“

Abbildung 29: Darstellung der Fehlerverhältnisse der chinesischen Probanden in Prozent in Experiment 3 ,Sprachproduktion“

Abbildung 30: Darstellung der Fehlerverhältnisse der deutschen Probanden in Prozent in Experiment 3 ,Sprachproduktion“

Abbildung 31: Mittlere Differenzen in den Reaktionszeiten mit Angabe des Standardfehlers von Durchgängen mit taxonomischen minus neutralen Distraktoren sowie relationalen minus neutralen Distraktoren in den SOA-Bedingungen SOA -150 ms und SOA $-300 \mathrm{~ms}$ ( $n=50$ chinesische Probanden, $n=51$ deutsche Probanden) $\ldots 100$

Abbildung 32: Interaktion „Distraktor x Nation“. Mittlere Differenzen in den Reaktionszeiten (ms) zwischen Durchgängen mit taxonomischen versus neutralen Distraktoren (blau) sowie relationalen versus neutralen Distraktoren (rot) in Abhängigkeit von der Nation der Versuchsteilnehmer

Abbildung 33: Interaktion „Distraktor x SOA. Mittlere Differenzen in den Reaktionszeiten (ms) zwischen Durchgängen mit taxonomischen versus neutralen Distraktoren (blau) sowie relationalen versus neutralen Distraktoren (rot) in Abhängigkeit von der SOA-Bedingung ( $n=50$ chinesische Probanden, $n=51$ deutsche Probanden) 


\section{Einleitung}

Die Fähigkeit, wahrgenommene Reize hinsichtlich gemeinsamer Merkmale zu organisieren, in Klassen zusammenzufassen und $\mathrm{zu}$ repräsentieren, stellt einen wesentlichen Vorgang der kognitiven Informationsverarbeitung dar. Kategorisierung ermöglicht es uns, neue Erfahrungen mit bereits vorhandenem Wissen in Verbindung zu bringen und uns auch in neuen Situationen angemessen zu verhalten.

Die zentrale, zugrundeliegende Idee des dargestellten Forschungsprojektes liegt in der Fragestellung, ob Menschen ${ }^{1}$ in verschiedenen Kulturen die gleichen Reize in gleicher Weise repräsentieren.

In frühen Arbeiten zu diesem Thema wurde vermutet, dass Menschen aus unterschiedlichen Kulturen ihre Umwelt unterschiedlich kategorisieren, weil sich ihre Sprachen und damit die Benennungen der zu klassifizierenden Objekte unterscheiden. Der linguistische Determinismus z.B. geht von der These aus, dass die Sprache die Art, wie jemand denkt oder die Welt repräsentiert, maßgeblich determiniert oder zumindest stark beeinflusst. So verfügen nach einem bekannten Beispiel von Whorf die Inuit über verschiedene Wörter für „Schnee“, die jeweils auch die Beschaffenheit und Qualität des Schnees widerspiegeln (Whorf, 1956). Der Einfluss von Sprache auf Kategorisierungspräferenzen soll an anderer Stelle noch ausführlich diskutiert werden (vgl. Abschnitt 2.4).

Ein augenscheinlicher Vorteil von Kategorisierungen ist es, kognitive Kapazität zu sparen. Hierbei werden Objekte auf unterschiedliche Art und Weise gruppiert, um somit einfach an Informationen über ein betreffendes Objekt zu gelangen. Objekte können dabei nach gemeinsamen Kategorien oder nach relationalen Verbindungen gruppiert werden. Zum Beispiel lassen sich die beiden Objekte Apfel und Banane in die Kategorie Obst einordnen. Zwischen den beiden Objekten Schaf und Wolle dagegen besteht eine relationale Verbindung, da das Schaf Wolle liefern kann. Genau in diesem Punkt konnten Unterschiede zwischen verschiedenen Kulturen festgestellt werden (vgl. Yan, Lüer, \& Lass, 2005) - es scheint also unterschiedliche Tendenzen zu geben, nach welchen Kriterien Objekte in Gruppen eingeteilt werden.

\footnotetext{
${ }^{1}$ Aufgrund der besseren Lesbarkeit wird auf die gleichzeitige Verwendung männlicher und weiblicher Sprachform verzichtet. Sämtliche Bezeichnungen von Personen gelten für beide Geschlechter.
} 
Viele Forschungsarbeiten beschäftigen sich mit Unterschieden zwischen der westlichen und der ostasiatischen Welt, vermutlich weil viele Unterschiedlichkeiten bei diesen beiden Kulturen besonders hervorstechen. Die kulturellen Differenzen weisen hier schon eine lange Historie auf und Gründe liegen vermutlich weit in der Vergangenheit. Unterschiede im Gruppierungsverhalten scheinen dabei ein zeitlich stabiles Phänomen zu sein und sind durch verschiedenste Arbeiten belegt (Yan et al., 2005; Ji, Zhang, \& Nisbett, 2004).

Die Art und Weise, wie Objekte gruppiert werden, liefert Informationen darüber, wie Personen die Welt und ihre Objekte sehen und organisieren, wie sie sich in der Welt bewegen und welche Gesichtspunkte bei der Verbindung von Objekten als entscheidend angesehen werden. Dies wiederum gibt Aufschluss über die kognitiven Strukturen von Personen aus unterschiedlichen Kulturen und ist somit ein aufschlussreiches und wichtiges Forschungsfeld. Die Forschung über kulturelle Unterschiede beinhaltet also das Verstehen und Kennenlernen fremder Kulturen und stellt somit eine Annäherung auf der Basis von Unterschieden dar. Dies ist besonders in einer Welt von sehr großer Bedeutung, in der durch Globalisierung und Informationstechnologien Entfernungen immer geringer $\mathrm{zu}$ werden scheinen und die Begegnung mit fremden Kulturen ein alltägliches Phänomen ist (Bell \& Kozlowski, 2002; Duarte \& Snyder, 2001).

Wenn nun, wie durch verschiedene Belege gezeigt, Unterschiede zwischen Kulturen hinsichtlich des Gruppierungsverhaltens bestehen, so stellt sich die Frage, worauf diese Unterschiede basieren und wie sie vermittelt werden. Dabei sind Einflüsse vieler Faktoren vorstellbar, wobei zu den stärksten wohl die jeweilige Kultur und die Sprache zu zählen sind. In den vorliegenden Experimenten mit deutschen und chinesischen Probanden wird die Fragestellung untersucht, ob beobachtbare Variationen im Kategorisierungsverhalten nicht in der Fähigkeit zur Kategorisierung allein zu suchen sind, sondern unter Einbezug von kulturellen Variablen interpretiert werden können.

Zunächst werden im Anfangsteil der Arbeit die theoretischen und empirischen Grundlagen erläutert, die als Basis für die Fragestellung und die Ableitung der Hypothesen dienen. Es folgt die Darstellung der Überprüfung der Hypothesen in den Experimenten 1 bis 3 und die Vorstellung der Ergebnisse. Abschließend werden die Ergebnisse diskutiert sowie Anregungen für weitere zukünftige Forschungen gegeben. 


\section{Theoretischer und empirischer Hintergrund}

Um die Grundkonzeption des zu berichtenden Forschungsprojektes darzustellen, wird zunächst ein Überblick gegeben mit einer ausführlichen Darstellung der relevanten Befunde. Zentral für die zu entwickelnde Fragestellung der vorliegenden Dissertation sind Befunde zu Unterschieden im Erleben und Verhalten von Personen ostasiatischer und westlicher Herkunft. Dabei wird eine kulturvergleichende Perspektive eingenommen. Es wird zunächst ein entwicklungsgeschichtlicher Überblick gegeben und anschließend werden die unterschiedlichen Denkstile näher betrachtet. Abschließend werden aus der aktuellen Befundlage die Forschungshypothesen abgeleitet.

\subsection{Entwicklungsgeschichtlicher Hintergrund}

Bei der Frage nach der Entwicklung unterschiedlicher Denkstile zwischen verschiedenen Kulturen können Spuren weit in die Vergangenheit zurückverfolgt werden. Schon zwischen den Menschen im antiken Griechenland und im damaligen China lassen sich Unterschiede beschreiben (Peng \& Nisbett, 1999). Dies bezieht sich nicht nur auf kognitive Prozesse, sondern auch auf die Philosophie, Gesellschaftsform sowie die wirtschaftliche Entwicklung.

Als Determinanten verschiedener Denkstile werden von Ji, Peng und Nisbett (2000) kulturelle und sozioökonomische Wurzeln der chinesischen und der europäischen Kultur aufgezeigt. Die europäische Kultur, fußend auf einem aristotelischen Verständnis von Logik, neige demnach eher zu einem analytischen Denkstil, wohingegen die frühe traditionelle chinesische Kultur eher den Weisheiten des Konfuzius, taoistischen Einflüssen und einem gewissen Volksglauben folgend zu einem holistischen, ganzheitlichen Denkstil neige. Verschiedene philosophische Strömungen waren und sind wohl auch durch diese Wurzeln erst in die Lage versetzt worden, ihre Erkenntnisse in der Gesellschaft durchzusetzen (Ji, Peng, \& Nisbett, 2000).

Griechische Philosophen betrachteten die Welt als eine Ansammlung von eigenständigen Objekten, die eigens innewohnende Eigenschaften aufweisen, mit deren Hilfe Kategorisierungen erst möglich werden. Die kleinste damals noch theoretisch 
postulierte Einheit stellt das sogenannte „Atom“ dar. Der Mensch wurde ebenfalls als ein autonomes Individuum angesehen. Das Systematisieren einzelner Objekte mit den ihnen innewohnenden spezifischen Eigenschaften führte dabei zu Kategorien. Es wurde möglich, Regeln und Vorhersagen abzuleiten. Der Mensch konnte mithilfe dieser Regeln seine Welt systematisieren und sich dadurch zunehmend besser in ihr zurechtfinden. Vergangene Ereignisse konnten erklärt und zukünftige Geschehnisse vorhergesagt werden (Hansen, 1983).

Die Menschen im traditionellen China hingegen verstanden die Welt als Sammlung von überlappenden und sich gegenseitig durchdringenden Stoffen und Energien (Nisbett, Peng, Choi, \& Norenzayan, 2001). Aus dieser Sichtweise heraus ist es nachvollziehbar, sich eher auf die Beziehungen zwischen Objekten zu konzentrieren, als sie jedes für sich zu kategorisieren. Eine Kategorisierung würde nur einen Teilaspekt der Wirklichkeit darstellen, da ein größerer Gesamtzusammenhang jenseits der Erfahrungswelt eines einzelnen Menschen liegt. Da die Komplexität der Welt zu groß ist, konzentriert man sich auf die Relationen zwischen Objekten. Es wurde davon ausgegangen, dass Geschehnisse vielmehr durch den Kontext als durch die Eigenschaften von Objekten beeinflusst werden. Aufgrund der vielfältigen Verknüpfungen und Relationen scheint ein permanenter Wandel der Dinge stattzufinden. Unterschiedlichste Einflussfaktoren scheinen so zahlreich zu sein, dass eine hastig aufgestellte Regel schnell wieder verworfen wird (Nisbett, 2003).

Als Alternative zu einem formal logischen Denkstil wird an dessen Stelle eine Dialektik gesetzt. Diese hat zum Ziel, nicht an Gegensätzen zu scheitern, sondern vielmehr eine Integration derselben anzustreben. Die Philosophen im traditionellen China strebten dementsprechend Verstehen durch Sinneserfahrungen und direkte Wahrnehmung an. Sie entwickelten folglich keine formalen logischen Systeme, sondern eine Dialektik. Diese versuchte, bestehende Gegensätze nebeneinander stehen zu lassen, einen Mittelweg zu finden oder die Gegensätze sogar zu integrieren und zur Erklärung von Beziehungen heranzuziehen (Lloyd, 1990). Für Philosophen der europäischen Antike wie Aristoteles hingegen konnten Gegensätze nicht parallel bestehen, da dieses ihrem Weltverständnis und Verständnis von Ästhetik widersprach. Somit mussten, wenn zwei oder mehr gegensätzliche Positionen aufeinander trafen, stets alle bis auf 
eine von ihnen ausgeschlossen werden, um so eine Auflösung des Konfliktes zu ermöglichen (Nisbett, 2003).

Aristoteles entwickelte z.B. erste Hypothesen zur Schwerkraft, erläuterte diese aber als eine Eigenschaft von Objekten, die Dinge zum Fallen bringt. Er versuchte also mit Hilfe von Objekteigenschaften das Verhalten ebendieses Objektes zu erklären. Im Gegensatz dazu erbrachten die Philosophen im traditionellen China z.B. Wissen über Magnetismus oder akustische Resonanz, also Phänomene, die mit einem ganzen Feld von Kräften, die sich gegenseitig durchdringen, zusammenhängen (Needham, 1962).

Im europäischen Denken, besonders in der hellenistischen Tradition, wird die Erkenntnis geradezu absolutiert. Ein Gott des antiken griechischen Olymps - Apollon steht für Licht und Erkenntnis in seiner reinsten Form. Soll eine Erkenntnis demnach rein sein, so erstrahlt sie in hellstem Licht und soll durch nichts getrübt werden.

Für Chinesen würde diese Allegorie nur einen sehr bruchstückhaften Teil der Wirklichkeit abbilden. Am deutlichsten wird die geradezu diametral entgegensetzte ostasiatische Denkrichtung in der metaphorischen Verschmelzung von Licht und Dunkelheit im Yin-Yang-Symbol, das eine andere Weltsicht allegorisch dargestellt.

Beide Gegensätze Yin und Yang als Symbole für Männlichkeit und Weiblichkeit, Licht und Schatten, Gut und Böse sind in einem Zeichen zusammen vereinigt. Sie bedingen einander, ein Teil des Einen ist sogar im Zentrum des Anderen wieder $\mathrm{zu}$ finden. Wird diese taoistische Allegorie auf die Lebenswirklichkeit eines Menschen aus einem asiatisch geprägten Umfeld übertragen, könnte dies bedeuten, dass jede Wirklichkeit mehr als bloß eine determinierte Sichtweise haben kann. Der Realitätsbegriff bekommt somit einen konstruktivistischen Charakter und entzieht sich dem Erkenntnishorizont des einzelnen Individuums.

Ein weiterer entscheidender Unterschied zwischen dem antiken Griechenland und dem traditionellen China war das politische System. In Griechenland entwickelten sich die Stadtstaaten als Staatsform, wobei die Bürger aufgefordert waren, am politischen Leben teilzunehmen und zu wählen. Hier entstand also die erste bekannte Demokratie. In Versammlungen sollte das Volk mit Hilfe von Argumenten überzeugt werden. Sprachliche Auseinandersetzungen sowie Debatten gehörten zum täglichen politischen Leben. Auseinandersetzungen zwischen Personen hatten für den Einzelnen meist wenige Konsequenzen, sie stellten geradezu eine Kunstform dar. Die eigene 
Meinung konnte in der Regel gefahrlos geäußert werden, zumal Griechenland für Andersdenkende im Zweifelsfall eine Ausreisemöglichkeit in einen anderen Stadtstaat oder über das Meer bot (Bleicken, 1995). Eine solche unabhängige Lebenssituation könnte zur Entwicklung eines analytischen Weltbildes beigetragen haben (Nisbett et al., 2001), zumal auch die meisten Menschen mehr Kontrolle über ihr Leben und ihr Land hatten. Sie nahmen sich als Individuen getrennt von anderen und mit Einfluss auf das eigene Schicksal wahr, was der analytischen Denkweise entspricht. Im damaligen China waren die Menschen im Gegensatz dazu in vielerlei Form abhängig. In einer Monarchie unter den verschiedenen Kaisern hatten sie kein Mitbestimmungsrecht, das Leben wurde kontrolliert durch den Monarchen und das Streben nach Harmonie und Frieden bestimmte den Alltag (Gernet, 1988). Alle Objekte und Geschehnisse wurden als verknüpft angesehen, so dass nichts verändert werden konnte, ohne eine Kettenreaktion in Gang zu setzten. Möglicherweise wirkte diese politische und gesellschaftliche Situation auf die Formung einer ganzheitlichen Weltsicht. Die unterschiedlich geprägten Gesellschaften Chinas und Griechenlands könnten somit das Denken der Personen in holistischer beziehungsweise analytischer Weise beeinflusst haben (Nisbett, 2003).

Durch seine geographische Lage ist China als Land vor allem geprägt durch weite fruchtbare Felder und befahrbare Flüsse, jedoch relativ arm an hohen Bergen. Somit überwog eine großangelegte Landwirtschaft (Staiger, Friedrich, \& Schütte, 2006) - es sei beispielsweise an die gigantischen chinesischen Reisterrassen erinnert. Deshalb wurden wahrscheinlich intakte Beziehungen und eine friedliche Nachbarschaft für den wirtschaftlichen Erfolg gepflegt (Nisbett, 2003). Die großen Felder konnten häufig nur gemeinsam ertragreich bewirtschaftet werden. Somit lag es nahe, harmonischen Beziehungen und einem intakten Netzwerk eine große Bedeutung beizumessen und sich selbst als Teil dieses Netzwerkes zu sehen. Individualistische Gesellschaften, wie zum Beispiel die griechische, entstanden im Gegensatz dazu überwiegend basierend auf Fischen und Jagen und weniger auf großangelegter Landwirtschaft. Der Grund dafür lag primär in der geographischen Lage Griechenlands, reich an hohen Bergen und Meer, aber arm an weiten bebaubaren Feldern (Jacobs \& Curtius, 2011). Kooperation untereinander war für diese Art des Erwerbs nur bedingt notwendig, wodurch die Menschen im antiken Griechenland in großem Ausmaß selbständig handeln konnten (Nisbett, 2003). Somit spielten Beziehungen eine weniger große Rolle und der Fokus 
lag eher auf der eigenen Person und dem Erreichen der eigenen Ziele. Die Personen hatten mehr Kontrolle und verschiedene Chancen hinsichtlich ihrer Erwerbstätigkeit (Masuda \& Nisbett, 2001). Im Vordergrund stand der eigene Profit, und um diesen zu erreichen, gab es unterschiedliche Möglichkeiten, das heißt, es mussten eigene Entscheidungen getroffen werden, zum Beispiel hinsichtlich Investitionen in das eigene Gewerbe. Es machte also Sinn, Objekte zu klassifizieren und anhand ihrer Eigenschaften ihr Verhalten vorherzusagen, da diese Methode den größten persönlichen Gewinn versprach (Nisbett, 2003). Das wiederum förderte möglicherweise die Entwicklung einer analytischen Denkweise. So könnten also neben der politischen und gesellschaftlichen Situation auch die geographische Lage und die ökonomischen Möglichkeiten der damaligen Zeit einen Einfluss auf die Menschen gehabt haben.

Eine vielleicht auf den ersten Blick triviale Hypothese, aber in Konsequenz von außerordentlich großer Tragweite ist die Annahme, dass diese postulierten Unterschiede der damaligen Kulturen bis heute noch Einflüsse auf die Menschen sowie deren kognitive Prozesse ausüben (Ji et al., 2000).

\subsection{Denkstile in westlichen und ostasiatischen Kulturen}

Ein wichtiges Konzept bei der Frage nach kulturellen Einflüssen auf kognitive Prozesse in der jüngeren Forschung ist, wie bereits oben angeklungen, die Unterscheidung von analytischem und holistischem Denken. Diese traditionell bedingten Unterschiede greift Nisbett (2003) auf und äußert sich über eine von asiatischen Kultureinflüssen geprägte, ganzheitliche Weltsicht, insbesondere bei Menschen mit chinesischen Sozialisationserfahrungen, folgendermaßen:

[...] we've seen that modern Asians, like the ancient Chinese, view the world in holistic terms: They see a great deal of the field, especially background events; they are skilled in observing relationships between events; they regard the world as complex and highly changeable and its opponents as interrelated; they see events as moving in circle between extremes, and they feel that control over events requires coordination with others. (S. 109) 
Holistisches Denken ist also dadurch charakterisiert, dass es am Gesamtzusammenhang orientiert ist und damit Beziehungen zwischen Objekten und dem Kontext in hohem Maße berücksichtigt werden. Ereignisse werden demnach anhand von Relationen prognostiziert. Aus einem holistischen Denkstil ergeben sich zahlreiche Implikationen für unterschiedlichste Bereiche des Erlebens. So neigen Ostasiaten zu einer ganzheitlichen Feldwahrnehmung und ziehen diese sogar in Kausalitätsschlüsse mit ein. Daraus resultiere ein nur reduzierter Gebrauch von Kategorien. Auch soll dieser Denkstil dialektische Argumentationsweise fördern (Nisbett et al., 2001).

Analytisches Denken, wie es u.a. einigen Philosophen der europäischen Antike zugeschrieben wird, beinhaltet hingegen stärker die Separierung eines Objektes von seinem Kontext, womit durch die Analyse seiner Eigenschaften und durch das Einordnen in eine Kategorie das Ableiten von Regeln und logischen Schlüssen ermöglicht wird. Auf der Ebene der Wahrnehmung wird der Kontext eher ignoriert. Dieses Denken zielt darauf ab, die Komplexität der Wirklichkeit auf kleinere handhabbare und damit besser verarbeitbare Einheiten zu reduzieren. Zusammenhänge und Hintergründe erschließen sich oft leichter, wenn es ein klares Kategorisierungsprinzip gibt, mit dessen Hilfe ein Individuum sich in seiner Lebenswirklichkeit bewegen kann (Nisbett et al., 2001).

Die Möglichkeit zur eindeutigen Kategorisierung mag für den Einen oder Anderen lebensnotwendig sein und den Charakter einer Wahrheit annehmen, da Einiges, was den persönlichen Erfahrungsrahmen sprengt, tiefsten Argwohn und Gefühle der Angst auf sich ziehen kann. In der Maslowschen Bedürfnishierarchie stellt Sicherheit nach den physiologischen Grundmotiven das zweitwichtigste Bedürfnis dar (Maslow, 1943). Wird dieses Bedürfnis dauerhaft verletzt, so kann dies schwerwiegende Folgen für das körperliche und seelische Wohlbefinden haben. Dies spiegelt sich z.B. in dem auf der Maslowschen Theorie aufbauenden Kontrollmotiv nach Grawe wider (Grawe, 2000). Erleiden Menschen einen Kontrollentzug oder verspüren keinerlei Selbstwirksamkeit mehr, so kann dies empfindliche psychische Konsequenzen nach sich ziehen, falls keinerlei Bewältigungsstrategien oder Ressourcen 
zur Verfügung stehen (Maslow, 1943). Dies kann sogar in einer erlernten Hilflosigkeit münden, die es vermag, einer Depression den Weg zu ebnen (Seligman, 1975).

Nach der Schilderung des theoretischen Hintergrundes soll sich nun die weitere Darstellung den empirischen Befunden zuwenden. Der folgende Überblick orientiert sich an den Arbeiten, die eine Aussage bezüglich der interessierenden Frage erlauben, ob nämlich in der ostasiatischen Kultur von einem zugrunde liegenden holistischen Denkmodell auszugehen ist, in der westlichen Kultur dagegen von einem analytischen Denkmodell.

Bereits Hsu (1953) argumentiert, dass der „fundamentale Attributionsfehler“ häufiger bei individualistischen Kulturen auftritt und demnach bei Amerikanern häufiger anzutreffen sei als bei Chinesen. Unter dem „fundamentalen Attributionsfehler" versteht man, dass bei Personen beobachtetes Verhalten eher dispositional attribuiert wird (z.B.: „Der ist eben so faul“), als dass die Situation berücksichtigt wird (z.B.: „Er musste sich um seine kranke Großmutter kümmern und hatte deswegen weniger Zeit"“). Letzteres Attributionsmuster steht in Übereinstimmung mit der ganzheitlichen Sichtweise des holistischen Denkens, da außer der Disposition auch situative Aspekte zur Erklärung des Verhaltens herangezogen werden (vgl. Hsu, 1953).

Morris und Peng (1994) präzisieren diese These dahingehend, dass kulturspezifische Attributionsmuster über implizite Theorien entstehen und anhand von Induktion und Sozialisation erworben werden. In individualistischen Kulturen lassen sich Personen eher $\mathrm{zu}$ einer dispositionsbezogenen Attribution verleiten als in kollektivistischen Kulturen. In der angesprochenen Studie von Morris und Peng wurden Zeitungsartikel über schwere Verbrechen, zumeist Amokläufe, in englisch- und chinesischsprachigen Zeitungen verglichen. Das Resultat war, dass in englischsprachigen Zeitungen mehr dispositionale und in chinesischsprachigen Zeitungen mehr situative Erklärungen für die gleichen Verbrechen gewählt wurden. In einer weiteren Untersuchung wurde deutlich, dass chinesische Probanden eher mit kontrafaktischen Urteilen bezüglich der Einschätzung eines Verbrechens hinsichtlich der zugrunde gelegten Attribution von Disposition und Situation umgehen konnten. So fanden es chinesische Probanden nicht widersprüchlich, wenn postuliert wurde, dass 
eine Person z.B. eigentlich ein netter Ehemann gewesen sei, trotzdem aber ein Gewaltverbrechen begangen habe. Außerdem zeigte sich, dass chinesische Probanden situative Gründe stärker gewichteten, während amerikanische Probanden mehr Gewicht auf dispositionale Gründe legten (Morris \& Peng, 1994).

Nisbett und Kollegen (2001) differenzieren kulturspezifische Denkstile dahingehend, dass Ostasiaten $\mathrm{zu}$ ganzheitlicher Feldwahrnehmung neigen (d.h. Umgebungsreize werden stark in die Wahrnehmung einbezogen) und sogar Kausalität diesem Denken unterordnen. Sie machen eher wenig Gebrauch von Kategorien und formaler Logik, sondern denken vielmehr dialektisch und verhalten sich dementsprechend. Dies sei unter anderem auf eine spezifische Agrikultur, Sesshaftigkeit und eine Großfamilienstruktur zurückzuführen. Feldabhängigkeit könne sich z.B. in einem starken Familienzugehörigkeitsgefühl und daraus resultierendem sozialen Engagement äußern (Nisbett et al., 2001).

Die westlich geprägten Menschen hingegen neigen dazu, mit analytischen, formal logischen Regeln, die auf Objekten und Kategorien beruhen, ihr Verhalten zu begreifen. Dies manifestiere sich in einem nomadischen Lebensstil mit einer zentralen Kernfamilie und einem hohen Bedürfnis nach Selbstverwirklichung (Wong, 2006).

Der Umgang mit Widersprüchlichkeit steht bei einer Untersuchung von Peng und Nisbett (1999) im Mittelpunkt. Chinesische Probanden zeigten in verschiedenen Studien ein dialektisches, kompromissfähiges Problemlöseverhalten, wohingegen amerikanische und europäische Probanden sich in ihrem Handeln eher vom dem Ziel der Wahrheitsfindung im Sinne einer aristotelischen Logik leiten ließen. In einer ersten Studie äußerte sich dies bei Chinesen in der bevorzugten Verwendung dialektischer Sprichwörter. So wurden den Probanden Sprichwörter vorgelegt, deren Vertrautheit bewertet werden sollte. Hierbei ordneten die chinesischen Probanden vorgelegten Sprichwörtern in ihrer Sprache einen höheren Bekanntheitsgrad zu als amerikanische Probanden denselben Sprichwörtern in englischer Sprache. In einer zweiten Studie wurde dieses Ergebnismuster repliziert mit Probanden, die einen jüdischen Hintergrund hatten und jiddische Sprichwörter beurteilen sollten. In einer dritten Studie wurde nach Lösungen für soziale Fragestellungen gefragt. Dialektische Lösungen für soziale Konflikte und die generelle Bevorzugung dialektischer Argumente fielen bei den chinesischen Versuchsteilnehmern auf (zum Beispiel „both the mothers and the 
daughters have failed to understand each other"). Wurden zwei widersprüchliche Aussagen präsentiert, entschieden sich amerikanische Probanden eher dafür, Stellung für ein Argument zu beziehen. Dagegen akzeptierten chinesische Probanden meist beide Positionen (Peng \& Nisbett, 1999; vgl. auch Ji et al., 2000).

\subsection{Kategorisierungsleistungen}

Im Folgenden sollen Kategorisierungsleistungen näher betrachtet werden. Dabei wird zunächst die Rolle der Erziehung ausgeführt und anschließend auf die verschiedenen kulturellen Kategorisierungspräferenzen eingegangen.

\subsubsection{Rolle der Erziehung}

Durch das Heranwachsen in einer Kultur, in der viel Wert auf zwischenmenschliche Beziehungen gelegt und eine Sicht des Selbst als Bestandteil eines großen Ganzen vermittelt wird, entwickelt sich auch eine Wahrnehmung in Bezug auf die Umwelt in entsprechender Weise. Dieser Denkstil kann sich dann bis in das Verhalten auswirken. Vermutlich geschieht dies vor allem durch die Erziehung und die dabei vermittelten Werte, Normen und sozialen Verhaltensweisen, wie die nachfolgend exemplarisch angeführten Untersuchungen belegen.

Fernald und Morikawa (1993) untersuchten japanische und amerikanische Mütter hinsichtlich der Art, mit ihren Kindern zu spielen und vor allem auch zu sprechen. Es wurde das Spielverhalten mit zwei Stofftieren - einem Hund und einem Schwein - und einem Holzauto beobachtet. Dabei stellten sie folgende Unterschiede fest: Amerikanische Mütter sagten häufiger den Namen von Objekten in den spielerischen Interaktionen mit ihrem Kind, sowohl beim Stofftier als auch beim Auto, während japanische Mütter Spielzeuge eher dazu benutzten, Beziehungen und soziale Konventionen zu erklären. Beispielsweise demonstrierten japanische Mütter signifikant häufiger höfliche Handlungsroutinen (z.B. Begrüßen/Verabschieden oder nach dem Befinden erkundigen). Der Name des Spielzeugs oder die Kategorie, in die das Objekt gehört, waren dafür nur wenig relevant. Amerikanische Mütter hingegen wiederholten oftmalig den Spielzeugnamen (,Das ist ein Hund“, oder auch onomatopoetisch: „Wuff- 
Wuff") oder den zugehörigen Oberbegriff wie „Auto“ oder „Tier“. Diese Ergebnisse legen nahe, dass amerikanische Kinder eher dazu erzogen werden, der Kategorisierung von Objekten Aufmerksamkeit zu schenken, und japanische Kinder eher dazu, ihre Aufmerksamkeit auf die Beziehungen zwischen den Objekten zu lenken (Fernald \& Morikawa, 1993).

Neben der Kultur, in der ein Mensch heranwächst, beeinflussen auch die Merkmale der jeweiligen Sprache, die man spricht, die kognitive Entwicklung. Ostasiatische Sprachen (z.B. Chinesisch oder Japanisch) sind stark kontextorientiert, d.h. die Wörter sind oft mehrdeutig und müssen somit im Kontext erfasst und interpretiert werden (Freeman \& Habermann, 1996). Hier wird also schon bei der Sprache gelernt, dass Dinge nicht für sich alleine stehen, sondern immer in Verbindung mit anderen Faktoren betrachtet und interpretiert werden müssen. Beispielsweise gibt es im Japanischen verschiedene Wörter für ,ich“, die die jeweilige Beziehung des Selbst zu anderen und somit die soziale Stellung präzisieren (Nisbett, 2003). Im Gegensatz dazu versuchen zum Beispiel amerikanische Eltern, ihren Kindern Wörter unabhängig vom Kontext verständlich zu machen (Heath, 1982). Somit entwickelt sich eher ein Verständnis dafür, dass Dinge für sich alleine stehen und unabhängig vom Kontext, in den sie eingebettet sind, beurteilt werden können (Nisbett et al., 2001).

Ein weiteres Kennzeichen der chinesischen Sprache ist, dass Nomen eine untergeordnete Rolle in Sätzen spielen. Im Chinesischen können Nomen in den Sätzen zum Teil optional sein und sogar weggelassen werden, da sie für die Kommunikation nicht unbedingt benötigt werden. Sätze mit Oberbegriffen, also solchen Nomen, die sich auf Kategorien und Arten beziehen, sollten somit beispielsweise im Englischen üblicher sein als im Chinesischen. Diese Hypothese wurde von Gelman und Tardif (1998) untersucht. Es wurden chinesisch- und englischsprechende Familien beobachtet, und ihre Konversation wurde analysiert. Die Ergebnisse zeigten, dass Oberbegriffe zwar sowohl in der englischen als auch in der chinesischen Sprache vorkamen, aber in der englischsprachigen Konversation häufiger auftraten als in der chinesischsprachigen.

Die zitierten Arbeiten zeigen, wie sich Erziehungsstil und sprachliche Kommunikation auf die kognitive Entwicklung auswirken und sich schließlich in unterschiedlichen Gruppierungs- oder Kategorisierungstendenzen niederschlagen können. 


\subsubsection{Kategorisierungspräferenzen}

Wie oben ausgeführt legen die beschriebenen Unterschiede im Denkstil zwischen Angehörigen der westlichen und der ostasiatischen Kultur entsprechende Unterschiede auch beim Kategorisieren nah. Um die hier relevanten Arbeiten angemessen darstellen zu können, müssen zunächst die Begriffe taxonomische und relationale Beziehung eingeführt werden.

Eine taxonomische Beziehung liegt vor, wenn die Objekte einer gemeinsamen Kategorie zugeordnet werden können. Zum Beispiel haben Kuh und Ente eine taxonomische Beziehung, da beide Mitglieder der Kategorie Tier sind. Dagegen handelt es sich um eine relationale Beziehung, wenn eine logische, thematische oder funktionale Verbindung zwischen den Objekten hergestellt werden kann, wie zum Beispiel zwischen Kuh und Gras, da die Kuh das Gras frisst.

In einer Untersuchung von Norenzayan, Smith, Kim und Nisbett (2002) wurde geprüft, ob sich ein kultureller Unterschied bei Kategorisierungsentscheidungen nachweisen lässt. Als Ergebnis zeigte sich, dass amerikanische Probanden dazu tendieren, die Zielobjekte auf der Basis von formalen Regeln zu kategorisieren, während asiatische Probanden sie eher auf der Basis von allgemeiner Ähnlichkeit ordnen. So sollten z.B. Sonnenblumen anhand ihrer Beschaffenheit (Anzahl der Blätter, Blütenform, Länge des Stängels) einer bestimmten Art zugeordnet werden. Hierbei wurden auch Mischformen präsentiert, die nicht eindeutig zuzuordnen waren. In einer weiteren Untersuchung von Norenzayan und Kollegen (2002) sollten von den Probanden Fabeltiere und Phantasietiere anhand von bestimmten Kriterien (Ein- oder Zweibeiner, Form des Hauptes, etc.) einer Kategorie zugeordnet werden. Diese im Experiment erworbenen Strategien sollten die Versuchsteilnehmer anschließend auf bisher unbekannte Phantasietiere anwenden. Die Autoren der Studie identifizierten zwei Strategien beim Vorgehen, eine regelbasierte und eine erfahrungsbasierte. Regelbasiert bedeutet hier, dass entschieden wird, ob ein Objekt in eine Kategorie gehört, indem die Regeln für diese Kategorie angewendet werden und so bestimmt wird, ob das Objekt die erforderlichen Kriterien erfüllt. Es handelt sich nach der oben eingeführten Definition um eine taxonomische Kategorienbildung. Erfahrungsbasierte Strategie meint in diesem Zusammenhang, es wird entschieden, ob ein Objekt in eine 
Kategorie gehört, indem die Ähnlichkeit der Objekte mit gespeicherten Objekten dieser Kategorie verglichen wird. Ist das unbekannte Tier einem bekannten ähnlich, wird es eher in dieselbe Kategorie eingeordnet. Durch geschickte Konstruktion des Versuchsmaterials konnten Schlüsse auf die von den Probanden verwendete Strategie gezogen werden. Wie vorhergesagt wurde die regelbasierte Strategie eher von den amerikanischen als von den ostasiatischen Probenden verwendet, die eine erfahrungsbasierte Strategie anwendeten (Norenzayan et al, 2002).

Auch bei Gedächtnisleistungen haben sich Unterschiede in der Verwendung von Kategorisierungen nachweisen lassen. Gutchess et al. (2006) fanden, dass die Anzahl verwendeter Kategorien beim Einprägen von Wortlisten eine zentrale Rolle spielt. Die Wörter einer Liste konnten als Gedächtnisstütze in eine gemeinsame Kategorie eingeordnet werden. So ist es z.B. möglich, die Wörter „Möbel“, „Pflanzen“ und „Büroartikel“ der Kategorie „Büro“ zu zuordnen. Aufgabe der Probanden war es, sich eine Liste von Wörtern einzuprägen, die in unterschiedliche Kategorien einteilbar waren. Insgesamt benutzten chinesische Probanden Kategorien als Strategie seltener als amerikanische Probanden (Gutchess et al., 2006).

Unsworth, Sears und Pexman (2005) gingen explizit der These nach, dass Chinesen eher aufgrund von relationalen Beziehungen, Kanadier dagegen aufgrund taxonomischer Beziehungen kategorisieren. Das Versuchsmaterial war so gestaltet, dass den Probanden jeweils drei Bilder vorgelegt wurden und diese entscheiden mussten, welche beiden jeweils am besten zusammenpassten. Die Ergebnisse dieser Untersuchung zeigen, dass die Gruppe der kanadischen Probanden dazu tendierte, ihre Urteile auf der Basis gemeinsamer Kategorien $\mathrm{zu}$ fällen und seltener als die chinesischen Probanden Objekte aufgrund von relationalen Beziehungen zu gruppieren. Die chinesischen Probanden zeigten keine Präferenz für relationale Gruppierungen, sondern entschieden sich nahezu gleich häufig für relationale und taxonomische Gruppierungen. Ihr Verhalten wird von Unsworth und Kollegen so erklärt, dass die chinesischen Probanden in Kanada lebten und somit neben ihrer ursprünglichen auch von der westlichen Kultur beeinflusst worden waren.

Aus dem Dargestellten lässt sich die erste Forschungshypothese ableiten: 
Hypothese 1: Bei der Beurteilung von Bildpaaren ist die Reaktionszeit von chinesischen Probanden bei Bildpaaren mit relationalen Beziehungen geringer als bei Bildpaaren mit taxonomischen Beziehungen. Bei deutschen Probanden hingegen ist die Reaktionszeit bei der Beurteilung von Bildpaaren mit taxonomischen Beziehungen geringer als bei Bildpaaren mit relationalen Beziehungen.

Die der Hypothese 1 zugrundeliegende Annahme, dass sich chinesische und deutsche Probanden in ihren Kategorisierungspräferenzen unterscheiden, kann auch im Rahmen des semantischen Primings geprüft werden.

Beim semantischen Priming wird davon ausgegangen, dass die Verarbeitung eines Wortes die Verarbeitung eines zweiten nachfolgenden Wortes insbesondere dann beeinflusst, wenn zwischen den beiden Wörtern durch die Wörter bezeichneten Konzepte eine semantische Beziehung besteht.

Die vorherige Darbietung eines Reizes (der sogenannte Prime) beeinflusst somit die Verarbeitungszeit eines Zielreizes (das Target). Semantische Primingeffekte werden unter anderem mittels sogenannter lexikalischer Entscheidungsaufgaben untersucht. Bei dieser Aufgabe muss der Proband entscheiden, ob eine Buchstabenreihenfolge ein Wort seiner Sprache ist oder nicht (Wolle: ja, Wulle: nein).Wenn z.B. als Prime das Wort „Schaf“ gegeben wird, kann die lexikalische Entscheidung beim Target „Wolle“ (ist Wolle ein Wort des Deutschen?) schneller getroffen werden, als beim Target „Auto“.

Zur Erklärung von semantischen Primingeffekten wird in der Regel ein assoziatives Netzwerk postuliert, in dem Konzepte in Form von mentalen Repräsentationen gespeichert und organisiert sind. Das Modell von Collins und Loftus (1975) zur Aktivierungsausbreitung (Spreading Activation Network) findet unter anderem in der Sprachpsychologie und beim semantischen Priming seine Anwendung und dient als theoretisches Modell zur Veranschaulichung der Prozesse, welche bei der Auswahl eines Konzeptes beziehungsweise eines Wortes im Gedächtnis ablaufen. Das Modell geht von einem neuronalen Netzwerk aus, in dem sich die Aktivierung eines Konzeptes über die assoziativen Verbindungen mit anderen Konzepten ausbreitet. Die einzelnen Konzepte fungieren als Knoten innerhalb eines assoziativen Netzwerkes. Nach Aktivierung eines Konzeptes breitet sich die Aktivierung auf weitere, mit dem Konzept assoziierte Knoten aus. Die Ausbreitung erfolgt gleichzeitig in alle verfügbaren 
Richtungen. Die Stärke der Aktivierung wird dabei durch die Stärke der Assoziation moduliert. Bereits aktivierte Konzepte können schneller verwendet werden als nicht aktivierte (Collins \& Loftus, 1975; Neely \& James, 1991).

Dies führt zur zweiten Forschungshypothese, die den Grundgedanken der ersten Hypothese aufgreift:

Hypothese 2: Die Darbietung eines sprachlichen Primes, welcher in einer relationalen Beziehung zu einem darauf folgenden relational verbundenen Bildpaar steht, sollte bei chinesischen Probanden zu einer Verringerung der Reaktionszeit führen verglichen mit einem sprachlichen Prime, welcher in einer taxonomischen oder neutralen Beziehung zu einem darauf folgenden relational verbundenen Bildpaar steht.

Bei deutschen Probanden sollte die Darbietung eines sprachlichen Primes, welcher in einer taxonomischen Beziehung zu einem darauf folgenden taxonomisch verbundenen Bildpaar steht, zu einer Verringerung der Reaktionszeit führen verglichen mit einem sprachlichen Prime, der in einer relationalen oder einer neutralen Verbindung zu einem taxonomisch verbundenen Bildpaar steht.

\subsection{Interaktion von Sprache und Kognition - eine Frage des Informationsverarbeitungsniveaus}

Die bereits vorgestellten Untersuchungen belegen, dass sich kulturbedingte Unterschiede in den Denkweisen von Personen unterschiedlicher kultureller Herkunft finden lassen. Angenommen wird, dass sich die beschriebenen Unterschiede im Verhalten durch die verschiedenen Kulturen, in denen Menschen aufwachsen, erklären lassen. Mit dem Begriff der Kultur sind primär die spezifischen Werte und Ideale assoziiert sowie Vorstellungen darüber, wie ein Mensch sein und sich verhalten sollte und was Recht und Unrecht ist. Diese Einstellungen spiegeln sich dann zum Beispiel in kulturspezifischen Erzählungen, Symbolen, Gewohnheiten oder sozialen Verhaltensweisen wider (Heine, Lehman, Markus, \& Kitayama, 1999). Aber auch die Sprache, die ein Mensch spricht, ist eng mit der Kultur, in der er lebt, verbunden, da Sprache ein Ausdrucksmittel von Kultur darstellt (Ji et al., 2004). Das führt zu der 
Annahme, dass nicht nur die klassischen kuturbedingten Variablen, wie philosophische Traditionen, kognitive Prozesse und Kategorisierungsleistungen beeinflussen können, sondern auch die Sprache selbst einen entsprechenden Einfluss ausüben kann.

Hunt und Agnoli (1991) schlagen folgende Bezeichnung zur Unterscheidung von Spracheffekten auf die Interpretation der Realität vor: „high-level effects“ und „low-level effects“ der Sprache.

Der bekannte und viel diskutierte Ansatz von Whorf (1956) geht von einem „high-level effect“ der Sprache aus. Denn der sogenannte Whorfsche Determinismus besagt, dass die Grenzen unserer Sprache auch die Grenzen unseres Denkens sind. Whorf vertritt den Standpunkt, dass damit Sprache zu einem determinierenden Faktor unserer Wahrnehmung wird. Dies wird an dem klassischen Beispiel der Inuit, die über eine Vielzahl an Wörtern für „Schnee“ verfügen, illustriert.

Als ein jüngeres Beispiel für die Interaktion von Sprache und Denken seien hier Untersuchungen von Levinson (1996) aufgeführt. Es wurden zwei unterschiedliche Volksgruppen - Niederländer und Angehörige einer mexikanischen Ethnie, die den sogenannten Tzeltal-Dialekt sprechen - hinsichtlich ihrer Raumwahrnehmung miteinander verglichen. Im Holländischen, wie auch im Deutschen, werden räumliche Verhältnisse eher situationsspezifisch veranschaulicht. Die Position eines Objektes wird demnach relativ zu der Person des Betrachters beschrieben: „Der Mann steht links vom Motorrad.“ Oder die Position wird relativ zu anderen Objekten beschrieben: „Vor dem Haus befindet sich ein Garten." Im Tzeltal-Dialekt hingegen gibt es keine Begriffe wie links, rechts, vorne oder hinten. Es werden vielmehr absolute Koordinaten zur Beschreibung verwendet: „Der Mann steht südlich vom Motorrad.“ Solche Beschreibungen sind zwar auch im Holländischen möglich, im Gebrauch aber eher ungewöhnlich.

Levinson (1996) konnte zeigen, dass die sprachlichen Unterschiede nicht nur einen Einfluss auf die visuelle räumliche Vorstellung und gedächtnismäßige Speicherung hatten, sondern auch auf die Schlussfolgerungen, die aus den verschiedenen Repräsentationen entstanden. In einem weiteren Experiment von Levinson sollten vier unterschiedliche Tierfiguren (ein Schwein, eine Kuh, ein Pferd und ein Schaf), die vor dem Probanden nebeneinander auf einem Tisch 1 standen, auf einem anderen Tisch 2 hinter dem Versuchsteilnehmer in gleicher Weise angeordnet 
werden. Der Proband musste sich also zu diesem Zweck umdrehen und veränderte damit auch seine Orientierung im Raum in Hinblick auf die absoluten Koordinaten. Die Holländer bauten die Figuren auf Tisch 2 relativ zur eigenen Perspektive unter Bezugnahme auf links-rechts-Relationen in gleicher Anordnung auf. Die Mexikaner dagegen bauten die Figuren auf Tisch 2 so auf, dass die Positionen der Figuren in Bezug auf die Himmelsrichtungen erhalten blieben, wodurch sich deren Positionen relativ zum Betrachter veränderten.

In einem weiteren Experiment konnte Levinson (1996) zeigen, dass die unterschiedliche Enkodierung der räumlichen Verhältnisse sich auch beim Schlussfolgern auswirkt. Die Probanden sahen als erste Szene auf Tisch 1 z.B. einen blauen Kegel A neben einem gelben Würfel B (Prämisse 1). Nachdem sie sich umgedreht hatten, sahen sie auf Tisch 2 als zweite Szene einen gelben Würfel B neben einem roten Zylinder C (Prämisse 2). Dann drehten sie sich wieder zu Tisch $1 \mathrm{um}$, auf dem der blaue Kegel A in derselben Position wie in der ersten Szene zu sehen war. Ihre Aufgabe war es, den roten Zylinder $\mathrm{C}$ in Übereinstimmung mit den zuvor gesehenen beiden Prämissen zu platzieren. Es resultieren unterschiedliche Lösungen, je nachdem ob die Objekte relativ zur Perspektive des Betrachters oder unter Bezugnahme auf die Himmelsrichtungen enkodiert wurden (Levinson, 1996).

In einer anderen Untersuchung von Haun und Kollegen unter der Mitwirkung von Levinson aus dem Jahr 2011 konnten die oben geschilderten Befunde mit anderen Volksgruppen repliziert werden. Es wurde erneut eine cross-culture Studie mit zwei Populationen durchgeführt, die sich hinsichtlich der sprachlichen Klassifizierung räumlicher Anordnungen unterscheiden. Niederländische und namibische Grundschulkinder wurden hinsichtlich ihrer Fähigkeit zur räumlichen Rekonstruktion verglichen. Die niederländischen Kinder präferierten wie zu erwarten einen relativen Bezugsrahmen bei räumlichen Anordnungen, wohingegen die namibischen Grundschüler einen absoluten Bezugsrahmen für räumliche Anordnungen verwendeten. In diesem Fall sollten die Kinder Anordnungen von drei Tieren rekonstruieren, nachdem sie sich um ein Schulgebäude herum begeben hatten und damit den Winkel zum Tisch, auf dem sie die Tiere in derselben Anordnung wieder aufbauen sollten, gegenüber der Ausgangsposition um 90 Grad verändert hatten. Es konnte in der Untersuchung ein Zusammenhang des jeweilig kulturell präferierten Bezugsrahmens 
und der räumlichen Kognitionsleistung aufgezeigt werden. Es fiel sowohl den niederländischen als auch den namibischen Kindern schwer, einen anderen als den bevorzugten Bezugsrahmen zu verwenden. Versuchten sie es dennoch, führte das zu einer verminderten Rekonstruktionsleistung (Haun, Rapold, Janzen, \& Levinson, 2011).

Die vorgestellten Untersuchungsbefunde zu ,high-level effects“ der Sprache beziehungsweise Kultur von Levinson und seiner Forschergruppe sind wesentlich differenzierter als der von Whorf (1957) postulierte Determinismus. Dass die Annahme einer deterministischen Beziehung zwischen Sprache und Kultur einerseits und Kognition andererseits nicht zutreffend ist, zeigt zum Beispiel die Arbeit von Malt Sloman, Gennari, Shi und Wang (1999). Es wurde untersucht, wie Englisch, Chinesisch und Spanisch sprechende Personen 60 unterschiedliche Behälter kategorisierten. Diese Kategorisierung sollte entweder nach der Bezeichnung der Behälter oder nach der subjektiv wahrgenommenen Ähnlichkeit vollzogen werden. Die Ergebnisse zeigten, dass es zwischen den Sprachgruppen signifikante Unterschiede darin gab, wie die Behälter bezeichnet wurden und welche Behälter dieselbe Bezeichnung erhielten. Demgegenüber konnten zwischen den Sprachgruppen nur geringe Unterschiede bezüglich der Kategorisierung nach der wahrgenommenen Ähnlichkeit festgestellt werden (Malt, Sloman, Gennari, Shi, \& Wang, 1999). Nach der Whorfschen Hypothese hätten die sprachspezifischen Unterschiede in den Bezeichnungen jedoch dazu führen müssen, dass sich auch die Kategorisierungen nach Ähnlichkeiten zwischen den Sprachgruppen unterscheiden sollten. Die vorgestellte Untersuchung legt dagegen vielmehr nah, dass die Benennung von Objekten auch anderen Faktoren als der bloßen Wahrnehmung, wie zum Beispiel kulturellen Einflüssen, unterliegen kann.

Unter „low-level effect“" verstehen Hunt und Agoloio (1991), dass durch Eigenarten von Sprachen unterschiedlich hohe Kosten für die kognitive Informationsverarbeitung entstehen können. Ein solcher „low-level effect“ konnte zum Beispiel in der Arbeit von Maass und Russo (2003) nachgewiesen werden. Die Autoren prüften die Annahme, dass die räumliche Interpretation von Aussagen, die eine Handlung beschreiben wie „Das Kind gibt der Mutter einen Blumenstrauß“, durch die Lese- und Schreibgewohnheiten beeinflusst wird. Untersucht wurde, ob sich arabischund italienischsprachige Probanden, die jeweils eine andere Lese- und Schreibrichtung erlernt haben, hinsichtlich der Präferenz für räumliche Agent-Rezipient-Anordnungen 
unterschieden. Es konnte gezeigt werden, dass es einen sprachbedingten Einfluss auf die räumlich-visuelle Kognition gab und je nach erlernter Lese- und Schreibrichtung eine andere räumliche Anordnung präferiert wurde. Die italienischsprachigen Probanden sahen den Agenten, das Kind, links vom Rezipienten, der Mutter, die arabischsprachigen hingegen sahen den Agenten rechts vom Rezipienten.

Die zitierten Arbeiten zu high-level und low-level Effekten der Sprache machen deutlich, dass Sprache auf sehr unterschiedliche Weise zu kulturellen Unterschieden bei kognitiven Anforderungen beitragen kann.

\subsection{Sprachproduktion}

Nach den vorgestellten Überlegungen könnte sich also ein Einfluss des Denkstils nicht nur beim Kategorisierungsverhalten selbst, sondern auch bei der Sprache oder genauer der Sprachproduktion zeigen, wenn der Sprachproduktion Kategorisierungen voraus gehen.

Sprachproduktion setzt sich nach den gegenwärtigen Theorien aus mehreren Teilprozessen zusammen. Wesentliche Teilprozesse sind die Bereitstellung von semantischen Inhalten, welche enkodiert werden sollen, sowie die Auffindung von Wortformen (und anderen sprachlichen Elementen) in einer Sprache, durch die die semantischen Inhalte dargestellt werden (Dell \& O'Seaghdha, 1992; Grabowski \& Herrmann, 1994; Levelt, 1993; Levelt, Roelofs, \& Meyer, 1999). Diese beiden Teilprozesse werden oft als semantischer oder auch konzeptueller und als phonologischer oder auch lexikalischer oder phonetischer Teilprozess bezeichnet. Sieht man eine Katze und will man sie mittels der englischen Sprache benennen, so aktiviert man zunächst das Konzept für „Katze“ und produziert dann die zugehörige Wortform „cat“. Wird nun ein Konzept in konkrete Sprache umgesetzt, so spricht man von Objektbenennung (Herrmann \& Deutsch, 1976; Weiß \& Baratelli, 2003).

Ein klassisches Paradigma zur Differenzierung der genannten Teilprozesse ist das sogenannte Bild-Wort-Interferenz-Paradigma, das im Folgenden anhand der viel zitierten Untersuchung von Schriefers, Meyer und Levelt (1990) näher beschrieben werden soll. Schriefers und Kollegen ließen Objekte von Versuchsteilnehmern benennen. Gleichzeitig mit dem Zielobjekt wurden zusätzliche Reize dargeboten, 
sogenannte Distraktoren, die als irrelevant dargestellt wurden. Schriefers und Kollegen verwendeten auditive Distraktoren, die den Probanden über Kopfhörer während der Objektbenennung dargeboten wurden. Es kamen Distraktoren zur Anwendung, die dem Objekt entweder semantisch oder phonologisch ähnlich waren oder auch in keinerlei Beziehung zu dem Objekt standen. Eine Kontrollbedingung wurde ohne Distraktoren realisiert. Die Distraktoren wurden in variablem zeitlichen Abstand (SOA, „stimulus onset asynchrony“) vor oder nach dem Zeitpunkt der Präsentation des zu benennenden Objekts dargeboten. Eines der Ergebnisse der Studie von Schriefers et al. war, dass die semantischen Distraktoren im Mittel zu einer längeren Benennungszeit der Objekte führten. Dies wird als Hemmung des Benennungsprozesses interpretiert, welche besonders stark $150 \mathrm{~ms}$ vor der Darbietung des Objekts auftrat. Die phonologischen Distraktoren erleichterten die Benennung, wenn sie 150 ms nach dem zu benennenden Objekt erschienen. Hingegen fehlte ein markanter Einfluss der semantischen Distraktoren nach der Objektdarbietung und der phonologischen Distraktoren vor der Objektdarbietung. Daraus wird von Schriefers und Kollegen gefolgert, dass der semantische dem phonologischen Teilprozess der Sprachproduktion vorausgeht. Beide Teilprozesse werden von den Autoren als Module aufgefasst, die strikt seriell aktiviert werden und bei denen es keine Rückkopplung gibt (Schriefers, Meyer, \& Levelt, 1990, 2003).

Schriefers und Kollegen argumentieren zudem für eine separate, zeitlich zwischen der semantisch-konzeptuellen und phonologischen Prozessebene (Wortformebene) angeordnete „Lemma-Ebene“ (Levelt, 1993; Levelt u. a., 1999). Lemmata sind bei Levelt (1993) eine Komponente seiner Dreistufenauffassung, nach der eine konzeptuelle Produktionsebene von einer Lemma-Ebene sowie von mehreren Wortform-Ebenen getrennt ist. Andere Modellklassen wie zum Beispiel die Aktivationsausbreitungs- beziehungsweise Netzwerkmodelle der Sprachproduktion entsprechen dagegen einer Zweistufenauffassung. Nach dieser unterscheidet man nur eine konzeptuell-semantische von einer phonologischen (Wortform-) Ebene (Caramazza, 1997; Dell, Burger, \& Svec, 1997; Herrmann, 2005; Rickheit, Herrmann, $\&$ Deutsch, 2003).

Die genaue Prozessstruktur der Sprachproduktion und damit auch des Objektbenennungsvorgangs ist bis heute umstritten (Rickheit et al., 2003; Weekes, 
Robert, \& Chen, 2002). Neben das serielle Modell der Sprachproduktion von Levelt, Schriefers und anderen Autoren - vgl. auch das WEAVER++-Modell (Roelofs, 1997) treten andere Modelle wie die soeben genannten Aktivationsausbreitungsbeziehungsweise Netzwerkmodelle. Ihnen zufolge verläuft die Sprachproduktion nicht streng seriell, sondern entweder unidirektional-kaskadenförmig oder bidirektional, d.h. mit Rückkopplung von der phonologischen Verarbeitung zur semantischen (Caramazza, 1997). Inzwischen gibt es sowohl empirische Studien, welche die serielle Auffassung der Sprachproduktion unterstützten, als auch andere Untersuchungen welche eher für Netzwerkmodelle mit Rückkopplung sprechen (vgl. u.a. Dell \& O’Seaghdha, 1992; Grabowski \& Herrmann, 1994; Schriefers et al., 2003).

Die Sprachproduktion ist demnach kein ausschließlich serieller Prozess, sondern auch mit Rückkopplung von der phonologischen Verarbeitung zur semantischen Ebene versehen. Interessanterweise sprechen die Ergebnisse einer Untersuchung, in der die Bild-Wort-Interferenz unter Verwendung der chinesischen Sprache mit chinesischen Probanden eingesetzt wurde, für die Annahme von seriellen Prozessen (Weekes et al., 2002).

Zhang und Yang (2005) haben mit dem Bild-Wort-Interferenz-Paradigma die Rolle des Tones im Chinesischen bei der Sprachproduktion untersucht. Eine Besonderheit der chinesischen Sprache ist nämlich, dass sie eine Tonsprache beziehungsweise eine tonale Sprache ist (Norman, 1988). Im Deutschen zum Beispiel bilden bekanntlich Lautklassen die Phoneme, durch die Bedeutungsunterschiede signalisiert werden. Bei Verwendung des Chinesischen steht daneben eine weitere Klasse von diskriminativen phonologischen Merkmalen zur Verfügung: Jede Silbe kann mit bis zu vier alternativen Tonhöhen und Tonverläufen ausgesprochen werden und erhält damit auch bei sonst gleicher Lautung unterschiedliche Bedeutungen. So hat zum Beispiel die Silbe „ma“ bei hohem gleichmäßigem Tonhöhen-Niveau (= Ton 1: „mā“) die Bedeutung „Mutter“. Bei einem von mittlerem Niveau aufsteigenden Ton (= Ton 2: „mác) bedeutet „ma“ „Hanf“. Wenn der Ton zunächst von einem knapp mittleren Tonhöhen-Niveau absinkt und dann steil ansteigt (= Ton 3: „mă“), so hat „ma“ die Bedeutung „Pferd“. Bei einem scharf abfallenden Ton (= Ton 4: „mà“) hat „ma“ die Bedeutung „schimpfen“. Welche Bedeutung die Silbe „ma“ also nun hat, entscheidet sich durch die Verwendung eines spezifischen Tons. 
In der oben genannten Untersuchung interessierten sich Zhang und Yang (2005) insbesondere für die Entstehungsbedingungen des phonologischen Erleichterungseffektes. Ihre Befunde belegen, dass im Chinesischen der Ton eine zentrale Rolle spielt, aber vor allem im Zusammenhang mit der Silbe. Wenn sich Distraktor und die Benennung des Objektes reimten, stellten die Autoren eine Erleichterung fest, und zwar auch dann, wenn die beiden Wörter nicht im Ton übereinstimmten. Die Erleichterung fiel aber bei zusätzlicher Übereinstimmung im Ton deutlich höher aus.

Insgesamt ist festzustellen, dass die weiter oben beschriebenen Untersuchungen von Schriefers und Kollegen (1990) bisher kaum unter Verwendung der chinesischen Sprache repliziert worden sind. Es wurde dabei in der Regel auf die Verwendung eines zeitlich variablen SOA-Intervall verzichtet. Bei SOA = 0 wurden sowohl phonologische als auch graphemische Erleichterungseffekte gefunden (Weekes et al., 2002; Zhou, Zhuang, Wu, \& Yang, 2003). Zhang und Yang (2005) fanden nur einen phonologischen Erleichterungseffekt. Zhou und Kollegen (2003) erhielten sowohl einen phonologischen als auch einen graphemischen Erleichterungseffekt bei einer SOA von +150 . Die Studie von Zhang und Yang (2004) erbrachte dieselben Effekte bei einer SOA von +100 .

Wenn sich ein Einfluss des Denkstils nicht nur im Kategorisierungsverhalten selbst, sondern auch in der Sprachproduktion zeigt, soweit nämlich Kategorisierungsprozesse für die Sprachproduktion relevant sind, dann sollte sich ein Einfluss des Denkstils auch im Bild-Wort-Interferenz-Paradigma nachweisen lassen.

Es wird folgende Hypothese abgeleitet:

Hypothese 3: Die Darbietung eines sprachlichen Distraktors, welcher in einer relationalen Beziehung zu einem darauf folgenden Target steht, sollte bei chinesischen Probanden innerhalb des Bild-Wort-Interferenz-Paradigmas zu einer größeren Interferenz führen, verglichen mit einem sprachlichen Distraktor, welcher in einer taxonomischen oder neutralen Beziehung zu einem darauf folgenden Target steht.

Bei deutschen Probanden sollte die Darbietung eines sprachlichen Distraktors, welcher in einer taxonomischen Beziehung zu einem darauf folgenden Target steht, zu einer größeren Interferenz führen, verglichen mit einem sprachlichen Distraktor, der in 
einer relationalen oder einer neutralen Verbindung zu einem darauf folgendem Target steht.

\subsection{Zusammenfassungen der Hypothesen}

In den im Folgenden vorgestellten Experimenten 1 und 2 wurde ein möglicher kulturbedingter Einfluss des Denkstils auf Entscheidungen bei Kategorisierungsaufgaben untersucht. Im Experiment 3 stand die Sprachproduktion im Fokus. Die zugrundeliegende Überlegung ist hier, dass sich der Denkstil auf die Sprachproduktion auswirken sollte (im Sinne eines „high-level effects“ der Sprache, vgl. 2.5), wenn interferierende Verarbeitungsprozesse wirksam werden. Diese Aufgabenanforderung wurde mit dem Bild-Wort-Interferenz-Paradigma realisiert.

Aus diesen Überlegungen heraus werden die drei in den nachfolgenden Experimenten explizit formulierbaren Hypothesen verfolgt:

Hypothese 1: Bei der Beurteilung von Bildpaaren ist die Reaktionszeit von chinesischen Probanden bei Bildpaaren mit relationalen Beziehungen geringer als bei Bildpaaren mit taxonomischen Beziehungen. Bei deutschen Probanden hingegen ist die Reaktionszeit bei der Beurteilung von Bildpaaren mit taxonomischen Beziehungen geringer als bei Bildpaaren mit relationalen Beziehungen.

Hypothese 2: Die Darbietung eines sprachlichen Primes, welcher in einer relationalen Beziehung zu einem darauf folgenden relational verbundenen Bildpaar steht, sollte bei chinesischen Probanden zu einer Verringerung der Reaktionszeit führen verglichen mit einem sprachlichen Prime, welcher in einer taxonomischen oder neutralen Beziehung zu einem darauf folgenden relational verbundenen Bildpaar steht.

Bei deutschen Probanden sollte die Darbietung eines sprachlichen Primes, welcher in einer taxonomischen Beziehung $\mathrm{zu}$ einem darauf folgenden taxonomisch verbundenen Bildpaar steht, zu einer Verringerung der Reaktionszeit führen verglichen mit einem sprachlichen Prime, der in einer relationalen oder einer neutralen Verbindung zu einem taxonomisch verbundenen Bildpaar steht. 
Hypothese 3: Die Darbietung eines sprachlichen Distraktors, welcher in einer relationalen Beziehung zu einem darauf folgenden Target steht, sollte bei chinesischen Probanden innerhalb des Bild-Wort-Interferenz-Paradigmas zu einer größeren Interferenz führen, verglichen mit einem sprachlichen Distraktor, welcher in einer taxonomischen oder neutralen Beziehung zu einem darauf folgenden Target steht.

Bei deutschen Probanden sollte die Darbietung eines sprachlichen Distraktors, welcher in einer taxonomischen Beziehung zu einem darauf folgenden Target steht, zu einer größeren Interferenz führen, verglichen mit einem sprachlichen Distraktor, der in einer relationalen oder einer neutralen Verbindung zu einem darauf folgendem Target steht. 


\section{Methode Experiment 1 „Kategorisierung über Bildpaare“}

Die Experimente 1 und 2 wurden im Mai 2009 in China an der East China Normal University Shanghai und von Juni bis November 2009 in Deutschland am Georg-EliasMüller-Institut für Psychologie der Georg-August-Universität Göttingen durchgeführt. Das Experiment 3 wurde ein Jahr später im Mai 2010 in China an der East China Normal University Shanghai und von Juni bis November 2010 in Deutschland am Georg-Elias-Müller-Institut für Psychologie der Georg-August-Universität Göttingen durchgeführt.

Zunächst werden die spezifischen Details von Experiment 1 geschildert. Es sei bereits an dieser Stelle darauf verwiesen, dass an Experiment 1 und Experiment 2 dieselben Probanden teilgenommen haben und zum Teil auch identische technische Hilfsmittel wie Notebook und Software zum Einsatz kamen. Die Gemeinsamkeiten in den methodischen Details, wie die Charakteristika der Stichprobenzusammensetzung und die Konstruktion des Versuchsmaterials werden deshalb im Methodenteil zu Experiment 1 ausführlich beschrieben. Bei der Darstellung des Experiments 2 wird an den entsprechenden Stellen auf diese Beschreibung verwiesen.

\subsection{Probanden}

An der Untersuchung nahmen 52 chinesische Studierende, 8 männliche und 44 weibliche, im Alter von 22 bis 28 Jahren $(M=23,83$ Jahre; $S D=1,23)$ und 51 deutsche Studierende im Alter von 19 bis 31 Jahren $(M=23,55$ Jahre; $S D=2,83)$ teil, von denen 10 männlich und 41 weiblich waren. Die Mehrzahl der chinesischen Probanden studierte im Hauptfach Psychologie $(n=42)$. Die anderen chinesischen Teilnehmer waren Studierende folgender Fachrichtungen: Kunst $(n=4)$, Erziehungswissenschaften $(n=3)$, Chinesisch für Ausländer $(n=2)$ und Chemie $(n=1)$. Auch die meisten deutschen Probanden studierten Psychologie $(n=35)$. Die restlichen Teilnehmer kamen aus den Rechts-, Wirtschafts- und Sozialwissenschaften $(n=10)$, aus der Philosophischen Fakultät $(n=5)$ und aus den Agrarwissenschaften $\quad(n=1)$.

Die Probanden wurden über einen Aushang oder durch persönliches Ansprechen rekrutiert. Ausschlusskriterium für die chinesischen Teilnehmer war, wenn sie bereits 
eine Reise außerhalb von China gemacht hatten. Die deutschen Probanden durften über keinerlei chinesische Sprachkenntnisse verfügen. Diese Ausschlusskriterien wurden gewählt, da über intensivere Auseinandersetzung mit der fremden Kultur bereits eine Beeinflussung des Denkstils hätte stattfinden können.

\subsection{Versuchsmaterial und Aufgabenanforderung}

Mit Hilfe der kommerziellen Software Presentation ${ }^{\circledR}$ wurde ein eigens für diese experimentelle Durchführung konzipiertes Programm realisiert. Das Programm lief auf einem Notebook mit Windows XP, Intel Celeron 1,6 GHZ und 2 GB Arbeitsspeicher. Bei der Durchführung der Versuche wurde dasselbe Notebook sowohl in China als auch in Deutschland verwendet.

Aufgabe des Probanden war es, paarweise angeordnete Bilder, die auf dem Bildschirm des Notebooks dargeboten wurden, hinsichtlich der Frage zu beurteilen, ob sie in irgendeiner Art und Weise zusammenpassten. Bei den Bildern handelte es sich um farbige Zeichnungen von Objekten (siehe Abbildung 1), die gleichzeitig auf dem Bildschirm erschienen. Eine vollständige Liste der verwendenden Bilder befindet sich im Anhang (siehe Anhang A).

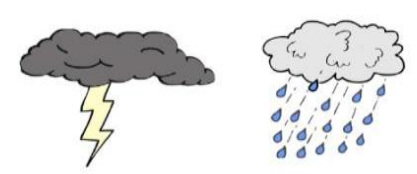

Abbildung 1: Beispiel eines zu bewertenden Bildpaares. In diesem Fall handelt es sich um eine taxonomische Beziehung zwischen den Bildern.

Das Bildmaterial und die als semantische Primes verwendeten Wörter wurden zuvor in einem Vorversuch auf ihre Eignung untersucht. An dem Vorversuch nahmen fünf deutsche und fünf chinesisches Probanden teil. Im ersten Teil des Vorversuchs sollten die Probanden beurteilen, ob es ihnen leicht fiel, die gezeigten Bilder zu identifizieren. Dies wurde anhand einer Power Point Präsentation realisiert, bei der die Probanden gebeten wurden, die Bilder namentlich zu benennen. Im zweiten Teil des Vorversuchs wurden Kombinationen von jeweils zwei Bildern gezeigt, welche auf ihre taxonomische oder relationale Kategorisierbarkeit bewertet werden sollten. Hier wurden 
die Probanden gefragt, ob sich die zwei Bilder in eine gemeinsame Kategorie einordnen lassen oder ob es einen logischen oder funktionalen Zusammenhang gibt.

Gab es Schwierigkeiten bei einzelnen Items, also konnten z.B. zwei Vorversuchsteilnehmer keine Identifizierung leisten oder keinen Zusammenhang herstellen, wurden entsprechende Items oder deren Kombination verworfen. Traten außerdem Abweichungen vom Gruppenmittel in der Kategorisierung von über 20\% auf, so wurde das Item ebenfalls nicht weiter verwendet. Aufgrund der Größe des Itempools von 300 Items war dies eine aufwendige Prozedur. Ausgewählt wurden schließlich 240 Bildpaare, die im Experiment in randomisierter Reihenfolge dargeboten wurden. Die Randomisierung wurde dabei durch die Software geleistet. Dadurch wurde sichergestellt, dass jeder Proband eine neue Reihenfolge erhielt.

Folgende experimentelle Variation wurde vorgenommen: Die Bildpaare konnten sich darin unterscheiden, ob die beiden Bilder überhaupt eine wie auch immer geartete Beziehung zueinander besaßen (Unabhängige Variable 1, UV1). Falls eine Beziehung vorlag, konnte diese entweder taxonomischer oder relationaler Natur sein. So lassen sich z.B. die Bilder „Hände“ und „Handschuhe“ in einen funktionalen Zusammenhang bringen, so dass diese als relational zusammenhängend bewertet werden können.

Bei der Hälfte der 240 Bildpaare bestand eine Beziehung zwischen den Bildern, die bei 60 Bildpaaren taxonomisch und bei 60 Bildpaaren relational (vgl. Anhang A) war. Um zu vermeiden, dass die Probanden durch ausschließlich zusammenpassende Bildpaare automatisch mit „Ja“ antworteten, bestand bei den verbleibenden 120 Bildpaaren keine Beziehung, so dass mit „Nein“ geantwortet werden musste. Diese sogenannten neutralen Bildpaare wurden überwiegend aus derselben Bilderserie kombiniert, aus der auch die taxonomisch und relational zusammenpassenden Bildpaare erstellt wurden.

Um eine unterschwellige Beeinflussung des Kategorisierungsverhaltens hervorzurufen, wurden vor der Darbietung des Bildpaares ein deutsches Wort bei deutschen Probanden oder chinesische Schriftzeichen bei chinesischen Probanden als semantischer Prime eingeblendet (vgl. Anhang A). Hierbei wurde variiert, welche Beziehung der sprachliche Prime zu dem Bildpaar aufwies (UV 2): Die Beziehung des Primes zu einem Bild konnte entweder kongruent oder inkongruent sein hinsichtlich der Beziehung der beiden Bilder zueinander. Eine weitere Variante war, dass der Prime 
keinerlei Beziehung $\mathrm{zu}$ den beiden Bildern aufwies. Außerdem wurden Versuchsdurchgänge ohne Prime realisiert.

Kongruent bedeutete konkret entweder, dass vor einem taxonomisch verbundenen Bildpaar ein Wort erschien, welches in einer taxonomischen Beziehung zu einem Bild des Paares stand, oder dass einem relational verbundenen Bildpaar ein sprachlicher Prime vorgeschaltet war, der ebenfalls in einer relationalen Beziehung zu einem Bild des Paares stand. Beispielsweise wäre ein kongruenter Prime bei der Darbietung der Bilder eines Hundes und einer Katze, die in einer taxonomischen Beziehung zueinander stehen, da beide Begriffe in die Kategorie Tier eingeordnet werden können, z.B. das Wort Pferd. Denn ein Pferd ist ebenfalls der Kategorie Tier zuzuordnen.

Als inkongruent wurden Primewörter bezeichnet, deren Beziehung zu einem Bild des Paares nicht der Beziehung der beiden Bilder untereinander entsprach. Bezogen auf das oben genannte Beispiel der Bilder eines Hundes und einer Katze wäre ein inkongruenter Prime beispielsweise das Wort Leine, da der Begriff Leine in einer relationalen Beziehung zum Begriff Hund steht, während die Bilder untereinander taxonomisch miteinander verbunden sind.

Weiterhin enthielten einige Versuchsdurchgänge ein neutrales Wort als Prime, welches in keiner besonderen Beziehung zu dem Bildpaar stand. Bezüglich des bereits verwendeten Beispiels wäre das Wort Stuhl ein neutraler Prime. Eine Kontrollbedingung für die Wirkung des Primes wurde realisiert, indem bei 30 Versuchsdurchgängen anstelle des Primes nur ein leeres Feld gezeigt wurde, das einen identischen Zeitraum wie die verwendeten Primes beanspruchte. Die zeitliche Abfolge sollte auf diesem Wege in allen Bedingungen konstant gehalten werden.

Auf die einzelnen Primebedingungen (kongruent, inkongruent, neutral, kein Prime) entfielen jeweils 15 Bildpaare innerhalb der variierten Beziehung (taxonomisch oder relational). Tabelle 1 gibt eine Übersicht über die hypothesenrelevanten Bedingungen, die sich aus der Kombination der beiden unabhängigen Variablen UV1 und UV2 ergeben. 
Tabelle 1: Kombinationen kongruenter und inkongruenter Primes mit taxonomisch und relational verbundenen Bildpaaren

\begin{tabular}{|l|l|l|}
\hline \multicolumn{1}{|l|}{} & \multicolumn{2}{|l|}{ Beziehung zwischen den Bildern in den Bildpaaren } \\
\hline $\begin{array}{l}\text { Beziehung des Primes zu } \\
\text { einem Bild des } \\
\text { Bildpaares }\end{array}$ & taxonomisch & relational \\
\hline taxonomisch & $\begin{array}{l}\text { taxonomische Beziehung, } \\
\text { kongruenter Prime }\end{array}$ & $\begin{array}{l}\text { relationale Beziehung, } \\
\text { inkongruenter Prime }\end{array}$ \\
\hline relational & $\begin{array}{l}\text { taxonomische Beziehung, } \\
\text { inkongruenter Prime }\end{array}$ & $\begin{array}{l}\text { relationale Beziehung, } \\
\text { kongruenter Prime }\end{array}$ \\
\hline neutral & $\begin{array}{l}\text { taxonomische Beziehung, } \\
\text { neutraler Prime }\end{array}$ & $\begin{array}{l}\text { relationale Beziehung, } \\
\text { neutraler Prime }\end{array}$ \\
\hline keine & $\begin{array}{l}\text { taxonomische Beziehung, } \\
\text { kein Prime }\end{array}$ & $\begin{array}{l}\text { relationale Beziehung, } \\
\text { kein Prime }\end{array}$ \\
\hline
\end{tabular}

\subsection{Versuchsablauf}

Nach einer kurzen Begrüßung wurden die Probanden gebeten, sich an das bereits vorbereitete Notebook zu setzen, auf dessen Bildschirm die Versuchsinstruktion zu sehen war. Die Probanden wurden aufgefordert, sich die Anweisungen zunächst aufmerksam durchzulesen. Bevor das eigentliche Experiment startete, konnten Verständnisfragen gestellt werden. Für das Durchlesen der Instruktionen wurde den Probanden soviel Zeit gegeben, wie diese dafür benötigten. Anschließend konnten sie selbständig mit der Leertaste das Experiment starten. Die Instruktion war im Falle der deutschen Studierenden in deutscher Sprache, im Falle der chinesischen Studierenden auf Mandarin verfasst (vgl. Anhang B). In der Instruktion wurden die Probanden aufgefordert, die anschließend gezeigten Bildpaare danach $\mathrm{zu}$ bewerten, ob die abgebildeten Objekte in irgendeiner Art und Weise zusammenpassten oder nicht. Die Probanden wurden angewiesen, ihre Antwort über die Computertastatur einzugeben, wobei das Drücken der Taste L ein „Ja“, das Drücken der Taste S ein „Nein“ bedeutete. Die Tasten befinden sich von der Leertaste gleich weit entfernt, an der linken und rechten Seite der Tastatur. Die Tasten wurden mit einem Sticker mit der Aufschrift „Ja“ oder „Nein“ versehen. Die Probanden wurden aufgefordert, die Finger auf den Tasten liegen zu lassen, um eine schnellere Antwortabgabe zu ermöglichen, falls der Proband unabsichtlich die Hände von den Tasten entfernen oder anderweitig abschweifen sollte. 
Die Instruktion beinhaltete jeweils ein Beispiel für relationale und taxonomische Beziehungen und verdeutlichte einen Versuchsdurchgang.

Ein einzelner Versuchsdurchgang gestaltete sich nun wie folgt: Vor jedem dargebotenen Bildpaar erschien auf dem Notebookbildschirm für die Dauer von $850 \mathrm{~ms}$ ein Fixationskreuz, welches die Probanden fixieren sollten. Das Fixationskreuz diente dazu, die Aufmerksamkeit der Probanden an die Stelle des Bildschirms zu lenken, an welcher der Prime und das nachfolgende Bildpaar erschienen. Nach Verschwinden des Fixationskreuzes erschien der sprachliche Prime beziehungsweise das leere Feld für den Zeitraum von 9,9 ms. Dies entspricht einer Bildschirmrate auf einem TFTNotebookbildschirm und ist damit die aufgrund der technischen Gegebenheiten kürzest mögliche Präsentationszeit. Als Prime erschien entweder für die deutschen Probanden ein deutsches Wort oder für chinesische Probanden ein chinesisches Schriftzeichen. Der Prime wurde durch eine Reihe von X-Zeichen (,XXXXXX“) für 20 ms maskiert. Diese Maskierung wird in Metaanalysen als ideal für semantisches Priming angesehen (Kinoshita \& Lupker, 2003). Es folgte danach für 200 ms das Fixationskreuz, bevor das zu bewertende Bildpaar dargeboten wurde, das bis zur Antwortabgabe der Probanden sichtbar blieb. Die Probanden konnten dann die Antwort über die Tastatur eingeben, was erst nach der Präsentation des Bildpaares möglich war. Nach der Abgabe der Antwort startete der nächste Versuchsdurchgang mit der Darbietung des Fixationskreuzes. Die zeitlichen Ereignisse in einem Versuchsdurchgang werden in Abbildung 2 anhand eines Beispiels verdeutlicht.

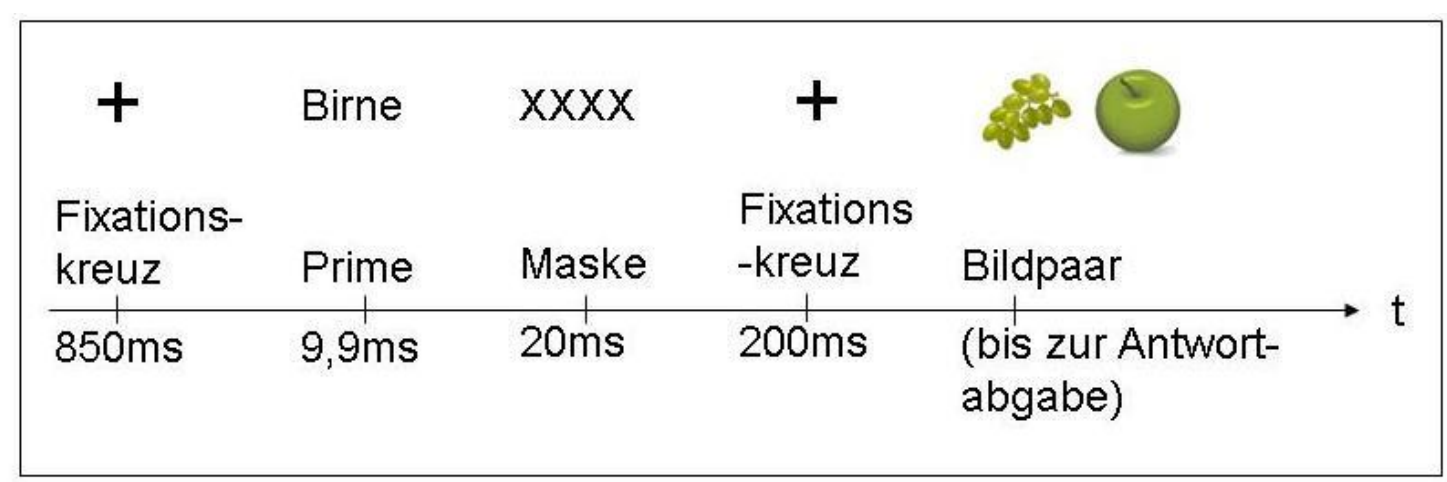

Abbildung 2: Zeitliche Sequenz der Ereignisse in einem Versuchsdurchgang in Experiment 1 „Kategorisierung über Bildpaare“ anhand eines Beispiels 
Die 240 Bildpaare wurden den Probanden in vier Blöcken von jeweils 60 Items präsentiert. Nach jedem Block bestand für die Probanden die Möglichkeit, eine Pause von beliebiger Länge zu machen und anschließend den nächsten Block selbständig über die Leertaste zu starten. Sobald alle Versuchsdurchgänge durchlaufen waren, d.h. nach den vier Blöcken, wurde den Probanden auf dem Bildschirm das Ende des am Computer durchgeführten ersten Teils des Experimentes 1 angezeigt. Nach einer Pause wurde anschließend das Experiment 2 („Kategorisierung über Bildtripel“) durchgeführt, das weiter unten dargestellt wird.

Nach der Bearbeitung der Kategorisierungsaufgabe am Computer wurden die Probanden gebeten, einen Fragebogen auszufüllen. Je nach Herkunft des Versuchsteilnehmers war der Fragebogen auf Deutsch oder auf Mandarin (vgl. Anhang C) verfasst. Er diente der Prüfung der Frage, ob die Antworten, welche die Probanden zuvor am Computer gegeben hatten, mit den Antworten übereinstimmten, die sie beim Lesen der den Bildern entsprechenden Wörter gegeben hätten. Auf diese Weise sollte die Eindeutigkeit der Interpretation der verwendeten Bilder kontrolliert werden. Den Teilnehmern wurden alle im Experiment dargebotenen Bildpaare, die durch eine taxonomische oder relationale Beziehung verbunden waren, als Wortpaare in Form einer Liste vorgelegt. Sie bekamen die schriftliche Instruktion zu beurteilen, ob die Wortpaare auf irgendeine Art und Weise zusammen passten. Ihre Antwort sollten die Teilnehmer diesmal abgeben, indem sie in der entsprechenden Spalte der Liste ein „Ja“ oder „Nein“ ankreuzten. Zusätzlich wurden einige der Wortpaare in die Liste aufgenommen, die keine besondere Beziehung enthielten, um ein möglicherweise auftretendes automatisches Antwortmuster durch ausschließlich mit „Ja“ zu beantwortenden Items zu vermeiden.

Mit Hilfe eines weiteren Fragebogens wurden am Ende der Versuchssitzung die demografischen Daten erhoben, und die Probanden hatten die Möglichkeit, Anmerkungen und Kommentare abzugeben (siehe Anhang C).

Alle Probanden, die in Deutschland an dem Experiment teilnahmen, wurden in demselben Experimentalraum des Georg-Elias-Müller-Instituts für Psychologie in Göttingen untersucht. In China stand ein Labor in einem Beratungskomplex auf dem Gelände der East China Normal University, Shanghai zur Versuchsdurchführung zur 
Verfügung. Alle Erhebungen fanden in diesem Labor statt. Die räumlichen Bedingungen in Göttingen und Shanghai können als vergleichbar angesehen werden.

Das Experiment wurde in Deutschland und China von demselben Versuchsleiter, dem Autor dieser Arbeit, durchgeführt. Alle Instruktionen wurden schriftlich in der jeweiligen Landessprache dargeboten, und die Probanden wurden angewiesen, das Experiment selbständig am Computer durchzuführen. Für die Durchführung in Shanghai stand eine chinesische Hilfskraft zur Beantwortung eventueller Fragen zur Verfügung. 


\section{Ergebnisse Experiment 1 „Kategorisierung über Bildpaare“}

Im ersten Experiment sollten die chinesischen und deutschen Probanden entscheiden, ob es eine, wie auch immer geartete, Beziehung zwischen zwei in Bildern dargestellten Objekten gibt. Gemessen wurde dabei die Reaktionszeit von der Darbietung des Bildpaares bis zur Antwortabgabe. Erwartet wurde, dass sich kulturbedingte Präferenzen in einer kürzeren Reaktionszeit niederschlagen sollten. Deutsche Probanden sollten bei taxonomischen Beziehungen schnellere Reaktionszeiten zeigen. Chinesische Probanden dagegen sollten bei relationalen Beziehungen kürzere Reaktionszeiten zeigen (Hypothese 1). Vorgeschaltete semantische Primes sollten die Antwortgeschwindigkeit bei Kongruenz zur Beziehung des Bildpaares erhöhen und entsprechend zu einer Verkürzung der Reaktionszeiten führen, bei Inkongruenz sollte es zu einer Verlängerung der Reaktionszeiten kommen. Der Kongruenzeffekt sollte bei chinesischen Probanden im Falle von relational verbundenen Bildpaaren auftreten und bei deutschen Probanden im Falle von taxonomisch verbundenen Bildpaaren (Hypothese 2).

\subsection{Overall-Analyse der Reaktionszeiten}

Zur ersten Übersicht über die Reaktionszeiten wurde eine Varianzanalyse mit wiederholten Messungen und den beiden Innersubjektfaktoren „Bildpaarbeziehung“ (2 Stufen: relational und taxonomisch) und „Prime“ (4 Stufen: relational, taxonomisch, neutral und kein Prime) sowie dem Zwischensubjektfaktor „Nation“ (2 Stufen: China und Deutschland) durchgeführt. Tabelle 2 enthält eine Zusammenstellung der Reaktionszeitdaten, in Abbildung 3 sind die Daten für deutsche und chinesische Probanden gegenübergestellt. Da die nicht zusammenpassenden Bildpaare lediglich in den Versuch aufgenommen worden waren, um ein automatisiertes Antwortverhalten der Probanden zu unterbinden, wurden die bei der Beurteilung dieser Paare gemessenen Reaktionszeiten und Fehler nicht in der Auswertung berücksichtigt. Wie in der Abbildung $3 \mathrm{zu}$ erkennen ist, fielen die Reaktionszeiten der chinesischen Probanden durchweg länger aus als die der deutschen. Es sei an dieser Stelle bereits angemerkt, dass diese Diskrepanz noch zu diskutieren sein wird (siehe Abschnitt 9.1). 
Die Varianzanalyse ergab einen signifikanten Haupteffekt des Faktors „Bildpaarbeziehung“ $\left(F(1,101)=19.15, p<.001, \varepsilon^{2}=.16\right)$. Auch für den Faktor „Prime“ wurde ein signifikanter Haupteffekt gefunden $\left(F(3,99)=9,53, p<.001, \varepsilon^{2}=\right.$ .22). Demnach hatten sowohl die Variation der Bildpaarbeziehung als auch die Variation der Beziehung zwischen Prime und Bildpaar einen systematischen Effekt auf die Reaktionszeiten. Als Zwischensubjekteffekt erwies sich „Nation“ als signifikant ( $F(1$, $\left.101)=30.02, p<.001, \varepsilon^{2}=.23\right)$. Das heißt, der schon angesprochene Unterschied zwischen chinesischen und deutschen Versuchsteilnehmern erwies sich als signifikant (siehe Abbildung 3).

Die nach Hypothese 1 erwartete Interaktion „Bildpaarbeziehung x Nation“ erwies sich als nicht signifikant $(F(1,101)=.88, p<.349)$. Es gab zwei andere signifikante Interkationen. Dies war zum Einen die Interaktion zwischen der Bildpaarbeziehung und dem Primetyp $\left(F(3,99)=8.40, p<.001, \varepsilon^{2}=.20\right)$. Zum Anderen erwies sich die dreifache Interaktion zwischen Bildpaarbeziehung, Primetyp und Nation ebenfalls als signifikant $\left(F(3,99)=3.15, p=.028, \varepsilon^{2}=.09\right)$. 
Tabelle 2: Mittlere Rektionszeiten in Abhängigkeit der Bildpaarbeziehung (taxonomisch versus relational) und des Primes (inkongruent, kongruent, neutral, kein) von $n=52$ chinesischen Probanden und $n=51$ deutschen Probanden. Mittelwerte sind in Millisekunden (ms) angegeben.

\begin{tabular}{|c|c|c|c|}
\hline $\begin{array}{l}\text { Bidlpaarbeziehung/ } \\
\text { Prime }\end{array}$ & Nation & $\begin{array}{l}\text { Mittelwert } \\
\text { in ms }\end{array}$ & $\begin{array}{c}\text { Standardfehler } \\
\text { des } \\
\text { Mittelwertes }\end{array}$ \\
\hline \multirow{3}{*}{$\begin{array}{l}\text { taxonomisch/ } \\
\text { kongruenter Prime }\end{array}$} & China & 1470 & $\overline{100}$ \\
\hline & Deutschland & 1021 & 30 \\
\hline & Gesamt & 1248 & 58 \\
\hline \multirow{3}{*}{$\begin{array}{l}\text { taxonomisch/ } \\
\text { inkongruenter } \\
\text { Prime }\end{array}$} & China & 1340 & 31 \\
\hline & Deutschland & 1034 & 47 \\
\hline & Gesamt & 1189 & 27 \\
\hline \multirow{3}{*}{$\begin{array}{l}\text { taxonomisch/ } \\
\text { neutraler Prime }\end{array}$} & China & 1209 & 78 \\
\hline & Deutschland & 944 & 31 \\
\hline & Gesamt & 1078 & $\overline{61}$ \\
\hline \multirow{3}{*}{$\begin{array}{l}\text { taxonomisch/ } \\
\text { kein Prime }\end{array}$} & China & 1342 & $\overline{34}$ \\
\hline & Deutschland & 984 & 80 \\
\hline & Gesamt & 1165 & 33 \\
\hline \multirow{3}{*}{$\begin{array}{l}\text { relational/ } \\
\text { kongruenter Prime }\end{array}$} & China & 1379 & 63 \\
\hline & Deutschland & 1042 & $\overline{44}$ \\
\hline & Gesamt & 1212 & $\overline{47}$ \\
\hline \multirow{3}{*}{$\begin{array}{l}\text { relational/ } \\
\text { inkongruenter } \\
\text { Prime }\end{array}$} & China & 1536 & $\overline{31}$ \\
\hline & Deutschland & 1087 & 100 \\
\hline & Gesamt & 1314 & 34 \\
\hline \multirow{3}{*}{$\begin{array}{l}\text { relational/ } \\
\text { neutraler Prime }\end{array}$} & China & 1490 & 58 \\
\hline & Deutschland & 1062 & 31 \\
\hline & Gesamt & 1278 & $\overline{47}$ \\
\hline \multirow{3}{*}{$\begin{array}{l}\text { relational/ } \\
\text { kein Prime }\end{array}$} & China & 1336 & 27 \\
\hline & Deutschland & 1039 & 78 \\
\hline & Gesamt & 1189 & 31 \\
\hline
\end{tabular}




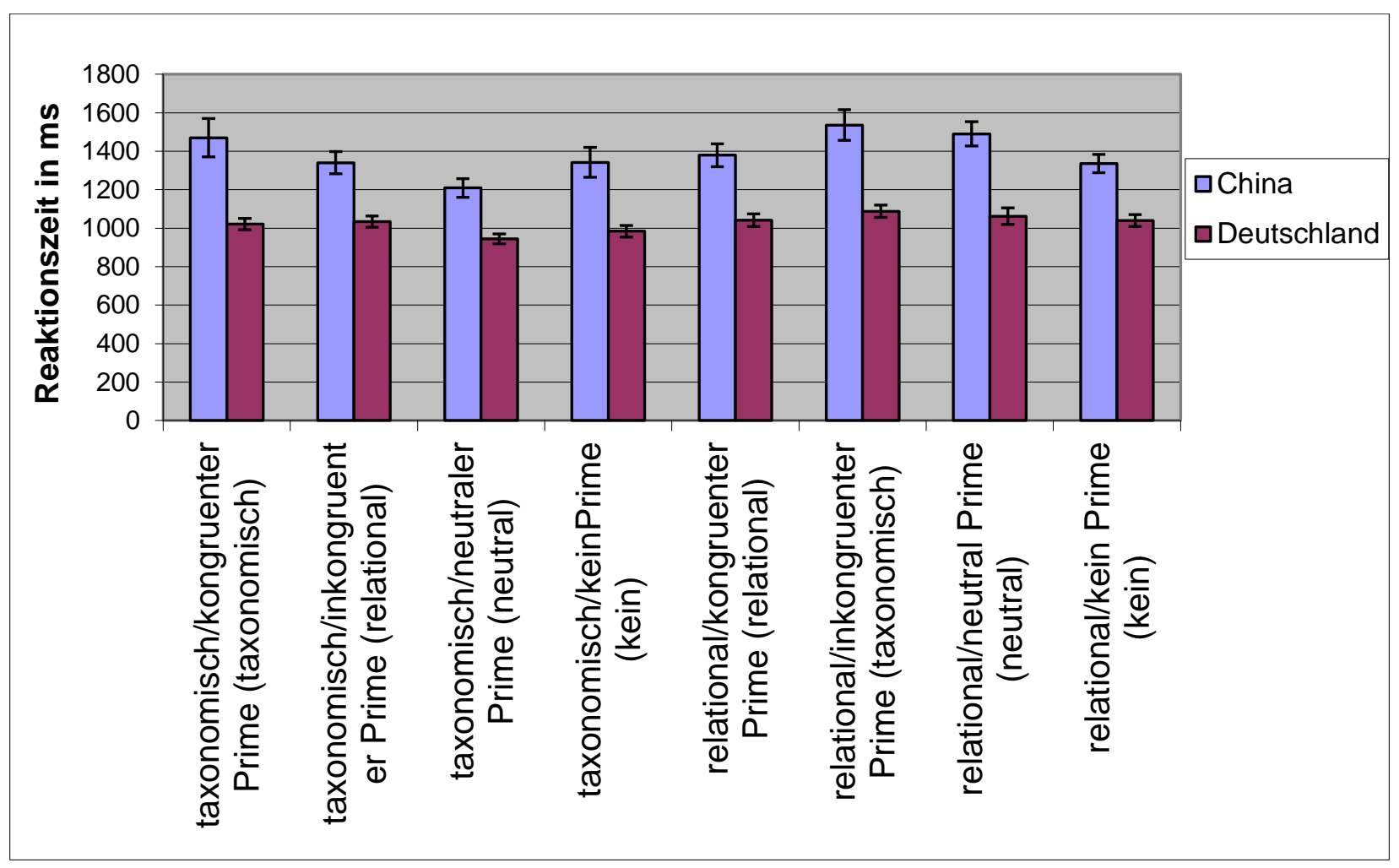

Abbildung 3: Darstellung der mittleren Reaktionszeiten (ms) in Abhängigkeit von Bildpaarbeziehung (Angabe vor dem Schrägstrich) und Prime (Angabe hinter dem Schrägstrich). Die Werte für deutsche Probanden $(n=51)$ und chinesische Probanden $(n$ =52) sind einander gegenüber gestellt und der Standardfehler abgetragen.

\subsection{Betrachtung der Bildpaarbeziehung}

Von theoretischem Interesse war der Effekt der Bildpaarbeziehung auf die Reaktionszeit. Zur näheren Analyse dieses Effektes wurde der Kontrast zwischen den mittleren Reaktionszeiten zwischen taxonomischen Bildpaaren versus relationalen Bildpaaren berechnet. Der T-Test für abhängige Stichproben ergab für die Gesamtstichprobe ein signifikantes Ergebnis $(t(102)=-4.388, p<.001)$. Dies ist in der Abbildung 4 verdeutlicht. Es zeigt sich also, dass über alle Probanden hinweg die taxonomische Beziehung im Mittel schneller erkannt wurde. Angehörige beider Nationen erzielten kürzere Reaktionszeiten, wenn die Bildpaare in taxonomischer Beziehung zueinander standen, als wenn die Beziehung relationaler Art war. 


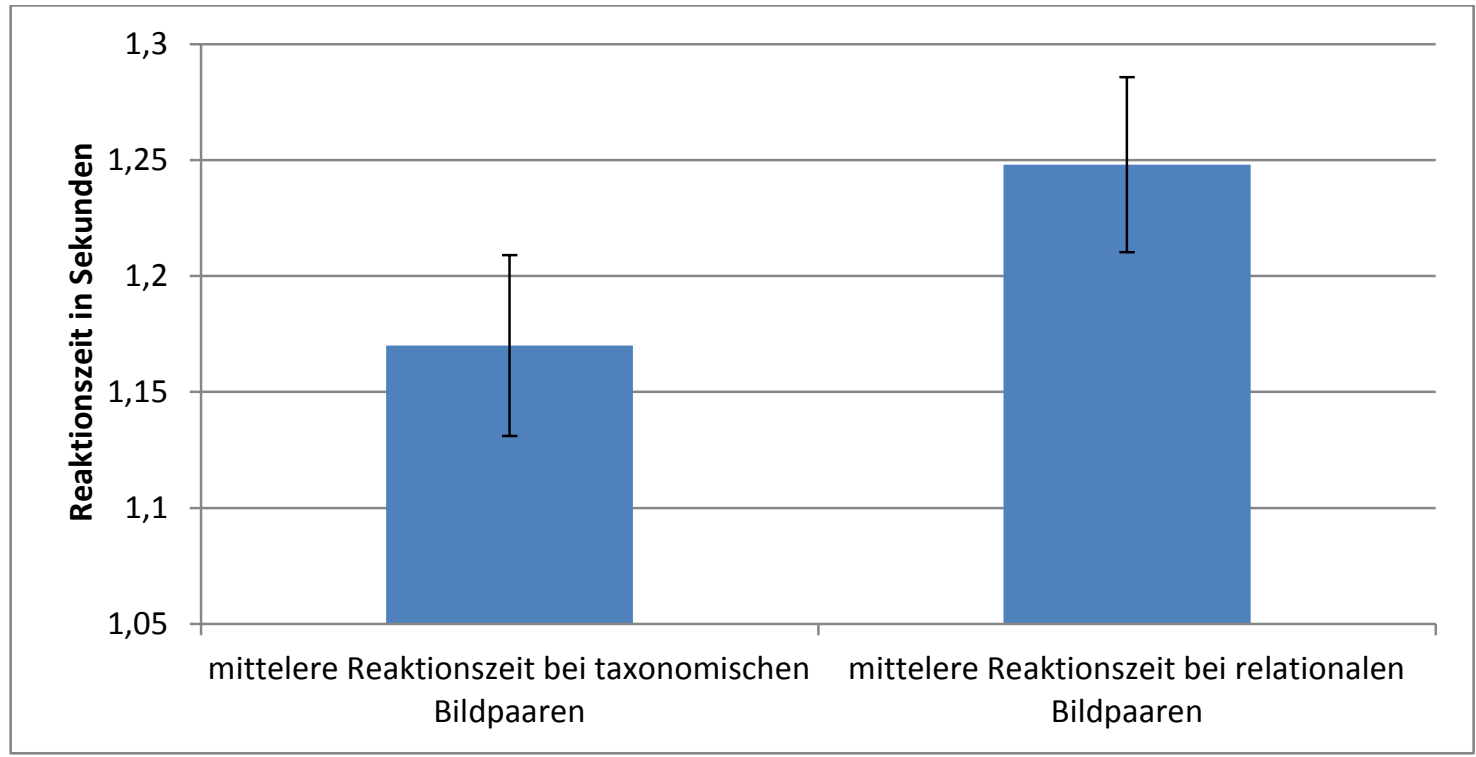

Abbildung 4: Mittlere Reaktionszeiten in Sekunden bei taxonomischen Bildpaaren gegenüber mittleren Reaktionszeiten bei relationalen Bildpaaren innerhalb der gesamten Stichprobe mit Standardfehlern.

Dieses Muster lässt sich auch innerhalb der beiden Nationen wieder finden. Sowohl bei den chinesischen Probanden $(t(51)=-3.010, p=.004)$ als auch bei den deutschen Probanden $(t(50)=-3.762, p<.001)$ unterscheiden sich die Reaktionszeiten bei taxonomischen Bildpaaren signifikant von denen bei relationalen Bildpaaren (siehe Abbildung 5). 


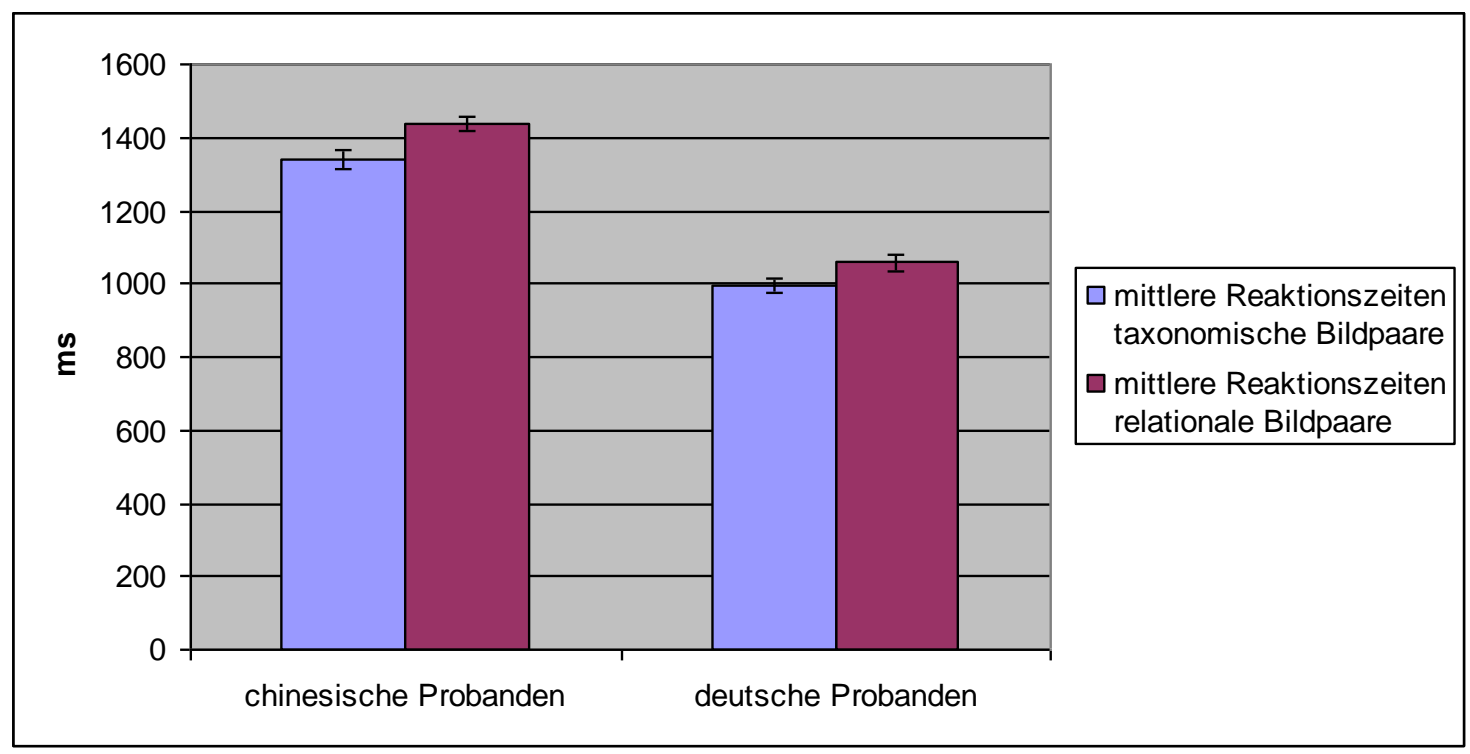

Abbildung 5: Mittlere Reaktionszeiten (ms) bei taxonomischen Bildpaaren versus mittlere Reaktionszeiten bei relationalen Bildpaaren, getrennt für deutsche $(n=51)$ und chinesische Probanden $(n=52)$ mit Angabe des Standardfehlers des Mittelwertes.

Bildet man einen Kontrast, der die Mittelwerte der Differenz der Bildpaarbeziehungen - taxonomisch versus relational - zwischen den beiden Nationen vergleicht, so ergibt sich kein signifikanter Unterschied zwischen chinesischen und deutschen Probanden $(t(51)=0.904, p=.349)$. Somit erklärt sich der signifikante Zwischensubjekteffekt „Nation“ nicht aus den unterschiedlichen Effekten der Bildpaarbeziehung.

\subsection{Wirkung der Primes}

Der Prime wurde in vier Variationen in Abhängigkeit zu den Bildpaaren dargeboten. Er konnte in kongruenter, inkongruenter oder neutraler Beziehung zu dem Bildpaar stehen, oder es wurde kein Prime dargeboten. Abbildung 6 zeigt die Reaktionszeiten in Abhängigkeit von dieser Variation für die chinesische Stichprobe, Abbildung 7 zeigt die Daten für die deutsche Stichprobe. 


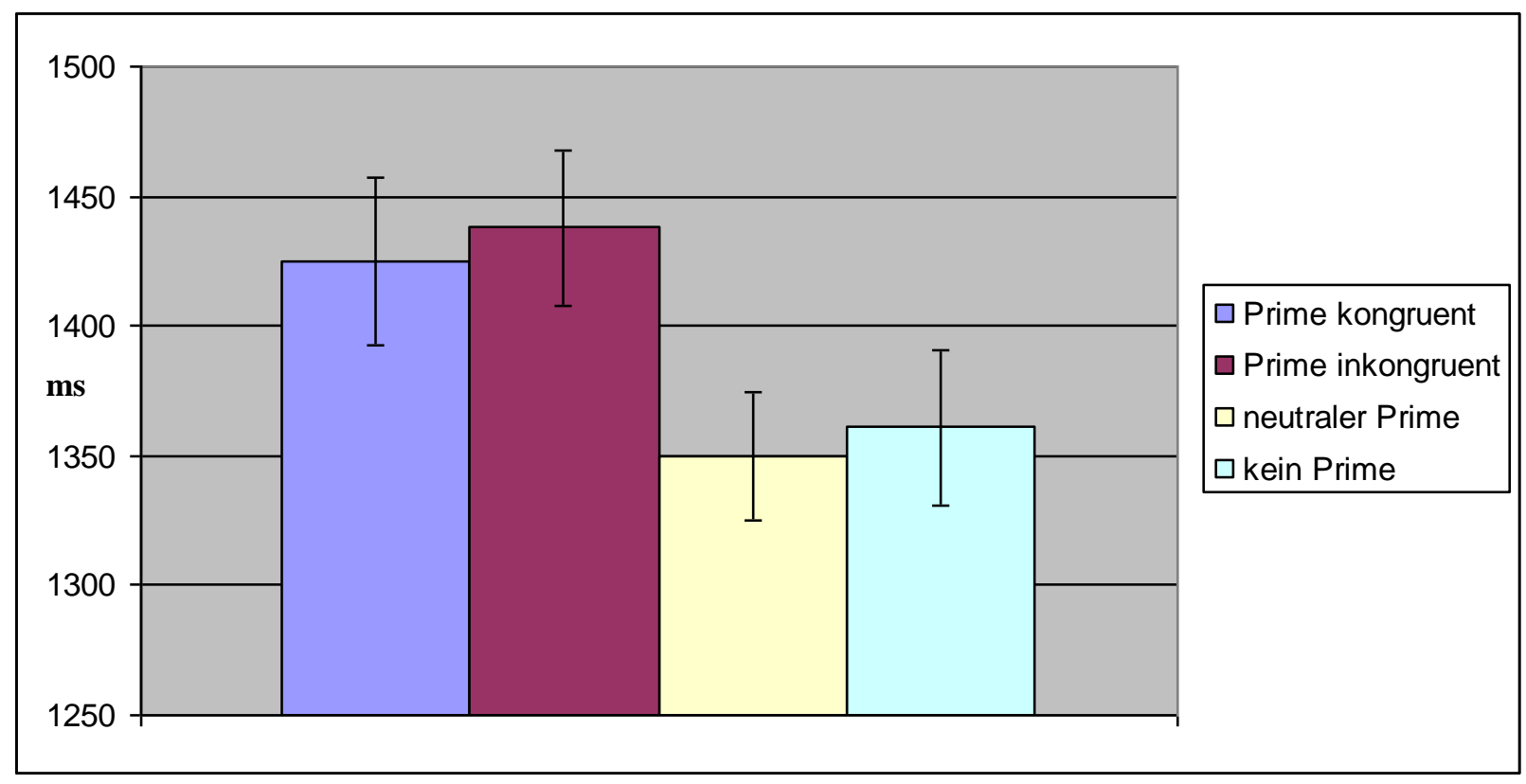

Abbildung 6: Darstellung der mittleren Reaktionszeiten (ms) in Abhängigkeit von der Primebeziehung in der chinesischen Stichprobe $(n=52)$ mit Angabe des Standardfehlers.

Eine Berechnung der Kontraste für die chinesische Stichprobe ergab signifikante Unterschiede zwischen den folgenden Bedingungen: kongruenter Prime versus kein Prime $(t(51)=2.687, p=.005)$, inkongruenter Prime versus neutraler Prime $(t(51)=$ 2.804, $p<.001)$, inkongruenter Prime versus kein Prime $(t(51)=2.906, p<.005)$.

Die Reaktionszeiten der Bedingung mit den neutralen Primes heben sich nicht signifikant von der Bedingung ohne Primes ab $(t(51)=-0.300, p=.765)$. Neutrale Primes, die keine besondere Beziehung zu dem Bildpaar aufwiesen, haben also bei den chinesischen Probanden keinen signifikanten Effekt auf die Reaktionszeit. Dies entspricht den Erwartungen, da in dieser Bedingung kein semantischer Effekt auftreten sollte beziehungsweise keine semantische Aktivierung oder Hemmung stattfinden sollte. Allerdings hebt sich die kongruente Bedingung nicht von der inkongruenten Bedingung ab $(t(51)=-0.338, p=.737)$, wie es nach Hypothese 2 postuliert wurde. Inkongruente und kongruente Primes führen gleichermaßen zu einer Verlängerung der Reaktionszeit. Offensichtlich hat eine vergleichbare semantische Aktivierung in den beiden Bedingungen stattgefunden, so dass die erwarteten spezifischen Unterschiede ausblieben. 


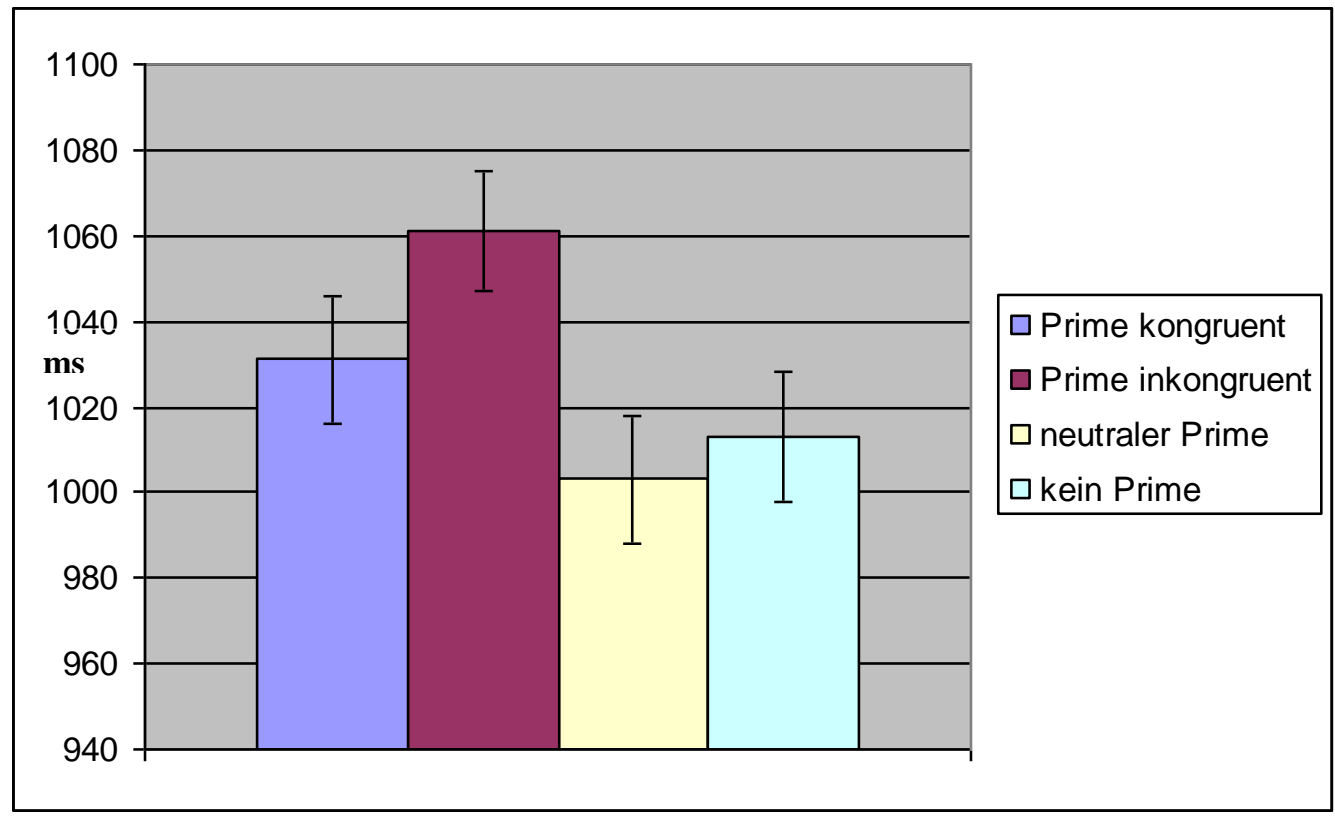

Abbildung 7: Darstellung der mittleren Reaktionszeiten (ms) in Abhängigkeit von der Primebeziehung innerhalb der deutschen Stichprobe $(n=51)$ mit Angabe des Standardfehlers.

Innerhalb der deutschen Stichprobe zeichnet sich folgendes Bild ab. Eine Berechnung der Kontraste ergab signifikante Unterschiede zwischen den folgenden Bedingungen: kongruenter Prime versus inkongruenter Prime $(t(50)=-2.092, p=.042)$, inkongruenter versus neutraler Prime $(t(50)=3.712, p=.003)$ und inkongruenter versus kein Prime $(t(50)=2.048, p=.036)$.

Die Reaktionszeiten der Bedingung mit den neutralen Primes heben sich nicht signifikant von der Bedingung ohne Primes ab $(t(50)=-0.410, p=.684)$. Neutrale Primes haben auch bei den deutschen Probanden keinen signifikanten Effekt auf die Reaktionszeit. Dies entspricht den Erwartungen, da in dieser Bedingung kein semantischer Effekt auftreten sollte beziehungsweise keine semantische Aktivierung stattfinden sollte.

Die Reaktionszeiten mit inkongruenten Primes sind signifikant länger als die Reaktionszeiten der kongruenten Bedingung $(t(50)=-2.09, p=.042)$ und auch länger als die der Bedingung mit neutralen Primes $(t(50)=3.172, p=.003)$ sowie der Bedingung ohne Primes $(t(50)=2.048, p=.036)$. Anders als bei den chinesischen Probanden führten also bei deutschen Probanden inkongruente Primes zu einer Verlängerung der 
Reaktionszeit, was aufgrund der vermuteten Interferenz nach Hypothese 2 erwartet worden war.

Aufgrund des beobachteten Kongruenzeffektes soll in einem letzten Schritt noch näher analysiert werden, ob es systematische Unterschiede innerhalb der kongruenten Primebedingung bei den deutschen Probanden gibt. Die Frage, die näher betrachtet werden soll, ist, ob taxonomische Primes bei taxonomischen Bildpaarbeziehungen oder relationale Primes bei relationalen Bildpaarbeziehungen zu schnelleren Reaktionszeiten führen. Bildet man einen Kontrast, der genau die besagten Bedingungen vergleicht, so lässt sich kein signifikanter Unterschied feststellen $(t(50)=-0.864, p=.392)$. Dies bedeutet, dass die deutschen Probanden uneingeschränkt von den kongruenten Primes profitieren konnten und zwar unabhängig von der Art der Bildpaarbeziehung, was nach Hypothese 2 anders erwartet worden war.

\subsection{Auswertung der Fehlerprozente}

Analog zur Reaktionszeit sollen im Folgenden nun auch die Fehlerprozente analysiert werden. Hierbei handelt es sich um eine post hoc-Betrachtung. Zur ersten Übersicht über die Fehlerprozente wurde eine Varianzanalyse mit wiederholten Messungen und den beiden Innersubjektfaktoren „Bildpaarbeziehung“ (2 Stufen: relational und taxonomisch) und „Prime“ (4 Stufen: relational, taxonomisch, neutral und kein Prime) sowie dem Zwischensubjektfaktor „Nation“ (2 Stufen: China und Deutschland) durchgeführt Tabelle 3 enthält eine Zusammenstellung der Fehlerprozente.

In der Varianzanalyse zeigte sich ein signifikanter Haupteffekt des Faktors „Prime“ $\left(F(3,99)=10.22, p<.001, \varepsilon^{2}=.236\right)$. In Abbildung 8 , in der die Daten in Abhängigkeit von der Primebeziehung dargestellt sind, ist dieser Effekt veranschaulicht. Der Zwischensubjekteffekt „Nation“ erwies sich außerdem als hoch signifikant ( $F(1$, 101) $\left.=10173, p<.0001, \varepsilon^{2}=.99\right)$. Wie Tabelle $3 \mathrm{zu}$ entnehmen ist, liegen die Fehlerprozentwerte der chinesischen Probanden durchweg unter den der deutschen Probanden. 
Tabelle 3: Mittlere Fehlerprozente in Abhängigkeit der Bildpaarbeziehung (taxonomisch versus relational) und des Primes (inkongruent, kongruent, neutral, kein) von $n=52$ chinesischen Probanden und $n=51$ deutschen Probanden.

\begin{tabular}{|c|c|c|c|}
\hline $\begin{array}{l}\text { Bildpaarbeziehung } \\
\text { /Prime }\end{array}$ & Nation & Fehlerprozente & $\begin{array}{c}\text { Standardfehler } \\
\text { des } \\
\text { Mittelwertes }\end{array}$ \\
\hline \multirow{3}{*}{$\begin{array}{l}\text { taxonomisch/ } \\
\text { kongruenter Prime }\end{array}$} & China & 17.44 & 2.24 \\
\hline & Deutschland & 6.54 & 0.78 \\
\hline & Gesamt & 12.04 & 1.30 \\
\hline \multirow{3}{*}{$\begin{array}{l}\text { taxonomisch/ } \\
\text { inkongruenter } \\
\text { Prime }\end{array}$} & China & 18.08 & 1.97 \\
\hline & Deutschland & 11.63 & 1.05 \\
\hline & Gesamt & 14.89 & 1.16 \\
\hline \multirow{3}{*}{$\begin{array}{l}\text { taxonomisch/ } \\
\text { neutraler Prime }\end{array}$} & China & 17.56 & 2.08 \\
\hline & \begin{tabular}{|l|} 
Deutschland \\
\end{tabular} & 10.98 & 1.21 \\
\hline & Gesamt & 14.30 & 1.25 \\
\hline \multirow{3}{*}{$\begin{array}{l}\text { taxonomisch/ } \\
\text { kein Prime }\end{array}$} & China & 13.33 & 2.06 \\
\hline & \begin{tabular}{|l} 
Deutschland \\
\end{tabular} & 8.10 & 1.14 \\
\hline & Gesamt & 10.74 & 1.20 \\
\hline \multirow{3}{*}{$\begin{array}{l}\text { relational/ } \\
\text { kongruenter Prime }\end{array}$} & China & 17.95 & 2.04 \\
\hline & \begin{tabular}{|l|} 
Deutschland \\
\end{tabular} & 9.02 & 2.04 \\
\hline & Gesamt & 13.53 & 1.50 \\
\hline \multirow{3}{*}{$\begin{array}{l}\text { relational/ } \\
\text { inkongruenter } \\
\text { Prime }\end{array}$} & China & 19.23 & 2.54 \\
\hline & Deutschland & 11.50 & 2.08 \\
\hline & Gesamt & 15.40 & 1.68 \\
\hline \multirow{3}{*}{$\begin{array}{l}\text { relational/ } \\
\text { neutraler Prime }\end{array}$} & China & 19.62 & 2.25 \\
\hline & Deutschland & 10.46 & 1.28 \\
\hline & Gesamt & 15.08 & 1.37 \\
\hline \multirow{3}{*}{$\begin{array}{l}\text { relational/ } \\
\text { kein Prime }\end{array}$} & China & 15.90 & 2.21 \\
\hline & Deutschland & 8.63 & 1.90 \\
\hline & Gesamt & 12.30 & 1.50 \\
\hline
\end{tabular}




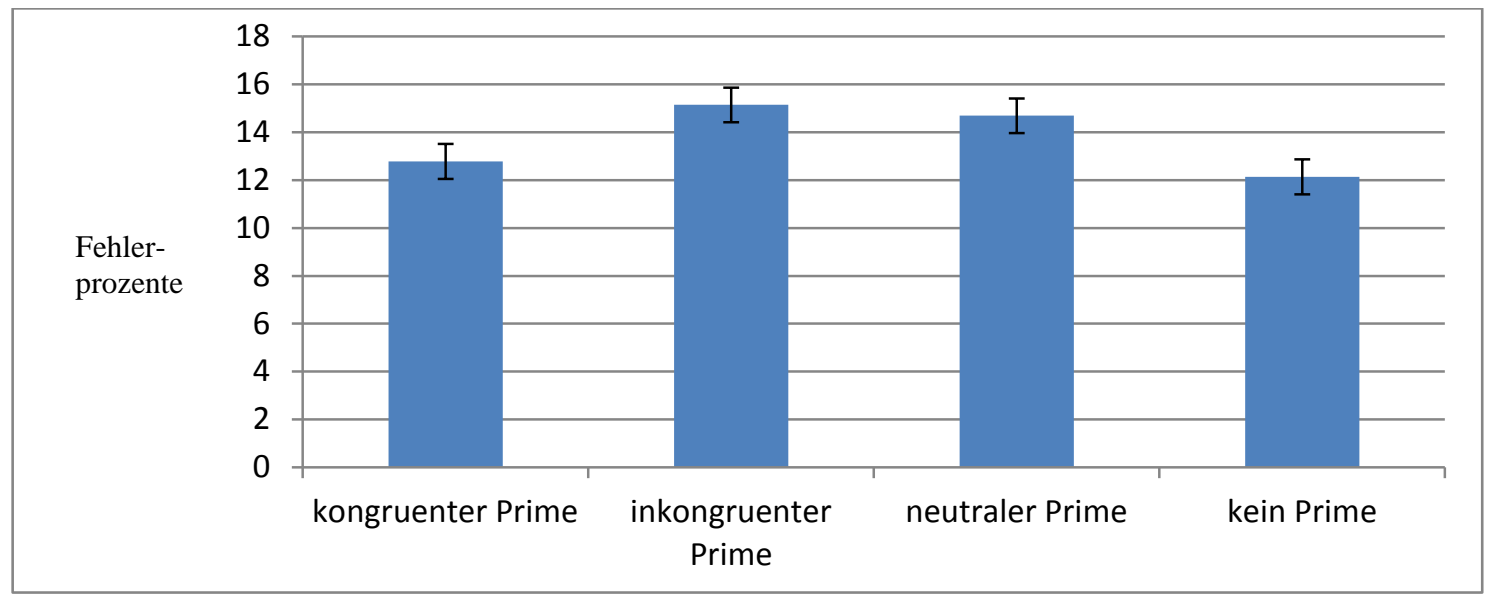

Abbildung 8: Darstellung der Fehlerprozente in Abhängigkeit von der Primebeziehung über die gesamte Stichprobe $(N=103)$.

Um den Haupteffekt des Faktors „Prime“ genauer zu analysieren, wurden die Fehlerprozentwerte in Abhängigkeit von der Primebedingung betrachtet und entsprechende Kontraste berechnet. Die kongruenten Primes führten zu signifikant kleineren Fehlerprozenten als die inkongruenten $(t(102)=2.847, p=.005)$ und die Bedingung der neutralen Primes $(t(102)=2.391, p=.019)$. Die Bedingung ohne Primes führte zu signifikant kleineren Fehlerprozenten als die inkongruenten Primes $(t(102)=-$ 3.846, $p<.001)$ und die Bedingung der neutralen Primes $(t(102)=-3.381, p=.001)$.

Betrachtet man die Daten der chinesischen Stichprobe genauer zeichnet sich nur ein unspezifischer Primeeffekt ab (siehe Abbildung 9). Eine Berechnung der entsprechenden Kontraste ergab signifikante Unterschiede zwischen den folgenden Bedingungen: kein Prime versus kongruenter Prime $(t(51)=-2.602, p=.012)$, kein Prime versus inkongruenter Prime $(t(51)=-2.929, p=.005)$ kein Prime versus neutraler Prime $(t(51)=-2.915, p=.004)$. 


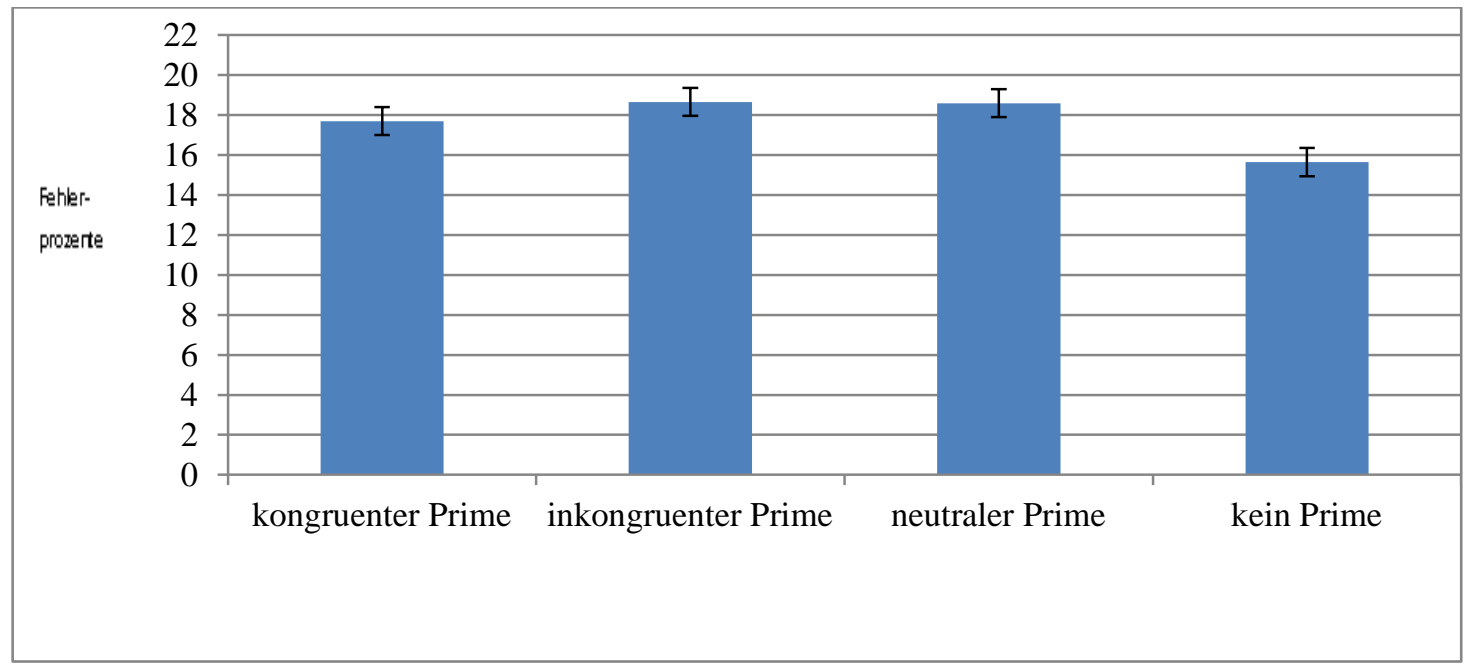

Abbildung 9: Darstellung der Fehlerprozente in Abhängigkeit von der Primebeziehung innerhalb der chinesischen Stichprobe $(n=52)$.

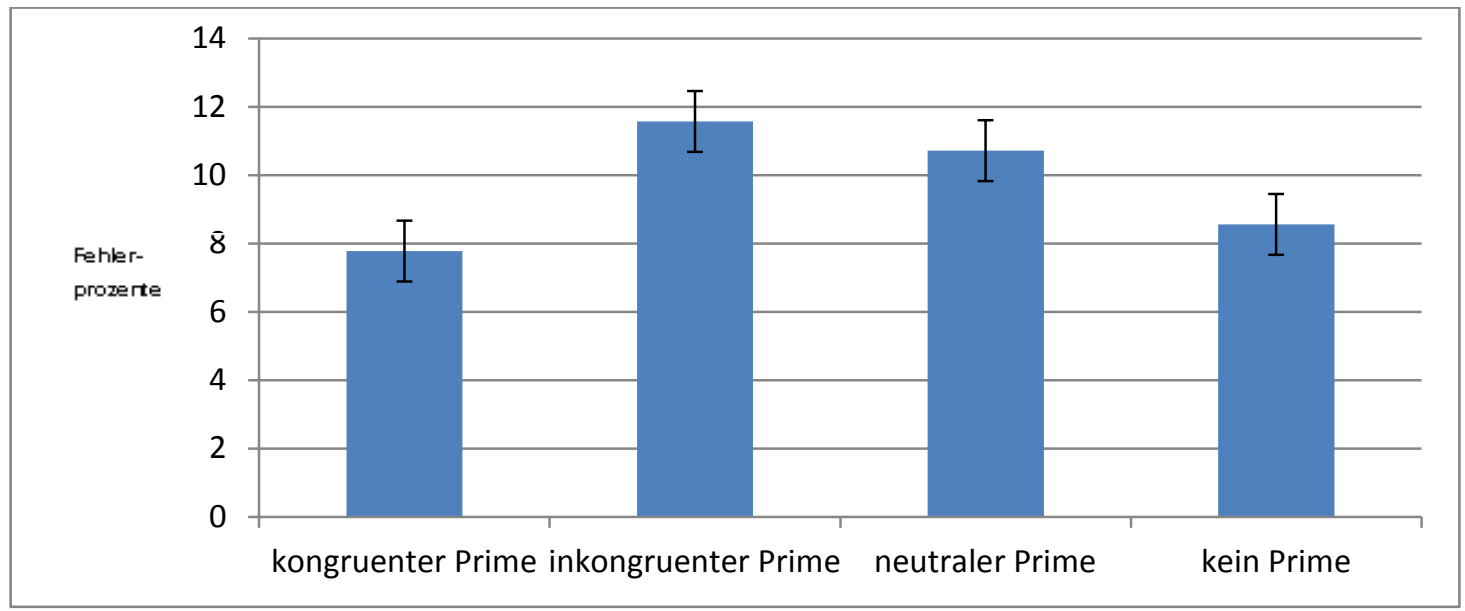

Abbildung 10: Darstellung der Fehlerprozente in Abhängigkeit von der Primebeziehung innerhalb der deutschen Stichprobe $(n=51)$.

In der deutschen Stichprobe zeigt sich ein anderes Bild (siehe Abbildung 10). Signifikante Unterschiede ergaben sich zwischen den folgenden Bedingungen: kongruenter versus inkongruenter Prime $(t(50)=3.82, \mathrm{p}<.001)$, kongruenter versus neutraler Prime $(t(50)=2.67, \mathrm{p}=.010)$, inkongruenter Prime versus kein Prime $(t(50)=$ $-2.52, \mathrm{p}=.015)$.

Vergleicht man die beiden Nationen, so fällt besonders auf, dass die deutschen Probanden in der Bedingung mit kongruenten Primes im Vergleich zu den chinesischen Probanden signifikant weniger Fehler machten $(t(101)=-5.05, p<.001)$. Wie bereits 
erwähnt und auch Tabelle $3 \mathrm{zu}$ entnehmen ist, liegen allerdings die Fehlerprozentwerte der deutschen Probanden durchweg unter denen der chinesischen Probanden, so dass sich auch die anderen Bedingungen signifikant voneinander unterscheiden $(p<.05)$. Dieser Effekt wird zu diskutieren sein.

Zusammengefasst ergibt sich folgendes Bild. Bei den Chinesen gibt es weniger Fehler in der Bedingung ohne Prime im Vergleich mit den anderen Bedingungen. Gleichzeitig lässt sich feststellen, dass sich die Bedingungen mit inkongruenten, kongruenten und neutralen Primes nicht unterscheiden. Bei den Deutschen finden sich weniger Fehler bei den kongruenten Primes im Vergleich $\mathrm{zu}$ den anderen Primebedingungen. Es lassen sich keine Unterschiede zwischen den Bedingungen mit inkongruenten und neutralen Primes sowie ohne Prime verzeichnen. Das heißt, dass bei den Chinesen der Prime ein starker Ablenker ist, der unabhängig von der Beziehung seine Wirkung entfaltet und offensichtlich zusätzliche kognitive Kapazitäten verbraucht. Chinesische Schriftzeichen stellen somit unter der realisierten Aufgabenanforderung einen Störreiz dar.

Während bei den deutschen Versuchsteilnehmern der inhaltliche Aspekt des Primes relevant wird, indem ein kongruenter Prime eine Bahnung auszulösen vermag, trifft dies offenbar auf die chinesischen Probanden nicht zu. Die chinesischen Schriftzeichen scheinen vielmehr in Konkurrenz zu der verlangten Kategorisierung und Verarbeitung der Bilder zu treten.

\subsection{Nachtestfragebogen}

Zur Kontrolle des eingesetzten Bildmaterials wurde zusätzlich ein Nachtestfragebogen eingesetzt. Bei diesem sollten die Probanden die während des Computerexperimentes dargebotenen Itempaare erneut beurteilen als zusammenpassend oder nicht zusammenpassend, diesmal allerdings nicht als Bildstimuli, sondern in Form von Wortpaaren. Analog zum Computerexperiment wurde die Fehlerquote ermittelt.

Über die Fehlerprozente wurde eine Varianzanalyse mit wiederholten Messungen und dem Innersubjektfaktor „Bildpaarbeziehung“ (2 Stufen: relational und taxonomisch) sowie dem Zwischensubjektfaktor „Nation“ (2 Stufen: China und Deutschland) 
durchgeführt. Tabelle 4 enthält eine Zusammenstellung der Fehlerprozente, in Abbildung 11 und 12 sind die Daten für deutsche und chinesische Probanden dargestellt.

Die Varianzanalyse ergab einen signifikanten Haupteffekt des Zwischensubjektfaktors „Nation“ $(F(1,101)=49.29, p<.001)$, aber keinen signifikanten Haupteffekt des Faktors „Bildpaarbeziehung“ $(F(1,101)=2.02, p=.157)$. Allerdings war die Interaktion „Bildpaarbeziehung x Nation“ signifikant $(F(1,101)=$ 15.35, $p<.001)$. Es handelt sich um eine disordinale Interaktion. So machen die chinesischen Probanden bei den taxonomischen Wortpaaren mehr Fehler, beurteilten die beiden bezeichneten Objekte also als nicht zusammen passend. Die deutschen Probanden dagegen machten mehr Fehler bei den relationalen Bildpaaren. Dieses Ergebnismuster wurde zwar nicht erwartet, steht aber in Einklang mit der Hypothese 1.

Tabelle 4: Mittlere Fehlerprozente im Nachtestfragebogen in Abhängigkeit der Bildpaarbeziehung (taxonomisch versus relational) von $n=52$ chinesischen Probanden und $n=51$ deutschen Probanden.

\begin{tabular}{|l|l|r|r|}
\hline Bildpaarbeziehung & Nation & Fehlerprozente & $\begin{array}{c}\text { Standardfehler } \\
\text { des } \\
\text { Mittelwertes }\end{array}$ \\
\hline taxonomisch & China & 3.79 & 0.64 \\
\cline { 2 - 4 } & Deutschland & 1.18 & 0.18 \\
\hline relational & China & 0.50 & 0.32 \\
\cline { 2 - 4 } & Deutschland & 1.72 & 0.72 \\
\hline
\end{tabular}




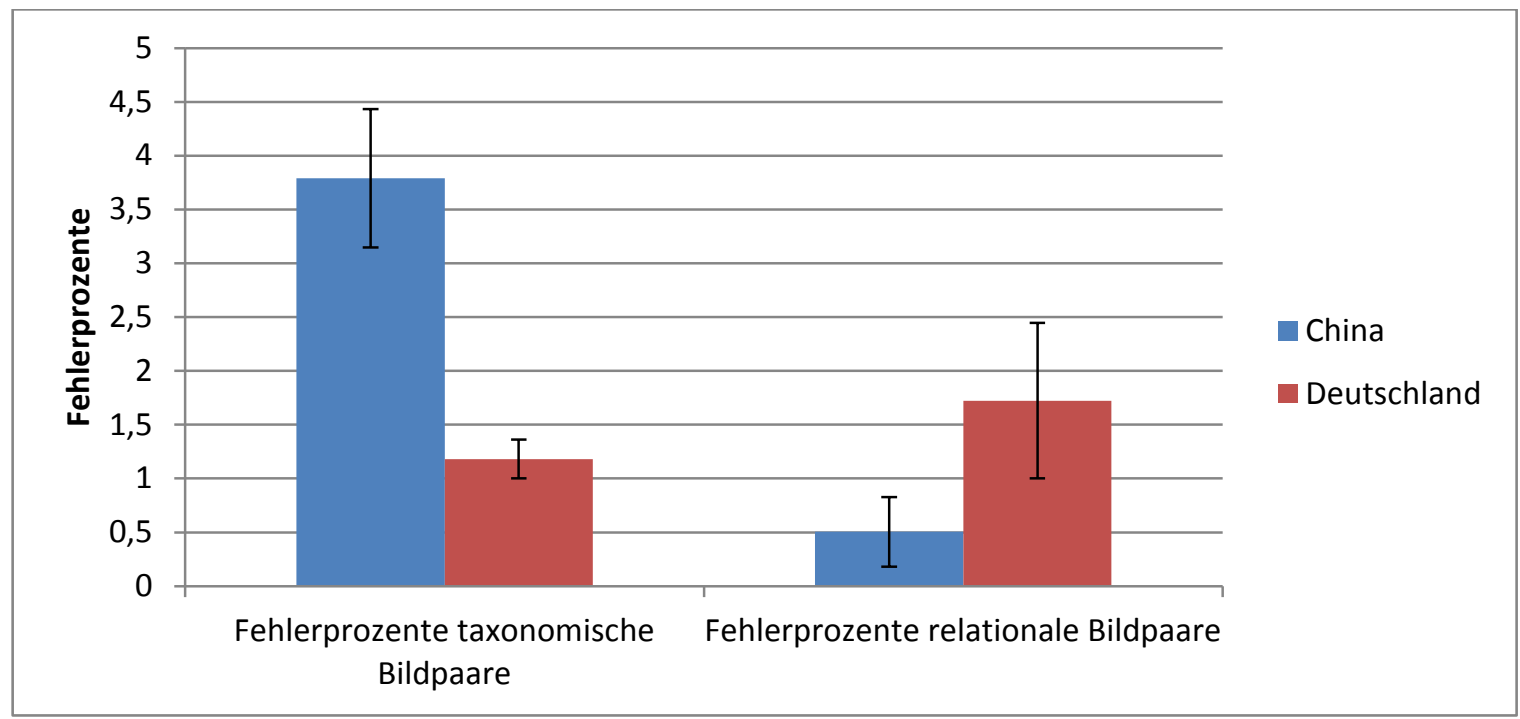

Abbildung 11: Darstellung der Fehlerprozente im Nachtestfragebogen von $n=52$ chinesischen Probanden und $n=51$ deutschen Probanden.

Außerdem zeigte sich, dass die Fehlerquote bei allen Probanden im Nachtestfragebogen niedriger war als im Computerexperiment (vgl. Abbildung 12). Bei den deutschen Probanden zeigte sich im Nachtestfragebogen $(M=1,11 \%)$ im Vergleich zum Computerexperiment $(M=9,6 \%)$ ein signifikanter Unterschied $(t(50)=-8.59, p<$ $.001)$. Auch bei den chinesischen Probanden zeigte sich im Nachtestfragebogen $(M=$ 2,49\%) im Vergleich zum Computerexperiment $(M=17,39 \%)$ ein signifikanter Unterschied $(t(51)=-11.96, p<.001)$. 


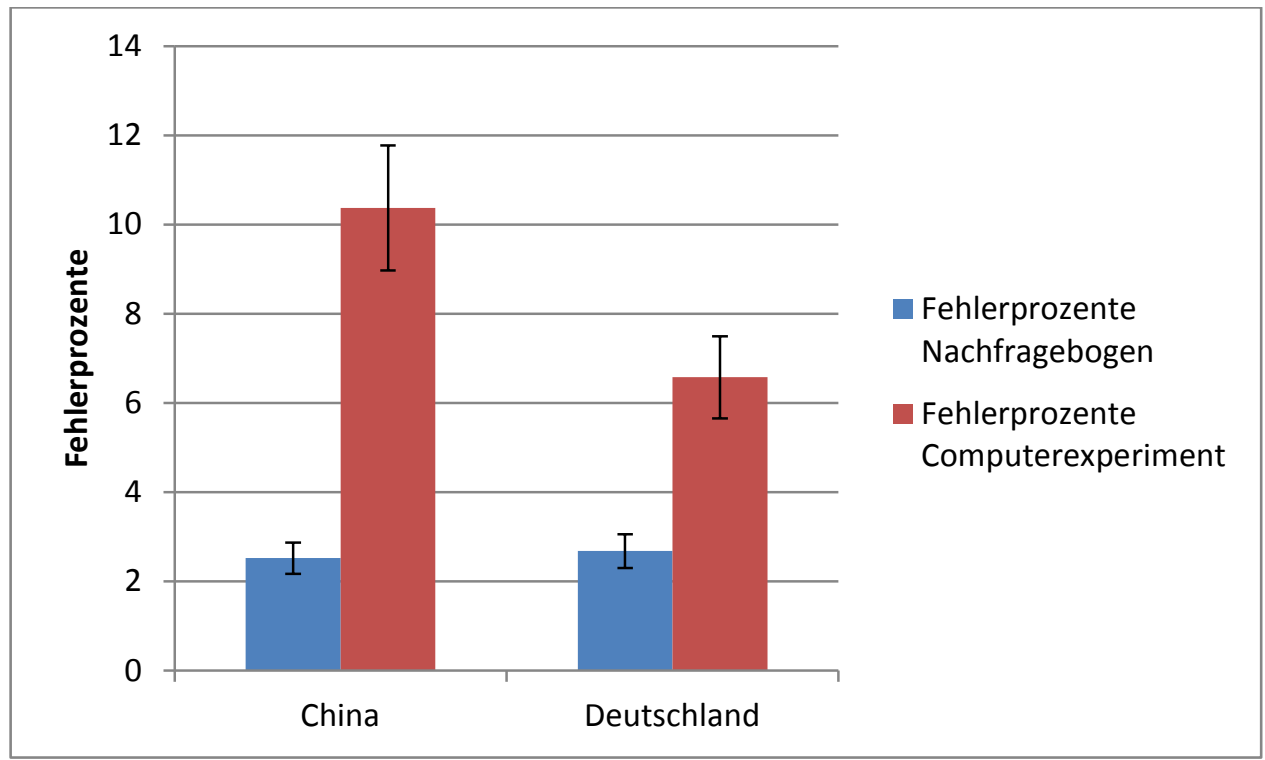

Abbildung 12: Darstellung der Fehlerprozente im Nachfragebogen und Computerexperiment von $n=52$ chinesischen Probanden und $n=51$ deutschen Probanden

Diese Befunde legen zusammen genommen den Schluss nahe, dass Konzepte über Wörter eindeutiger zu identifizieren sind als über bildliche Darstellungen und sich daher bei sprachlichen Stimuli die postulierten Effekte nachweisen lassen. Eventuell ist es auch kognitiv aufwendiger, Konzepte über Bilder zu identifizieren, wodurch die postulierten Effekte ebenfalls beeinflusst werden könnten.

\subsection{Fazit Experiment 1 „Kategorisierung über Bildpaare“}

Eine eindeutige Kategorisierungspräferenz im Sinne des analytischen und holistischen Denkstils (vgl. Nisbett, 2003) nach Hypothese 1und 2 wird durch die Daten nicht bestätigt. Beide Probandengruppen, Chinesen und Deutsche, zeigten schnellere Reaktionszeiten, wenn die zu beurteilenden Bilder in einer taxonomischen Beziehung zueinander standen. Dem Ansatz von Nisbett folgend wäre dagegen zu erwarten gewesen, dass sich bei den chinesischen Probanden kürzere Reaktionszeiten bei den relationalen Bildpaarbeziehungen finden lassen. Auch das nach Hypothese 2 erwartete spezifische Datenmuster bezüglich der Primewirkung ließ sich nicht nachweisen. Allerdings machten die chinesischen Probanden bei den taxonomischen Wortpaaren 
mehr Fehler, beurteilten die beiden bezeichneten Objekte also als nicht zusammen passend. Die deutschen Probanden dagegen machten mehr Fehler bei den relationalen Bildpaaren.

Außerdem zeigte sich, dass die chinesischen Probanden langsamer waren als die deutschen Probanden, aber dennoch nicht weniger Fehler produzierten. Geht man von der Präferenz eines analytischen Denkstils bei den deutschen Probanden und eines holistischen Denkstils bei den chinesischen Probanden aus, so könnte dies ein Hinweis sein, dass es mehr Zeit benötigt, aus einem holistischen Präferenzrahmen heraus, Entscheidungen zu treffen (vgl. auch Abschnitt 9.1).

Das verwendete Design - die Erhebung von Entscheidungszeiten bei einer Kategorisierungsaufgabe - scheint die postulierten Kategorisierungspräferenzen in diesem Fall nicht eindeutig abzubilden. Bisher gab es allerdings auch noch keine Studien, in denen eine abhängige Variable auf Intervallskalenniveau verwendet worden ist. Meistens wurde das Kategorisierungsverhalten über Häufigkeiten von Wahlalternativen erfasst (z.B. Ji et al., 2004; Norenzayan et al., 2002; Unsworth et al., 2005). Das verwendete Material scheint aufgrund der Ergebnisse im Nachtestfragebogen prinzipiell geeignet $\mathrm{zu}$ sein, um Kategorisierungspräferenzen abbilden zu können. Denkbar ist, dass sich Kategorisierungspräferenzen auch bei leicht unterschiedlichen Anforderungen auf verschiedene Weise manifestieren. So könnte eine Entscheidung über Bildpaare im Gegensatz zu Wortpaaren andere kognitive Verarbeitungsprozesse implizieren, die sich dann auf die Reaktionszeiten auswirken.

Zur Erhöhung der internen Validität im Sinne einer Minimierung von Kontexteffekten könnte das verwendete Bildmaterial auch schematischer, als Strichzeichnungen, dargestellt werden. Im Sinne der externen Validität bleibt darauf zu achten, Bilder zu wählen, die für beide Probandengruppen gleichermaßen einfach zu identifizieren sind. Entsprechende Voruntersuchungen könnten breiter und differenzierter angelegt sein, als dies in dem vorliegenden Vortest realisiert werden konnte. 


\section{Methode Experiment 2 „Kategorisierung über Bildtripel“}

In einem zweiten Experiment steht erneut der Nachweis von Kategorisierungspräferenzen im Mittelpunkt. Das Vorgehen lehnt sich dabei an das Experiment von Unsworth, Sears und Pexman (2005) an (vgl. Abschnitt 2.3.2).

\subsection{Probanden}

Am Experiment 2 nahmen dieselben Probanden teil, wie am Experiment 1. Es wird an dieser Stelle auf die Beschreibung der Stichprobe des ersten Experiments verwiesen (vgl. Abschnitt 3.1).

\subsection{Versuchsmaterial und Aufgabenanforderung}

Mit Hilfe der Software Presentation ${ }^{\circledR}$ wurde erneut eine Softwarelösung zur experimentellen Durchführung realisiert. Die Durchführung fand auf demselben Notebook statt wie auch beim ersten Experiment.

Aufgabe des Probanden war es, drei Bilder, die auf dem Bildschirm des Notebooks dargeboten wurden, hinsichtlich der Frage zu beurteilen, welche zwei der drei Bilder am ehesten in irgendeiner Art und Weise zusammenpassten. Bei den Bildern handelte es sich um farbige Zeichnungen von Objekten. Eine vollständige Liste der verwendenden Bilder befindet sich im Anhang (vgl. Anhang D).

Das Bildmaterial und die als semantischen Primes verwendeten Wörter wurden wie beim ersten Experiment in einem Vorversuch auf ihre Eignung untersucht. An dem Vorversuch nahmen dieselben Probanden teil wie im Vorversuch des Experiments 1. Im ersten Teil des Vorversuchs sollten die Probanden beurteilen, ob es ihnen leicht fiel, die gezeigten Bilder zu identifizieren. Dies wurde anhand einer Power Point Präsentation realisiert, bei der die Probanden gebeten wurden, die Bilder namentlich zu benennen. Im zweiten Teil des Vorversuchs wurden Kombinationen von jeweils drei Bildern gezeigt, welche auf ihre taxonomische oder relationale Kategorisierbarkeit bewertet werden sollten. Hier wurden die Probanden gefragt, ob sich zwei der drei Bilder in eine 
gemeinsame Kategorie einordnen lassen oder ob es einen logischen oder funktionalen Zusammenhang gibt.

Gab es Schwierigkeiten bei einzelnen Items, also konnten z.B. zwei Vorversuchsteilnehmer keinen Identifizierung leisten oder keinen Zusammenhang herstellen, wurden entsprechende Items oder deren Kombination verworfen. Traten außerdem Abweichungen vom Gruppenmittel in der Kategorisierung von über 20\% auf, so wurde das Item ebenfalls nicht weiter verwendet. Ausgewählt wurden schließlich 80 Bildtripel, die im Experiment in randomisierter Reihenfolge dargeboten wurden. Die Randomisierung wurde dabei durch die Software geleistet. Dadurch wurde sichergestellt, dass jeder Proband eine neue Reihenfolge erhielt.

Folgende experimentelle Variation wurde vorgenommen: Innerhalb der Bildtripel konnten immer je zwei Bilder entweder in taxonomischer, relationaler oder neutraler Beziehung zueinander stehen (Unabhängige Variable 1,UV1). Die Anzahl und Art der Beziehungen innerhalb der Bildtripel wurden in 3 Itemsets variiert (siehe unten). Falls eine Beziehung vorlag, konnte diese entweder taxonomischer oder relationaler Natur sein. So lassen sich z.B. die Bilder „Regen“ und „Regenjacke“ in einen funktionalen Zusammenhang bringen, so dass diese als relational zusammenhängend bewertet werden können. Außerdem wurde die Relation variiert, in der ein sprachlicher Prime zu den beiden Bildern eines Tripels stand, die ihrerseits eine taxonomische, relationale oder neutrale Beziehung aufwiesen. Zusätzlich gab es Versuchsdurchgänge, in denen kein Prime dargeboten wurde. Die Primevariationen (UV2) waren analog zu Experiment 1 gestaltet (siehe Abschnitt 3.2).

Es gab drei verschiedene Itemsets. Im Itemset 1 (80 Bildtripel) waren die Items so gestaltet, dass es jeweils zwei Bilder eines Tripels gab, die in taxonomischer Beziehung zueinander standen, und zwei Bilder, die in relationaler Bedingung standen. Eines der drei Bilder konnte demnach zwei verschiedenen Beziehungen zugeordnet werden. In dem in Abbildung 13 gezeigtem Beispiel besteht eine taxonomische Beziehung zwischen der Fliege und der Biene und eine eher relationale Beziehung zwischen der Biene und dem Bienenstock. Fliege und Bienenstock stehen in einem neutralen Zusammenhang. 


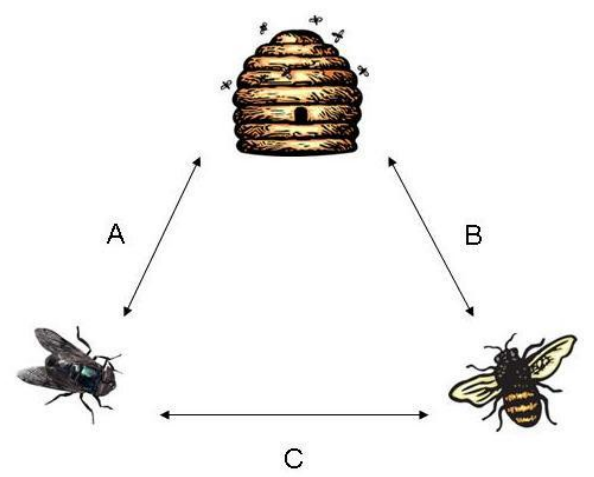

Abbildung 13: Stimulus aus Itemset 1 von Experiment 2. „A“, „B“ und „C“ markieren die Beziehung, die über eine Tastatur gewählt werden konnte. „B“ würde bedeuten, die Biene und der Bienenstock gehören zusammen; „C“ würde bedeuten, die Fliege und die Biene werden als zusammengehörig empfunden.

Hier ein paar Beispiele für die vorgenommene Variationen der Primes: Für das Bildtripel „Schiff, Auto, Straße“ ist „Flugzeug“ ein taxonomischer Prime, da das Flugzeug auch ein Fortbewegungsmittel wie das Auto und das Schiff ist und sich diese drei Objekte einer Kategorie zuordnen lassen. Das Wort „Papier“ ist ein relationaler Prime für das Tripel „Bleistift, Kugelschreiber, Radiergummi“, da zwei der genannten Gegenstände auf Papier schreiben. „Bär“ ist ein neutraler Prime für das Bildtripel „Hut, Hose, Kopf“, da es keinen Zusammenhang gibt.

Insgesamt gab es im Itemset 120 Grundkonstellationen von Bildtripels, die unter Variation des Primes (taxonomisch, relational, neutral oder kein Prime) und der Position innerhalb der Dreiecksanordnung (unterschiedliche räumliche Anordnung) jeweils viermal dargeboten wurden. Insgesamt umfasste das Itemset 180 Items. Tabelle 5 gibt eine Übersicht über die Kombination von Primes und Bildtripeln im Itemset 1. 
Tabelle 5: Kombinationen kongruenter und inkongruenter Primes mit taxonomisch und relational verbundenen Beziehungen aus einem Bildtripel

\begin{tabular}{|l|l|l|}
\hline \multicolumn{2}{|l|}{} & \multicolumn{2}{|l|}{ Beziehung zwischen zwei Bildern aus dem Bildtripel } \\
\hline $\begin{array}{l}\text { Beziehung des Primes zu } \\
\text { einem Bildpaar des } \\
\text { Bildtripels }\end{array}$ & taxonomisch & relational \\
\hline taxonomisch & \begin{tabular}{l|l|} 
taxonomische Beziehung, \\
kongruenter Prime
\end{tabular} & $\begin{array}{l}\text { relationale Beziehung, } \\
\text { inkongruenter Prime }\end{array}$ \\
\hline relational & $\begin{array}{l}\text { taxonomische Beziehung, } \\
\text { inkongruenter Prime }\end{array}$ & $\begin{array}{l}\text { relationale Beziehung, } \\
\text { kongruenter Prime }\end{array}$ \\
\hline neutral & $\begin{array}{l}\text { taxonomische Beziehung, } \\
\text { neutraler Prime }\end{array}$ & $\begin{array}{l}\text { relationale Beziehung, } \\
\text { neutraler Prime }\end{array}$ \\
\hline keine & $\begin{array}{l}\text { taxonomische Beziehung, } \\
\text { kein Prime }\end{array}$ & $\begin{array}{l}\text { relationale Beziehung, } \\
\text { kein Prime }\end{array}$ \\
\hline
\end{tabular}

Im Itemset 2 wurden Bildtripel dargeboten, bei denen jeweils zwei Bilder in taxonomischer Beziehung standen, während das dritte Bild eine neutrale Beziehung zu den beiden anderen Bildern aufwies. Die Zitrone und die Orange in Abbildung 14 stehen in einer taxonomischen Beziehung zueinander. Der Bleistift steht in neutraler Beziehung zu den anderen beiden Objekten.

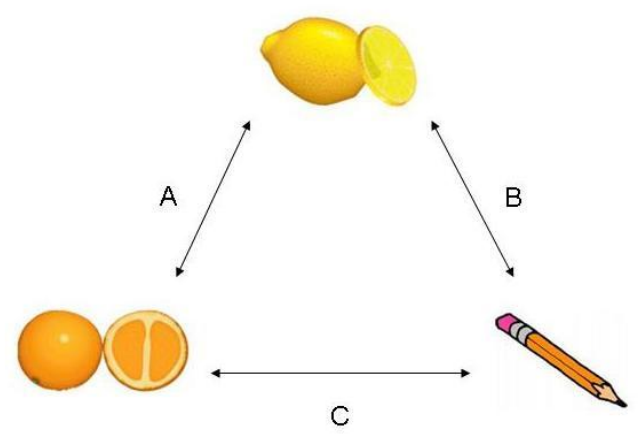

Abbildung 14: Stimulus aus Itemset 2 von Experiment 2. „A“, „B“ und „C“ markieren die Beziehung, die über eine Tastatur gewählt werden konnte. „A“ würde bedeuten, die Zitrone und die Orange gehören zusammen; „C“ würde bedeuten, die Orange und der Bleistift werden als zusammengehörig empfunden. 
Bei einer Gesamtzahl von 20 verschiedenen Grundkonstellationen wurden die oben bereits beschriebenen Variationen des Primes (taxonomisch, relational, neutral oder kein Prime) und der räumlichen Anordnung vorgenommen. Tabelle 6 gibt eine Übersicht über die Kombination von Primes und Bildtripeln im Itemset 2. Insgesamt gab es im Set 2 ebenfalls 80 Items.

Tabelle 6: Kombinationen kongruenter und inkongruenter Primes mit taxonomisch und neutral verbundenen Beziehungen aus einem Bildtripel

\begin{tabular}{|l|l|l|}
\hline \multicolumn{1}{|l|}{} & \multicolumn{2}{|l|}{ Beziehung zwischen zwei Bildern aus dem Bildtripel } \\
\hline $\begin{array}{l}\text { Beziehung des Primes zu } \\
\text { einem Bildpaar des } \\
\text { Bildtripels }\end{array}$ & taxonomisch & neutral \\
\hline taxonomisch & $\begin{array}{l}\text { taxonomische Beziehung, } \\
\text { kongruenter Prime }\end{array}$ & $\begin{array}{l}\text { keine Beziehung, neutraler } \\
\text { Prime }\end{array}$ \\
\hline relational & $\begin{array}{l}\text { taxonomische Beziehung, } \\
\text { inkongruenter Prime }\end{array}$ & $\begin{array}{l}\text { keine Beziehung, neutraler } \\
\text { Prime }\end{array}$ \\
\hline neutral & $\begin{array}{l}\text { taxonomische Beziehung, } \\
\text { neutraler Prime }\end{array}$ & $\begin{array}{l}\text { keine Beziehung, neutraler } \\
\text { Prime }\end{array}$ \\
\hline keine & $\begin{array}{l}\text { taxonomische Beziehung, } \\
\text { kein Prime }\end{array}$ & $\begin{array}{l}\text { keine Beziehung, kein } \\
\text { Prime }\end{array}$ \\
\hline
\end{tabular}

Itemset 3 umfasste 20 Bildtripel, bei denen jeweils zwei Bilder in relationaler Beziehung standen, während das dritte Bild eine neutrale Beziehung zu den anderen beiden Bildern aufwies. Bei dem in Abbildung 15 gezeigten Beispiel stehen der Schraubenzieher und die Schraube eher in relationaler Beziehung. Die Banane soll sich $\mathrm{zu}$ diesen beiden Objekten in einer neutralen Beziehung befinden. 


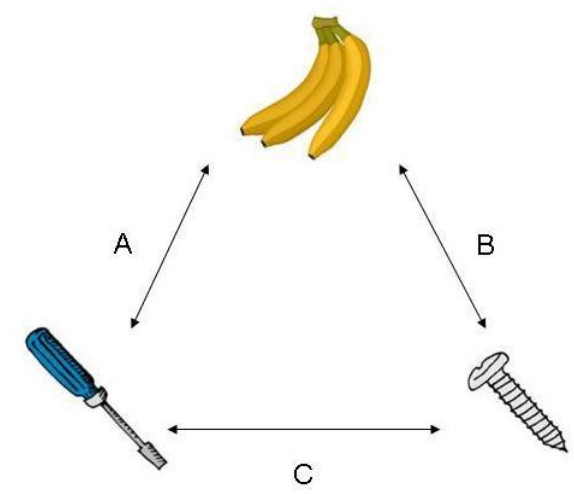

Abbildung 15: Stimulus aus Itemset 3 von „Experiment 2. „A““, „B“ und „C“ markieren die Beziehung, die über eine Tastatur gewählt werden konnte. „A“ würde bedeuten, der Schraubenzieher und die Banane gehören zusammen; „B“ würde bedeuten, die Banane und die Schraube gehören zusammen; „C“ würde bedeuten, der Schraubenzieher und die Schraube werden als zusammengehörig empfunden.

Hinsichtlich des Primes und der räumlichen Anordnung wurden die gleichen Variationen vorgenommen wie in den anderen Itemsets, so dass Set 3 auch 80 Items umfasste. Tabelle 7 gibt eine Übersicht über die Kombination von Primes und Bildertripel von Itemset 3.

Tabelle 7: Kombinationen kongruenter und inkongruenter Primes mit relationalen und neutral verbundenen Beziehungen aus einem Bildtripel

\begin{tabular}{|l|l|l|}
\hline \multicolumn{1}{|l|}{} & \multicolumn{2}{|l|}{ Beziehung zwischen zwei Bildern aus dem Bildtripel } \\
\hline $\begin{array}{l}\text { Beziehung des Primes zu } \\
\text { einem Bildpaar des } \\
\text { Bildtripels }\end{array}$ & relational & neutral \\
\hline taxonomisch & $\begin{array}{l}\text { relationaler Beziehung, } \\
\text { inkongruenter Prime }\end{array}$ & $\begin{array}{l}\text { keine Beziehung, neutraler } \\
\text { Prime }\end{array}$ \\
\hline relational & $\begin{array}{l}\text { relationaler Beziehung, } \\
\text { kongruenter Prime }\end{array}$ & $\begin{array}{l}\text { keine Beziehung, neutraler } \\
\text { Prime }\end{array}$ \\
\hline neutral & $\begin{array}{l}\text { relationaler Beziehung, } \\
\text { neutraler Prime }\end{array}$ & $\begin{array}{l}\text { keine Beziehung, neutraler } \\
\text { Prime }\end{array}$ \\
\hline keine & $\begin{array}{l}\text { relationaler Beziehung, } \\
\text { kein Prime }\end{array}$ & $\begin{array}{l}\text { relationale Beziehung, } \\
\text { kein Prime }\end{array}$ \\
\hline
\end{tabular}


Die insgesamt 240 Items in Experiment 2 verwendeten Items wurden in randomisierter Reihenfolge kontrolliert durch die Software dargeboten. Durch die Variation der räumlichen Anordnung der Bilder innerhalb der Dreieckskonstellation sollten Positionseffekte verhindert und .stereotype Antwortmuster weitgehend unterbunden werden.

\subsection{Versuchsablauf}

Nach einer kurzen Begrüßung wurden die Probanden gebeten, sich an das bereits vorbereitete Notebook zu setzen, auf dessen Bildschirm die Versuchsinstruktion zu sehen war. Die Probanden wurden aufgefordert, sich die Anweisungen zunächst aufmerksam durchzulesen. Bevor das eigentliche Experiment startete, konnten Verständnisfragen gestellt werden. Für das Durchlesen der Instruktionen wurde den Probanden soviel Zeit gegeben, wie diese dafür benötigten. Anschließend konnten sie selbständig mit der Leertaste das Experiment starten. Die Instruktion war im Falle der deutschen Studierenden in deutscher Sprache, im Falle der chinesischen Studierenden auf Mandarin verfasst (Anhang E).

In der Instruktion wurden die Probanden aufgefordert, die anschließend gezeigten Bildtripel danach zu bewerten, ob zwei der abgebildeten Objekte als zusammengehörig empfunden werden. Die Probanden wurden angewiesen, ihre Antwort über Tastendruck auf einem externen Ziffernblock (einer Computertastatur) einzugeben. Hierfür wurden die Tasten 2, 4 und 6 gewählt, da die räumlich Anordnung auf dem Ziffernblock der räumlichen Anordnung der Stimuli entspricht und so die Antwortabgabe erleichtert werden sollte. Die jeweiligen Tasten waren mit den betreffenden Buchstaben A, B oder C überklebt, die auf je eine der entsprechenden Kombinationen verwiesen (vgl. Abbildung 13). Die Probanden wurden außerdem erneut aufgefordert die Finger auf den Tasten liegen zu lassen, um eine schnellere Antwortabgabe zu ermöglichen. Die Instruktion beinhaltete ein Beispiel für einen Versuchsdurchgang. 


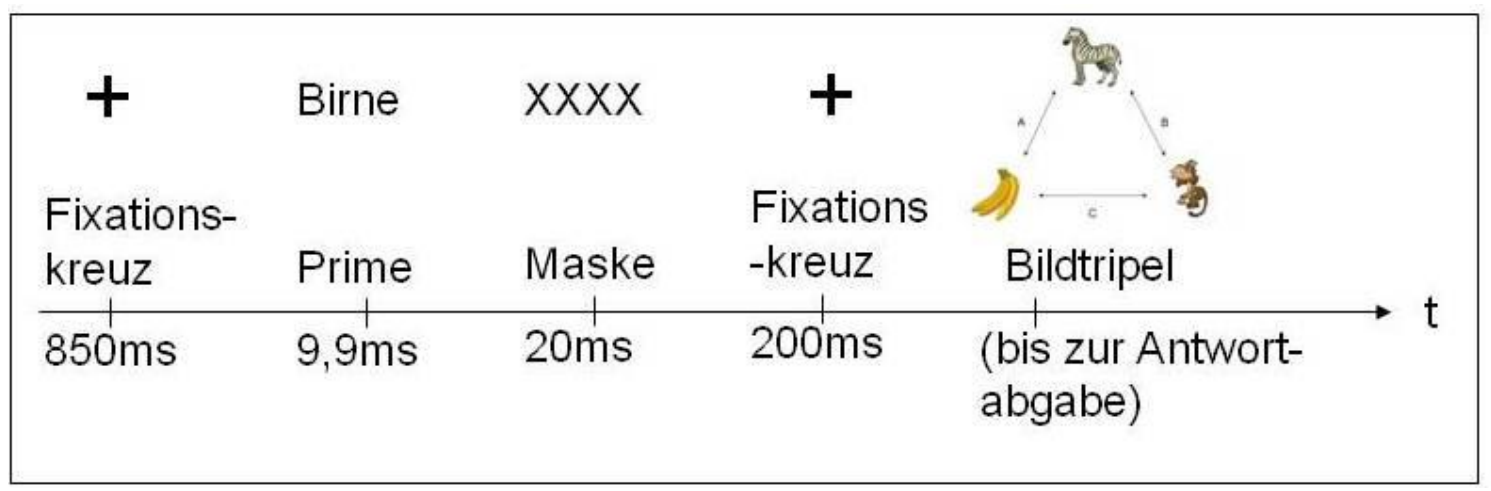

Abbildung 16: Zeitliche Sequenz der Ereignisse in einem Versuchsdurchgang in Experiment 2 , Kategorisierung über Bildtripel“" anhand eines Beispiel

Abbildung 16 veranschaulicht die Ereignisse in einem Versuchsdurchgang. Vor jedem dargebotenen Bildtripel erschien auf dem Notebookbildschirm für die Dauer von $850 \mathrm{~ms}$ ein Fixationskreuz, welches die Probanden fixieren sollten. Das Fixationskreuz diente dazu, die Aufmerksamkeit der Probanden an die Stelle des Bildschirms zu lenken, an welcher später der Prime und das nachfolgende Bildtripel erscheinen würden. Nach Verschwinden des Fixationskreuzes wurde der sprachliche Prime beziehungsweise das leere Feld für den Zeitraum von 9.9 ms präsentiert. Dies entspricht einer Bildschirmrate auf einem TFT-Notebookbildschirm und ist damit die aufgrund der technischen Gegebenheiten kürzest mögliche Präsentationszeit. Als Prime wurde entweder für die deutschen Probanden ein deutsches Wort oder für chinesische Probenden ein chinesisches Schriftzeichen verwendet. Der Prime wurde wie beim ersten Experiment durch eine Reihe von X-Zeichen (,XXXXXX“) für 20 ms maskiert. Es folgte danach für 200 ms das Fixationskreuz, bevor das zu bewertende Bildtripel dargeboten wurde, das bis zur Antwortabgabe der Probanden sichtbar blieb. Die Probanden konnten dann die Antwort über den externen Ziffernblock eingeben. Nach der Abgabe der Antwort startete der nächste Versuchsdurchgang mit der Darbietung des Fixationskreuzes.

Die insgesamt 240 Bildtripel der drei Itemsets wurden den Probanden in vier Blöcken von jeweils 60 Items präsentiert. Nach jedem Block bestand für die Probanden die Möglichkeit, eine Pause von beliebiger Länge zu machen, und anschließend den nächsten Block selbständig über die Leertaste $\mathrm{zu}$ starten. Sobald alle Versuchsdurchgänge durchlaufen waren, d.h. nach den vier Blöcken, wurde den Probanden auf dem Bildschirm das Ende des am Computer durchgeführten ersten Teils 
des Experiments 2 angezeigt. Im Anschluss wurden die Probanden gebeten, die bereits erwähnten Fragebögen auszufüllen (siehe Abschnitt 3.2).

Nach dem Ausfüllen der Fragebögen bedankte sich der Versuchsleiter bei den Probanden und übergab die vereinbarte Aufwandsentschädigung oder füllte die Bescheinigung über erworbene Versuchspersonenstunden aus. Die Probanden wurden über Sinn und Zweck der Experimente aufgeklärt und sie wurden gebeten, die Informationen nicht weiter $\mathrm{zu}$ geben, um eine Voreingenommenheit bei weiteren Probanden zu vermeiden.

Es herrschten dieselben Rahmenbedingungen wie bei Experiment 1. Alle Probanden, die in Deutschland an dem Experiment teilnahmen, wurden in demselben Experimentalraum des Georg-Elias-Müller-Instituts für Psychologie in Göttingen untersucht. In China stand ein Labor in einem Beratungskomplex auf dem Gelände der East China Normal University, Shanghai zur Versuchsdurchführung zur Verfügung. Alle Erhebungen fanden in diesem Labor statt. Die Bedingungen können als weitestgehend vergleichbar angesehen werden.

Das Experiment wurde in Deutschland und China von demselben Versuchsleiter, dem Autor dieser Arbeit, durchgeführt. Alle Instruktionen wurden schriftlich in der jeweiligen Landessprache dargeboten, und die Probanden wurden angewiesen, das Experiment selbständig am Computer durchzuführen. Für die Durchführung in Shanghai stand eine chinesische Hilfskraft zur Beantwortung eventueller Fragen zur Verfügung. 


\section{Ergebnisse Experiment 2 „Kategorisierung über Bildtripel“}

Die mit den Itemsets 1, 2 und 3 erhobenen Daten wurden getrennt ausgewertet, um eine übersichtlichere Darstellung der Ergebnisse zu erreichen. Neben einem möglichen Effekt des Faktors Nation steht bei der Analyse der Daten zum Itemset 1 die Entscheidung des Probanden zwischen der taxonomischen und relationalen Bildpaarbeziehung im Fokus, während bei den anderen beiden Itemsets die Wirkung des Primes auf die Identifikation einer taxonomischen Bildpaarbeziehung (Itemset 2) und einer relationalen Bildpaarbeziehung (Itemset 3) im Vordergrund steht.

\subsection{Itemset 1}

Bei den Items des Set 1 mussten sich die Probanden für eine von drei möglichen Konstellationen - taxonomisch, relational, neutral - innerhalb einer Dreierkonstellation entscheiden. Als Indikator für das Vorliegen einer Präferenz wurde zum einen die mittlere Reaktionszeit herangezogen: Es wurde analysiert, ob Entscheidungen für die eine Art der Bildpaarbeziehung schneller getroffen wurden, als für die alternative Bildpaarbeziehung. Zum anderen wurde die Häufigkeit der Entscheidungen für eine bestimmte Art der Bildpaarbeziehung analysiert.

\subsubsection{Analyse der Reaktionszeiten}

Tabelle 8 enthält die interessierenden Mittelwerte der Reaktionszeiten, die in Abbildung 17 veranschaulicht werden. Abbildung 17 zeigt die Reaktionszeiten für Deutsche und Chinesen in Abhängigkeit von der getroffenen Entscheidung für eine Bildpaarbeziehung und vom verwendeten Primetyp.

Es wurde eine Varianzanalyse mit wiederholten Messungen durchgeführt mit den beiden Innersubjektfaktoren „Entscheidung“ (2 Stufen: relational und taxonomisch) und „Prime“ (4 Stufen: relational, taxonomisch, neutral und kein Prime) sowie dem Zwischensubjektfaktor „Nation“ (2 Stufen: China und Deutschland). Es ergab sich kein signifikanter Haupteffekt des Faktors „Entscheidung“ $(F(1,101)=0.891, p=.348$, $\left.\varepsilon^{2}=.009\right)$, jedoch ein signifikanter Haupteffekt des Faktors „Prime“ $(F(3,99)=8.564, p$ $\left.=.000, \varepsilon^{2}=.206\right)$. Demnach hatte die Variation der Beziehung zwischen Prime und gewähltem Bildpaar einen systematischen Einfluss auf die Reaktionszeit. Als 
Zwischensubjekteffekt erwies sich „Nation“ als signifikant $(F(1,101)=17.42, p=.000$, $\left.\varepsilon^{2}=.147\right)$. Wie in Abbildung $17 \mathrm{zu}$ erkennen ist, wurde damit ein Ergebnis des Experiments 1 repliziert: Die Reaktionszeiten der chinesischen Probanden fielen länger aus als die der deutschen Probanden. Die Interaktion „Prime x Nation“ war nicht signifikant $(F(1,99)=0.711, p=.547)$.

Tabelle 8: Mittlere Reaktionszeiten in Abhängigkeit der Entscheidung (taxonomisch versus relational) und des Primes (inkongruent, kongruent, neutral, kein) von $n=52$ chinesischen Probanden und $n=51$ deutschen Probanden. Mittelwerte sind in Millisekunden (ms) angegeben.

\begin{tabular}{|c|c|c|c|}
\hline $\begin{array}{l}\text { Entscheidung/ } \\
\text { Prime }\end{array}$ & Nation & $\begin{array}{l}\text { Reaktionszeit } \\
\quad \text { in } \mathrm{ms}\end{array}$ & $\begin{array}{c}\text { Standardfehler } \\
\text { des } \\
\text { Mittelwertes }\end{array}$ \\
\hline \multirow{3}{*}{$\begin{array}{l}\text { Entscheidung } \\
\text { taxonomisch / } \\
\text { Prime taxonomisch }\end{array}$} & China & 205.39 & 9.46 \\
\hline & Deutschland & 160.62 & 5.86 \\
\hline & Gesamt & 183.22 & 5.99 \\
\hline \multirow{3}{*}{$\begin{array}{l}\text { Entscheidung } \\
\text { taxonomisch / } \\
\text { Prime relational }\end{array}$} & China & 207.18 & 10.47 \\
\hline & Deutschland & 172.40 & 6.90 \\
\hline & Gesamt & 189.96 & 6.50 \\
\hline \multirow{3}{*}{$\begin{array}{l}\text { Entscheidung } \\
\text { taxonomisch / } \\
\text { Prime neutral }\end{array}$} & China & 198.71 & 7.91 \\
\hline & Deutschland & 148.62 & 5.37 \\
\hline & Gesamt & 166.40 & 5.08 \\
\hline \multirow{3}{*}{$\begin{array}{l}\text { Entscheidung } \\
\text { taxonomisch / } \\
\text { Prime kein }\end{array}$} & China & 183.83 & 9.12 \\
\hline & Deutschland & 165.89 & 6.88 \\
\hline & Gesamt & 182.46 & 5.93 \\
\hline \multirow{3}{*}{$\begin{array}{l}\text { Entscheidung } \\
\text { relational/ } \\
\text { Prime taxonomisch }\end{array}$} & China & 205.72 & 9.22 \\
\hline & Deutschland & 164.51 & 6.13 \\
\hline & Gesamt & 185.31 & 5.89 \\
\hline \multirow{3}{*}{$\begin{array}{l}\text { Entscheidung } \\
\text { relational/ } \\
\text { Prime relational }\end{array}$} & China & 203.00 & 8.77 \\
\hline & Deutschland & 169.25 & 6.23 \\
\hline & Gesamt & 186.29 & 5.63 \\
\hline \multirow{3}{*}{$\begin{array}{l}\text { Entscheidung } \\
\text { relational/ } \\
\text { Prime neutral }\end{array}$} & China & 203.08 & 8.21 \\
\hline & Deutschland & 162.84 & 5.76 \\
\hline & Gesamt & 180.08 & 5.28 \\
\hline \multirow{3}{*}{$\begin{array}{l}\text { Entscheidung } \\
\text { relational/ Prime kein }\end{array}$} & China & 196.99 & 10.35 \\
\hline & Deutschland & 159.96 & 4.75 \\
\hline & Gesamt & 181.73 & 6.09 \\
\hline
\end{tabular}




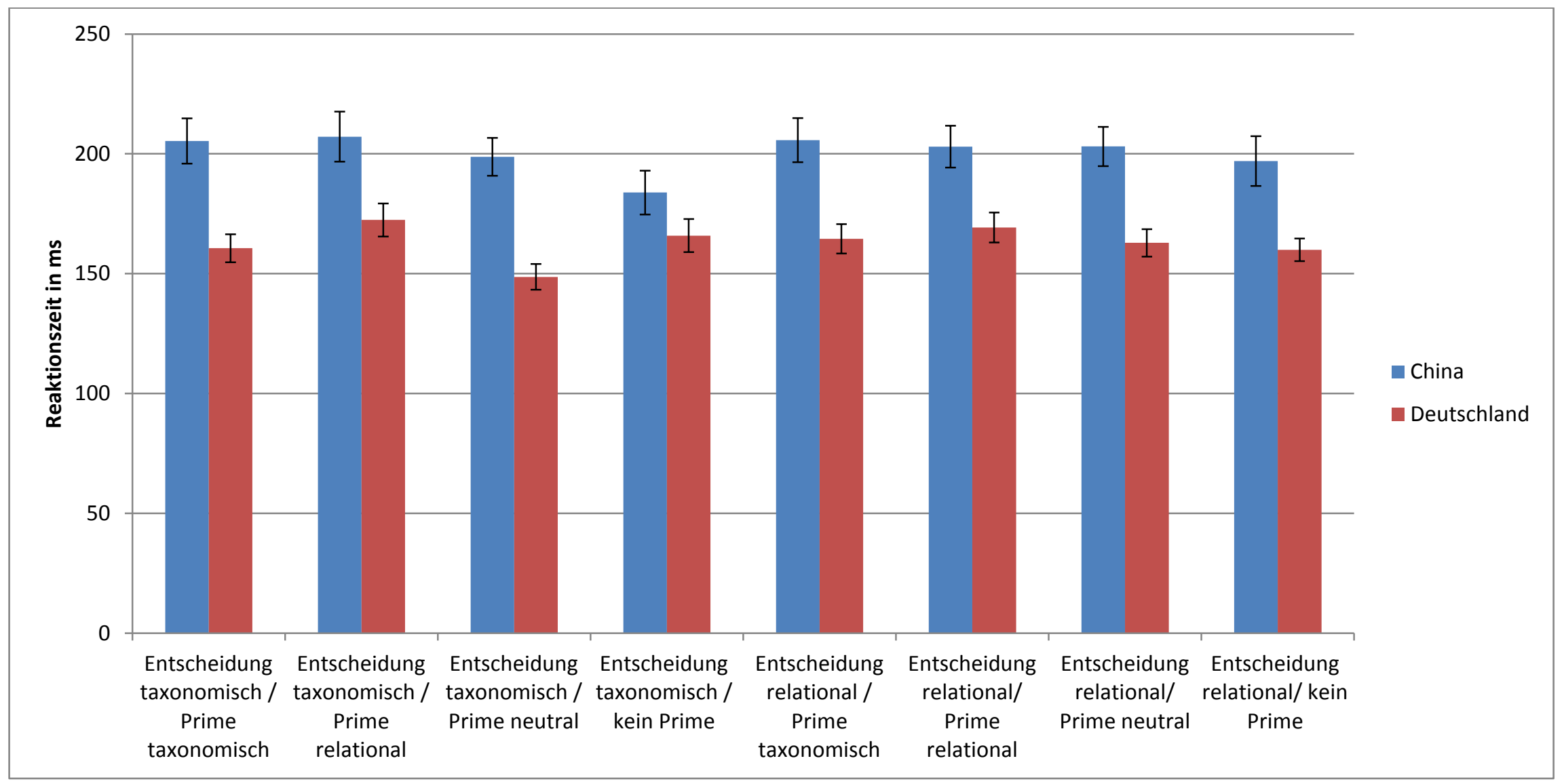

Abbildung 17: Mittlere Reaktionszeiten in Abhängigkeit der Entscheidung (taxonomisch versus relational) und des Primes (inkongruent, kongruent, neutral, kein) von $n=52$ chinesischen Probanden und $n=51$ deutschen Probanden. Mittelwerte sind in Millisekunden (ms) angegeben und der Standardfehler ist abgetragen. 


\subsubsection{Wirkung der Primes}

In Tabelle 10 sind die Reaktionszeitdaten in Abhängigkeit vom Primetyp für chinesische und deutsche Probanden einander gegenübergestellt. Abbildung 19 veranschaulicht die Daten.

Tabelle 9: Mittlere Reaktionszeiten in Abhängigkeit des Primes (inkongruent, kongruent, neutral, kein) der Gesamtstichprobe $(N=103)$. Mittelwerte sind in Millisekunden (ms) angegeben.

\begin{tabular}{|l|r|r|}
\hline Prime & $\begin{array}{c}\text { Reaktionszeit } \\
\text { in ms }\end{array}$ & Standardfehler \\
\hline $\begin{array}{l}\text { taxonomischer } \\
\text { Prime }\end{array}$ & 184.27 & 5.37 \\
\hline $\begin{array}{l}\text { relationaler } \\
\text { Prime }\end{array}$ & 188.12 & 5.27 \\
\hline $\begin{array}{l}\text { neutraler } \\
\text { Prime }\end{array}$ & 182.10 & 5.50 \\
\hline $\begin{array}{l}\text { kein } \\
\text { Prime }\end{array}$ & 173.24 & 4.76 \\
\hline
\end{tabular}

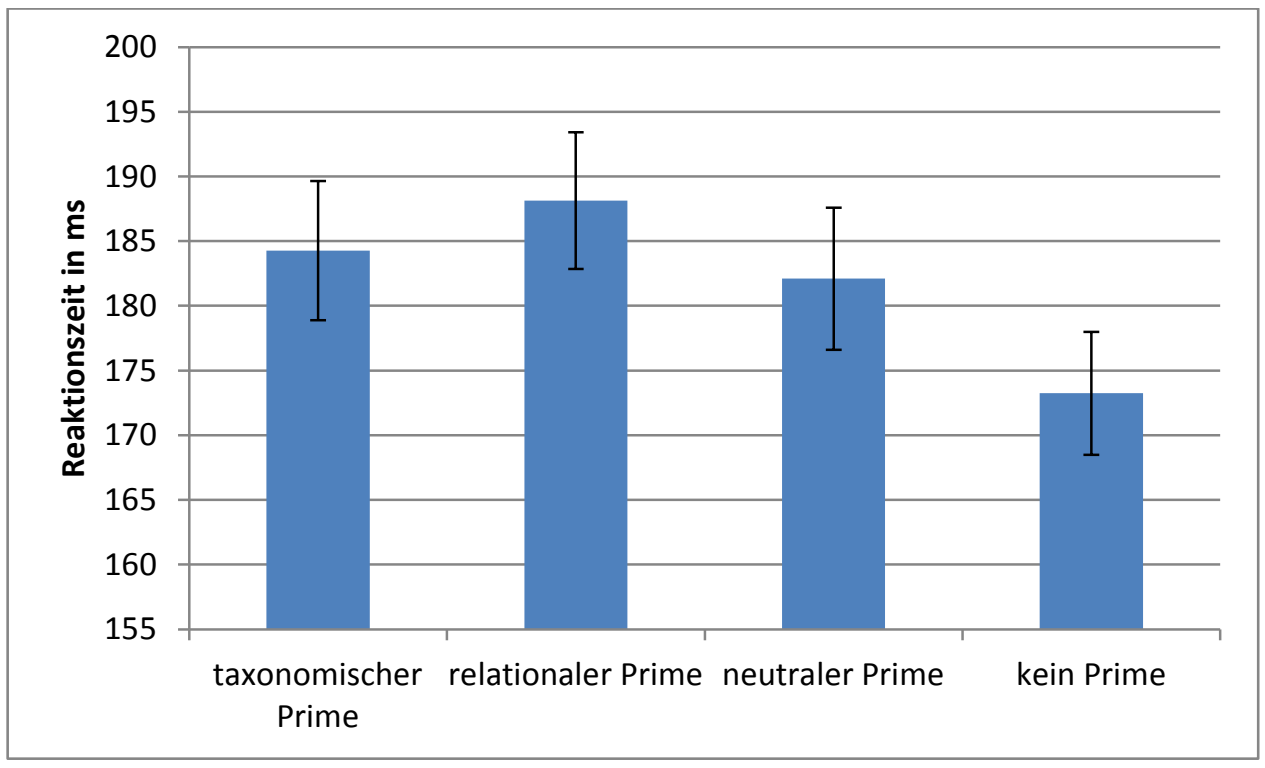

Abbildung 18: Mittlere Reaktionszeiten in Abhängigkeit des Primes (inkongruent, kongruent, neutral, kein) der Gesamtstichprobe $(N=103)$. Mittelwerte sind in Millisekunden (ms) angegeben und der Standardfehler ist abgetragen.

Es zeigte sich, dass die Bedingungen mit keinem Prime $(M=173.24 \mathrm{~ms}) \mathrm{zu}$ signifikant kürzeren Reaktionszeiten führten als die Bedingungen mit relationalem Prime $(M=$ 
$188.12 \mathrm{~ms} ; t(102)=1.90, p=.490)$. Die weiteren Bedingungen, inklusive taxonomischer Prime $(M=184.27 \mathrm{~ms})$ und neutraler Prime $(M=182.10 \mathrm{~ms})$, unterschieden sich nicht signifikant $(F(2,101)=0.606, p=.205)$.

Innerhalb der Nationen zeigt sich folgendes Bild:

Tabelle 10: Mittlere Reaktionszeiten in Abhängigkeit des Primes (inkongruent, kongruent, neutral, kein) von $n=52$ chinesischen Probanden und $n=51$ deutschen Probanden. Mittelwerte sind in Millisekunden (ms) angegeben.

\begin{tabular}{|l|r|r|l|l|}
\hline & $\begin{array}{l}\text { Reaktionszeit } \\
\text { China } \\
\text { (ms) }\end{array}$ & $\begin{array}{l}\text { Reaktionszeit } \\
\text { Deutschland } \\
\text { (ms) }\end{array}$ & $\begin{array}{l}\text { Standardfehler } \\
\text { China }\end{array}$ & $\begin{array}{l}\text { Standardfehler } \\
\text { Deutschland }\end{array}$ \\
\hline $\begin{array}{l}\text { taxonomischer } \\
\text { Prime }\end{array}$ & 205.55 & 162.56 & 5.40 & 8.27 \\
\hline $\begin{array}{l}\text { relationaler } \\
\text { Prime }\end{array}$ & 205.09 & 170.82 & 5.62 & 8.28 \\
\hline $\begin{array}{l}\text { neutraler } \\
\text { Prime }\end{array}$ & 200.90 & 162.93 & 5.24 & 8.90 \\
\hline $\begin{array}{l}\text { kein } \\
\text { Prime }\end{array}$ & 190.41 & 155.73 & 4.67 & 7.56 \\
\hline
\end{tabular}

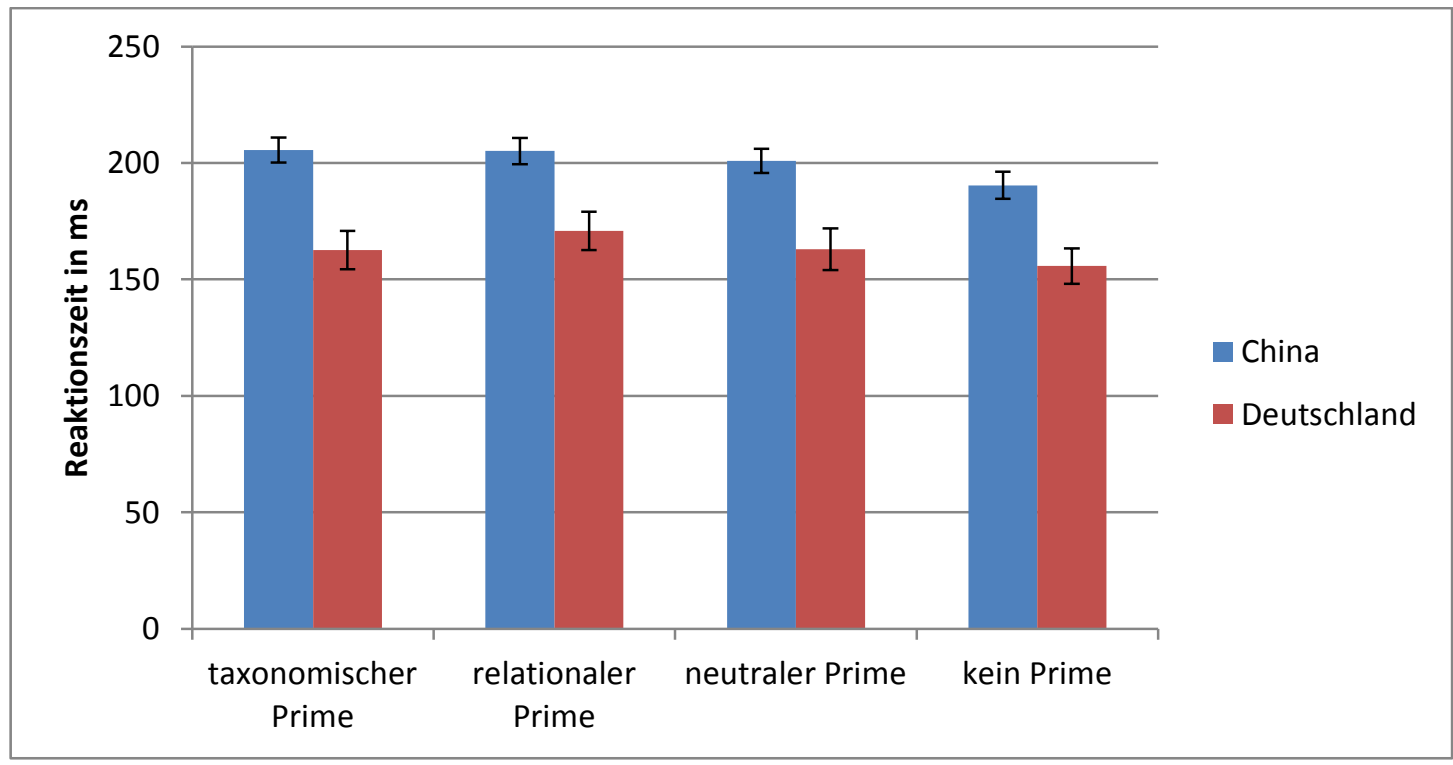

Abbildung 19: Mittlere Reaktionszeiten in Abhängigkeit des Primes (inkongruent, kongruent, neutral, kein) von $n=52$ chinesischen Probanden und $n=51$ deutschen Probanden. Mittelwerte sind in Millisekunden (ms) angegeben und der Standardfehler ist abgetragen. 
Die Gegenüberstellung der Daten der beiden Probandengruppen zeigt, dass die deutschen Probanden schneller antworteten als die chinesischen Probanden. Dies ist der bereits erwähnte Zwischensubjekteffekt „Nation“, der sich als signifikant herausstellte $\left(F(1,101)=17.42, p=.000, \varepsilon^{2}=.147\right)$. Die Reaktionszeiten der deutschen Probanden unterschieden sich nicht in den unterschiedlichen Primebedingungen $(F(4,101)=0.723$, $p=.328)$. Bei den chinesischen Probanden war die Reaktionszeit in der Bedingung ohne Prime $(M=190.41 \mathrm{~ms})$ signifikant schneller als bei einem taxonomischen $(M=205.55$ $\mathrm{ms} ; t(51)=2.86, p=.006)$ und relationalen Prime $(M=205,09 \mathrm{~ms} ; t(51)=2.677, p=$ $.01)$. Es gab keine weiteren signifikanten Unterschiede zwischen den Primebedingungen $(p>.05)$.

\subsubsection{Häufigkeit getroffener Entscheidungen}

Da sich die Reaktionszeit nur als bedingt aussagekräftig erwies, wurde zusätzlich die Häufigkeit analysiert mit der die Probanden sich für ein relational verbundenes Bildpaar beziehungsweise für ein taxonomisch verbundenes Bildpaar entschieden hatten. Im Folgenden wird die sprachliche Formulierung „relationale Entscheidung“ und „taxonomische Entscheidung“ verwendet. Dieses Vorgehen entspricht dem Ansatz der zitierten Vorgängerstudien (z.B. Norenzayan et al., 2002; Unsworth et al., 2005).

In einer Post-hoc-Analyse wurde also zur Kennzeichnung einer vorliegenden Entscheidungspräferenz ein Kontrast gebildet, der sich folgendermaßen zusammensetzt: Summe der Anzahl taxonomischer Entscheidungen minus Summe der Anzahl relationaler Entscheidungen.

Dieser Kontrast kann somit mögliche Entscheidungspräferenzen in den Konfliktbedingungen des Itemsets 2 abbilden, wenn der Proband sich zwischen einem relational und einem taxonomisch verbundenem Bildpaar entscheiden muss. Eine positive Differenz spiegelt also eine Tendenz zu taxonomischen Entscheidungen wider, während eine negative Differenz eine Tendenz zu relationalen Entscheidungen anzeigt. 


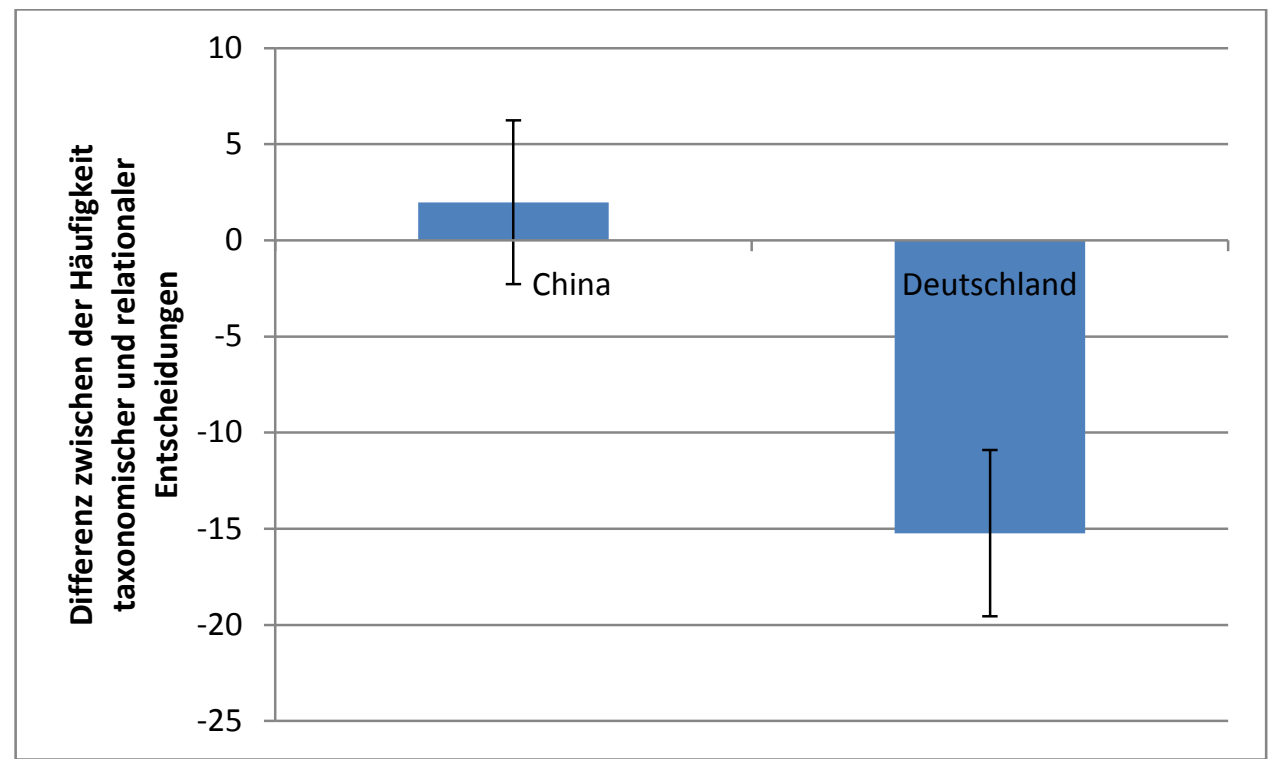

Abbildung 20: Kontrast über die getroffenen Entscheidungen von $n=52$ chinesischen Probanden und $n=51$ deutschen Probanden: Summe der Anzahl taxonomischer Entscheidungen minus Summe der Anzahl relationaler Entscheidungen.

Für die chinesische Probandenstichprobe ergab sich ein positiver Wert von +1.98 . Damit liegt eine leichte Tendenz zu taxonomischen Entscheidungen vor. Die deutschen Probanden wiesen bei der berechneten Differenz einen Wert von -15.24 auf und zeigten damit eine Tendenz zu relationalen Entscheidungen. Dieses Ergebnismuster entspricht nicht den Erwartungen. Es wurde erwartet, dass chinesische Probanden eher zu relationalen Entscheidungen tendieren und deutsche Probanden taxonomische Entscheidungen präferieren sollten. Das vorliegende Muster der chinesischen Probanden steht jedoch unter Berücksichtigung des vorliegenden Standardfehlers (siehe Tabelle 10) im Einklang mit Befunden von Unsworth und Kollegen (2005). In dieser Untersuchung waren ebenfalls Antwortmöglichkeiten ausgewertet worden, und die chinesischen Probanden neigten gleichermaßen zu taxonomische und relationalen Urteilen.

\subsection{Itemset 2}

Im Itemset 2 wurden den Probanden Bildtripel dargeboten, bei denen jeweils zwei Bilder in taxonomischer Beziehung standen, während das dritte Bild eine neutrale Beziehung zu den Bildern aufwies. D.h. die Entscheidung für eine taxonomische Beziehung war 
ohne Konkurrenz, wenn die Probanden der Instruktion folgten. Tatsächlich fiel auch in 91 Prozent der betreffenden Durchgänge innerhalb der Gesamtstichprobe die Entscheidung zu Gunsten der taxonomischen Beziehung.

Es wurde eine Varianzanalyse mit wiederholten Messungen mit dem Innersubjektfaktor „Prime“ (4 Stufen: relational, taxonomisch, neutral und kein Prime) sowie dem Zwischensubjektfaktor „Nation“ (2 Stufen: China und Deutschland) durchgeführt. Es gab einen signifikanten Haupteffekt des Faktors „Prime“ $\left(F(3,99)=9.710, p<.001, \varepsilon^{2}\right.$ $=.227)$. Demnach führte die Variation des Primetyps $\mathrm{zu}$ unterschiedlichen Reaktionszeiten. Als Zwischensubjekteffekt erwies sich „Nation“ als signifikant ( $F(1$, $\left.101)=15.92, p<.001, \varepsilon^{2}=.137\right)$. Die Interaktion „Prime $\mathrm{x}$ Nation“ war nicht signifikant $(F(3,99)=0.627, p=.599)$.

Um den Haupteffekt des Faktors „Prime“ genauer zu analysieren, wurden die Reaktionszeiten in Abhängigkeit von der Primebedingung betrachtet und entsprechende Kontraste berechnet. Tabelle 11 enthält die über die Gesamtstichprobe gemittelten Reaktionszeiten für jede der vier Primebedingungen, und Abbildung 21 veranschaulicht diese Daten.

I

Tabelle 11: Mittlere Reaktionszeiten im Itemset 2 in Abhängigkeit des Primes (inkongruent, kongruent, neutral, kein) der Gesamtstichprobe $(N=103)$. Mittelwerte sind in Millisekunden (ms) angegeben.

\begin{tabular}{|l|l|l|}
\hline Prime & $\begin{array}{l}\text { Reaktionszeit } \\
\text { in } \mathbf{~ m s}\end{array}$ & Standardfehler \\
\hline $\begin{array}{l}\text { taxonomischer } \\
\text { Prime }\end{array}$ & 146.85 & 4.77 \\
\hline $\begin{array}{l}\text { relationaler } \\
\text { Prime }\end{array}$ & 148.43 & 3.81 \\
\hline $\begin{array}{l}\text { neutraler } \\
\text { Prime }\end{array}$ & 150.17 & 4.39 \\
\hline $\begin{array}{l}\text { kein } \\
\text { Prime }\end{array}$ & 156.56 & 4.77 \\
\hline
\end{tabular}




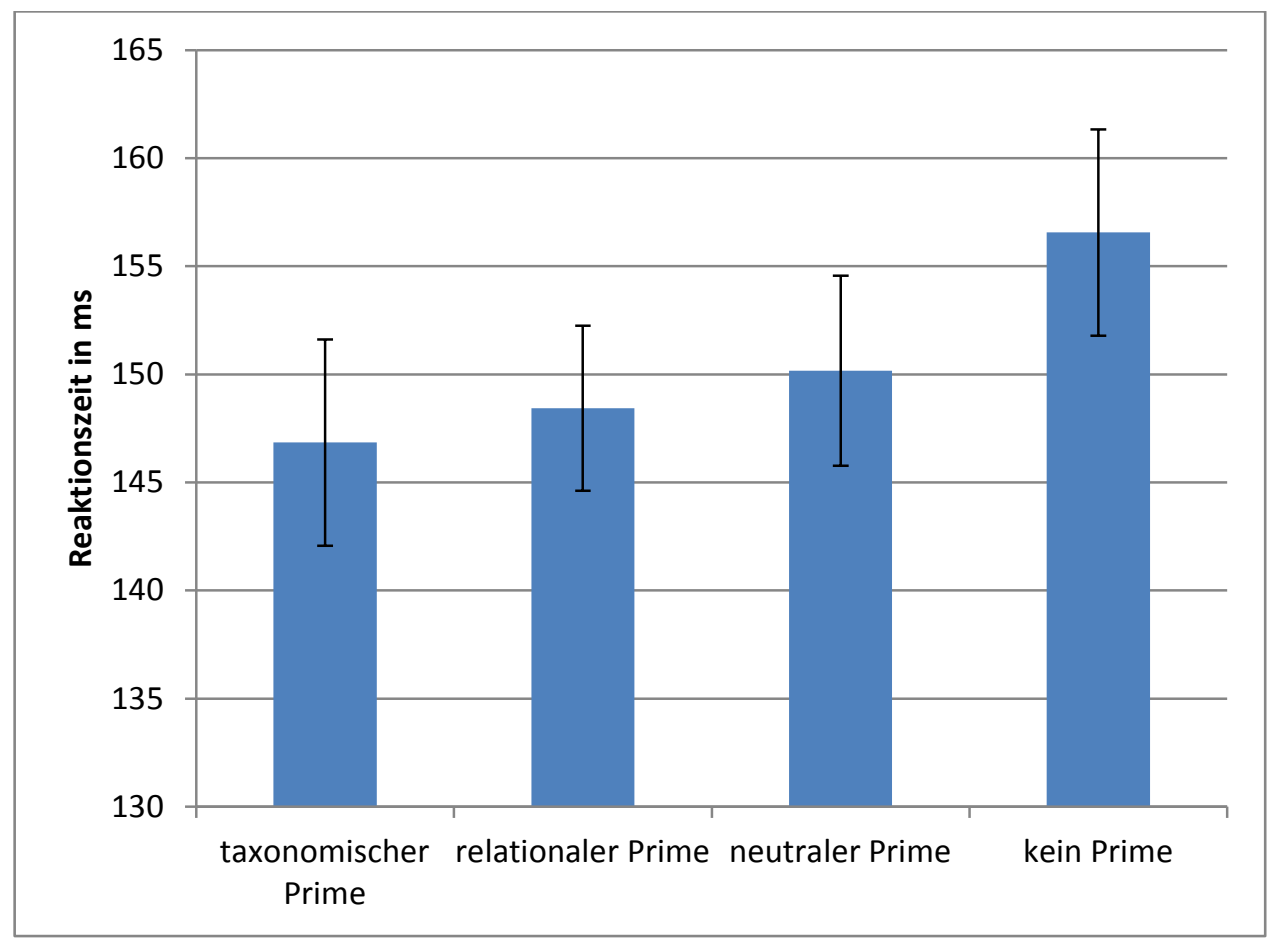

Abbildung 21: Mittlere Reaktionszeiten im Itemset 2 in Abhängigkeit des Primes (inkongruent, kongruent, neutral, kein) der Gesamtstichprobe $(N=103)$. Mittelwerte sind in Millisekunden (ms) angegeben und der Standardfehler ist abgetragen.

Es zeigte sich, dass die Bedingung ohne Prime $(M=156.56 \mathrm{~ms}) \mathrm{zu}$ signifikant längeren Reaktionszeiten führte als die Bedingungen mit taxonomischem Prime $(M=$ $146,85 \mathrm{~ms} ; t(102)=-4.617, p<.001)$, relationalem Prime $(M=148,43 \mathrm{~ms} ; t(102)=4.04$, $p<.001)$ und neutralem Prime $(M=150,17 \mathrm{~ms} ; t(102)=3.175, p=.002)$. Die übrigen Kontraste erwiesen sich als nicht signifikant ( $p>.05)$.

Zur genaueren Analyse des Zwischensubjekteffektes „Nation“ wurden die Daten der chinesischen und deutschen Probanden miteinander verglichen (siehe Tabelle 12 und Abbildung 22). Wie in Abbildung 22 deutlich zu erkennen ist, antworteten die deutschen Probanden $(M=134.46 \mathrm{~ms})$ schneller als die chinesischen Probanden ( $M=166.23 \mathrm{~ms})$. Dies ist der bereits erwähnte Zwischensubjekteffekt „Nation“, der sich als signifikant erwies $\left(F(1,101)=15.92, p<.001, \varepsilon^{2}=.137\right)$. 
Tabelle 12: Mittlere Reaktionszeiten in Abhängigkeit des Primes (inkongruent, kongruent, neutral, kein) von $n=52$ chinesischen Probanden und $n=51$ deutschen Probanden. Mittelwerte sind in Millisekunden (ms) angegeben.

\begin{tabular}{|l|r|r|l|l|}
\hline & $\begin{array}{l}\text { Reaktionszeit } \\
\text { China } \\
\text { (ms) }\end{array}$ & $\begin{array}{l}\text { Reaktionszeit } \\
\text { Deutschland } \\
\text { (ms) }\end{array}$ & $\begin{array}{l}\text { Standardfehler } \\
\text { China }\end{array}$ & $\begin{array}{l}\text { Standardfehler } \\
\text { Deutschland }\end{array}$ \\
\hline $\begin{array}{l}\text { taxonomischer } \\
\text { Prime }\end{array}$ & 162.23 & 131.17 & 8.13 & 3.90 \\
\hline $\begin{array}{l}\text { relationaler } \\
\text { Prime }\end{array}$ & 162.88 & 133.70 & 5.88 & 3.92 \\
\hline $\begin{array}{l}\text { neutraler } \\
\text { Prime }\end{array}$ & 166.50 & 133.51 & 6.94 & 4.28 \\
\hline $\begin{array}{l}\text { kein } \\
\text { Prime }\end{array}$ & 173.33 & 139.47 & 7.90 & 4.14 \\
\hline
\end{tabular}

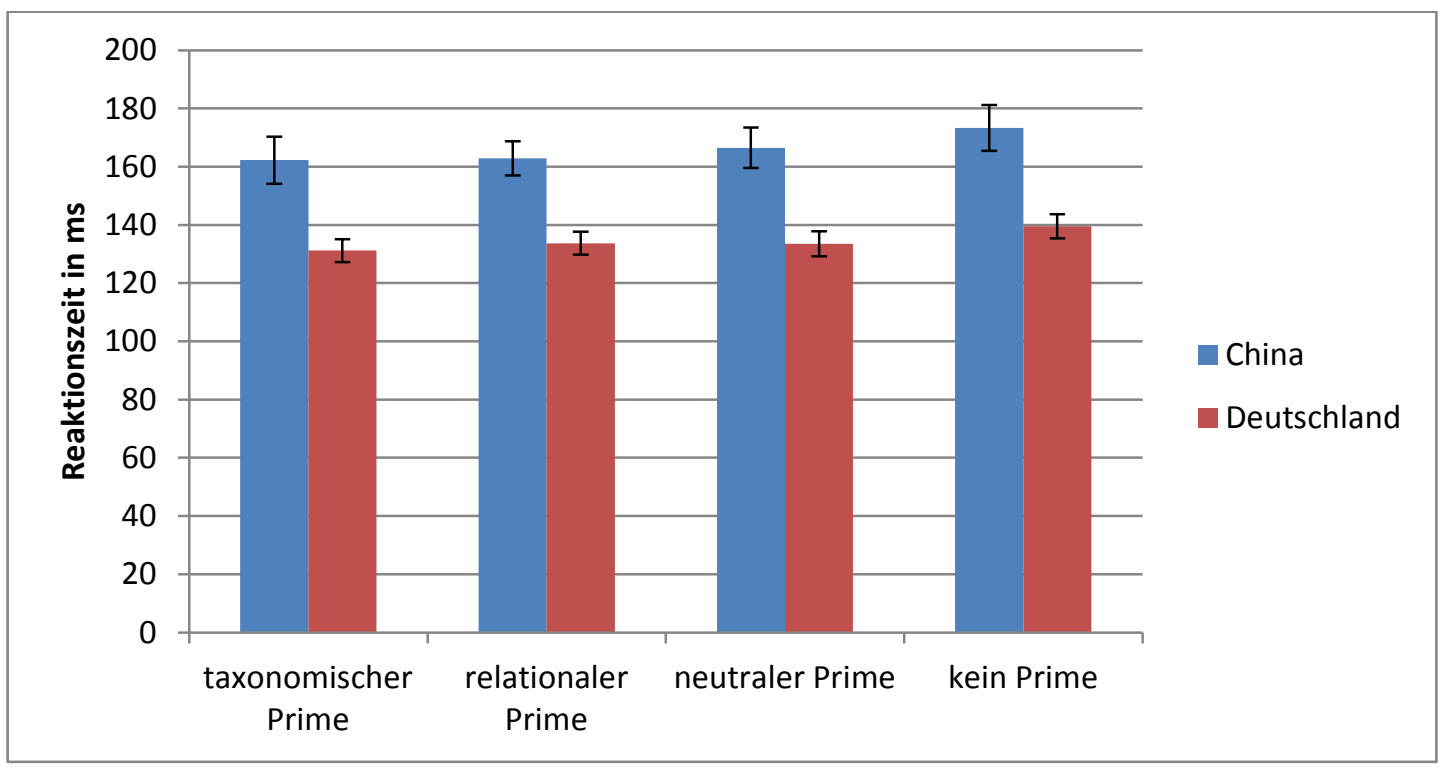

Abbildung 22: Mittlere Reaktionszeiten im Itemset 2 in Abhängigkeit des Primes (inkongruent, kongruent, neutral, kein) von $n=52$ chinesischen Probanden und $n=51$ deutschen Probanden. Mittelwerte sind in Millisekunden ( $\mathrm{ms}$ ) angegeben und der Standardfehler ist abgetragen.

Das Ergebnismuster sowohl für die chinesischen als auch für die deutschen Probanden entsprach, wie zu erwarten, dem der Gesamtstichprobe. Bei den chinesischen Probanden zeigte sich, dass die Bedingung ohne Prime $(M=173.33 \mathrm{~ms})$ zu signifikant längeren Reaktionszeiten führte als die Bedingungen mit taxonomischen Prime $(M=$ $162.23 \mathrm{~ms} ; t(51)=-3.20, p=.002)$, relationalem Prime $(M=162.88 \mathrm{~ms} ; t(51)=-3.18 p$ $=.002)$ und neutralem Prime $(M=166.50 \mathrm{~ms} ; t(51)=-2.10, p=.041)$. Die übrigen 
Kontraste erwiesen sich als nicht signifikant $(p>.05)$. Auch bei den deutschen Probanden zeigte sich ein entsprechendes Muster: Die Bedingung ohne Prime $(M=139.47 \mathrm{~ms})$ führte wieder $\mathrm{zu}$ signifikant längeren Reaktionszeiten als die Bedingungen mit taxonomischem Prime $(M=131.17 \mathrm{~ms} ; t(50)=-3.49, p=.001)$, relationalem Prime $(M=133.70 \mathrm{~ms} ; t(50)=-2.51, p=.015)$ und neutralem Prime $(M=$ $133,51 \mathrm{~ms} ; t(50)=-2.49, p=.016)$. Die übrigen Kontraste erwiesen sich als nicht signifikant $(p>.05)$.

\subsection{Itemset 3}

Im Itemset 3 wurden Bildtripel dargeboten, bei denen jeweils zwei Bilder in relationaler Beziehung standen, während das dritte Bild eine neutrale Beziehung zu den anderen beiden Bildern aufwies. Hier gab es keine Konkurrenz zu der Entscheidung für eine relationale Beziehung, wenn sich die Probanden nach der Instruktion richteten, was sie in 94 Prozent der Fälle auch taten.

Es wurde eine Varianzanalyse mit wiederholten Messungen mit dem Innersubjektfaktor „Prime“ (4 Stufen: relational, taxonomisch, neutral und kein Prime) sowie dem Zwischensubjektfaktor „Nation“ (2 Stufen: China und Deutschland) durchgeführt. Es zeigte sich auch in diesem Itemset ein signifikanter Haupteffekt des Faktors „Prime“ $\left(F(3,99)=21.751, p<.001, \varepsilon^{2}=.397\right)$. Wie bei den Itemsets 1 und 2 erwies sich der Zwischensubjekteffekt „Nation“ als signifikant $(F(1,101)=33,361, p<$ $\left..001, \varepsilon^{2}=.248\right)$. Die Interaktionen „Prime $\mathrm{x}$ Nation“ war nicht signifikant $(F(3,99)=$ $0.563, p=.641)$.

Um den Haupteffekt des Faktors „Prime“ genauer zu analysieren, wurden die Reaktionszeiten in Abhängigkeit von der Primebedingung betrachtet und entsprechende Kontraste berechnet. Tabelle 13 enthält die über die Gesamtstichprobe gemittelten Reaktionszeiten für jede der vier Primebedingungen, und Abbildung 23 veranschaulicht diese Daten. 
Tabelle 13: Mittlere Reaktionszeiten im Itemset 3 in Abhängigkeit des Primes (inkongruent, kongruent, neutral, kein) der Gesamtstichprobe $(\mathrm{N}=103)$. Mittelwerte sind in Millisekunden (ms) angegeben.

\begin{tabular}{|l|r|r|}
\hline Prime & $\begin{array}{c}\text { Reaktionszeit } \\
\text { in ms }\end{array}$ & Standardfehler \\
\hline $\begin{array}{l}\text { taxonomischer } \\
\text { Prime }\end{array}$ & 161.15 & 3,88 \\
\hline $\begin{array}{l}\text { relationaler } \\
\text { Prime }\end{array}$ & 158.24 & 4.18 \\
\hline $\begin{array}{l}\text { neutraler } \\
\text { Prime }\end{array}$ & 149.14 & 3.76 \\
\hline $\begin{array}{l}\text { kein } \\
\text { Prime }\end{array}$ & 163.83 & 4.60 \\
\hline
\end{tabular}

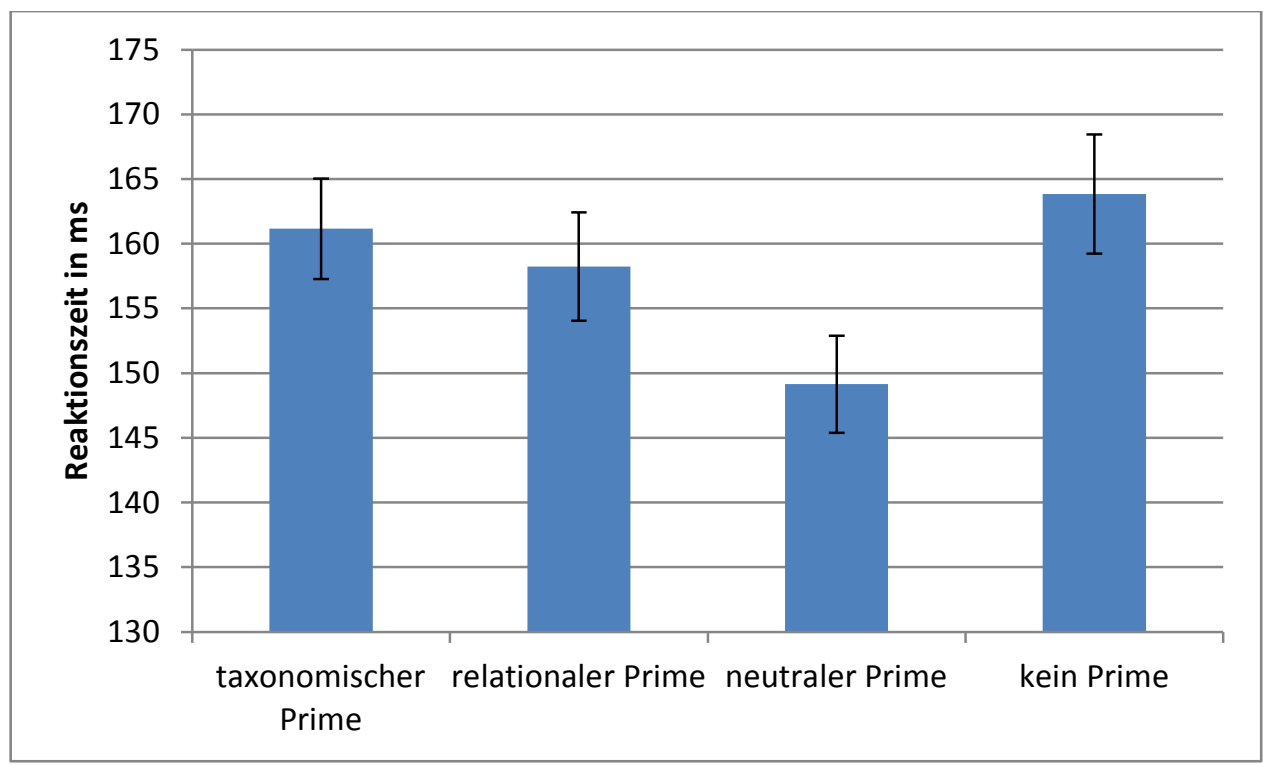

Abbildung 23: Mittlere Reaktionszeiten im Itemset 3 in Abhängigkeit des Primes (inkongruent, kongruent, neutral, kein) der Gesamtstichprobe $(N=103)$. Mittelwerte sind in Millisekunden (ms) angegeben und der Standardfehler ist abgetragen.

Es zeigte sich, dass die Bedingung mit neutralem Prime $(M=149.14 \mathrm{~ms}) \mathrm{zu}$ signifikant kürzeren Reaktionszeiten führte als die Bedingungen mit taxonomischem Prime $(M=161.15 \mathrm{~ms} ; t(102)=14.46, p<.001)$, relationalem Prime $(M=158.24 \mathrm{~ms}$; $t(102)=4.835, p<.001)$ und ohne Prime $(M=163.83 \mathrm{~ms} ; t(102)=-7.218, p<.001)$. Die Bedingung mit relationalem Prime unterschied sich außerdem von der Bedingung ohne Prime $(t(102)=2.509, p=.014)$. Die übrigen Kontraste erwiesen sich als nicht signifikant $(p>.05)$ 
Zur genaueren Analyse des Zwischensubjekteffektes „Nation“ wurden die Daten der chinesischen und deutschen Probanden miteinander verglichen (siehe Tabelle 14 und Abbildung 24).

Tabelle 14: Mittlere Reaktionszeiten im Itemset 3 in Abhängigkeit des Primes (inkongruent, kongruent, neutral, kein) von $n=52$ chinesischen Probanden und $n=51$ deutschen Probanden. Mittelwerte sind in Millisekunden (ms) angegeben.

\begin{tabular}{|l|r|r|l|l|}
\hline & $\begin{array}{l}\text { Reaktionszeit } \\
\text { China } \\
\text { (ms) }\end{array}$ & $\begin{array}{l}\text { Reaktionszeit } \\
\text { Deutschland } \\
\text { (ms) }\end{array}$ & $\begin{array}{l}\text { Standardfehler } \\
\text { China }\end{array}$ & $\begin{array}{l}\text { Standardfehler } \\
\text { Deutschland }\end{array}$ \\
\hline $\begin{array}{l}\text { taxonomischer } \\
\text { Prime }\end{array}$ & 180.48 & 141.45 & 5.69 & 3.60 \\
\hline $\begin{array}{l}\text { relationaler } \\
\text { Prime }\end{array}$ & 177.88 & 138.22 & 6.36 & 3.74 \\
\hline $\begin{array}{l}\text { neutraler } \\
\text { Prime }\end{array}$ & 167.39 & 130.53 & 5.67 & 3.33 \\
\hline $\begin{array}{l}\text { kein } \\
\text { Prime }\end{array}$ & 184.65 & 142.61 & 7.26 & 3.82 \\
\hline
\end{tabular}

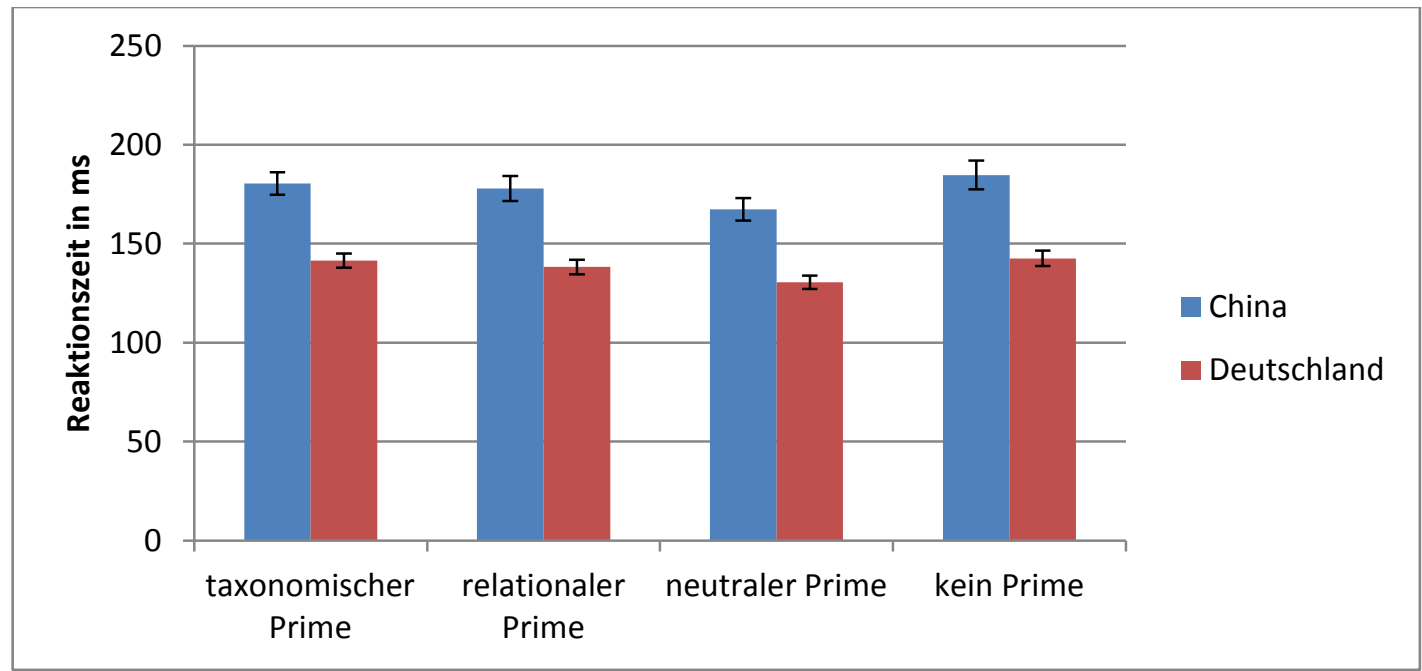

Abbildung 24: Mittlere Reaktionszeiten im Itemset 3 in Abhängigkeit des Primes (inkongruent, kongruent, neutral, kein) von $n=52$ chinesischen Probanden und $n=51$ deutschen Probanden. Mittelwerte sind in Millisekunden (ms) angegeben und der Standardfehler ist abgetragen.

Wie in Abbildung 24 deutlich zu erkennen antworteten die deutschen Probanden $(\mathrm{M}=138,2 \mathrm{~ms})$ schneller als die chinesischen Probanden $(\mathrm{M}=177,6)$. Dies ist der bereits erwähnte Zwischensubjekteffekt „Nation“, der sich als signifikant erwies ( $F(1$, $\left.101)=33.361, p=.000, \varepsilon^{2}=.248\right)$. 
Die Ergebnismuster sowohl für die chinesische als auch die deutsche Probandenstichprobe entsprachen, wie zu erwarten, dem der Gesamtstichprobe. Bei den chinesischen Probanden führte die Bedingung mit neutralem Prime $(M=167.39 \mathrm{~ms}) \mathrm{zu}$ signifikant kürzeren Reaktionszeiten als die Bedingung mit taxonomischem Prime $(M=$ $180.48 \mathrm{~ms} ; t(51)=37.67, p<.001)$, relationalem Prime $(M=177.88 \mathrm{~ms} ; t(51)=3.322$, $p=.002)$ und ohne Prime $(M=184,65 \mathrm{~ms} ; t(51)=-5.05, p<.001)$. Die anderen Kontraste erwiesen sich als nicht signifikant $(p>.05)$.

Bei den deutschen Probanden führten ganz analog die Bedingungen mit neutralem Prime $(M=167.39 \mathrm{~ms})$ zu signifikant kürzeren Reaktionszeiten als die Bedingungen mit taxonomischem Prime $(M=141.45 \mathrm{~ms} ; t(50)=37.18, p<.001)$, relationalem Prime $(M=138.22 \mathrm{~ms} ; t(50)=3.77, p<.001)$ und ohne Prime $(M=142.61$ ms; $t(50)=-5.86, p<.001)$. Auch hier erwiesen sich die übrigen Kontraste als nicht signifikant.

\subsection{Fazit Experiment 2 „Kategorisierung über Bildtripel““}

In Experiment 2 sollte eine Kategorisierungspräferenz über die Auswahl eines von drei möglichen Bildpaaren erfasst werden. Dazu wurden drei Itemsets realisiert. Bei Itemset 1 stand die Entscheidung des Probanden zwischen der taxonomischen und relationalen Bildpaarbeziehung im Fokus. Hierbei zeigte sich, dass sich über die Reaktionszeit keine Unterschiede in der Kategorisierungspräferenz abbilden ließen. Die Art der Entscheidung, die die Probanden trafen, ob sie das taxonomisch verbundene Bildpaar wählten oder das relational verbundene Bildpaar hatte also keinen Einfluss auf die Reaktionszeit.

Da die Reaktionszeit bei den geforderten Entscheidungen offenbar keinen sehr aussagekräftigen Indikator darstellte, wurde post hoc ein Kontrast über die Häufigkeit der getroffenen Entscheidung gebildet, indem die Anzahl der relationalen Entscheidungen von der Anzahl taxonomischer Entscheidungen subtrahiert wurde. Mit diesem Parameter konnte annähernd ein Ergebnis von Unsworth und Kollegen repliziert werden, nämlich dass die Chinesen etwa gleich viele taxonomische wie relationalen Entscheidungen trafen (Unsworth et al., 2005). Allerdings zeigten die deutschen Probanden nach diesem Parameter eine klare Präferenz zu relationalen Beziehungen. 
Dieses post hoc gefundene Ergebnis entspricht nicht den Erwartungen (vgl. Hypothese 1 und 2). Basierend auf dem Ansatz von Nisbett wäre zu erwarten gewesen, dass sich bei den deutschen Probanden eine Tendenz zu taxonomischen Entscheidungen zeigen sollte (Ji et al.., 2000; Nisbett, 2003).

Möglicherweise könnte die Schwierigkeit über Reaktionszeiten eine Differenzierung zwischen taxonomisch und relational verbundenen Konzepten sichtbar zu machen, auch an dem Itemmaterial gelegen haben. Wie bereits erwähnt, scheint das verwendetet Material aufgrund der Ergebnisse im Nachtest zwar prinzipiell geeignet zu sein, um Kategorisierungspräferenzen abbilden zu können. Denkbar ist aber, dass sich Kategorisierungspräferenzen auch bei leicht unterschiedlichen Anforderungen auf verschiedene Weise manifestieren. So könnte eine Entscheidung auf der Basis von Bildstimuli im Gegensatz zu Wortstimuli andere kognitive Verarbeitungsprozesse implizieren, die sich dann auf die Reaktionszeit auswirken.

Außerdem zeigte sich erneut, dass die chinesischen Probanden insgesamt langsamer antworteten als die deutschen Probanden, aber dennoch nicht weniger Fehler produzierten. Geht man von der Präferenz eines analytischen Denkstils bei den deutschen Probanden und eines holistischen Denkstils bei den chinesischen Probanden aus, so könnte dies ein Hinweis sein, dass es mehr Zeit benötigt aus einem holistischen Präferenzrahmen heraus Entscheidungen zu treffen - bei Bildpaaren ebenso wie bei Bildtripeln.

Die Wirkung des Primes war insgesamt sehr heterogen. Bei Itemset 1, wo eine taxonomische Bildpaarbeziehung mit einer relationalen konkurrierte, konnten die chinesischen Probanden am schnellsten antworten, wenn kein Prime dargeboten wurde. Dies zeigt erneut den starken Einfluss der als Primes verwendeten chinesischen Schriftzeichen. Bei den deutschen Probanden ließ sich kein derartiger Effekt auffinden. Dies spricht dafür, dass chinesische Schriftzeichen unter den realisierten Aufgabenanforderungen eine andere Primewirkung erzielten als Wörter in alphabetischer Schreibweise. Die chinesischen Schriftzeichen scheinen vielmehr in Konkurrenz zur verlangten Kategorisierung und der Verarbeitung der Bildstimuli zu treten.

Bei den beiden Itemsets 2 und 3 stand die Wirkung des Primes auf die Identifikation einer taxonomischen Bildpaarbeziehung (Itemset 2) beziehungsweise 
einer relationalen Bildpaarbeziehung (Itemset 3) im Vordergrund. Die deutschen und chinesischen Probanden verhielten sich recht ähnlich. Beim Itemset 3 zeigte sich überraschenderweise, dass es nicht $\mathrm{zu}$ schnelleren Antwortabgaben aufgrund eines semantischen Primings kam. Vielmehr schienen die Probanden durch die semantischen Primes im Vergleich zu einem neutralen Prime eine Hemmung zu erfahren. Wie ist das zu verstehen? Ein neutraler Prime könnte ein Netzwerk unspezifisch voraktivieren. Da es bei einem neutralen Prime in Vergleich zu der Bedingung ohne Prime zu einer Erleichterung kam, scheint das so voraktivierte Netzwerk effektiver genutzt werden zu können, um eine Entscheidung zu treffen und eine Beziehung zu identifizieren. Bildlich gesprochen wird das Netzwerk quasi ,,angewärmt. Dies galt offensichtlich nur, wenn die Entscheidung für eine relationale Bildpaarbeziehung (Itemset 3) anstand, und auch nur so lange, wie es keine konkurrierenden Beziehungen wie in Itemset 1gab. 


\section{Methode Experiment 3 ,Sprachproduktion“}

In den Experimenten 1 und 2 wurde ein möglicher kulturbedingter Einfluss des Denkstils auf Entscheidungen bei Kategorisierungsaufgaben untersucht. Im Experiment 3 steht nun die Sprachproduktion im Fokus. Die zugrundeliegende Überlegung ist, dass sich der Denkstil auf die Sprachproduktion auswirken sollte, wenn interferierende semantische Verarbeitungsprozesse wirksam werden.

Die Aufgabenanforderung wurde im Bild-Wort-Interferenz-Paradigma (siehe Abschnitt 2.5) realisiert. Die Probanden sollten so schnell wie möglich die graphische Darstellung eines konkreten Objektes (Target) benennen. Die Distraktoren wiesen eine taxonomische, relationale oder neutrale Beziehung zum Target auf (UV1). Es wurde z.B. bei dem Target „Bank“ „Stuhl“ als taxonomischer Distraktor, „Park“ als relationaler Distraktor und „Hemd“ als neutraler Distraktor verwendet. Da der Fokus auf der Beteiligung semantischer Prozesse bei der Sprachproduktion lag, wurde der Distraktor vor dem Target dargeboten, und zwar in den beiden SOA-Stufen -150 ms und -300 ms (UV2).

\subsection{Itemmaterial}

Als Itemmaterial dienten 30 ausgewählte lizenzfreie Bilder, die aus der Datenbank des „International Picture Naming Project“ abgerufen wurden ${ }^{2}$. Es handelt sich um schwarzweiße Strichzeichnungen mit 300 Pixeln (vgl. Anhang F). Das Itemmaterial wurde einigen Vortests unterzogen, die im Folgenden dargestellt werden sollen.

Die Geläufigkeit eines Wortes hat großen Einfluss sowohl auf die Schnelligkeit, mit der ein Wort gelesen wird, wie im Falle des Distraktorwortes, als auch auf die Schnelligkeit mit der die verbale Bezeichnung für ein Objekt produziert werden kann wie im Falle der Targets (Grabowski \& Herrmann, 1994; Herrmann, 2005; Herrmann \& Deutsch, 1976; Hörmann, 1991; Rickheit et al., 2003). Deshalb wurden für die Distraktorwörter und die verbalen Bezeichnungen der Bilder die Auftretenshäufigkeiten bestimmt.

\footnotetext{
${ }^{2}$ Abgerufen unter folgender Homepage: http://crl.ucsd.edu/experiments/ipnp/1database.html
} 
Eine weitere wichtige Variable, die einen Einfluss auf die semantische Verarbeitung von verbalen Items hat, ist die Bildhaftigkeit des Wortes (Herrmann \& Deutsch, 1976; Hörmann, 1991; Rickheit et al., 2003). Die Ausprägung der Bildhaftigkeit wurde über ein Ratingverfahren in einem Vorversuch erhoben. Außerdem sollten die Probanden in dem Vorversuch die im Hauptversuch verwendeten DistraktorTarget-Paare bewerten. Zum Einen sollten sie beurteilen, ob die Distraktor-Target-Paare sich auf zwei Objekte derselben Kategorie bezogen. Ihnen wurde jeweils das Distraktorwort mit der verbalen Bezeichnung des Targets vorgegeben. Zum Anderen sollten sie die Beziehung zwischen Distraktor und verbaler Bezeichnung des Targets danach beurteilen, ob zwischen den beiden bezeichneten Objekten eine logische oder funktionale Beziehung besteht.

\subsubsection{Worthäufigkeit}

Zunächst wurde betrachtet, ob sich die im Experiment verwendeten Items hinsichtlich ihrer Worthäufigkeit unterscheiden. Für die deutschen Items wurde auf die Datenbank „Cosmas $\mathrm{II}^{\text {‘3 }}$ zurückgegriffen, in der die Worthäufigkeit anhand einer Vielzahl repräsentativer Texte ermittelt wurde. Mit Hilfe einer Varianzanalyse wurden die verbalen Bezeichnungen der Bilder und die Distraktionen (taxonomische, relationale, neutrale) verglichen. Es zeigten sich bei den deutschen Items keine signifikanten Unterschiede zwischen den vier Itemgruppen $(F(3,119)=0.701, p=.554)$.

Auch bei den chinesischen Items wurde untersucht, ob sich die verwendeten Items hinsichtlich ihrer Worthäufigkeit unterscheiden. Die Worthäufigkeit wurde bestimmt mit Hilfe eines chinesischen Frequenzwörterbuchs (Wang \& Chang, 1986). Mit Hilfe einer Varianzanalyse wurden die verbalen Bezeichnungen der Bilder und die Distraktoren (taxonomische, relationale, neutrale) verglichen. Dabei zeigten sich keine signifikanten Unterschiede zwischen den vier Gruppen $(F(3,119)=2.701, p=.061)$. Hierbei wiesen die relationalen und neutralen Distraktoren der Tendenz nach eine

\footnotetext{
${ }^{3}$ COSMAS II: Eine am Institut für Deutsche Sprache konzipierte Volltextdatenbank für das linguistisch motivierte Recherchieren in den Textsammlungen (= Korpora) des IDS. COSMAS II steht für: Corpus Search, Management and Analysis System und ist das Nachfolgesystem von COSMAS I (1991-2003) am Institut für Deutsche Sprache.
} 
niedrigere Worthäufigkeit auf, die sich aber nicht in einem signifikanten Unterschied niederschlug.

Ebenfalls wurde der Anzahl der verwendeten Striche als Besonderheit der chinesischen Sprache Rechnung getragen und diese im Detail betrachtet. Hierbei zeichneten sich Distraktoren und Targets nicht durch eine unterschiedliche Anzahl an Strichen aus $(F(3,27)=2.049, p=.131)$.

\subsubsection{Bildhaftigkeit}

Insgesamt beantworteten 41 deutsche und 40 chinesische Probanden einen Onlinefragebogen $\mathrm{zu}$ den verbalen Bezeichnungen der Targets und den Distraktorwörtern. Dabei wurden anhand von 6-stufigen Likert-Skalen Fragen zur Bildhaftigkeit gestellt. Die Probanden dieser Onlineuntersuchung sind nicht identisch mit den Teilnehmern der Hauptuntersuchung.

- Wie leicht lässt sich ein Vorstellungsbild zu den folgenden Wörtern erzeugen?

Der Skalenwert 1 bedeutet „sehr leicht“, der Skalenwert 6 „sehr schwer“. Die mittleren Skalierungswerte aller Items wurden über eine Varianzanalyse mit wiederholten Messungen untersucht. Hierbei zeigten sich weder bei den deutschen $(F(3,27)=0.701, p=.120)$ noch bei den chinesischen Probanden $(F(3,27)=0.01, p=$ .230) signifikante Unterschiede. Der Gesamtmittelwert betrug bei den deutschsprachigen Items $M=1,99(S D=0.60)$ und bei den chinesischsprachigen Items $M=1,65(S D=$ $0.32)$.

- Wie leicht kann man das Objekt über die Sinne erfahren?

Die mittleren Skalierungswerte aller Items wurden über eine Varianzanalayse mit wiederholten Messungen untersucht. Auch hier zeigten sich weder bei den deutschen Items $(F(3,27)=0.254, p=.857)$ noch bei den chinesischen Items $(F(3,27)=1.04, p=$ .389) signifikante Unterschiede. Der Gesamtmittelwert betrug bei den deutschsprachigen 
Items $(M=2.61 ; S D=0.61)$ und bei den chinesischsprachigen Items $(M=2.09 ; S D=$ $0.34)$.

\subsubsection{Kontrolle der Distraktorwahl}

Zur Kontrolle der Distraktorwahl wurden zwei weitere Fragen gestellt.

- Wie leicht lassen sich die beiden Dinge einer gemeinsamen Kategorie zuordnen?

Mithilfe dieser Frage wurde der taxonomische Zusammenhang geprüft, d.h. inwieweit sich Targets und Distraktoren in eine Kategorie einordnen lassen. Die Benennung jeden Targets wurde in Kombination mit jedem der drei Distraktoren (taxonomisch, relational, neutral) vorgegeben.

Die mittleren Skalierungswerte aller Items wurden über eine Varianzanalyse mit wiederholten Messungen untersucht. Hierbei zeigten sich sowohl bei den deutschen $(F(2,28)=1424, p<.001)$ als auch bei den chinesischen Probanden $(F(2,28)=678, p<$ .001) signifikante Unterschiede, die im Folgenden näher betrachtet werden sollen.

Bei den deutschen Probanden unterschieden sich sowohl taxonomische $(M=$ 1,51) als auch relationale Distraktoren $(M=2.07)$ hinsichtlich ihrer Beziehung zum Target signifikant voneinander $(t(29)=-7.391, p<.001)$, ebenso unterschieden sich relationale und neutrale Distraktoren signifikant $(M=5.32 ; t(29)=-50.20, p<.001)$. Dabei zeigte sich folgender Trend: taxonomische < relationale < neutrale Distraktoren: Es fiel den Probanden am leichtesten, die taxonomischen Distraktoren mit den Targets in eine Kategorie einzuordnen, dann folgten die relationalen Distraktoren und dann die neutralen Distraktoren (siehe Abbildung 25).

Bei den chinesischen Probanden zeichnete sich ein identisches Muster ab. Sowohl die taxonomischen $(M=2.01)$ als auch die relationale Distraktoren $(M=2.34)$ unterschieden sich hinsichtlich ihrer Beziehung zum Target signifikant voneinander $(t(29)=-4.74, p<.001)$, ebenso unterschieden sich relationale und neutrale Distraktoren $(M=4.62 ; t(29)=-36.54, p<.001)$. Es zeigte sich bei den chinesischen Probanden ein identischer Trend: taxonomische < relationale < neutrale Distraktoren: Es fiel den 
chinesischen Probanden ebenfalls am leichtesten, die taxonomischen Distraktoren mit den Targets in eine Kategorie einzuordnen, dann folgten die relationalen Distraktoren und dann die neutralen Distraktoren (siehe Abbildung 25). In Abbildung 25 ist gut zu erkennen, dass die Differenz zwischen den taxonomischen und relationalen Distraktoren vergleichsweise gering ausfiel, die Differenz zwischen den neutralen Distraktoren und den anderen beiden Distraktorklassen dagegen sehr deutlich.

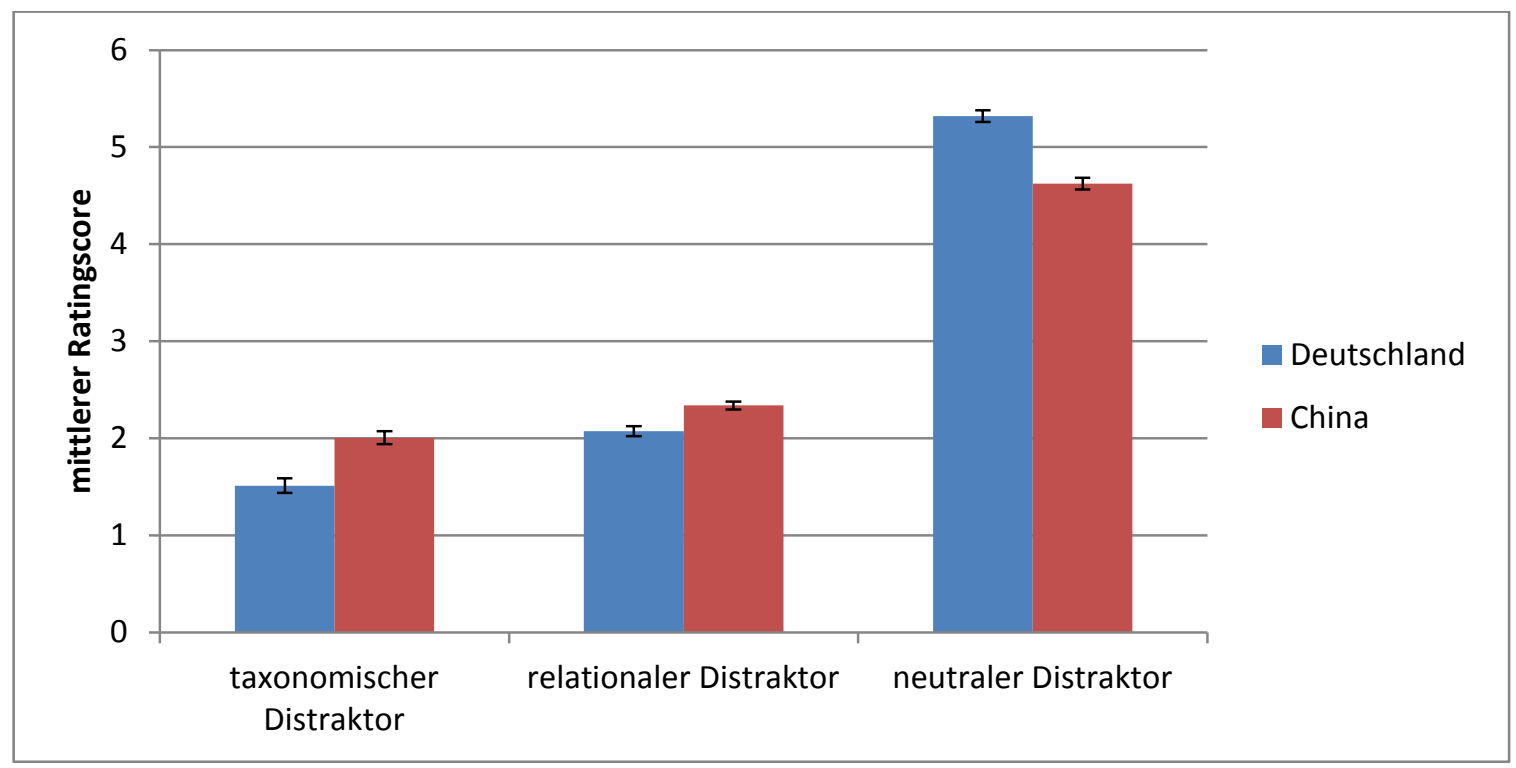

Abbildung 25: Mittlere Ratingscores mit Angabe des Standardfehlers für deutschsprachige Items (blau) und chinesischsprachige Items (rot) hinsichtlich der Frage, wie leicht sich die durch ein Distraktorwort und ein Targetwort bezeichneten beiden Objekte einer gemeinsamen Kategorie zuordnen lassen $(1=$ leicht, $6=$ schwer $)$. $n=41$ deutsche Probanden und $n=40$ chinesische Probanden.

- Wie leicht lässt sich eine logische oder funktionale Beziehung zwischen den beiden Objekten herstellen?

Mithilfe dieser Frage wurde näher betrachtet, inwieweit die Targets und Distraktoren in eine logische oder funktionale Beziehung, d.h. in einen relationalen Zusammenhang eingeordnet werden konnten. Auch in diesem Fall wurde die Benennung jeden Targets in Kombination mit jedem der drei Distraktorwörter vorgegeben.

Die mittleren Skalierungswerte aller Items wurden über eine Varianzanalyse mit wiederholten Messungen untersucht. Hierbei zeigten sich sowohl bei den deutschen 
$(F(2,28)=1244, p<.001)$ als auch bei den chinesischen Probanden $(F(2,28)=1106, p$ $<.001)$ signifikante Unterschiede.

Bei den deutschen Probanden unterschieden sich sowohl taxonomische $(M=$ 1.66) und relationale Distraktoren $(M=1.32)$ hinsichtlich ihrer Beziehung zum Target signifikant voneinander $(t(29)=4.53, p<.001)$ als auch relationale und neutrale Distraktoren $(M=5.24 ; t(29)=-50.20, p<.001)$. Dabei zeigte sich folgender Trend: relationale $<$ taxonomische $<$ neutrale Distraktoren. Dies bedeutet, es fiel den Probanden am leichtesten die relationalen Distraktoren mit den Targets in eine logische oder funktionale Beziehung zu setzen, dann folgten die taxonomischen Distraktoren und dann die neutralen Distraktoren (siehe Abbildung 26).

Bei den chinesischen Probanden unterschieden sich sowohl taxonomische $(M=$ 2.01) und relationale Distraktoren $(M=1.37)$ hinsichtlich ihrer Beziehung zum Target signifikant voneinander $(t(29)=9.06, p<.001)$ als auch relationale und neutrale Distraktoren $(M=4,44 ; t(29)=-47,05, p<.001)$. Dabei zeigte sich folgender Trend: relationale < taxonoimsche < neutrale Distraktoren: Es fiel auch den chinesischen Probanden am leichtesten, die relationalen Distraktoren mit den Targets in eine logische oder funktionale Beziehung zu setzen, dann folgten die taxonomischen Distraktoren und dann die neutralen Distraktoren (siehe auch Abbildung 26). In Abbildung 26 ist gut zu erkennen, dass die Differenz zwischen den taxonomischen und relationalen Distraktoren vergleichsweise gering ausfiel, die Differenz zwischen den neutralen Distraktoren und den anderen beiden Distraktorklassen dagegen sehr deutlich. 


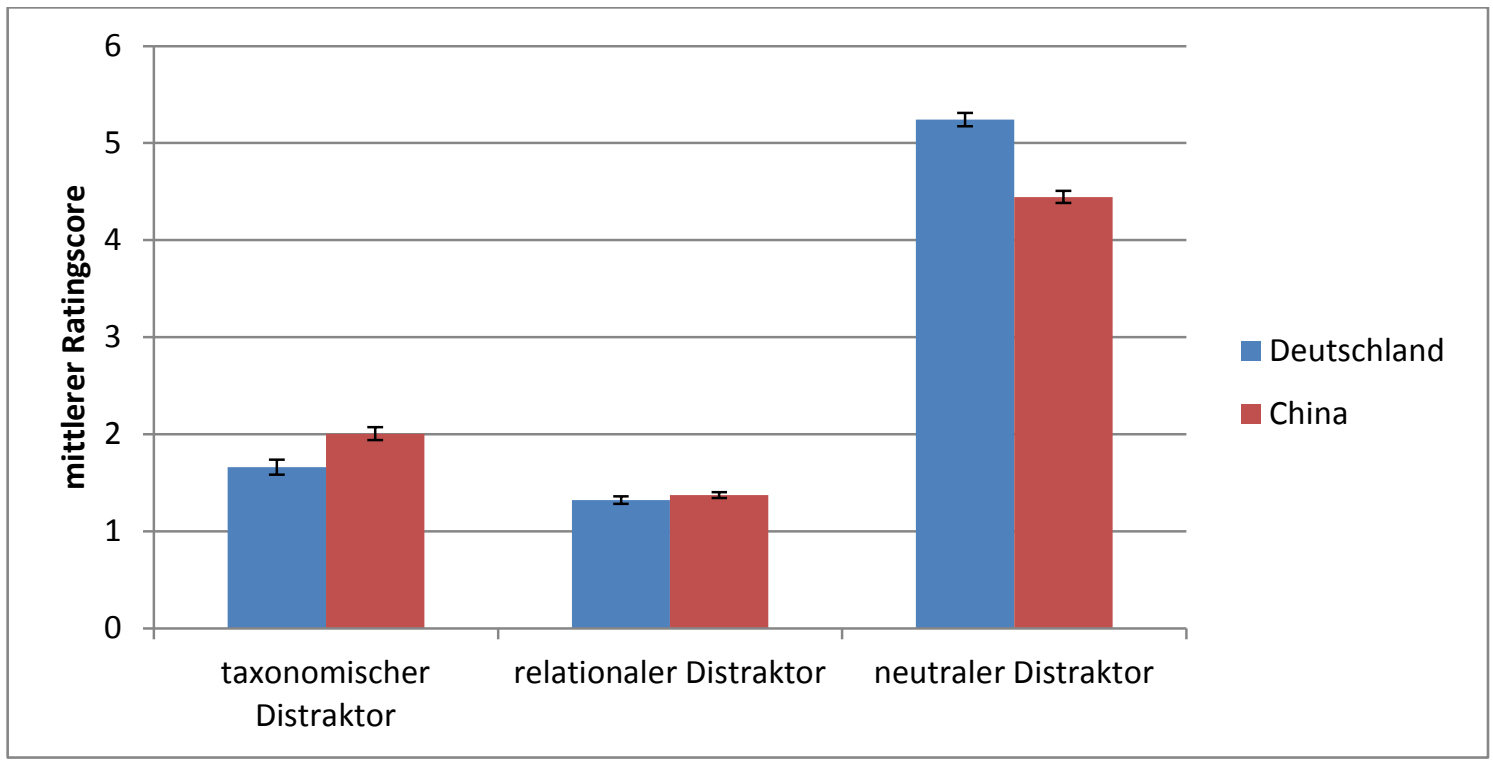

Abbildung 26: Mittlere Ratingscores mit Angabe des Standardfehlers für deutschsprachige Items (blau) und chinesischsprachige Items (rot) hinsichtlich der Frage, wie leicht sich für die durch ein Distraktorwort und ein Targetwort bezeichneten Objekte eine logische, funktionale Beziehung herstellen lässt $(1=$ leicht, $6=$ schwer $)$. $n=41$ deutsche Probanden und $n=40$ chinesische Probanden.

\subsubsection{Zusammenfassende Bewertung des Itemmaterials}

Die beschriebenen Analysen haben ergeben, dass das für die deutschen und chinesischen Probanden zusammengestellte Itemmaterial hinsichtlich der Vergleichbarkeit in Bezug auf die Variablen Auftretenshäufigkeit und Bildhaftigkeit mit Erfolg kontrolliert worden ist. Weder bei den deutsch- noch bei den chinesischsprachigen Items zeigten sich signifikante Unterschiede zwischen den vier Itemkategorien Targetwörter, taxonomische, relationale und neutrale Distraktorwörter.

Ebenfalls wurde die Auswahl der taxonomischen und relationalen Distraktoren als geeignet bestätigt. Bei der Frage, wie leicht sich zwei Objekte (ein Distraktorwort und ein Targetwort) in dieselbe Kategorie einordnen lassen, erhielten die taxonomischen Distraktoren und relationalen Distraktoren deutlich bessere Ratings im Vergleich zu den neutralen Distraktoren . Bei der Frage wie leicht sich zwischen den beiden durch die Wörter bezeichneten Objekten eine logische oder funktionale Beziehung herstellen ließ, erhielten die relationalen Distraktoren die besten Ratingscores, wobei relationale und taxonomische Distraktoren mit Abstand bessere Ratingscores als die neutralen 
Ratingscores aufwiesen. Das traf sowohl auf die deutsch- als auch auf die chinesischsprachigen Items zu.

\subsection{Probanden}

An der Untersuchung nahmen 50 chinesische Studierende teil, 27 männliche und 23 weibliche, im Alter von 19 bis 29 Jahren $(M=23.98$ Jahre; $S D=1.97)$ und 50 deutsche Studierende im Alter von 19 bis 31 Jahren $(M=20.92$ Jahre; $S D=2.17)$, von denen 15 männlich und 35 weiblich waren.

Die Mehrzahl der chinesischen Probanden studierte im Hauptfach Psychologie ( $n$ $=37$ ). Die anderen chinesischen Teilnehmer waren Studierende folgender Fachrichtungen: Softwareentwicklung $(n=3)$, Biomedizin $(n=3)$, Sprachen $(n=3)$, Betriebwirtschaftslehre $(n=2)$, Erziehungswissenschaften $(n=2)$. Auch die meisten deutschen Probanden studierten Psychologie $(n=48)$. Die restlichen deutschen Teilnehmer waren ein Student der Agrarwissenschaften und eine Hotelfachkraft in Ausbildung.

Die Probanden wurden über einen Aushang oder durch persönliches Ansprechen rekrutiert. Ausschlusskriterium für die chinesischen Teilnehmer war, wenn sie bereits eine Reise außerhalb von China gemacht hatten. Die deutschen Probanden durften über keinerlei chinesische Sprachkenntnisse verfügen. Diese Ausschlusskriterien wurden auch bei diesem Experiment gewählt, da über intensivere Auseinandersetzung mit der fremden Kultur bereits eine Beeinflussung des Denkstils hätte stattfinden können.

\subsection{Versuchsanordnung und Aufgabenanforderung}

Mit Hilfe der Software Presentation ${ }^{\circledR}$ wurde auch für das dritte Experiment ein eigens für die geplante experimentelle Durchführung konzipiertes Programm erstellt. Das Programm lief auf einem Notebook mit Windows XP, Intel Celeron 1.6 GHZ und 2 GB Arbeitsspeicher. Für die Durchführung der Versuche wurde dasselbe Notebook sowohl in China als auch in Deutschland verwendet.

Diesem Experiment liegt wie bereits erwähnt das Bild-Wort-InterferenzParadigma zu Grunde. Die Probanden sollten so schnell wie möglich eine graphische 
Darstellung eines konkreten Objektes (Target) benennen. Vor dem Erscheinen des Targets wurden Wörter (Distraktoren) unterhalb des Targets visuell eingeblendet. Untersucht wurde der Einfluss des Distraktortyps und der Stimulus-Onset-Asynchrony (SOA; Zeitintervall zwischen dem Onset des Targets und dem Onset des Distraktors) auf die Benennungszeit. Die SOA wurde in den Stufen -150 ms und -300 ms variiert. Das heißt, der Distraktor erschien entweder 150 ms oder 300 ms vor dem Target. Die Distraktoren unterschieden sich dahingehend, ob eine taxonomische, relationale oder neutrale Beziehung zum Target vorlag.

Es gab insgesamt 30 Target-Bilder und zu diesen fest zugeordnet jeweils einen taxonomischen, einen relationalen und einen neutralen Distraktor, insgesamt also 90 Target-Distraktor-Paare. Jedes der 90 Target-Distraktor-Paare wurde in den beiden genannten SOA-Stufen dargeboten, so dass sich insgesamt 180 Trials ergaben. Die Distraktoren wurden dabei in der Muttersprache des Probanden dargeboten (vgl. Anhang F). Die randomisierte Darbietung der Bildpaare wurde dabei durch die Software geleistet. Die Antwort des Probanden wurde über ein Mikrofon aufgezeichnet und durch einen in der Software „Presentation“ integrierten Voicekey im Anschluss an das Experiment ausgewertet.

\subsection{Versuchsdurchführung}

Der Hauptversuch gliederte sich in zwei verschiedene Abschnitte. Der erste Abschnitt bestand aus einer Lernphase, der zweite Abschnitt umfasste die Bild-WortInterferenzaufgabe. Die Probanden erhielten alle Instruktionen schriftlich, die deutschen Probanden in deutscher Sprache, die chinesischen Probanden in englischer Sprache (vgl. Anhang G). Für das Durchlesen konnten sich die Probanden beliebig viel Zeit nehmen und Rückfragen an den Versuchsleiter stellen.

Die Aufgabe im ersten Abschnitt des Versuchs bestand darin, für die 30 graphischen Darstellungen der Targets die entsprechenden standardisierten Bezeichnungen zu lernen. Dazu konnten die Probanden selbstständig eine Power-PointPräsentation durchblättern. Nach jedem Mausklick erschien eine neue graphische Darstellung mit Angabe der jeweils korrekten Bezeichnung unter dem abgebildeten Objekt (siehe Abbildung 27). 


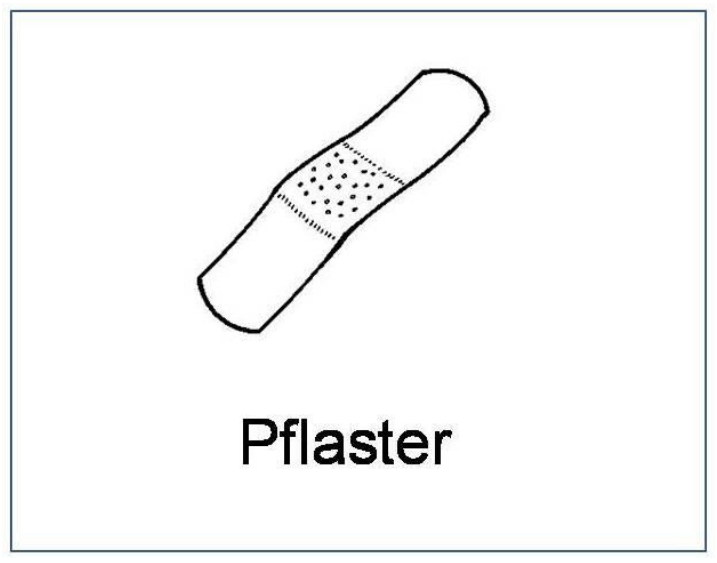

Abbildung 27: Darstellung eines Targets mitsamt standardisierter Bezeichnung in der Lernphase.

Die Probanden konnten die Power-Point-Präsentation so oft anschauen, bis sie glaubten, alle gezeigten Objekte richtig benennen zu können. Anschließend durchblätterten sie erneut die Präsentation, diesmal allerdings fehlten die Bezeichnungen, die unter den Abbildungen gestanden hatten. Die Probanden sollten die abgebildeten Objekte laut benennen und der Versuchsleiter kontrollierte anhand einer Liste, ob alle Objekte mit den korrekten Wörtern bezeichnet wurden. Wenn bei der Benennung Fehler auftraten, mussten die Probanden erneut einen Lerndurchgang absolvieren, bis sichergestellt war, dass alle abgebildeten Objekte mit Abschluss der Lernphase korrekt benannt werden konnten.

Der nachfolgende zweite Abschnitt des Versuchs umfasste die Bild-WortInterferenz-Aufgabe. Abbildung 28 veranschaulicht einen Versuchsdurchgang. Zu Beginn eines Durchgangs erschien auf dem Notebookbildschirm für die Dauer von 1 Sekunde ein Fixationskreuz, welches die Probanden nach Anweisung der Instruktion fixieren sollten. Das Fixationskreuz diente dazu, die Aufmerksamkeit der Probanden an die Stelle des Bildschirms zu lenken, an welcher das Target erscheinen würde. Nach Verschwinden des Fixationskreuzes erschien der Distraktor entweder 150 ms oder 300 ms vor dem Erscheinen des Targets. Aufgabe des Probanden war es, so schnell wie möglich die graphische Darstellung des Objektes (Target) zu benennen. Sie sollten dabei die Distraktoren ignorieren und sich auf die eingeblendeten Objekte konzentrieren und diese so schnell wie möglich mit dem richtigen Wort benennen. Dafür hatten die 
Probanden zwei Sekunden nach Erscheinen des Targets Zeit, bis erneut das Fixationskreuz erschien und ein neuer Versuchsdurchgang begann.

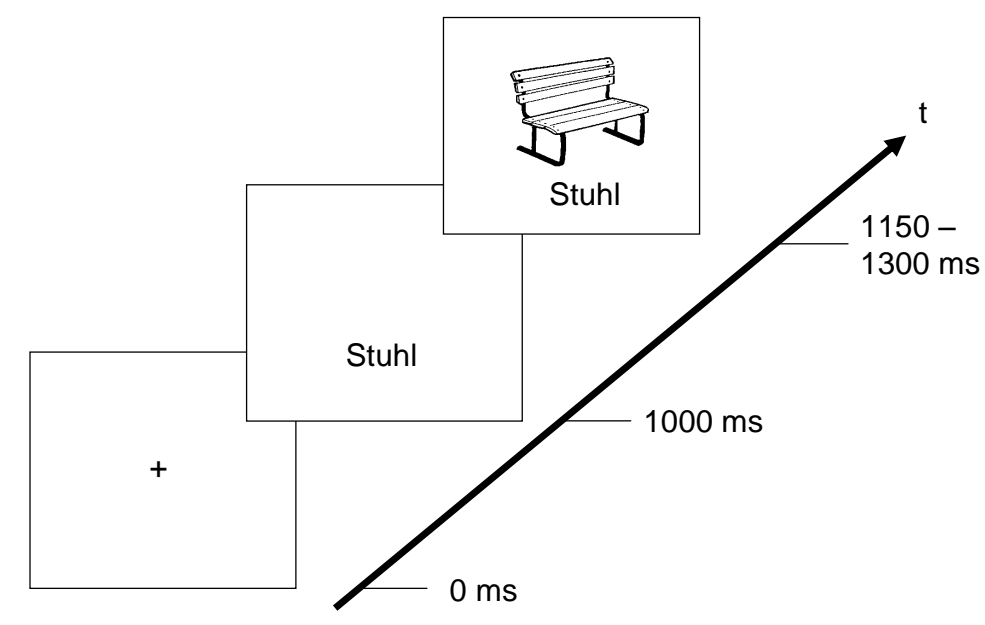

Abbildung 28: Zeitliche Sequenz der Ereignisse in einem Versuchsdurchgang mit taxonomischem Distraktor im Experiment 3 ,Sprachproduktion“.

Dieser Versuchsabschnitt dauerte insgesamt ca. 45 Minuten und war in zwei Blöcke unterteilt. Nach dem ersten Block hatten die Teilnehmer die Möglichkeit, eine Pause zu machen, die sie selbstständig durch Drücken der Leertaste beenden konnten, womit sie auch gleichzeitig den nächsten Versuchsdurchgang starteten. Im Anschluss an das Computerexperiment wurden die Probanden gebeten, einen Fragebogen auszufüllen. Je nach Herkunft des Versuchsteilnehmers war der Fragebogen auf Deutsch oder auf Englisch verfasst. Es wurde u.a. erfragt, welche Strategie die Probanden zur Benennung des Targets beziehungsweise zur Ausblendung des Distraktors einsetzten. Mit Hilfe desselben Fragebogens wurden am Ende des Experiments die demografischen Daten und etwaige Anmerkungen und Kommentare von beiden Versuchsgruppen erhoben (vgl. Anhang H). Im Anschluss daran wurden die Probanden über den Versuch aufgeklärt. 


\section{Ergebnisse Experiment 3 ,Sprachproduktion“"}

In dem folgenden Abschnitt werden die Ergebnisse der Fehleranalyse vorgestellt. Danach werden die Ergebnisse der Reaktionszeitdaten beschrieben.

\subsection{Fehleranalyse}

Zunächst wurden die fehlerhaften Trials identifiziert. Dazu wurden die mit einem Voicekey aufgenommenen verbalen Antworten abgehört und mit der Dokumentation der von der Software erzeugten Targetabfolge abgeglichen. Wurde keine korrekte Übereinstimmung zwischen der verbalen Antwort und der nach der Dokumentation zu erwartenden Bezeichnung des Targets festgestellt, so wurde der Versuchsdurchgang als fehlerhaft bewertet und ist nicht in die Auswertung der Daten eingeflossen. Es gab bei den chinesischen Probanden eine Fehlerquote von 3.6 Prozent. Bei den deutschen Probanden war eine Fehlerquote von 1.7 Prozent festzustellen.

Werden die Fehler genauer analysiert, so ergibt sich bei den chinesischen Probanden, dass vor allem störende Geräusche für die Mehrzahl der Fehler verantwortlich waren (siehe Abbildung 29). Diese störenden Geräusche entstanden z.B. wenn der Proband versehentlich gegen das Mikrofon kam oder sich räusperte. Zudem nahm das Mikrofon auch Störgeräusche der Netzaktivität von stummgeschalteten Mobiltelefonen wahr, wenn diese eine SMS oder einen Anruf erhielten. Zwar gab es kein akustisches Geräusch, aber das Mikrofon verzeichnete bei jeglicher Aktivität des Mobiltelefons ein lautes Rauschen, das sich über die akustische Antwort setzte und eine Diskriminierung zwischen Störgeräusch und Antwort nicht mehr ermöglichte. Aufgrund dessen wurde bei der Durchführung bei den deutschen Probanden darum gebeten, das Mobiltelefon auszuschalten. Bei den deutschen Probanden verteilen sich die Ursachen der Fehler gleichmäßig (siehe Abbildung 30). Aufgrund der geringen Anzahl von nicht durch störende Geräusche bedingten Fehlern über alle Versuchspersonen wurde post hoc von einer weiteren systematischen Analyse der Fehler abgesehen. 


\section{Fehleranalyse chinesische Probanden}

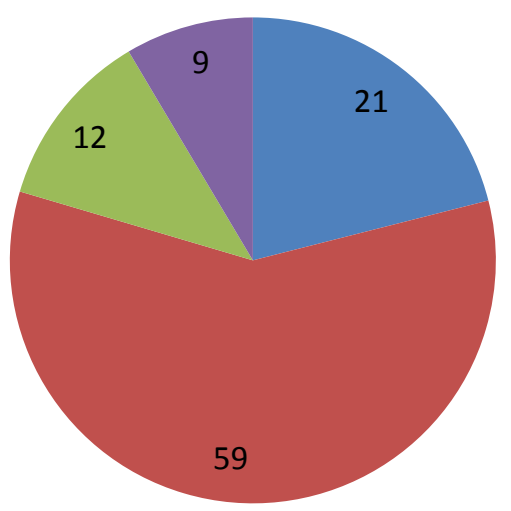

\section{falsche Benennung}

- Lärm/störende Geräusche

keine Antwort

zu späte Antwort

Abbildung 29: Darstellung der Fehlerverhältnisse der chinesischen Probanden in Prozent in Experiment 3 ,Sprachproduktion“.

\section{Fehleranalyse deutsche Probanden}

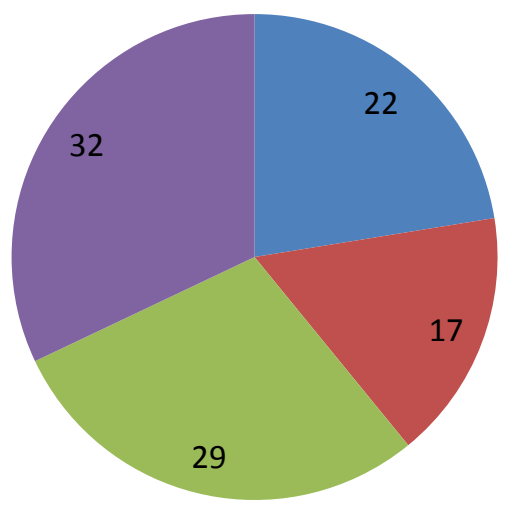

falsche Benennung

- Lärm/störende Geräusche

keine Antwort

zu späte Antwort

Abbildung 30: Darstellung der Fehlerverhältnisse der deutschen Probanden in Prozent in Experiment 3 ,Sprachproduktion“. 


\subsection{Analyse der Differenzwerte}

Gemessen wurde die Reaktionszeit vom Erscheinen des Targets bis $\mathrm{zu}$ seiner Benennung. Es wurden zunächst Differenzbeträge aus den Daten zweier DistraktorBedingungen gebildet. Für jedes Target wurde die Reaktionszeit mit neutralem Distraktor von der Reaktionszeit mit taxonomischem oder relationalem Distraktor abgezogen (dieses Vorgehen entspricht der Datenauswertung von Schriefers et al., 1990). Pro SOA-Bedingung (SOA -150 ms und SOA -300 ms) wurden also jeweils zwei mittlere Differenzbeträge generiert, einer für taxonomische und einer für relationale Targets. Wie Tabelle 15 und Abbildung $31 \mathrm{zu}$ entnehmen ist, fielen alle Mittelwerte positiv aus, d.h. die Distraktoren wirkten sich hemmend auf die Verarbeitung der Targets aus.

Tabelle 15: Mittlere Differenzen in den Reaktionszeiten mit Angabe des Standardfehlers von Durchgängen mit taxonomischen minus neutralen Distraktoren sowie relationalen minus neutralen Distraktoren in den SOA-Bedingungen SOA -150 ms und SOA -300 ms ( $n=50$ chinesische Probanden, $n=51$ deutsche Probanden).

\begin{tabular}{|c|c|c|c|}
\hline & Nation & Mittelwert ms & $\begin{array}{l}\text { Standardfehler } \\
\text { des Mittelwerts }\end{array}$ \\
\hline \multirow{2}{*}{$\begin{array}{l}\text { Differenz } \\
\text { taxonomischer } \\
\text { Distraktor } \\
\text { minus } \\
\text { neutraler } \\
\text { Distraktor; } \\
\text { SOA -300 }\end{array}$} & China & 27.74 & 8.10 \\
\hline & Deutschland & 11.80 & 5.94 \\
\hline \multirow{2}{*}{$\begin{array}{l}\text { Differenz } \\
\text { relationaler } \\
\text { Distraktor } \\
\text { minus } \\
\text { neutraler } \\
\text { Distraktor; } \\
\text { SOA -300 }\end{array}$} & China & 55.49 & 15.50 \\
\hline & Deutschland & 18.32 & 9.70 \\
\hline \multirow{2}{*}{$\begin{array}{l}\text { Differenz } \\
\text { taxonomischer } \\
\text { Distraktor } \\
\text { minus } \\
\text { neutraler } \\
\text { Distraktor; } \\
\text { SOA -150 }\end{array}$} & China & 30.15 & 8.53 \\
\hline & Deutschland & 32.01 & 7.59 \\
\hline \multirow{2}{*}{$\begin{array}{l}\text { Differenz } \\
\text { relationaler } \\
\text { Distraktor } \\
\text { minus } \\
\text { neutraler } \\
\text { Distraktor; } \\
\text { SOA -150 }\end{array}$} & China & 18.05 & 8.40 \\
\hline & Deutschland & 14.42 & 7.65 \\
\hline
\end{tabular}




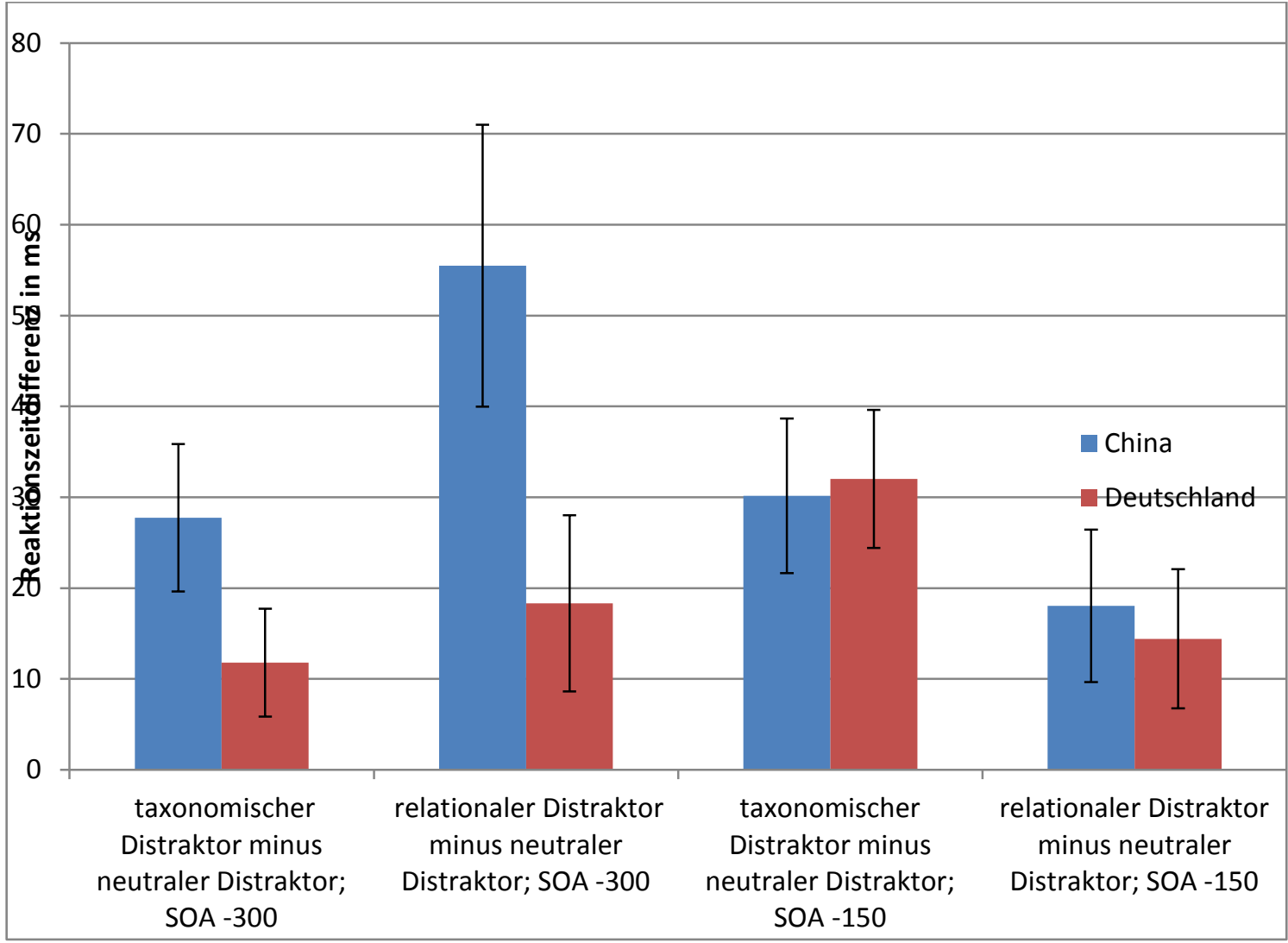

Abbildung 31: Mittlere Differenzen in den Reaktionszeiten mit Angabe des Standardfehlers von Durchgängen mit taxonomischen minus neutralen Distraktoren sowie relationalen minus neutralen Distraktoren in den SOA-Bedingungen SOA -150 ms und SOA -300 ms ( $n=50$ chinesische Probanden, $n=51$ deutsche Probanden)

Über diese Differenzwerte wurde eine Varianzanalyse mit wiederholten Messungen mit den beiden Innersubjektfaktoren „Distraktor“ (2 Stufen: relational und taxonomisch) und „SOA“ (2 Stufen: $-150 \mathrm{~ms}$ und $-300 \mathrm{~ms}$ ) sowie dem Zwischensubjektfaktor „Nation“ (2 Stufen: China und Deutschland) durchgeführt. Es gab keine signifikanten Haupteffekte, weder für den Faktor „Distraktor“ $(F(1,99)=$ $0.119, p=.730)$, noch für den Faktor, $\operatorname{SOA}^{“}(F(1,99)=0.383, p=.537)$. Es zeigten sich aber zwei signifikante Interaktionen „Distraktor x Nation“ $(F(1,99)=4.073, p=$ $\left..046, \varepsilon^{2}=.040\right)$ und „Distraktor x $\operatorname{SOA}^{\prime \prime}\left(F(1,99)=22.280, p=.000, \varepsilon^{2}=.184\right)$. Die dreifache Interaktion „Distraktor x SOA x Nation“ erwies sich als nicht signifikant $(F(1$, 99) $=1.349, p=.248)$.

Bei der Interaktion „Distraktor x Nation“ handelt es sich um eine disordinale Interaktion (siehe Abbildung 32). Die Differenzwerte der deutschen Probanden fielen 
bei taxonomischen Distraktoren höher aus als bei relationalen, während die Differenzwerte der chinesischen Probanden bei relationalen Distraktoren höher ausfielen als die bei taxonomischen. Dies bedeutet, dass der taxonomische Distraktor bei den deutschen Probanden einen größeren Ablenkungseffekt hatte, der relationale Distraktor dagegen hatte bei den chinesischen Probanden einen größeren Ablenkungseffekt.

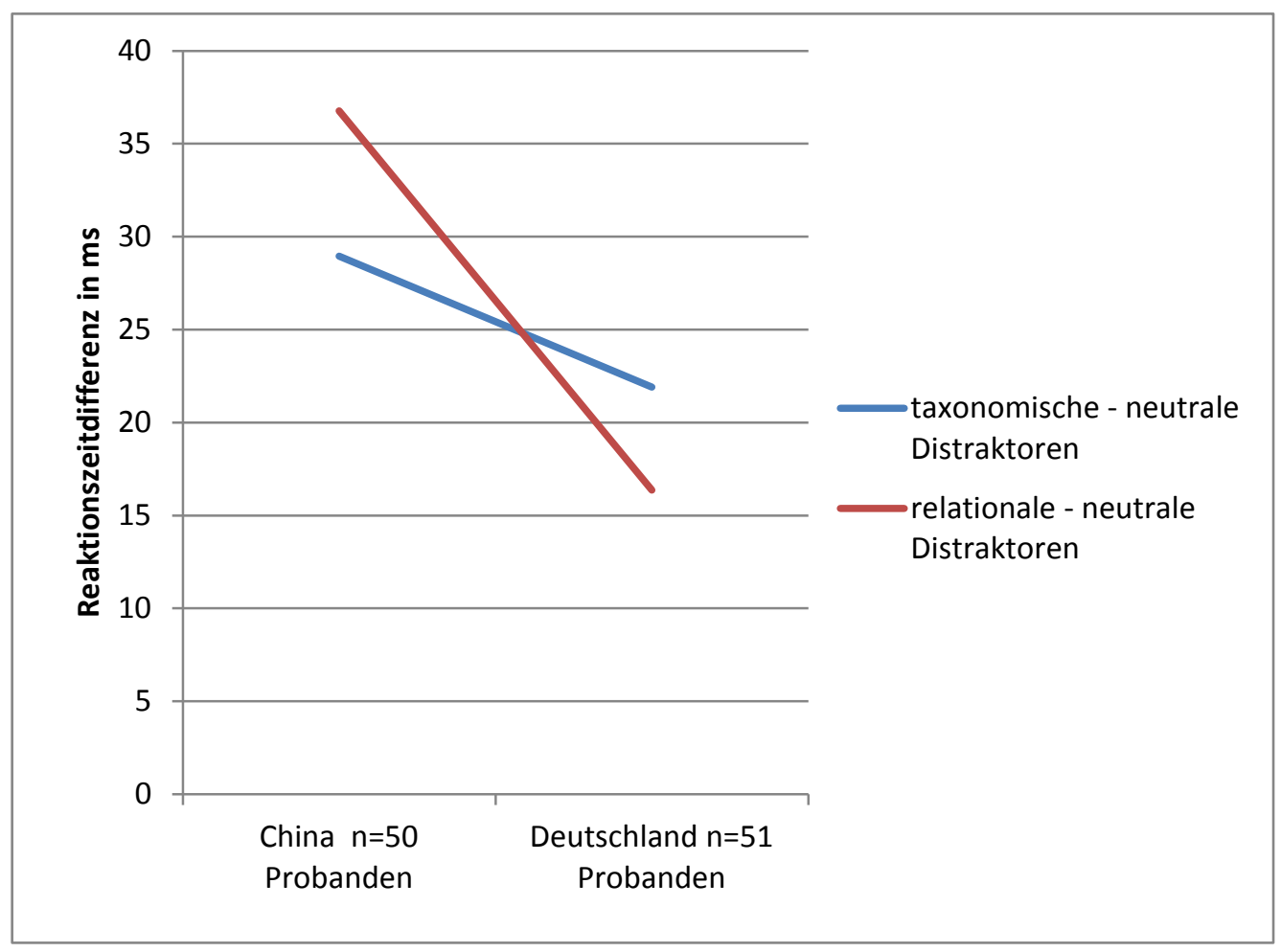

Abbildung 32: Interaktion „Distraktor $x$ Nation“. Mittlere Differenzen in den Reaktionszeiten (ms) zwischen Durchgängen mit taxonomischen versus neutralen Distraktoren (blau) sowie relationalen versus neutralen Distraktoren (rot) in Abhängigkeit von der Nation der Versuchsteilnehmer.

Dieses Ergebnismuster entspricht der Hypothese 3. Auf der Ebene der Sprachproduktion zeigte sich also die erwartete Kategoriserungspräferenz. Die Darbietung eines sprachlichen Distraktors, welcher in einer relationalen Beziehung zu einem darauf folgenden Target steht, hatte bei chinesischen Probanden innerhalb des Bild-Wort-Interferenz-Paradigmas zu einer größeren Interferenz geführt, verglichen mit einem sprachlichen Distraktor, welcher in einer taxonomischen Beziehung zu einem darauf folgenden Target steht. Im Gegensatz dazu hatte bei deutschen Probanden die Darbietung eines sprachlichen Distraktors, welcher in einer taxonomischen Beziehung 
zu einem darauf folgenden Target steht, zu einer größeren Interferenz geführt, verglichen mit einem sprachlichen Distraktor, der in einer relationalen Verbindung zu einem darauf folgendem Target steht.

Da das beschriebene Ergebnismuster auf der Aktivierung von Konzepten im semantischen Netz beruht ist von einem High-Level-Effekt der Sprache auszugehen (Hunt \& Agnoli, 1991). Dies soll später noch ausführlicher diskutiert werden.

Als ein weiteres Ergebnis ist die signifikante Interaktion „Distraktor x SOA“ festzuhalten. Auch diese Interaktion ist disordinal (siehe Abbildung 33). So zeigen sich bei der SOA von -300 ms im Mittel geringere Differenzen bei taxonomischen als bei relationalen Distraktoren. Bei einer SOA von $-150 \mathrm{~ms}$ zeigen sich hingegen im Mittel geringere Differenzen bei relationalen Distraktoren als bei taxonomischen Distraktoren. Dies bedeutet, dass der relationale Distraktor bei einer SOA von -300 ms einen größeren Ablenkungseffekt hatte als bei einer SOA von -150 ms. Der taxonomische Distraktor dagegen hat bei einer SOA von -150 ms einen größeren Ablenkungseffekt als bei -300 ms.

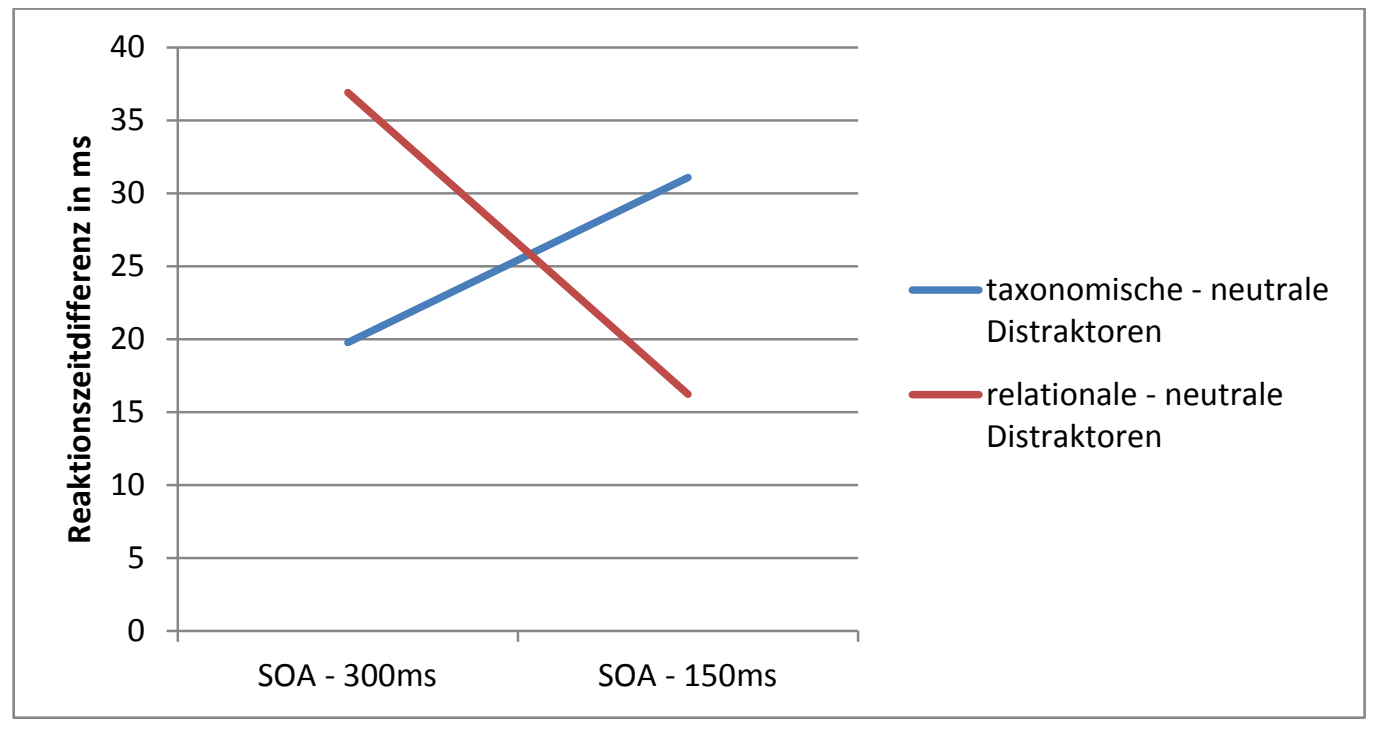

Abbildung 33: Interaktion „Distraktor $\mathrm{x}$ SOA. Mittlere Differenzen in den Reaktionszeiten (ms) zwischen Durchgängen mit taxonomischen versus neutralen Distraktoren (blau) sowie relationalen versus neutralen Distraktoren (rot) in Abhängigkeit von der SOA-Bedingung ( $n=50$ chinesische Probanden, $n=51$ deutsche Probanden). 
Dieses Ergebnismuster kann zur Präzisierung des zeitlichen Ablaufs von an der Sprachproduktion beteiligten Prozessen herangezogen werden. Schriefers et al. 1990 zeigten in ihrem Experiment, dass bei der Sprachproduktion ein semantischer Verarbeitungsprozess einem phonologischem vorgelagert ist. Die hier gefunden Ergebnisse legen den Schluss nah, dass ein relationaler Teilprozess einem taxonomischen voraus geht, womit die semantische Verarbeitungskomponente noch weiter ausdifferenziert werden würde. 


\section{Diskussion}

In der folgenden Diskussion werden zunächst die hypothesenrelevanten Ergebnisse behandelt. Im Anschluss daran werden die weiteren gefundenen Unterschiede zwischen chinesischen und deutschen Probanden erörtert. Schließlich erfolgt die Einordnung der Ergebnisse in den empirischen und theoretischen Rahmen. Abschließend werden die drei Experimente kritisch beleuchtet, Anregungen für weitere Forschungen aufgezeigt, und es wird versucht, den Ansatz der vorliegenden Arbeit in einen größeren Kontext zu stellen.

\subsection{Hypothesenrelevante Ergebnisse}

Hypothese 1 besagte, dass bei der Beurteilung von Bildpaaren die Reaktionszeit von chinesischen Probanden bei Bildpaaren mit relationalen Beziehungen geringer ist als bei Bildpaaren mit taxonomischen Beziehungen. Bei deutschen Probanden hingegen ist die Reaktionszeit bei der Beurteilung von Bildpaaren mit taxonomischen Beziehungen geringer als bei Bildpaaren mit relationalen Beziehungen. In Bezug auf diese Hypothese zeigte sich im ersten Experiment, dass von Vertretern beider Nationen bei einer taxonomischen Bildpaarbeziehung schnellere Reaktionszeiten abgegeben wurden. Eine hypothesenkonforme Kategorisierungspräferenz konnte für beide untersuchten Nationen somit nicht festgestellt werden.

Im zweiten Experiment zeigte sich sogar ein Muster, das der Hypothese 1 widerspricht. So tendierten die chinesischen Probanden in einer post hoc durchgeführten Auswertung mehr zu taxonomischen Entscheidungen als die deutschen Probanden. Die deutschen Probanden neigten dagegen mehr zu relationalen Entscheidungen. Auch in den Reaktionszeiten ließen sich keine signifikante Ergebnisse finden, welche die Hypothese stützen würden.

Nach Hypothese 2 wurde erwartet, dass die Darbietung eines sprachlichen Primes, welcher in einer relationalen Beziehung zu einem darauf folgenden relational verbundenen Bildpaar steht, bei chinesischen Probanden $\mathrm{zu}$ einer Verringerung der Reaktionszeit führen sollte, verglichen mit einem sprachlichen Prime, welcher in einer taxonomischen oder neutralen Beziehung $\mathrm{zu}$ einem darauf folgenden relational 
verbundenen Bildpaar steht. Bei deutschen Probanden sollte die Darbietung eines sprachlichen Primes, welcher in einer taxonomischen Beziehung zu einem darauf folgenden taxonomisch verbundenen Bildpaar steht, zu einer Verringerung der Reaktionszeit führen, verglichen mit einem sprachlichen Prime, der in einer relationalen oder einer neutralen Verbindung zu einem taxonomisch verbundenen Bildpaar steht. Das aufgrund dieser Hypothese postulierte Muster konnte in Experiment 1 nicht gefunden werden. Die deutschen Probanden profitierten besonders von kongruenten Primes, sowohl für taxonomische als auch für relationale Primes. Die chinesischen Probanden profitierten dagegen nicht von kongruenten Primes. Das semantische Priming wirkte in diesem Fall demnach nur bei den deutschen Probanden. Dieser Befund legt die Annahme nahe, dass die als Primes verwendeten chinesischen Schriftzeichen anders verarbeitet werden als alphabetische Wörter. Auch im zweiten Experiment ließ sich Hypothese 2 nicht bestätigen.

Die Wirkung des Primes war insgesamt sehr heterogen. Bei Itemset 1, wo eine taxonomische Bildpaarbeziehung mit einer relationalen konkurrierte, konnten die chinesischen Probanden am schnellsten antworten, wenn kein Prime dargeboten wurde. Dies zeigt erneut den starken Einfluss der als Primes verwendeten chinesischen Schriftzeichen. Bei den deutschen Probanden ließ sich kein derartiger Effekt auffinden. Dies spricht dafür, dass chinesische Schriftzeichen unter den realisierten Aufgabenanforderungen eine andere Primewirkung erzielten als Wörter in alphabetischer Schreibweise. Die chinesischen Schriftzeichen scheinen vielmehr in Konkurrenz zur verlangten Kategorisierung und der Verarbeitung der Bildstimuli zu treten.

Bei den beiden Itemsets 2 und 3 stand die Wirkung des Primes auf die Identifikation einer taxonomischen Bildpaarbeziehung (Itemset 2) beziehungsweise einer relationalen Bildpaarbeziehung (Itemset 3) im Vordergrund. Die deutschen und chinesischen Probanden verhielten sich recht ähnlich. Beim Itemset 3 zeigte sich überraschenderweise, dass es nicht zu schnelleren Antwortabgaben aufgrund eines semantischen Primings kam. Vielmehr schienen die Probanden durch die semantischen Primes im Vergleich zu einem neutralen Prime eine Hemmung zu erfahren.

Insgesamt zeigten sich in den ersten beiden Experimenten nicht die durch frühere Studien (z. B. Unsworth et al., 2005; Nisbett et al., 2001) belegten und vorhergesagten 
spezifischen Präferenzen der Probandengruppen für unterschiedliche Kategorisierungsarten, die sich in jeweils geringeren Reaktionszeiten bei der Beurteilung der präferierten Beziehungsart gegenüber der nicht präferierten niederschlagen sollten. Auch in einer post hoc durchgeführten Analyse der Antworthäufigkeiten zeigten weder chinesische Probanden Hinweise auf eine Präferenz für relationale Beziehungen, noch zeigten deutsche Probanden Hinweise auf eine Präferenz für taxonomische Beziehungen.

Ein möglicher Grund dafür, dass in diesem Experiment keine unterschiedlichen Präferenzen für relationale und taxonomische Beziehungen bei chinesischen und deutschen Probanden festgestellt werden konnten, könnte in der Art der Erfassung der Präferenz liegen. In früheren Experimenten (z.B. Nisbett et al., 2001; Unsworth et al., 2005) wurden die Probanden durch das Untersuchungsdesign gezwungen, sich bei Bildpaaren oder Bildtripeln zwischen relationaler und taxonomischer Kategorisierung zu entscheiden. Demnach konnte die Präferenz für eine Beziehungsart auch über die Häufigkeiten der entsprechenden Entscheidungen erfasst werden, was zu dem Befund führte, dass westliche Probanden eine Präferenz für taxonomische und asiatische Probanden hingegen eine Präferenz für relationale Beziehungen zeigen.

In Experiment 1 und 2 sollte hingegen die Präferenz über Unterschiede in den Reaktionszeiten bei der Beurteilung von Bildpaaren oder Bildtripeln mit relationaler und taxonomischer Beziehung erfasst werden. Dabei wurde davon ausgegangen, dass sich eine Präferenz in geringeren Reaktionszeiten bei der bevorzugten Beziehungsart niederschlagen würde, wobei die Präferenz bei chinesischen und deutschen Probanden bei unterschiedlichen Beziehungen liegen sollte. Diese erwarteten Unterschiede in den Reaktionszeiten konnten jedoch nicht festgestellt werden. Dies legt die Vermutung nahe, dass der Output eines kognitiven Verarbeitungsprozesses zwar kulturspezifische Präferenzen abbilden kann, möglicherweise aber Charakteristika wie seine Geschwindigkeit keine Indikatorfunktion haben.

Nach Hypothese 3 wurde erwartet, dass die Darbietung eines sprachlichen Distraktors, welcher in einer relationalen Beziehung zu einem darauf folgenden Target steht, bei chinesischen Probanden innerhalb des Bild-Wort-Interferenz-Paradigmas zu einer größeren Interferenz führen sollte, verglichen mit einem sprachlichen Distraktor, welcher in einer taxonomischen oder neutralen Beziehung zu einem darauf folgenden Target steht. Bei deutschen Probanden sollte die Darbietung eines sprachlichen 
Distraktors, welcher in einer taxonomischen Beziehung $\mathrm{zu}$ einem darauf folgenden Target steht, zu einer größeren Interferenz führen, verglichen mit einem sprachlichen Distraktor, der in einer relationalen oder einer neutralen Verbindung zu einem darauf folgendem Target steht.

Diese Hypothese ließ sich durch die signifikante disordinale Interaktion der Faktoren „Distraktor“ und „Nation“ in Experiment 3 bestätigen. Abhängige Variable war hier ein Differenzwert, der aus den Daten zweier Distraktorbedingungen gebildet wurde. Für jedes zu benenne Target wurde die Reaktionszeit mit neutralem Distraktor von der Reaktionszeit mit taxonomischem oder relationalem Distraktor abgezogen. Es zeigten sich bei den chinesischen Versuchsteilnehmern im Mittel geringere Differenzwerte bei taxonomischen als bei relationalen Distraktoren. Bei den deutschen Probanden zeigten sich hingegen im Mittel geringere Differenzwerte bei relationalen Distraktoren als bei taxonomischen Distraktoren. Das bedeutet, dass der taxonomische Distraktor bei den deutschen Probanden einen größeren Ablenkungseffekt hatte, der relationale Distraktor dagegen hatte bei den chinesischen Probanden einen größeren Ablenkungseffekt. Dieser Befund passt genau in den empirischen und theoretischen Rahmen zu kulturspezifischen Präferenzen bedingt durch einen analytischen oder holistischen Denkstil (Nisbett, 2003).

\subsection{Kultureffekte}

Deutsche Probanden zeigten, obwohl die Untersuchungsbedingungen in Göttingen und Shanghai vergleichbar waren und sich die beiden Probandengruppen in wichtigen soziodemographischen Variablen wie Alter und Bildung nicht unterschieden, über alle Bedingungen hinweg signifikant geringere Reaktionszeiten als die chinesischen Probanden. Da die schnelleren Reaktionszeiten der deutschen Probanden nicht mit einer im Vergleich zur chinesischen Probandenstichprobe höheren Fehlerrate einhergingen, können nicht unterschiedliche Bearbeitungsstrategien im Sinne eins Speed-AccuracyTrade-Off für diesen Effekt verantwortlich sein. Es muss nach anderen Gründen gesucht werden.

Denkbar ist, dass die Art des verwendeten Bildmaterials in Deutschland häufiger Verwendung findet und die Bilder somit für die deutschen Probanden geläufiger 
gewesen sein könnten. Das Bildmaterial wurde zwar durch einen Vortest hinsichtlich seiner kulturellen Salienz für beide Probandengruppen kontrolliert, jedoch lebten die Chinesen, die als Probanden im Vortest zur Verfügung standen, bereits eine Weile in Deutschland. Deshalb könnten ihnen die Darstellungen vertrauter gewesen sein als Probanden, die noch nie außerhalb von China gewesen sind. Sollte die Darstellungsart für deutsche Probanden geläufiger gewesen sein, könnten sie weniger Zeit benötigt haben, um die Objekte zu identifizieren und somit auch die Relationen zwischen den Bildpaaren zu beurteilen. Um die Eindeutigkeit des Itemmaterials sicher zu stellen, wurde außerdem ein Nachtestfragebogen eingesetzt. Bei diesem beurteilten die Probanden die während des Computerexperimentes dargebotenen Itempaare (Bilder!) erneut in Form von Wörtern als zusammenpassend oder nicht zusammenpassend, wobei sie sich so viel Zeit lassen konnten, wie sie benötigten. Hierbei zeigten sich bei deutschen und chinesischen Probanden signifikant weniger Fehler im Nachtestfragebogen als im Computerexperiment. Grund dafür kann in einer generell höheren Eindeutigkeit von Wörtern gegenüber Bildern gesehen werden. Während Wörter wenig Raum für unterschiedliche Interpretationen lassen, besteht bei Bildern immer die Möglichkeit, das dargestellte Objekt etwas anderes auszulegen und somit die intendierte Beziehung nicht als solche zu erkennen. Somit könnten Fehlinterpretationen der Bilder den Prozentsatz der richtigen Antworten im Computerexperiment bei allen Probanden gesenkt haben.

Um zu analysieren, ob die Bilder für chinesische und deutsche Probanden unterschiedlich eindeutig waren und somit die höheren Reaktionszeiten und höhere Fehlerprozente chinesischer Probanden erklärt werden könnten, wurden die Antworten von chinesischen und deutschen Probanden im Nachtestfragebogen verglichen. Sollte die im Computerexperiment gefundene Differenz zwischen deutschen und chinesischen Probanden dadurch zustande gekommen sein, dass das verwendete Versuchsmaterial für deutsche Probanden leichter zu bearbeiten war, sollten sich im Nachtestfragebogen keine Unterschiede mehr zwischen den Probandengruppen zeigen. Doch im Nachfragebogen stimmten deutsche Probanden taxonomischen zusammenpassenden Wortpaaren signifikant häufiger zu als chinesische Probanden. Chinesische Probanden hingegen stimmten relationalen Wortpaaren signifikant häufiger zu als deutsche Probanden. Die postulierten Differenzen zwischen deutschen und chinesischen Probanden ließen sich 
also erst im Nachtestfragebogen bei der Beurteilung von Wörtern aufzeigen. Das legt die Vermutung nahe, dass die erwarteten Effekte im Computerexperiment deshalb nicht zustande kamen, da das verwendete Bildmaterial für chinesische Probanden weniger vertraut und somit weniger eindeutig war.

Denkbar wäre es natürlich auch, dass nicht das verwendete Bildmaterial, sondern die realisierten relationalen und taxonomischen Beziehungen zwischen den dargebotenen Objekten für chinesische Probanden weniger gebräuchlich waren. In diesem Sinne wäre vorstellbar, dass die dargestellten Beziehungen in China seltener auftreten, zum Beispiel weil die Objekte an sich in China seltener Verwendung finden oder bestimmte Kombinationen von Objekten weniger häufig sind. Das könnte eine langsamere Antwortabgabe von chinesischen Probanden erklären.

Vorstellbar wäre aber auch, dass das Phänomen der Kategorisierung generell in einer westlichen Kultur ausgeprägter sein könnte als in der chinesischen - ganz im Sinne der postulierten kulturspezifischen Denkstile (Nisbett, 2003; Yan u. a., 2005). So könnten chinesische Probanden längere Reaktionszeiten benötigt haben, da der Vorgang der Kategorisierung und somit auch die Beurteilung einer Beziehung zwischen zwei Objekten für sie weniger geläufig ist und so mehr Bearbeitungszeit benötigt wird

\subsection{Kulturübergreifende Effekte}

Bei den Teilprozessen der Sprachproduktion sind die Bereitstellung von semantischen Inhalten, welche enkodiert werden sollen, sowie die Auffindung von Wortformen (und anderen sprachlichen Elementen) in einer Sprache, durch die die semantischen Inhalte dargestellt werden, von zentraler Bedeutung (Dell \& O’Seaghdha, 1992; Grabowski \& Herrmann, 1994; Levelt, 1993; Levelt et al. 1999). Diese beiden Teilprozesse werden oft als semantischer oder auch konzeptueller und als phonologischer oder auch lexikalischer oder phonetischer Teilprozess bezeichnet. Ein semantischer Distraktor sollte nun in eben diesen semantischen Teilprozess der Sprachproduktion eingreifen und ein phonologischer Distraktor in den phonologischen Teilprozess. Diese Logik liegt dem Bild-Wort-Interferenz-Paradigma zugrunde, wenn mit verschiedenen Distraktortypen unter Variation der SOA gearbeitet wird. 
Schriefers und Kollegen (1990) zeigten in ihrer vielzitierten Arbeit auf diesem Weg, dass bei der Sprachproduktion ein semantischer Verarbeitungsprozess einem phonologischen vorgelagert ist. Im Experiment 3 zeigte sich in beiden Probandengruppen bei der SOA von -300 ms eine geringere Interferenz bei taxonomischen als bei relationalen Distraktoren. Bei einer SOA von -150 ms zeigte sich hingegen eine geringere Interferenz bei relationalen Distraktoren als bei taxonomischen Distraktoren. Dieses Muster könnte den Schluss nahe legen, dass bei der Beurteilung von Assoziationen zwischen Objekten die Verarbeitung relationaler Beziehungen der Verarbeitung taxonomischer Beziehungen voraus geht. Und hierbei handelt sich ganz offenbar um einen kulturunabhängigen Effekt, der eine weitere Differenzierung der semantischen Verarbeitungskomponente bei der Sprachproduktion erlauben würde, wenn er sich als stabil herausstellt. Um diese Interpretation zu stützen, könnten in einem Anschlussexperiment zusätzliche SOA-Stufen eingeführt werden mit dem Ziel, die Wirkung der Distraktoren noch genauer differenzieren zu können. Hierbei wäre sowohl an eine SAO von Null zu denken, also ein gleichzeitiges Erscheinen des Distraktors mit dem Target, als auch an positive SOA-Stufen, wo der Distraktor erst nach dem Target dargeboten wird.

\subsection{Generelle Diskussion}

Ausgehend von der Annahme unterschiedlicher Denkweisen in asiatischen und westlichen Kulturen sollten sich differentielle Präferenzen für bestimmte zwischen Objekten bestehende Beziehungen bei chinesischen und deutschen Probanden zeigen. So sollten chinesische Probanden, denen eine holistische Denkweise zugesprochen wird, eine Präferenz für relationale Beziehungen haben. Deutsche Probanden hingegen sollten vor dem Hintergrund eines analytischen Denkstils taxonomische Beziehungen bevorzugen. Dies konnte bei der Sprachproduktion innerhalb des Bild-Wort-InterferenzParadigmas nachgewiesen werden, jedoch nicht bei Kategorisierungsanforderungen mit Bildpaaren oder Bildtripeln. Doch auch die vorliegenden Ergebnisse stützen zum Teil die Annahme eines zugrunde liegenden analytischen Denkmodells in westlichen und eines holistischen Denkmodells in asiatischen Kulturen und lassen sich wie folgt in diesen konzeptuellen Ansatz integrieren. 
Holistisches Denken beinhaltet im Gegensatz zu analytischem Denken die Wahrnehmung von allen Dingen als miteinander verknüpft und sich gegenseitig beeinflussend. Vermutlich entstanden aus der politischen Situation und der geographischen Lage des damaligen Chinas, entwickelte sich ein gruppenorientiertes System, in dem die Aufmerksamkeit vor allem auf den Beziehungen zwischen Objekten und Menschen liegt, da diese als entscheidend für die Bewältigung der Lebensanforderungen angesehen werden (Nisbett, 2003). Im Einklang mit diesem Ansatz steht die Annahme, dass die chinesische Kultur und Sprache Kategorisierung an sich deshalb weniger beinhaltet, da diese immer eine Form der Abgrenzung impliziert. So werden beim Kategorisieren Dinge als zusammengehörig und verbunden betrachtet und gleichzeitig andere als nicht zugehörig befunden und abgegrenzt. Dies könnte in einem Denkmodell, in dem alles als verknüpft und aufeinander einwirkend angesehen wird, als wenig sinnvoll erscheinen und somit weniger verbreitet sein. Durch die Ansicht der Verbundenheit und der gegenseitigen Beeinflussbarkeit aller Dinge könnte eine Abgrenzung von Objekten die Befürchtung wecken, die wichtigen Wirkeinflüsse zu vernachlässigen.

Das analytische Denkmodell hingegen beinhaltet die Annahme, die Welt sei eine Sammlung von eigenständigen Objekten, die auf der Basis ihrer Eigenschaften in Kategorien eingeteilt werden können. Der Fokus liegt hier also auf den Objekten an sich und ihren Eigenschaften (Nisbett, 2003). Dies könnte Kategorisierung generell stärker nahe legen und fördern als ein holistisches Denkmodell, da es auf der Basis dieser Annahmen mehr Sinn macht, Objekte als zusammengehörig und nicht zusammengehörig einzuteilen. Kategorisierung könnte hier stärker als wirksame Strategie angesehen werden, bei der Dinge gruppiert und organisiert werden, um so sinnvoll mit ihnen umgehen zu können.

Die Ergebnisse der vorgestellten Untersuchung stützen insgesamt die Annahme, dass es Unterschiede in den kognitiven Prozessen verschiedener Kulturen gibt. Es zeigte sich, dass Personen unterschiedlicher Kulturen verschiedene Arten haben, die Welt und ihre Objekte wahrzunehmen und zu organisieren. Diese Unterschiede scheinen sich durch kulturelle Einflüsse zu entwickeln.

Ziel der Untersuchungen war es, die Einflüsse von Kultur auf das Kategorisierungsverhalten mit unterschiedlichen experimentellen Paradigmen sichtbar 
zu machen. Die Ergebnisse legen nahe, dass das Kategorisierungsverhalten zwar anders als erwartet ausfiel, aber dennoch wie vermutet von Kultur beeinflusst wird. Die favorisierte Interpretation des Autors ist, dass es sich bei Kategorisierungspräferenzen um ein Spektrum handelt, dass je nach Aufgabenanforderung abrufbar ist und sich daher auch in unterschiedlichen Befunden manifestiert.

\subsection{Kritik und Anregung für weitere Forschung}

Um spezifische Effekte besser differenzieren zu können, sollte - wie in der vorliegenden Untersuchung geschehen - auf $\mathrm{zu}$ beurteilendes sprachliches Versuchsmaterial verzichtet werden, damit die Möglichkeit zur separaten Messung von Kultur- und Spracheinflüssen bestehen bleibt. Um Kultur- und Spracheffekte separat zu messen, müsste der in der vorgestellten Untersuchung durchgeführte Vergleich zwischen Probanden unterschiedlicher kultureller Herkunft sowie die Variation von Untersuchungsland und -sprache bei nachfolgenden Untersuchungen beibehalten werden. Der Vergleich von Probandengruppen unterschiedlicher kultureller Herkunft in unterschiedlichen Untersuchungsländern ermöglicht eine detaillierte Untersuchung eines möglichen Kultureffektes. Durch die Variation der Untersuchungssprache ließe sich ein möglicher Spracheffekt messbar machen.

Um den interessierenden Spracheffekt weitergehend zu untersuchen, könnten auch in nachfolgenden Untersuchungen sprachliche Primes in der jeweiligen Untersuchungssprache verwendet werden. Hierbei wäre zu untersuchen, inwieweit ein Prime, der in einer bestimmten Beziehung zum darauffolgenden Untersuchungsmaterial steht, die Entscheidung für eine relationale oder taxonomische Kategorisierung sowie die Art der assoziierten Objekte beeinflussen kann. Dabei wären den Besonderheiten des chinesischen Schriftzeichens allerdings stärker Rechnung zu tragen. Eventuell könnte ein Weg über das sogenannte „Pinyin“ gewählt werden. In Pinyin werden die chinesischen Schriftzeichen in lateinischen Buchstaben als Lautfolge dargestellt. Pinyin ist in China sehr geläufig, da SMS und Microsoft Office-Anwendungen darüber bedienbar sind. Unter Verwendung von Pinyin könnte der interferierende bildliche Charakter der chinesischen Schriftzeichen kontrolliert werden, ohne auf die chinesische Sprache an sich zu verzichten.

Zusätzlich wäre in einer nachfolgenden Untersuchung das Hinzufügen weiterer 
Probandengruppen sinnvoll. Diese könnten mit den vorhandenen Gruppen verglichen werden, um Kultur- und Spracheffekte noch genauer zu erfassen und differenzieren zu können. So wäre es möglich, zusätzlich deutsche Probanden in China zu untersuchen, um den Einfluss des Untersuchungslandes weitergehend zu bestimmen. Hierbei könnte außerdem variiert werden, ob diese auf Deutsch oder auf Chinesisch untersucht werden, um die Einflüsse der Untersuchungssprache genauer $\mathrm{zu}$ analysieren. Dies würde insgesamt eine stärkere Spezifizierung des Kategorisierungsverhaltens und darauf wirkender Einflüsse ermöglichen. Auch eine Ausweitung der Probandengruppe könnte hilfreich sein. Besonders die deutschen Probanden sind durch das Studium der Psychologie empirische Experimente gewohnt und so auch für Primingparadigmen sensibilisiert. Sie müssen zur Erlangung des Abschlusses an einer bestimmten Anzahl von Versuchen teilnehmen. Es könnte sich daher in den Reaktionszeiten widerspiegeln, dass die deutschen Studierenden diese Art von experimenteller Untersuchung eher gewohnt sind als die chinesischen Probanden.

Bezüglich des Versuchsmaterials müsste ähnlich dem Vortest des dritten Experiments zur Sprachproduktion sichergestellt werden, dass die verwendeten Bilder sowohl hinsichtlich der Gestaltungsart als auch hinsichtlich der dargestellten Objekte in China und in Deutschland gleichermaßen vertraut und eindeutig erscheinen. Ebenso müsste in beiden Ländern eine Absicherung erfolgen, dass die dargestellten relationalen und taxonomischen Beziehungen gleichermaßen häufig und alltäglich sind, so dass die Entscheidung für eine Beziehung nicht durch Unkenntnis der anderen zustande kommt und somit ein Effekt des Versuchsmaterials ausgeschlossen werden kann.

An dieser Stelle sei Kritik an der Theorie von Nisbett angeführt. Ratner und Hui (2003) kritisieren insbesondere, dass der Theorie ein pauschalisierender Kulturbegriff zugrunde liegen würde. Die chinesische Kultur sei sehr reduziert und mit Stereotypen dargestellt. Das Konzept der Dialektik, das Nisbett für den europäischen Raum postuliere, sei verkürzt bis schlichtweg falsch dargestellt. „Der mittlere Weg“ spiegele sich ebenfalls nicht in der chinesischen Geschichte wider, was z.B. durch das radikale Vorgehen während der „Kulturrevolution“ belegt werde. Insgesamt ließe sich die Theorie zusammenschrumpfen auf das bloße Generieren von operationalisierbaren Konstrukten, die anschließend einer Testung unterzogen werden könnten (Ratner \& Hui, 2003). 
Implizit steckt nach Meinung des Autors der Vorwurf von Ratner und Hui dahinter, dass der theoretische Rahmen erst nach dem Vorliegen der empirischen Befunde aufgestellt wurde. Nichtsdestotrotz zählt die Theorie von Nisbett heute immer noch zu den einflussreichsten Theorien innerhalb der kulturvergleichenden Forschung im ostasiatischen Raum. Auch die vorliegenden Untersuchungen lassen sich mit den aufgeführten Einschränkungen in diesen Rahmen einordnen. Sicherlich sollte durch weitere Forschungsbemühungen eine weitere Ausgestaltung und damit einhergehende Präzisierung des theoretischen Rahmens angestrebt werden.

\subsection{Ausblick}

In diesem letzten Unterabschnitt soll ein Ausblick weit über den bisherigen inhaltlichen und methodischen Rahmen hinaus gewagt werden, der die Bedeutung des in dieser Arbeit behandelten Themas verdeutlichen soll. Dass ein solcher Ausblick derzeit nur auf einem hypothetisch-spekulativen Weg vollzogen werden kann, bildet einen starken Kontrast zu der bisher in dieser Arbeit verfolgten streng experimentell-analytischen Vorgehensweise in den durchgeführten Untersuchungen. Da aber unsere Untersuchungen auch ganz neue Fragen aufgeworfen haben - so z.B. die Frage nach der Funktion von Primes in einer chinesisch-graphemischen Schriftweise - werden für weitergehende Forschungsarbeiten noch ganz neue Fragestellungen zu bearbeiten sein, für die angemessene Untersuchungsmethoden bisher nicht bekannt sind und deshalb erst gefunden werden müssen.

Nähern wir uns zunächst von ganz allgemeinen Überlegungen zu dem in Frage stehenden Untersuchungsgestand. Damit versuchen wir, die allgemeine Bedeutung der untersuchten Probleme zu begründen. Mit den beobachtbaren und gleichzeitig immer stärker voranschreitenden Tendenzen der Globalisierung in den verschiedensten Lebensbereichen rückt die Frage nach der Kommunikation zwischen sehr unterschiedlichen Kulturen in der Welt immer mehr in den Vordergrund. Dabei ist zunächst einmal die Wirtschaft die treibende Kraft, die diesen Prozess mir ihrem weltweiten Handel intensiv vorantreibt. Dabei tritt als erstes das Sprachproblem auf, das die Kommunikation behindert oder gar verunmöglicht. Mit Sprachkursen oder mit dem Rückzug auf eine Weltsprache - das Englische in unserer Zeit - ist das Problem einer 
interkulturellen Verständigung allein aber nicht zu lösen. Wenn es zwischen verschiedenen Kulturen z.B., wie in dieser Arbeit angenommen, auch noch unterschiedliche Denkstile gibt - und damit sind wir im Zentrum der in dieser Arbeit behandelten Thematik angekommen - dann ist deren Berücksichtigung auch noch ein Desiderat, das bei einer Kommunikation zwischen verschiedenen Kulturen zu berücksichtigen ist. Und dass es zwischen Europäern und Asiaten offenbar ganz unterschiedliche Denkstile gibt, haben Nisbett et al. mit ihren empirischen Untersuchungen hinreichend evident werden lassen. Nun könnte man allerdings gleich weiter fragen: Da Europäer bevorzugt analytisch denken und auf dieser Grundlage auch urteilen und Chinesen dagegen holistisch-relational vorgehen und daher die Umgebungsbedingungen stärker mit einbeziehen, ist damit nicht bereits ein ausreichendes Verständnis solcher interkulturellen kognitiven Stile erreicht?

In dieser Arbeit wird die Position vertreten, dass die alleinige Benennung von Unterschieden in Denkstilen, seien sie auch an noch so vielen Beispielen empirisch demonstriert worden, nicht ausreicht. Eine kognitive Wissenschaft muss vielmehr bis zu den Mechanismen, die diese Denkstile hervorbringen, mit ihren Untersuchungen vordringen. Mit dieser Überzeugung sind die drei in dieser Arbeit durchgeführten Experimente angelegt und durchgeführt worden. Erst aus der Kenntnis solcher Mechanismen heraus kann ein umfassendes Verständnis für Denkstile hervorgehen. Und erst aus einem Vergleich unterschiedlicher Denkstile auf der Ebene von kognitiven Mechanismen können subtile Verständnisse für Unterschiede zwischen kognitiven Gewohnheiten in verschiedenen Kulturen umfassend erklärt werden. Dabei besteht eine der größten Schwierigkeiten, hierfür passende experimentelle Untersuchungsparadigmen zu finden, mit denen die Mechanismen valide herausgearbeitet werden können. Wie in den vorangegangenen Experimenten gezeigt werden konnte, erweist sich z.B. das Paradigma des semantischen Primings, bezogen auf den Vergleich Europa mit Asien, hierfür offenbar als wenig bis nicht geeignet. So wird noch viel Forschungsarbeit zu leisten sein, um mit angemessenen experimentellen Paradigmen zielsicher diejenigen kognitiven Mechanismen heraus zu präparieren, die uns die Entstehung und Verwendung analytischer und holistischer Denkstile ausreichend verständlich machen können. Dabei wir auch zu bedenken sein, dass europäische Wissenschaftler, die in einem bevorzugt analytischen Denkstil erzogen und ausgebildet wurden und diese 
Vorgehensweise in ihrer eigenen Wissenschaft auch ausnahmslos praktizieren, mit ihren Denkgewohnheiten es nicht leicht haben werden, die notwendigen experimentellen Paradigmen zu entwickeln, die für solche interkulturell vergleichenden Untersuchungen unverzichtbar sind. 


\section{Zusammenfassung}

Ausgehend von der Annahme eines analytischen Denkmodells in westlichen und eines holistischen Denkmodells in (ost-)asiatischen Kulturen sind Unterschiede in den kognitiven Prozessen bei Menschen dieser unterschiedlichen Kulturen theoretisch und empirisch beschrieben worden (vgl. Nisbett, 2003; Yan et al., 2005). Die vorliegende Untersuchung widmete sich der Validierung von Kategorisierungspräferenzen. So belegen verschiedene Studien unter anderem Unterschiede im Kategorisierungsverhalten, die in einer Präferenz für taxonomische Beziehungen bei westlich geprägten Personen und in einer Präferenz für relationale Beziehungen bei Chinesen bestehen (vgl. Ji u. a., 2000; Norenzayan u. a., 2002; Unsworth u. a., 2005). In welchem Ausmaß diese unterschiedlichen Präferenzen durch die Kultur bestimmt werden, ist Untersuchungsgegenstand der vorliegenden Studien. Es wurde versucht, die Einflüsse von Kultur auf Kategorisierungspräferenzen mithilfe von unterschiedlichen experimentellen Paradigmen messbar zu machen.

In Experiment 1 wurden die Reaktionszeiten bei der Kategorisierung von Bildpaaren von chinesischen und deutschen Probanden erfasst. Es wurde davon ausgegangen, dass sich die Präferenz für eine Beziehungsart in geringeren Reaktionszeiten bei deren Beurteilung niederschlagen würde und dass die Variation von kultureller Herkunft der Probanden sowie die Variation eines eingeblendeten Primewortes zu Veränderungen in diesen Reaktionszeiten führen würden. Es stellte sich im ersten Experiment heraus, dass bei beiden Nationen bei einer taxonomischen Bildpaarbeziehung schnellere Reaktionszeiten resultierten. Die deutschen Probanden profitierten besonders von kongruenten Primes. Dies gilt sowohl für taxonomische wie auch für relationale Primes. Die chinesischen Probanden profitierten dagegen nicht von kongruenten Primes. Das semantische Priming wirkte in diesem Fall nur bei den deutschen Probanden. Die als Primes verwendeten chinesischen Schriftzeichen scheinen anders verarbeitet zu werden. Eine entsprechende Kategorisierungspräferenz für beide untersuchten Nationen nach der postulierten Hypothese lässt sich somit nicht feststellen.

In Experiment 2 wurden die Reaktionszeiten bei der Kategorisierung von Bildtripeln von chinesischen und deutschen Probanden ermittelt. Signifikanzen innerhalb der Reaktionszeiten, welche die Hypothese stützen würden, ließen sich nicht 
nachweisen. So tendierten die chinesischen Probanden in einer post-hoc-Auswertung mehr zu taxonomischen Entscheidungen als die deutschen Probanden. Die deutschen Probanden neigten dagegen mehr zu relationalen Entscheidungen.

Auch die Wirkung der Primes ließ sich nicht hypothesenkonform festhalten, da es bei den chinesischen Probanden zu schnelleren Reaktionszeiten bei der Bedingung ohne Prime kam. Dies zeigt erneut den starken Interferenzeffekt des chinesischen Schriftzeichens. Bei den deutschen Probanden ließ sich kein derartiger Effekt auffinden. Dies spricht dafür, dass chinesische Schriftzeichen eine andere Primewirkung erzielen als Wörter in europäischer Schreibweise. Es ist daher bei diesen beiden Schreibsystemen von einer unterschiedlichen Qualität auszugehen.

In Experiment 3 zur Sprachproduktion sollten die Kategorisierungspräferenzen über das Bild-Wort-Interferenz-Paradigma nachgewiesen werden. Die erwarteten Präferenzen ließen sich durch eine signifikante disordinale Interaktion der Faktoren „Distraktor“ und „Nation“ bestätigen. Es zeigten sich bei den chinesischen Versuchsteilnehmern geringere Interferenzen bei taxonomischen als bei relationalen Distraktoren. Bei den deutschen Probanden zeigten sich geringere Interferenzen bei relationalen Distraktoren als bei taxonomischen Distraktoren. Dieser Befund passt genau in den empirischen und theoretischen Rahmen des analytischen und holistischen Denkens (Nisbett, 2003).

Es wurden methodische Limitationen diskutiert und Anregungen für weiterführende Forschung aufgezeigt. Besonders die Verwendung der chinesischen Schriftzeichen als semantischer Prime wurde kritisch hinterfragt. Favorisiert wurde eine Interpretation der Daten dahingehend, dass je nach Aufgabenanforderung die Kategorisierungspräferenz innerhalb und zwischen den Kulturen variiert. 


\section{Literaturverzeichnis}

Baddeley, A. (1997). Human Memory: Theory and Practice, Revised Edition (Rev Sub.). Allyn \& Bacon.

Bell, B. S., \& Kozlowski, S. W. J. (2002). Adaptive Guidance: Enhancing Self-Regulation, Knowledge, and Performing in Technology-Based Training. Personal Psychology, 55(2), 267-306. doi:10.1111/j.1744-6570.2002.tb00111.x

Bleicken, J. (1995). Die athenische Demokratie (4., völlig überarb. u. wesentl. erw. A.). UTB, Stuttgart.

Caramazza, A. (1997). How Many Levels of Processing Are There in Lexical Access? Cognitive Neuropsychology, 14(1), 177-208. doi:10.1080/026432997381664

Collins, A. M., \& Loftus, E. F. (1975). A spreading-activation theory of semantic processing. Psychological Review, 82(6), 407-428. doi:10.1037/0033-295X.82.6.407

Dell, G. S., Burger, L. K., \& Svec, W. R. (1997). Language production and serial order: A functional analysis and a model. Psychological Review, 104(1), 123-147. doi:10.1037/0033-295X.104.1.123

Dell, G. S., \& O’Seaghdha, P. G. (1992). Stages of lexical access in language production. Cognition, 42(1-3), 287-314. doi:10.1016/0010-0277(92)90046-K

Duarte, D. L., \& Snyder, N. T. (2001). Mastering Virtual Teams: Strategies, Tools and Techniques That Succeed (2nd Bk\&Cdr.). Wiley \& Sons.

Fernald, A., \& Morikawa, H. (1993). Common Themes and Cultural Variations in Japanese and American Mothers' Speech to Infants. Child Development, 64(3), 637-656. doi:10.1111/j.1467-8624.1993.tb02933.x

Freeman, N. H., \& Habermann, G. M. (1996). Linguistic socialization: A Chinese perspective. The handbook of Chinese psychology (S. 79-92). New York, NY, US: Oxford University Press. 
Gelman, S. A., \& Tardif, T. (1998). A cross-linguistic comparison of generic noun phrases in English and Mandarin. Cognition, 66(3), 215-248. doi:10.1016/S00100277(98)00021-3

Gernet, J. (1988). Die chinesische Welt: Die chinesische Welt von den Anfängen bis zur Jetztzeit. Suhrkamp Verlag.

Grabowski, J., \& Herrmann, T. (1994). Sprechen: Psychologie der Sprachproduktion (1. Aufl.). Spektrum Akademischer Verlag.

Grawe, K. (2000). Psychologische Therapie (2., korr. A.). Hogrefe-Verlag.

Gutchess, A. H., Yoon, C., Luo, T., Feinberg, F., Hedden, T., Jing, Q., Nisbett, R. E., u. a. (2006). Categorical Organization in Free Recall across Culture and Age. Gerontology, 52(5), 314-323. doi:10.1159/000094613

Hansen, C. (1983). Language and logic in ancient China. Ann Arbor: University of Michigan Press.

Haun, D. B. M., Rapold, C. J., Janzen, G., \& Levinson, S. C. (2011). Plasticity of human spatial cognition: Spatial language and cognition covary across cultures. Cognition, 119(1), 70-80. doi:10.1016/j.cognition.2010.12.009

Heath, S. B. (1982). What no bedtime story means: Narrative skills at home and school. Language in Society, 11(01), 49-76. doi:10.1017/S0047404500009039

Heine, S. J., Lehman, D. R., Markus, H. R., \& Kitayama, S. (1999). Is there a universal need for positive self-regard? Psychological Review, 106(4), 766-794. doi:10.1037/0033295X.106.4.766

Herrmann, T. (2005). Grundriss der Psychologie: Sprache verwenden: Funktionen Evolution-Prozesse: BD 9 (1., Aufl.). Kohlhammer.

Herrmann, T., \& Deutsch, W. (1976). Psychologie der Objektbenennung. Bern, Stuttgart, Wien: Huber. 
Hörmann, H. (1991). Einführung in die Psycholinguistik (2., unveränd. A.). Wissenschaftliche Buchgesellschaft.

Hsu, F. L. K. (1953). Americans and Chinese: Two ways of life. New York: Schuman.

Hunt, E., \& Agnoli, F. (1991). The Whorfian hypothesis: A cognitive psychology perspective. Psychological Review, 98(3), 377-389. doi:10.1037/0033-295X.98.3.377

Jacobs, F., \& Curtius, C. (2011). Hellas: Geographie, Geschichte Und Litteratur Griechenlands... Nabu Press.

Ji, L. J., Peng, K., \& Nisbett, R. E. (2000). Culture, control, and perception of relationships in the environment. Journal of personality and social psychology, 78(5), 943.

Ji, L.-J., Zhang, Z., \& Nisbett, R. E. (2004). Is It Culture or Is It Language? Examination of Language Effects in Cross-Cultural Research on Categorization. Journal of Personality and Social Psychology, 87(1), 57-65. doi:10.1037/0022-3514.87.1.57

Kinoshita, S., \& Lupker, S. J. (2003). Masked Priming: The State of the Art. Psychology Press.

Lass, U., Yan, S., Yang, Y., Chen, G., Sun, P., Becker, D., Fang, Y., u. a. (2006). Recognition of briefly exposed digits, Latin letters, and Chinese characters: Zeitschrift für Psychologie, 214(1), 24-36. doi:10.1026/0044-3409.214.1.24

Levelt, W. J. M. (1993). Speaking: From Intention to Articulation (Reprint.). Mit Pr. Levelt, W. J., Roelofs, A., \& Meyer, A. S. (1999). A theory of lexical access in speech production. The Behavioral and brain sciences, 22(1), 1-38; discussion 38-75. Levinson, S. . (1996). Frames of reference and Mloyneux's question: Crosslinguistic'sevidence. In P. Bloom, M. . Peterson, L. Nadel, \& M. . Garrett (Hrsg.), Language and space. Cambridge, MA: MIT Press.

Lloyd, G. E. . (1990). Demystifying mentalities. New York: Cambridge University Press. 
Lüer, G., Becker, D., Lass, U., Yunqiu, F., Guopeng, C., \& Zhongming, W. (1998). Memory Span in German and Chinese. European Psychologist, 3(2), 102-112. doi:10.1027/1016-9040.3.2.102

Maass, A., \& Russo, A. (2003). Directional Bias in the Mental Representation of Spatial Events Nature or Culture? Psychological Science, 14(4), 296-301. doi:10.1111/14679280.14421

Malt, B. C., Sloman, S. A., Gennari, S., Shi, M., \& Wang, Y. (1999). Knowing versus Naming: Similarity and the Linguistic Categorization of Artifacts. Journal of Memory and Language, 40(2), 230-262. doi:10.1006/jmla.1998.2593

Maslow, A. H. (1943). A theory of human motivation. Psychological Review, 50(4), 370396. doi:10.1037/h0054346

Masuda, T., \& Nisbett, R. E. (2001). Attending holistically versus analytically: Comparing the context sensitivity of Japanese and Americans. Journal of Personality and Social Psychology, 81(5), 922-934. doi:10.1037/0022-3514.81.5.922

Morris, M. W., \& Peng, K. (1994). Culture and cause: American and Chinese attributions for social and physical events. Journal of Personality and Social Psychology, 67(6), 949971. doi:10.1037/0022-3514.67.6.949

Needham, J. (1962). Physics and physical technology. Science and civilization in China (Bd. 4). New York: Cambridge University Press.

Neely, James H., G. W. (1991). Semantic Priming Effects in Visual Word Recognition: A Selective Review of Current Findings and Theories. Basic Processes in Reading: Visual Word Recognition. Hillsdale: Routledge.

Nisbett, R. E. (2003). The Geography of Thought: How Asians and Westerners Think Differently... and Why. Free Press. 
Nisbett, R. E., Peng, K., Choi, I., \& Norenzayan, A. (2001). Culture and systems of thought: Holistic versus analytic cognition. Psychological Review, 108(2), 291-310. doi:10.1037/0033-295X.108.2.291

Norenzayan, A., Smith, E. E., Kim, B. J., \& Nisbett, R. E. (2002). Cultural preferences for formal versus intuitive reasoning. Cognitive Science, 26(5), 653-684.

Norman, J. (1988). Chinese. Cambridge University Press.

Peng, K., \& Nisbett, R. E. (1999). Culture, dialectics, and reasoning about contradiction. American Psychologist, 54(9), 741-754. doi:10.1037/0003-066X.54.9.741

Ratner, C., \& Hui, L. (2003). Theoretical and Methodological Problems in Cross-Cultural Psychology. Journal for the Theory of Social Behaviour, 33(1), 67-94. doi:10.1111/1468-5914.00206

Rickheit, G., Herrmann, T., \& Deutsch, W. (2003). Psycholinguistik. Ein Internationales Handbuch: An International Handbook (Bilingual.). Gruyter.

Roelofs, A. (1997). The WEAVER model of word-form encoding in speech production. Cognition, 64(3), 249-284. doi:10.1016/S0010-0277(97)00027-9

Schriefers, H., Meyer, A. S., \& Levelt, W. J. M. (1990). Exploring the time course of lexical access in language production: Picture-word interference studies. Journal of Memory and Language, 29(1), 86-102. doi:10.1016/0749-596X(90)90011-N

Schriefers, H., Meyer, A. S., \& Levelt, W. J. M. (2003). Exploring the time course of lexical access in language production: Picture word interference studies. Psycholinguistics: Critical Concepts in Psychology (S. 168-191). Londen: Routledge.

Seligman, M. E. P. (1975). Helplessness: On Depression, Development and Death. W.H.Freeman \& Co Ltd.

Sperling, G. (1967). Successive approximations to a model for short term memory. Acta Psychologica, 27(0), 285-292. doi:10.1016/0001-6918(67)90070-4 
Staiger, B., Friedrich, S., \& Schütte, H.-W. (2006). China: Lexikon zu Geographie und Wirtschaft (1., Aufl.). Primus Verlag.

Unsworth, S. J., Sears, C. R., \& Pexman, P. M. (2005). Cultural Influences on Categorization Processes. Journal of Cross-Cultural Psychology, 36(6), 662-688. doi:10.1177/0022022105280509

Wang, H., \& Chang, B.-Y. (1986). A frequency dictionary in modern Chinese. Beijing: Beijing Language Institute Press.

Weekes, B., Robert, D., \& Chen, M. J. C. (2002). Picture-Word Interference Effects on Naming in Chinese. In H. S. R. Kao, C. K. Leong, \& Ding-Guo Gao (Hrsg.), Cognitive Neuroscience Studies of the Chinese Language. Hong Kong: Hong Kong University Press.

Weiß, P., \& Baratelli, S. (2003). Das Benennen von Objekten. Sprachproduktion, ; Enzyklopädie der Psychologie (Bd. 1). Göttingen: Hogrefe.

Whorf, B. L. (1956). Language, thought and reality. Cambridge, MA: MIT Press. Wong, W. (2006). Understanding Dialectical Thinking from a Cultural-Historical Perspective. Philosophical Psychology, 19(2), 239-260. doi:10.1080/09515080500462420

Yan, S., Lüer, G., \& Lass, U. (2005). Kulturvergleichende Wahrnehmungs- und Kognitionsforschung. Enzyklopädie der Psychologie: Kulturvergleichende Psychologie (Bd. Band II). Göttingen: Hogrefe.

Zhang, Q., \& Yang, Y. (2005). The Phonological Planning Unit in Chinese Monosyllabic Word Production. Psychological Science, 28(2), 374 - 378.

Zhou, X., Zhuang, J., Wu, J., \& Yang, D. (2003). Phonological, orthographic and semantic activation in the speech production of Chinese. Acta Psychologica Sinica, (6), 712718. 


\section{Anhang}

Anhang A: Itemmaterial Experiment 1 ,Kategorisierung über Bildpaare“

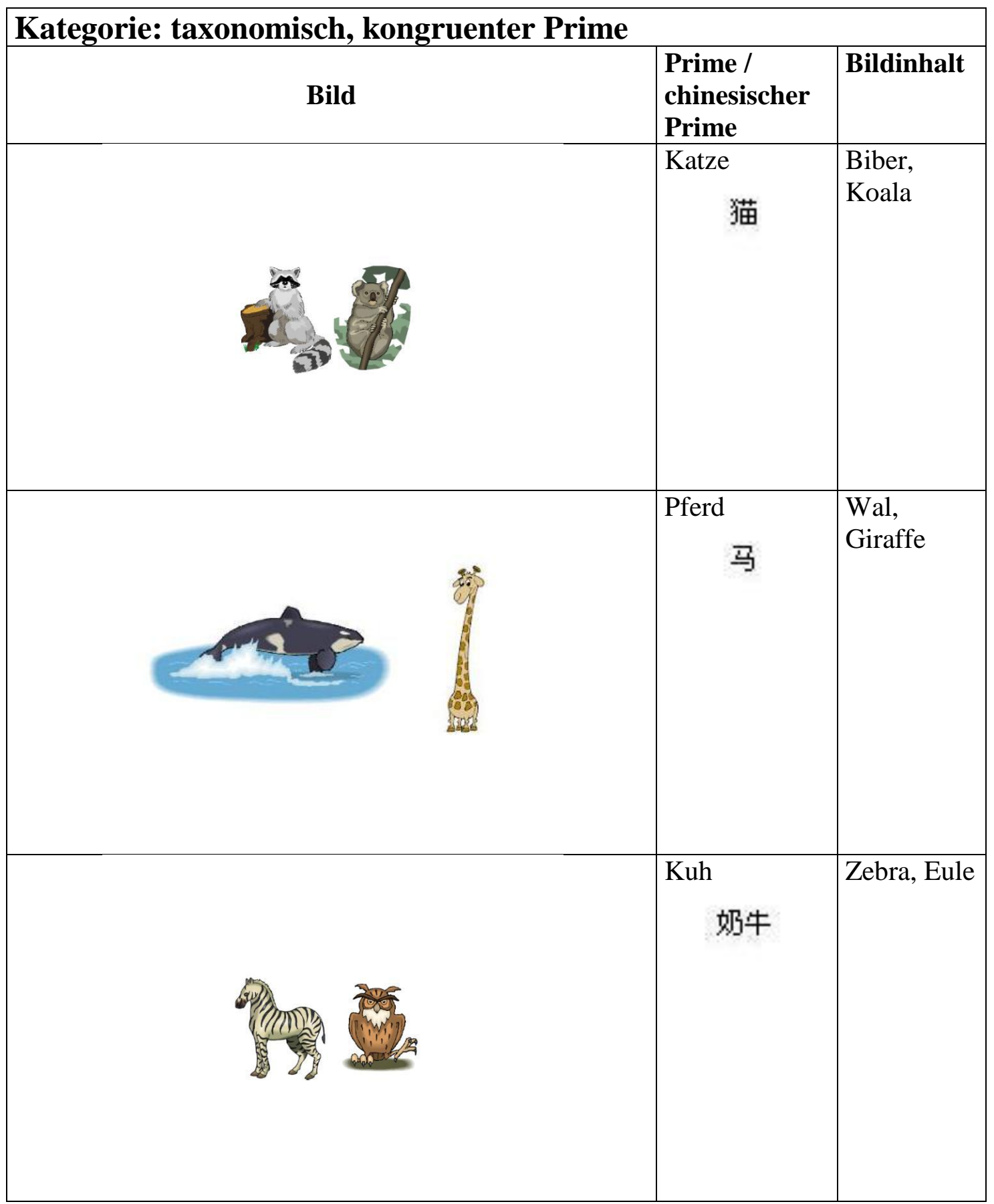




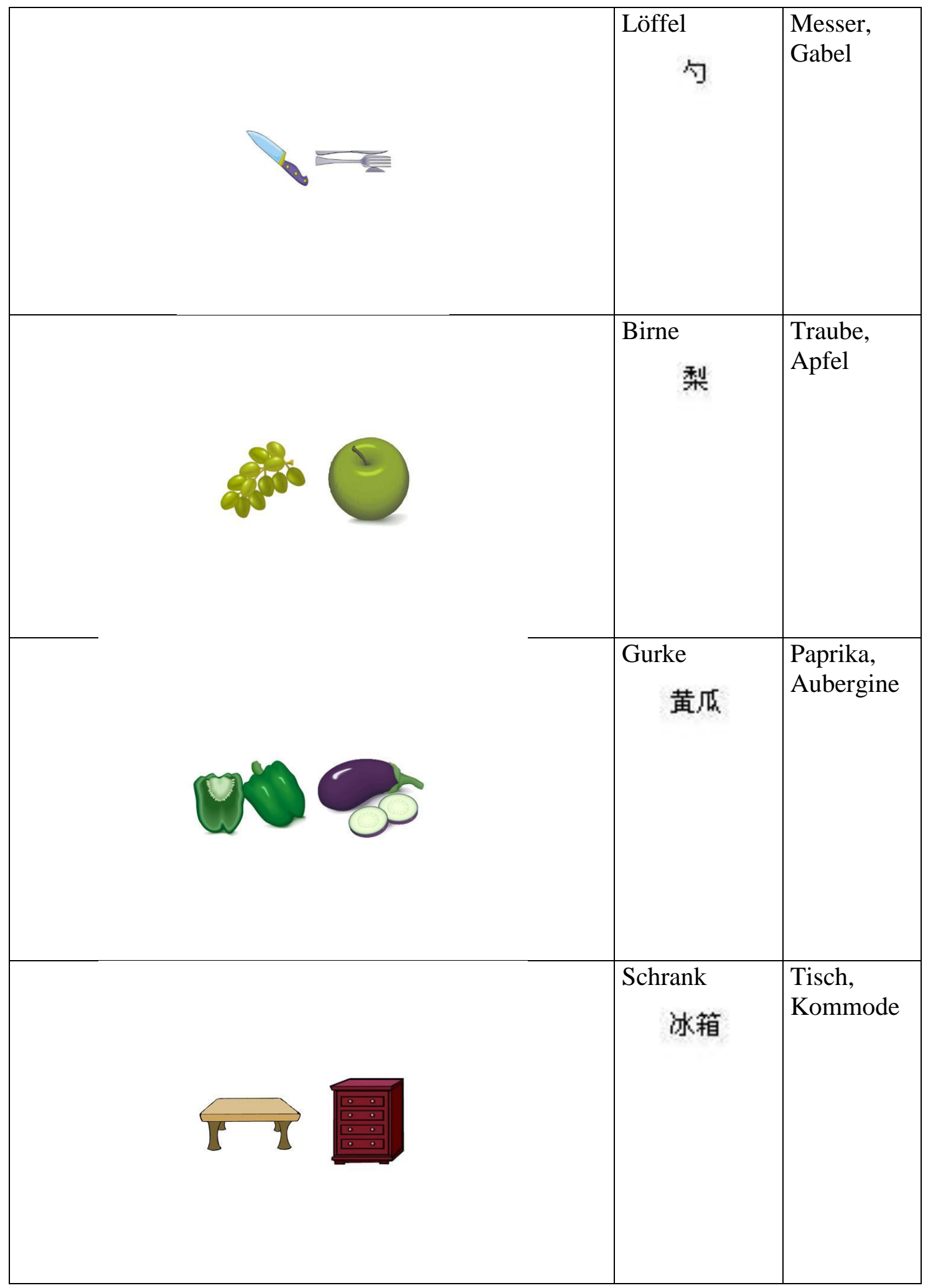




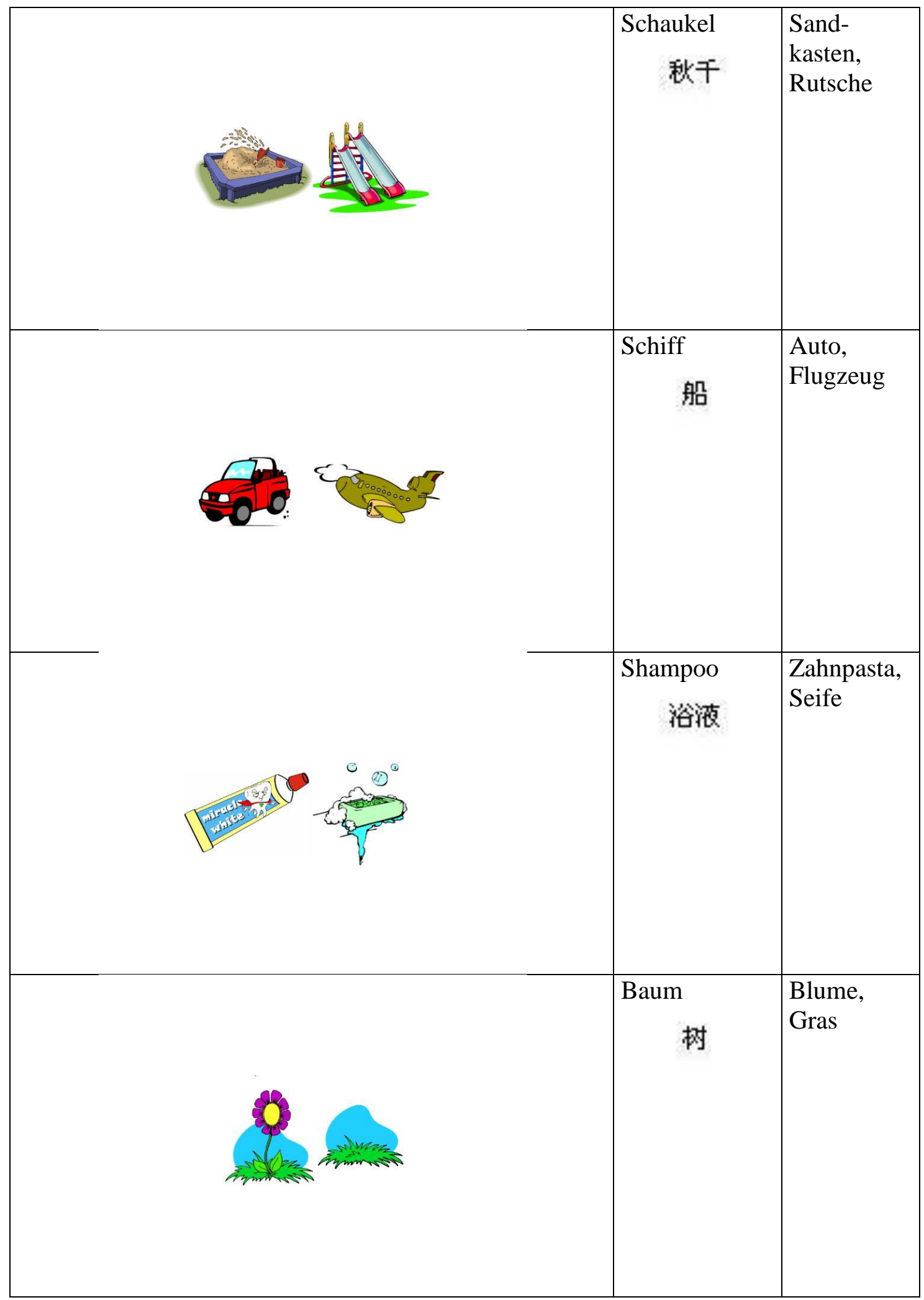




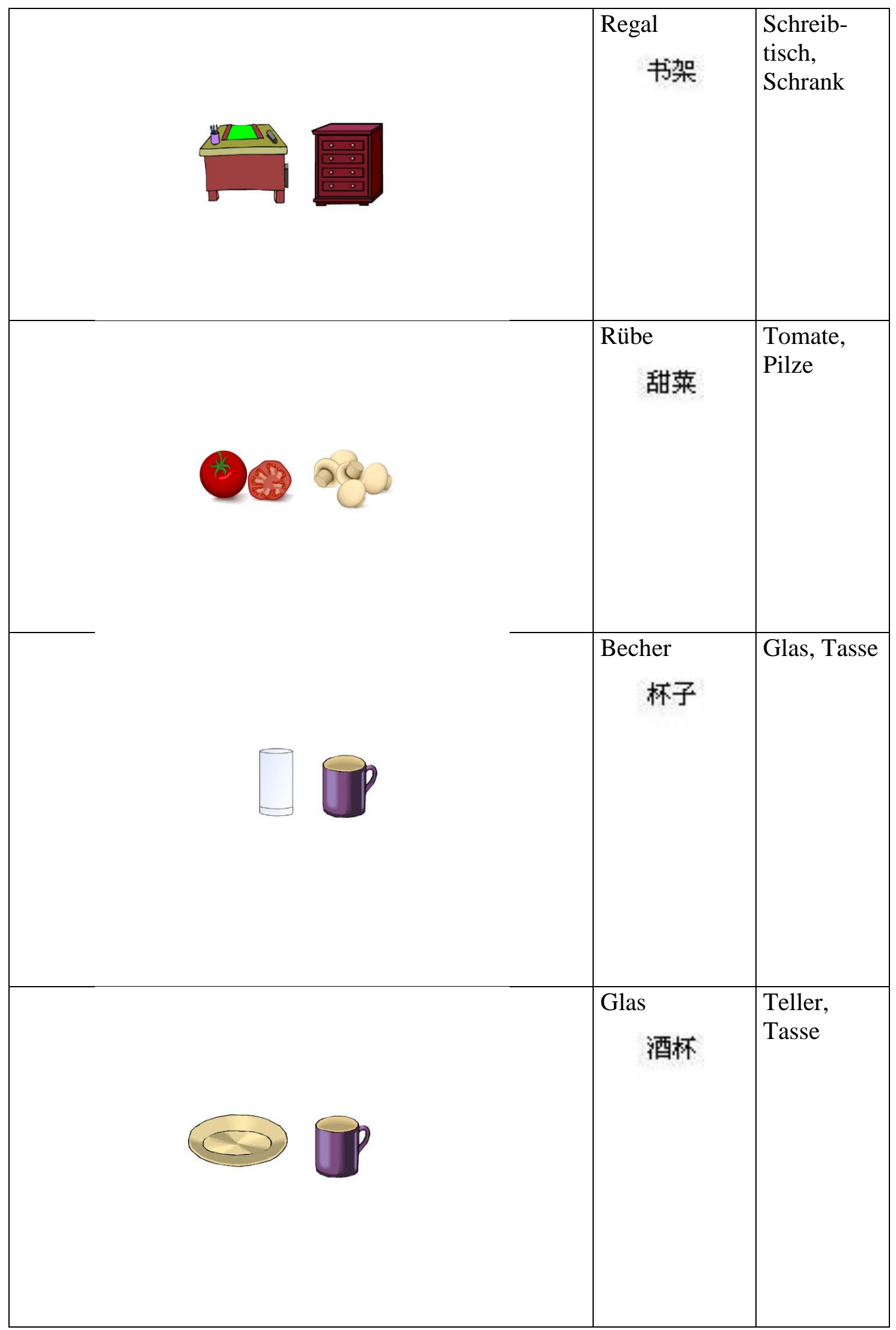


Kategorie: taxonomisch, inkongruenter Prime

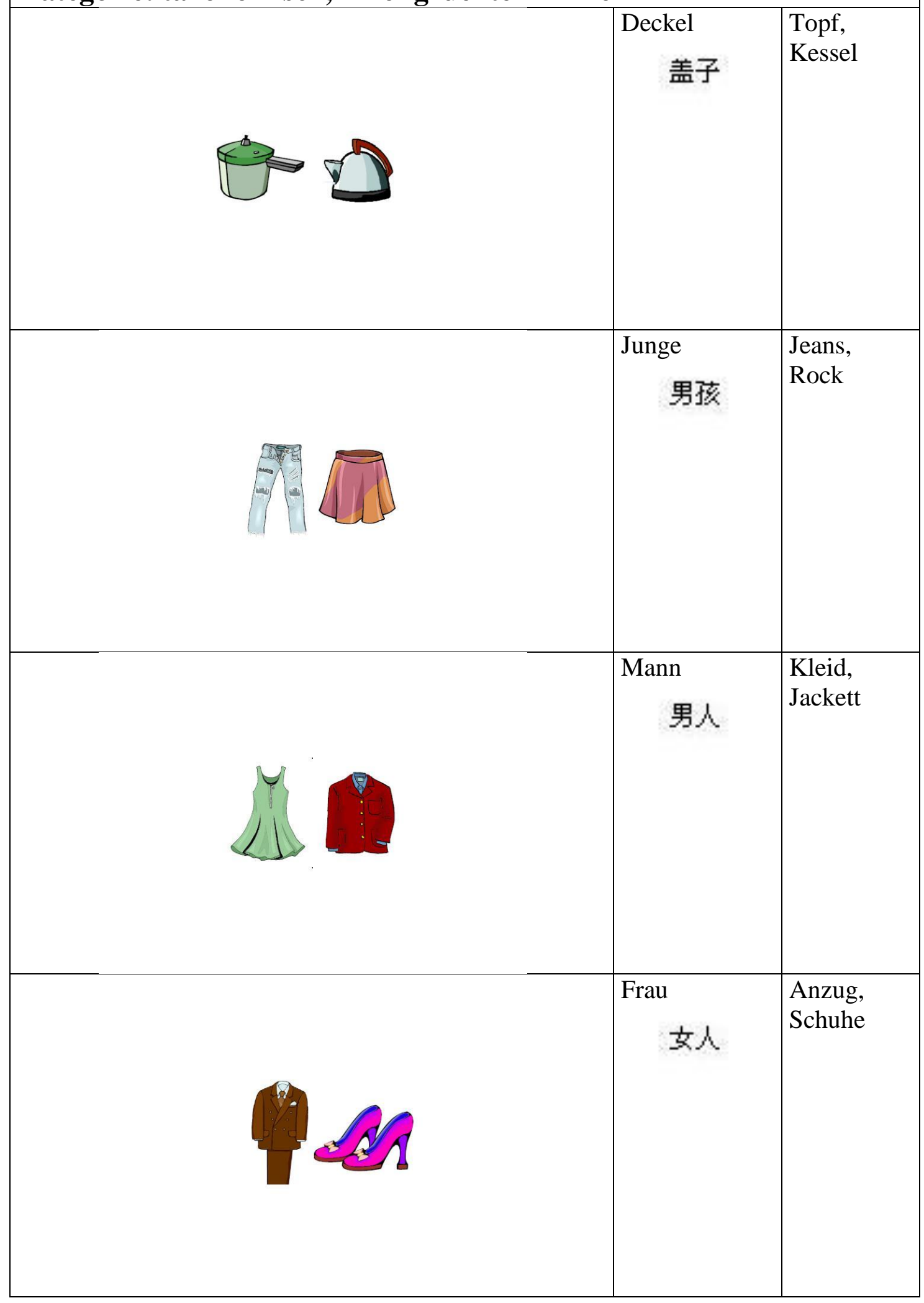




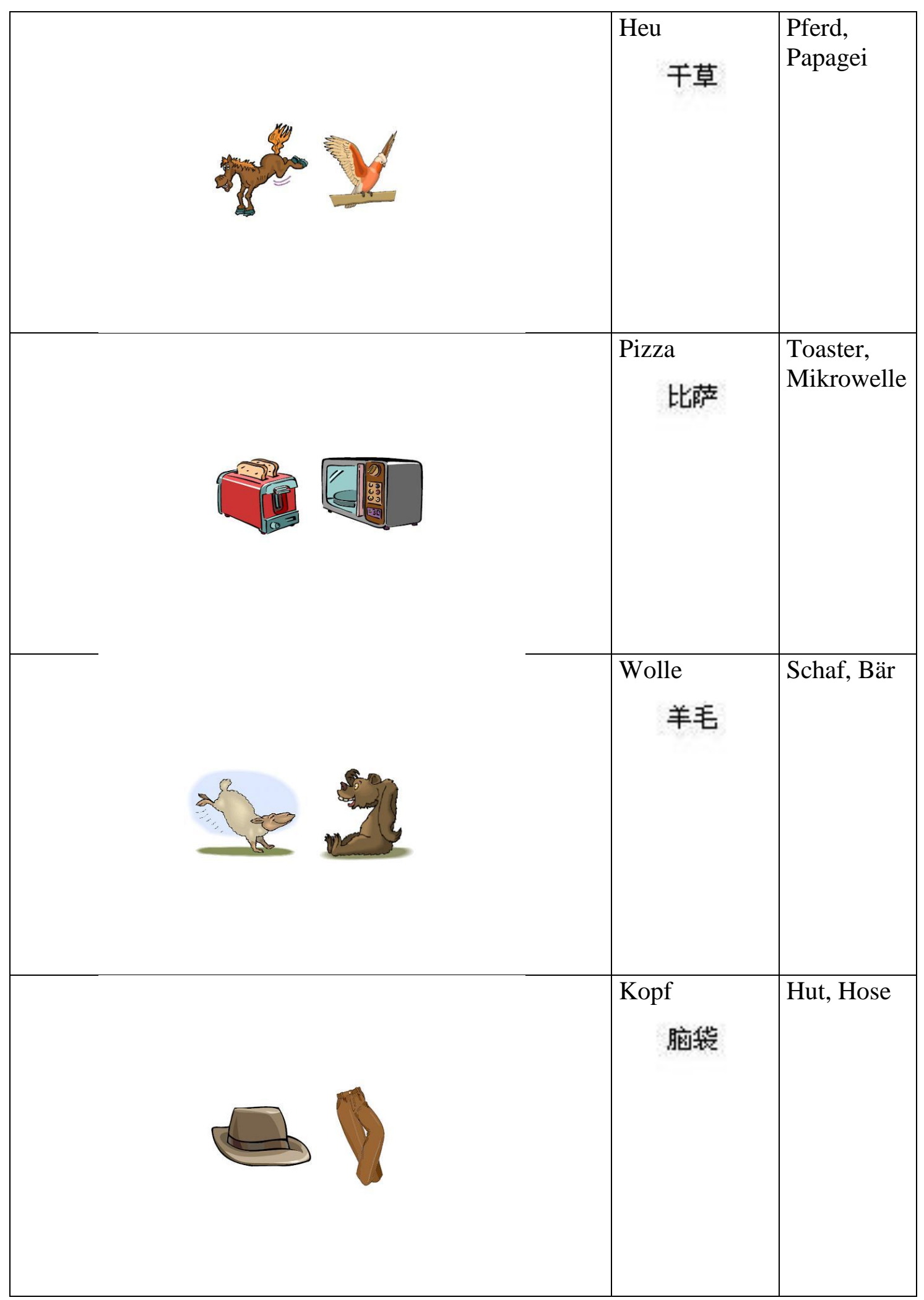




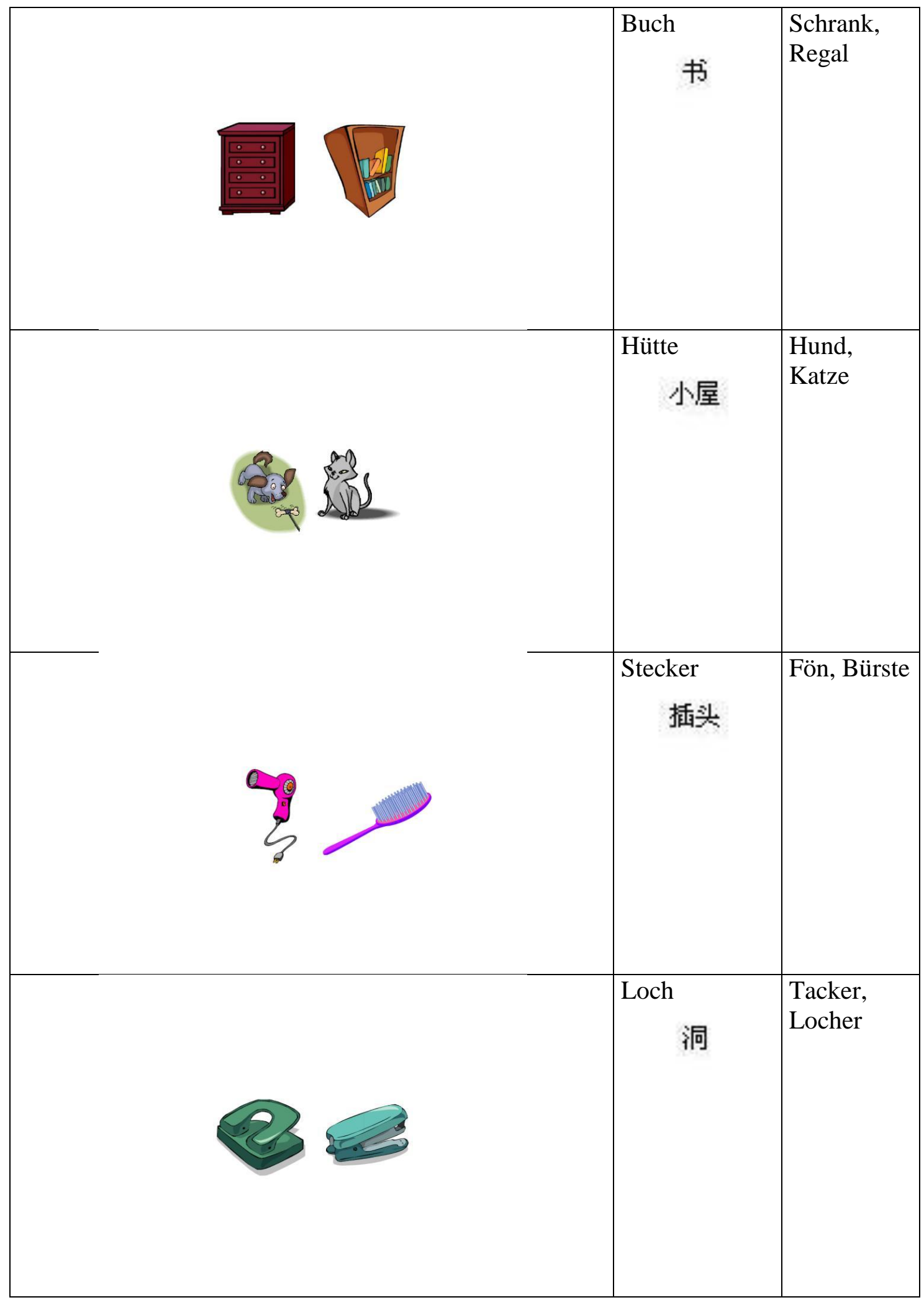




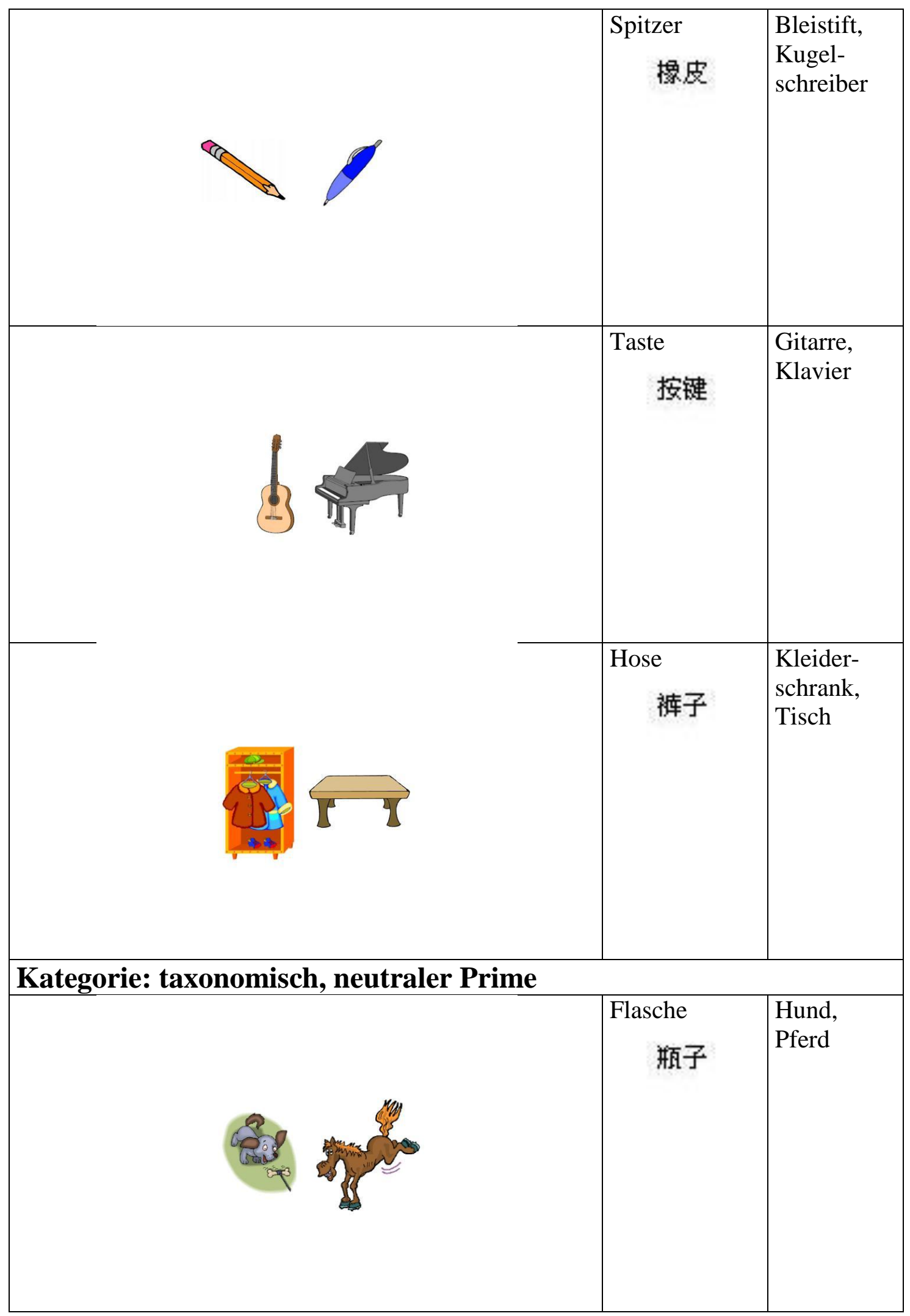




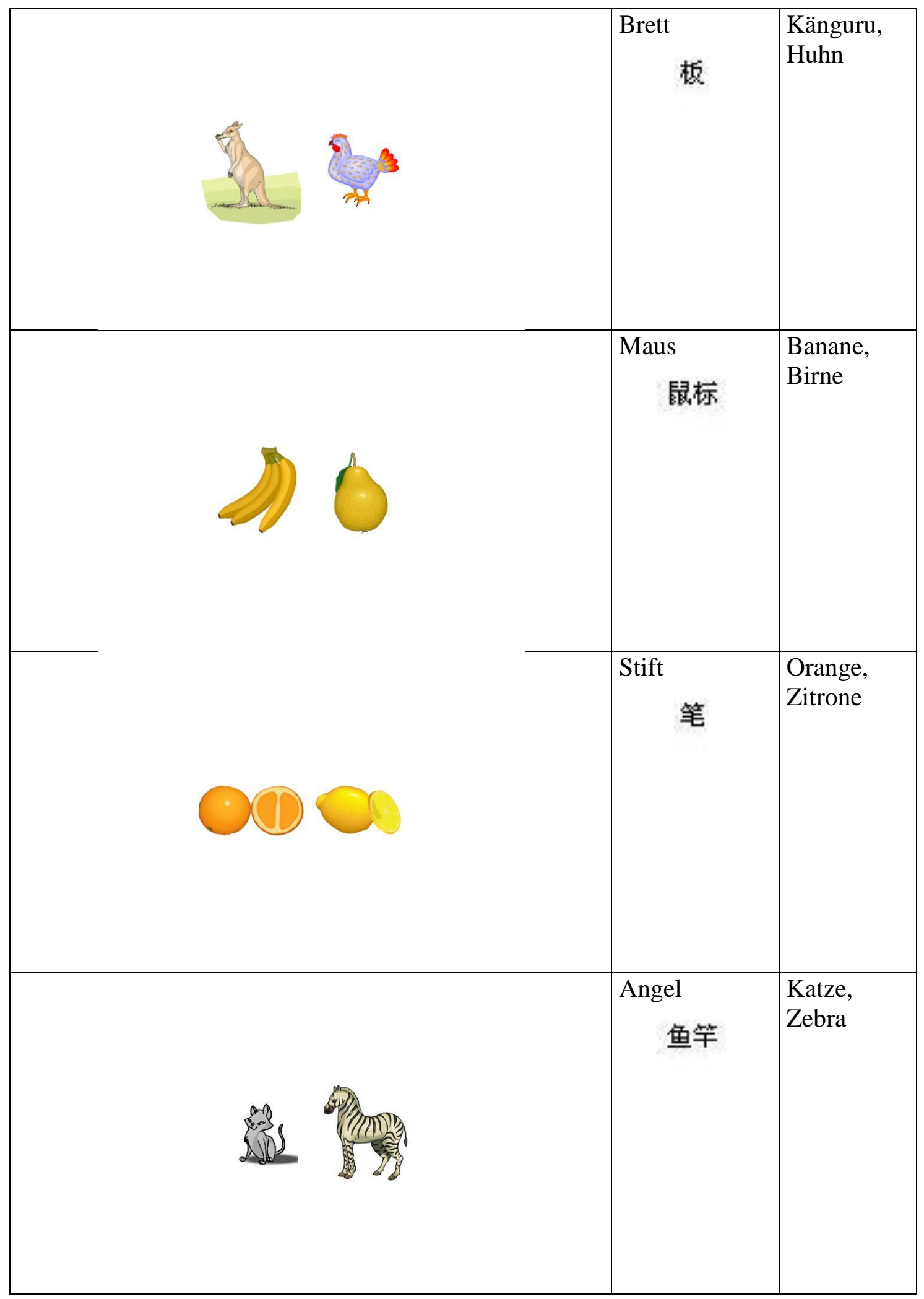




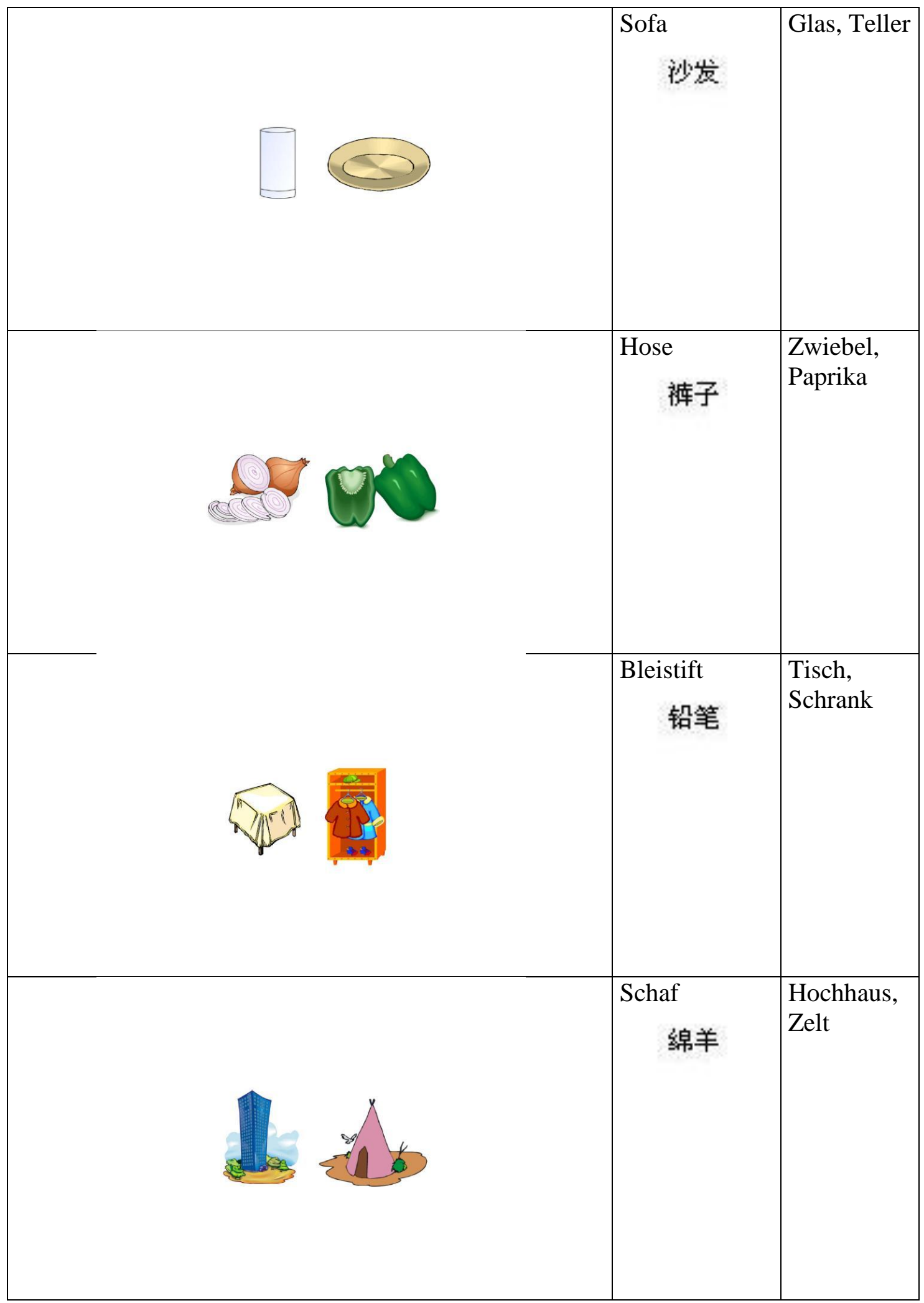




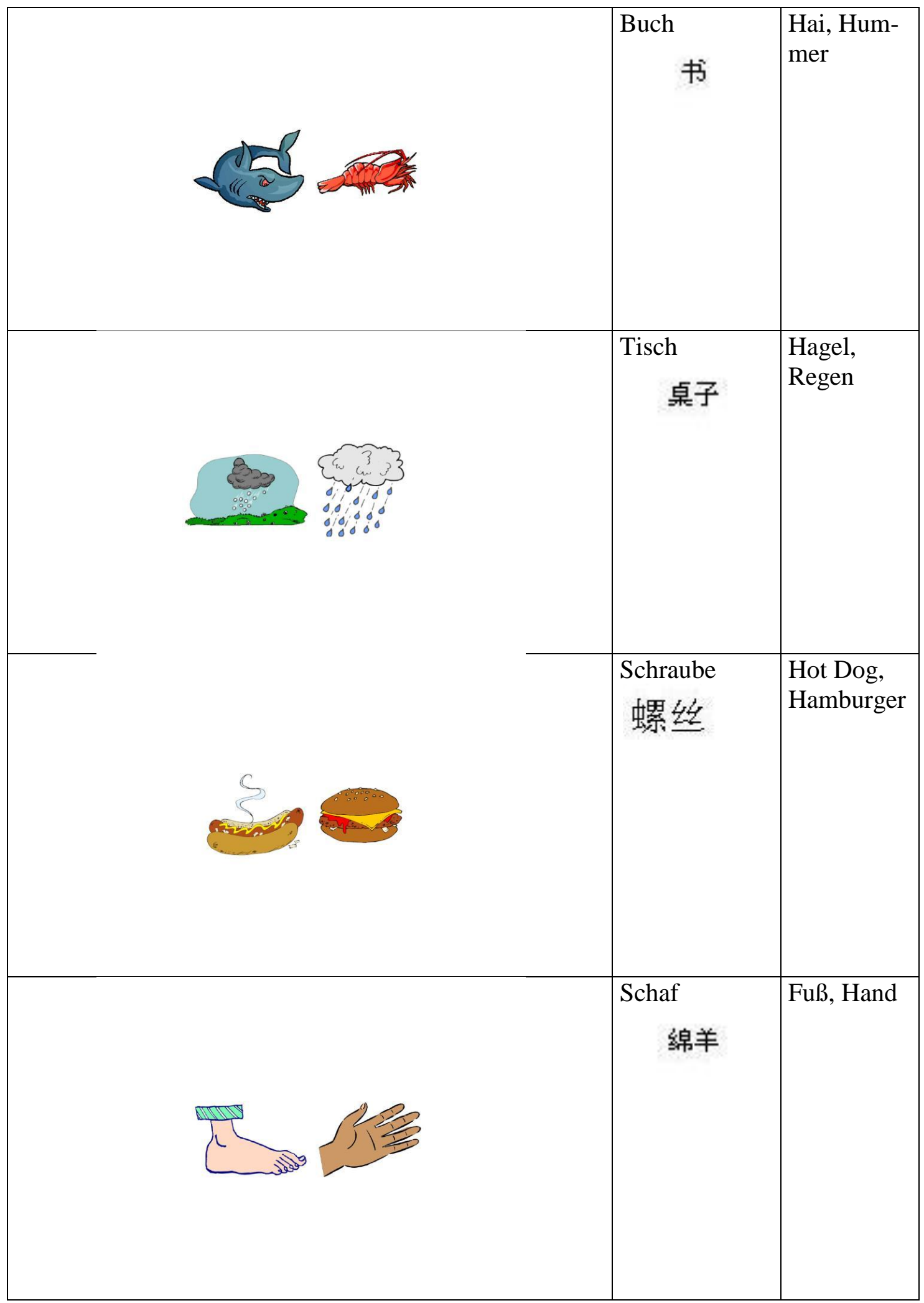




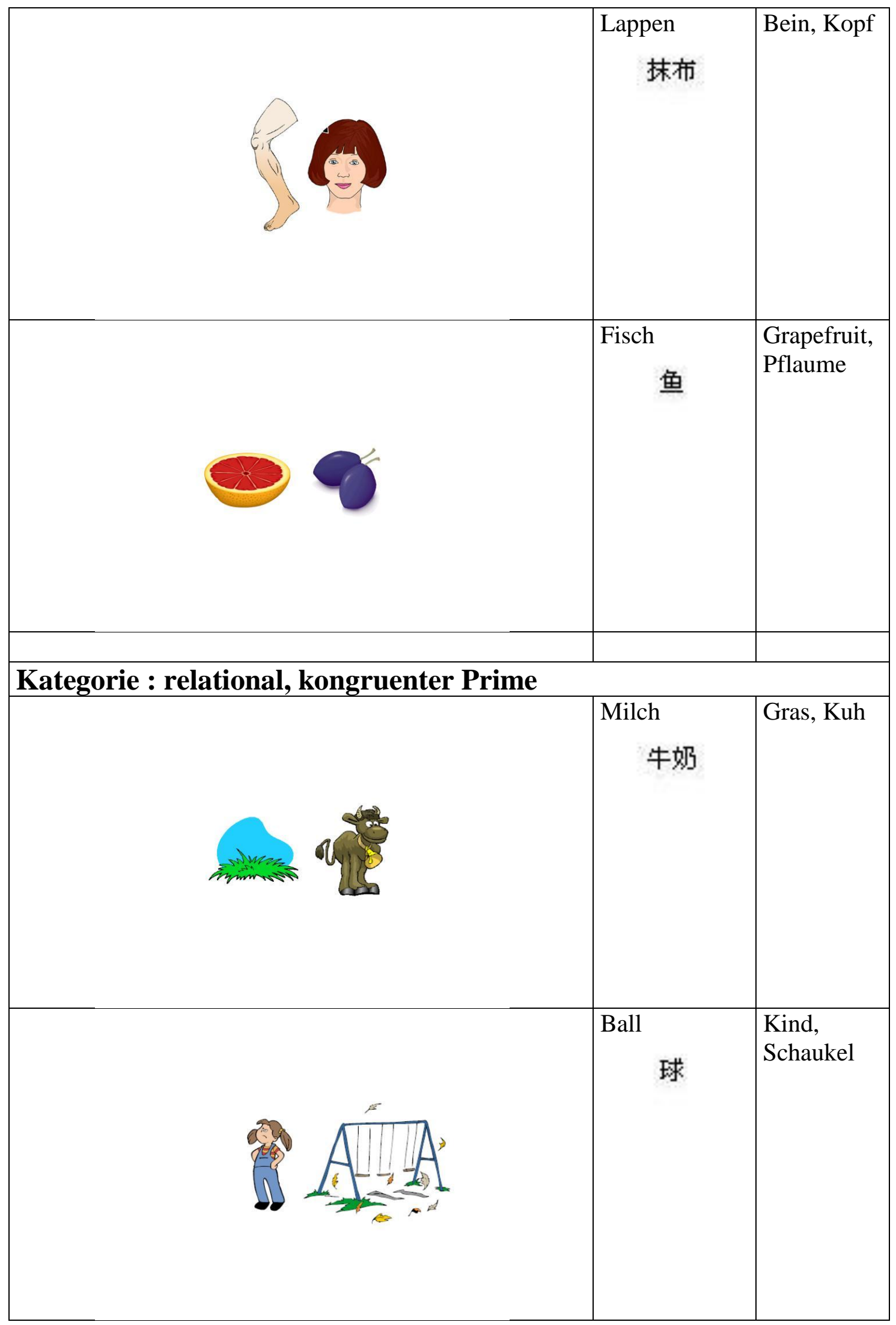




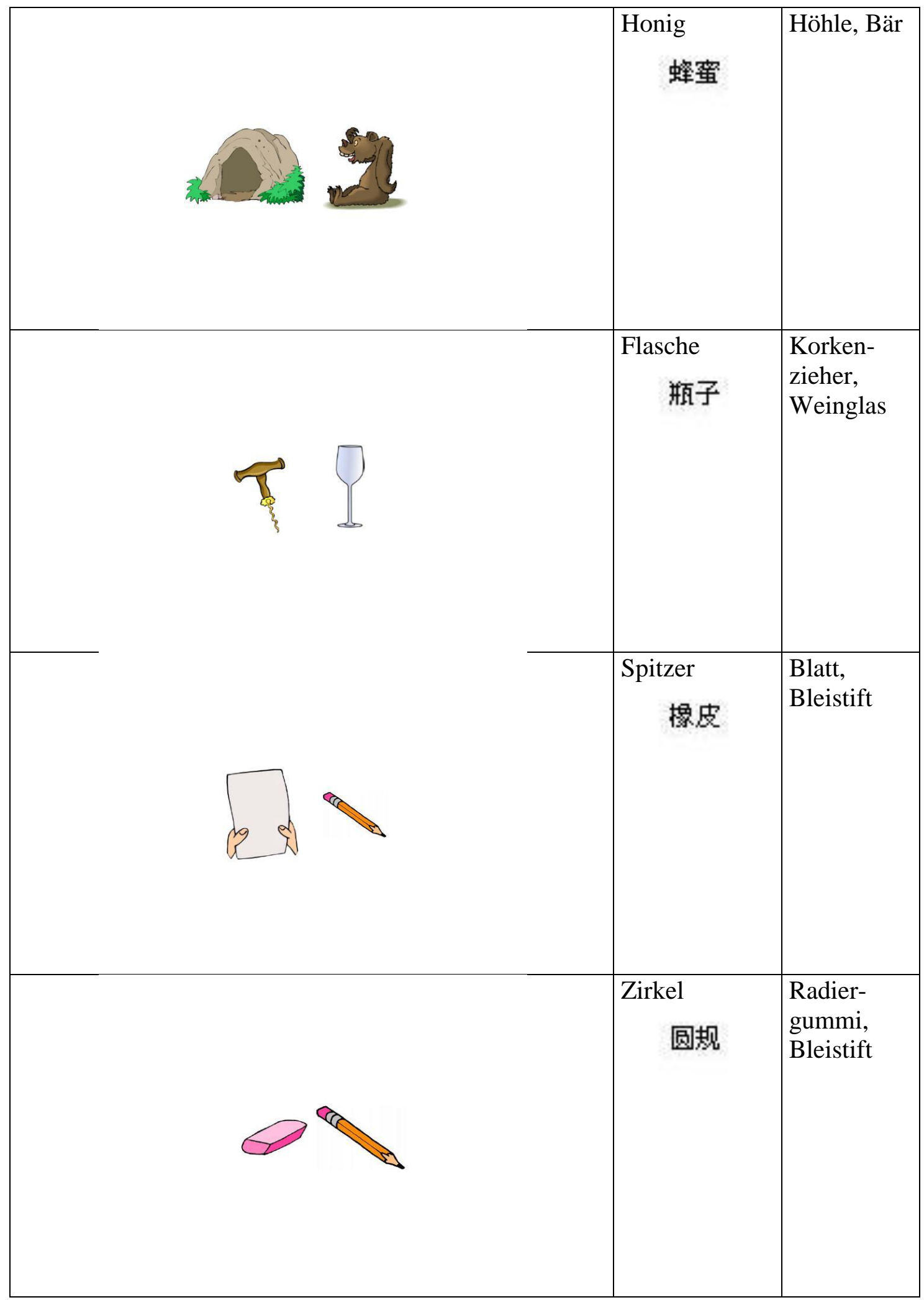




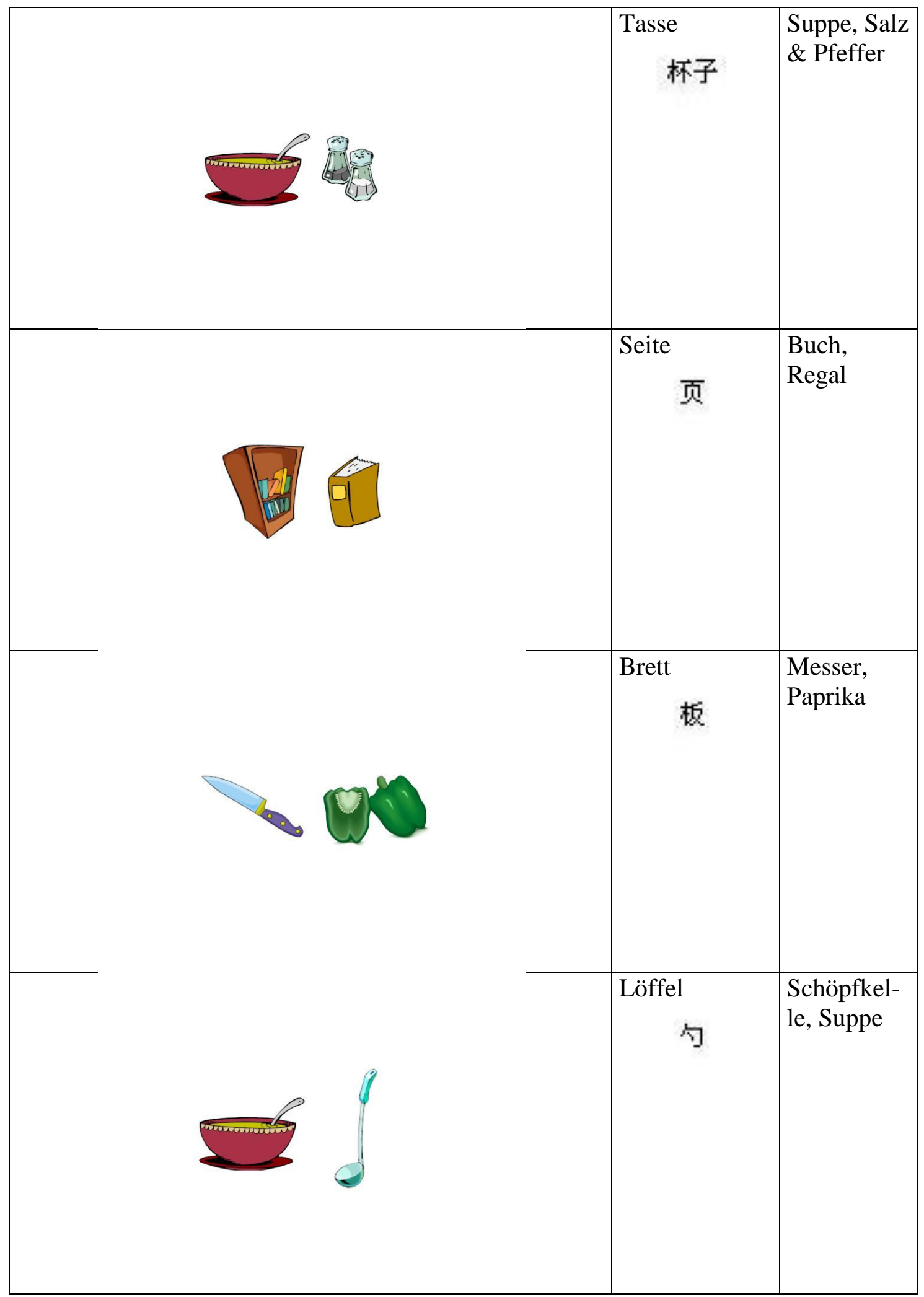




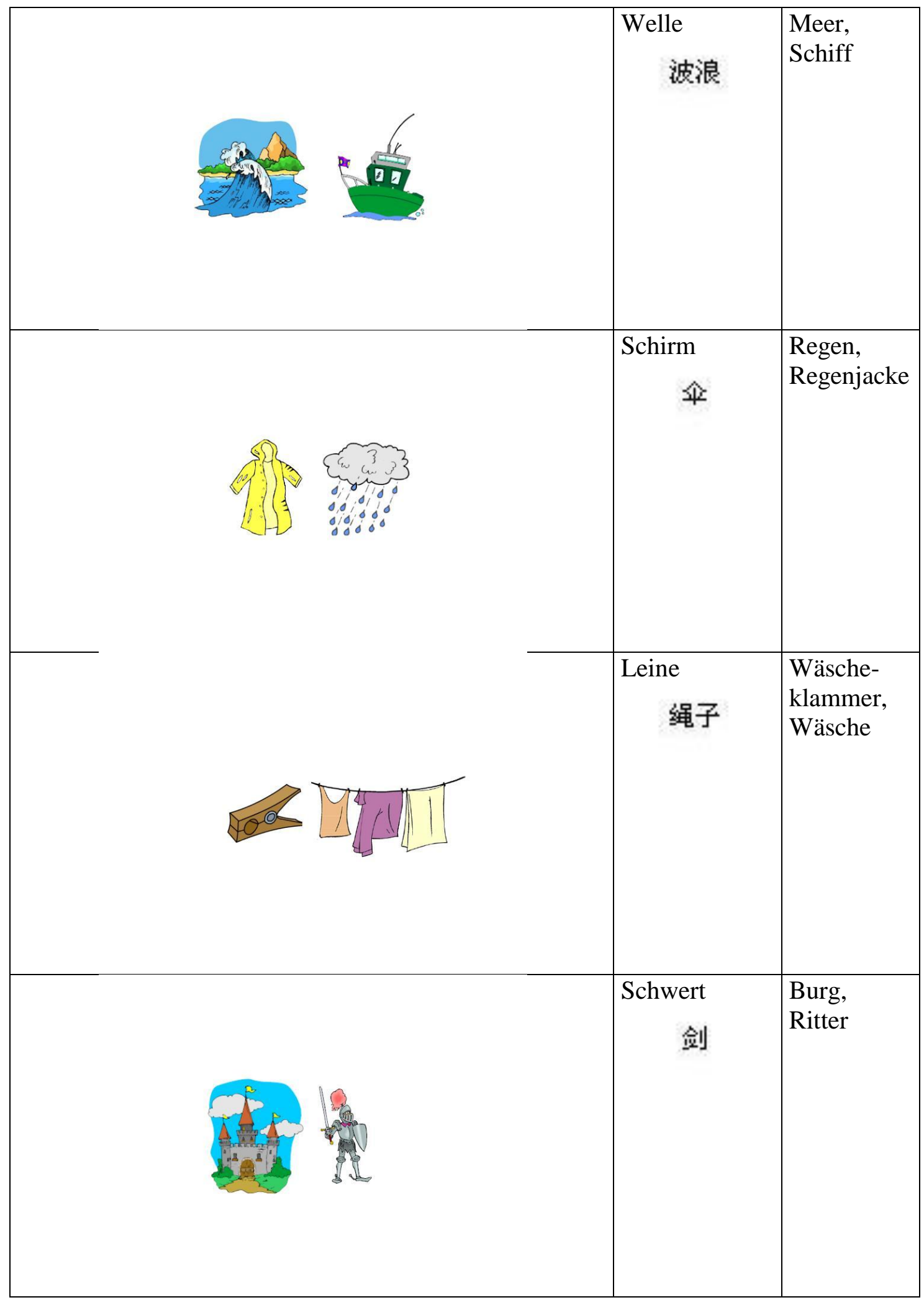




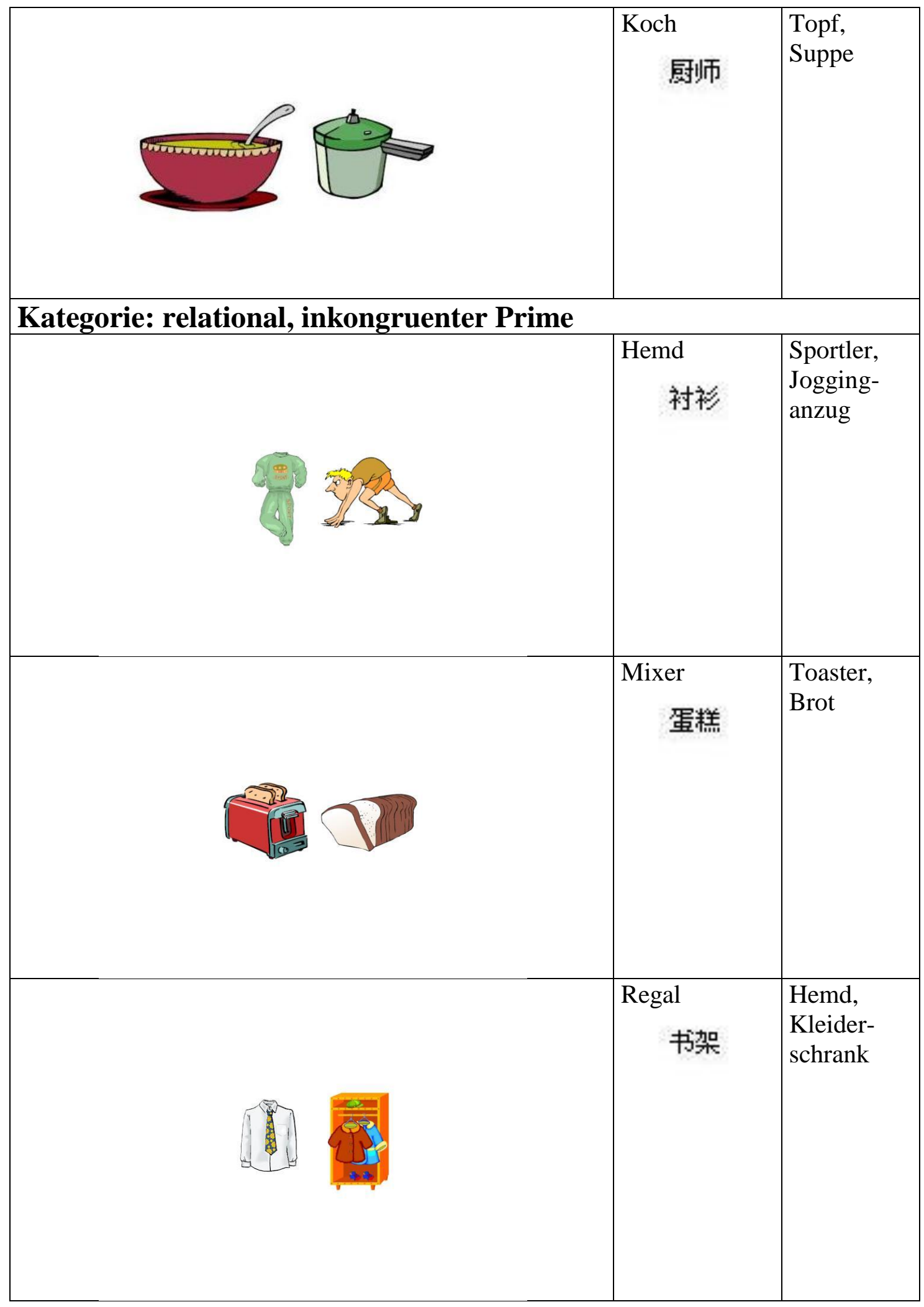




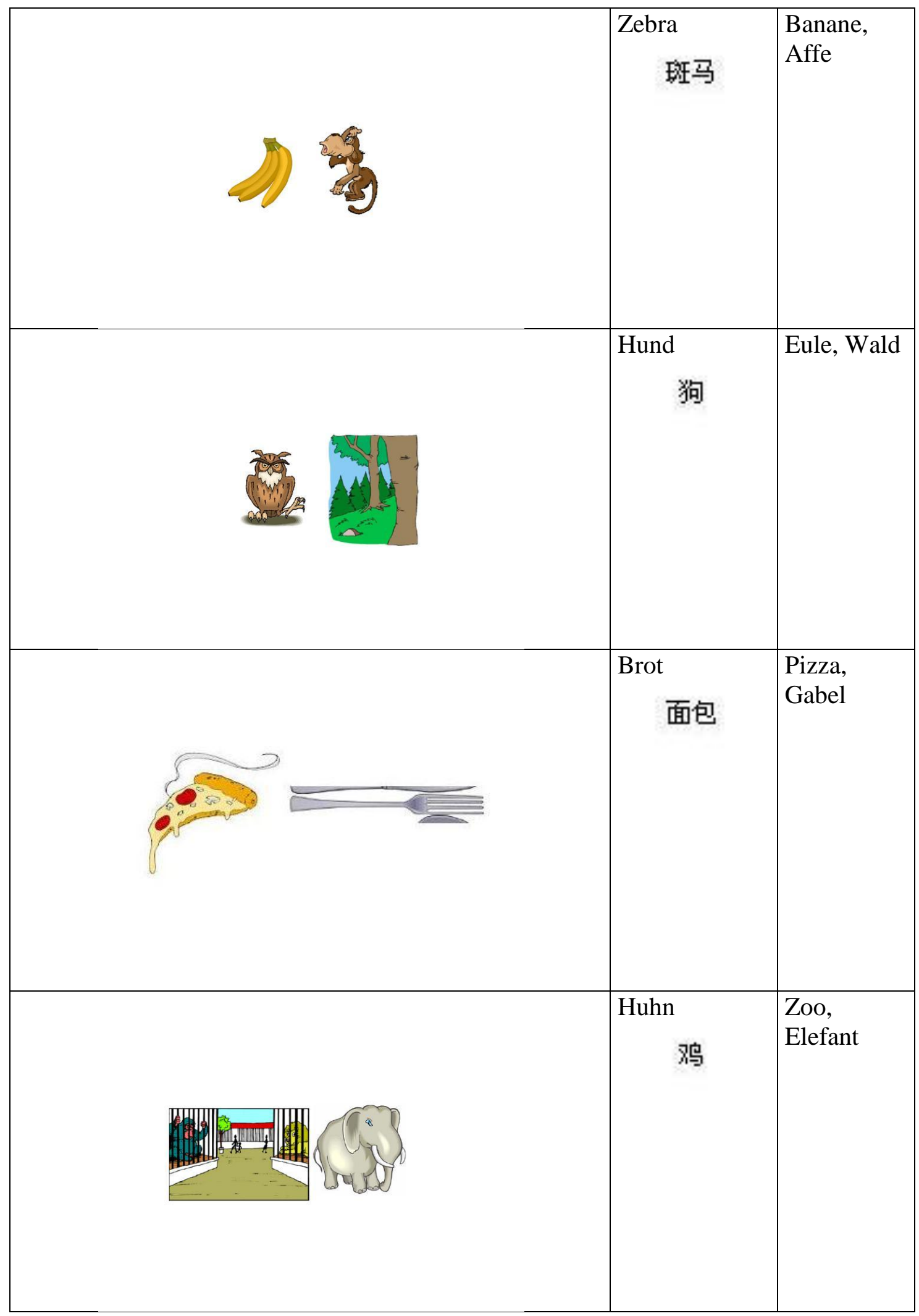




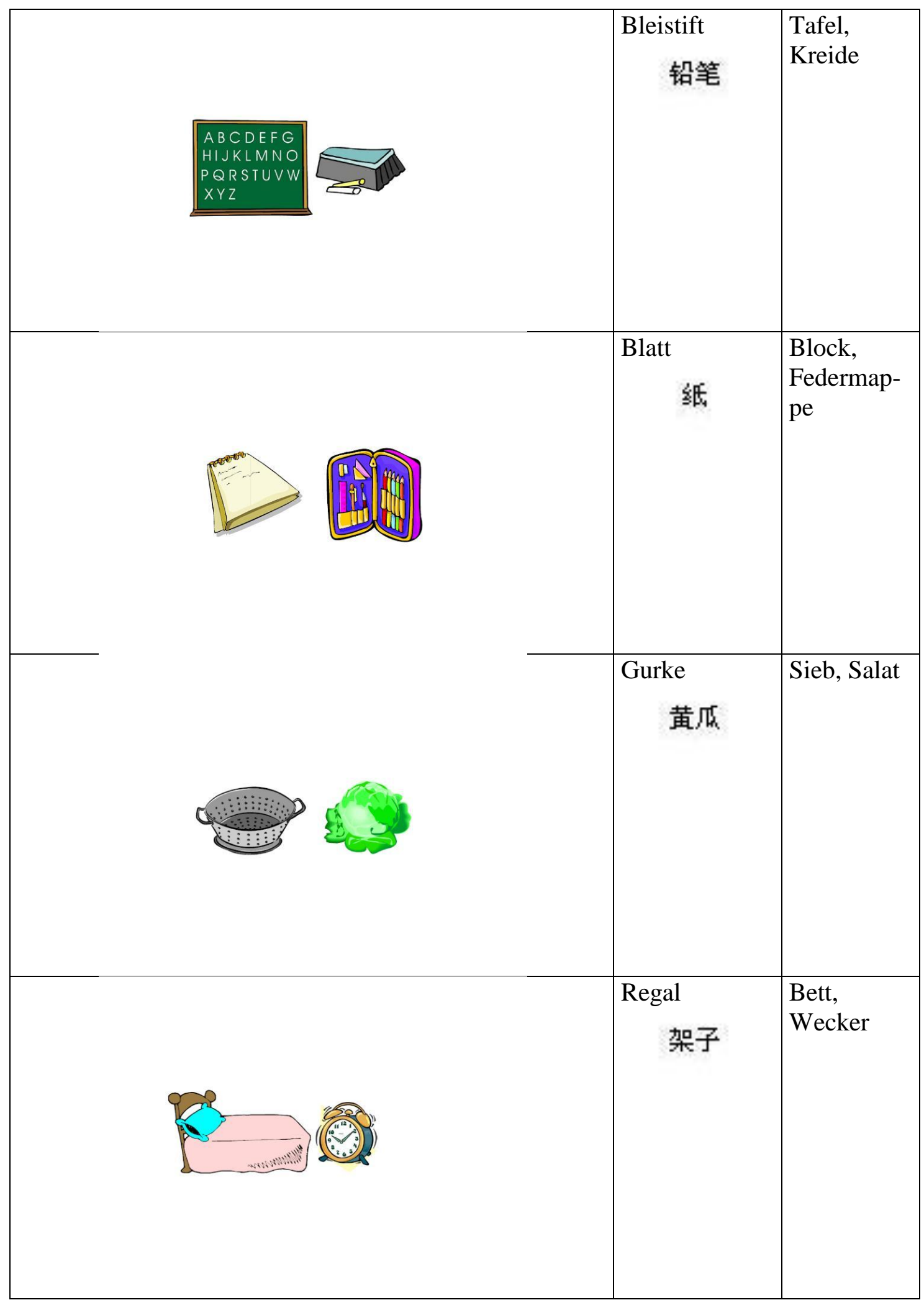




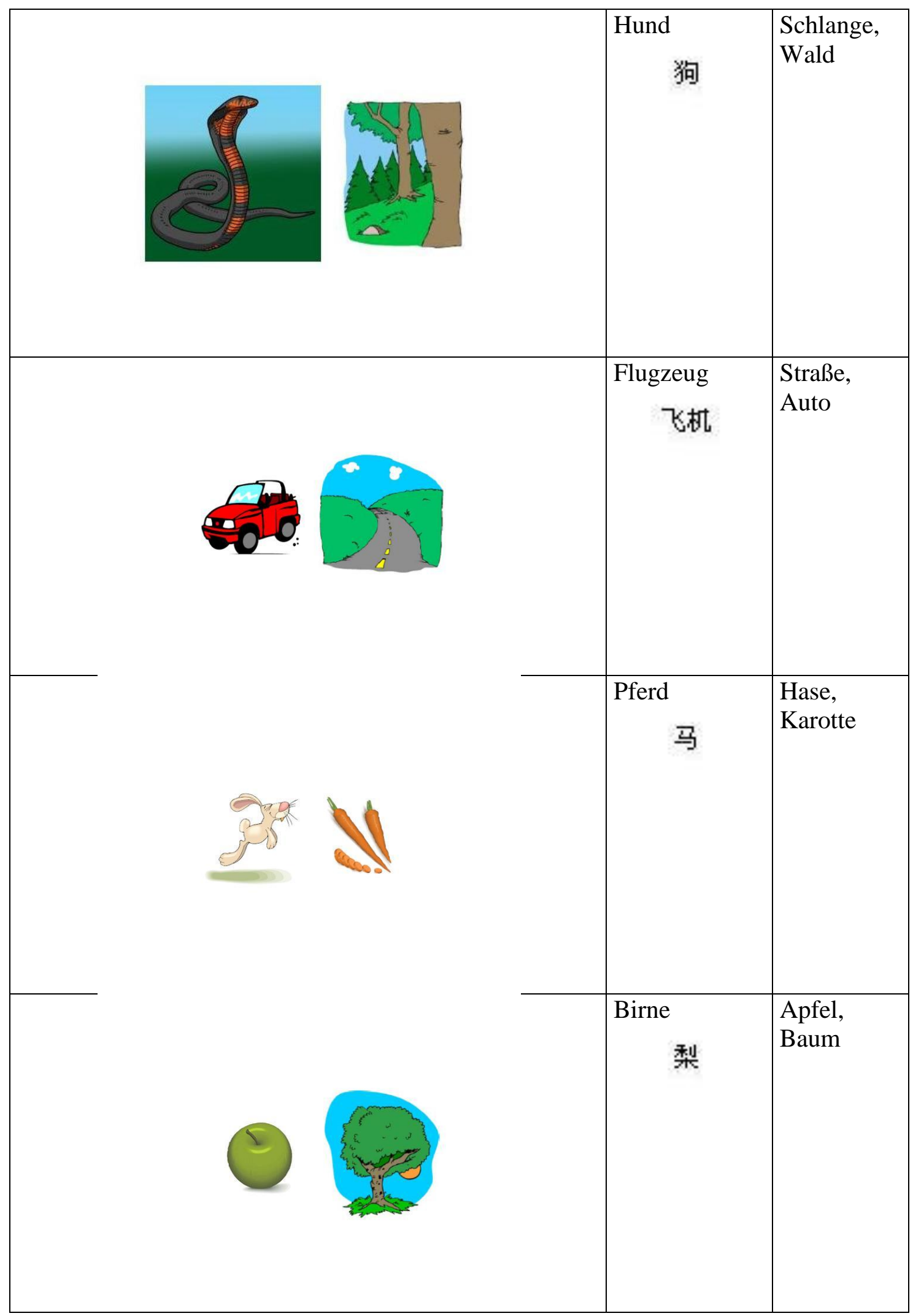




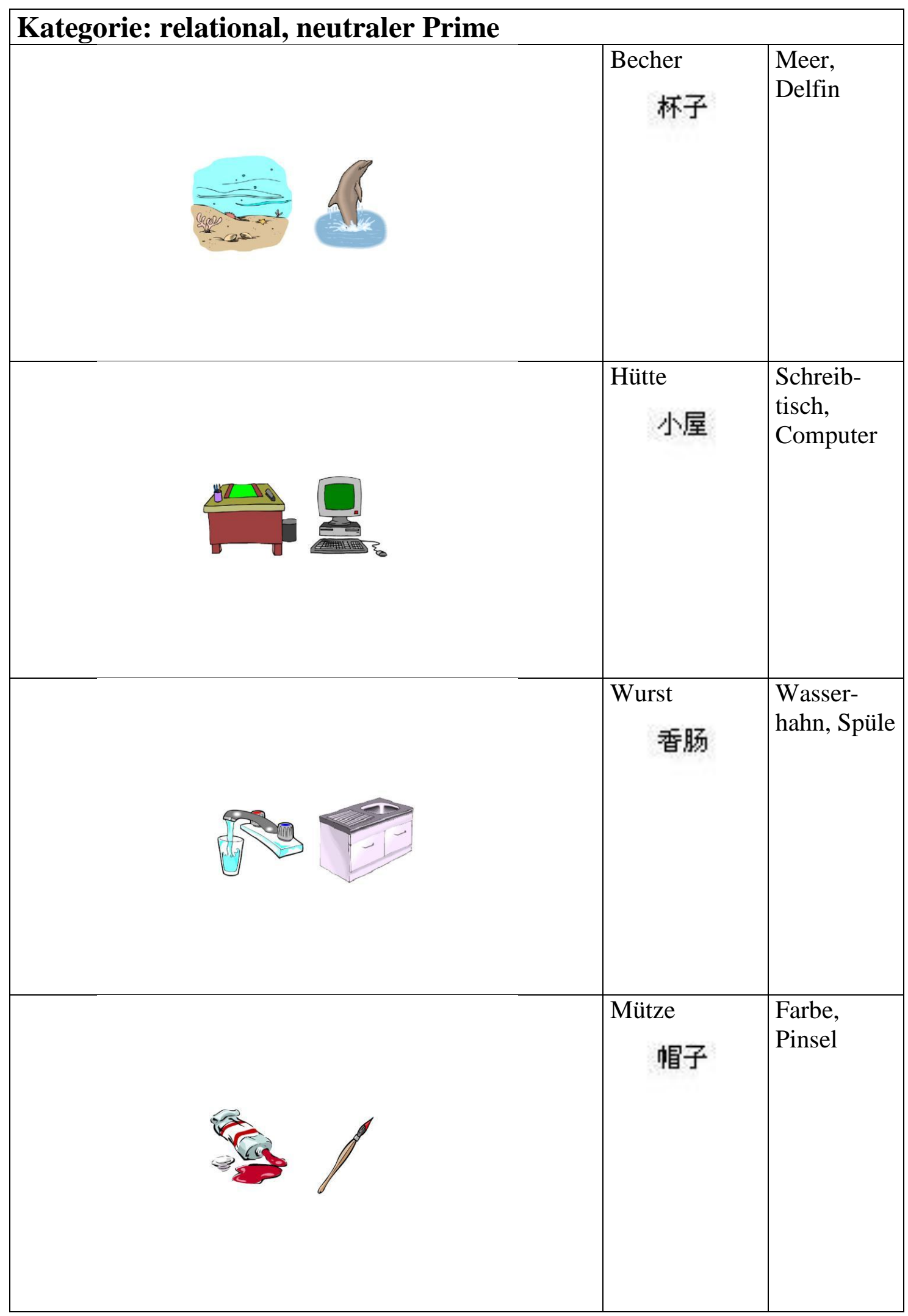




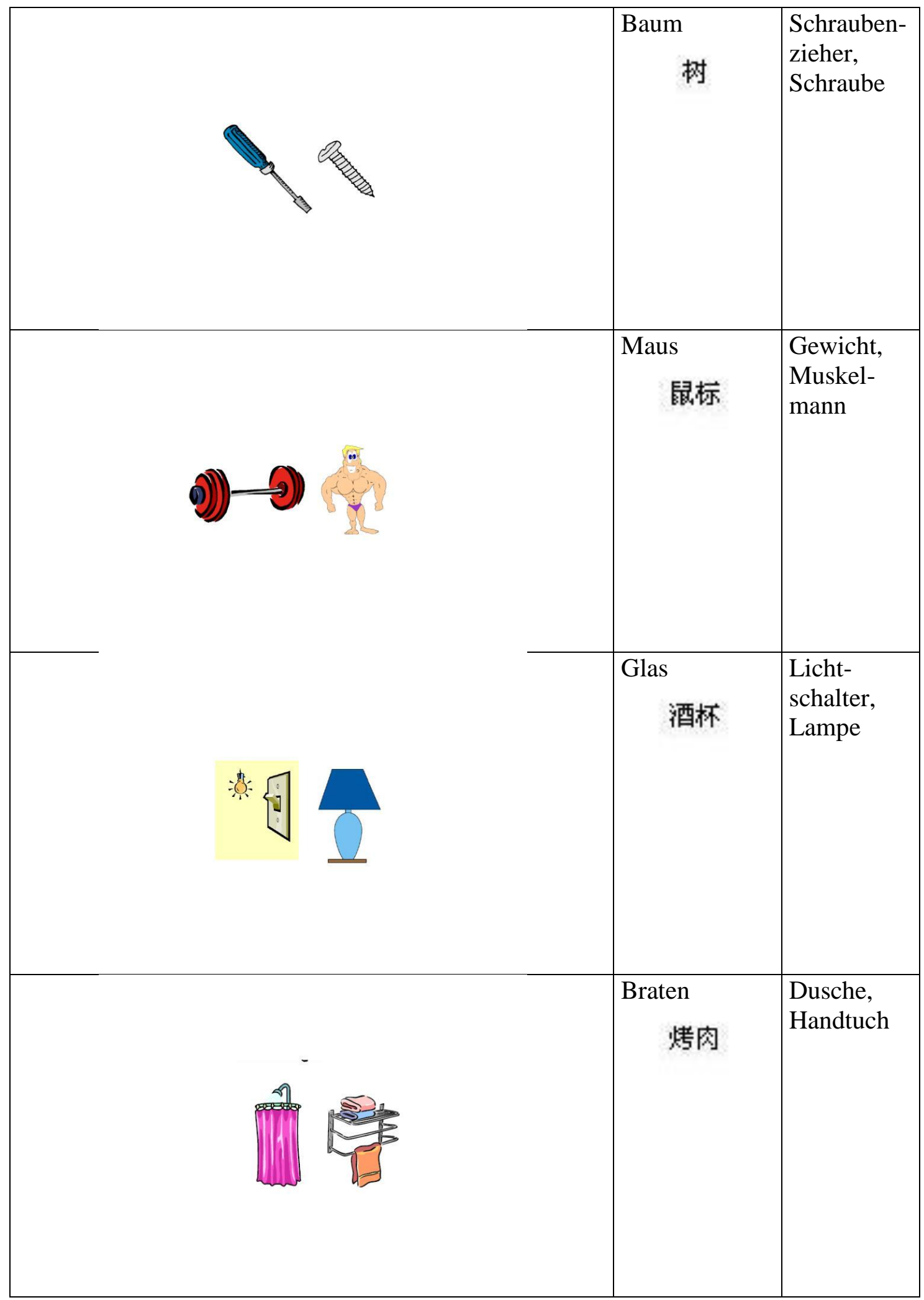




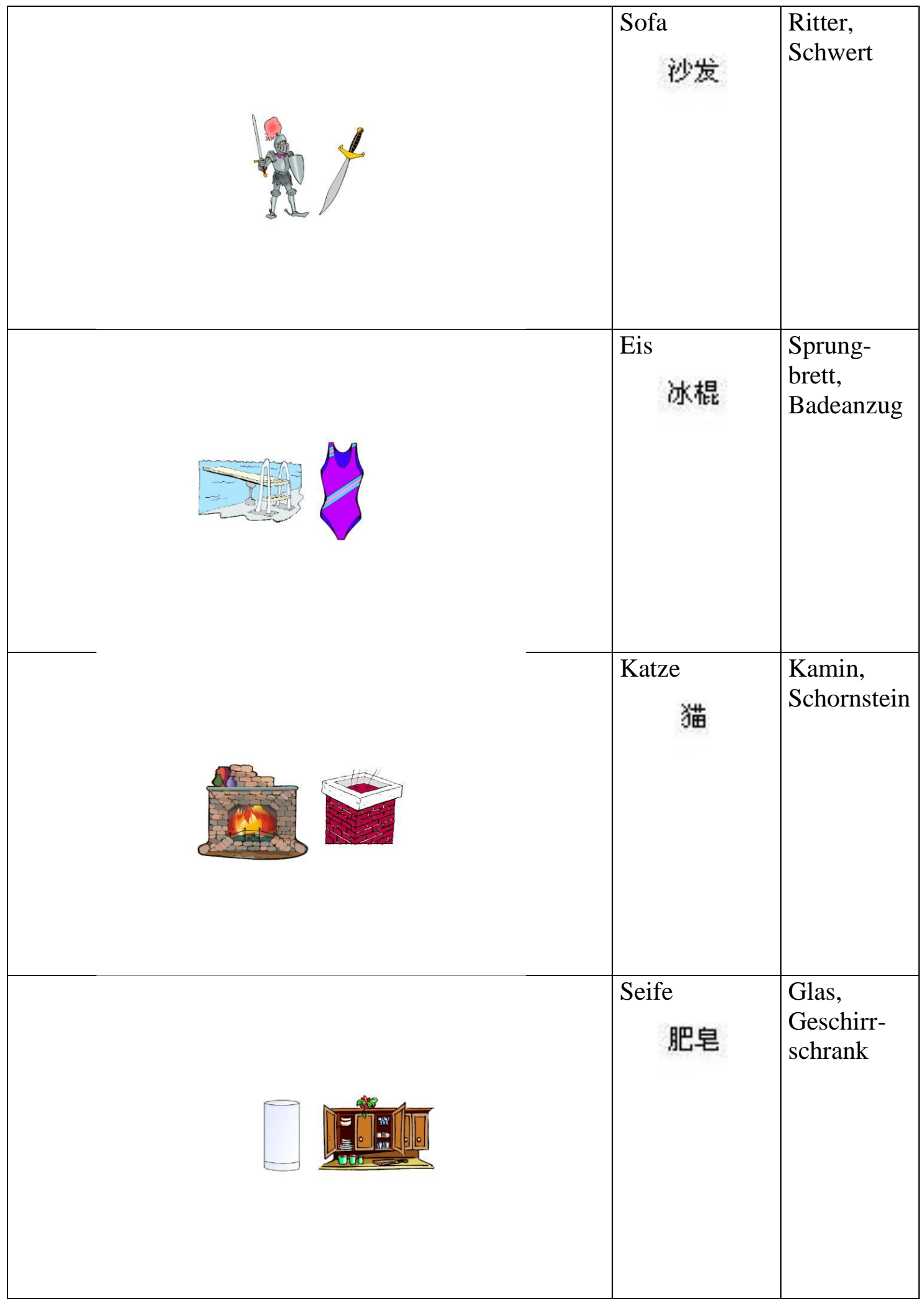




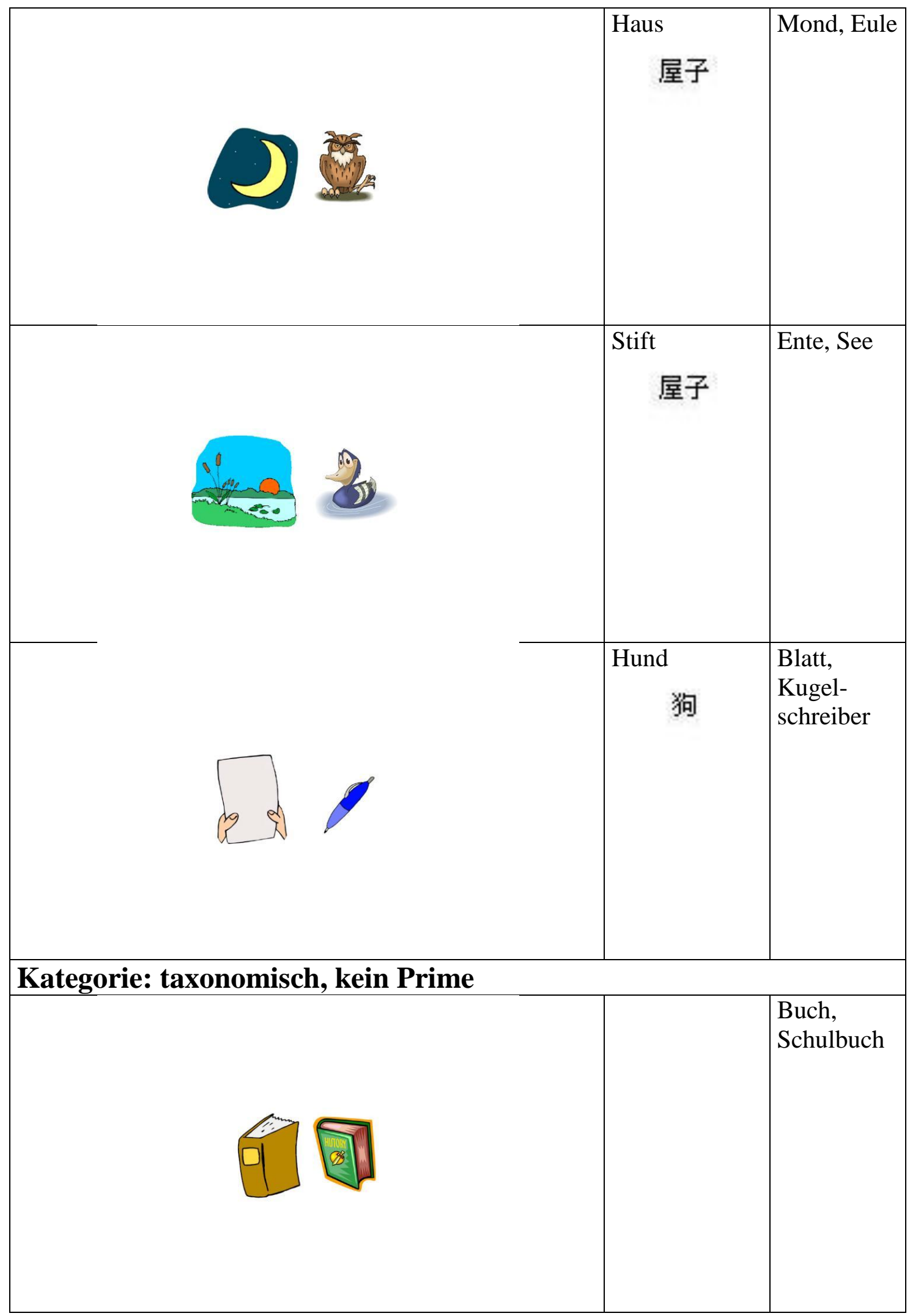




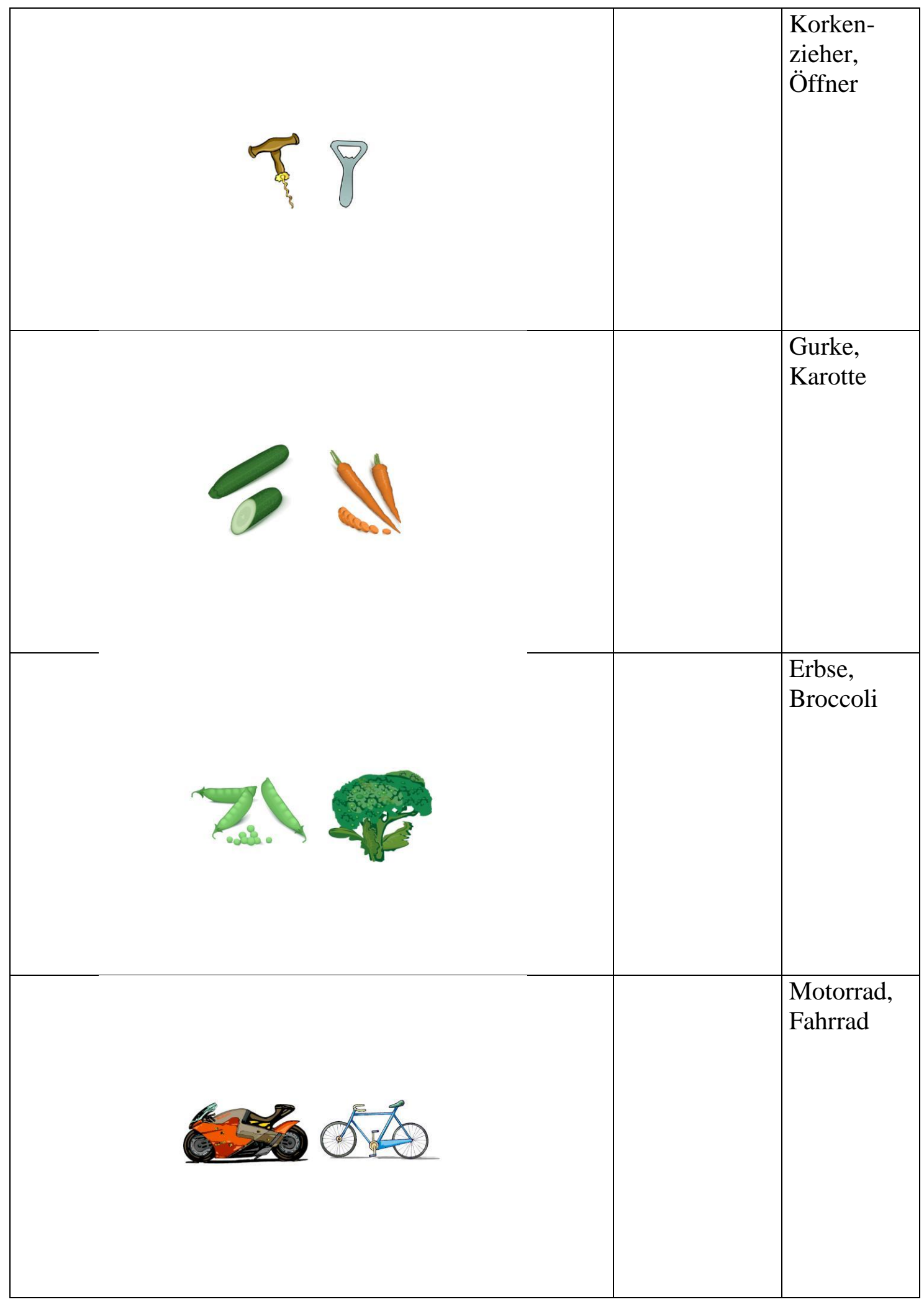




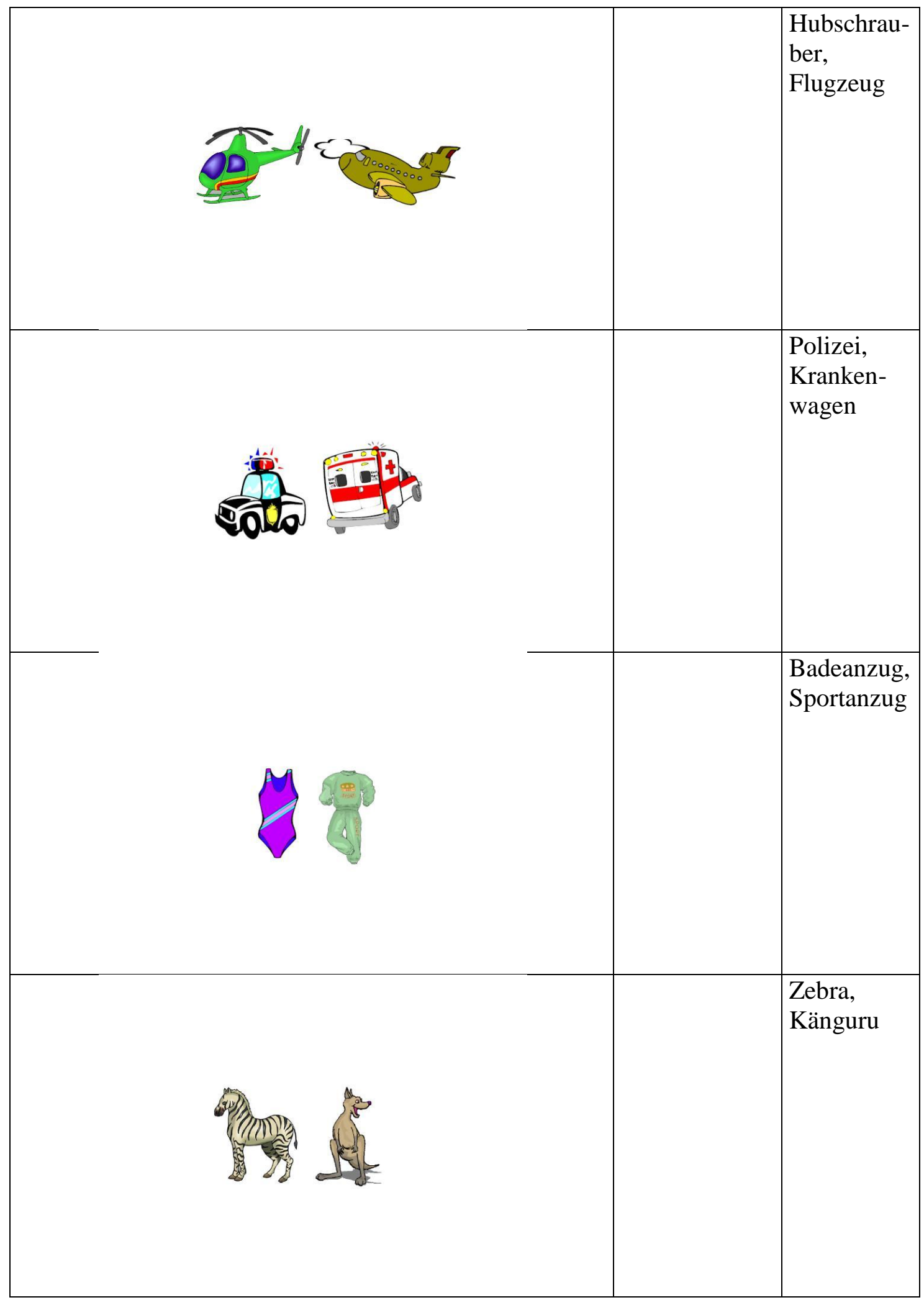




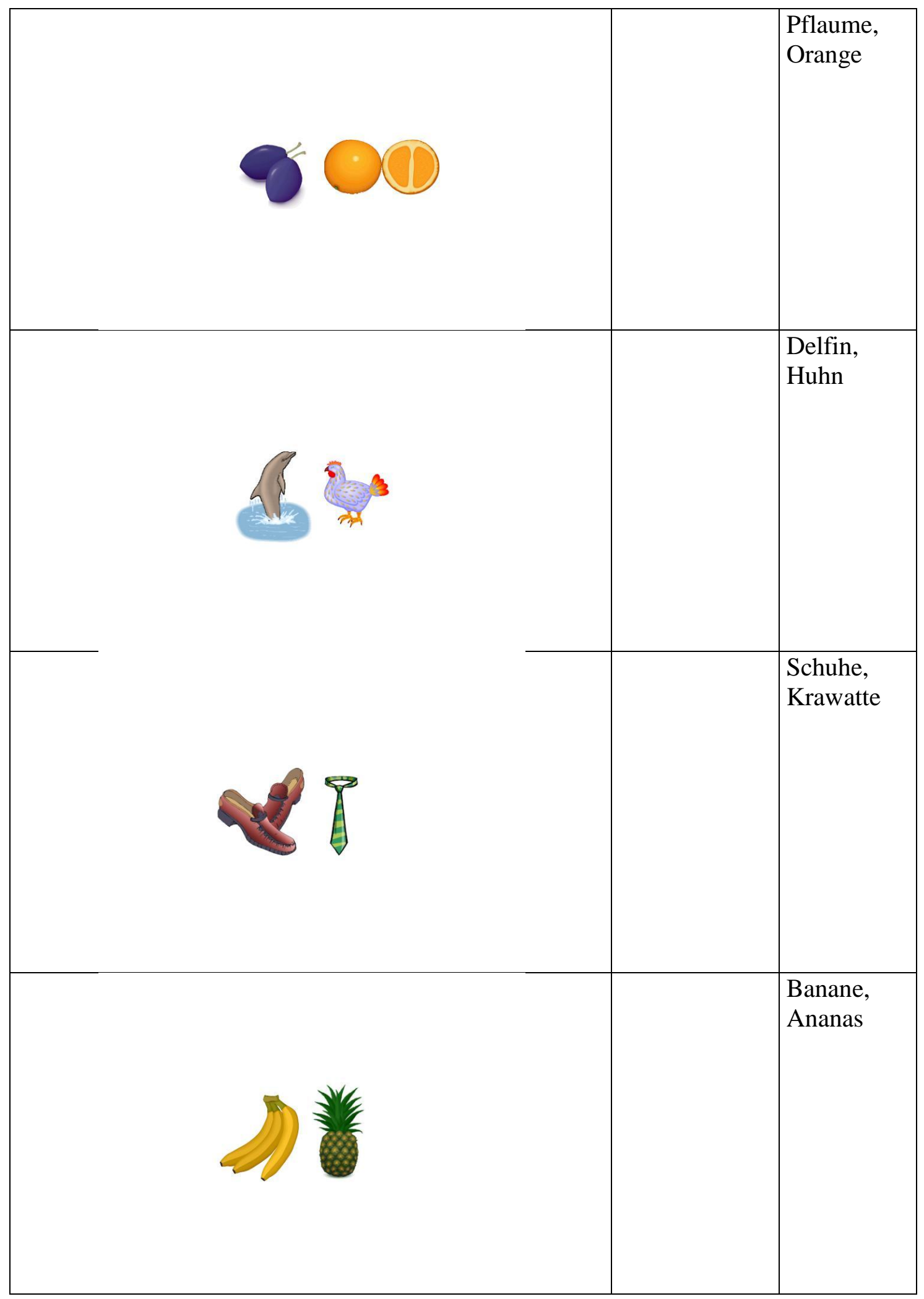




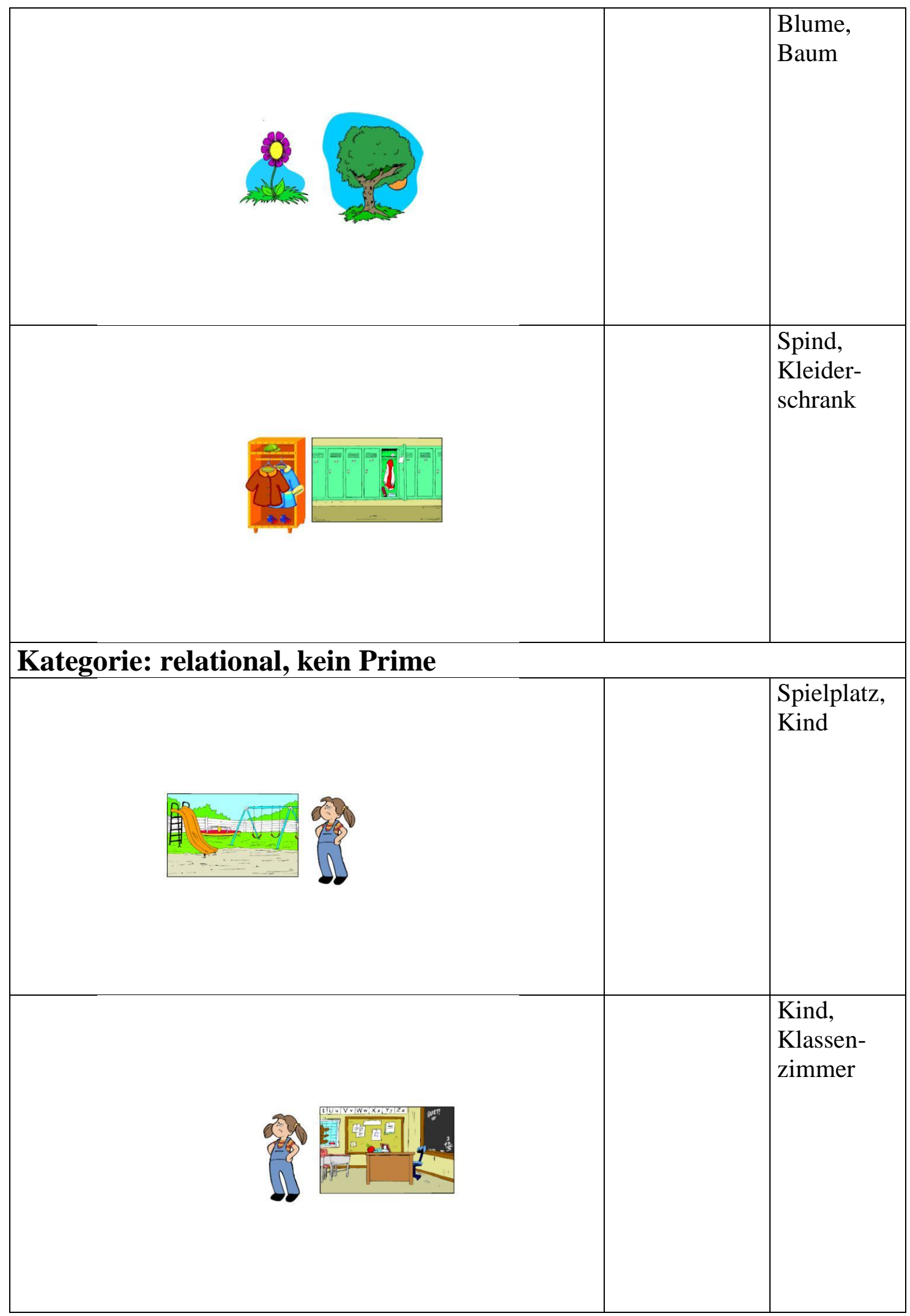




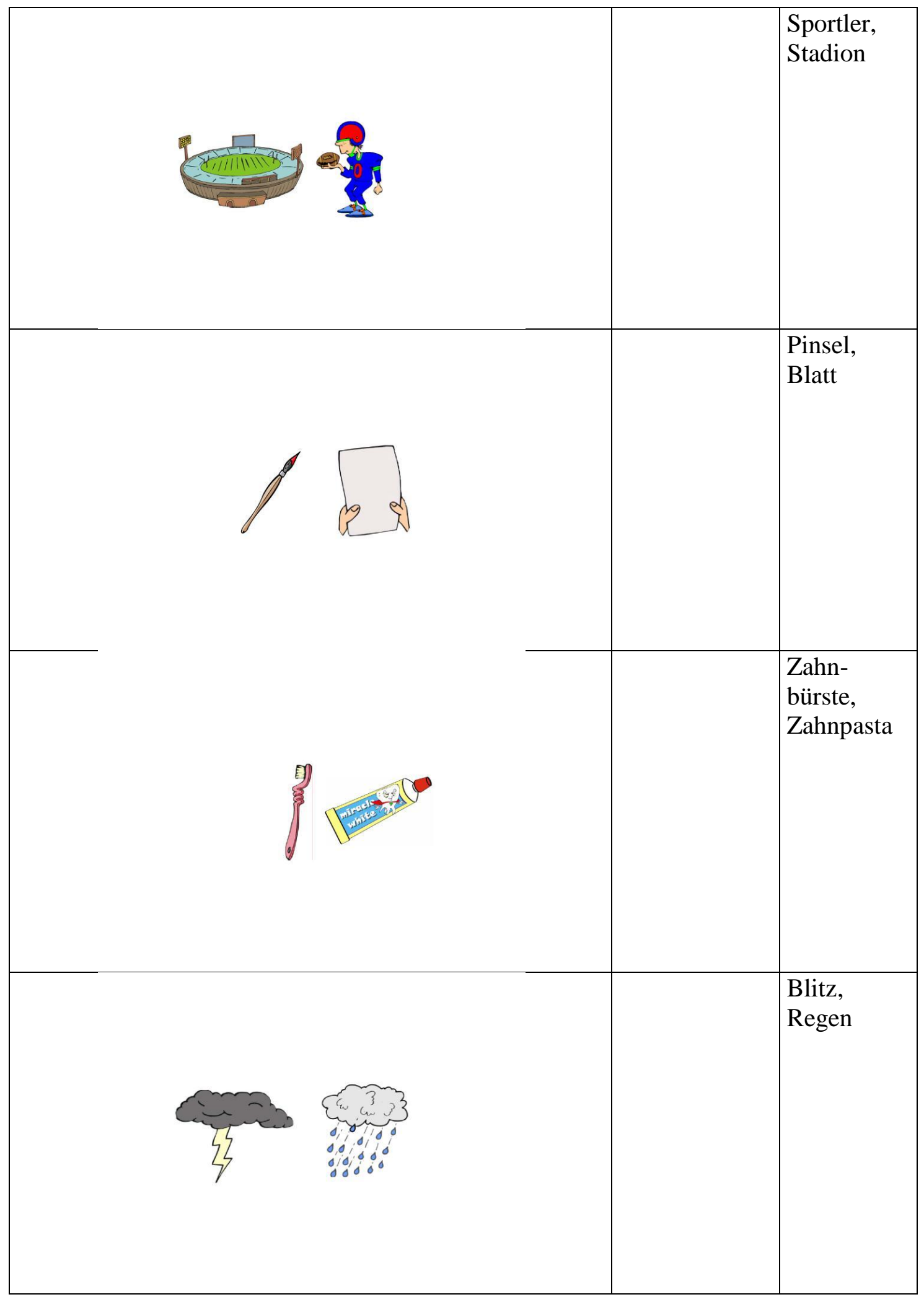




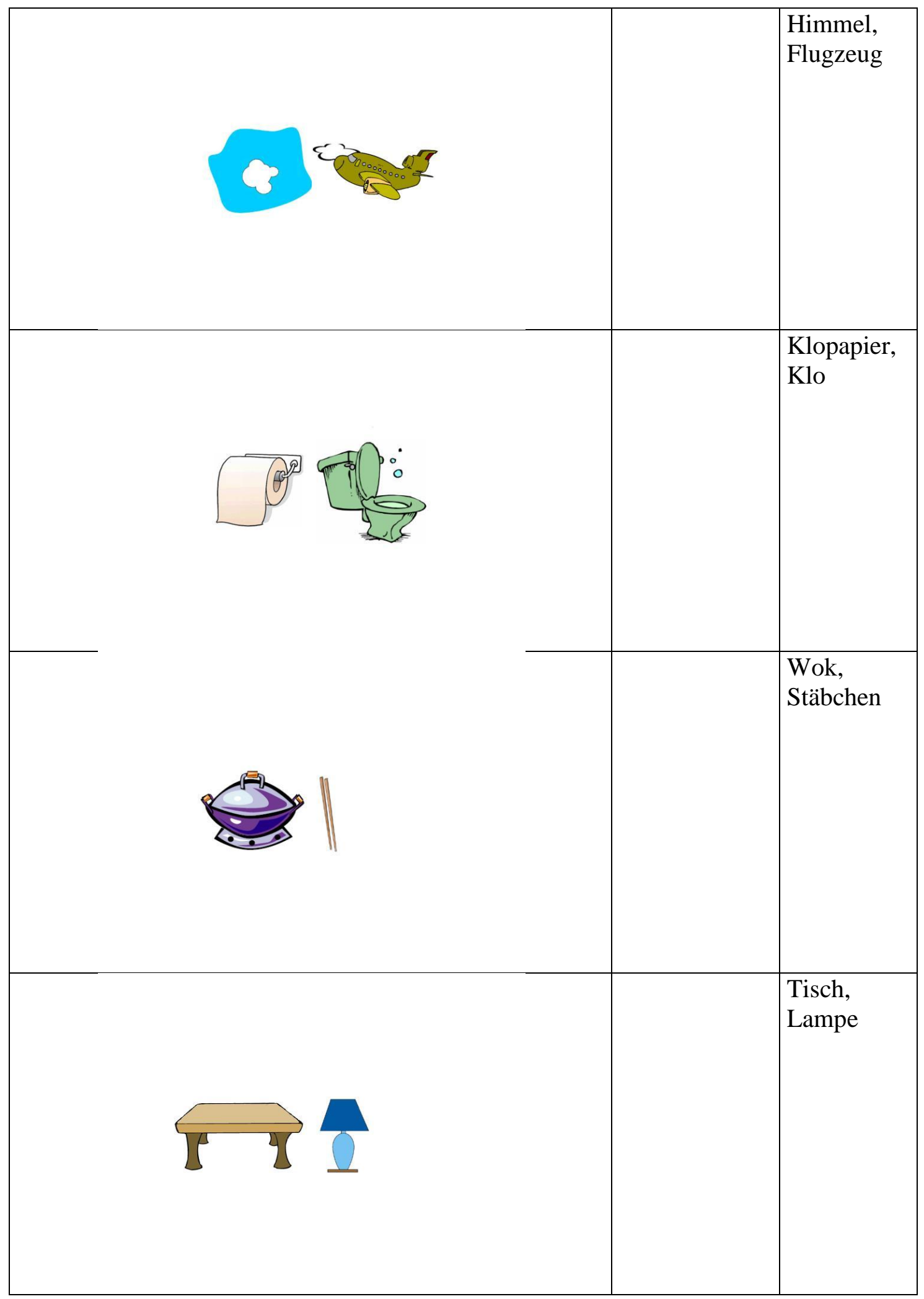




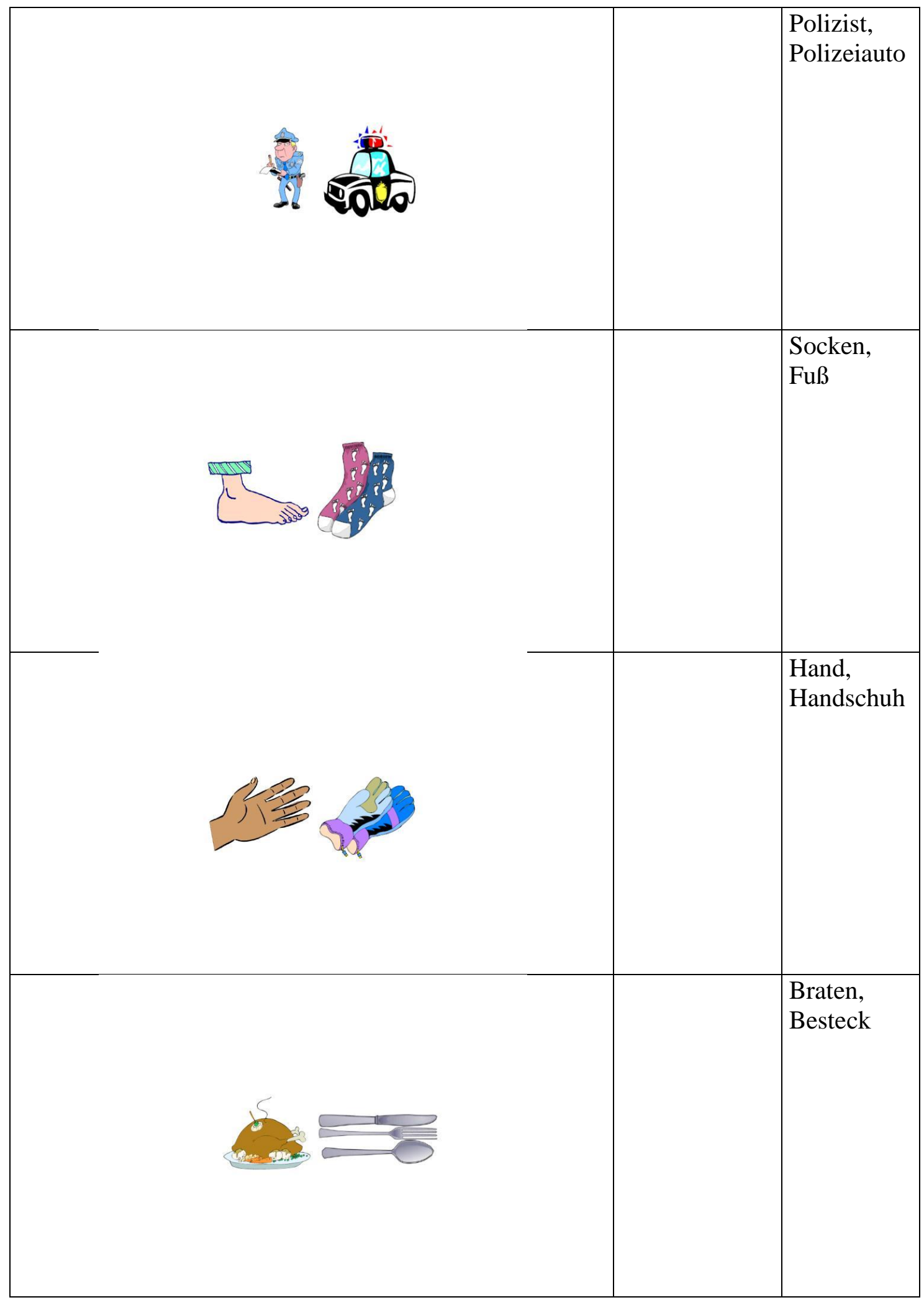



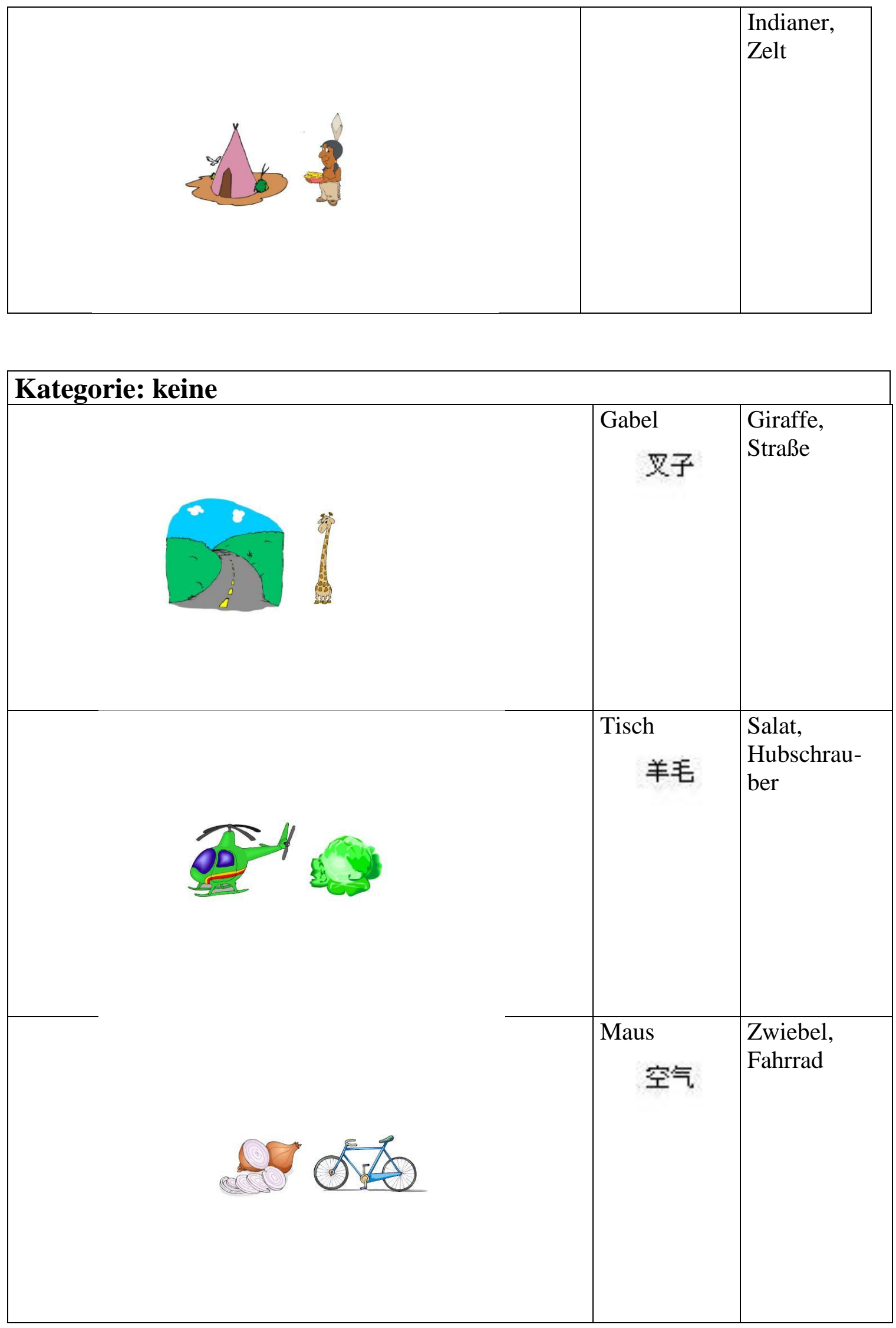


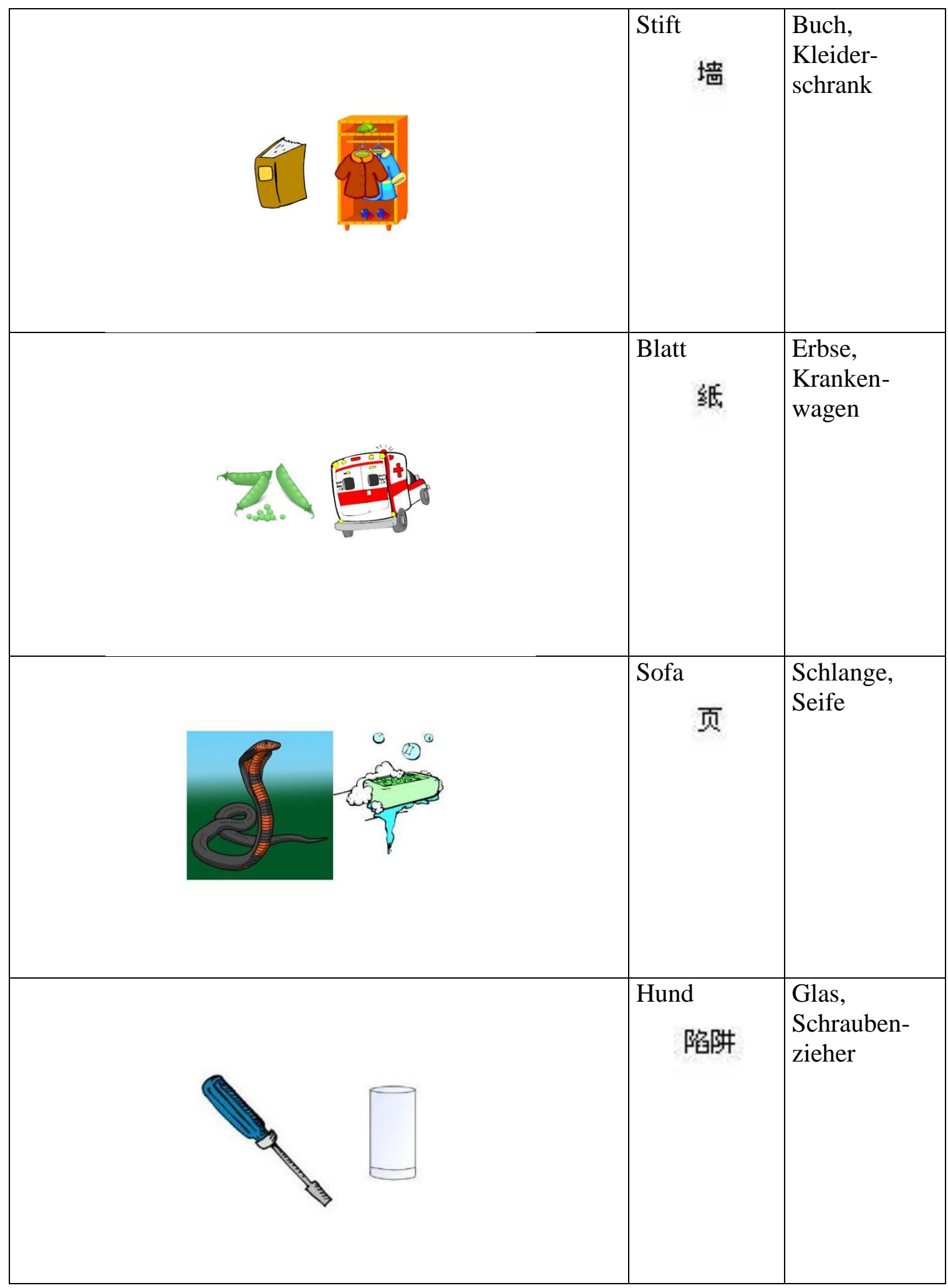




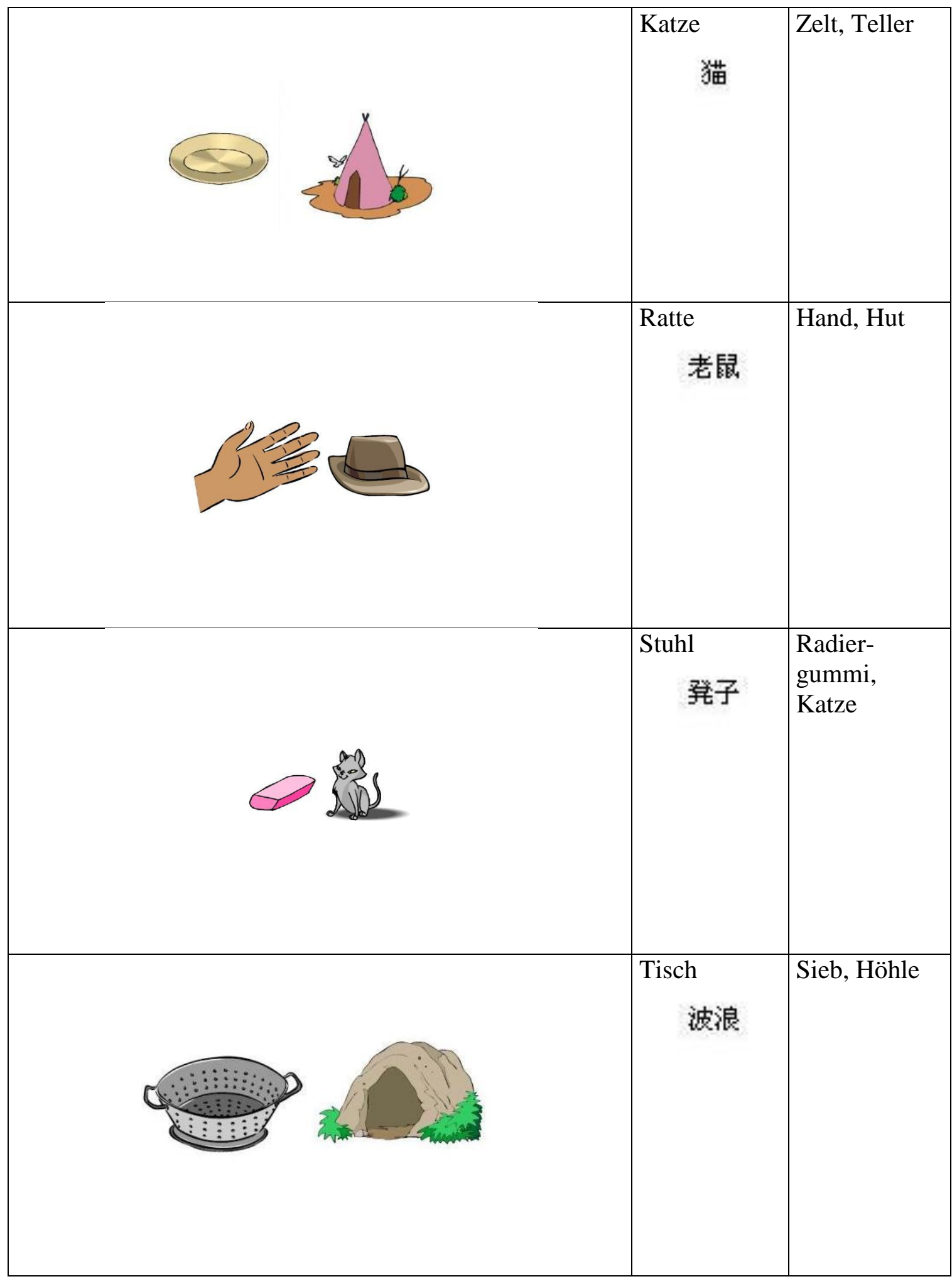




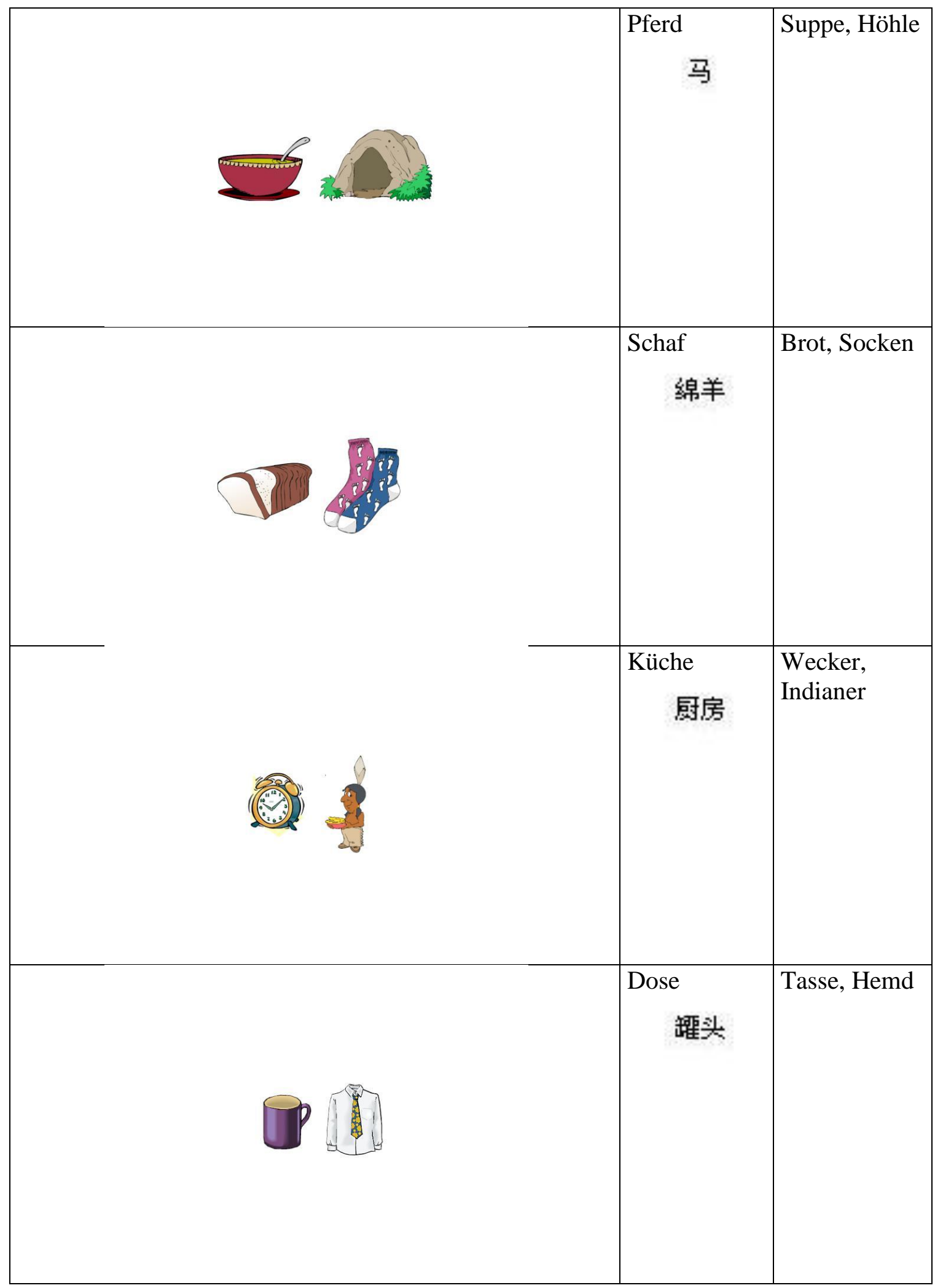




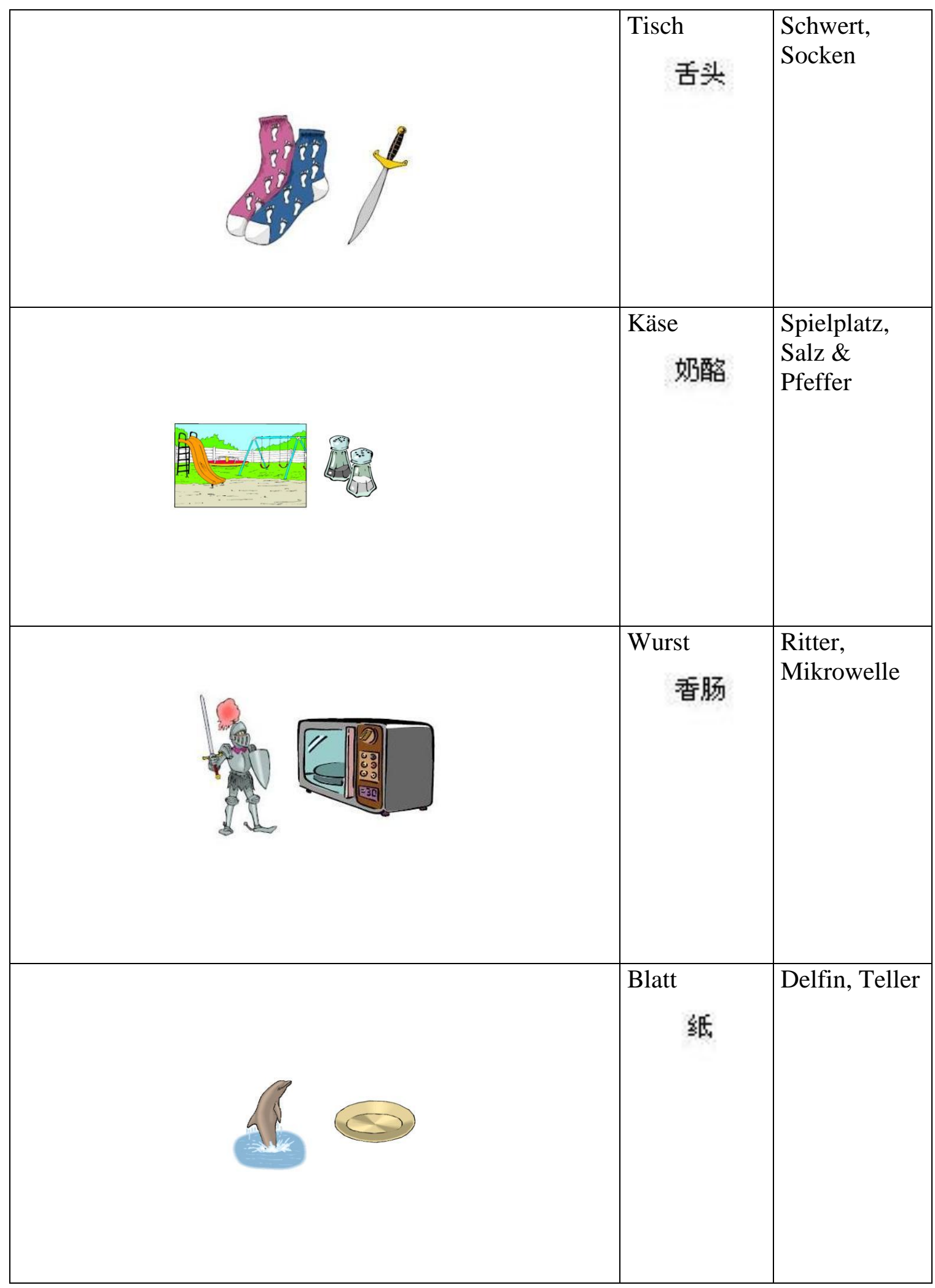




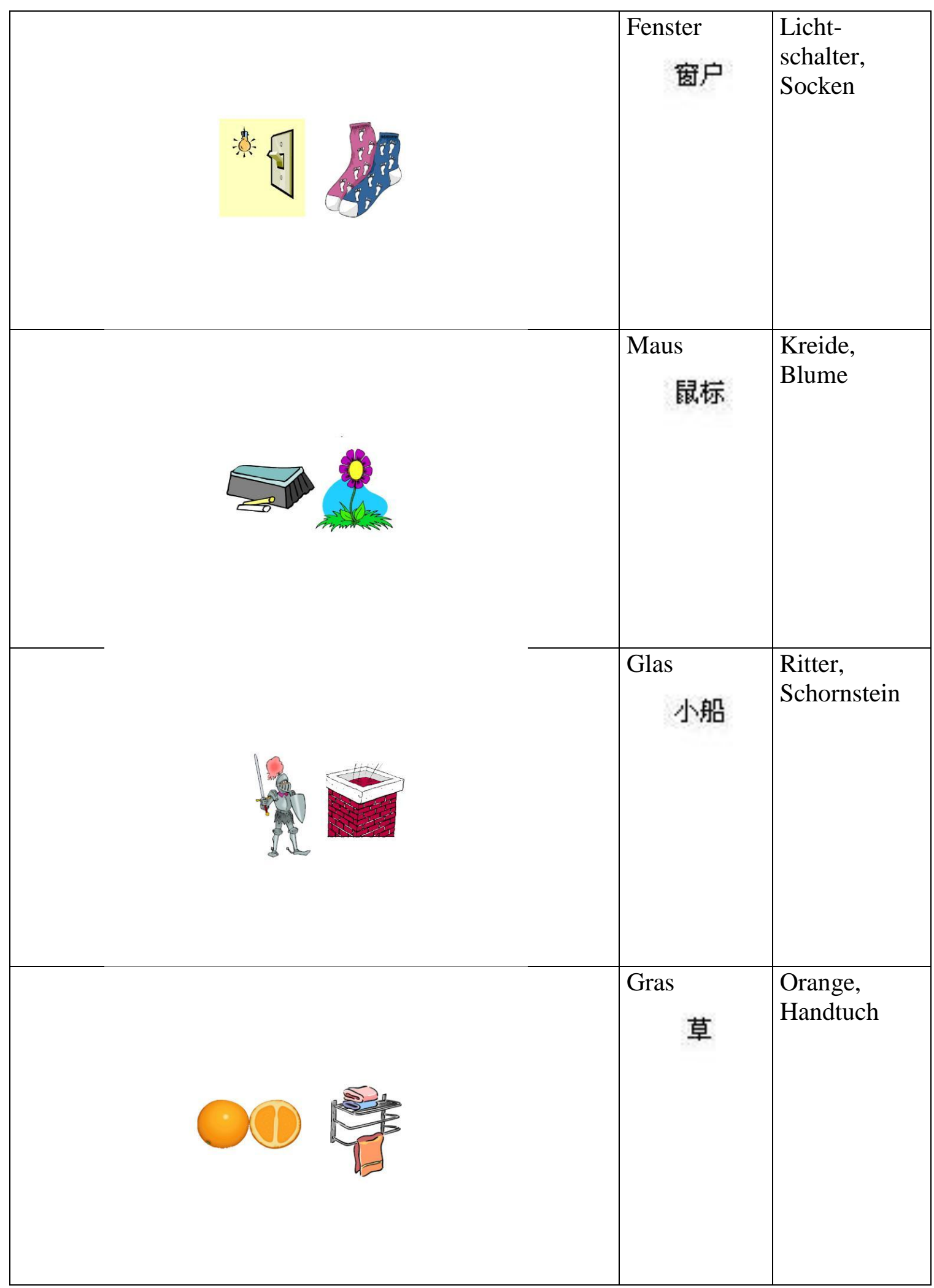




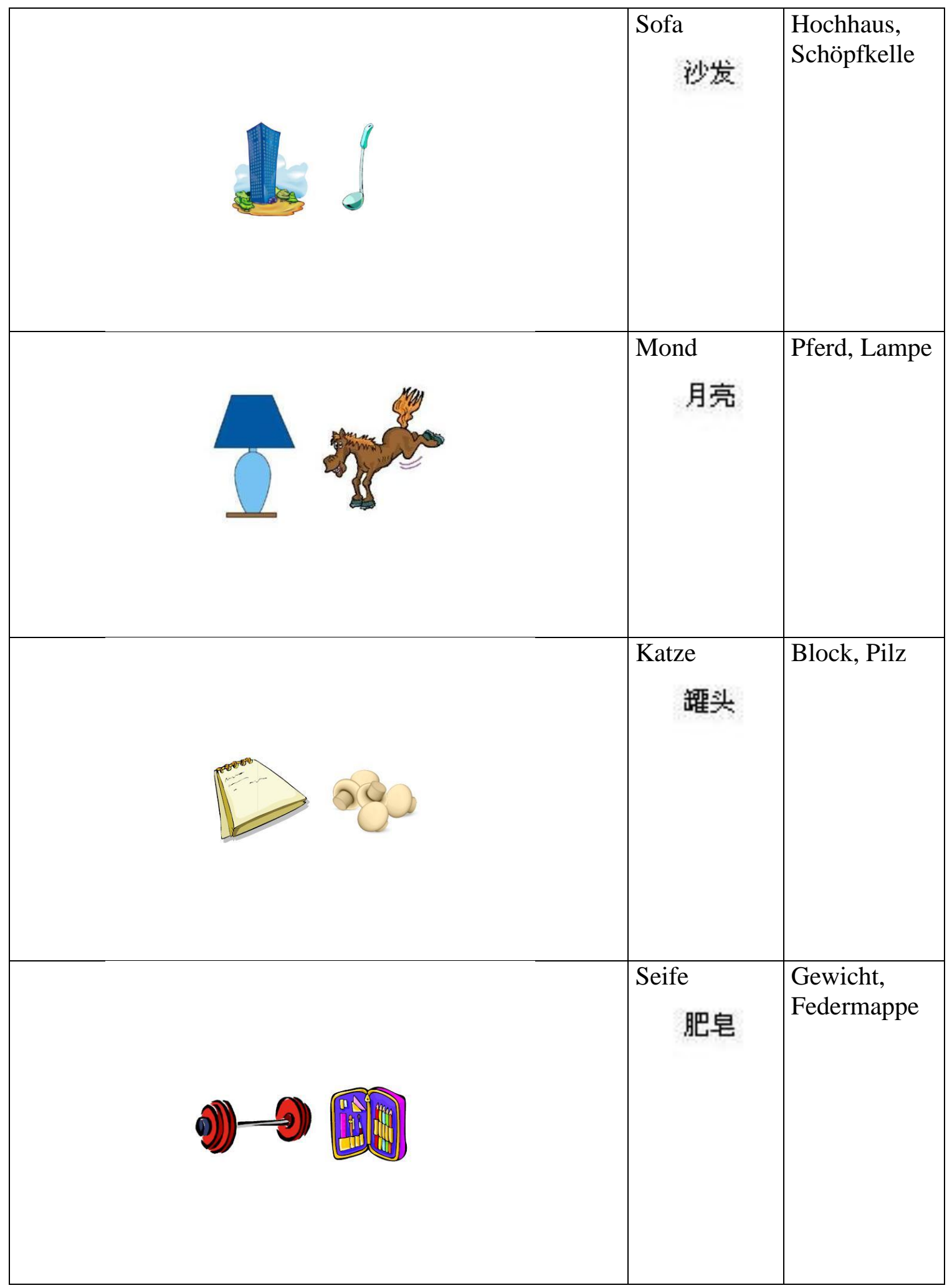




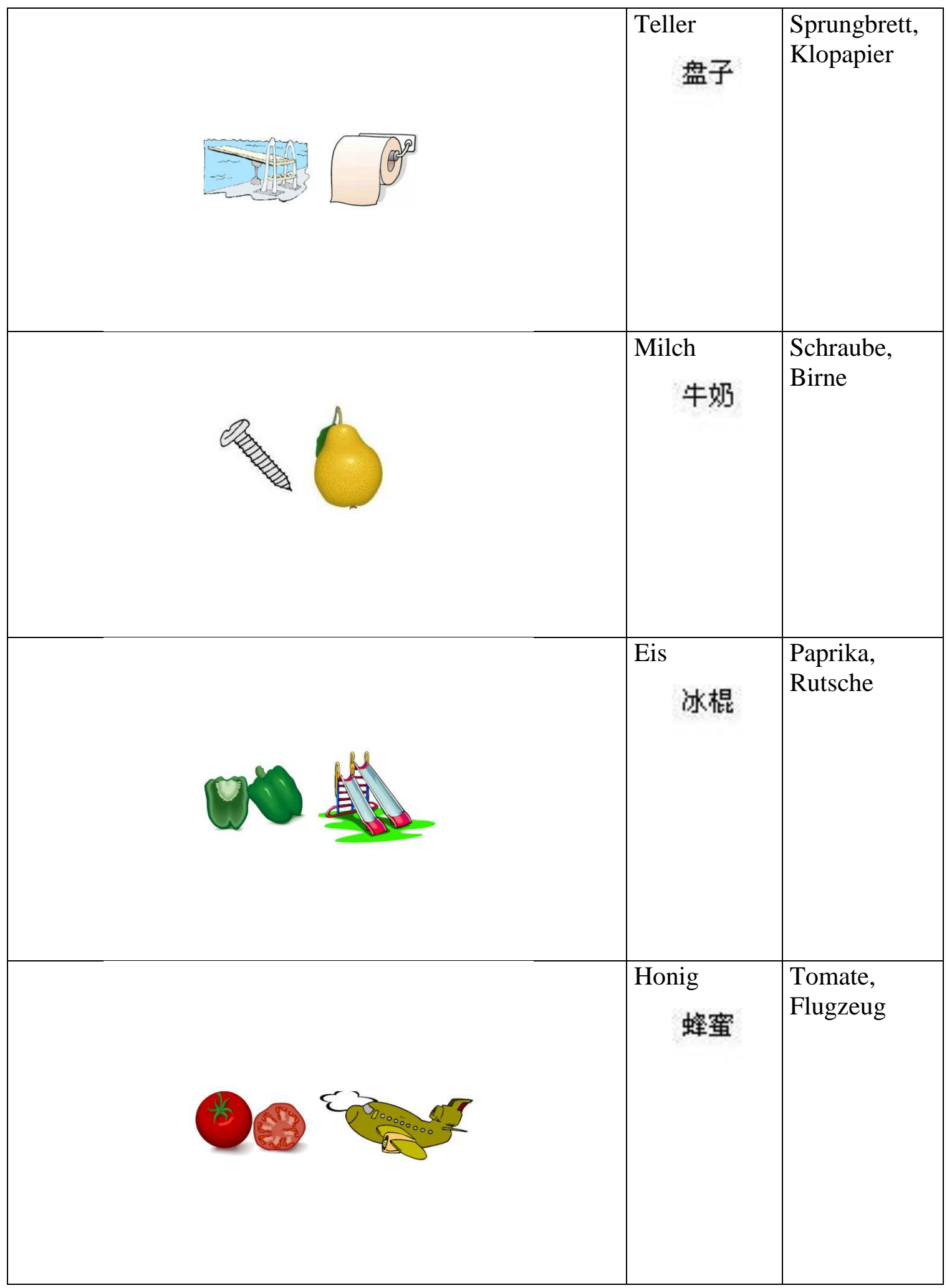




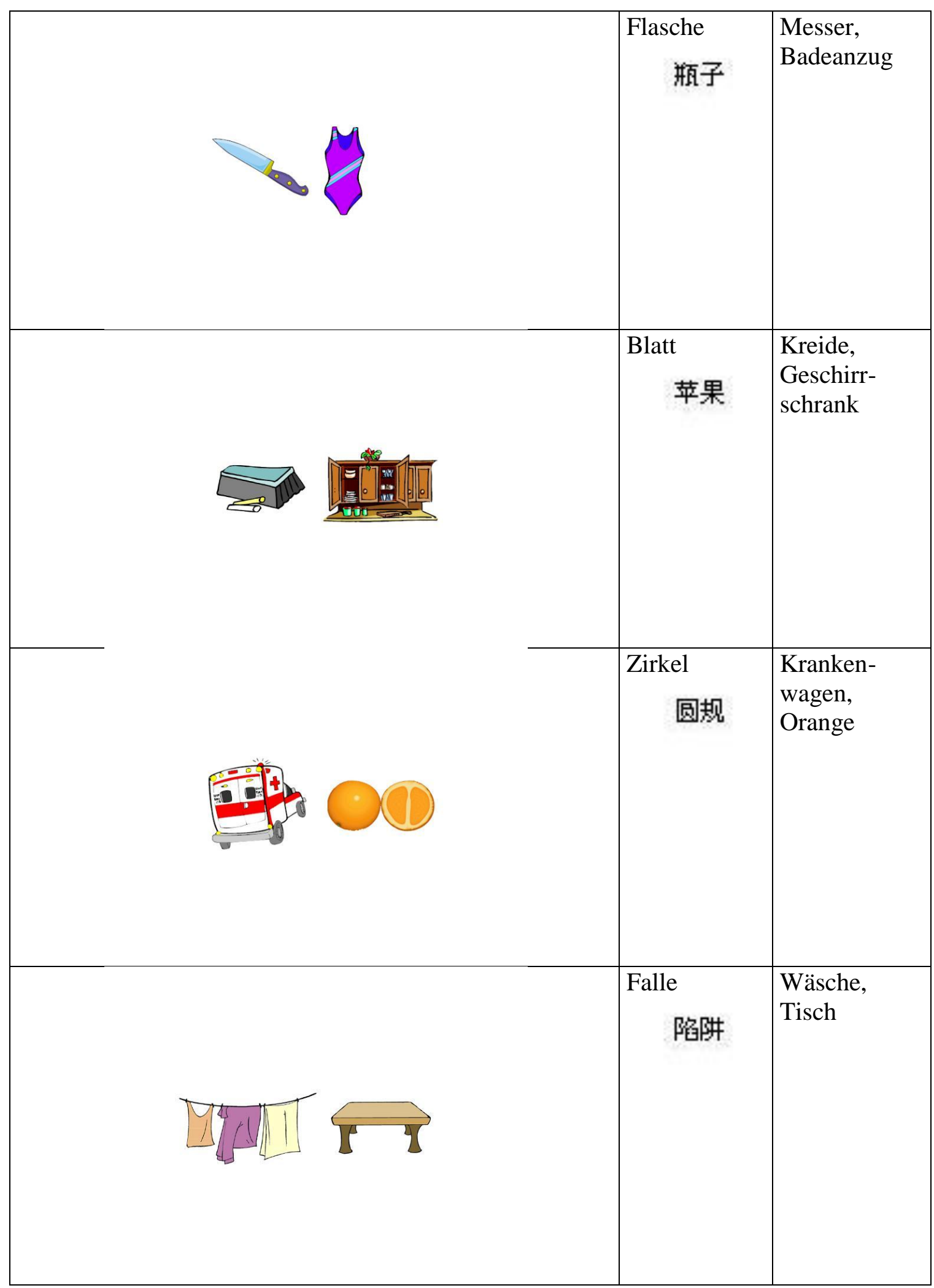




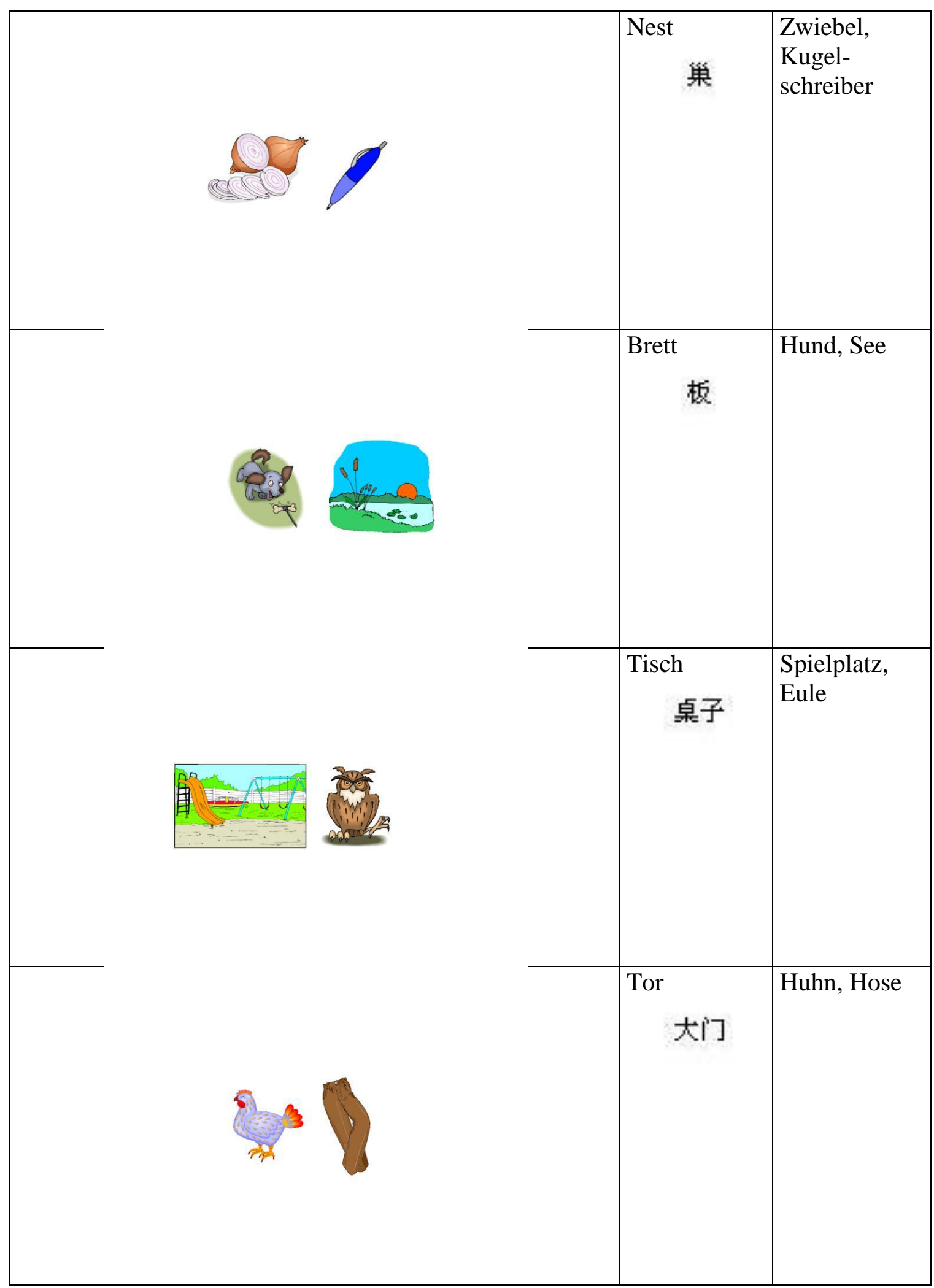




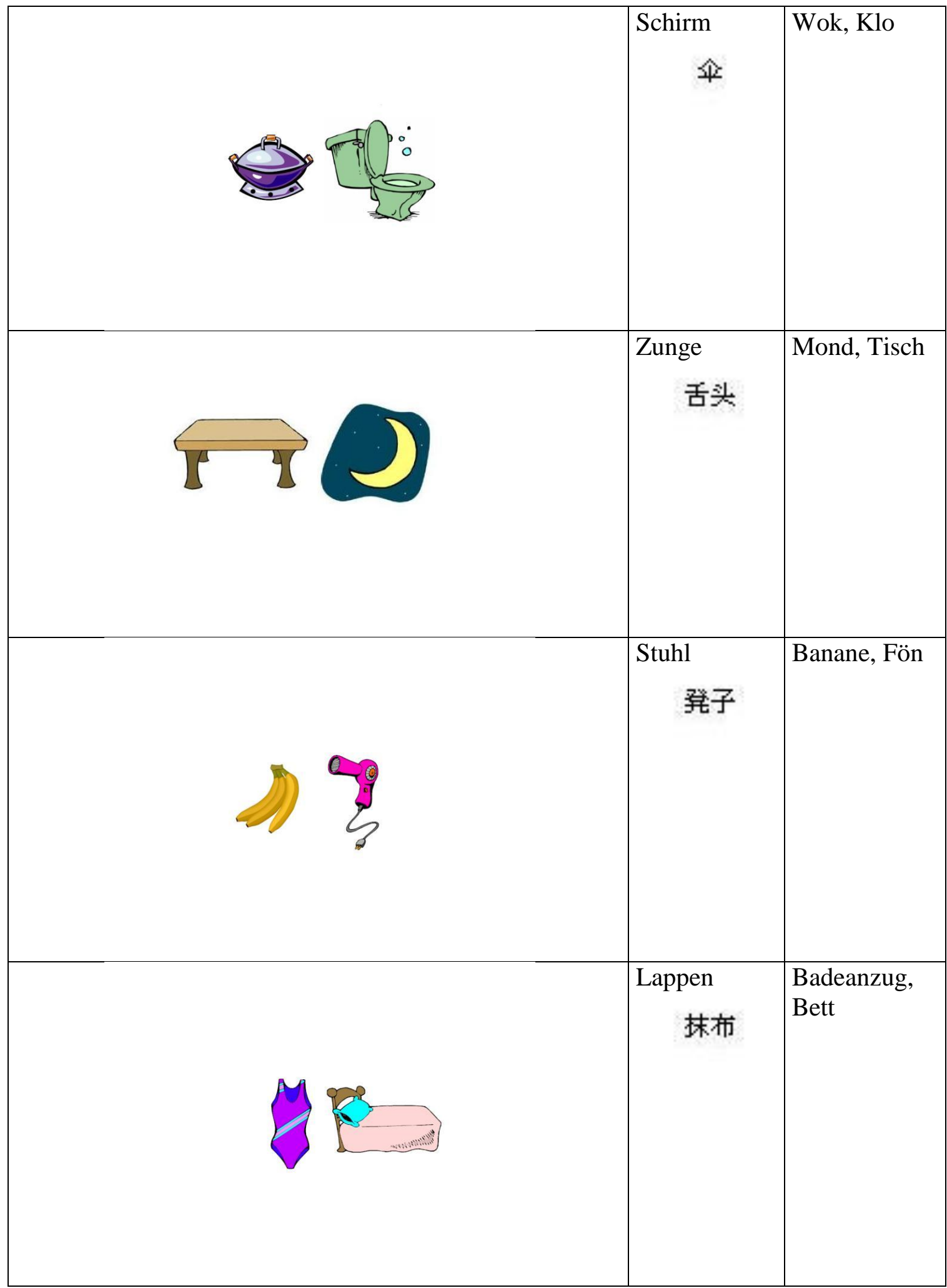




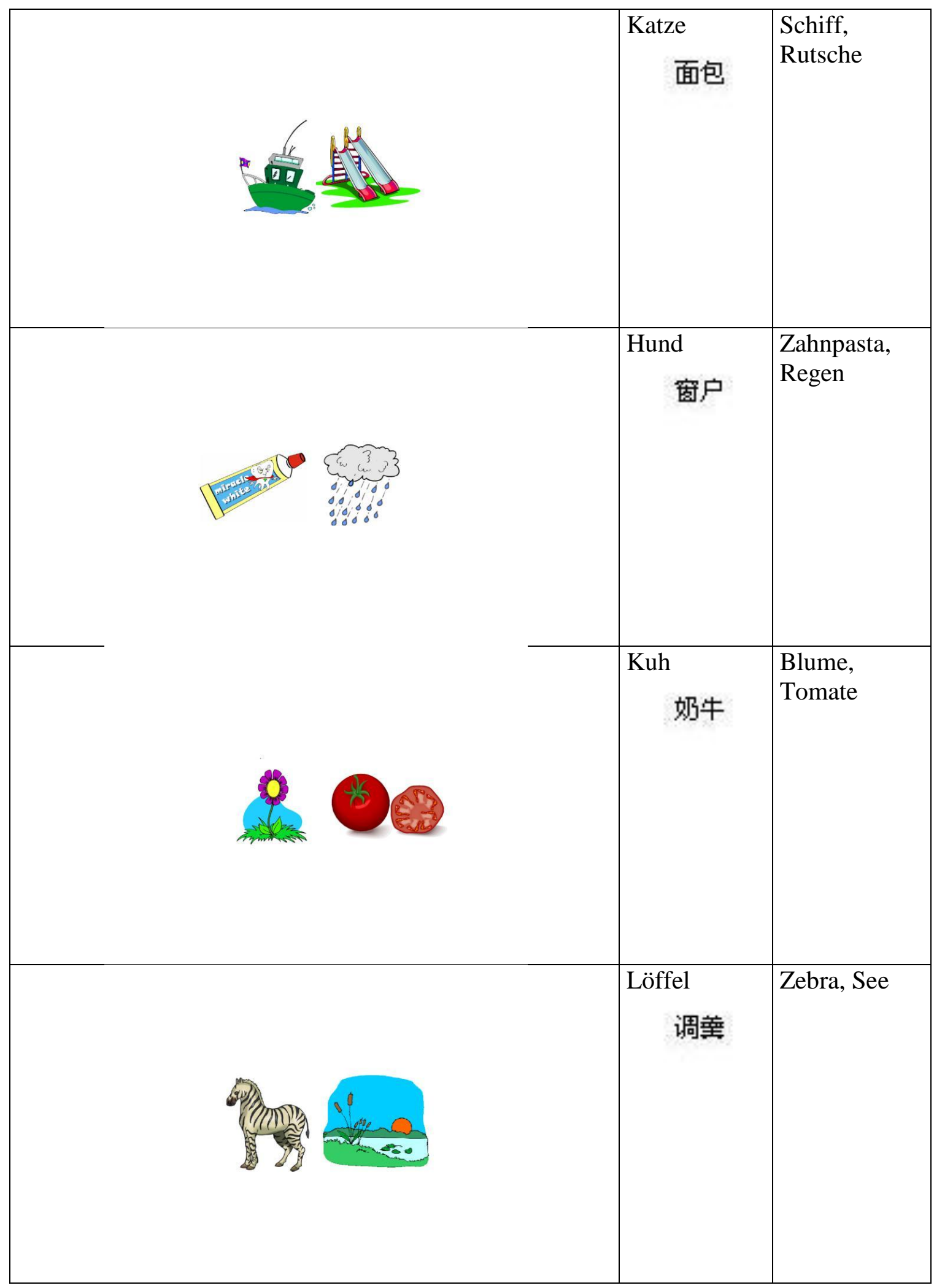




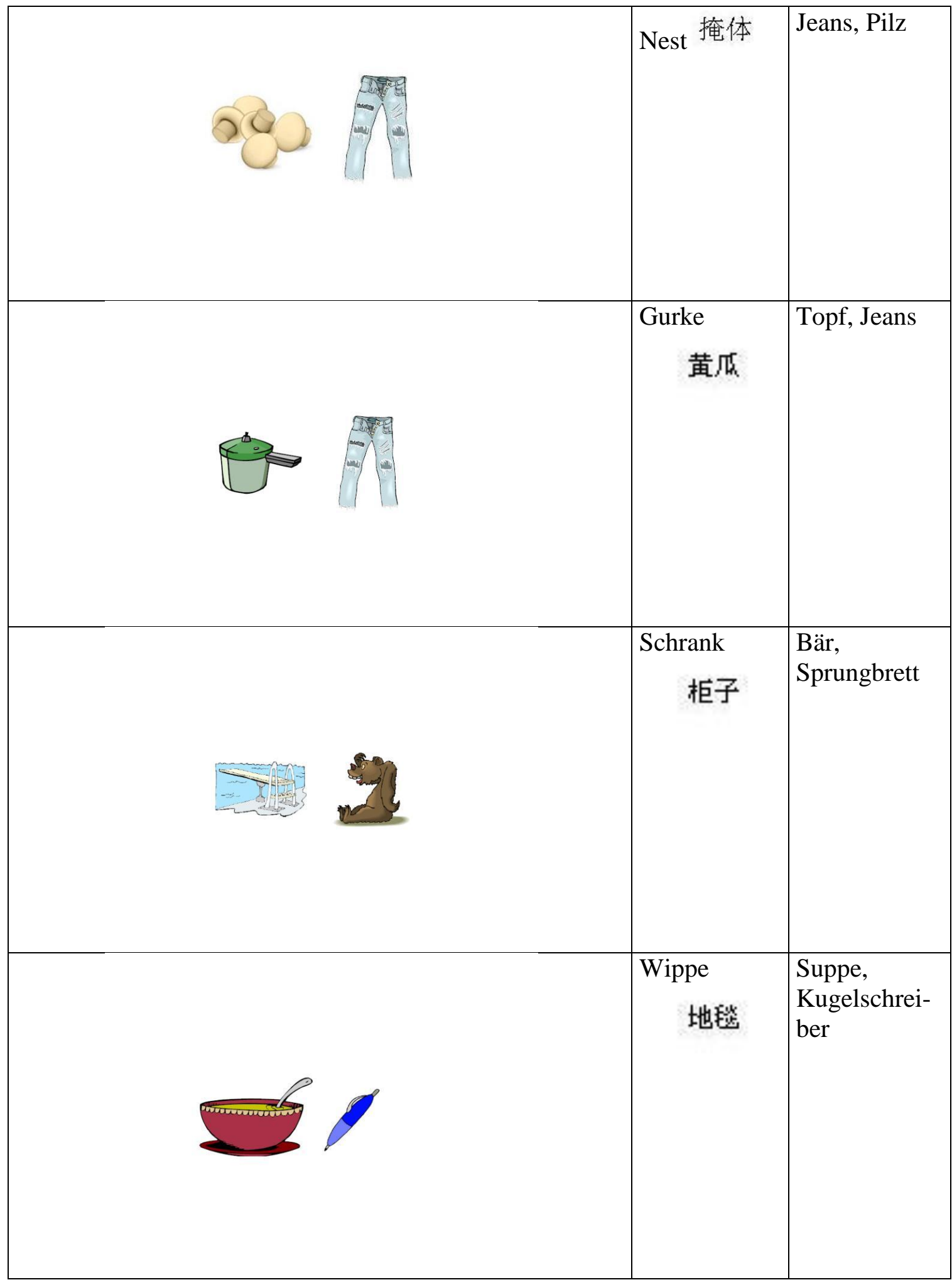




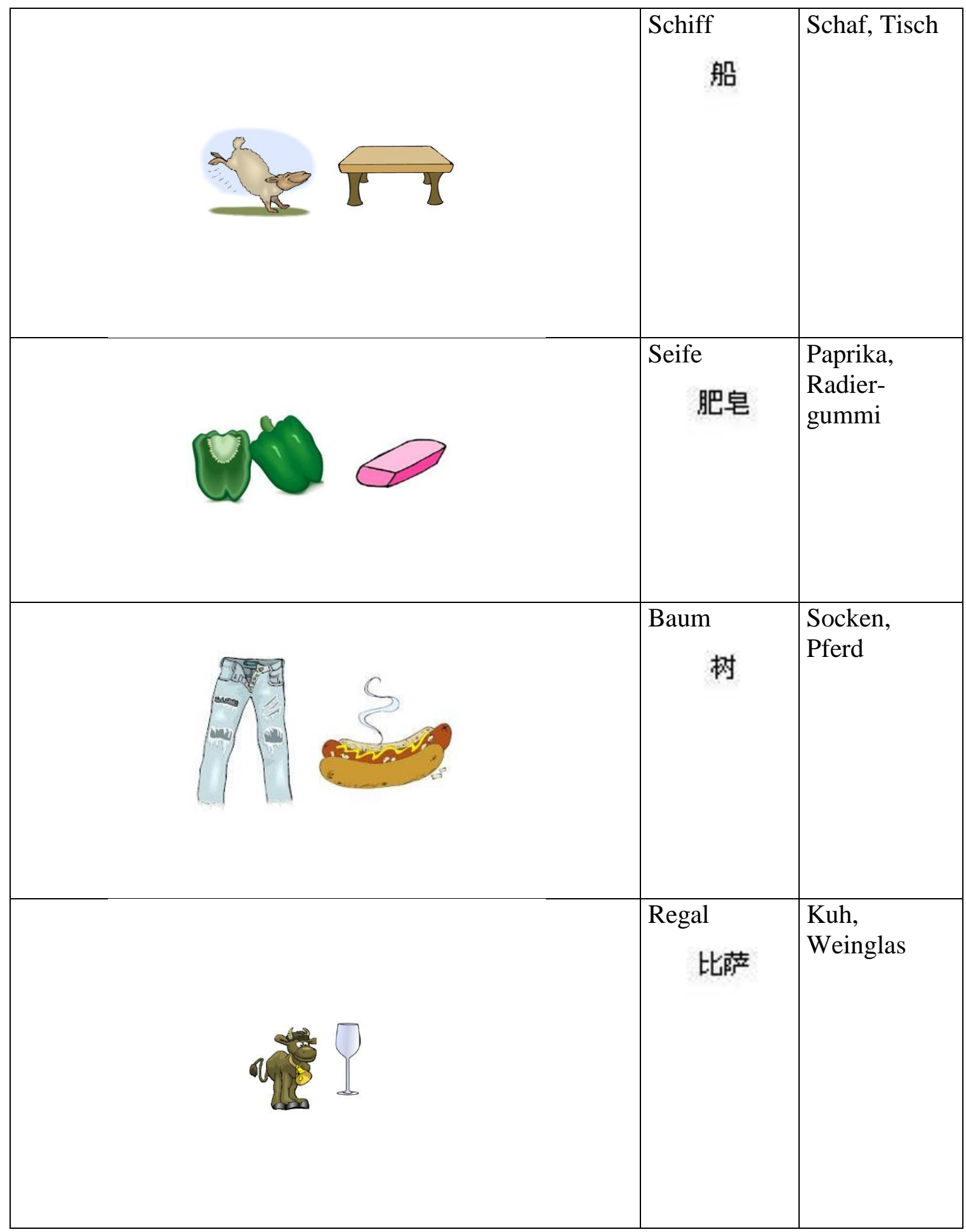




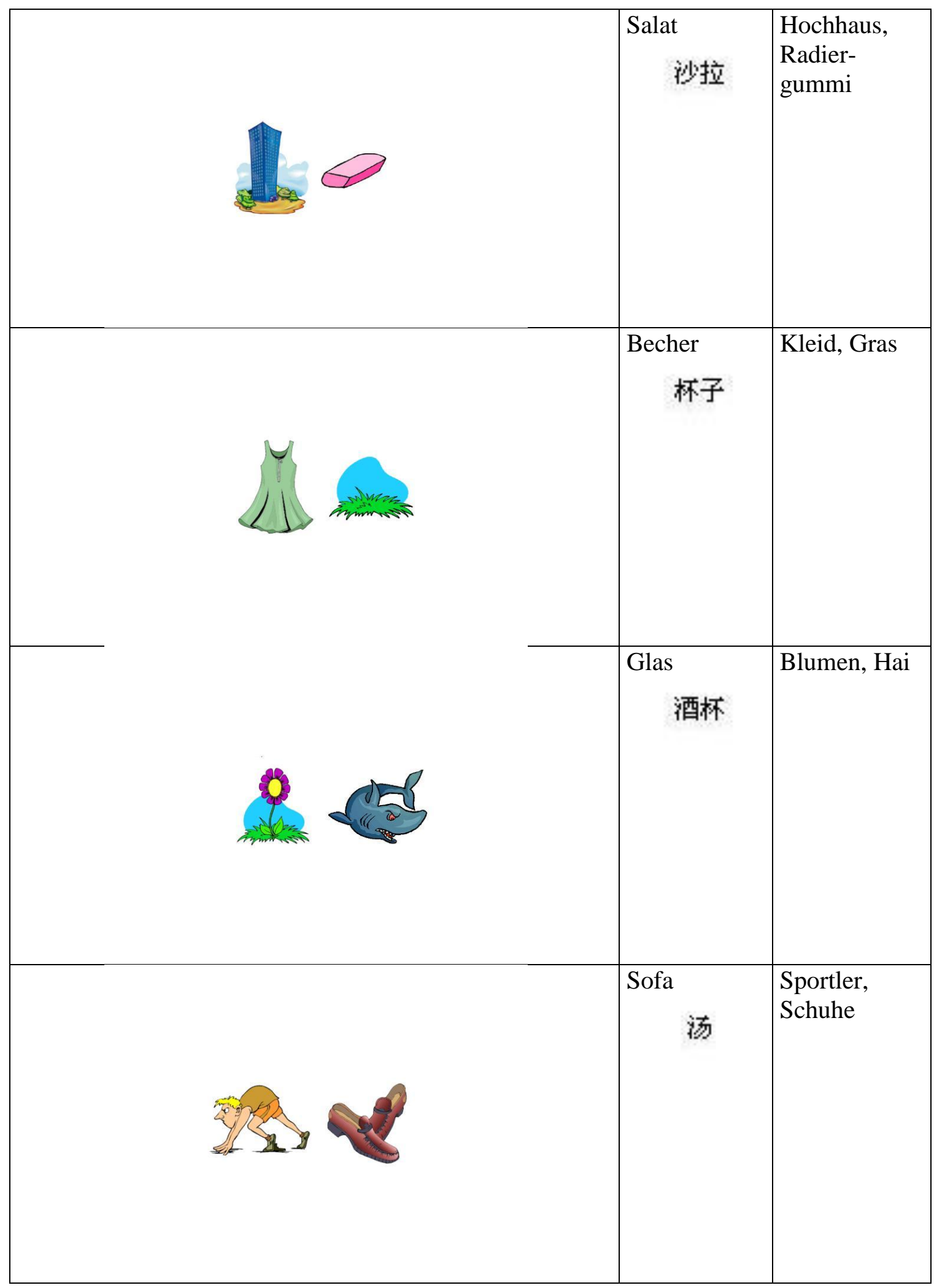




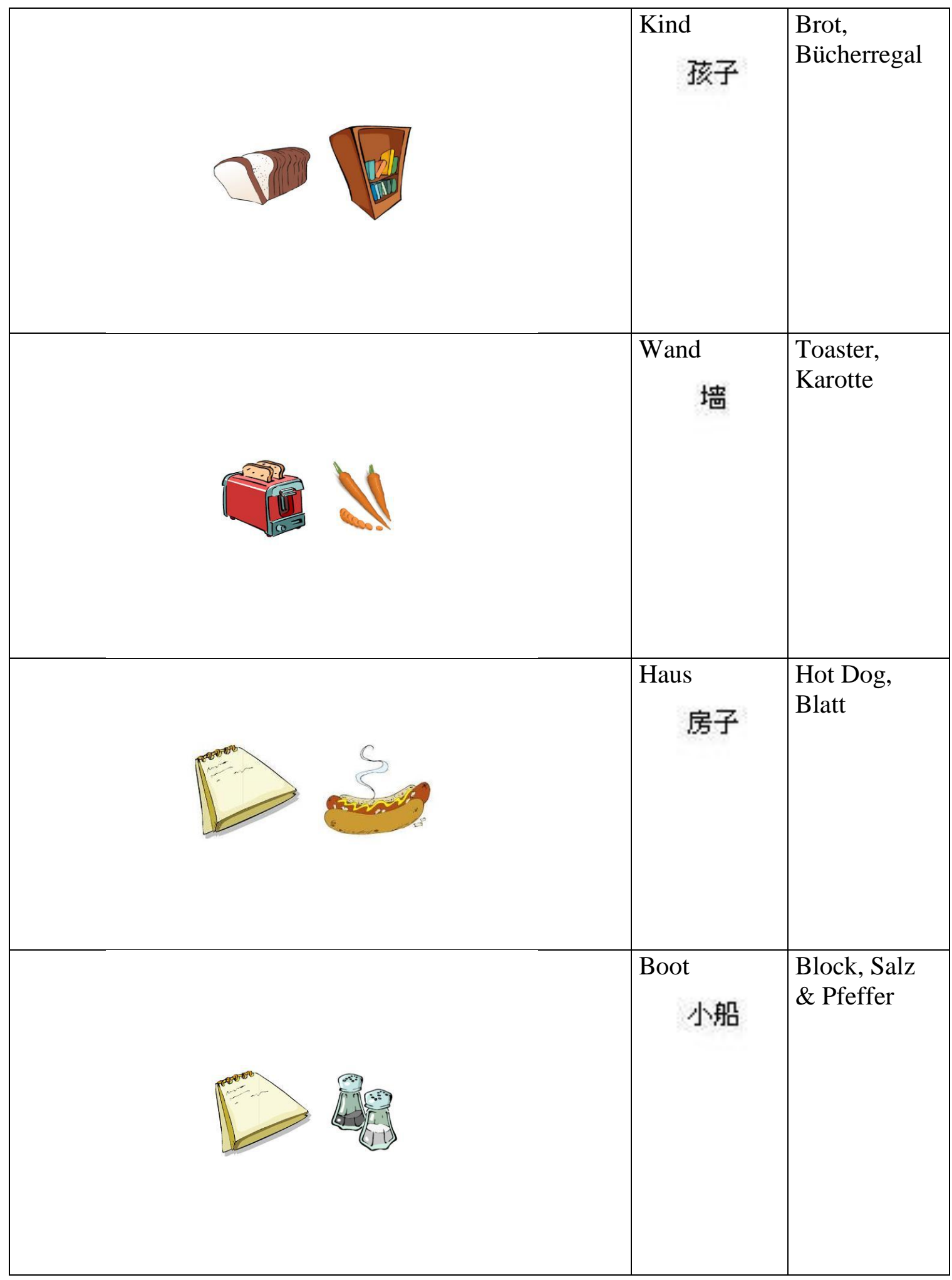




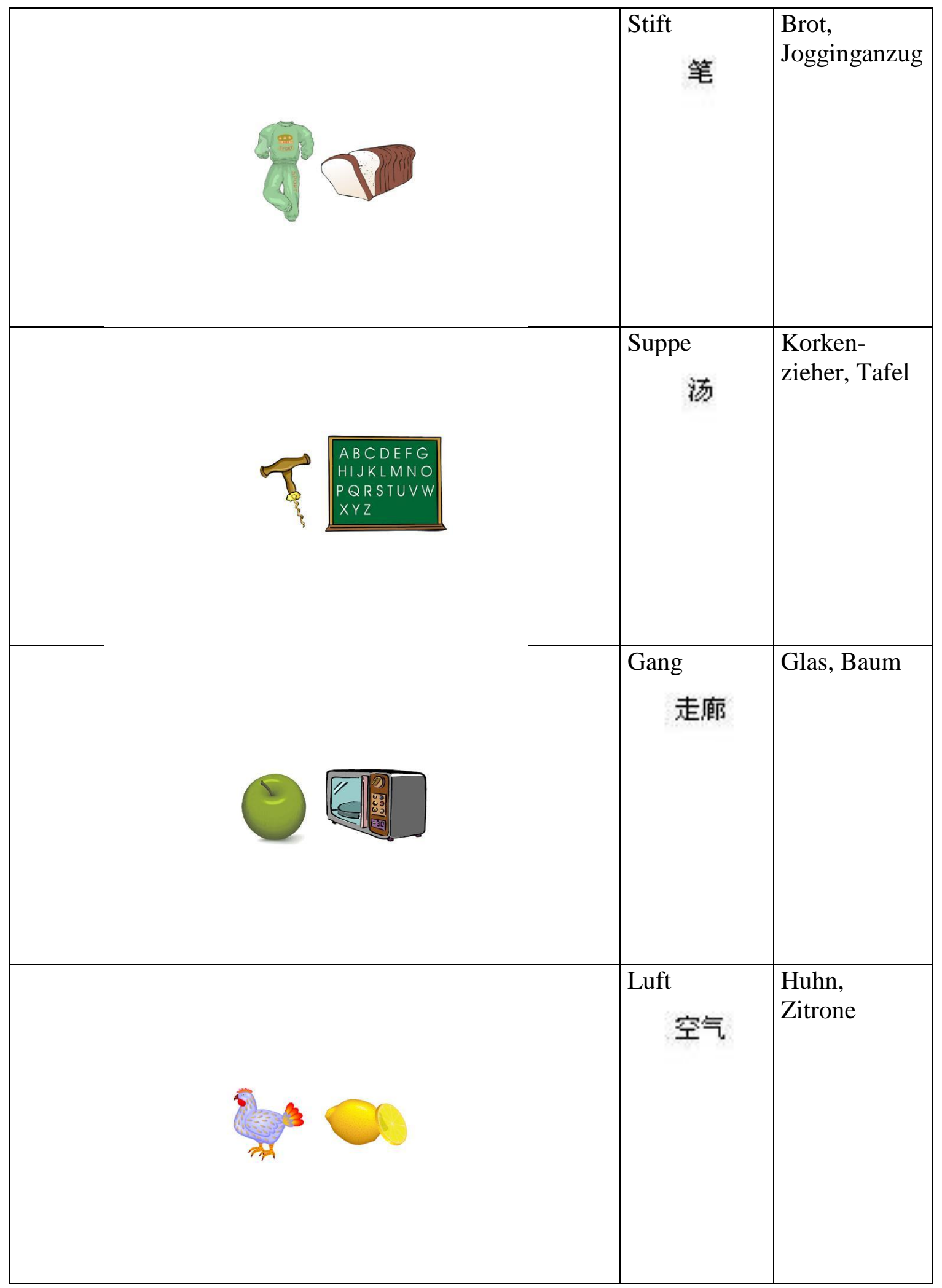




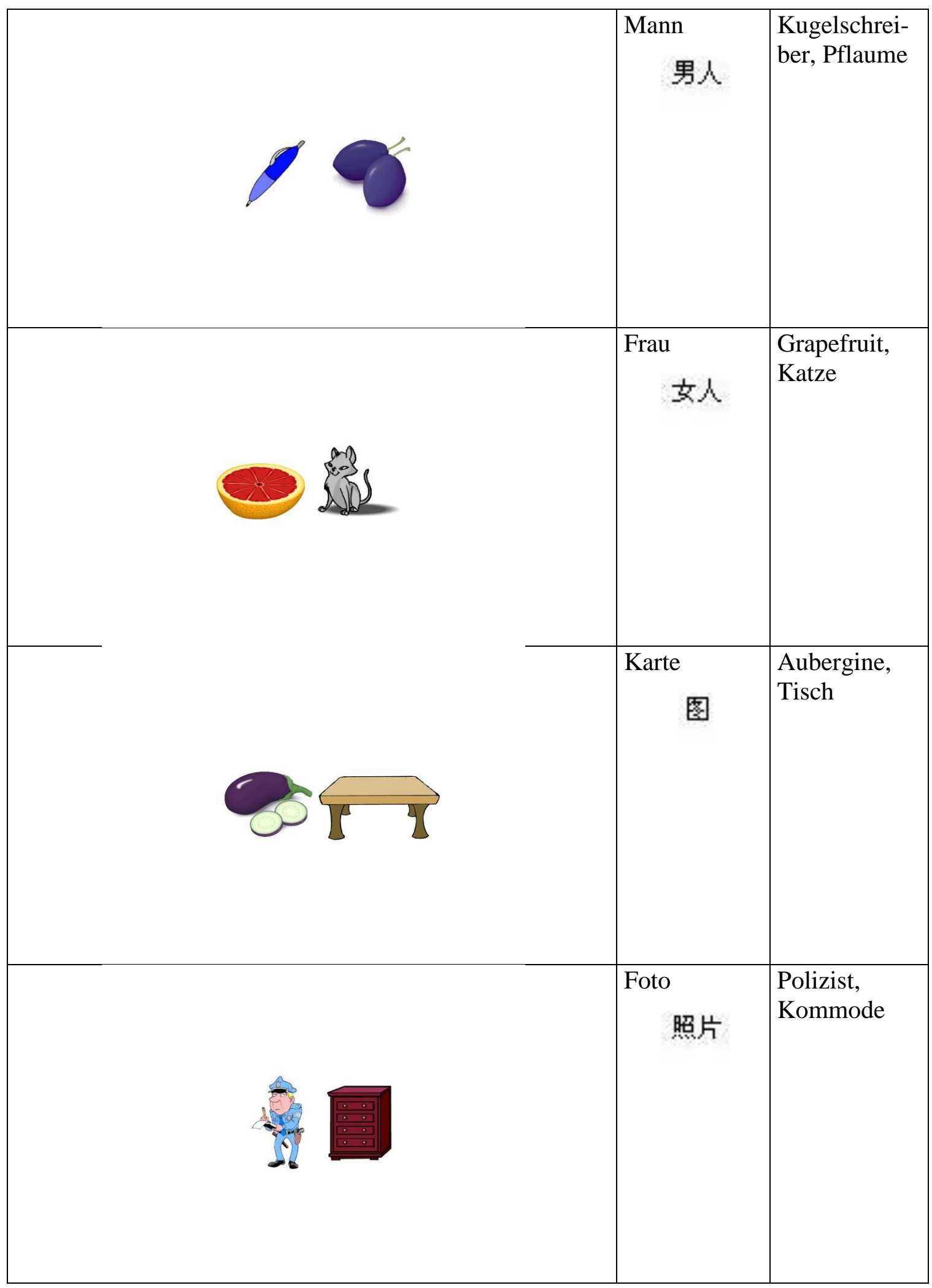




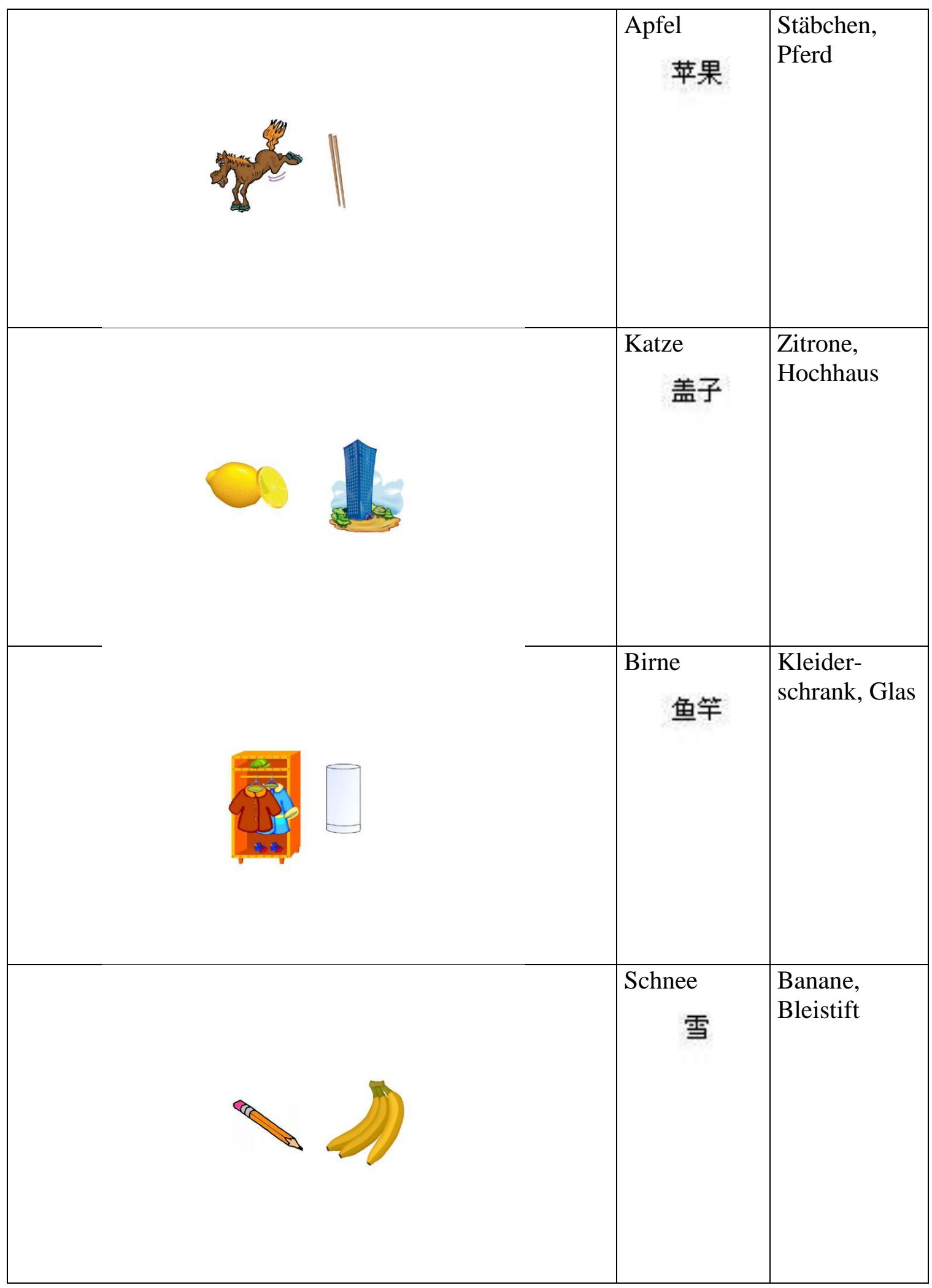




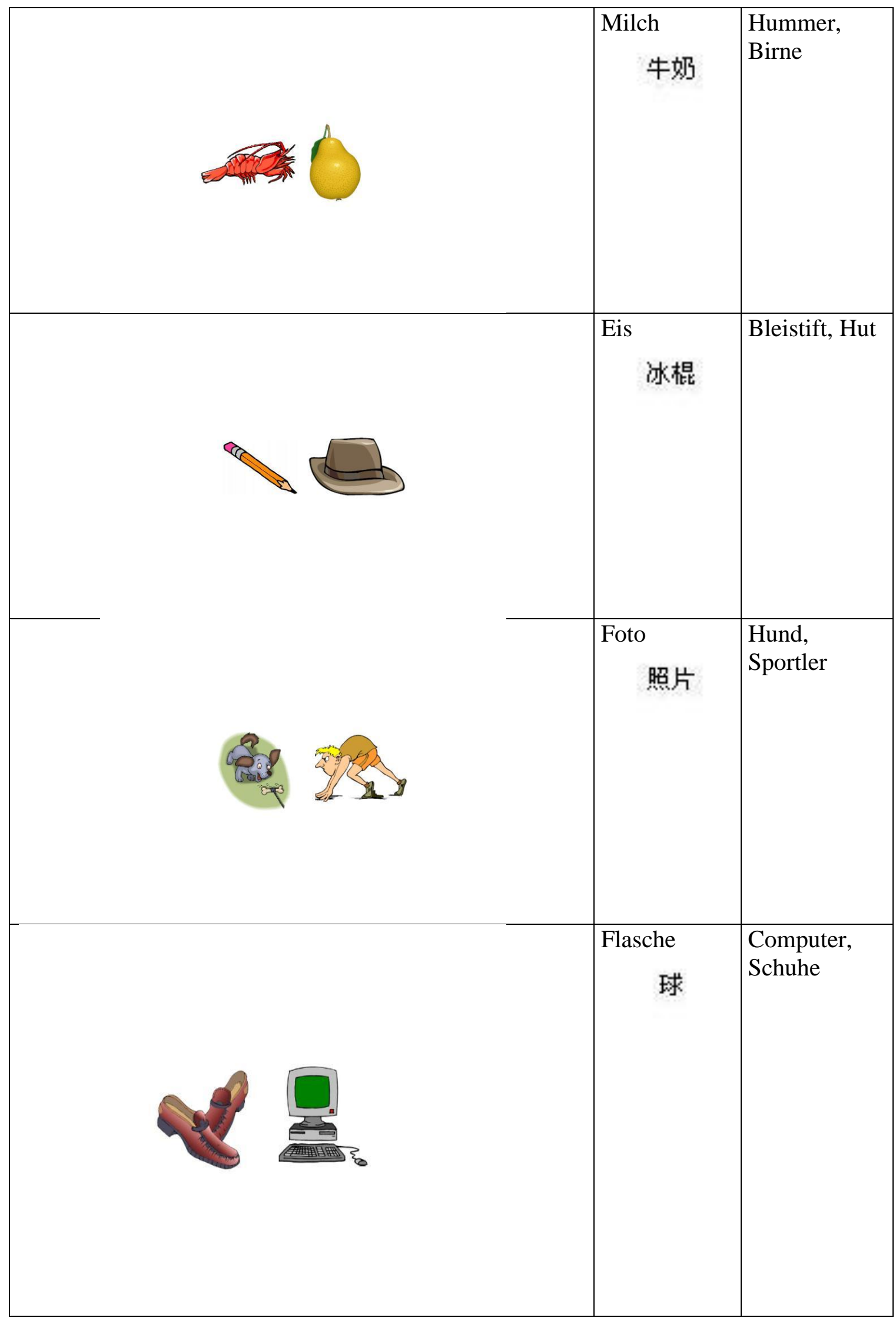




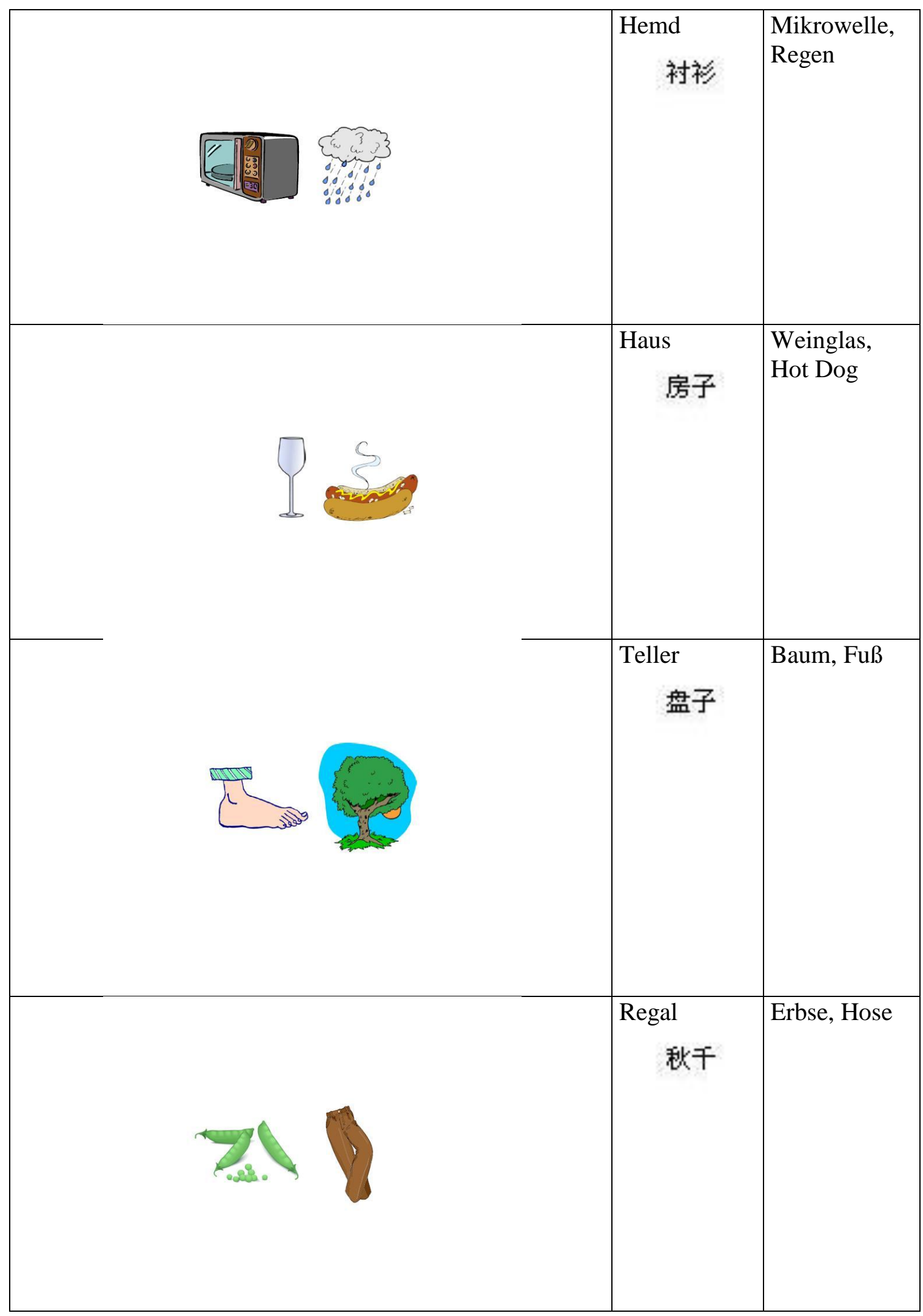




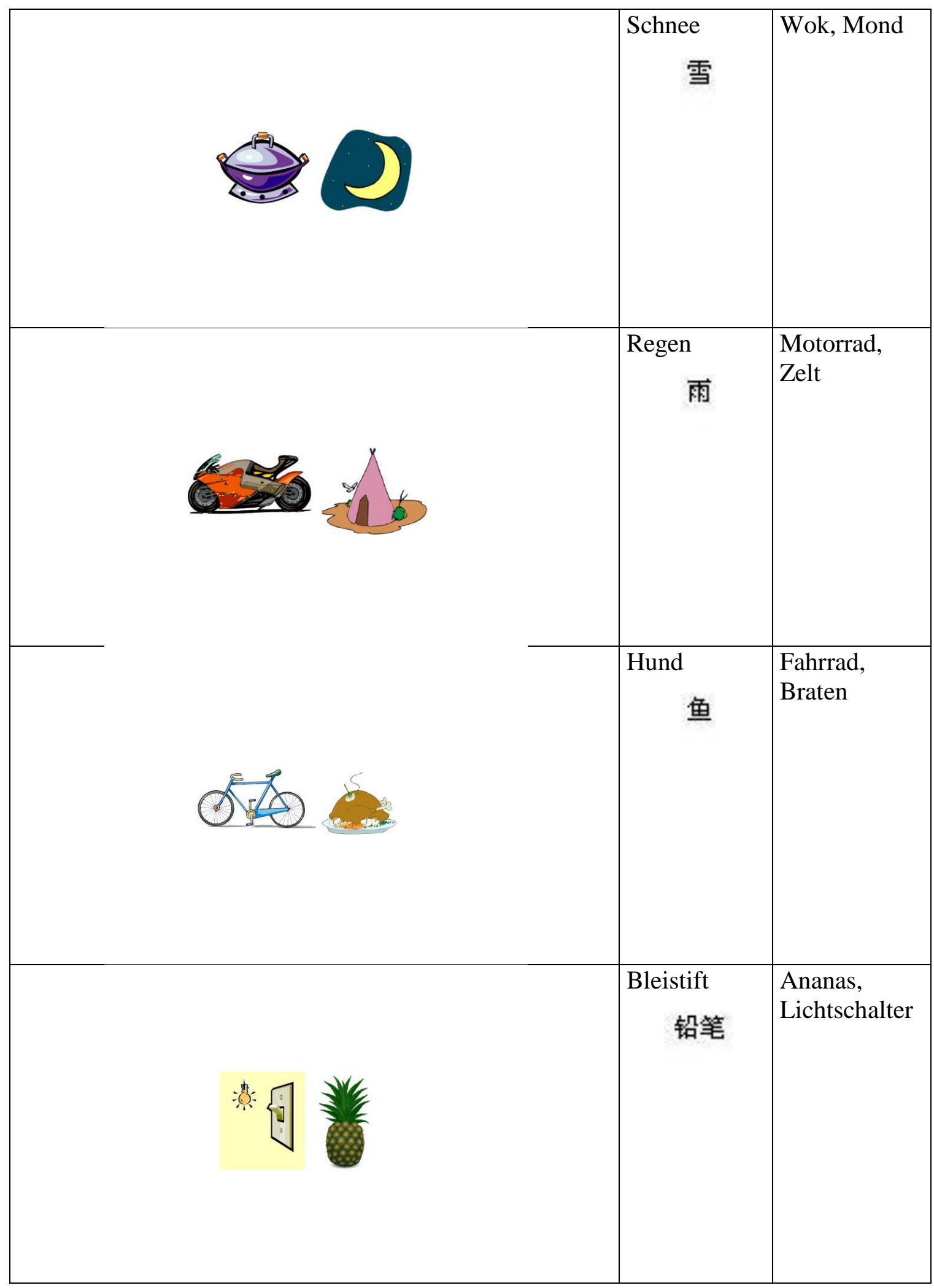




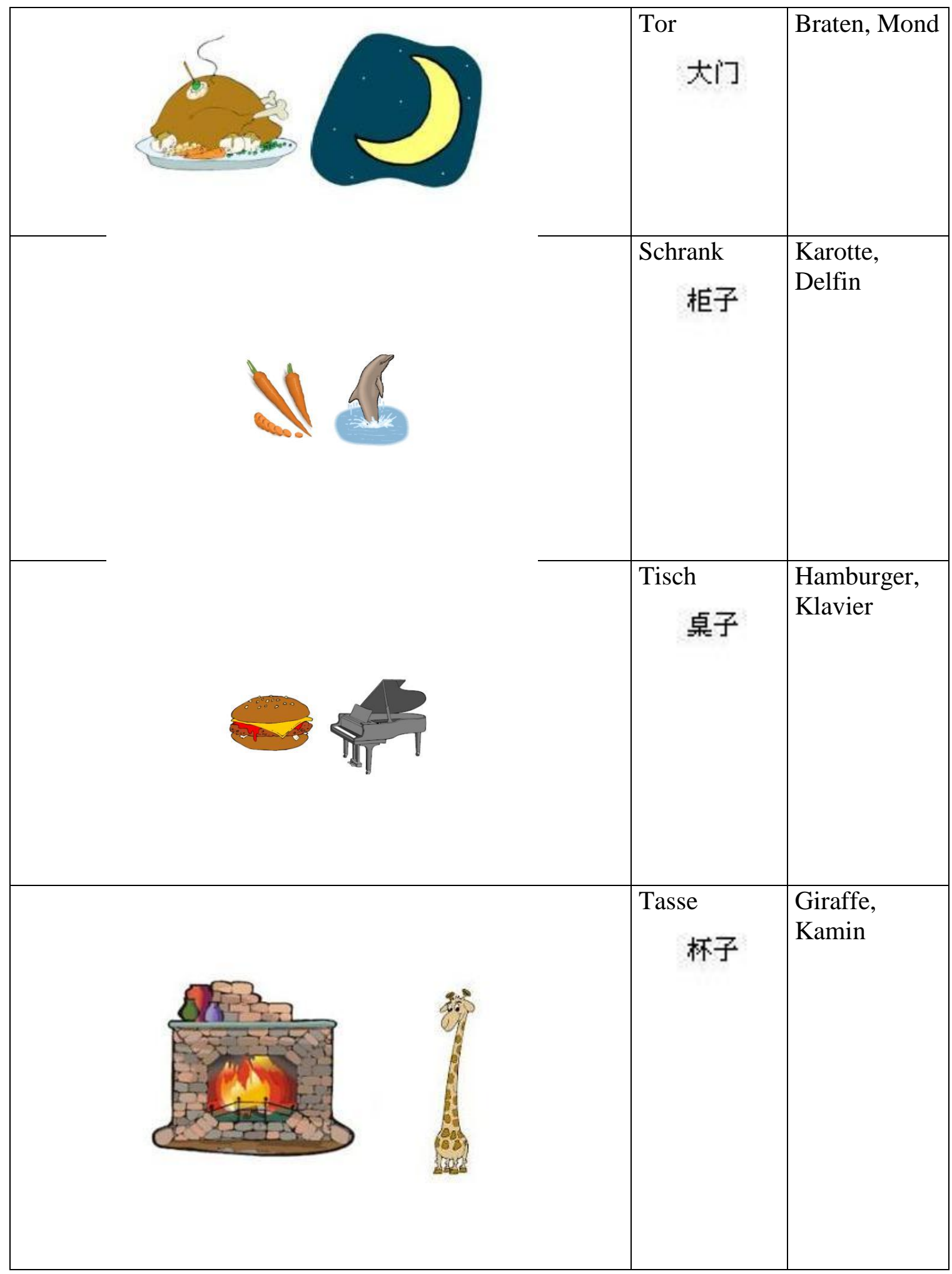




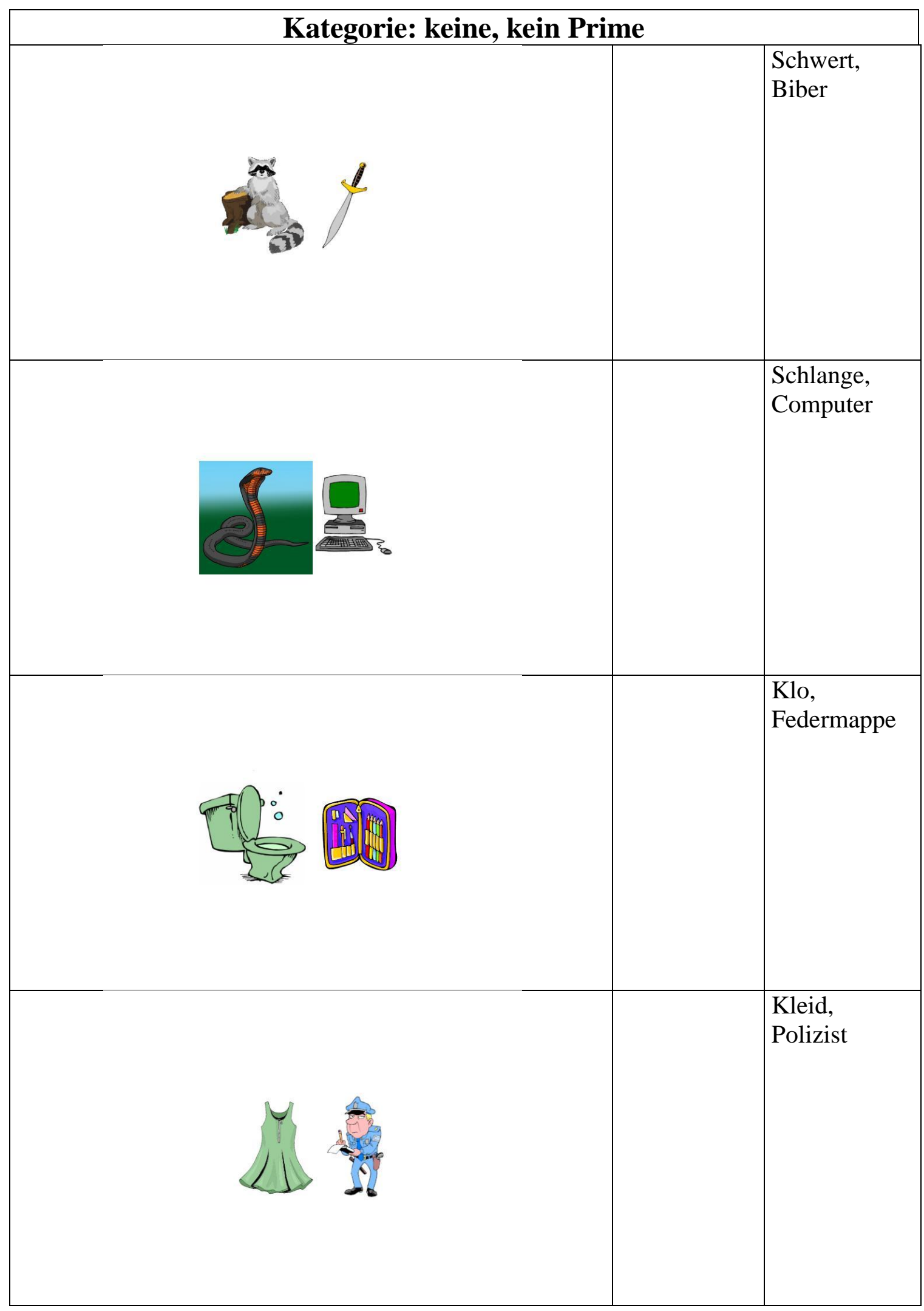




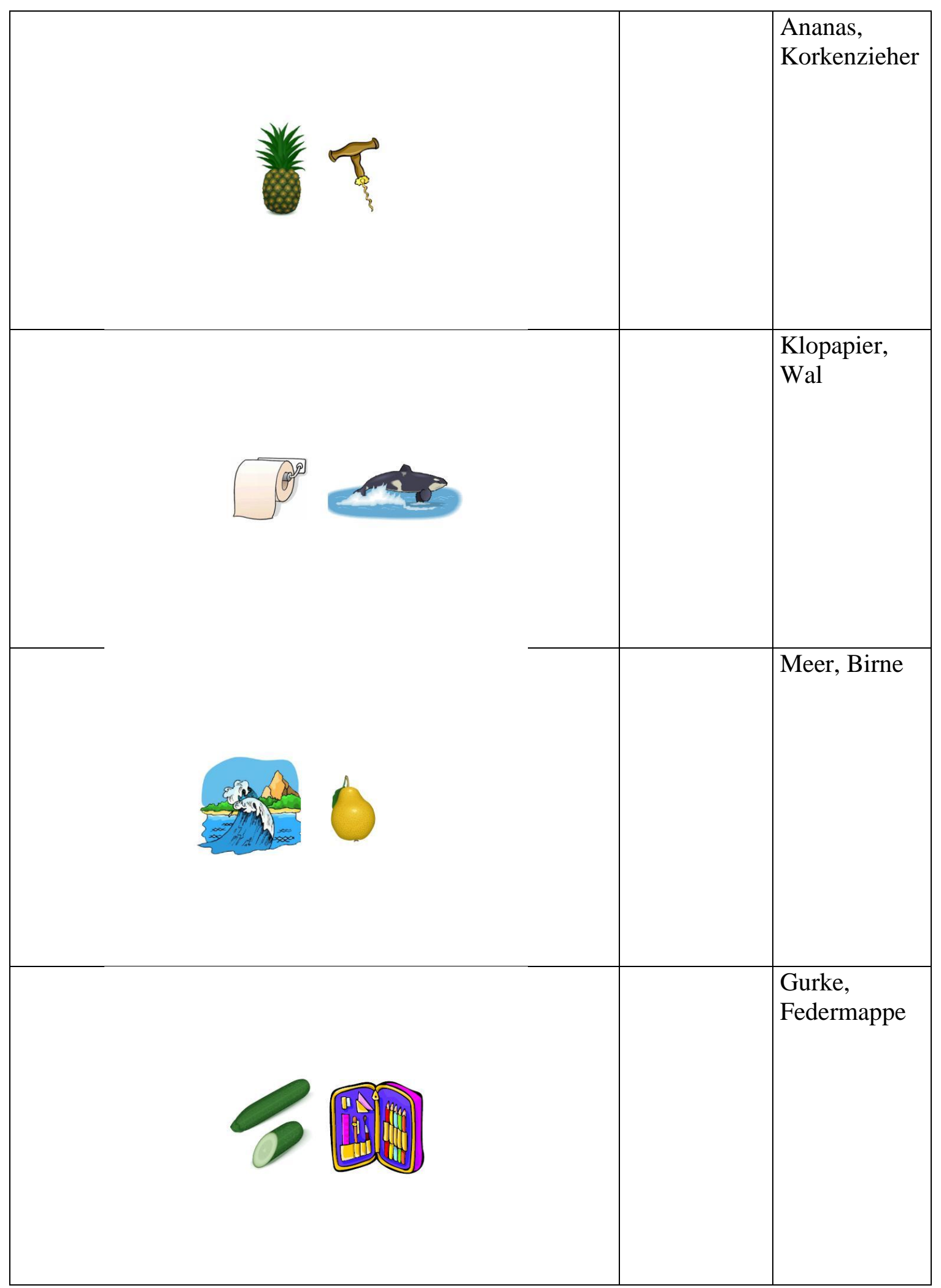




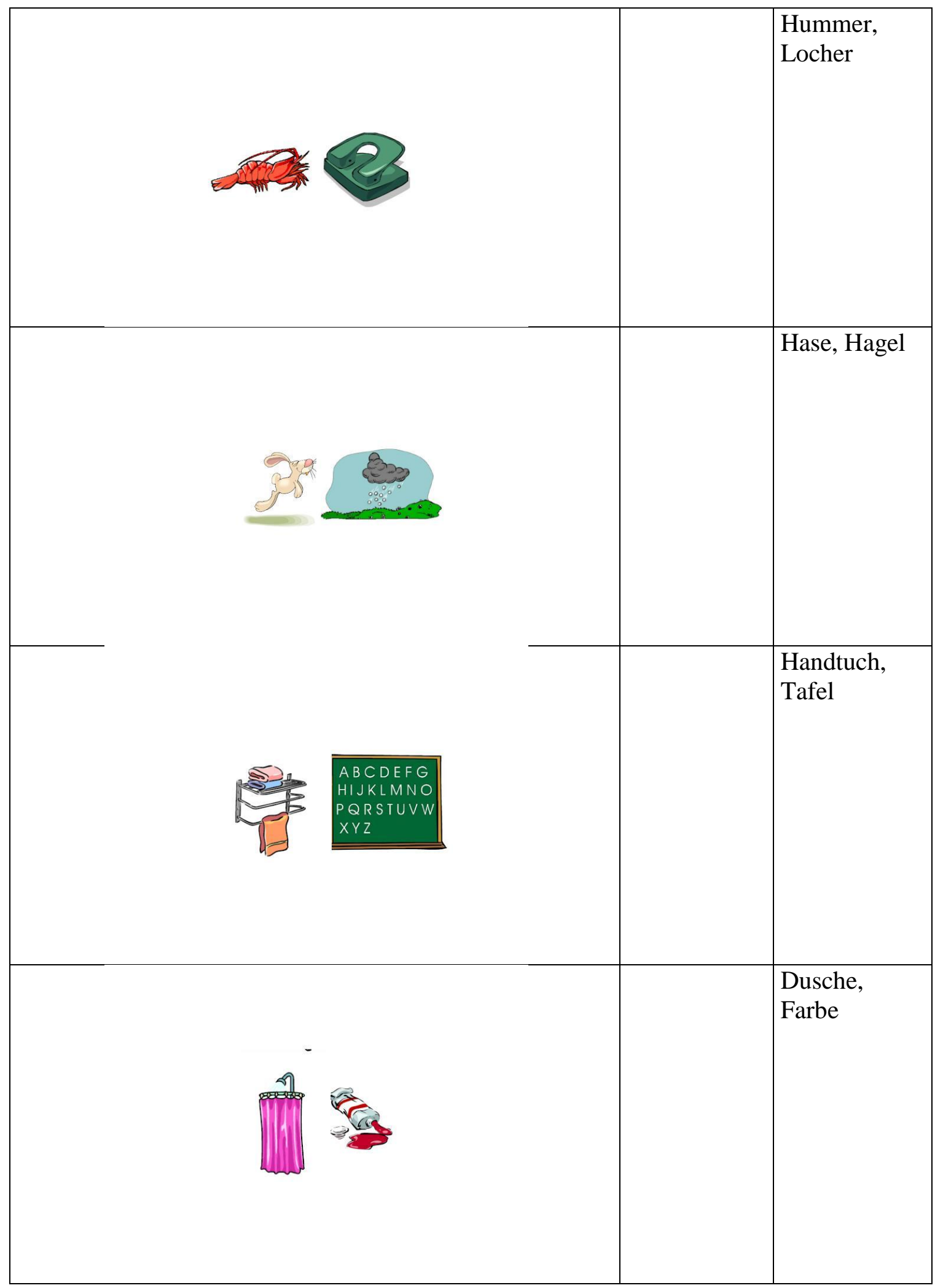




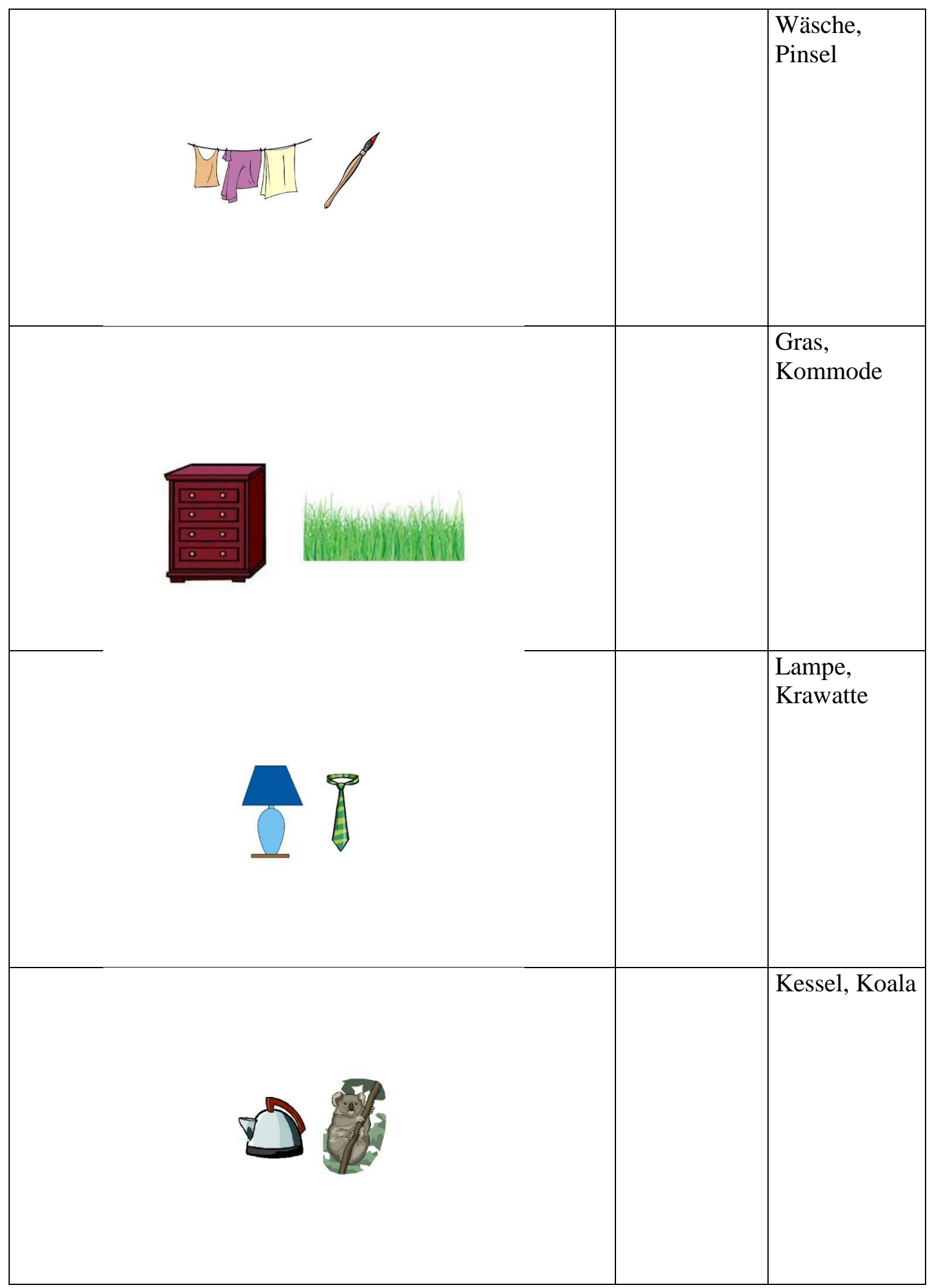




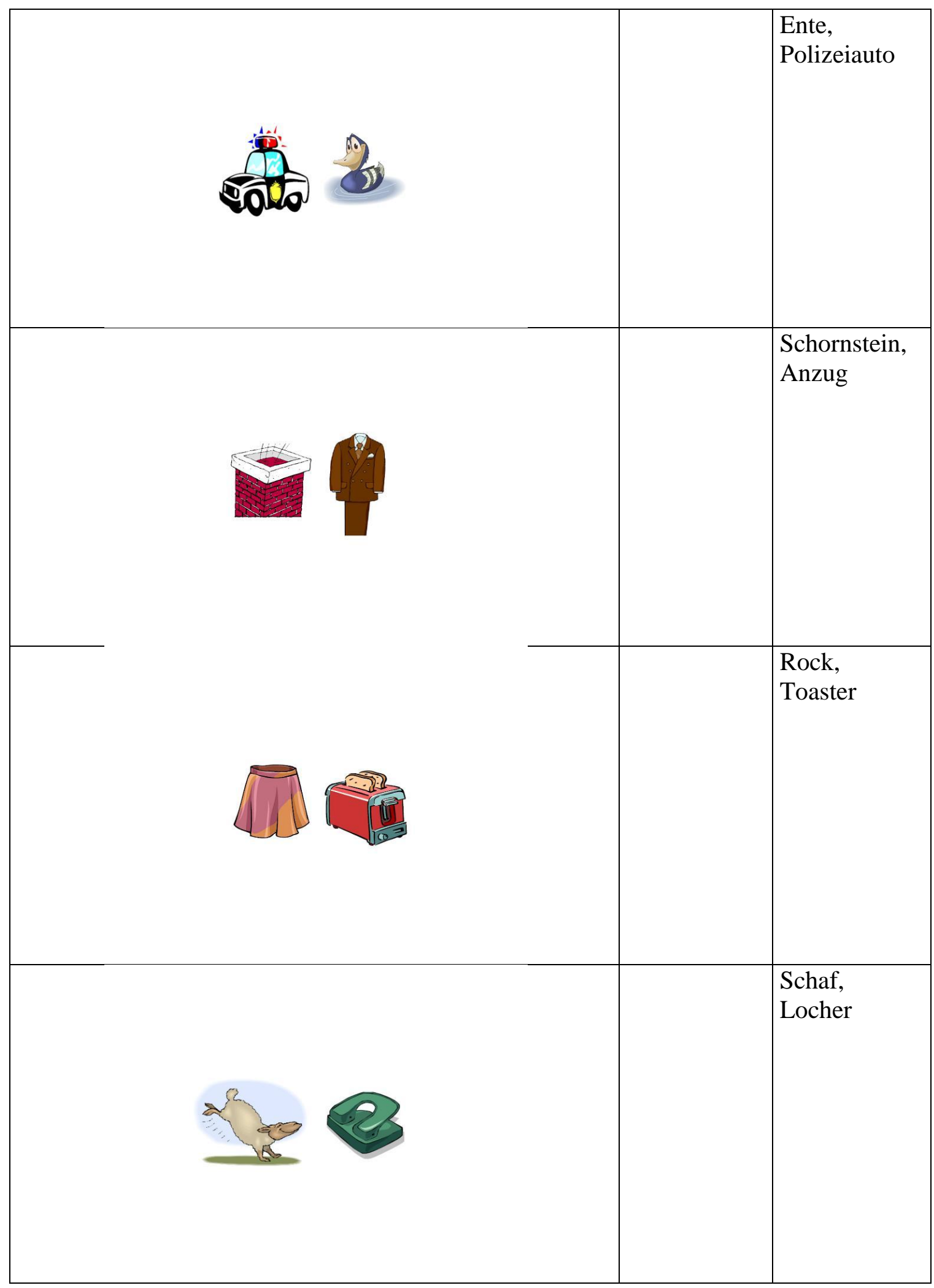




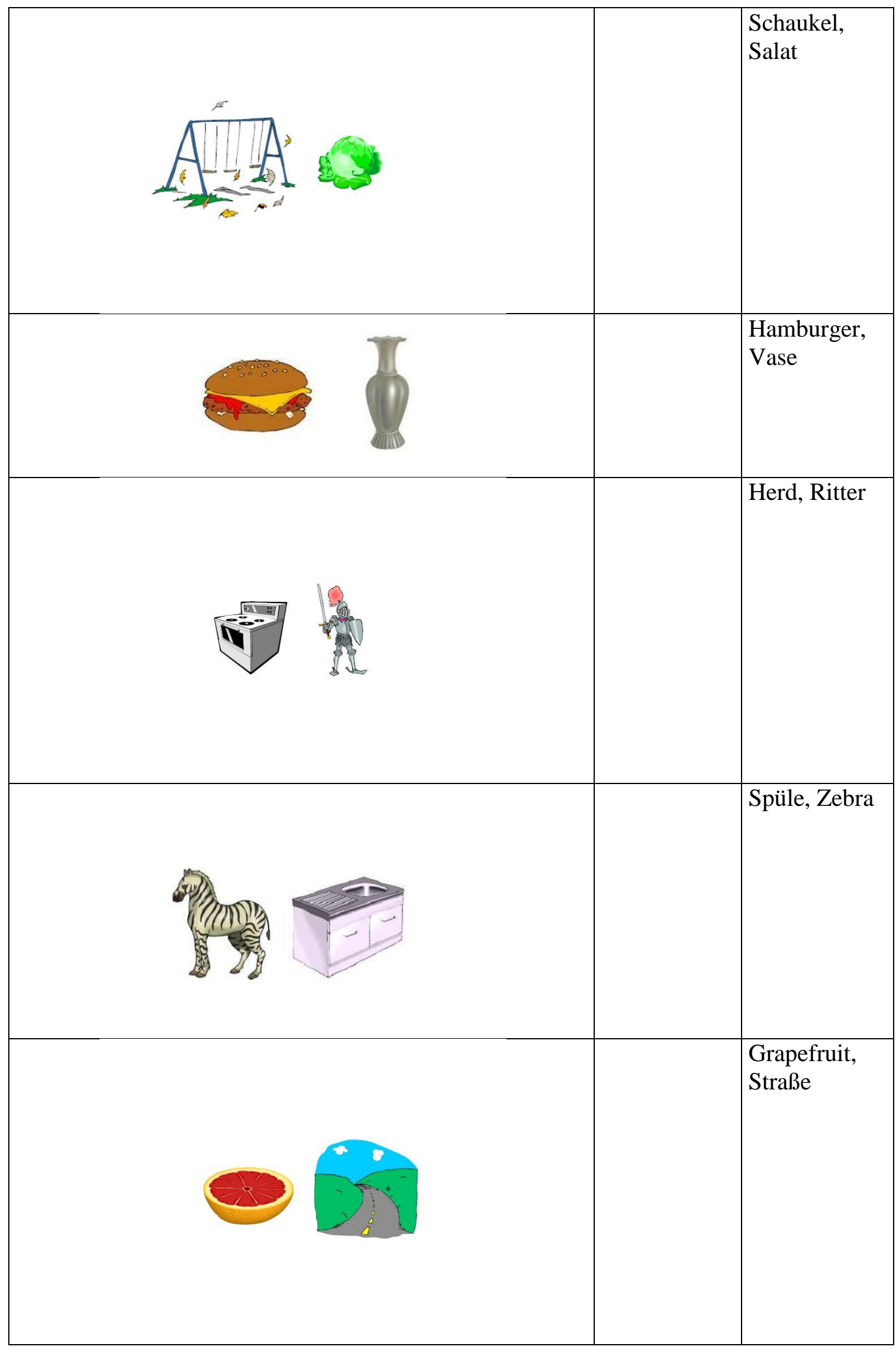




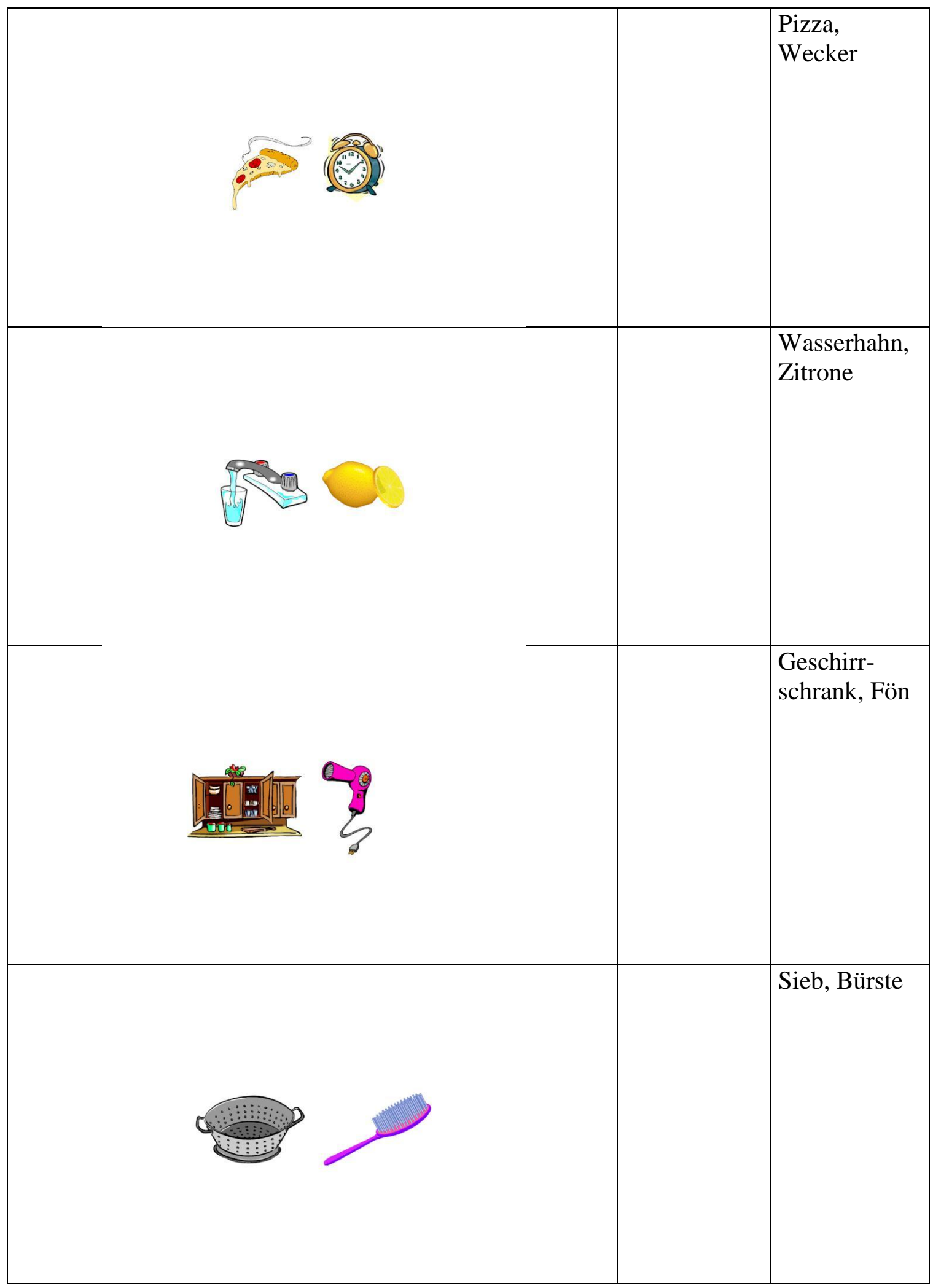


Anhang B: Instruktion Experiment 1 „Kategorisierung über Bildpaare“

$\underline{\text { Deutsche Instruktion }}$

Liebelr Versuchsteilnehmerlin, vielen Dank für Deine Bereitschaft an diesem Versuch teilzunehmen!

Bitte lies Dir die folgende Instruktion aufmerksam durch.

Im Verlauf des Versuchs werden Dir auf dem Bildschirm Paare von Bildern präsentiert. Bitte beurteile, ob die gezeigten Dinge in irgendeiner Art und Weise zusammen passen. Wenn man die beiden Bilder einer gemeinsamen Kategorie zuordnen kann, oder sie eine funktionale oder logische Verbindung zueinander besitzen, antworte mit JA.

Stehen die Dinge in keinerlei Beziehung zueinander, antworte mit NEIN. Deine Antwort kannst Du mit Hilfe der Tastatur über folgende Tasten eingeben:

JA: L

NEIN: $\mathbf{S}$

Versuche so schnell wie möglich zu antworten.

Der genaue Versuchsablauf wird im Folgenden anhand eines Beispiels verdeutlicht:

Richte Deinen Blick zunächst auf das Kreuz, welches auf dem Bildschirm erscheint!

$+$

Fixiere dieses Kreuz solange, bis die beiden Bilder präsentiert werden.

Beispiel:

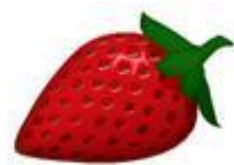

Gib Deine Antwort nun so schnell wie möglich über die Tastatur ein. Als nächstes erscheint wieder das Kreuz auf dem Bildschirm und der nächste Durchgang beginnt.

Das Experiment besteht aus mehreren Blöcken, wobei Dir nach Vollendung jeden Blocks die Möglichkeit gegeben wird, solange Pause zu machen, wie Du möchtest. Du kannst den nächsten Block selbständig starten, indem Du die Leertaste drückst.

Bei Fragen wende Dich bitte jetzt an die Versuchsleiterin! 


\section{Chinesische Instruktion}

亲爱的参试者:

非常感谢你参与这个实验！请你仔细阅读以下说明。

在实检过程中你将会每次在屏幕上看到两幅同时呈现的图片。请你直觉地判断，图上所画的 两个东西是否在某个方面或者某种关系上相互匹配。如果两张图片可以被归为一类，或者他 们之间存在功能上的或者逻辑上的联系, 请回答"是"。如果两發图片毫无联系，请回答"不 是"。你可以埇过敲击键盤上的下列键输入你的回答:

是: $L$

不是: $S$

\section{请你尽可能快速回答。}

下面是这样一个任务的例子已让你实验的具体过程：请你先注意屏幕上出现的十字！

请注视这个十字, 直到这两幅图片出现。 举例图片:
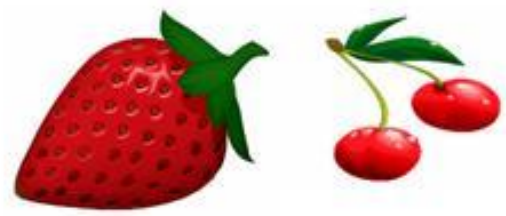

这时请尽可能快地通过键盤输入你的回答。你将会从随后的反馈信息中得知，接下去屏幕上 又会出现十字，下一轮回答又开始了。

这个试检分为几个部分。每一个部分完成后，你都能暂停休息一下。通过按空格键屌动你可 以下一部分实验, 继续进行回答。若有问题请现在向主试提出来! 
Anhang C: Nachtestfragebögen Experiment 1 „Kategorisierung über Bildpaare“

\section{Deutscher Nachtestfragebogen}

\section{Lie be/r Versuchsteilnehmer/in,}

Abschlie Bend beurteile bitte bei den folgenden Wortpaaren zunachst, ob die beiden Dinge in einer taxonomischen Beziehung zueinander stehen (das heibt, ob sie der gleichen Kate gorie angehören) und danach, ob sie in einer relationalen Beziehung zueinander stehen (das heiBt, ob sie eine logische oder funktionale Verbindung zueinander besitzen).

\begin{tabular}{l}
\begin{tabular}{l} 
Kann man sagen, dass folgende Dinge einer germeinsamen Kategorie zugeordnet \\
werden kbnnen (taxonomische Beziehung)? \\
\hline
\end{tabular} JA \\
\hline
\end{tabular}




\begin{tabular}{|c|c|c|c|c|c|}
\hline Kugelschreiber & & & & & \\
\hline $\begin{array}{l}\text { Polinei, } \\
\text { Krankenwagen }\end{array}$ & & & Blume, Baum & & \\
\hline Hochhaus, Loffel & & & Spind, Kleiderschrank & & \\
\hline Ful, Hand & & & Hund, Pferd & & \\
\hline Bein, Kopf & & & Orange, Fisch & & \\
\hline $\begin{array}{l}\text { Grapefruit, } \\
\text { Pflaume }\end{array}$ & & & Zahnpasta, Seife & & \\
\hline Buch, Schulbuch & & & Hubschrauber, Flugneug & & \\
\hline $\begin{array}{l}\text { Kann man sagen, } d: \\
\text { zueinander besitwen }\end{array}$ & $\begin{array}{l}15 s \mathrm{fi} \\
(\mathrm{rell})\end{array}$ & $\begin{array}{l}2 \text { Dinge } \\
\text { e Berieh }\end{array}$ & $\begin{array}{l}\text { ne logische oder funktional } \\
\text { ngy? }\end{array}$ & & \\
\hline & $\mathrm{JA}$ & NEIN & & $\mathrm{JA}$ & NEIN \\
\hline Braten, Besteck & & & Tisch, Lampe & & \\
\hline Moer, Delfin & & & Polizist, Polineiauto & & \\
\hline $\begin{array}{l}\text { Schreibtisch, } \\
\text { Computer }\end{array}$ & & & Socken, Fub & & \\
\hline Waserhahn, Spule & & & Hand, Handschuh & & \\
\hline Farbe, Pinsel & & & Suppe, Salz \& Pfeffer & & \\
\hline Ritter, Schornstein & & & Buch, Regal & & \\
\hline $\begin{array}{l}\text { Korlkentieher, } \\
\text { Weinglas }\end{array}$ & & & Messer, Paprika & & \\
\hline Blatt, Bleistift & & & Schöpfkelle, Suppe & & \\
\hline $\begin{array}{l}\text { Radiergummi, } \\
\text { Bleistift }\end{array}$ & & & Schrauberrieher, Schraube & & \\
\hline $\begin{array}{l}\text { Radiergummi, } \\
\text { Katre }\end{array}$ & & & Himme L, Flugreug & & \\
\hline Gras, Kuh & & & Eisw Liffel, Blech & & \\
\hline Kind, Schaukel & & & Zahnburste, Zahnpasta & & \\
\hline Hohle, Bur & & & Blit, Regen & & \\
\hline Pinsel, Blatt & & & Ball, Hut & & \\
\hline Indianer, Zelt & & & Bett, Wecker & & \\
\hline $\begin{array}{l}\text { Regen, } \\
\text { Regenjacke }\end{array}$ & & & Schlange, Apfel & & \\
\hline $\begin{array}{l}\text { Wascheklammer, } \\
\text { Wasche }\end{array}$ & & & Sirafe, Auto & & \\
\hline Topf, Hend & & & Hase, Karotte & & \\
\hline Wok, Stabchen & & & Apfel, Baum & & \\
\hline $\begin{array}{l}\text { Salatkopf, } \\
\text { Hubshrauber }\end{array}$ & & & Banane, Affe & & \\
\hline $\begin{array}{l}\text { Blatt, } \\
\text { Kuselschreiber }\end{array}$ & & & Gewicht, Bodybuilder & & \\
\hline Spielplatz, Kind & & & Lichitschalter, Lampe & & \\
\hline Burg, Ritter & & & Dusche, Handtuch & & \\
\hline Moer, Schiff & & & Ritter, Schwert & & \\
\hline Eule, Wald & & & Mond, Gras & & \\
\hline Piza, Milorowelle & & & Tafel, Kreide & & \\
\hline Zoo, Elefant & & & Block, Fedenmappe & & \\
\hline $\begin{array}{l}\text { Sprungbrett, } \\
\text { Badearrug. }\end{array}$ & & & Sieb, Salat & & \\
\hline
\end{tabular}




\begin{tabular}{|l|l|l|l|l|l|}
\hline Zwisbel, Fahmad & & & Delfin, Teller & & \\
\hline Mond, Eule & & & Sportler, Jogginganrug & & \\
\hline Ente, See & & & Kind, Klasserzimmer & & \\
\hline Sportler, Stadion & & & Tocster, Brot & & \\
\hline Kind, Wuste & & & Hend, Kleiderschrank & & \\
\hline Klopapier, Klo & & & Kreide, Blumen & & \\
\hline $\begin{array}{l}\text { Glas, } \\
\text { Geschirmschrank }\end{array}$ & & & Kamin, Schomsinin & & \\
\hline
\end{tabular}




\section{Chinesischer Nachtestfragebogen}

臬要的搭试：

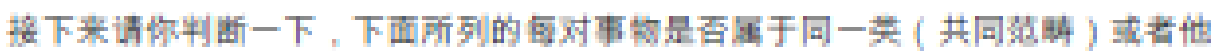

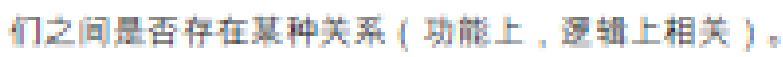

\begin{tabular}{|c|c|c|c|c|c|}
\hline & 量 & 吾 & & 量 & 百 \\
\hline 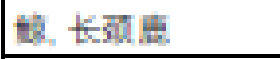 & & & 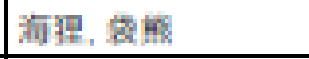 & & \\
\hline 长现底, 猫头注 & & & 睡豆，转护率 & & \\
\hline 吉他, 视等 & & & 点子，柜子 & & \\
\hline 否蒡，愁 & & & 西红枯, 留站 & & \\
\hline 相子, 缷根 & & & 玻䎸柾，莱柇 & & \\
\hline 淂，杉子椒 & & & 盘子，莱林 & & \\
\hline 力, 夌 & & & 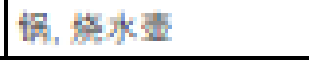 & & \\
\hline 裸子, 上衣 & & & 屏托车, 白行车 & & \\
\hline 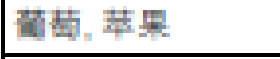 & & & 牛仔神，短裸 & & \\
\hline 牟子, 箱子 & & & 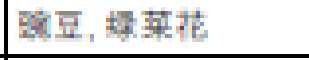 & & \\
\hline 杶子被，薪子 & & & 开关，措子 & & \\
\hline 黄盛, 胡要卜 & & & 故柜，兵子 & & \\
\hline 程返, 机头 & & & 洋要，胡子椒 & & \\
\hline 木垔起子、酒起子 & & & 点子，柜子 & & \\
\hline 秄子, 柦子 & & & 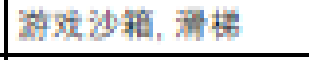 & & \\
\hline 流㴍, 鸡 & & & 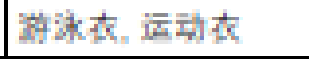 & & \\
\hline 71. 领带 & & & 科马，求是 & & \\
\hline 香蓓, 溒覀 & & & 小珑，教肉 & & \\
\hline 花, 草 & & & 深车, 飞机 & & \\
\hline 事, 夜柜 & & & 柜子，书繁 & & \\
\hline 猜，珘马 & & & 弱，䡒 & & \\
\hline 玻域杯，盘子 & & & 吹风，头剧 & & \\
\hline 戴最，鸡 & & & 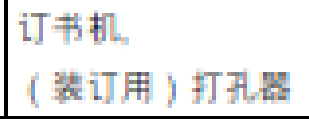 & & \\
\hline 裹望, 理 & & & 长殁底, 海㨁 & & \\
\hline 马, 野福 & & & 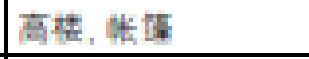 & & \\
\hline
\end{tabular}




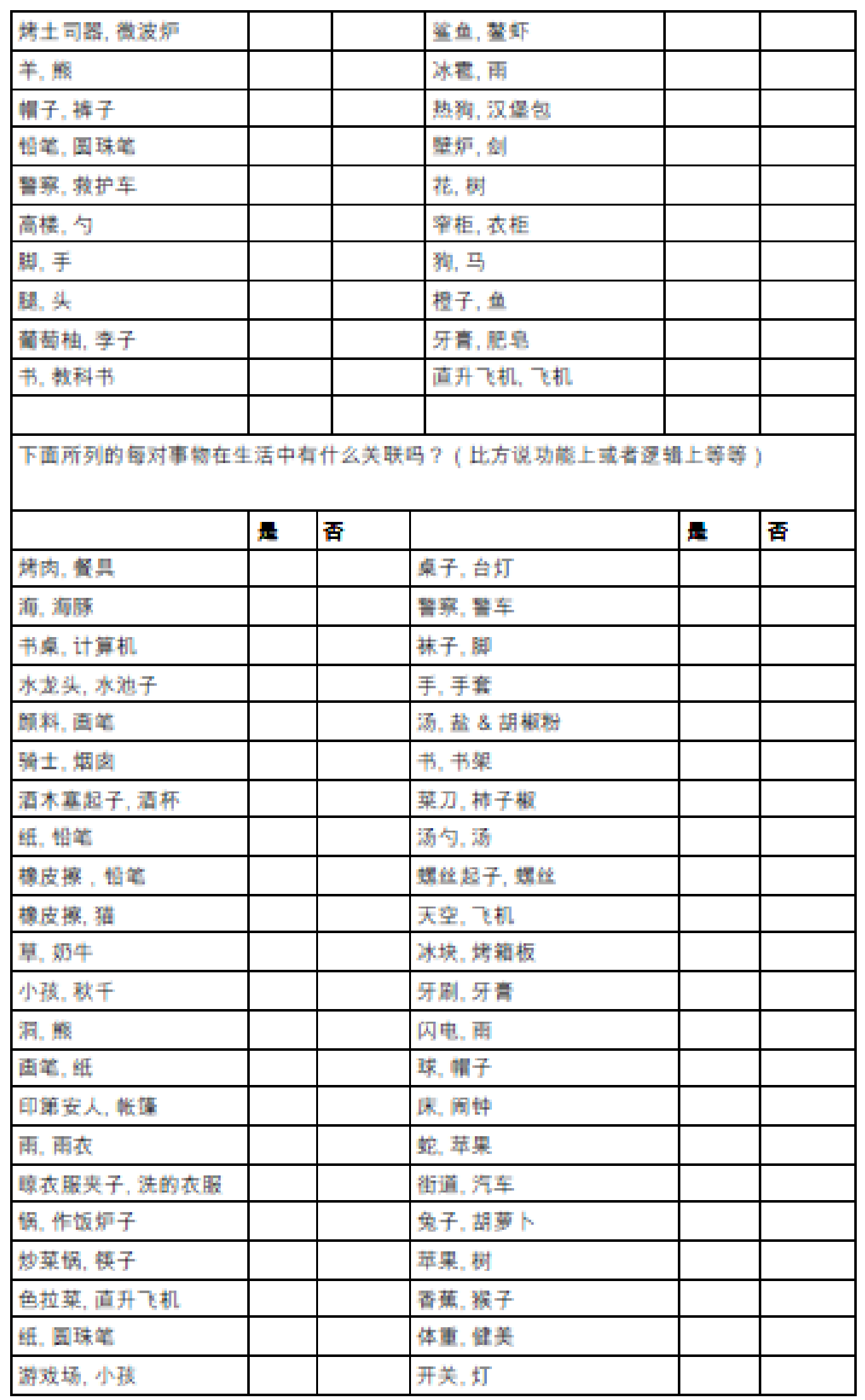




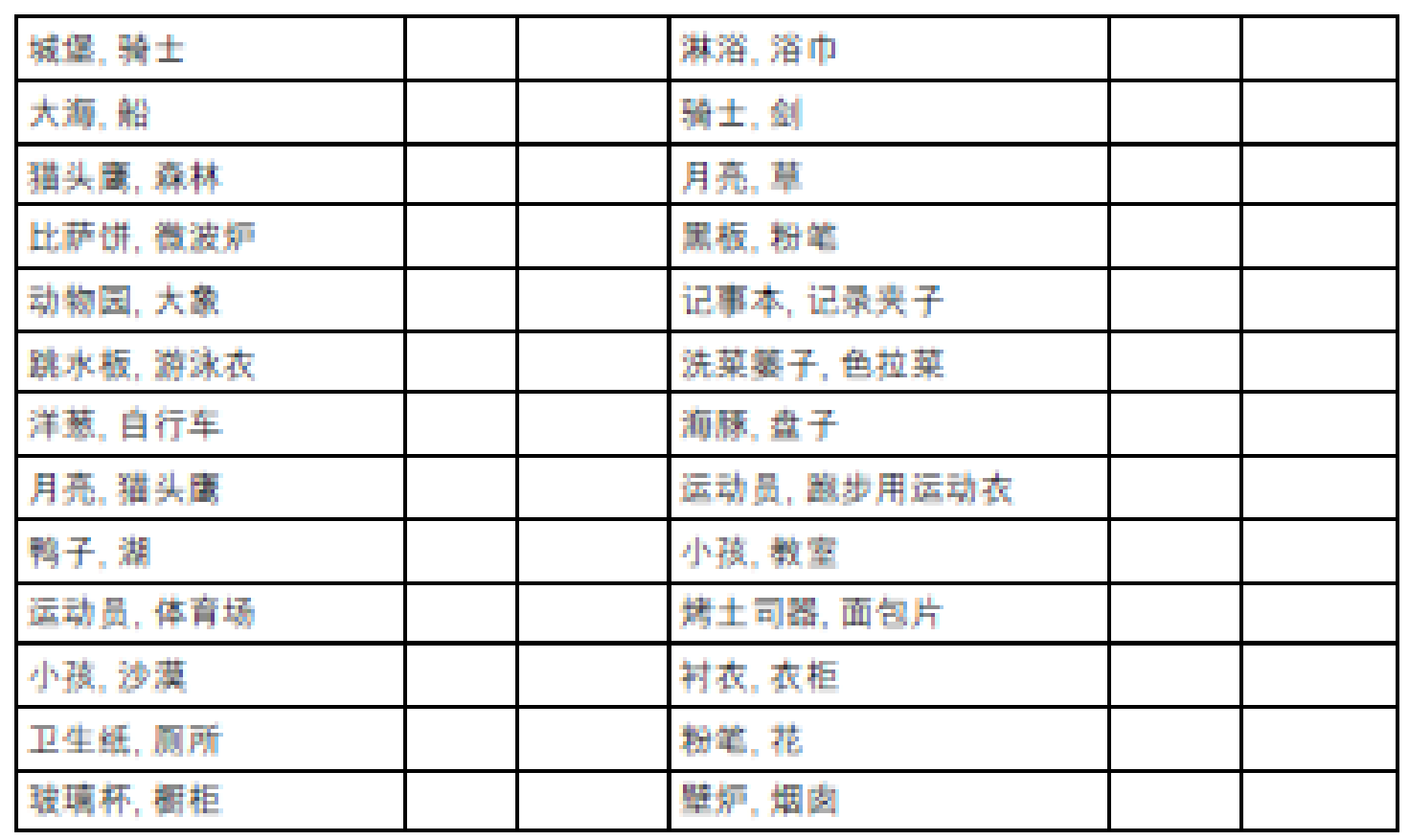


$\underline{\text { Fragebogen zur Erfassung der demographischen Daten in Deutschland }}$

Vielen Dank für Deine Teilnahme an diesem Experiment!

Abschließend werden noch einige Informationen zu Deiner Person erfasst. Deine Angaben werden natürlich anonym behandelt.

Geschlecht:

Alter:

Beruf/Studiengang:

Nationalitảt:

Muttersprache:

Geburtsland:

Falls Dein Geburtsland nicht Deutschland sein sollte, wie lange bist Du schon in Deutschland?

Hast Du noch Anmerkungen zum

Experiment?. 


\section{Fragebogen zur Erfassung der demographischen Daten in China}

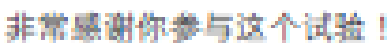

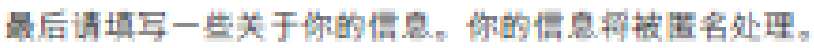

性别

年龄

职业/所学专业

因籍

Si证

出生国家:

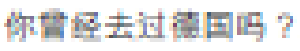

䐈果去过，你在郡员了多久?

你对这个实验还有什么评论吗? 
Anhang D: Itemmaterial Experiment 2 ,Kategorisierung über Bildtripel““

\begin{tabular}{|c|c|c|}
\hline $\begin{array}{l}\text { Beziehung: taxonomisch versus } \\
\text { relational }\end{array}$ & Prime: taxonomisch & \\
\hline Bild & Inhalt & Prime \\
\hline $\operatorname{li}^{3}$. & Gras, Kuh, Huhn & $\begin{array}{l}\text { Ziege } \\
\text { 拜 }\end{array}$ \\
\hline 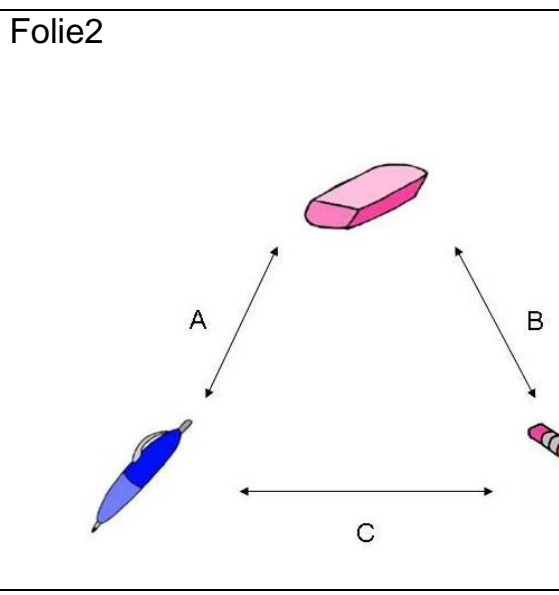 & \begin{tabular}{|l} 
\\
Bleistift, Kugelschreiber, \\
Radiergummi
\end{tabular} & $\begin{array}{l} \\
\text { Pinsel } \\
\text { 笔 }\end{array}$ \\
\hline Folie3 & \begin{tabular}{|l} 
\\
Banane, Affe, Zebra
\end{tabular} & $\begin{array}{l}\text { Giraffe } \\
\text { 长颈鹿 }\end{array}$ \\
\hline
\end{tabular}




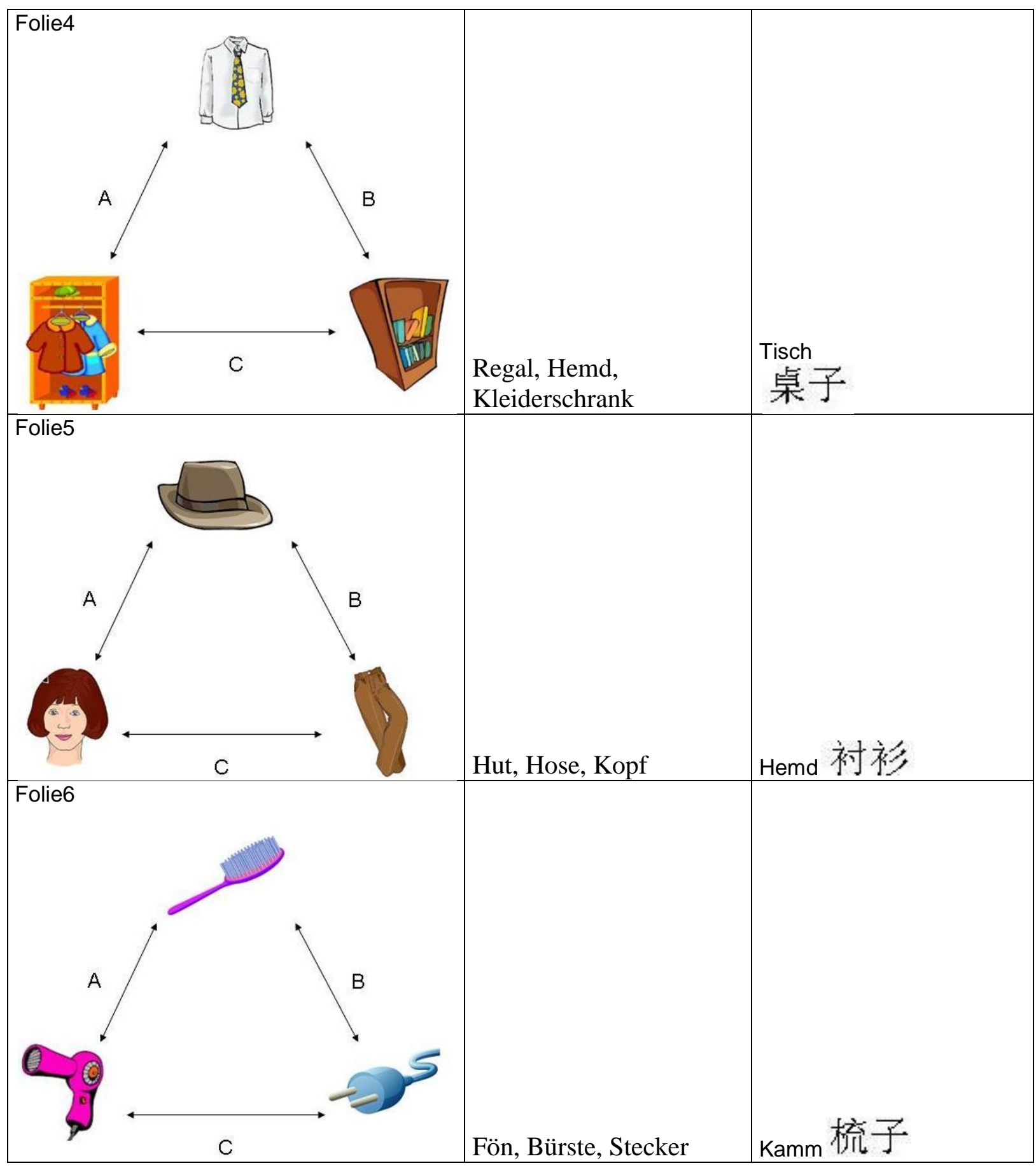




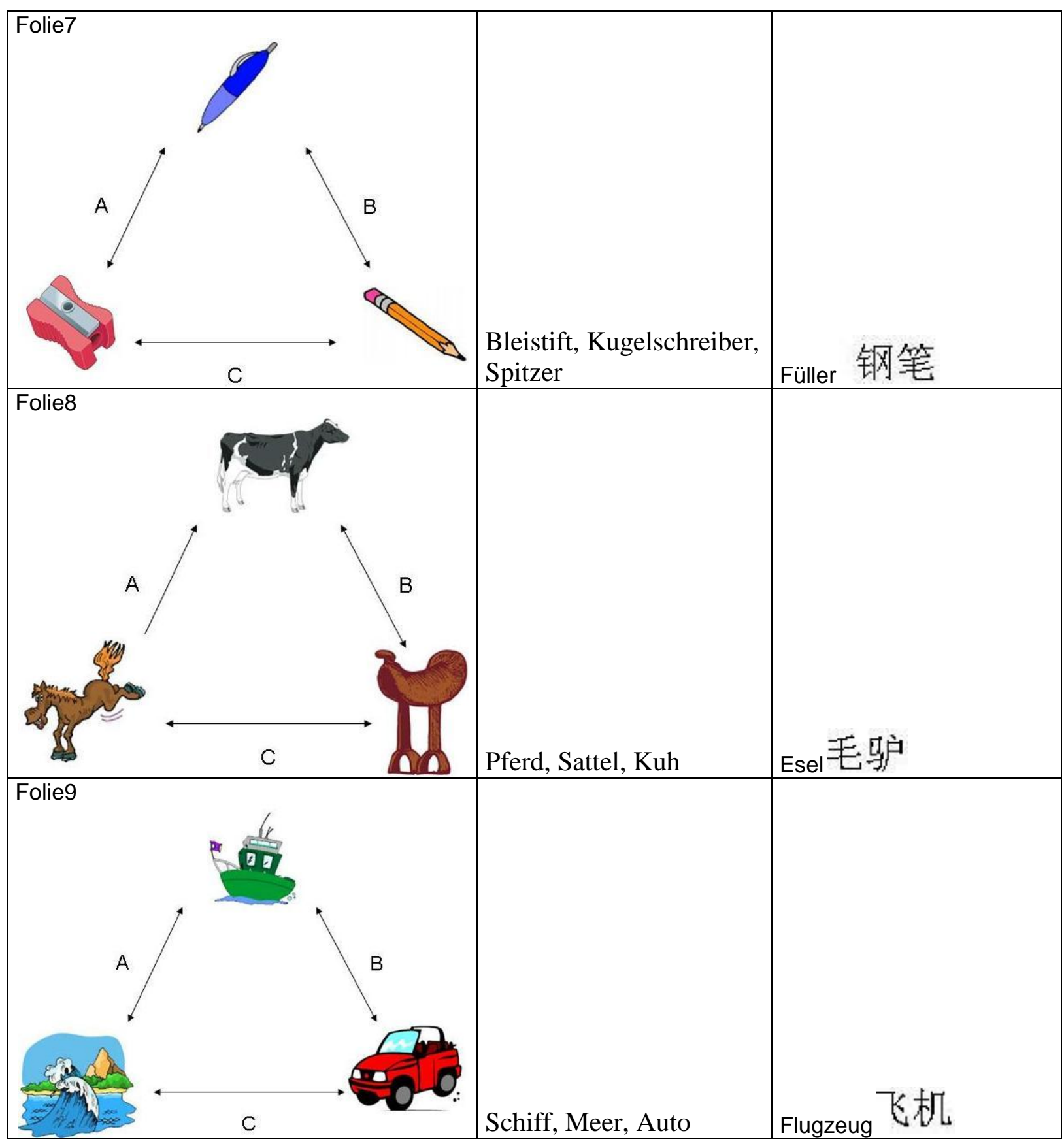




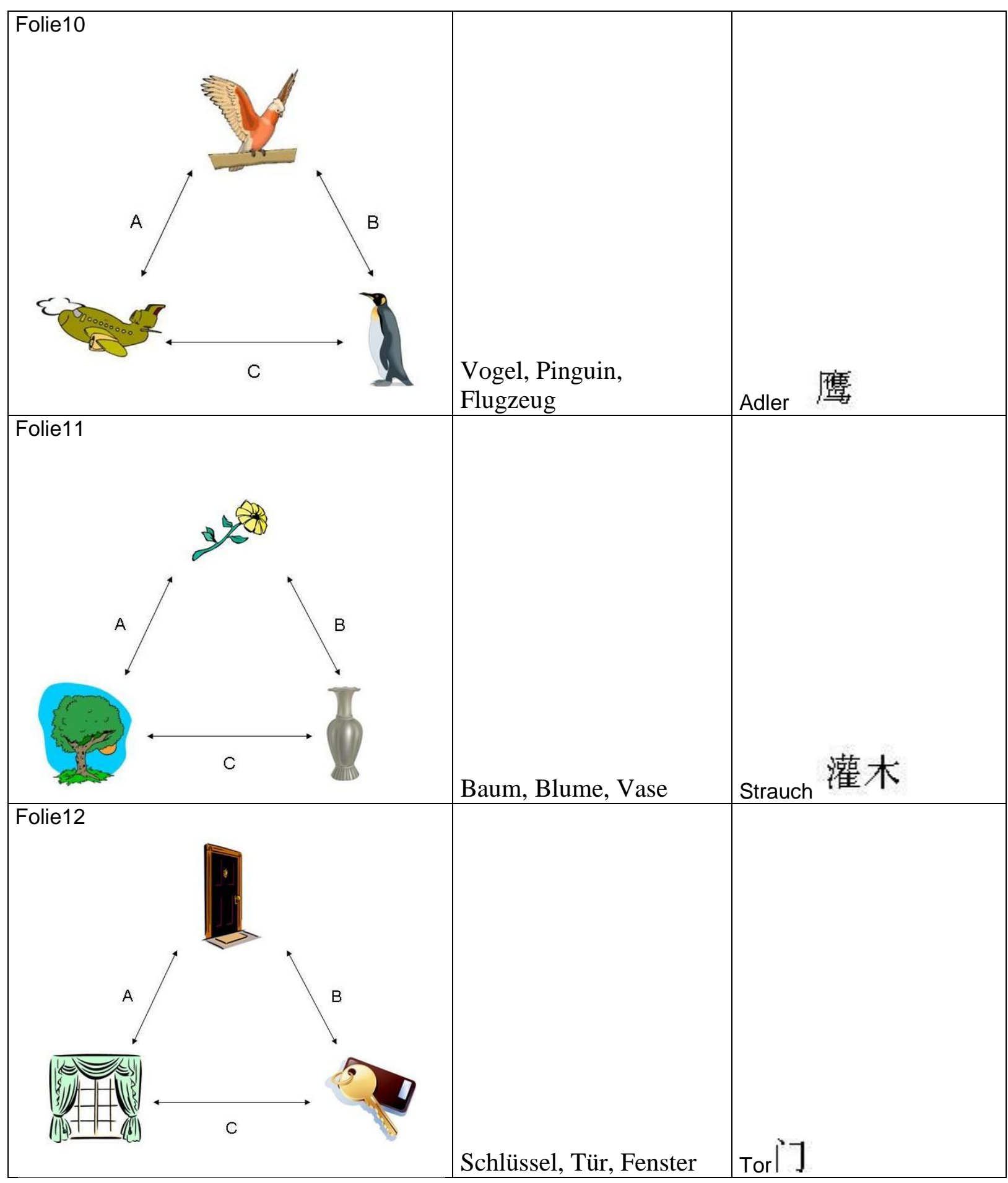




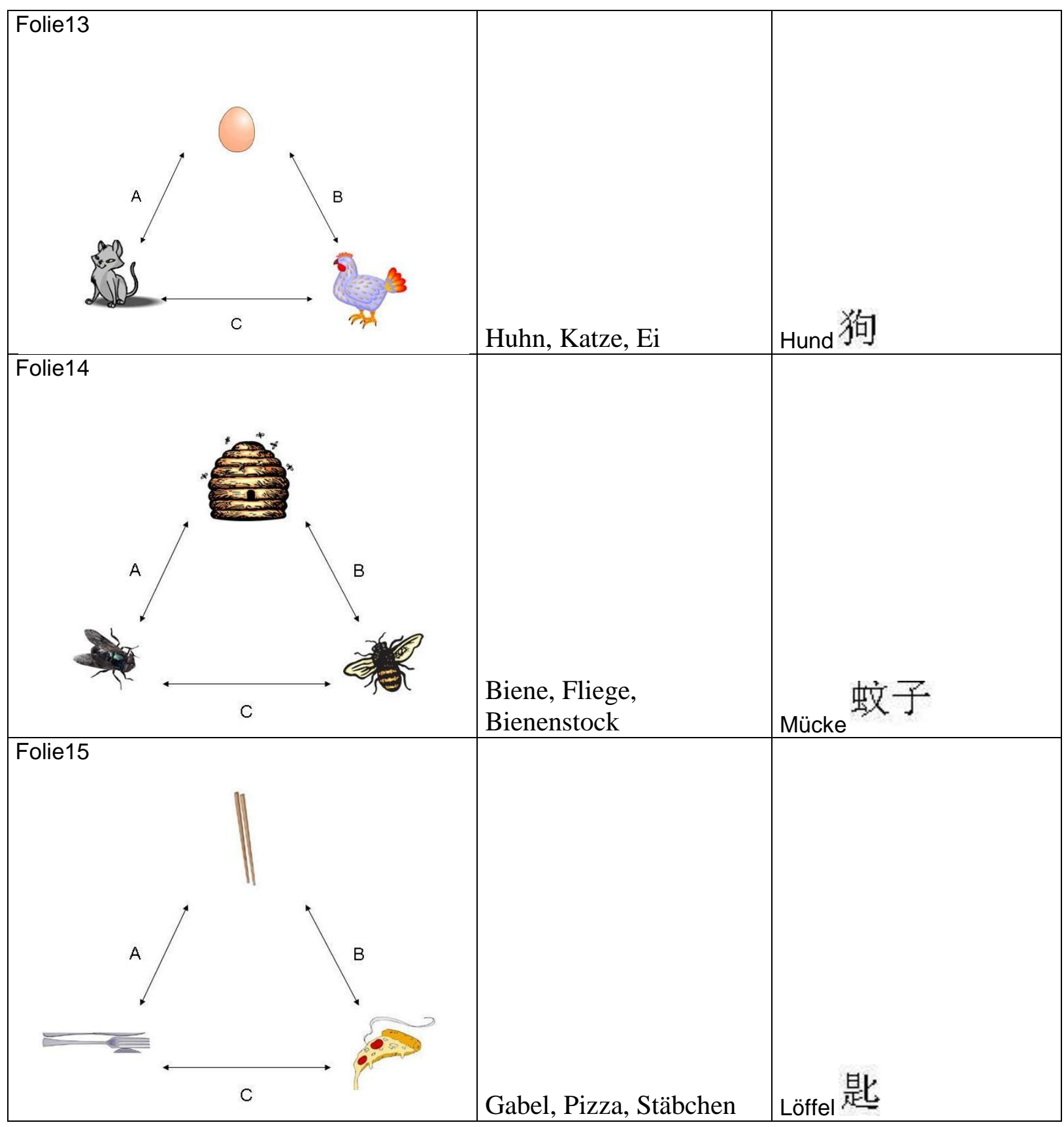




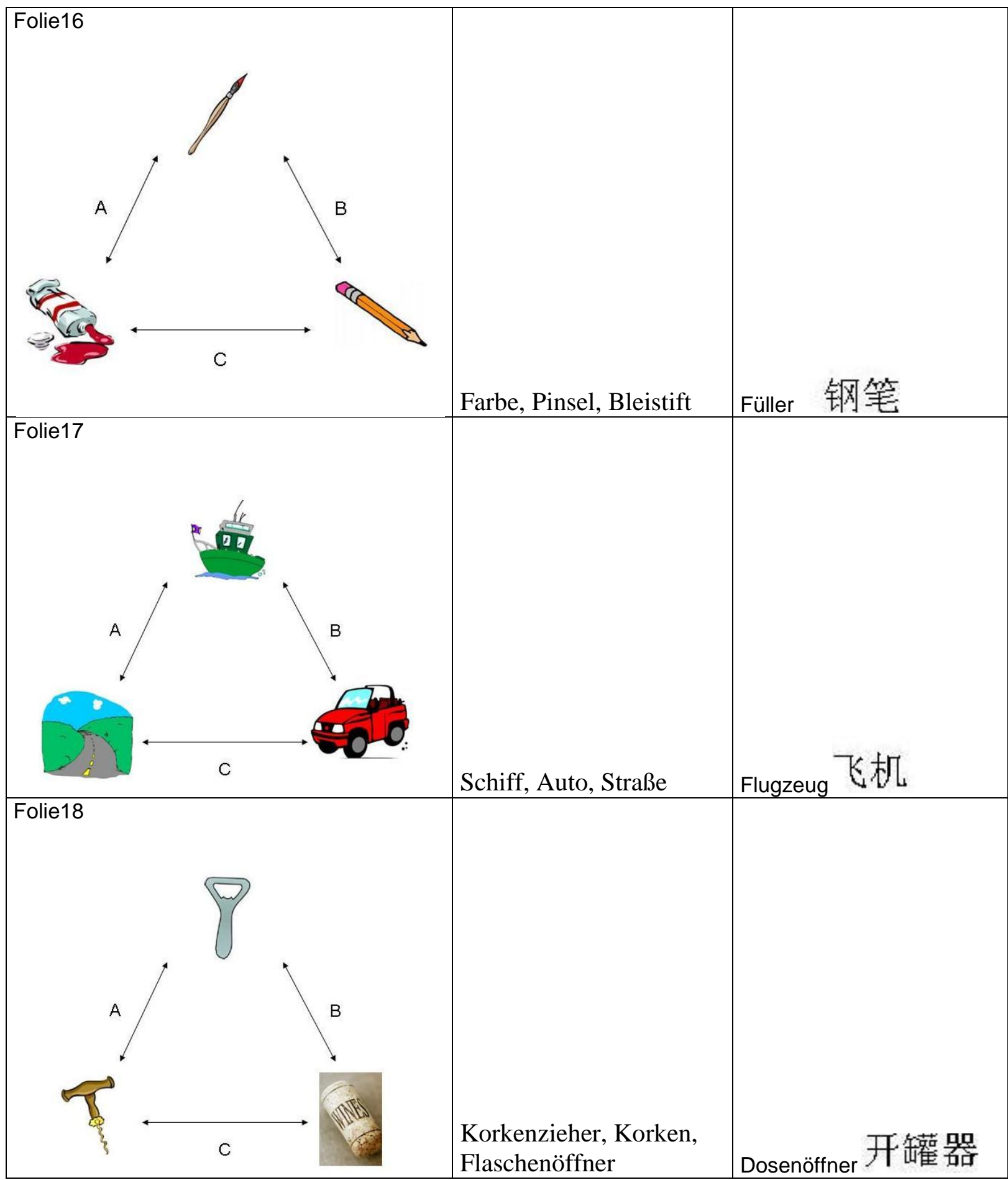




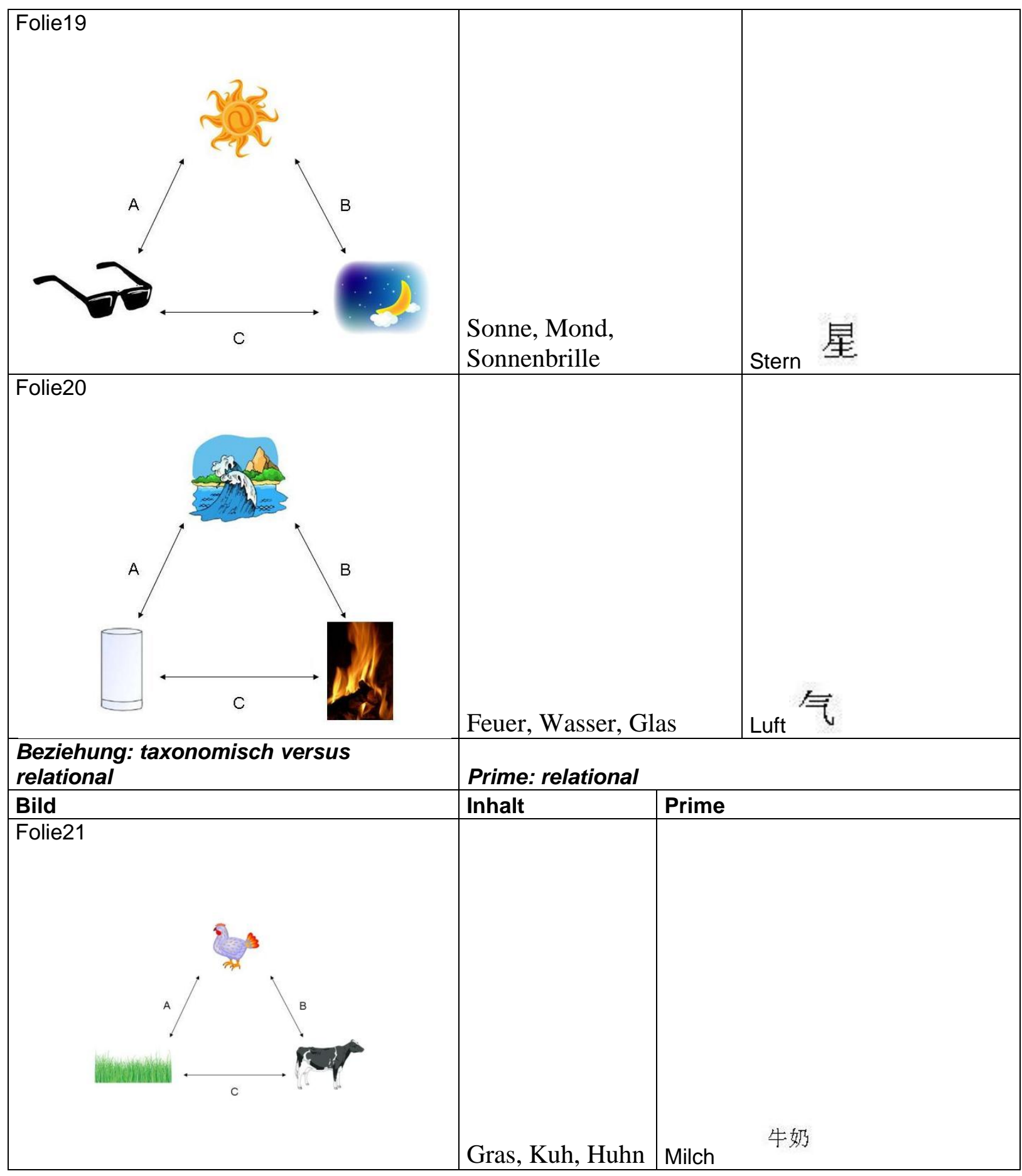




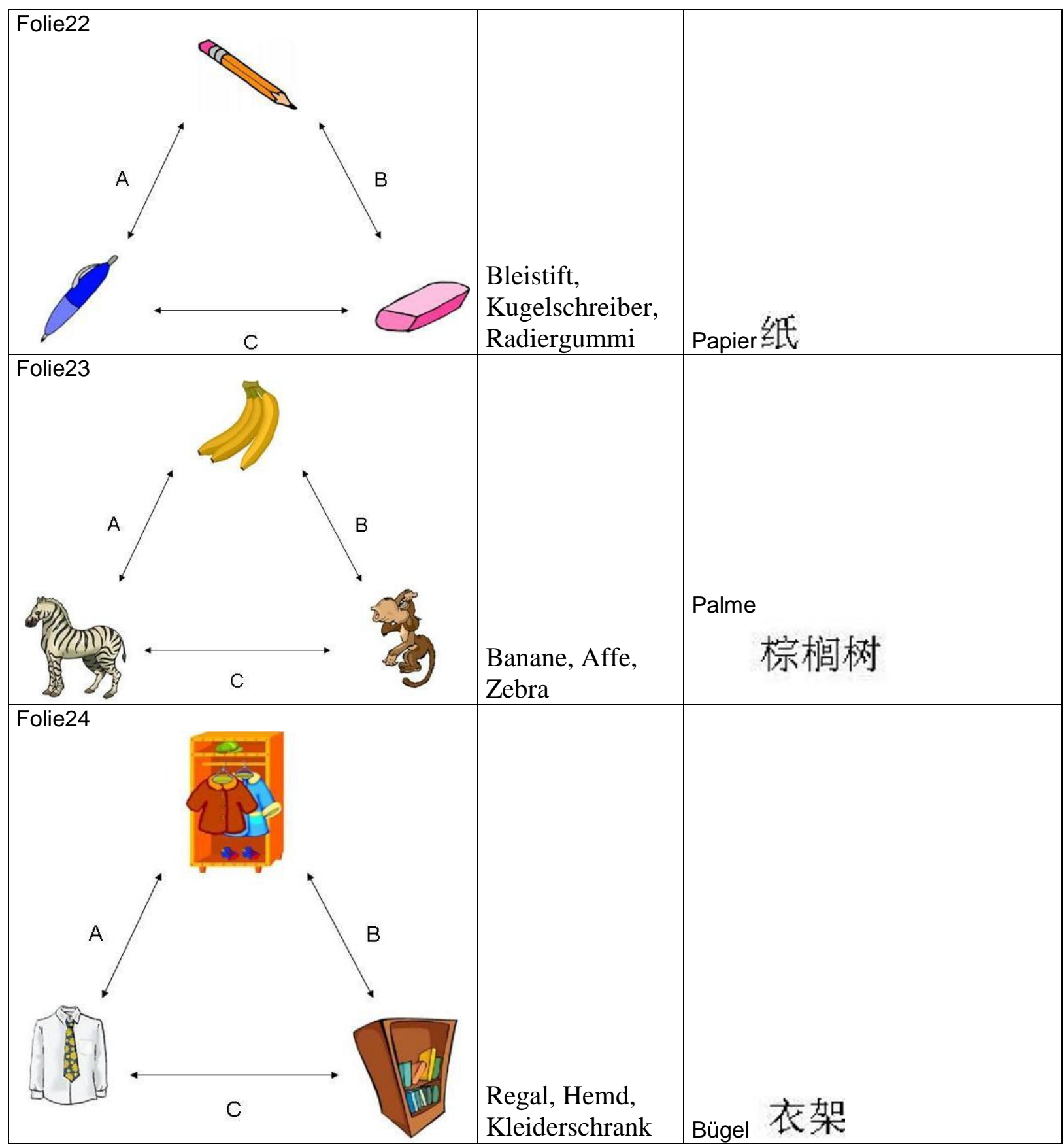




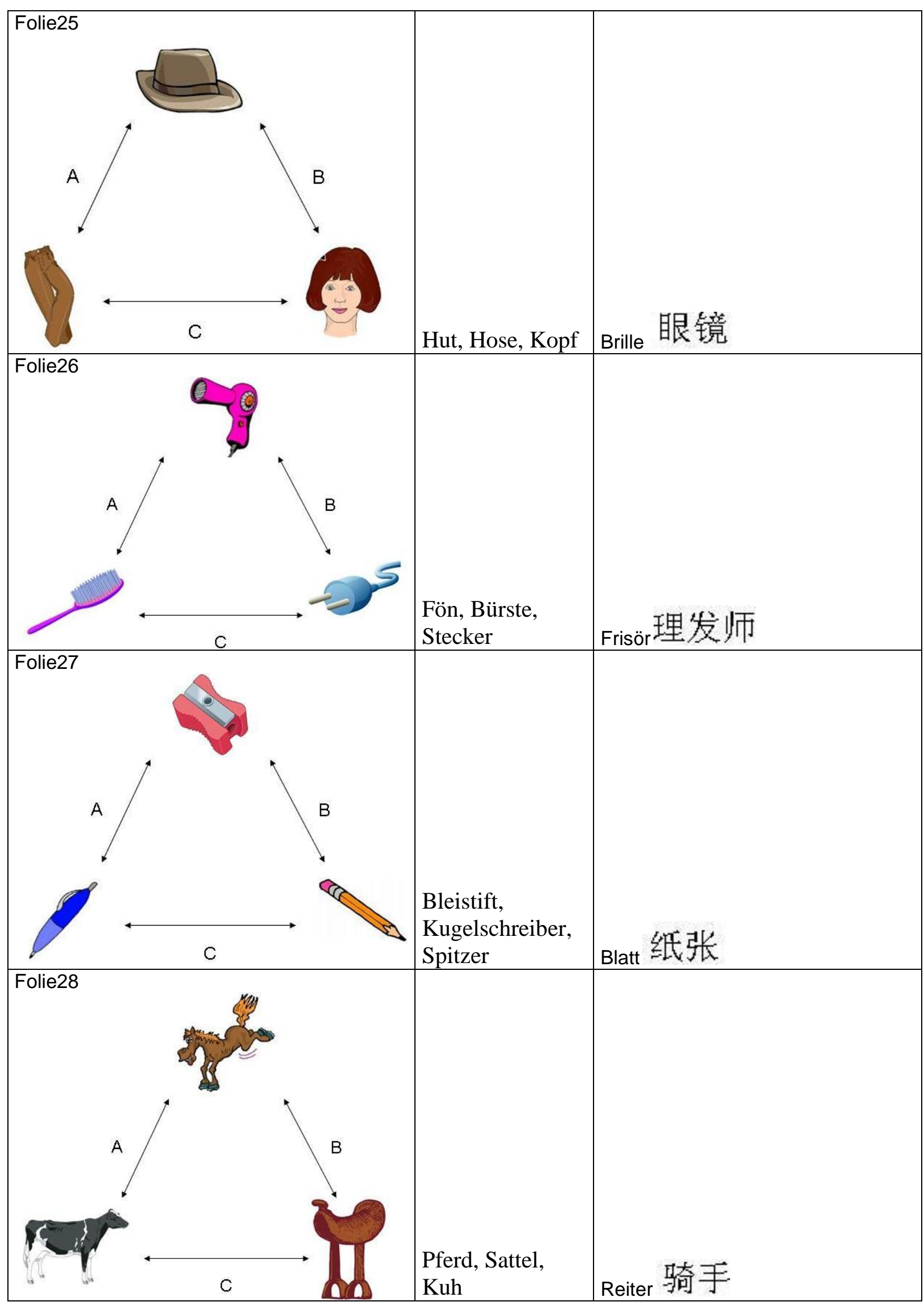




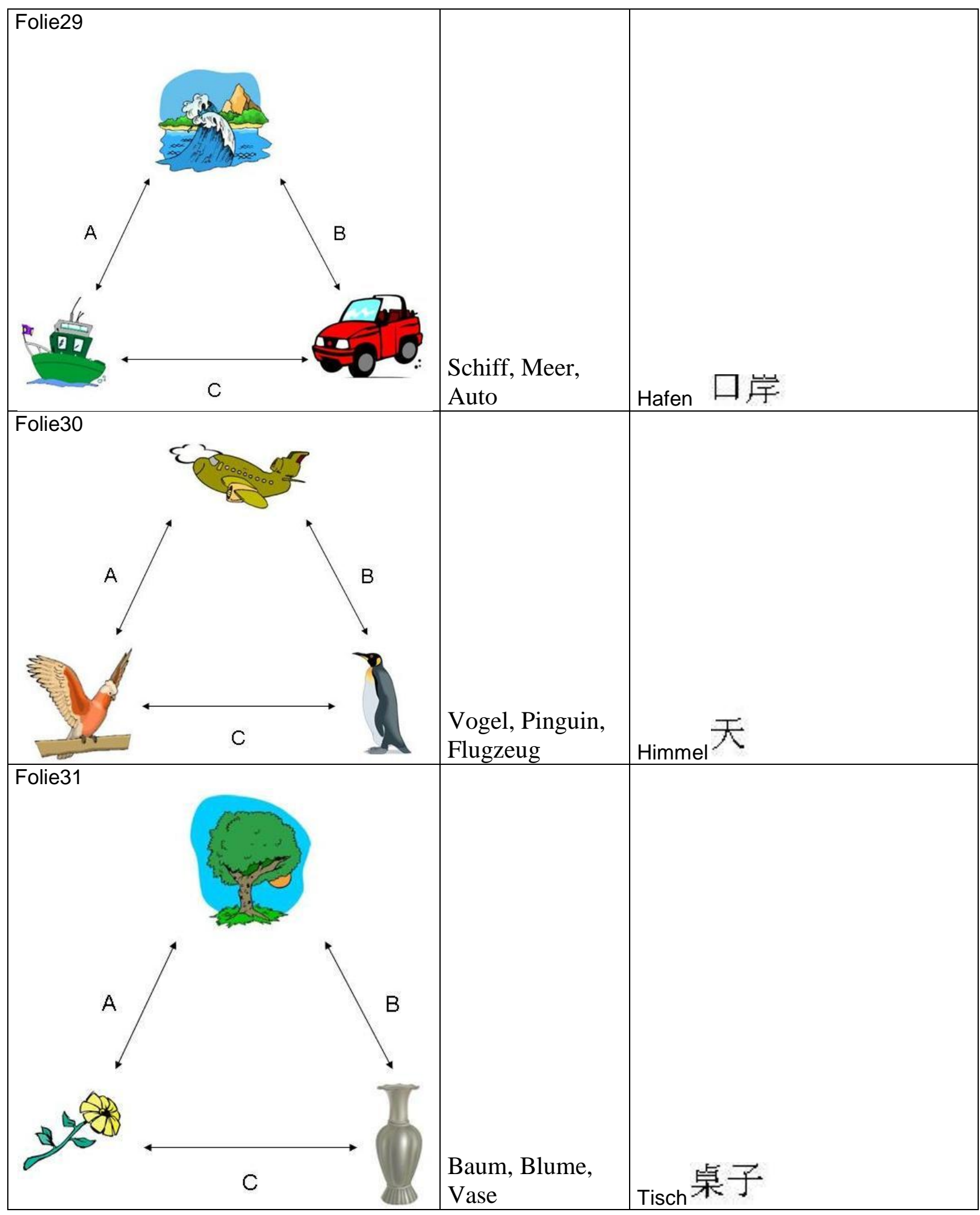




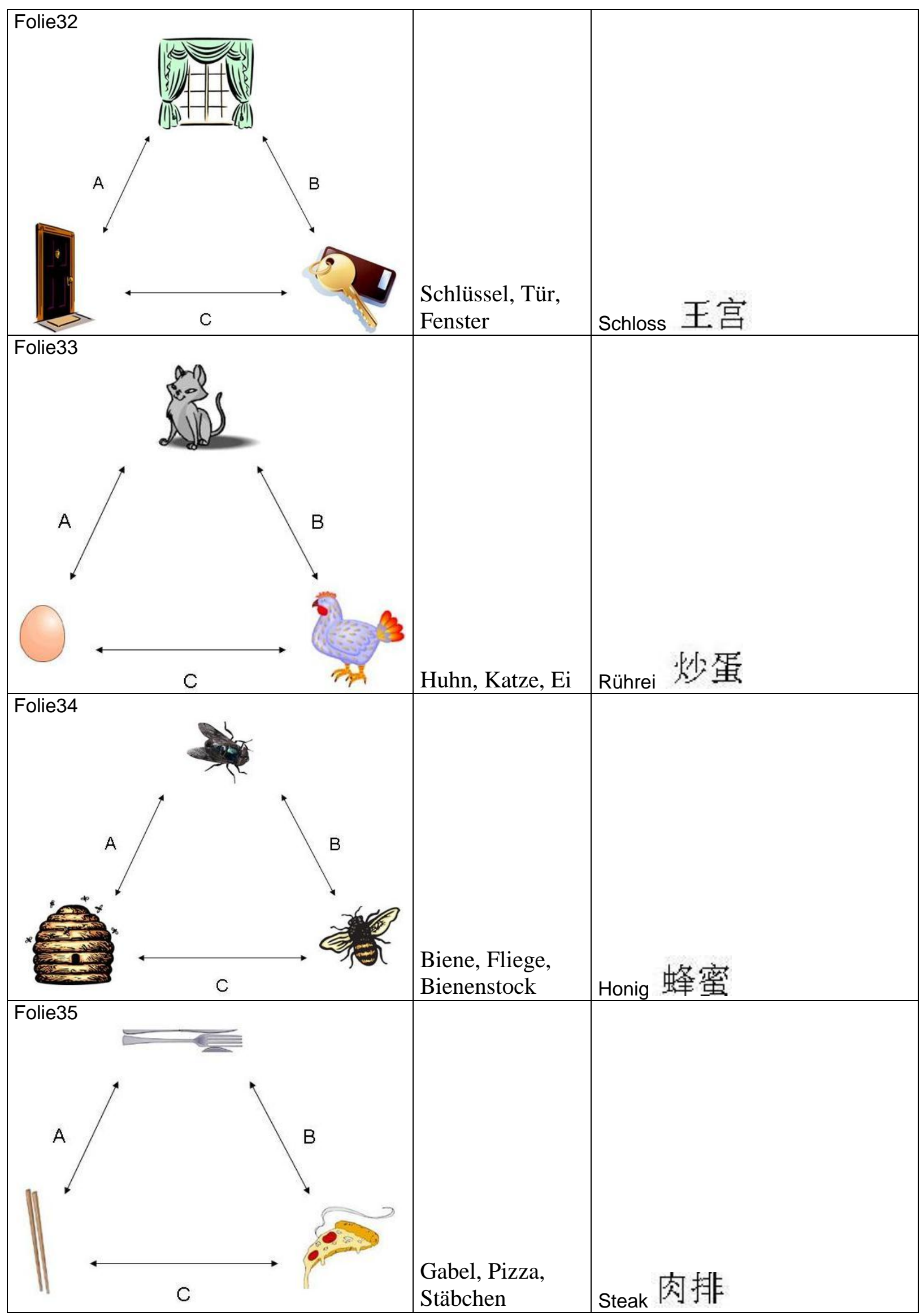




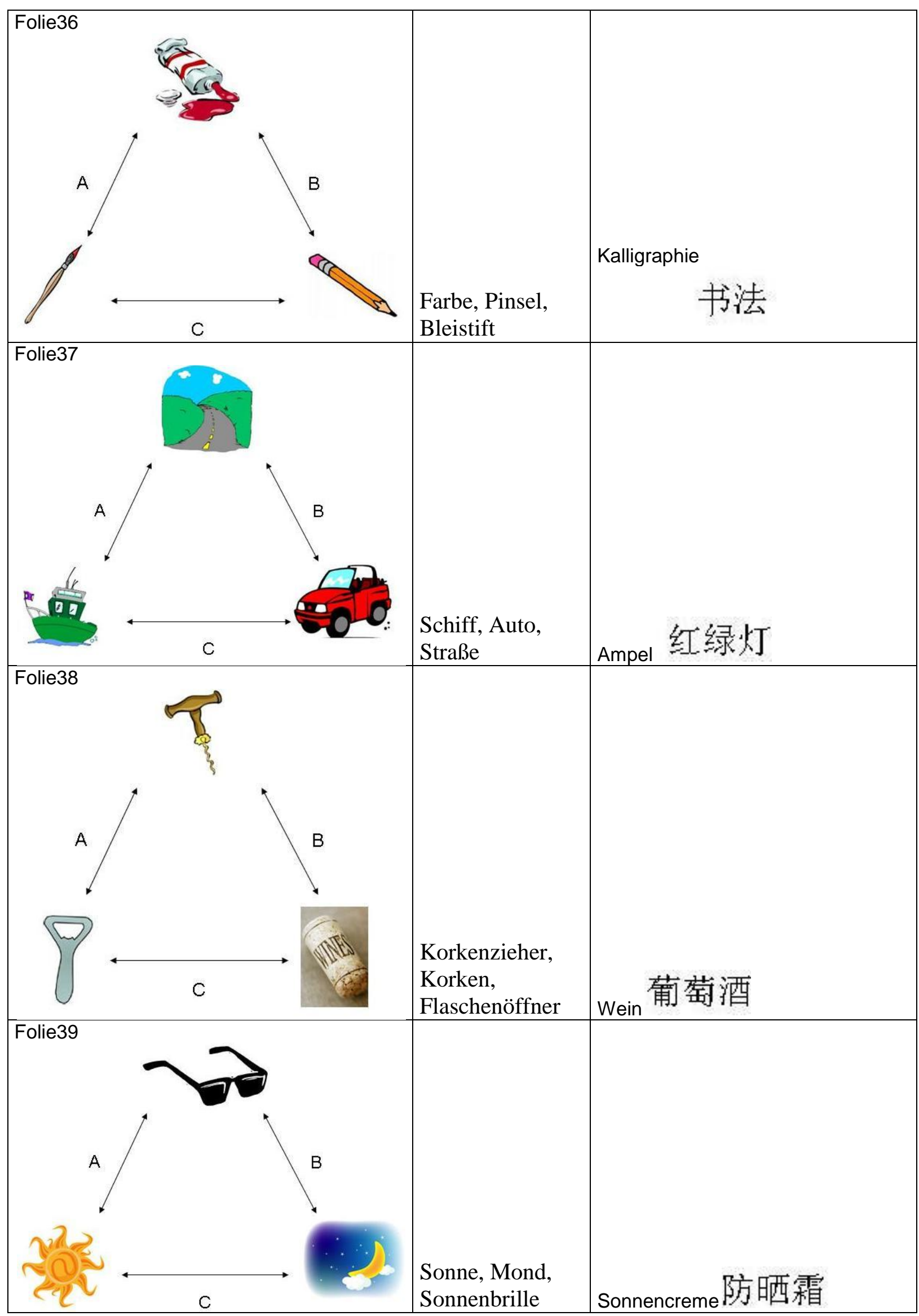




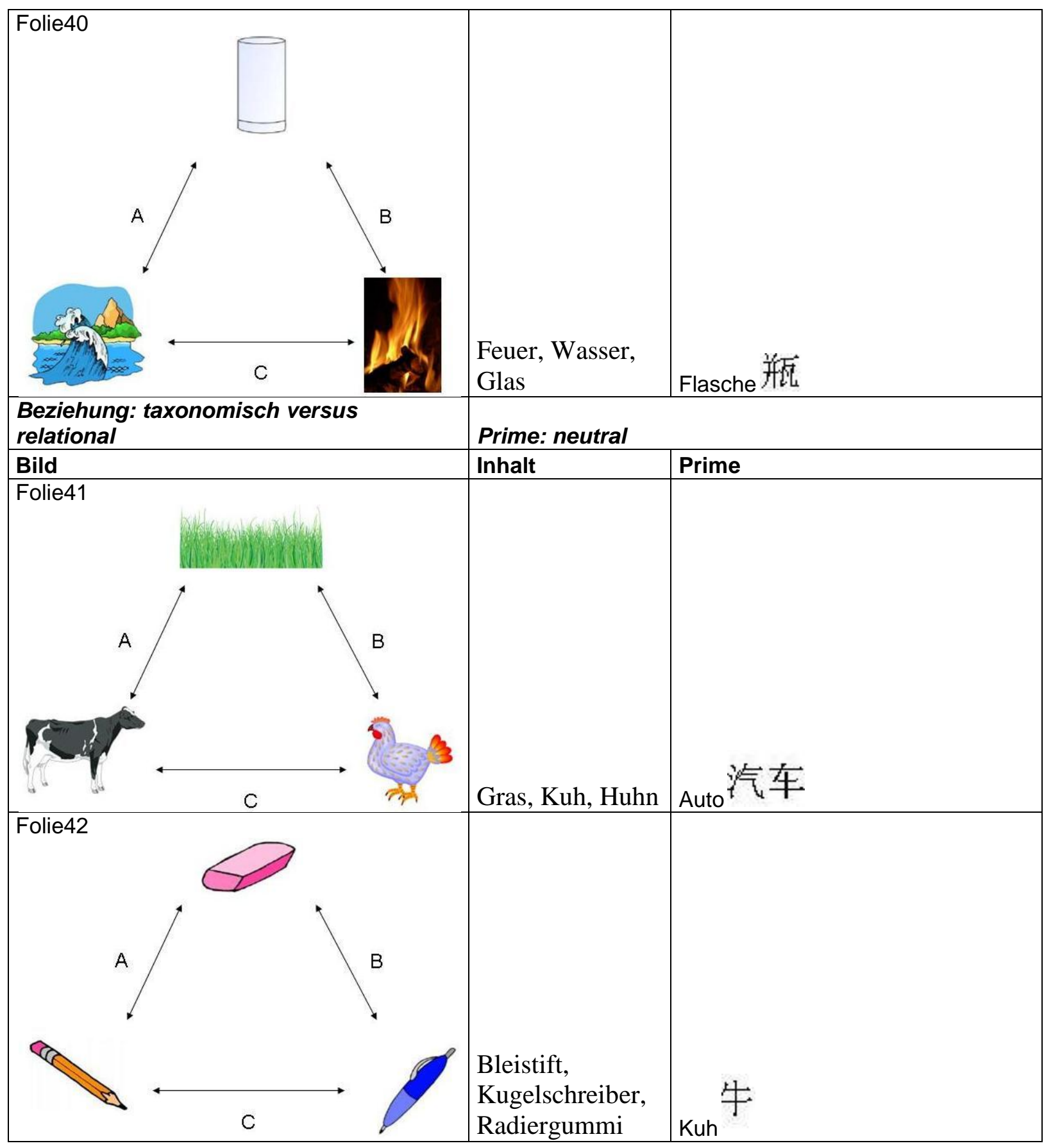




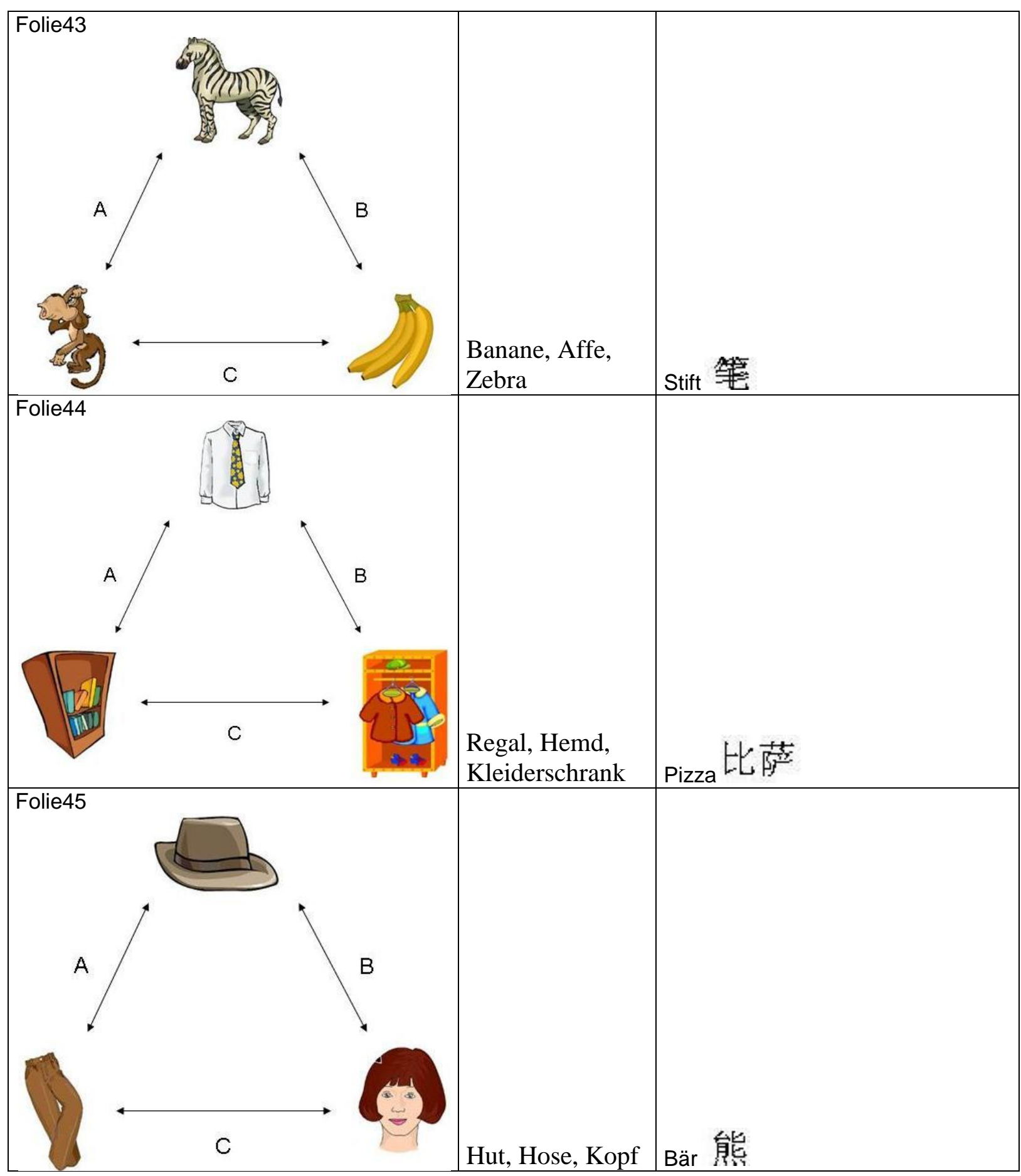




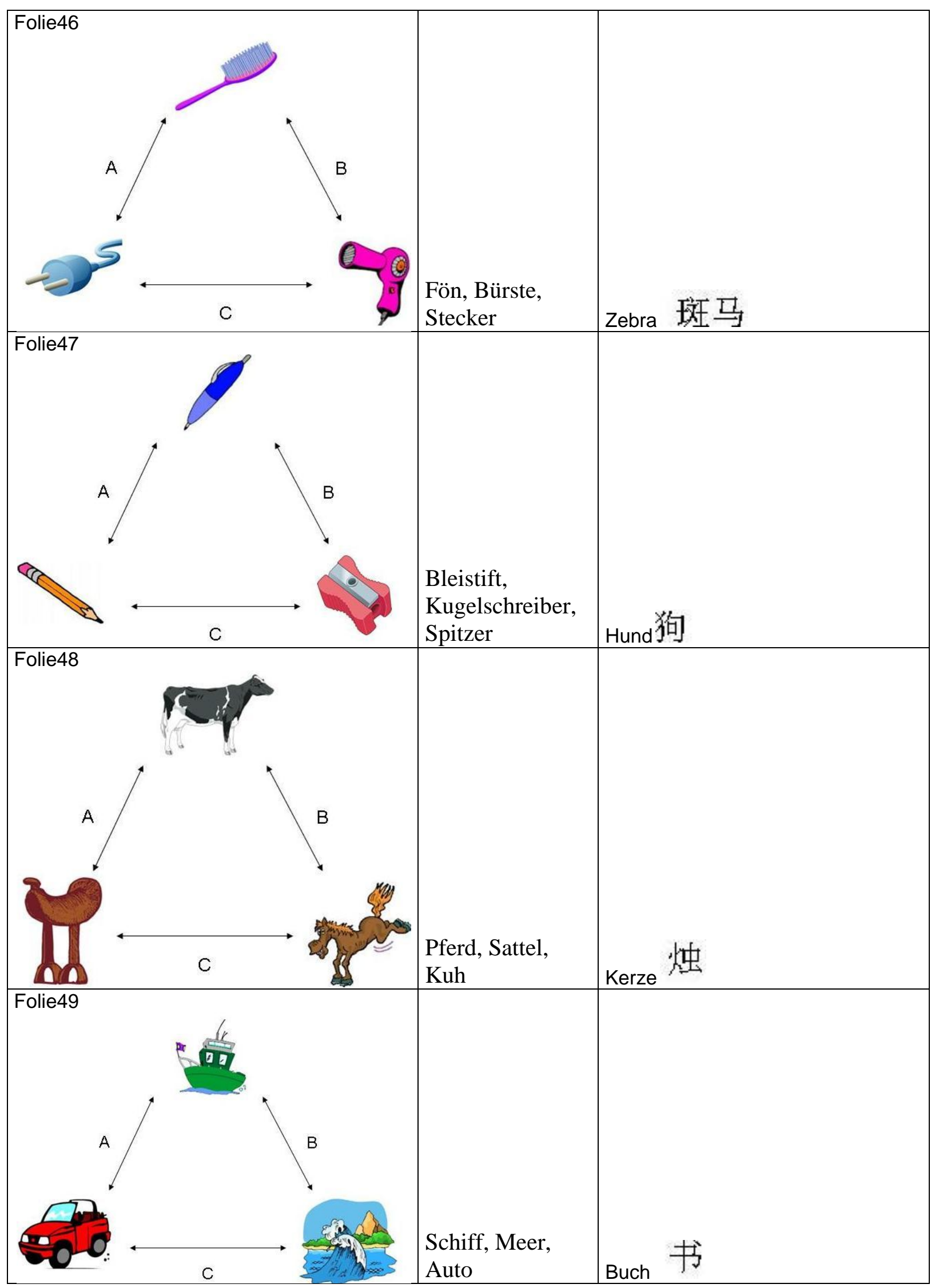




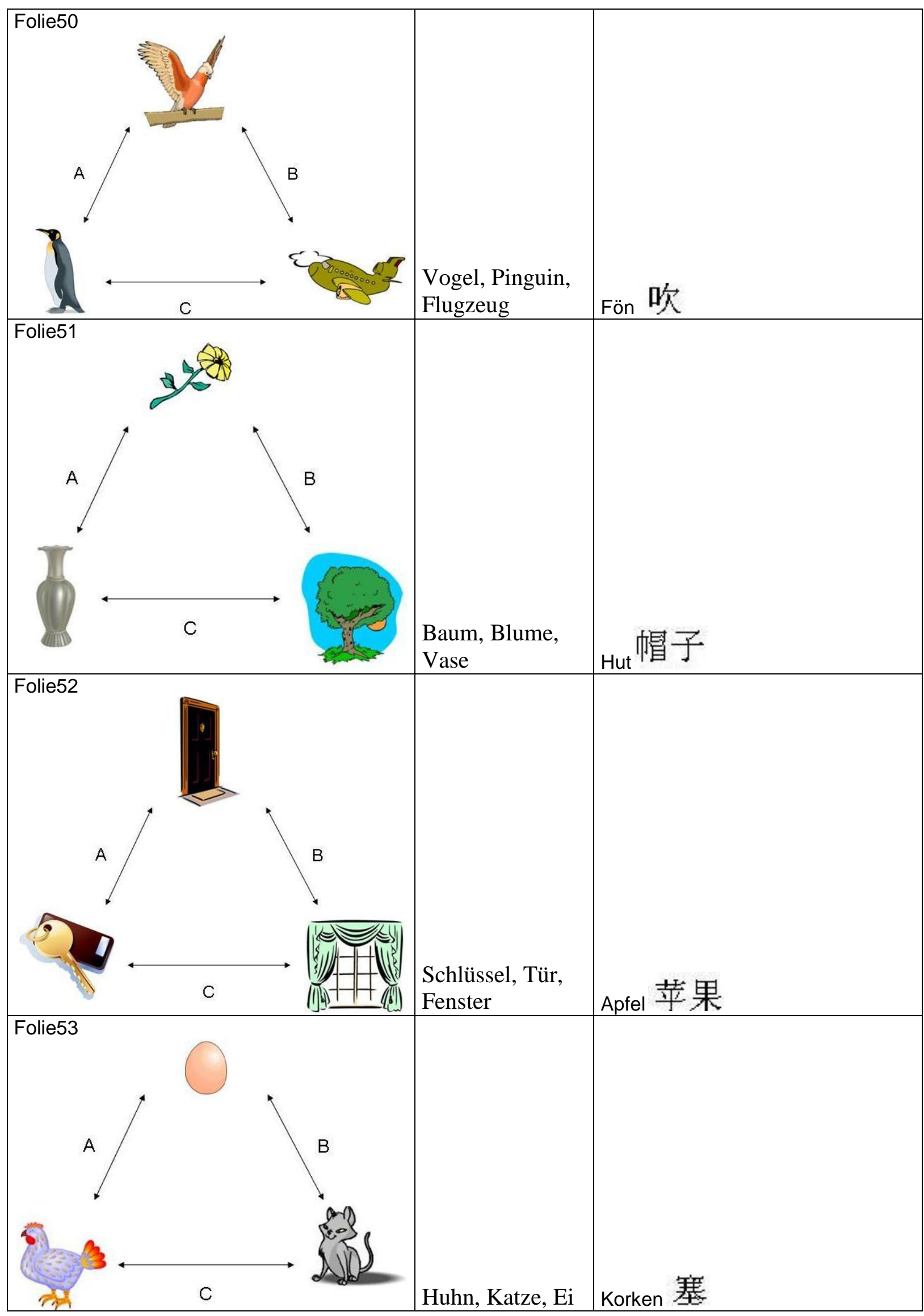




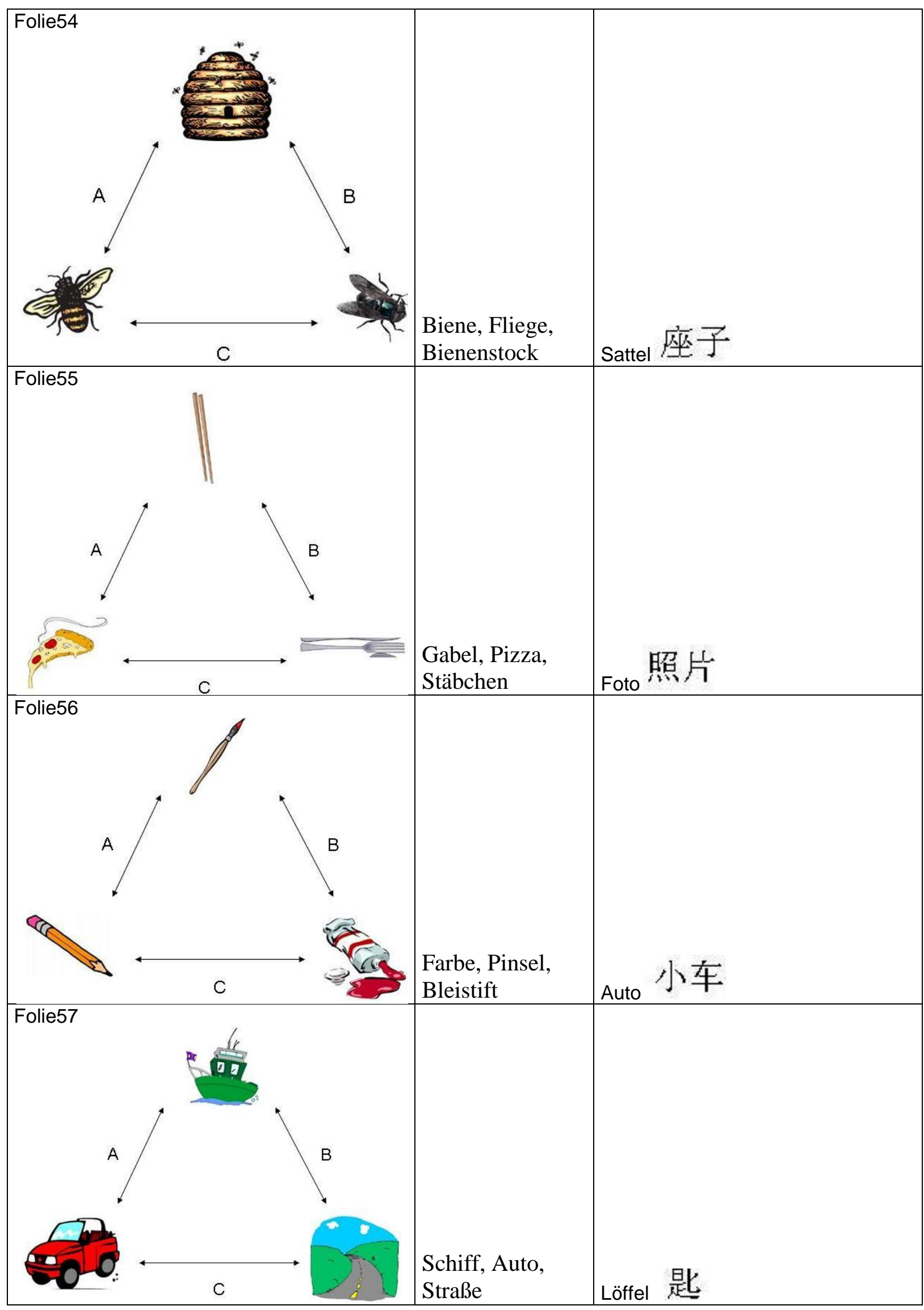




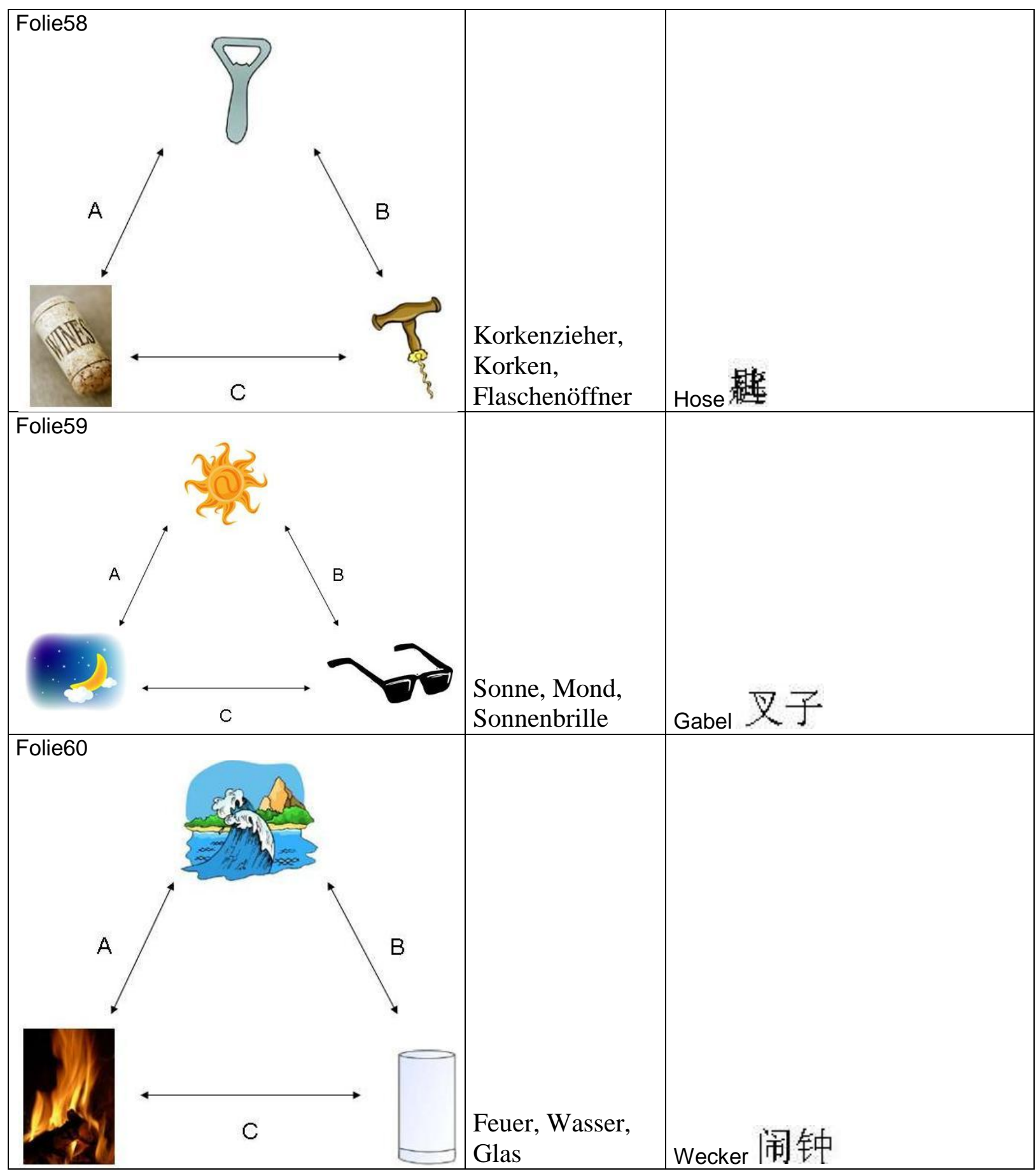




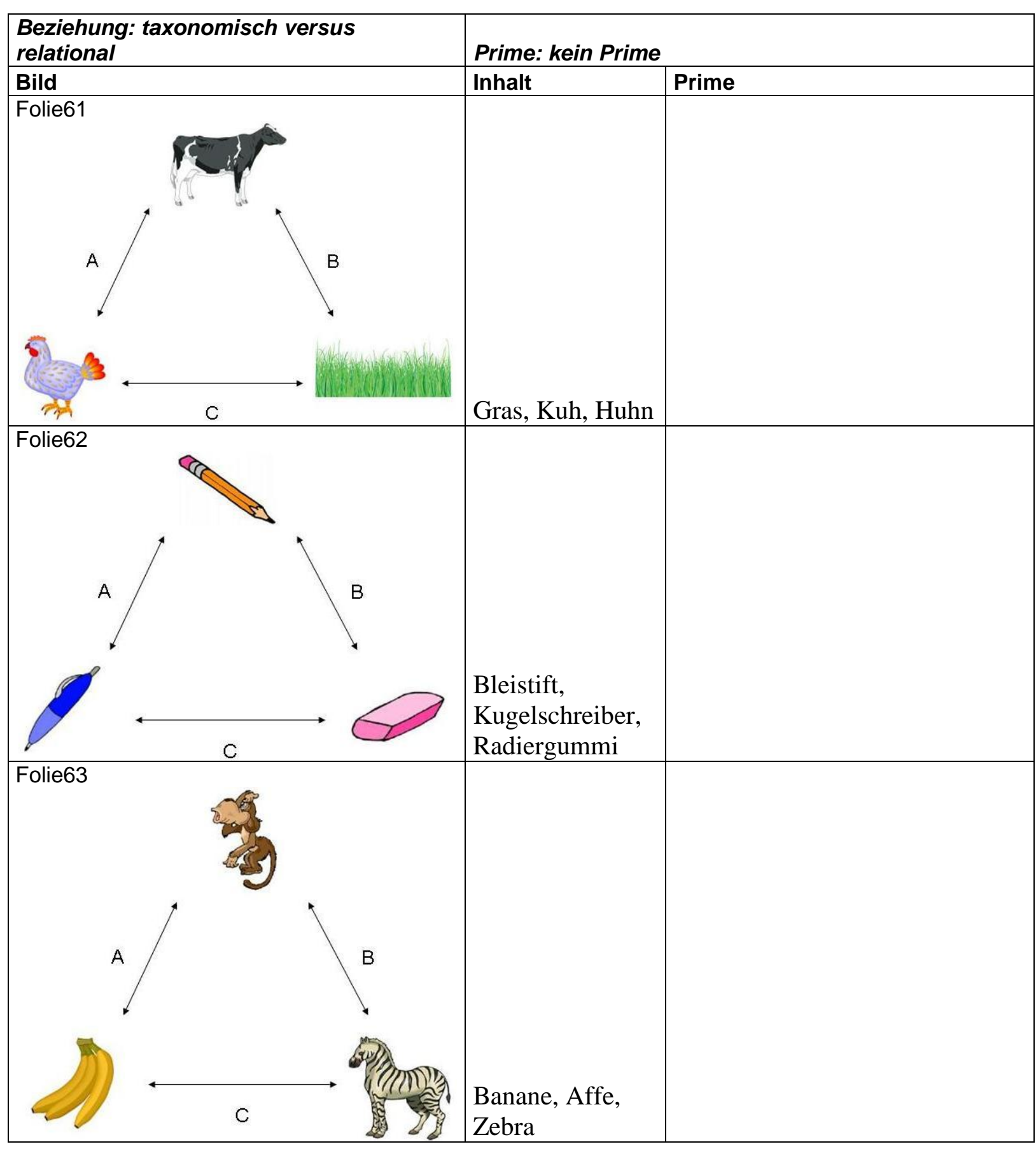




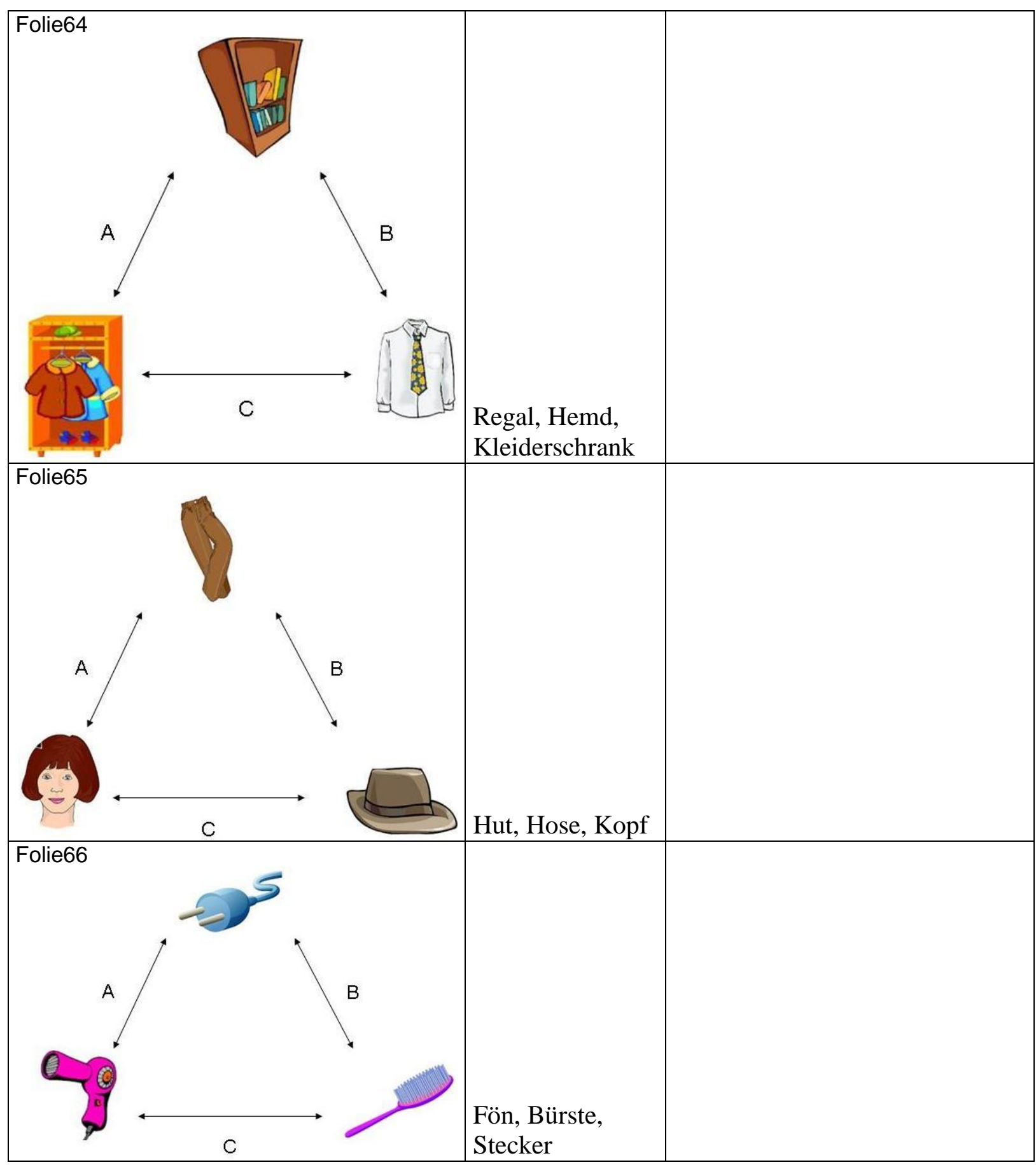




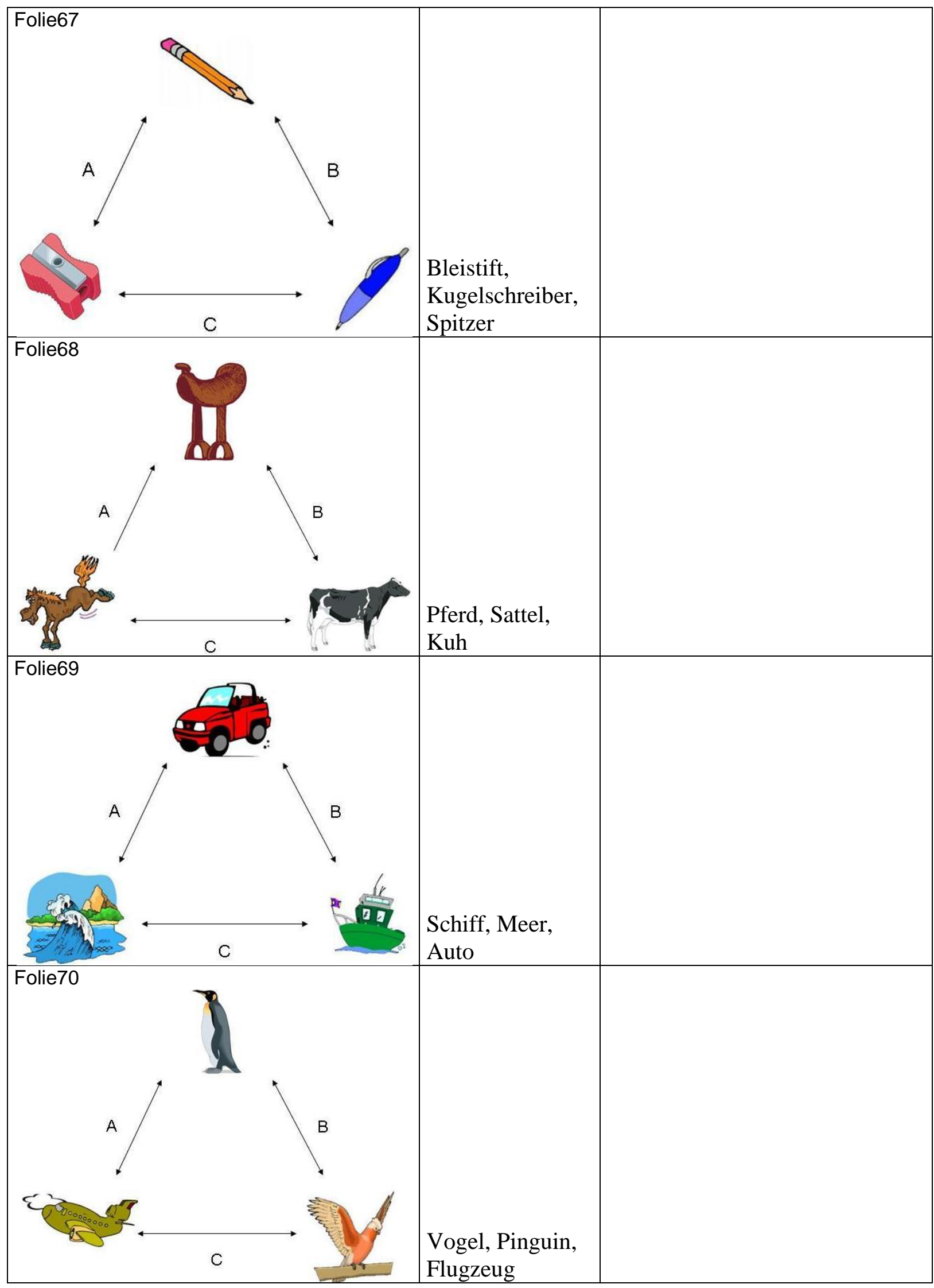




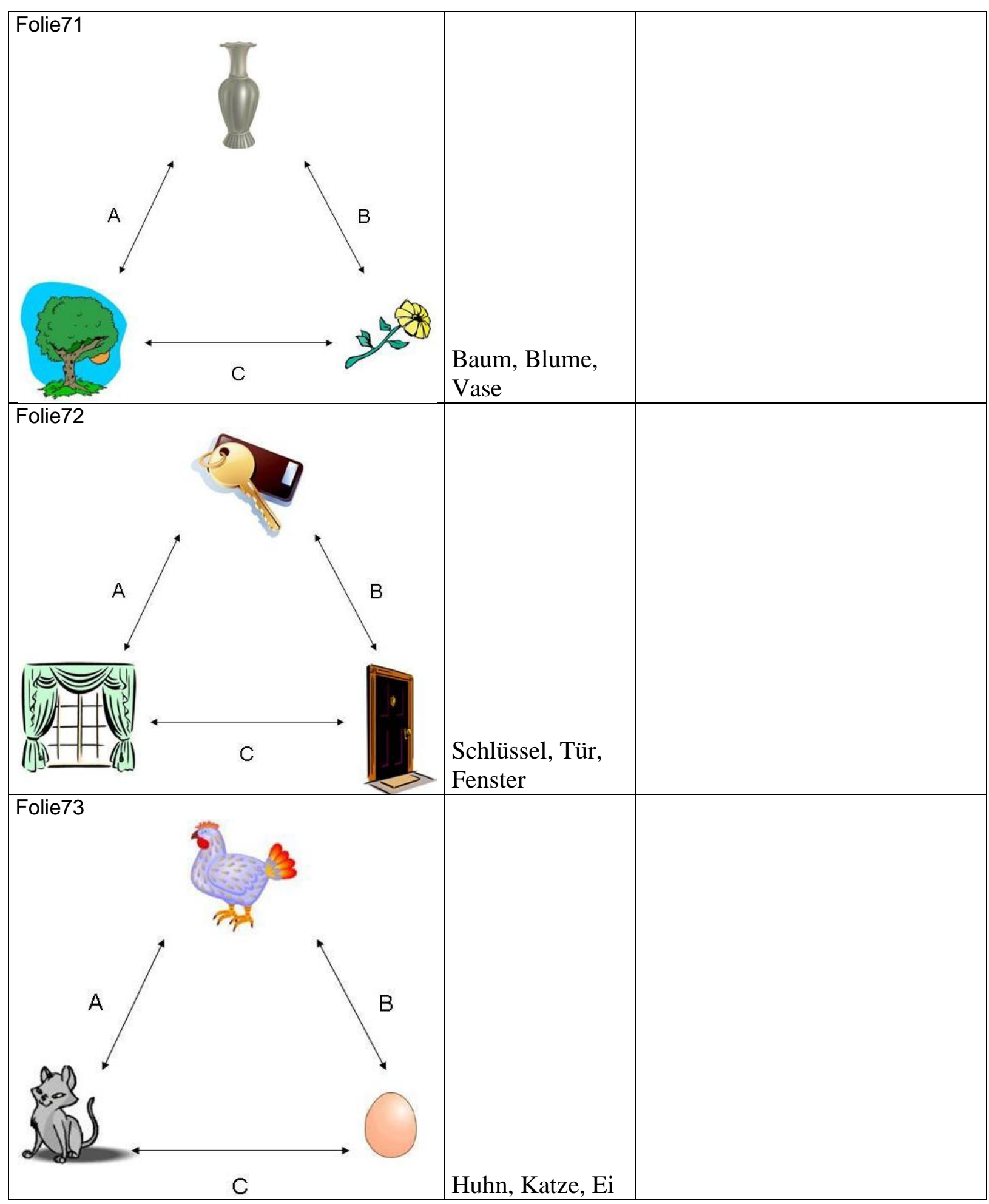




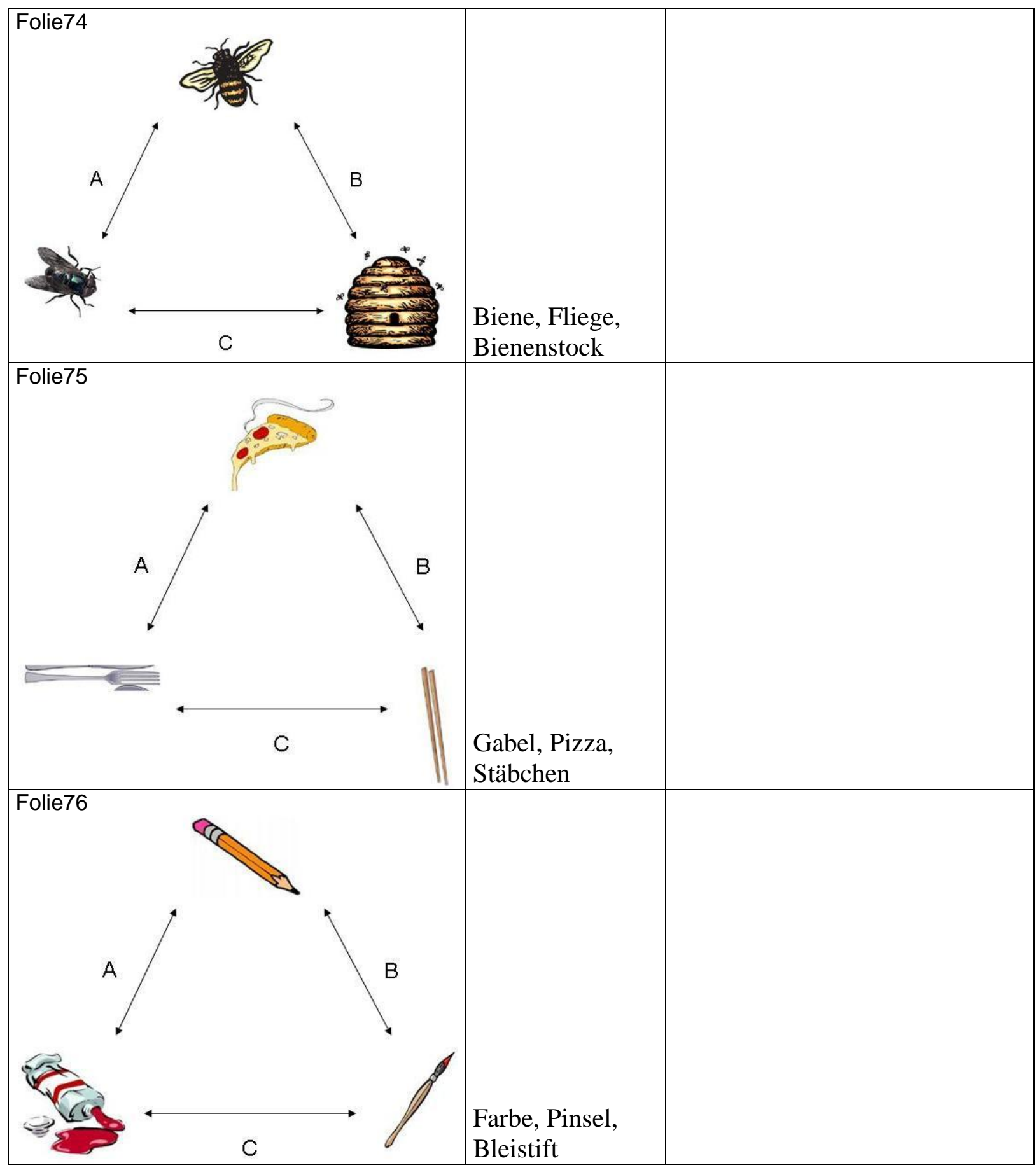




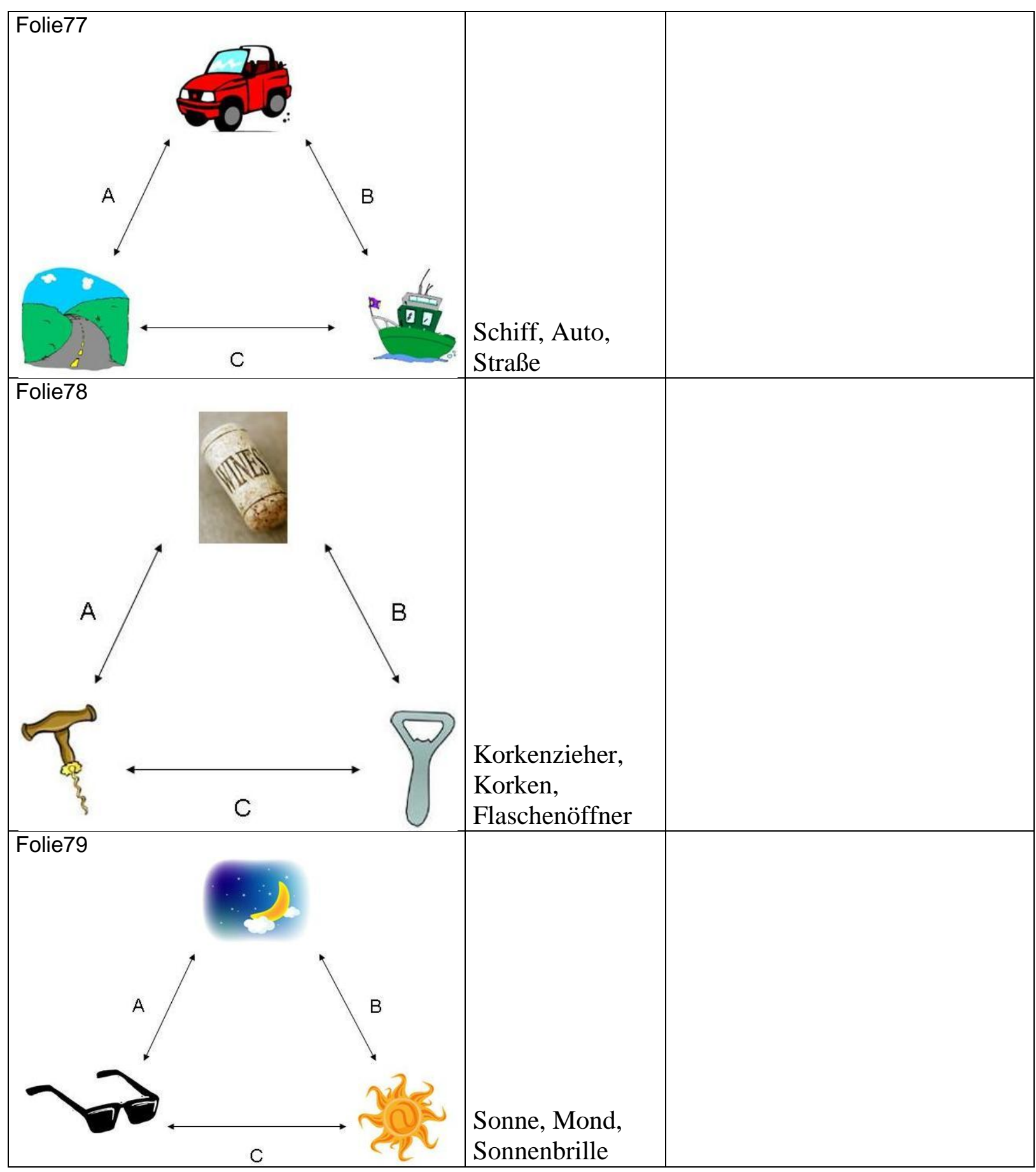




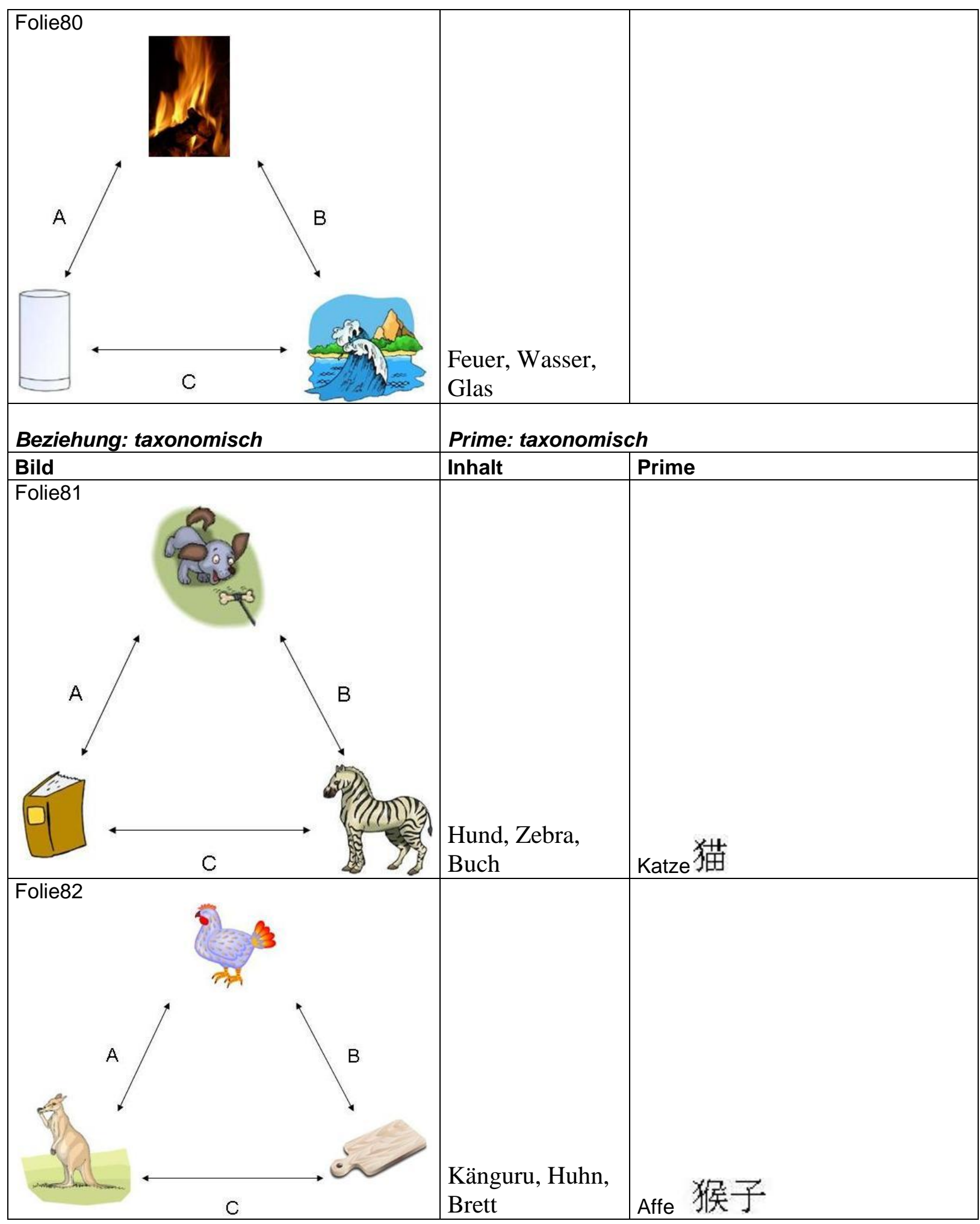




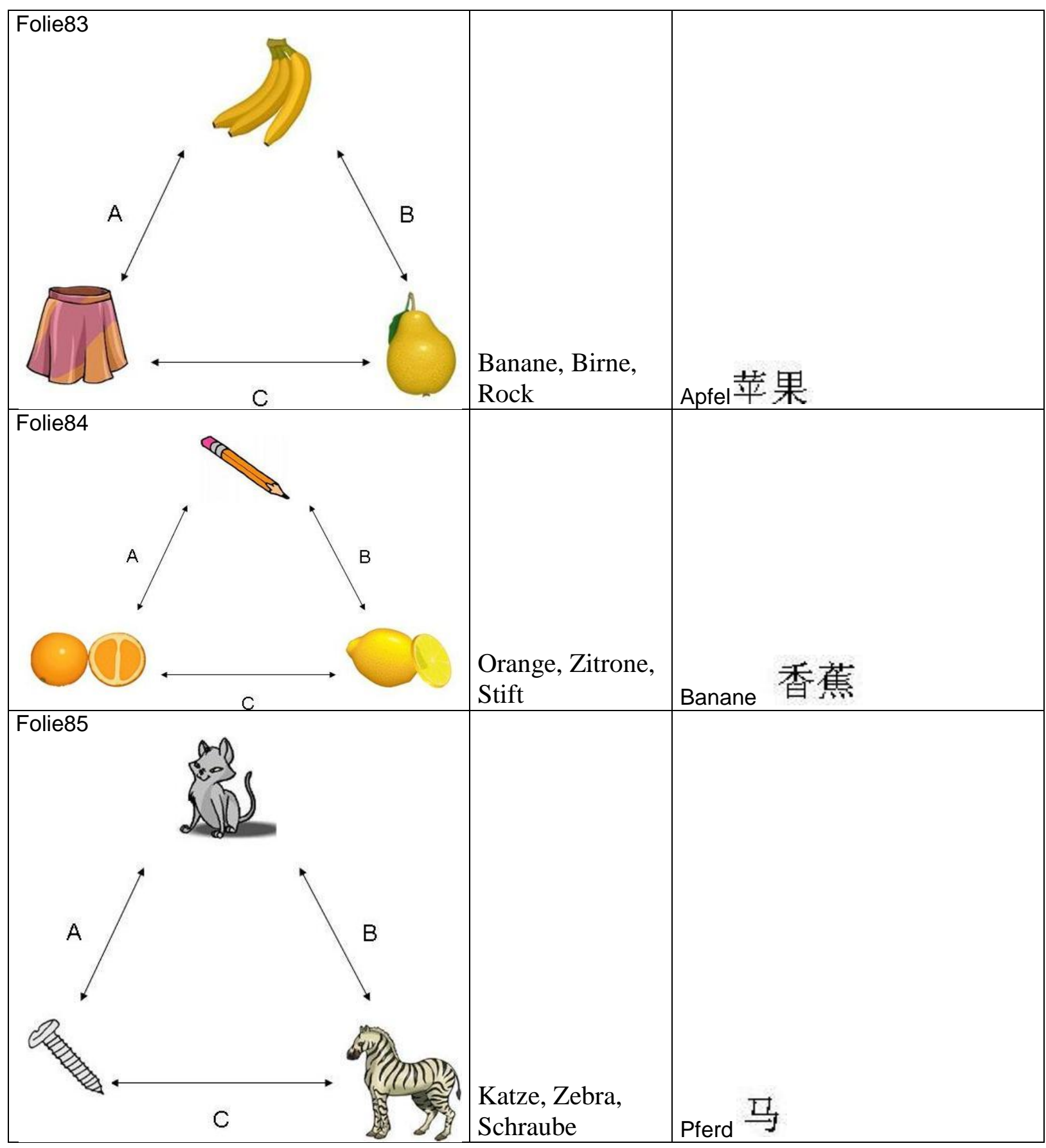




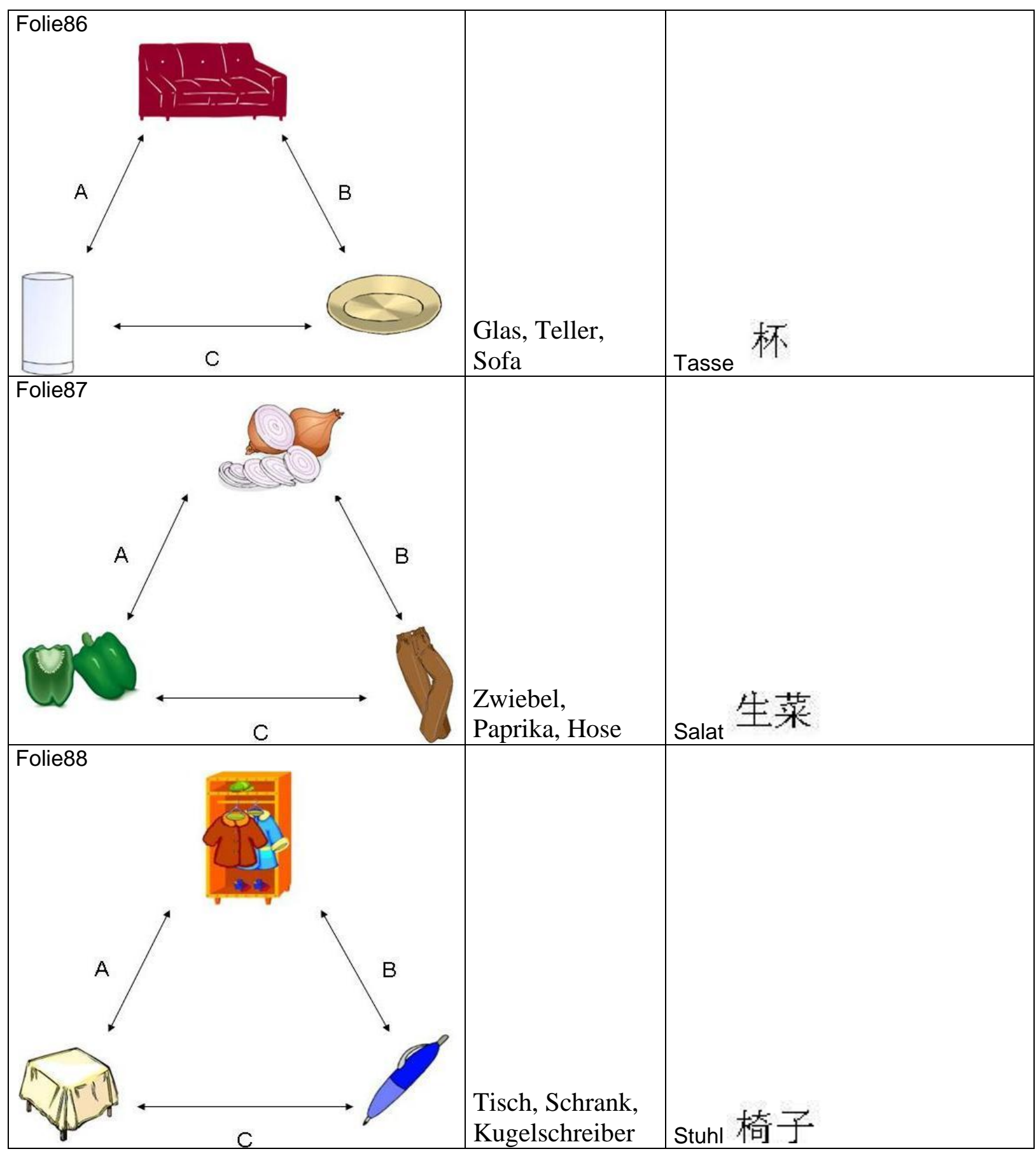




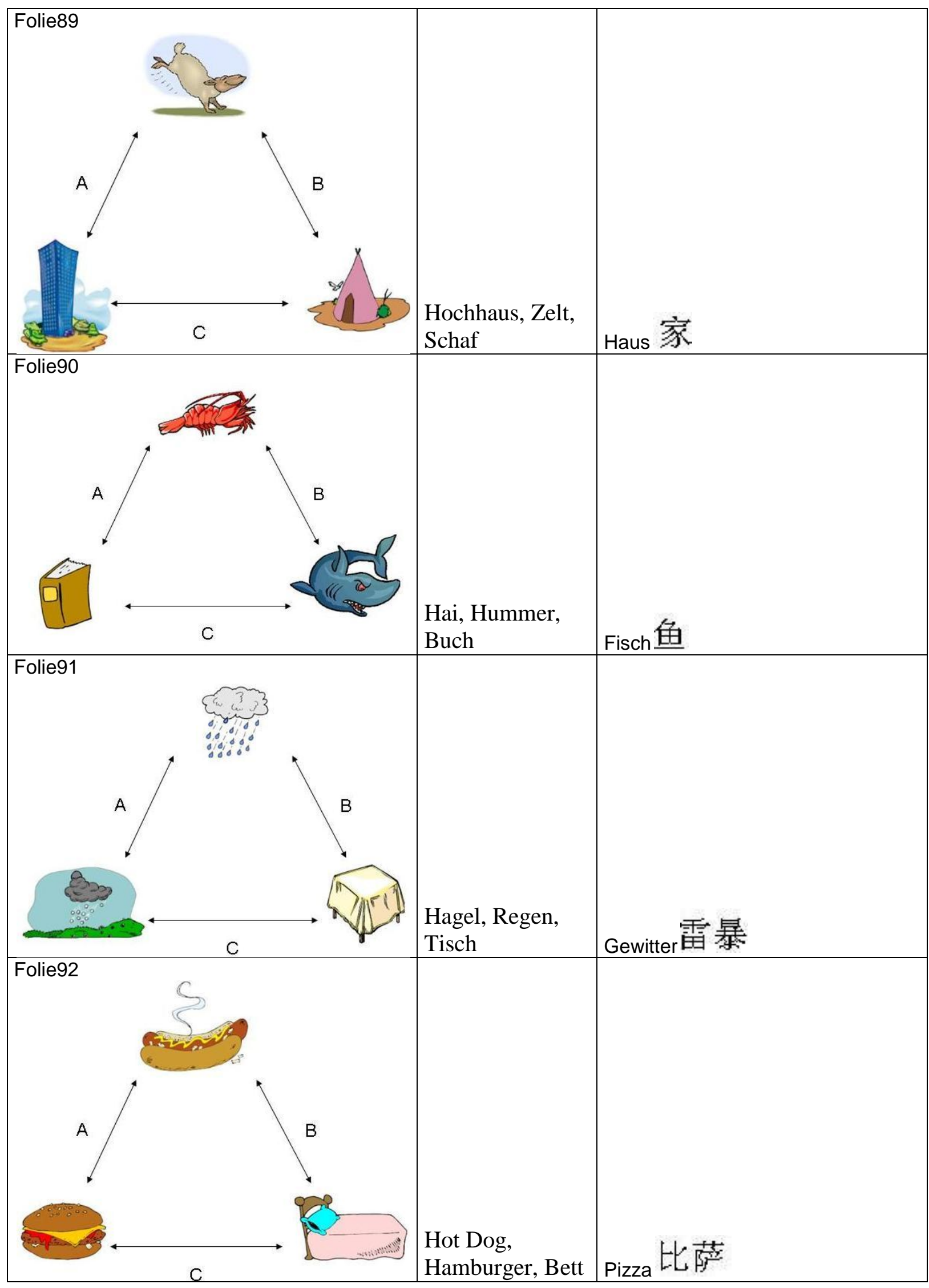




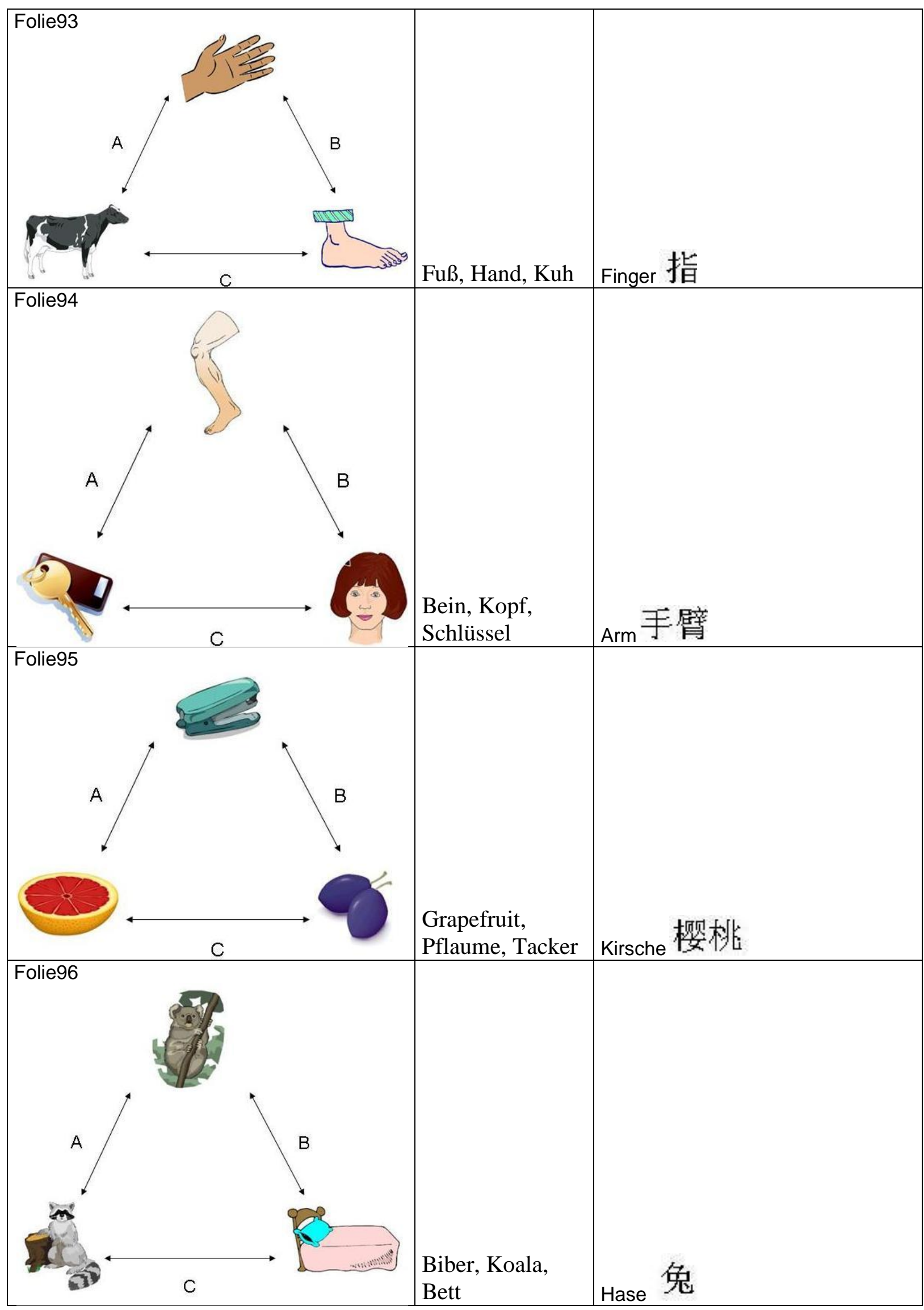




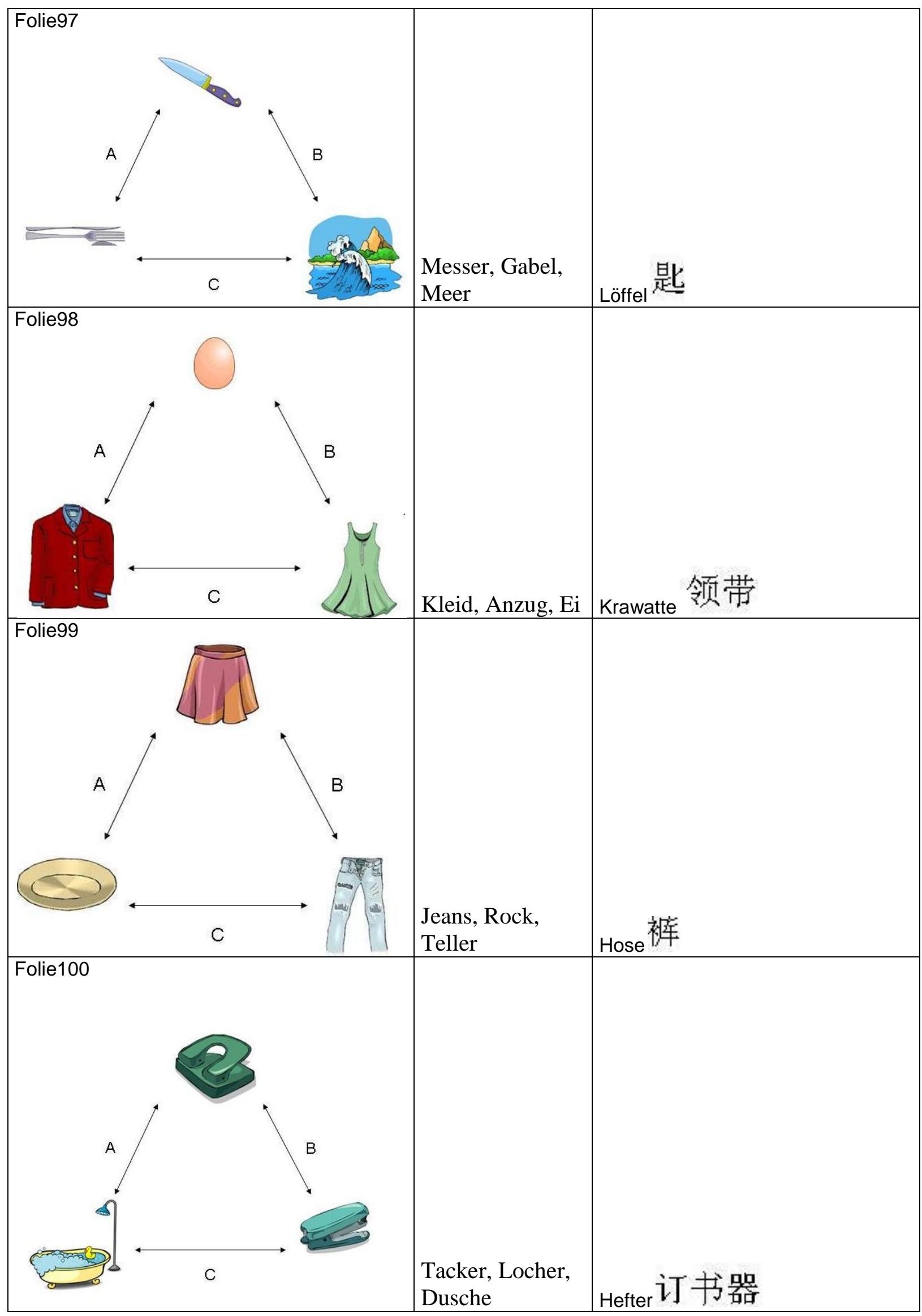




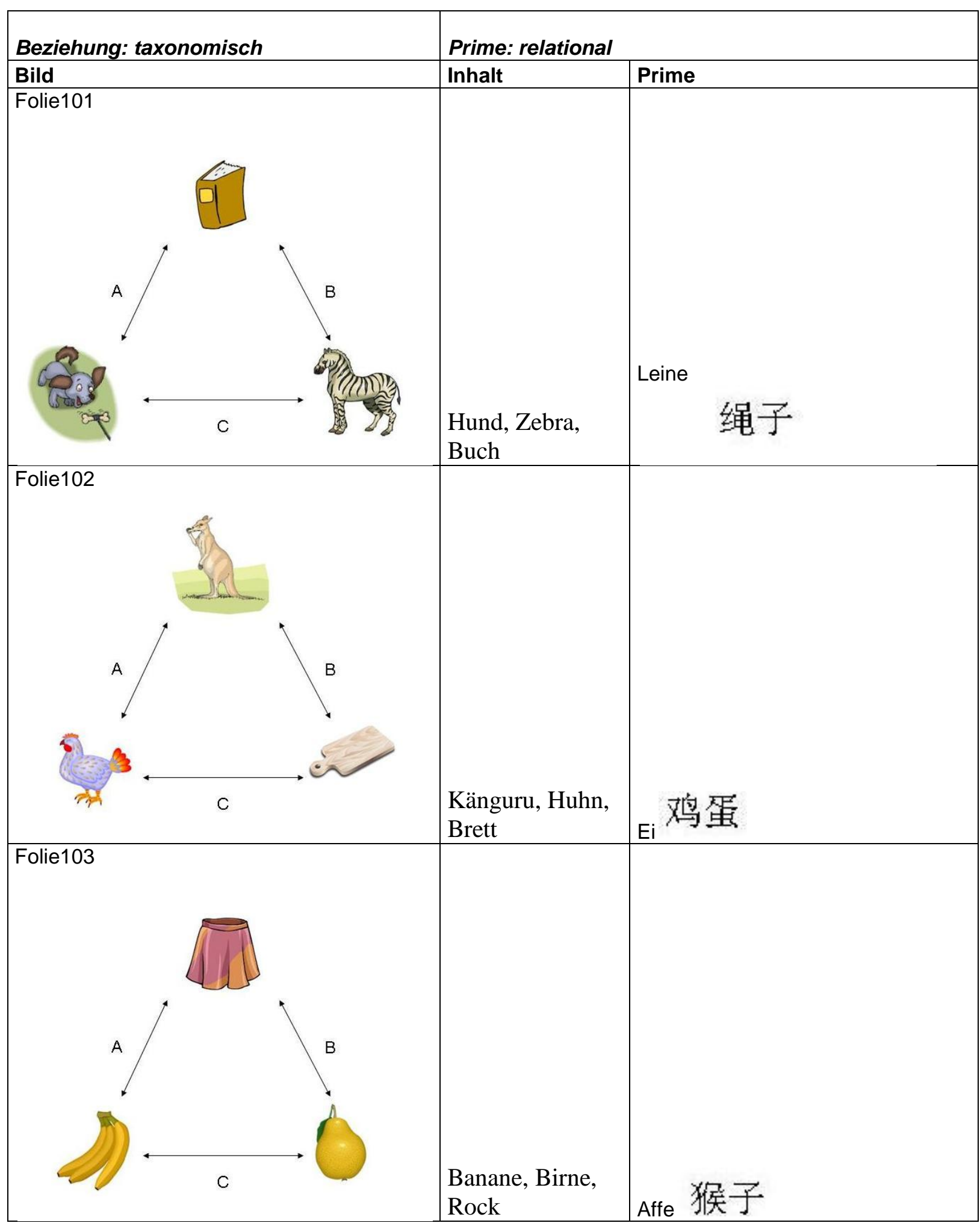




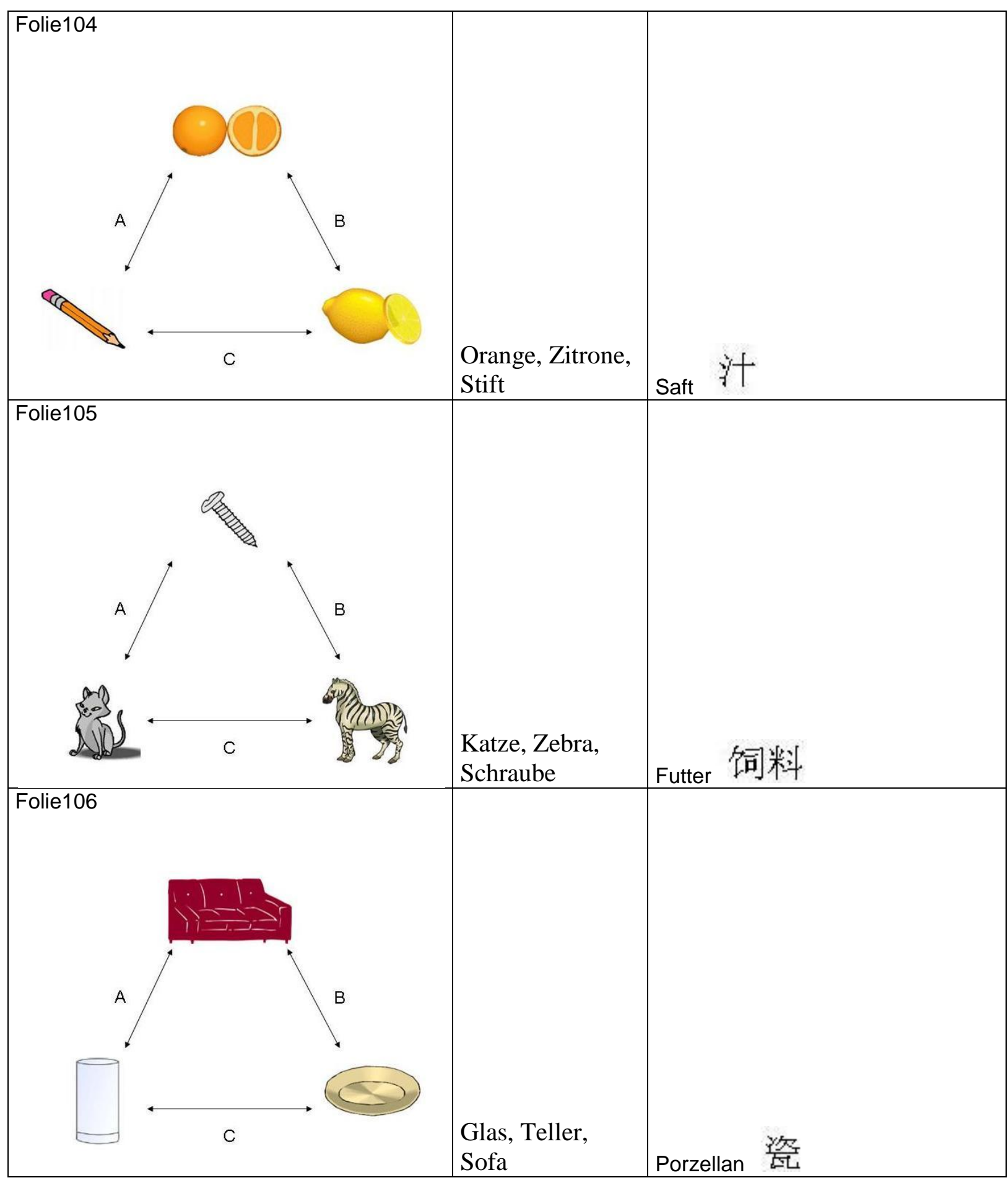




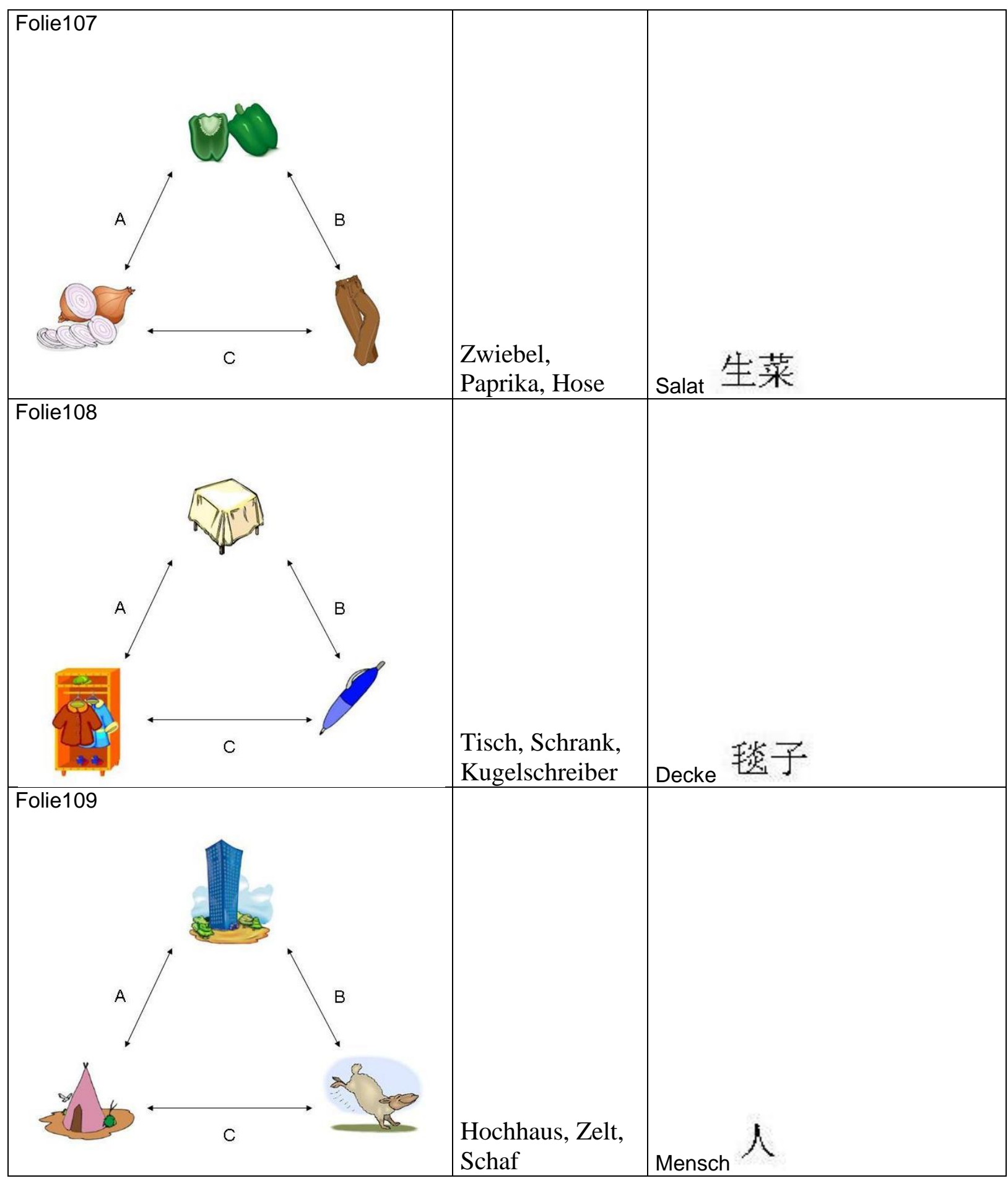




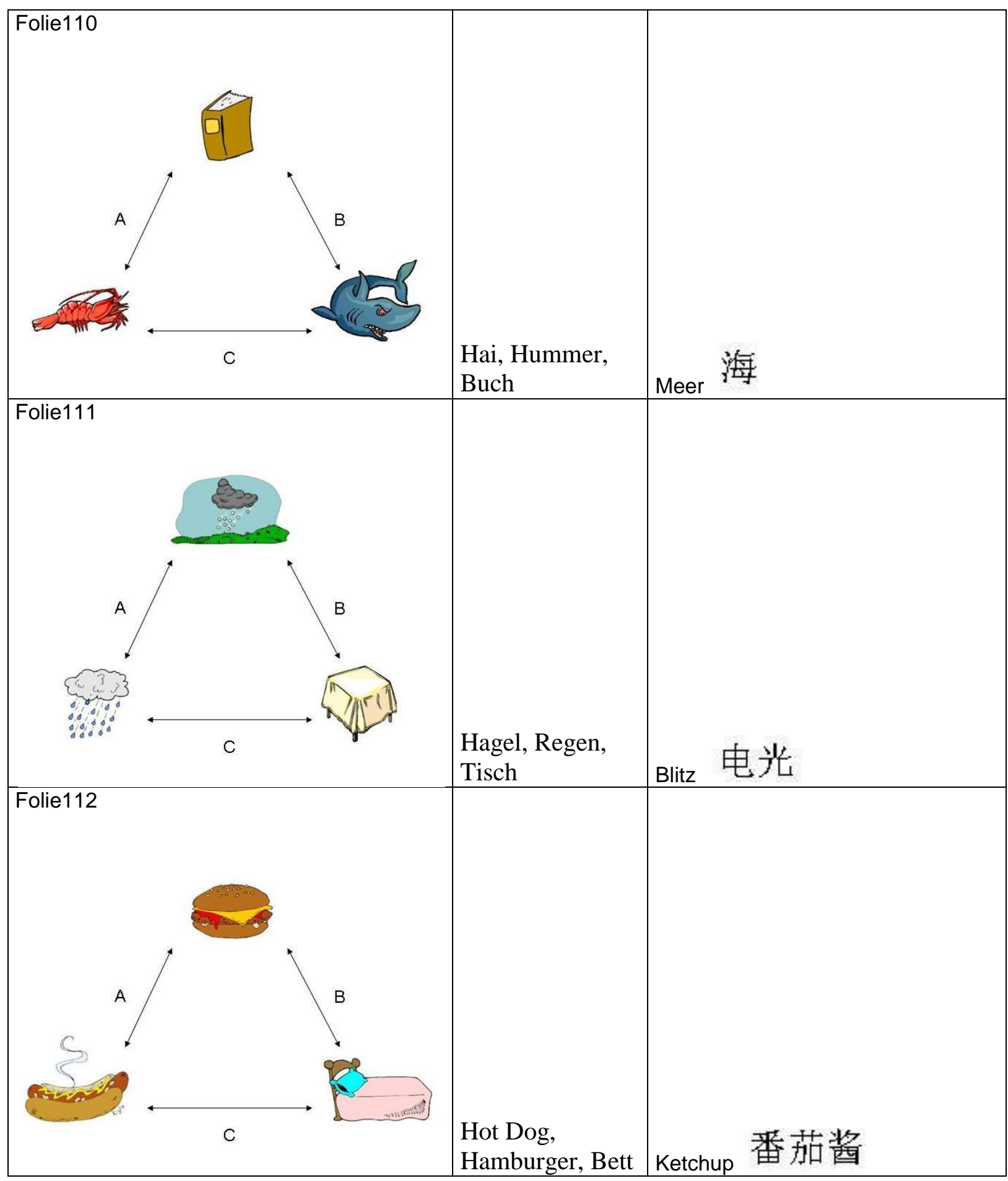




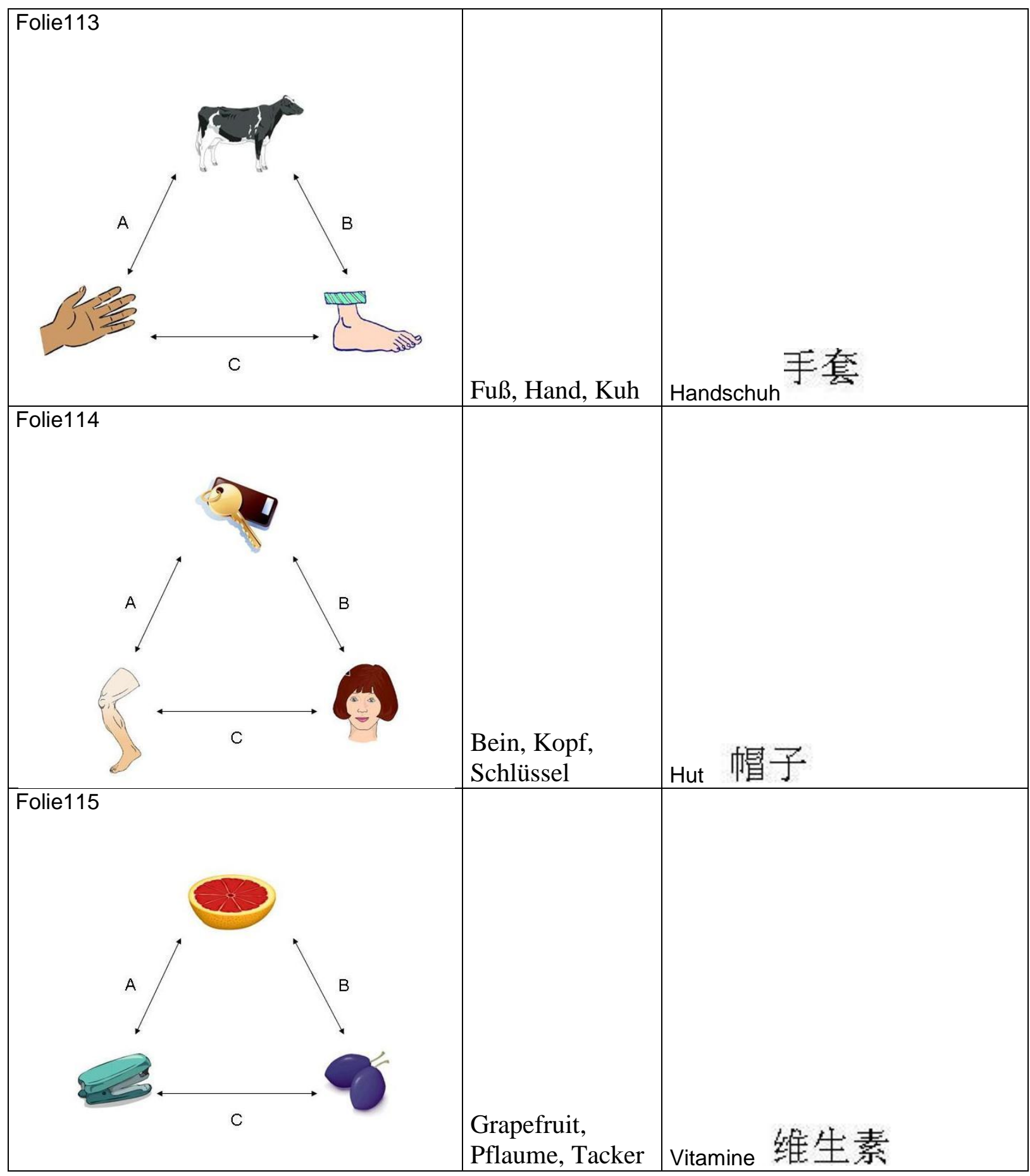




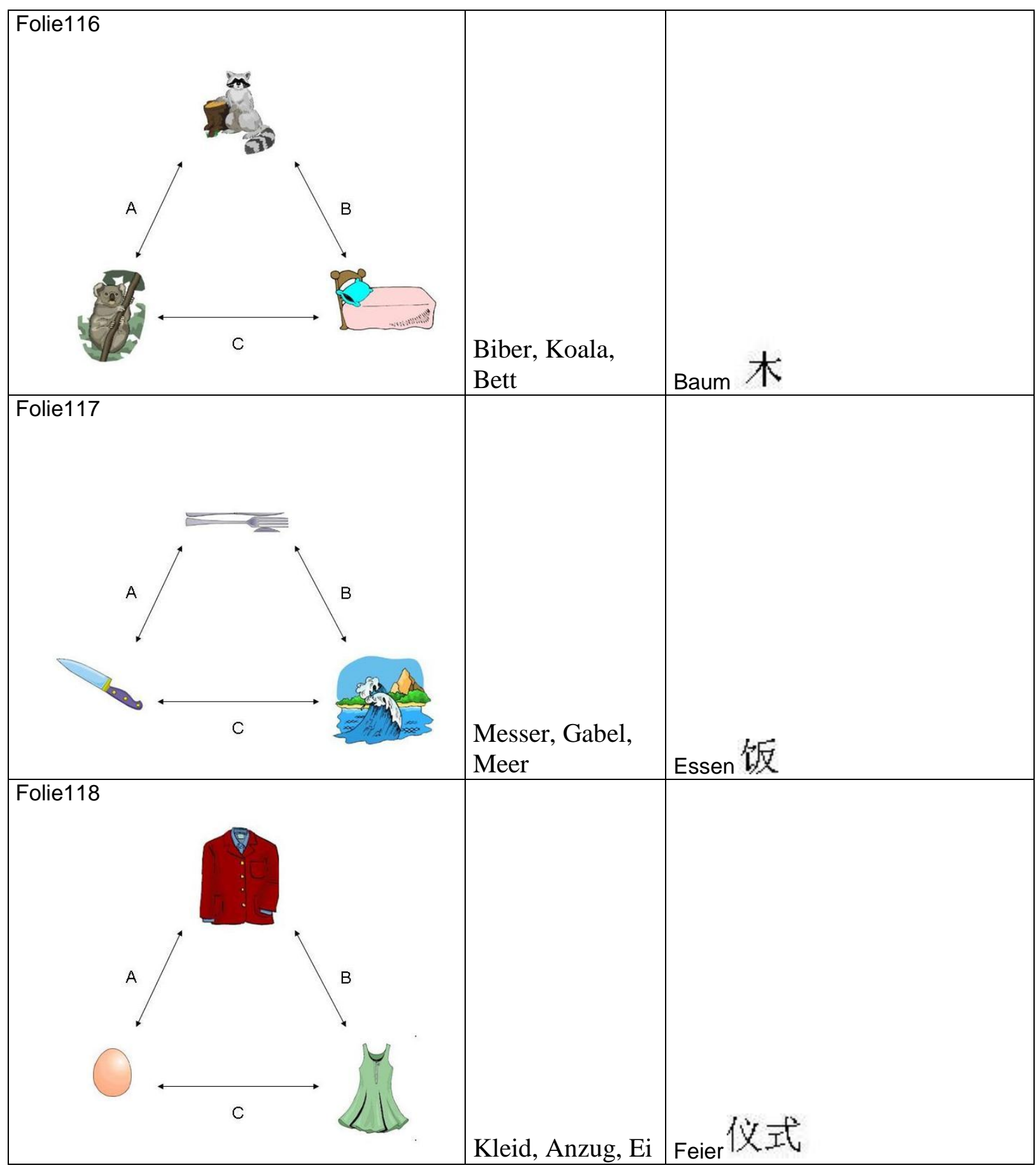




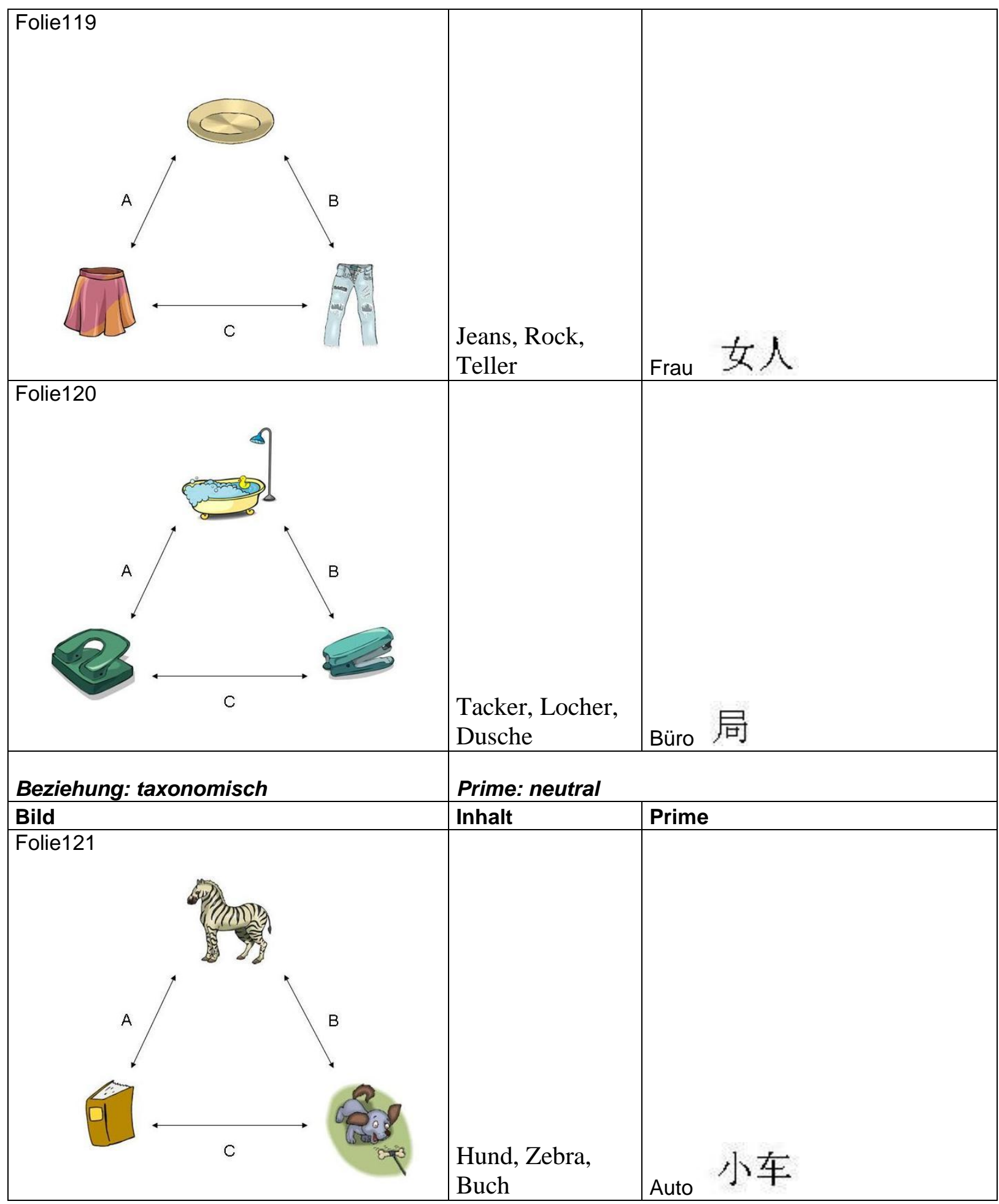




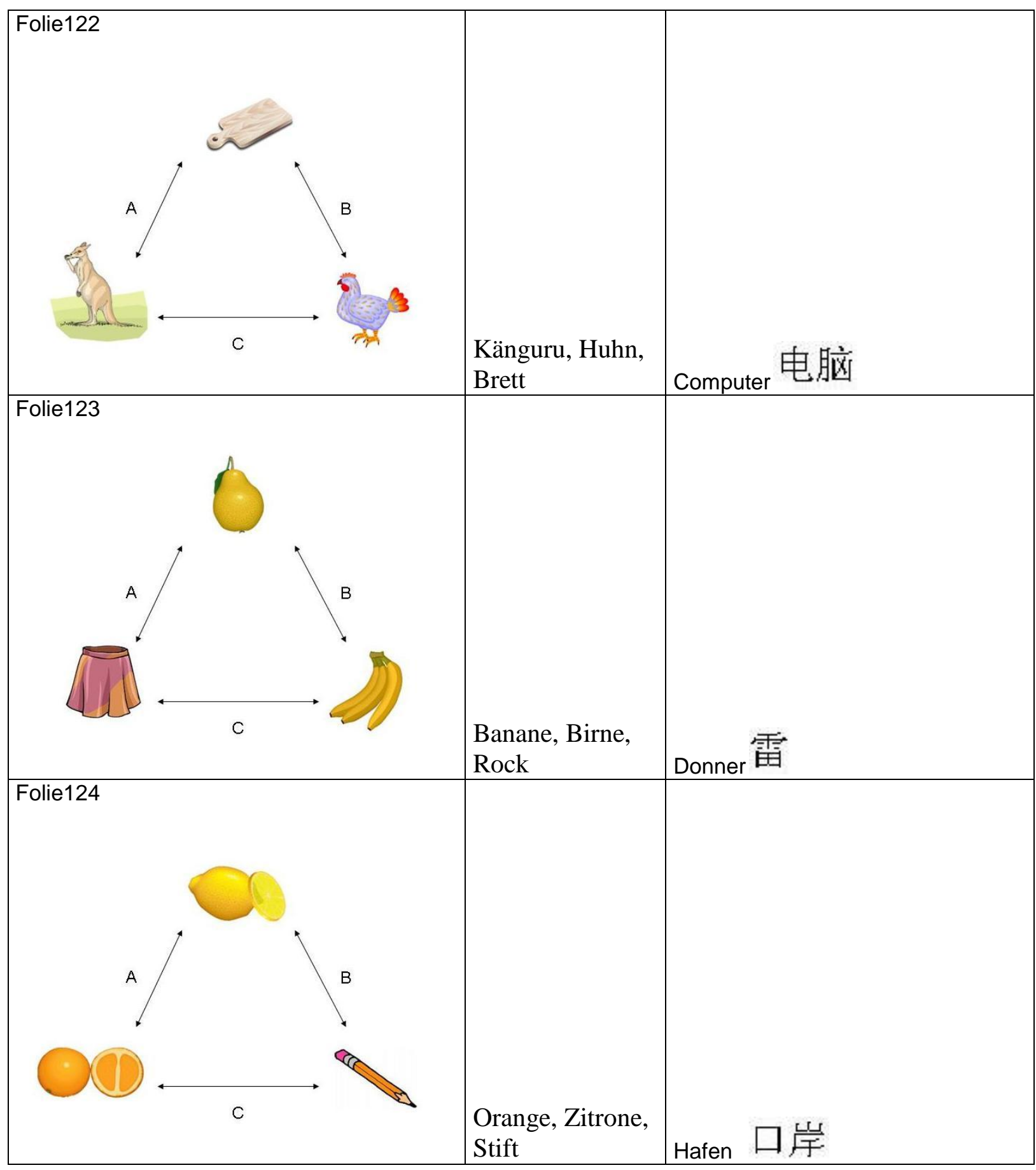




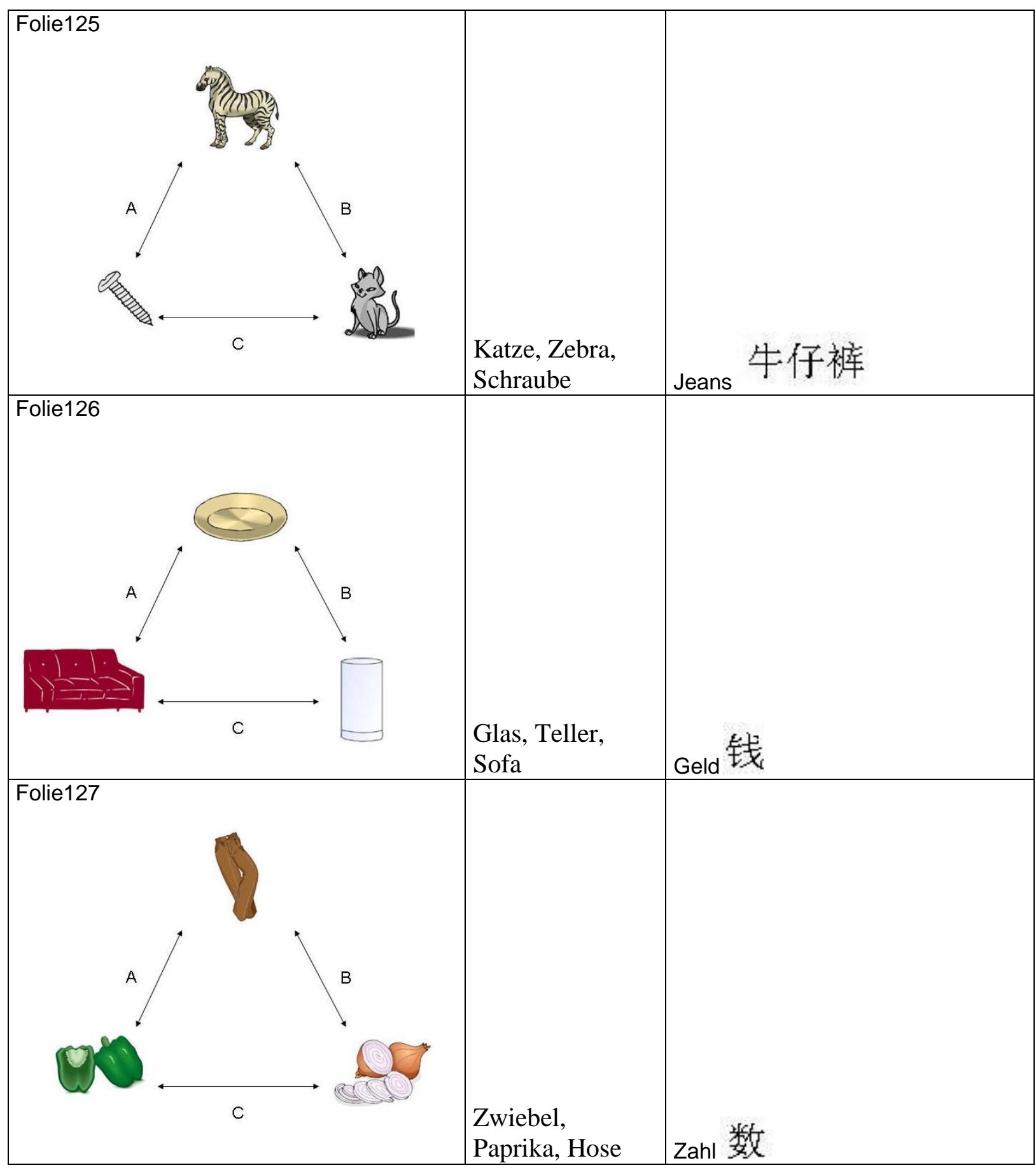




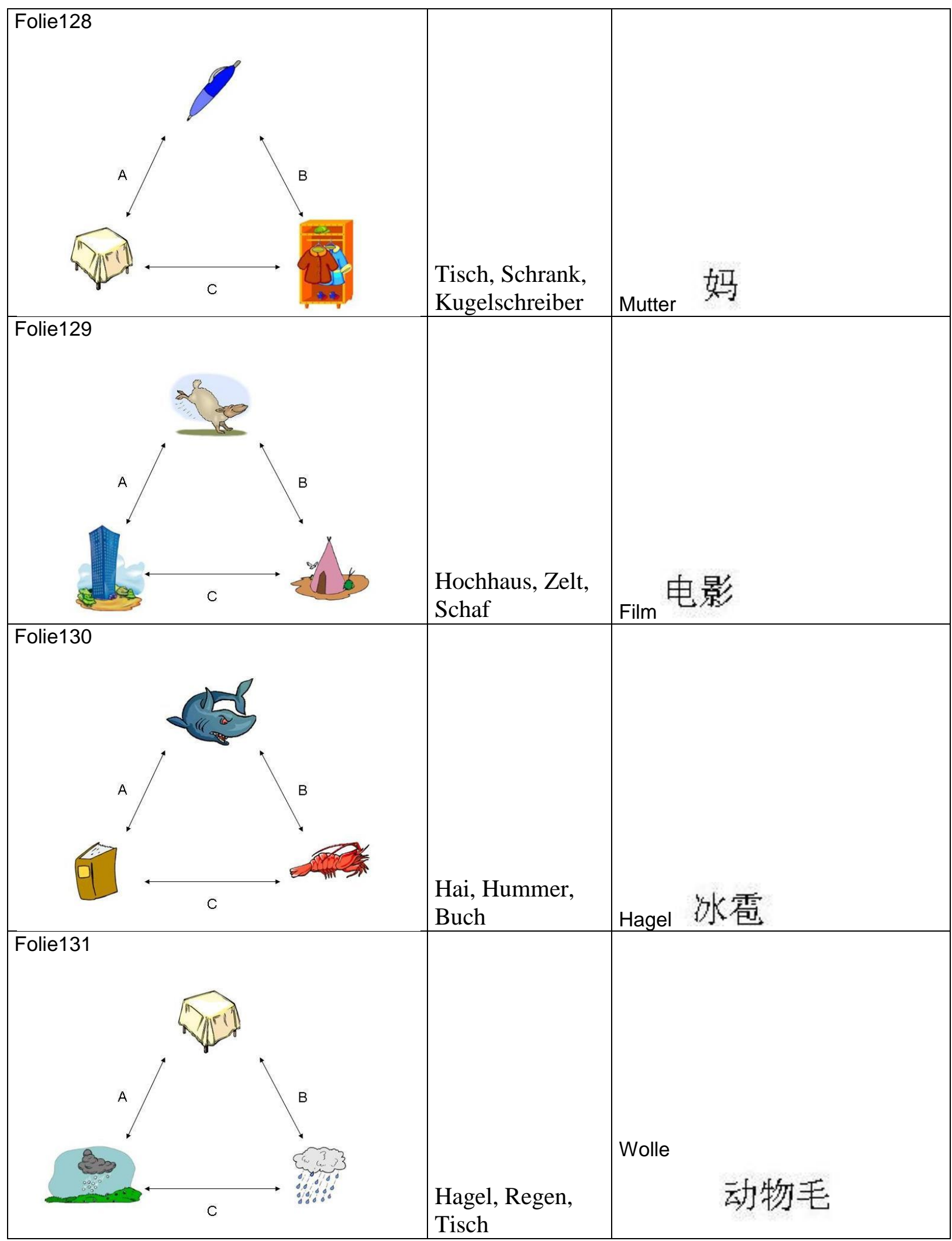




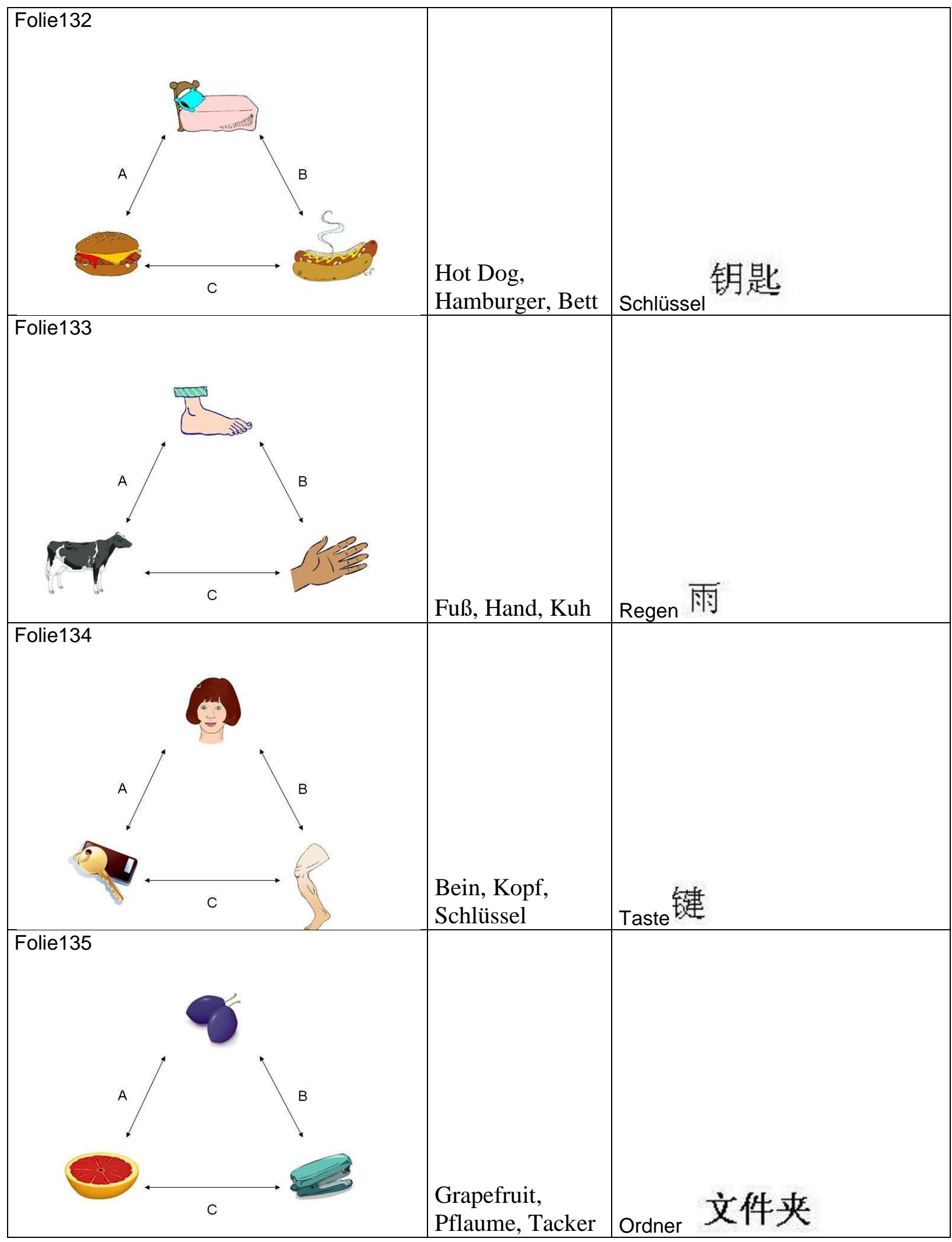




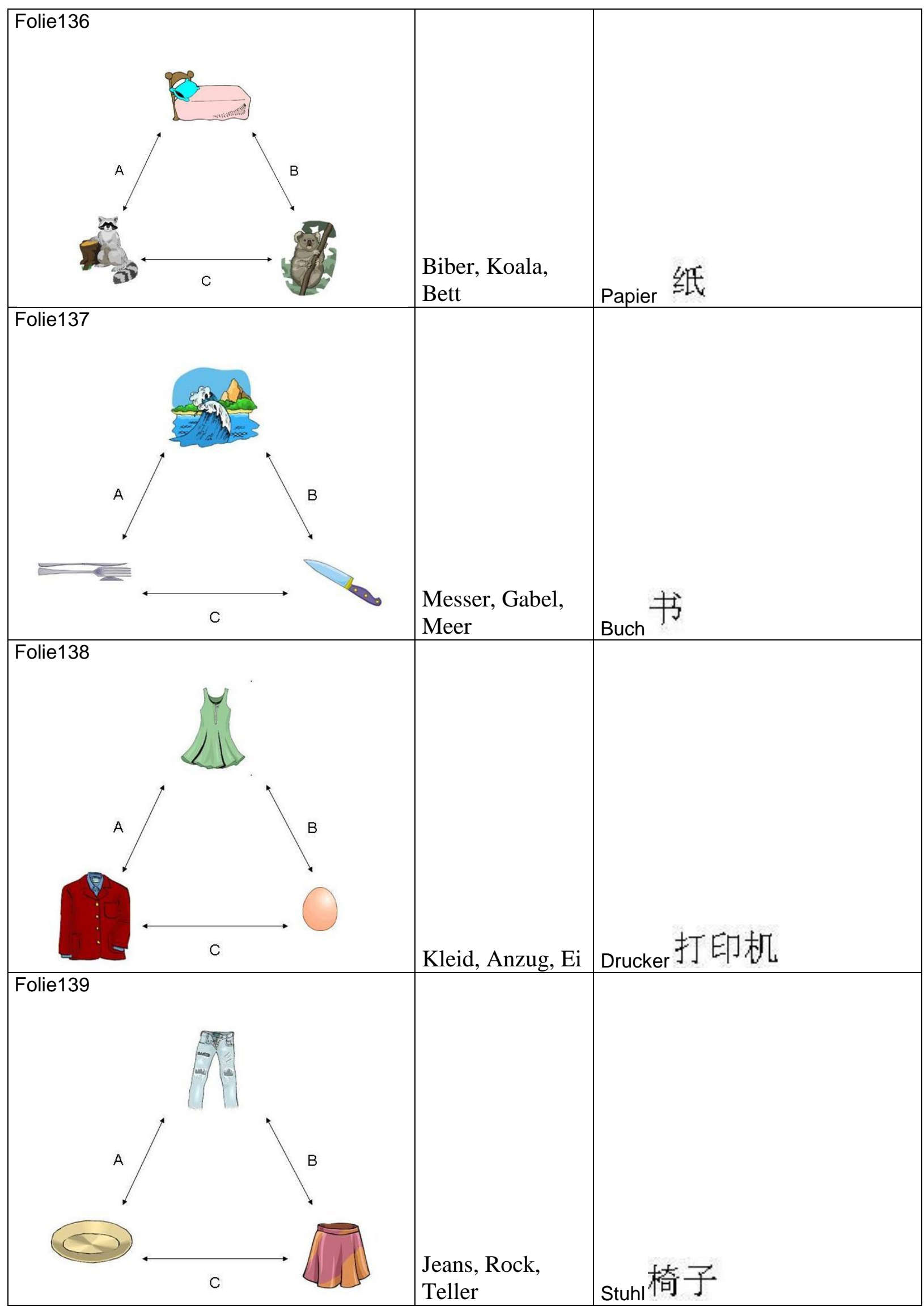




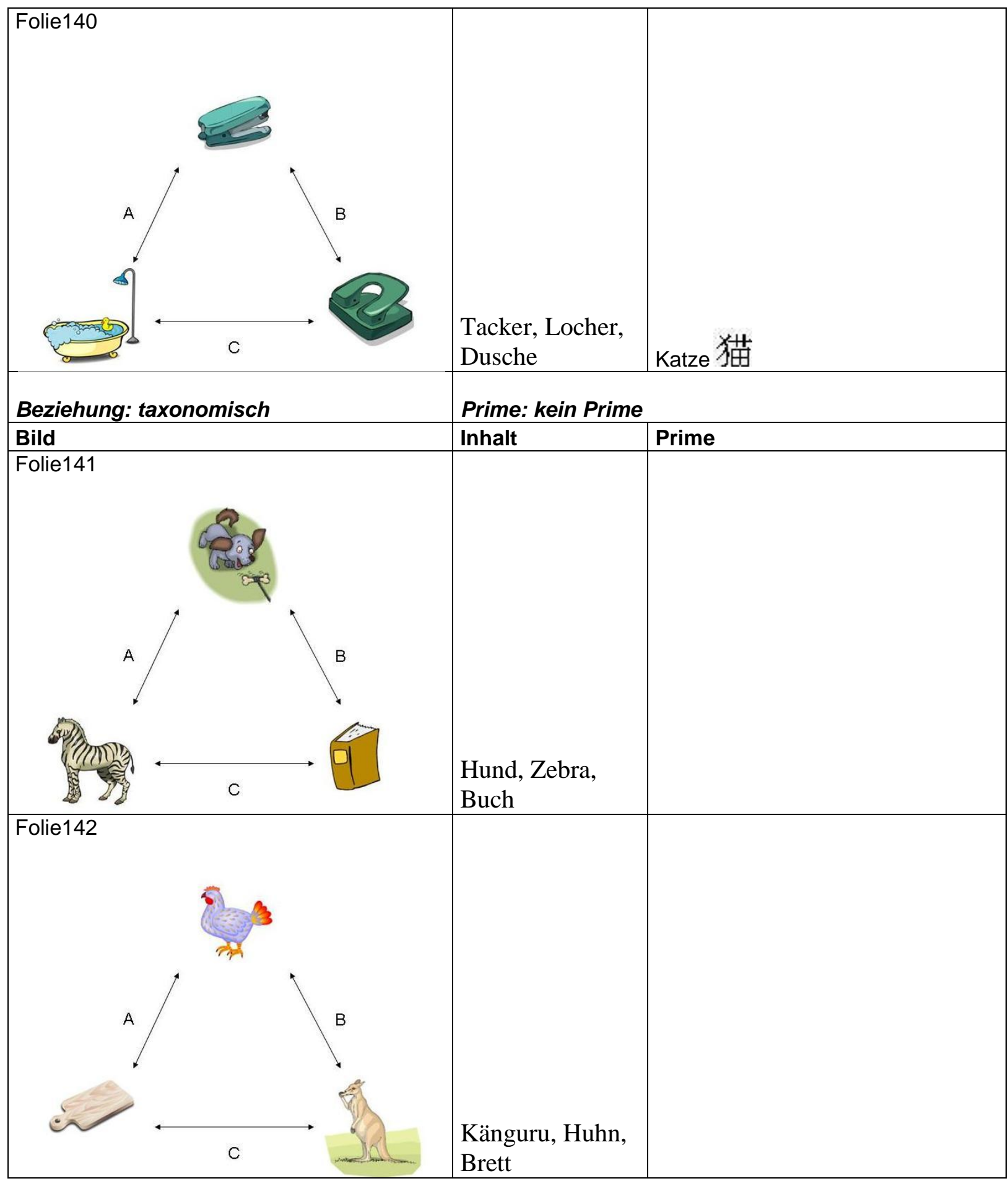




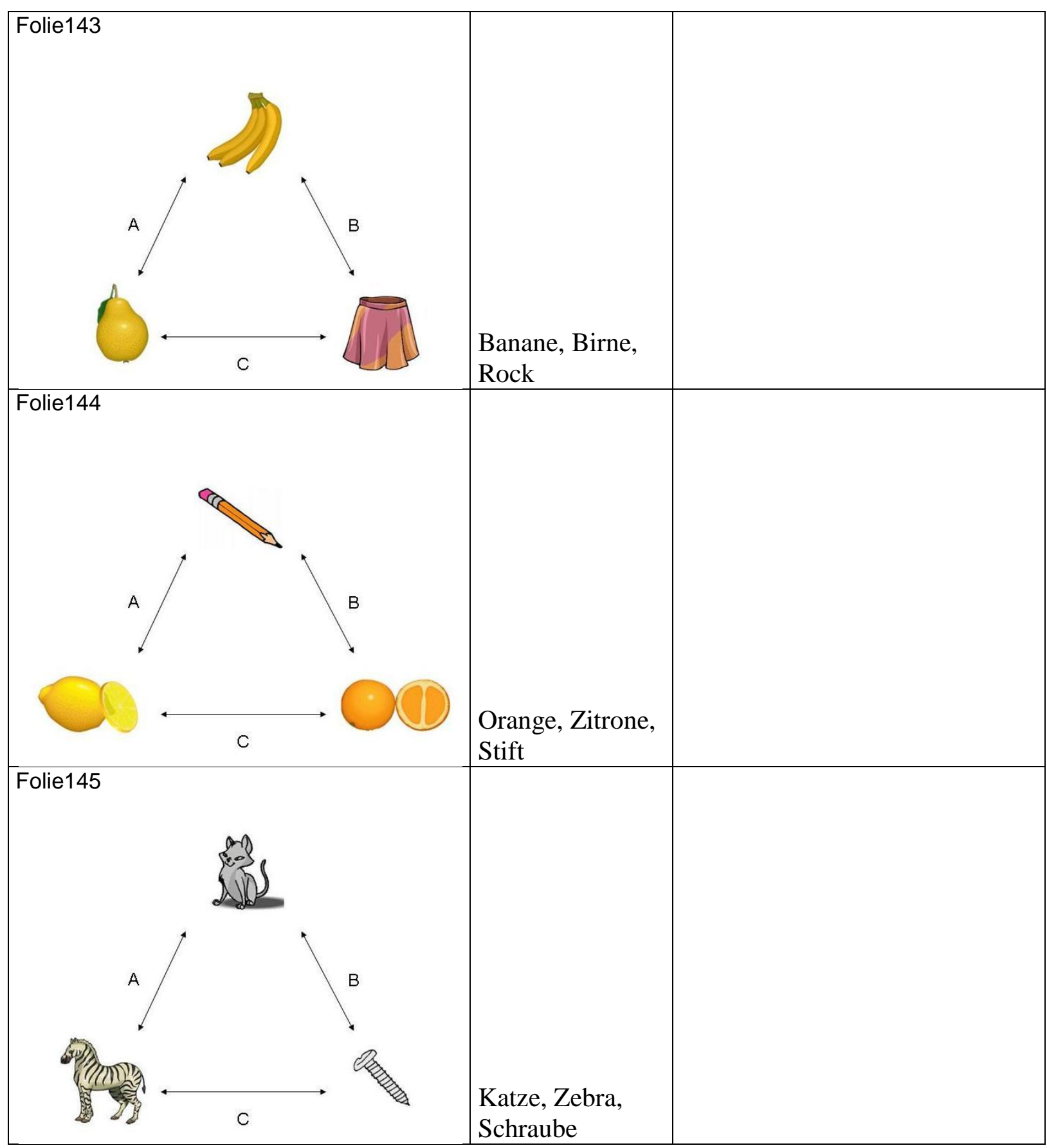




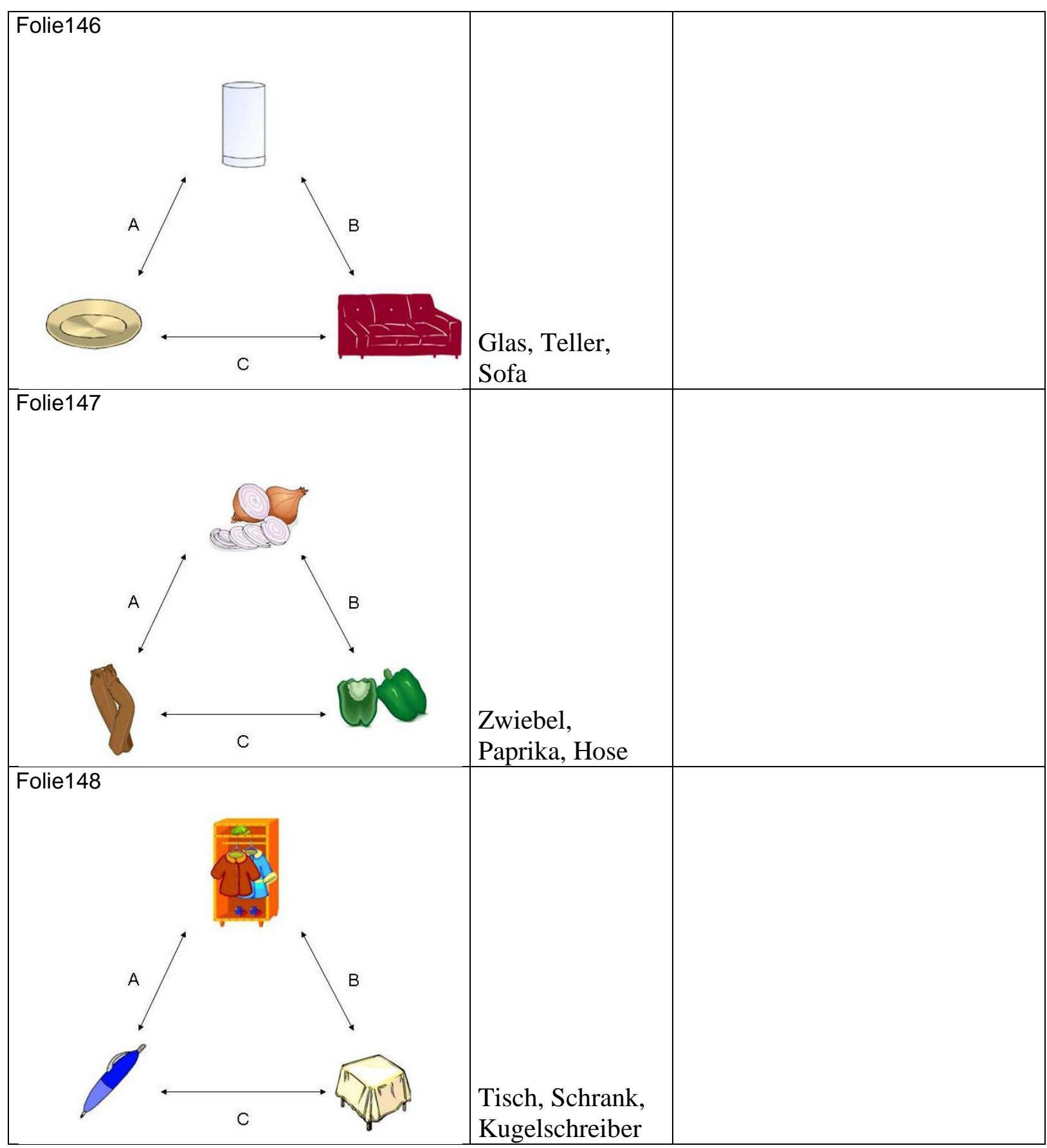




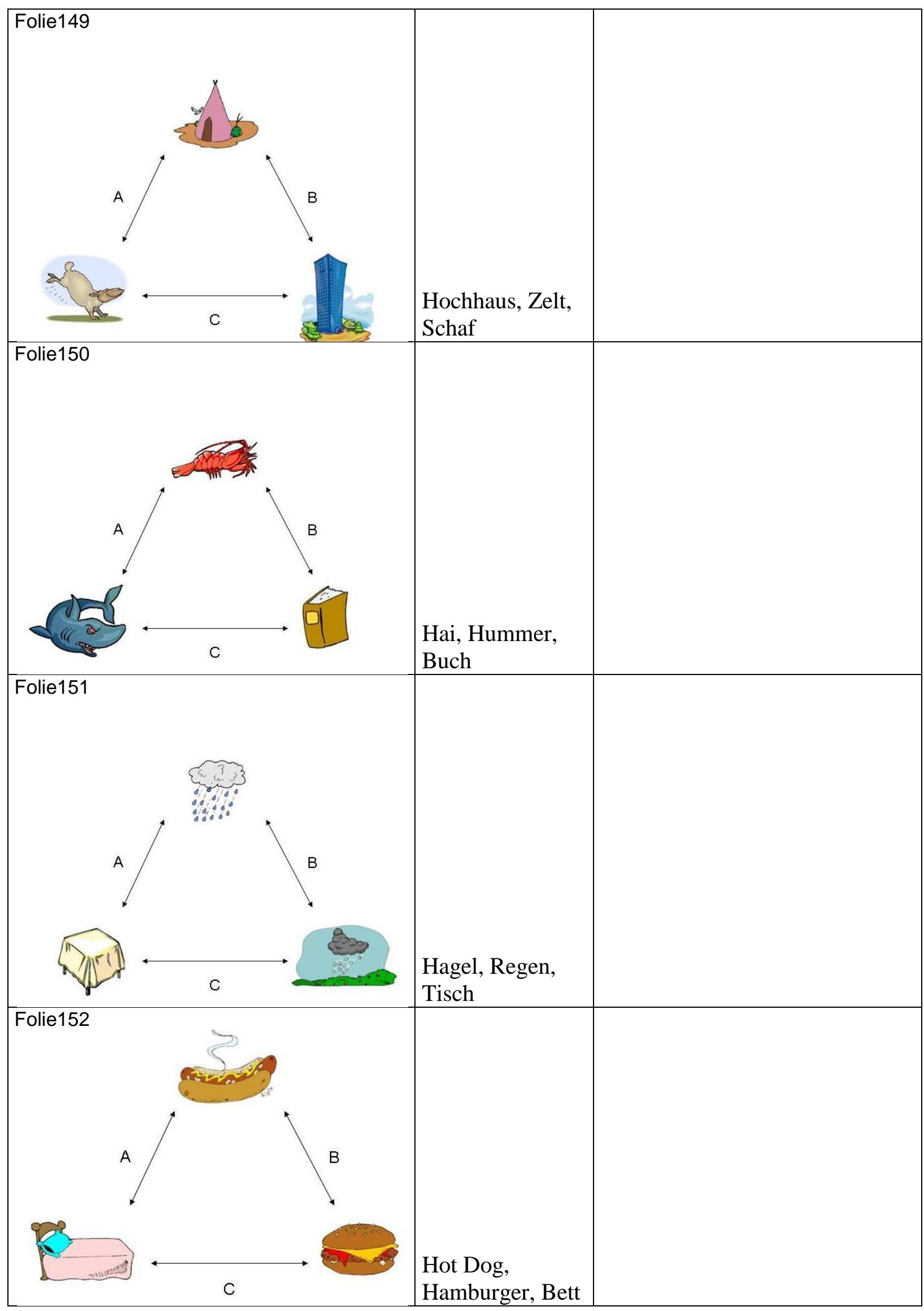




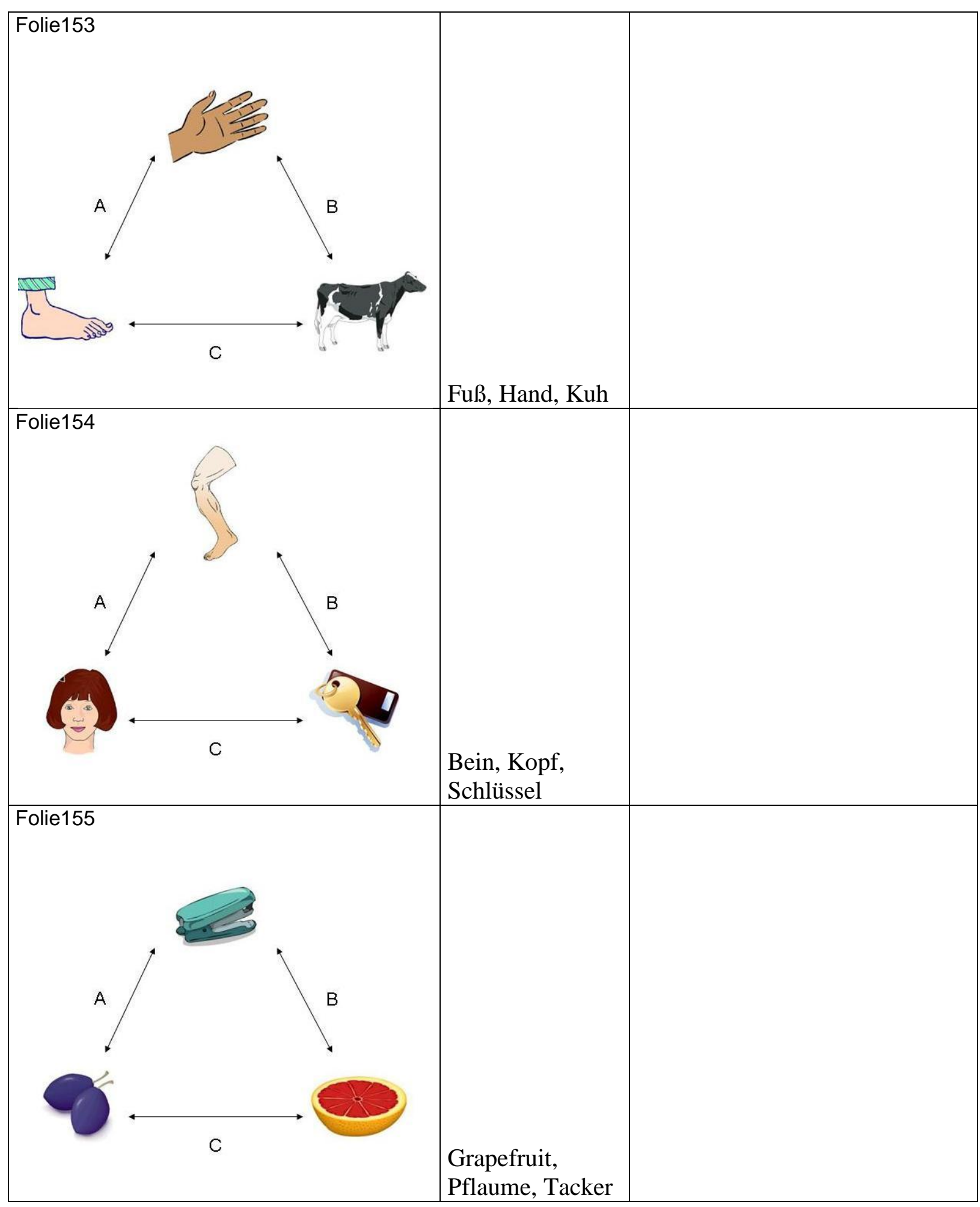




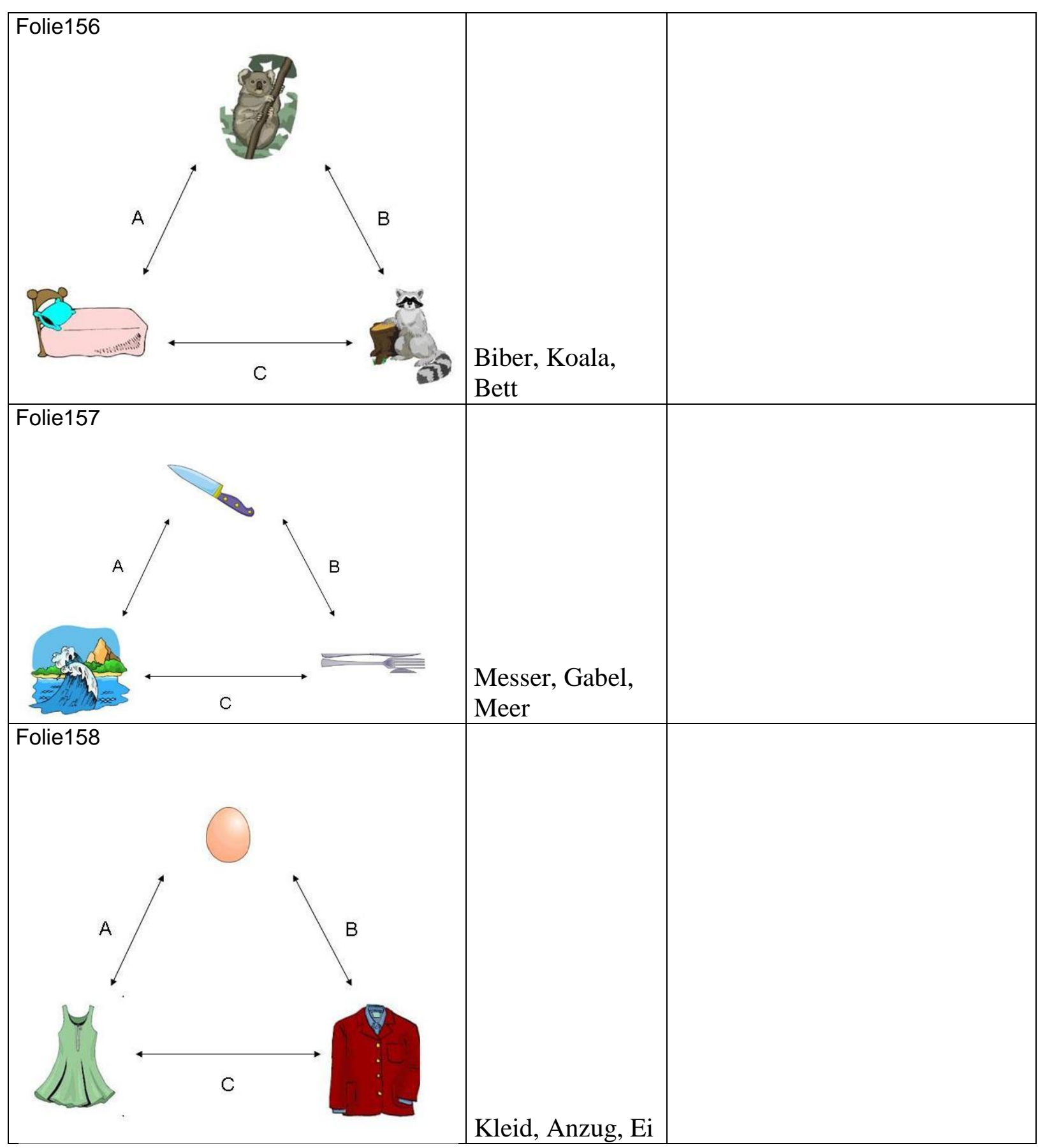




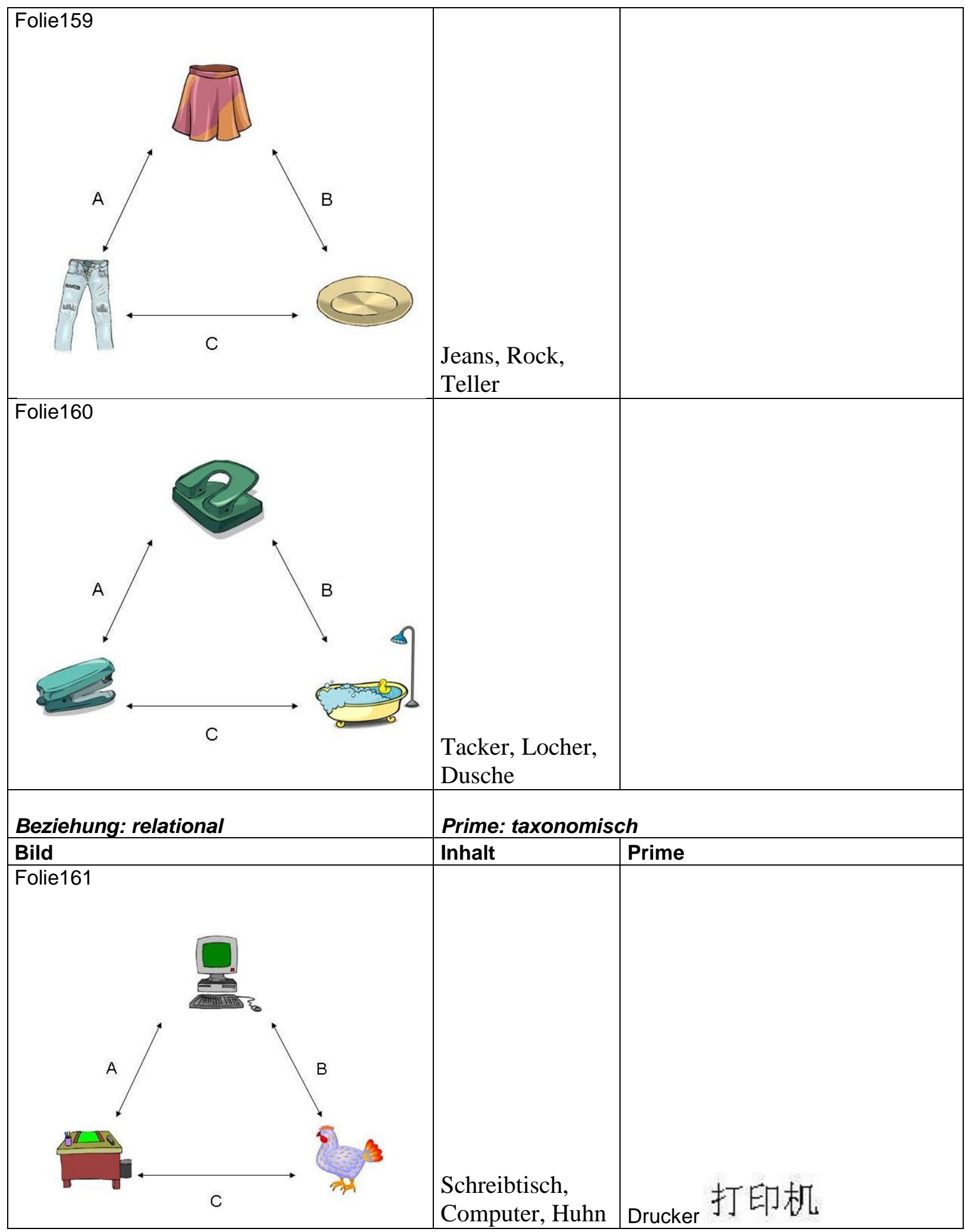




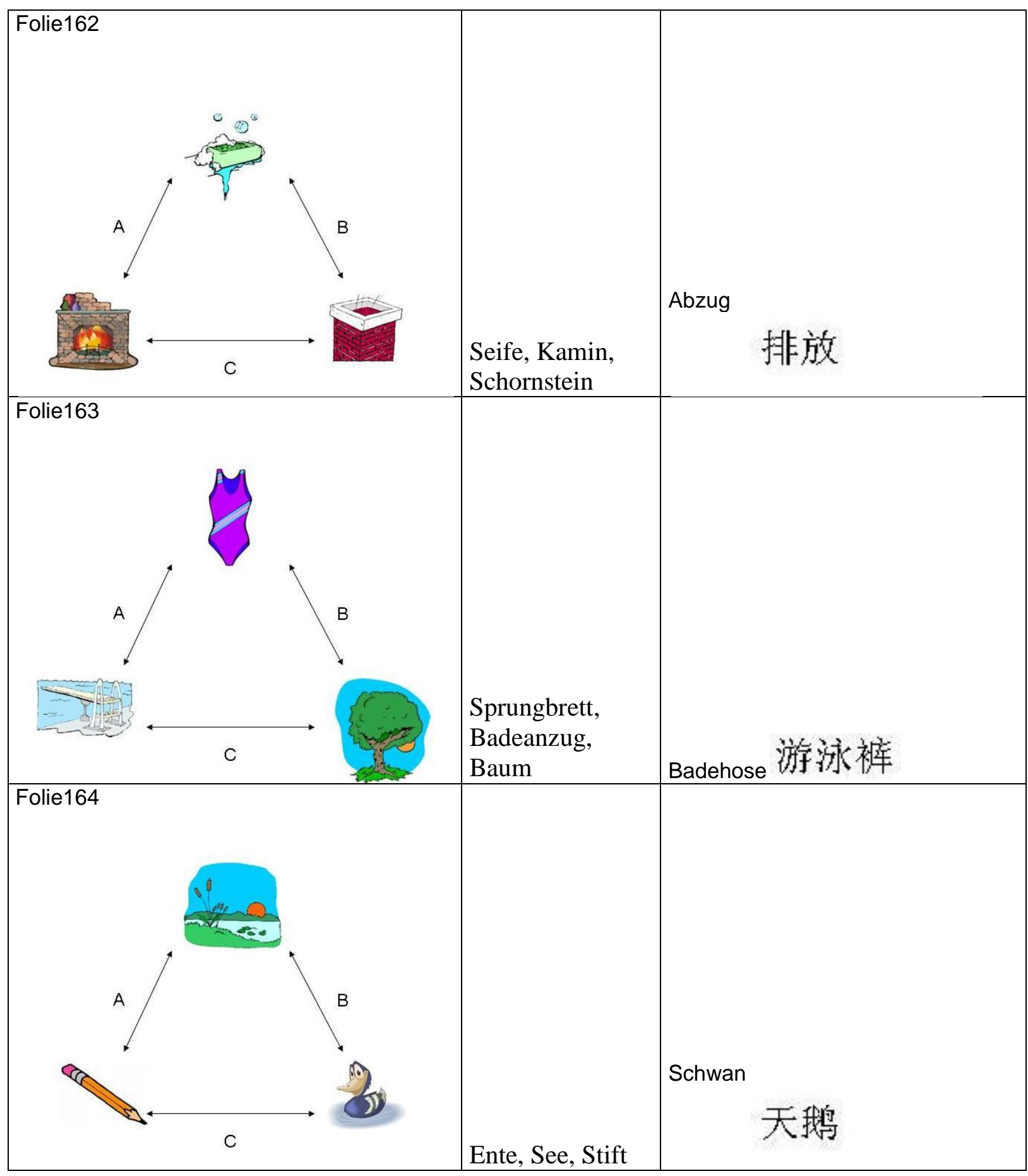




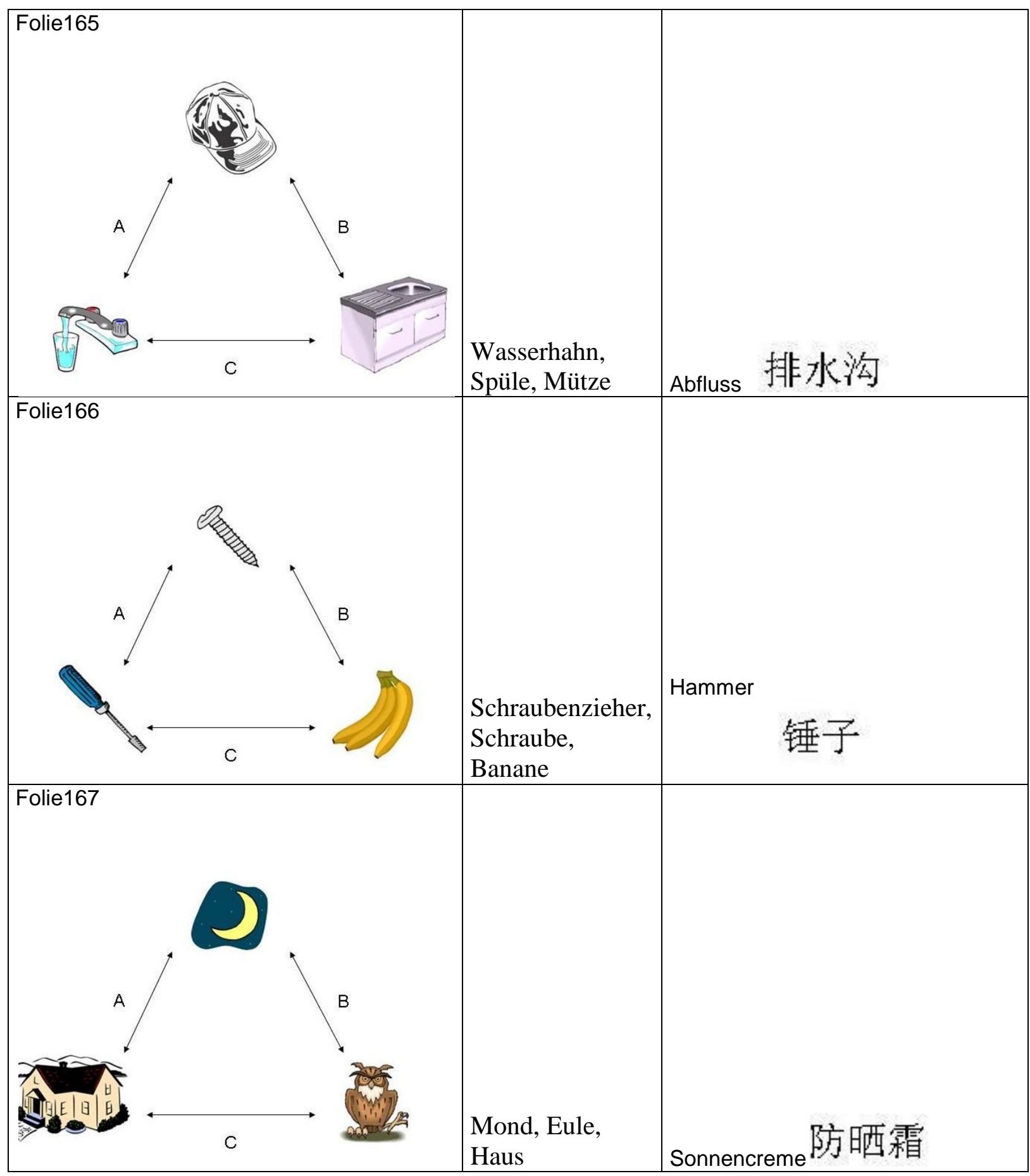




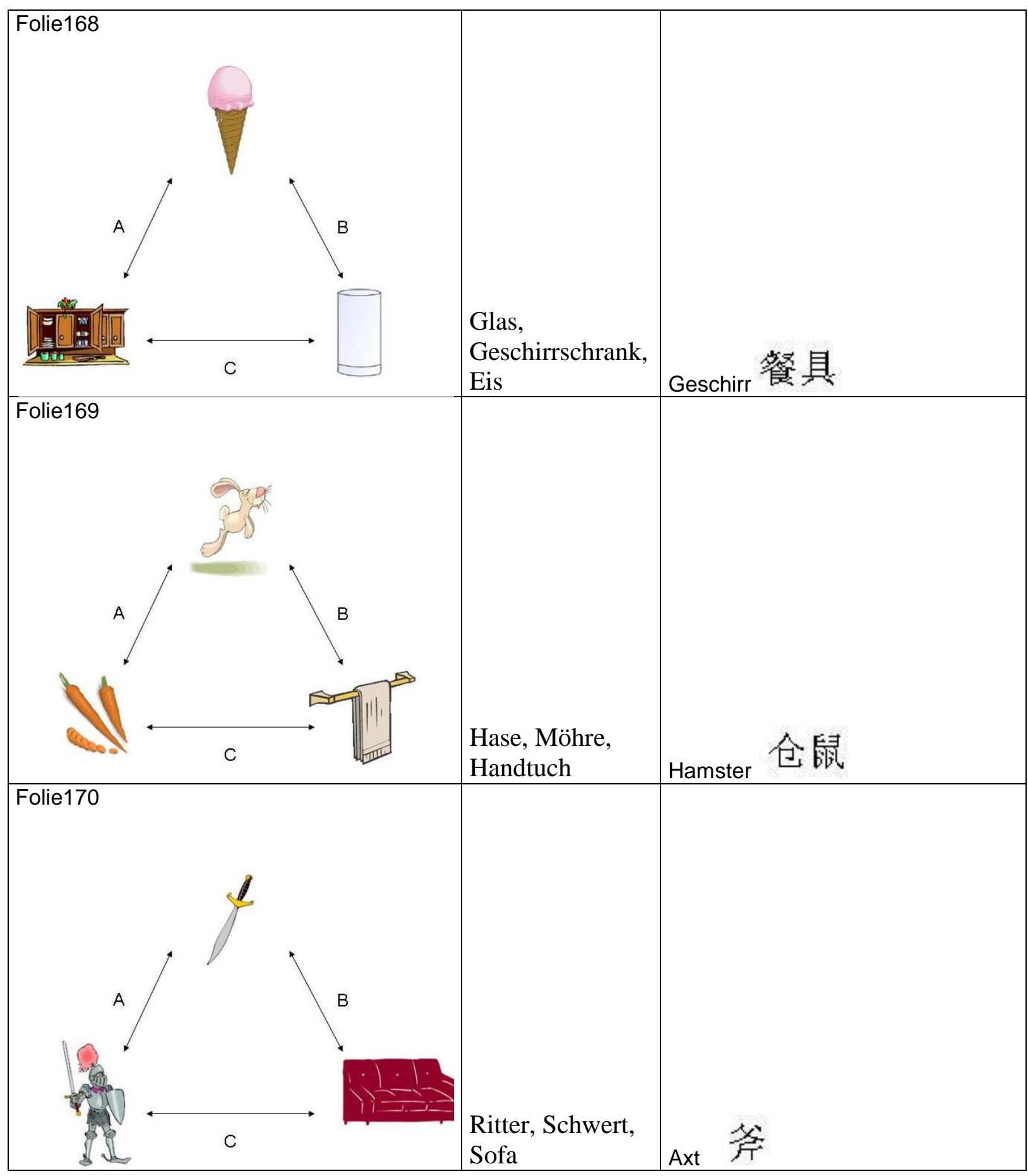




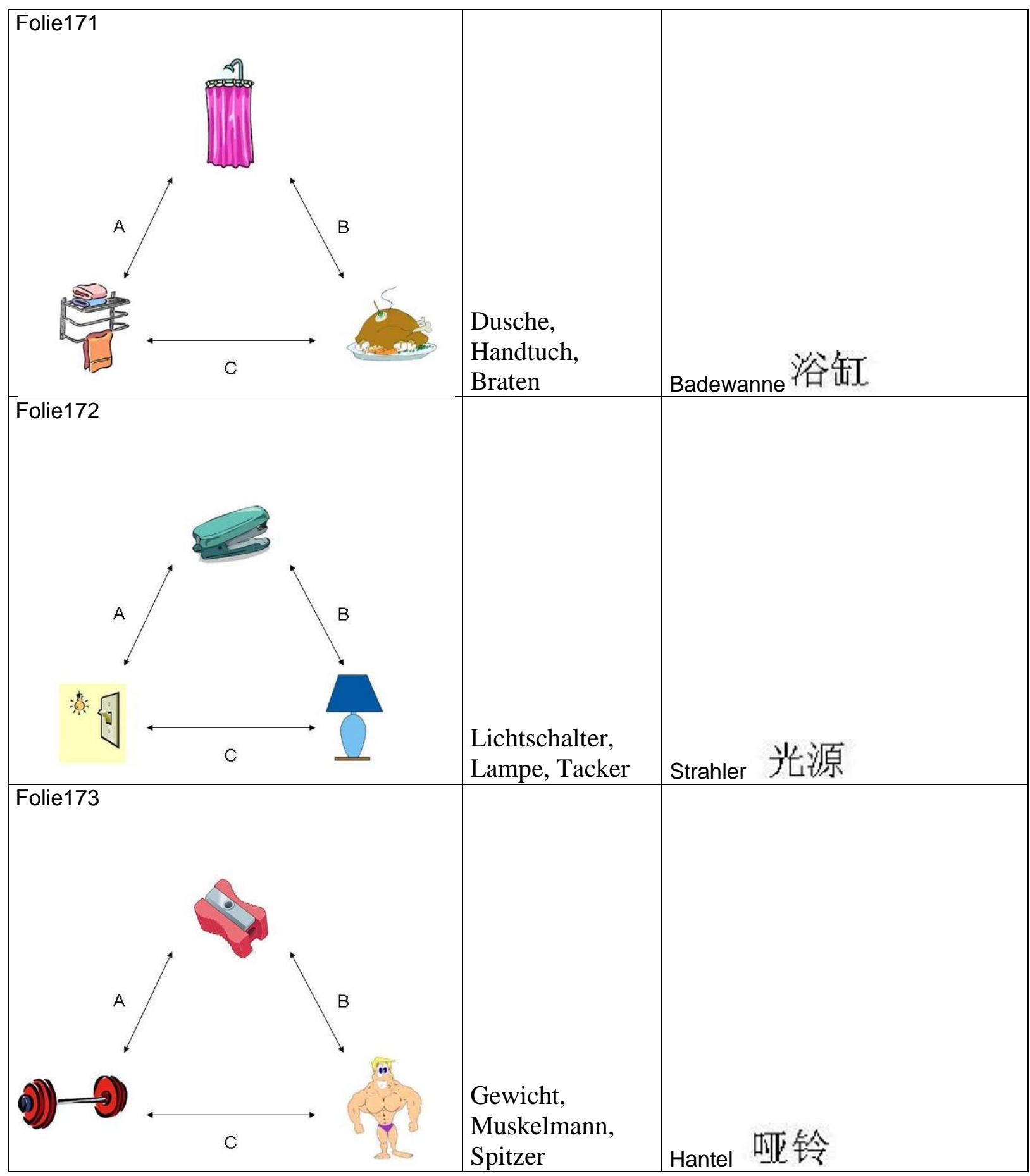




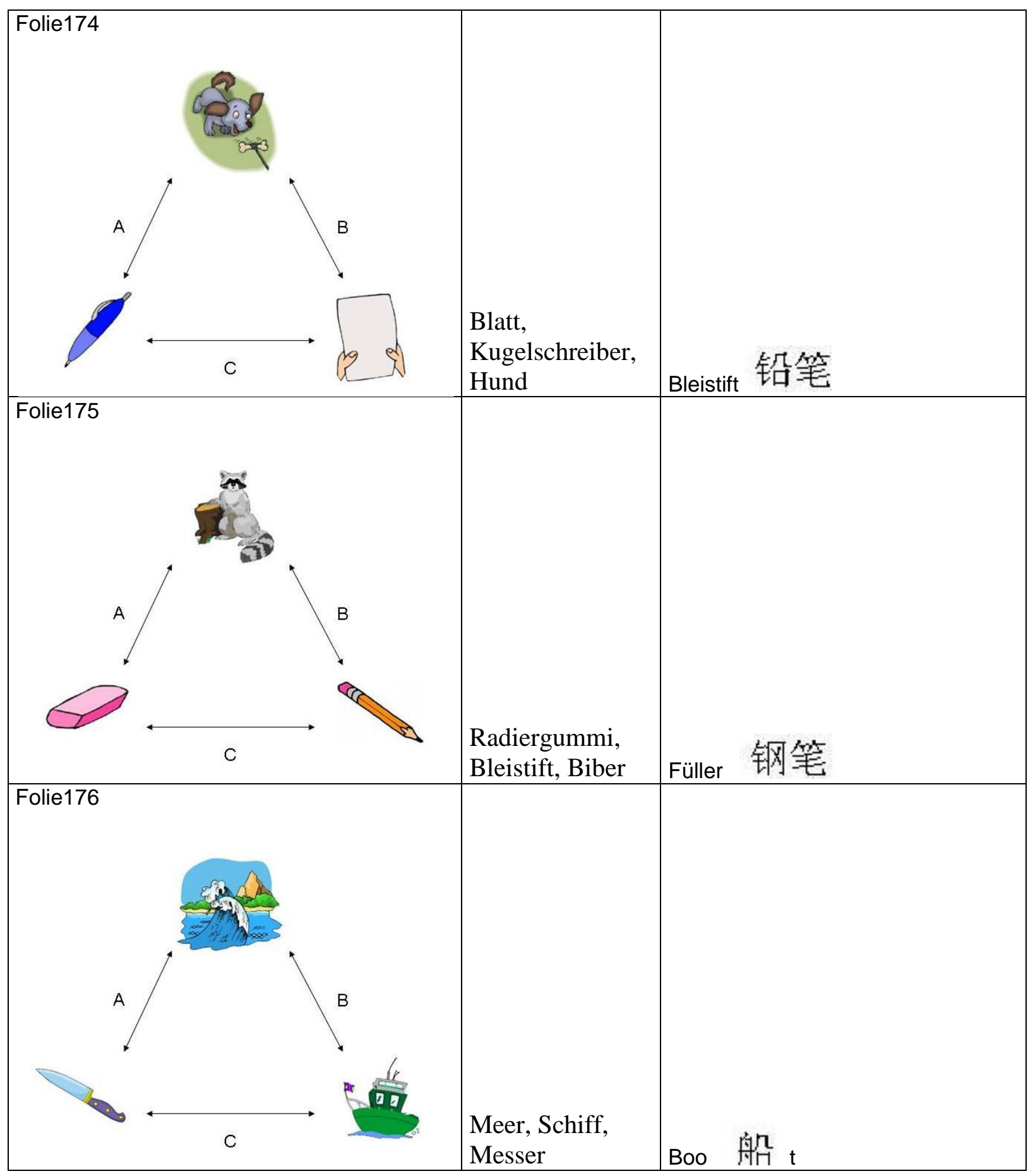




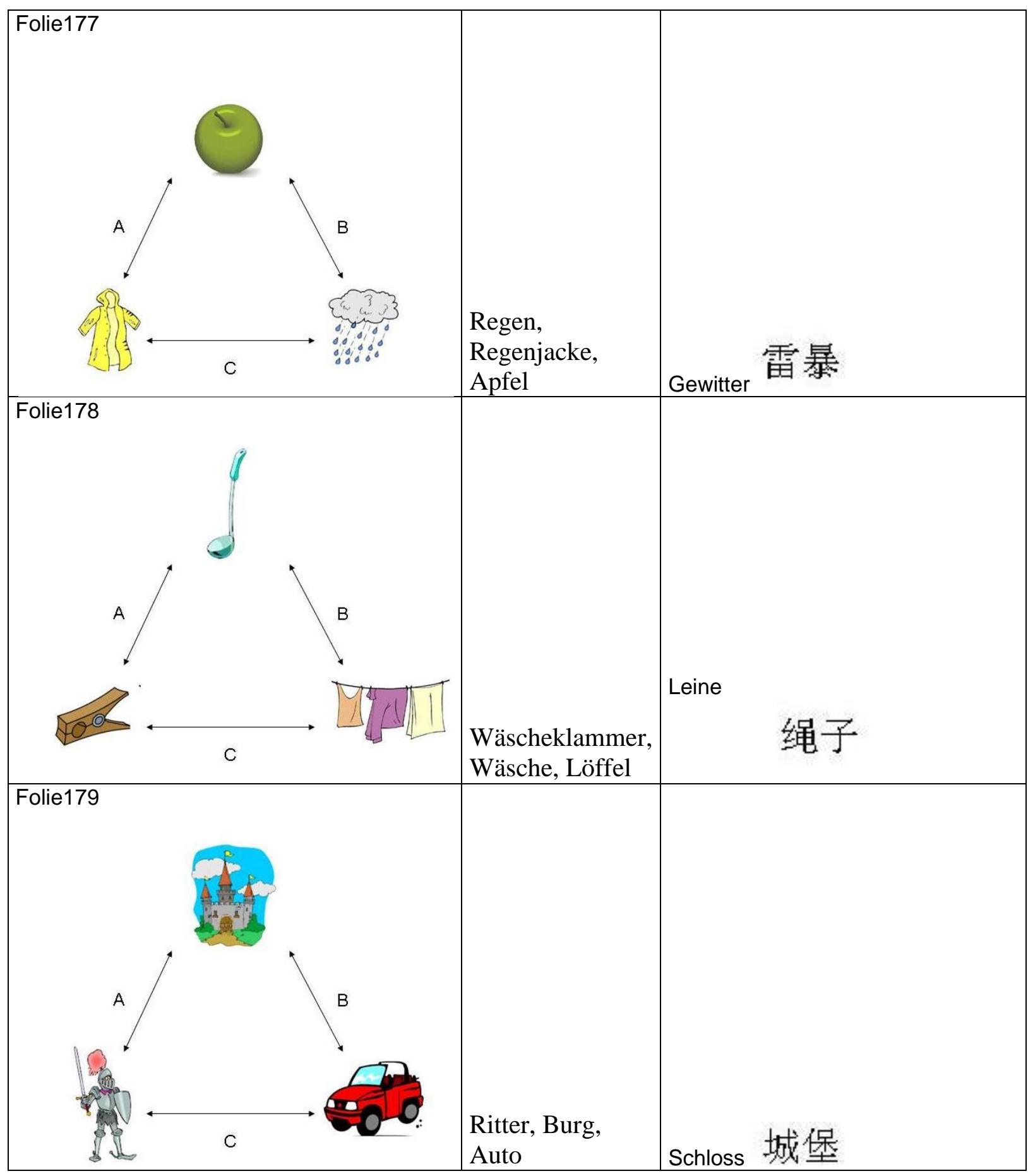




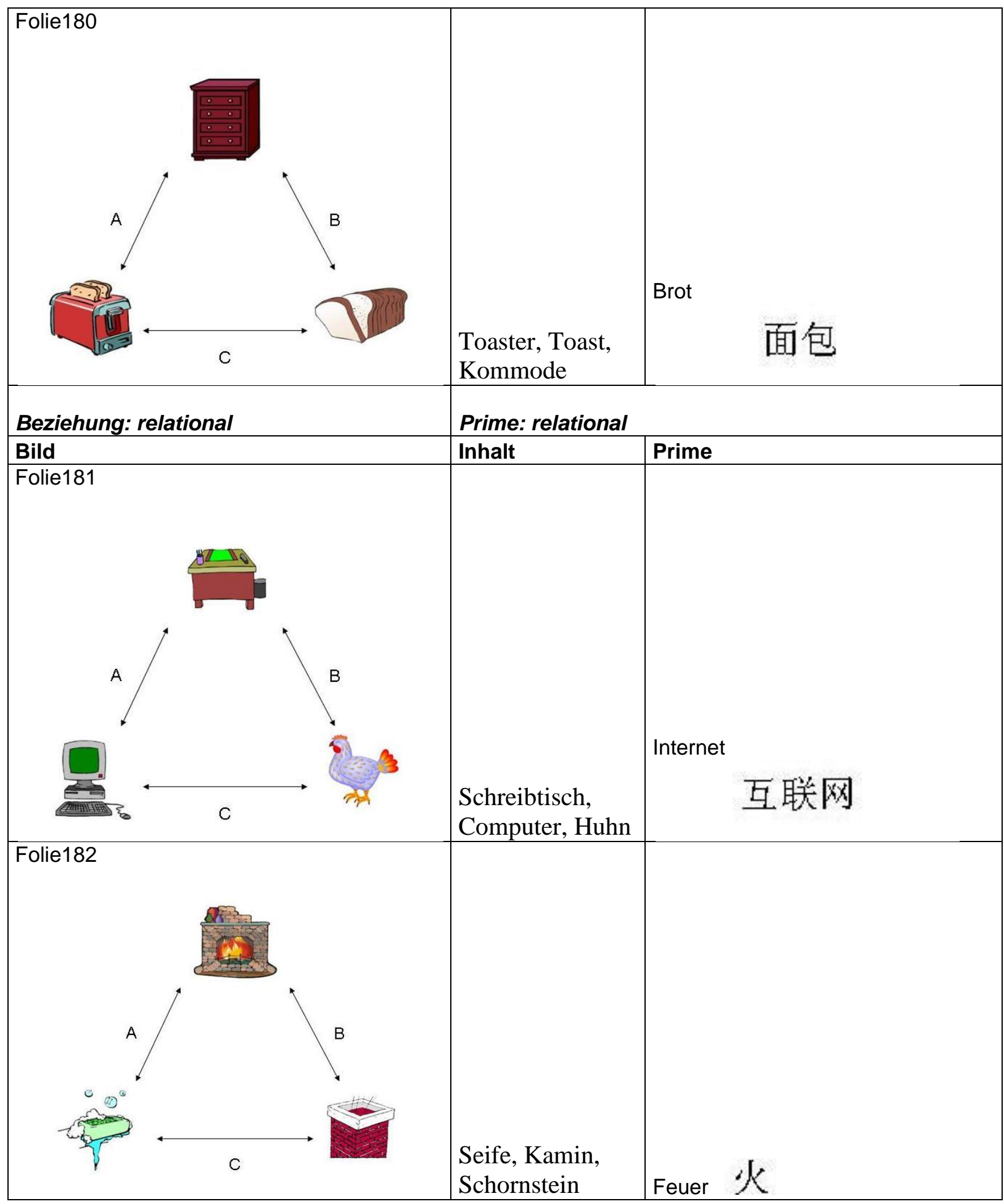




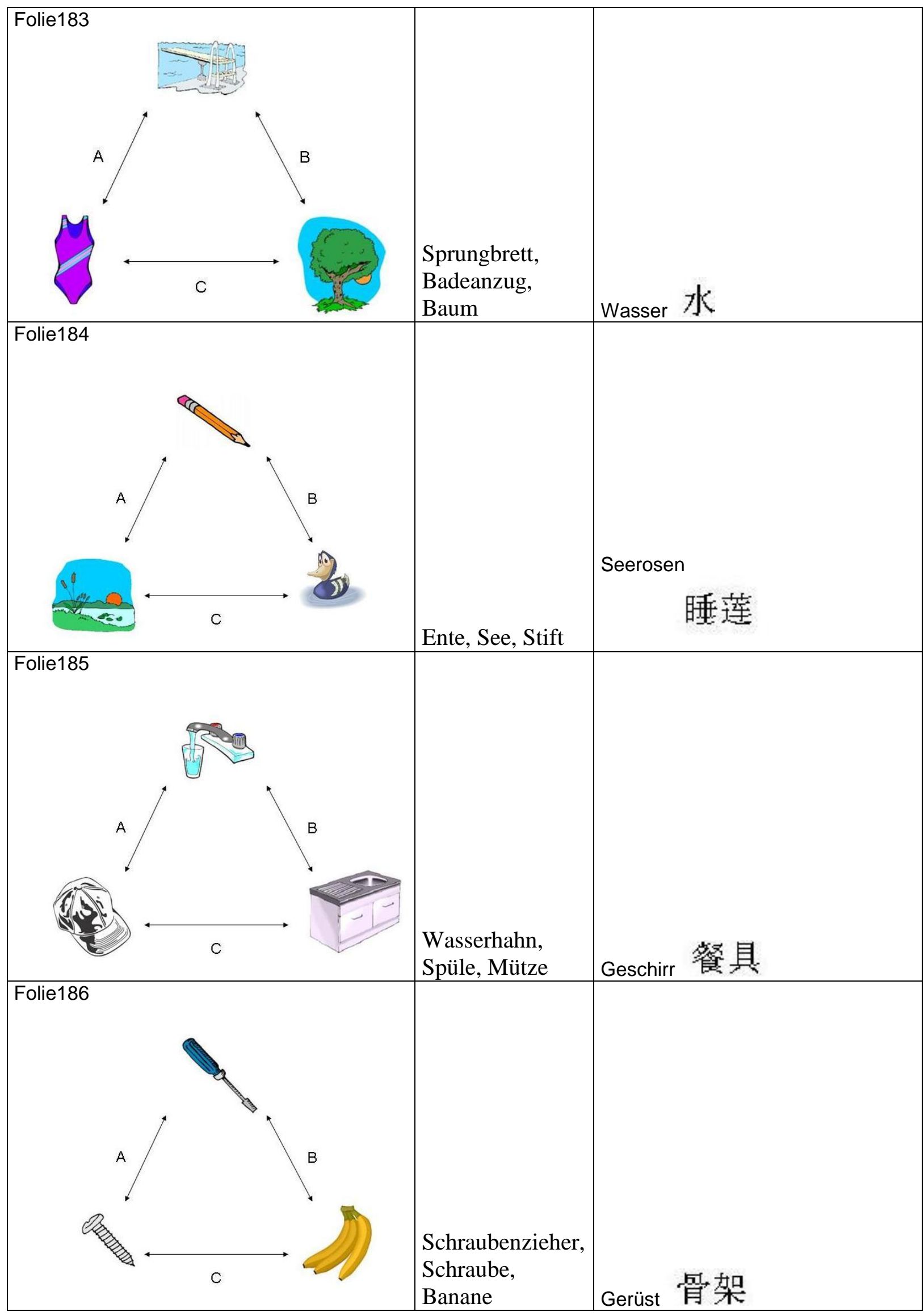




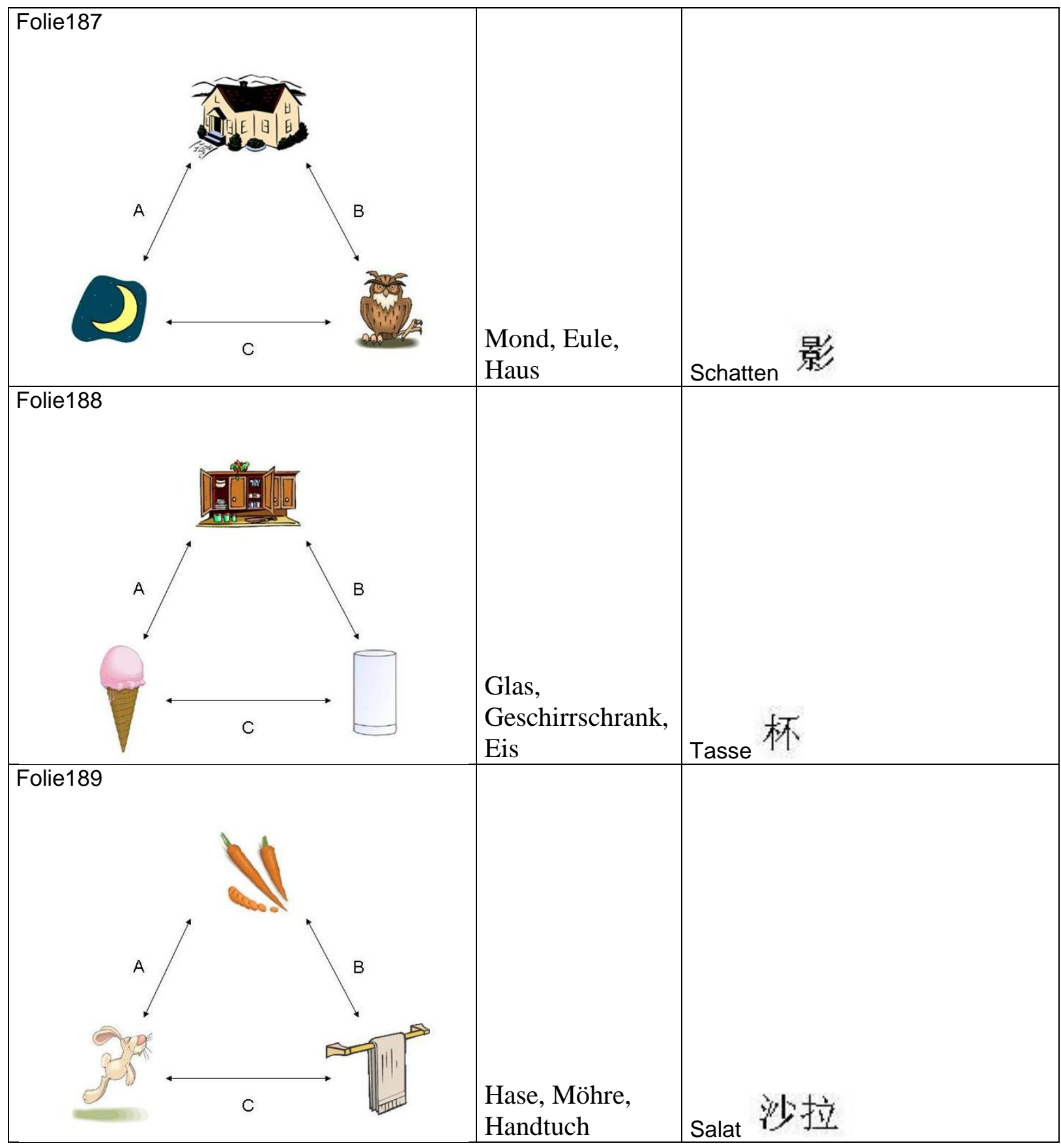




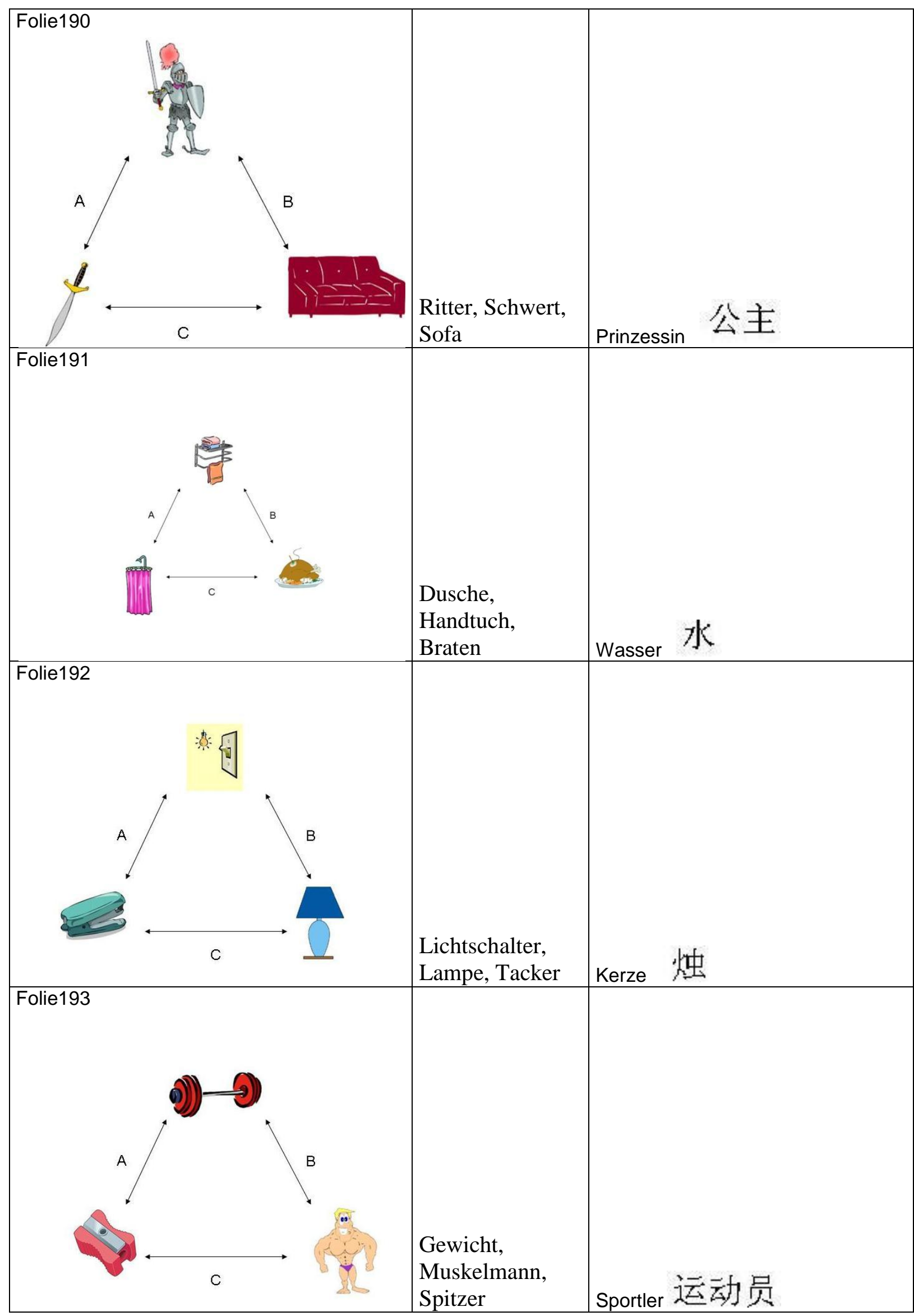




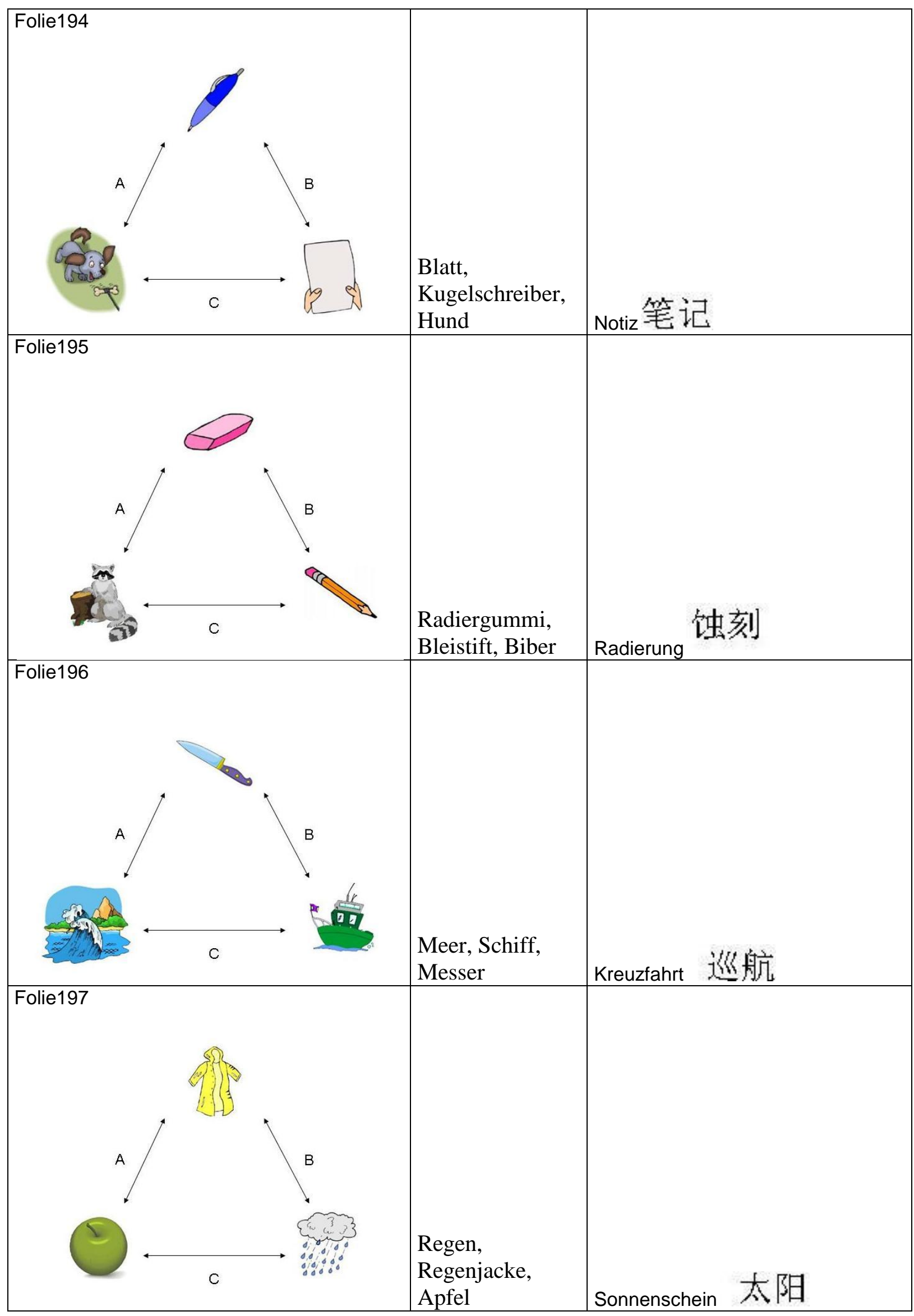




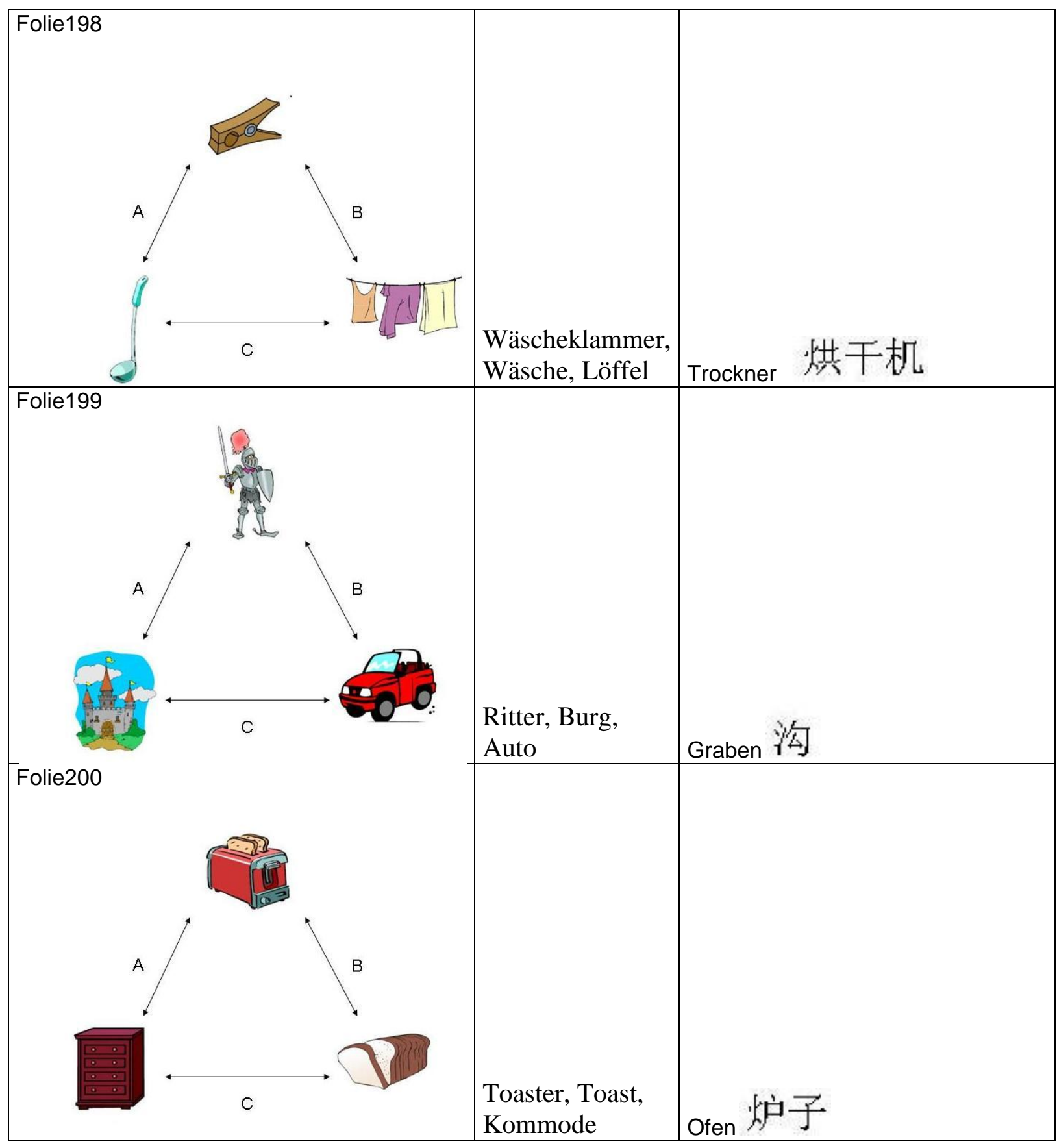




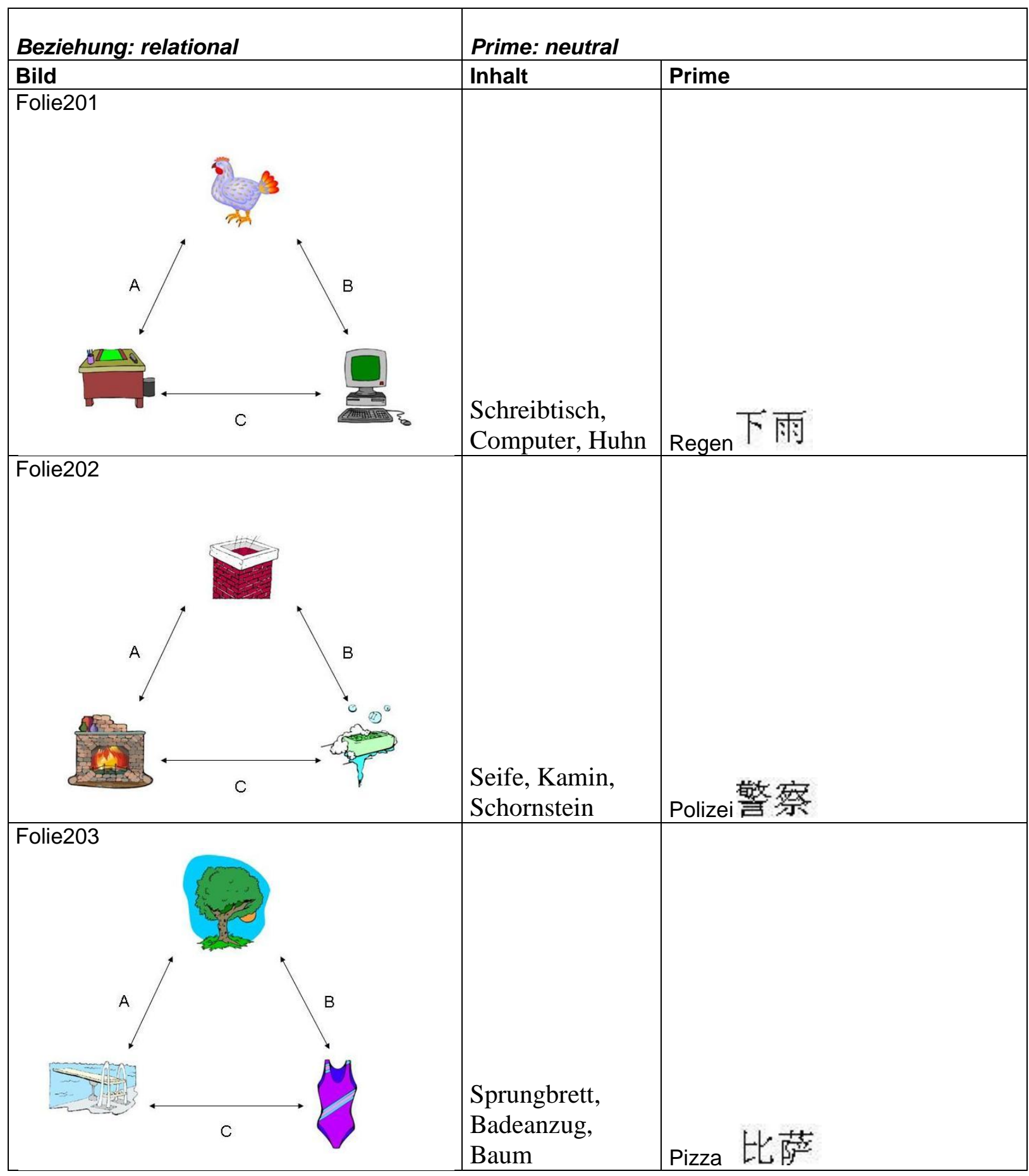




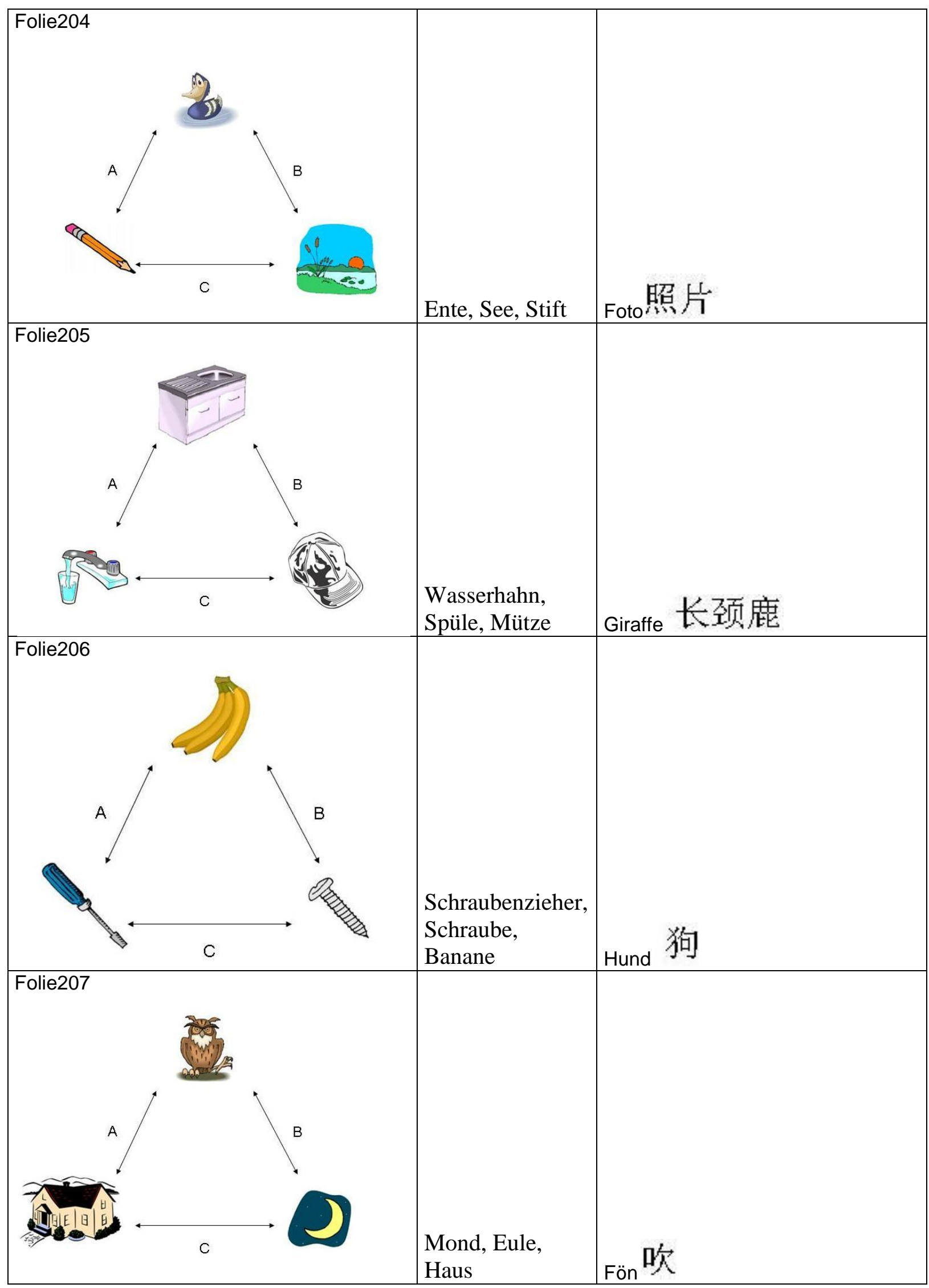




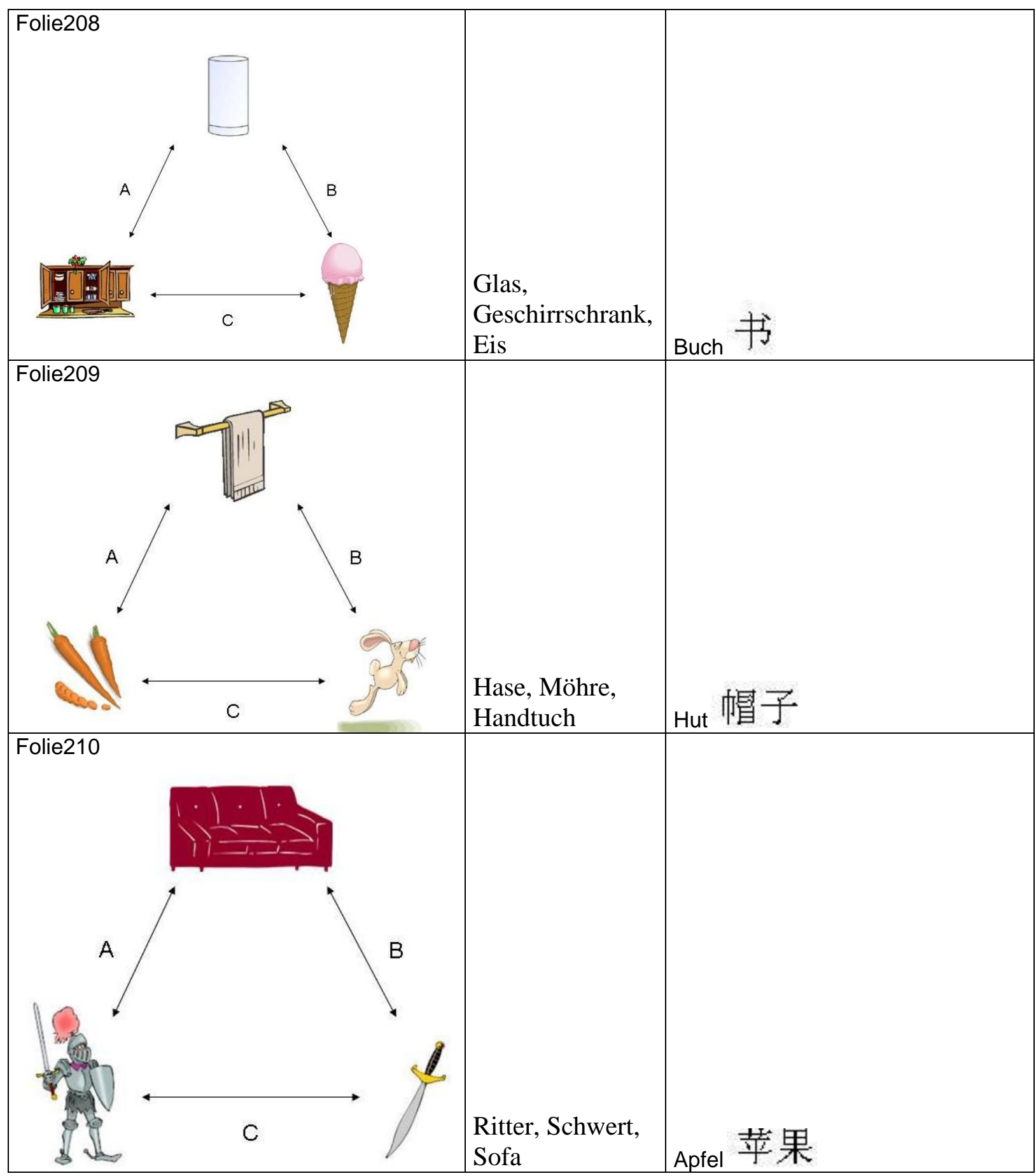




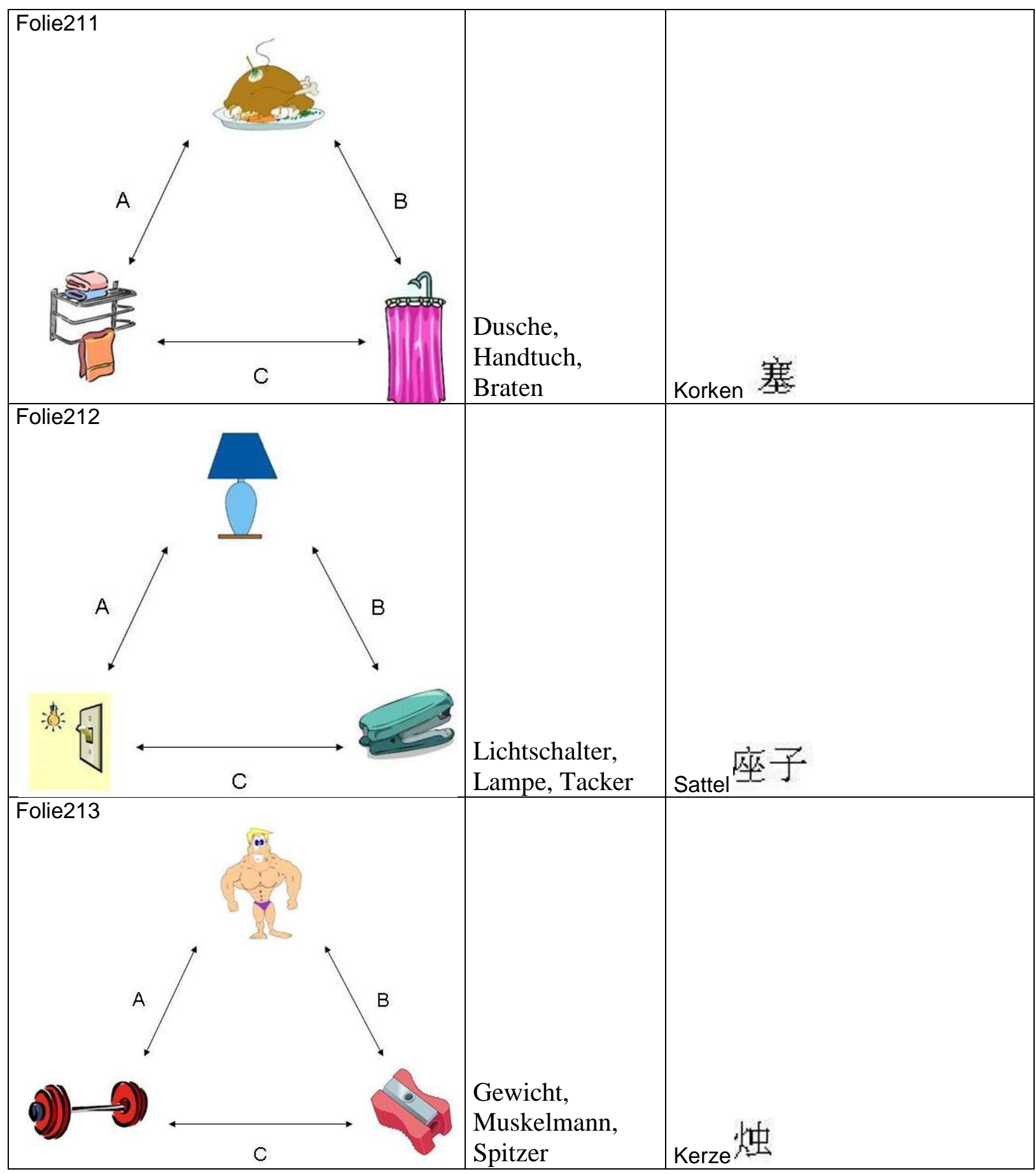


Anhang

260

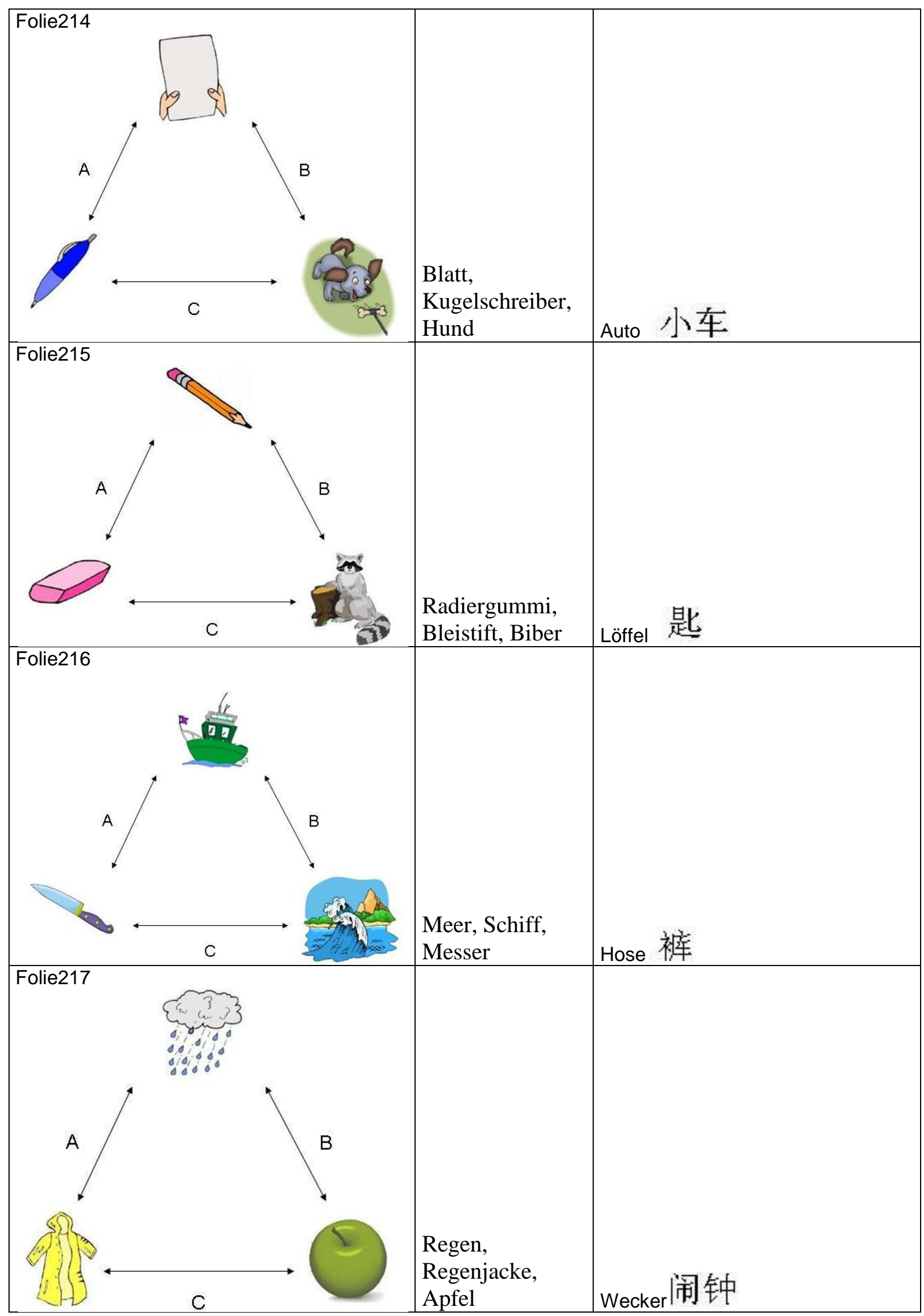




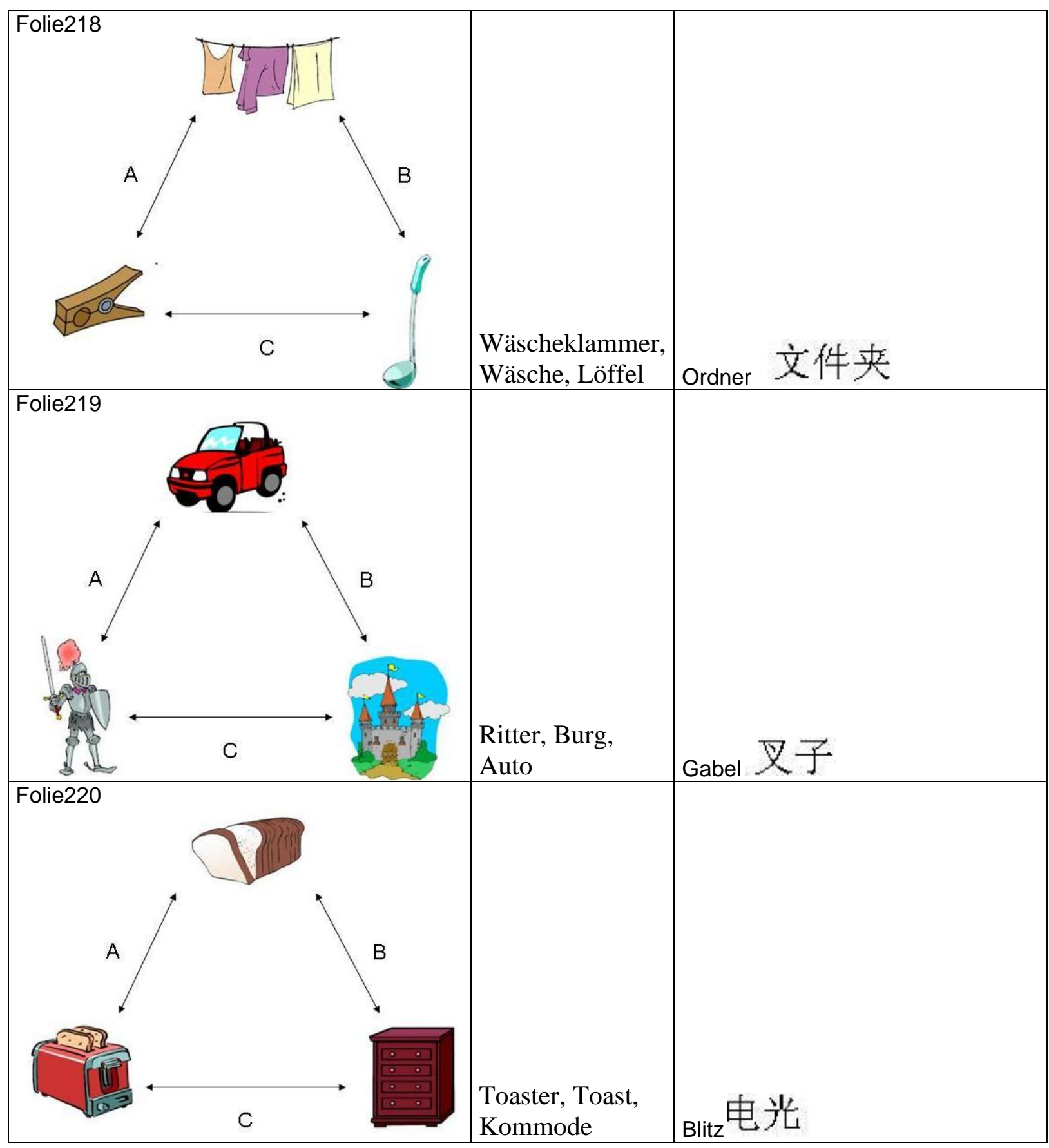




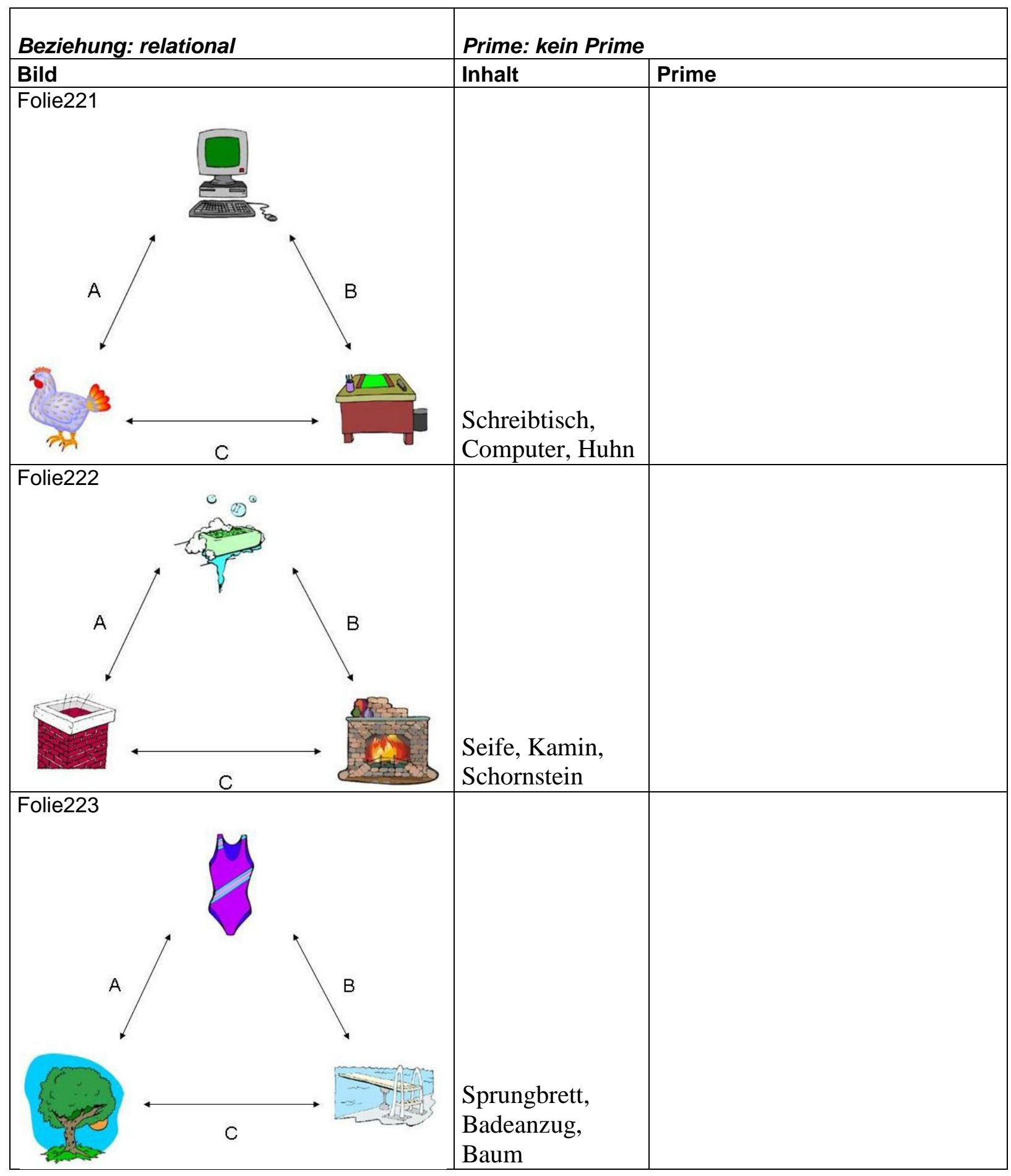




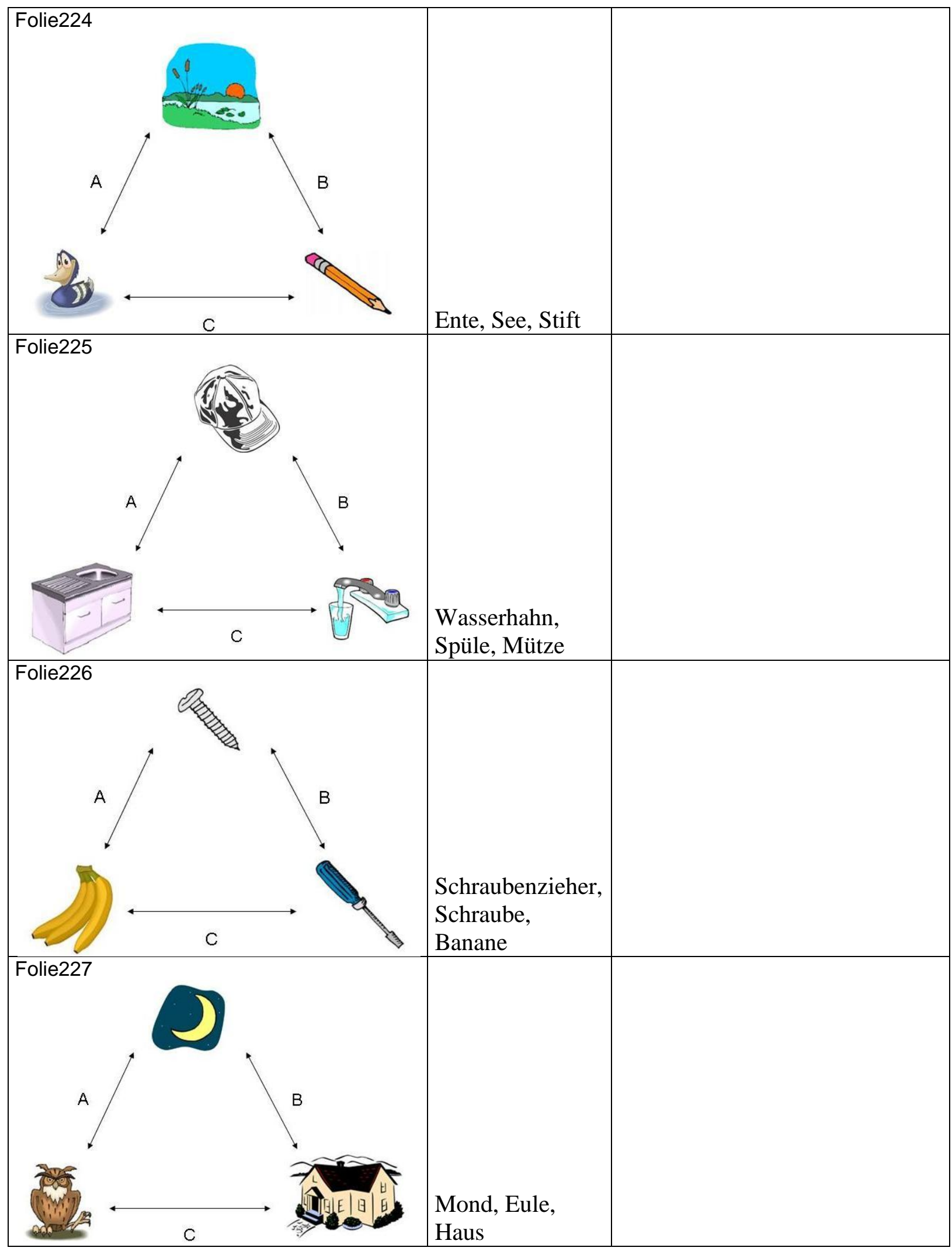




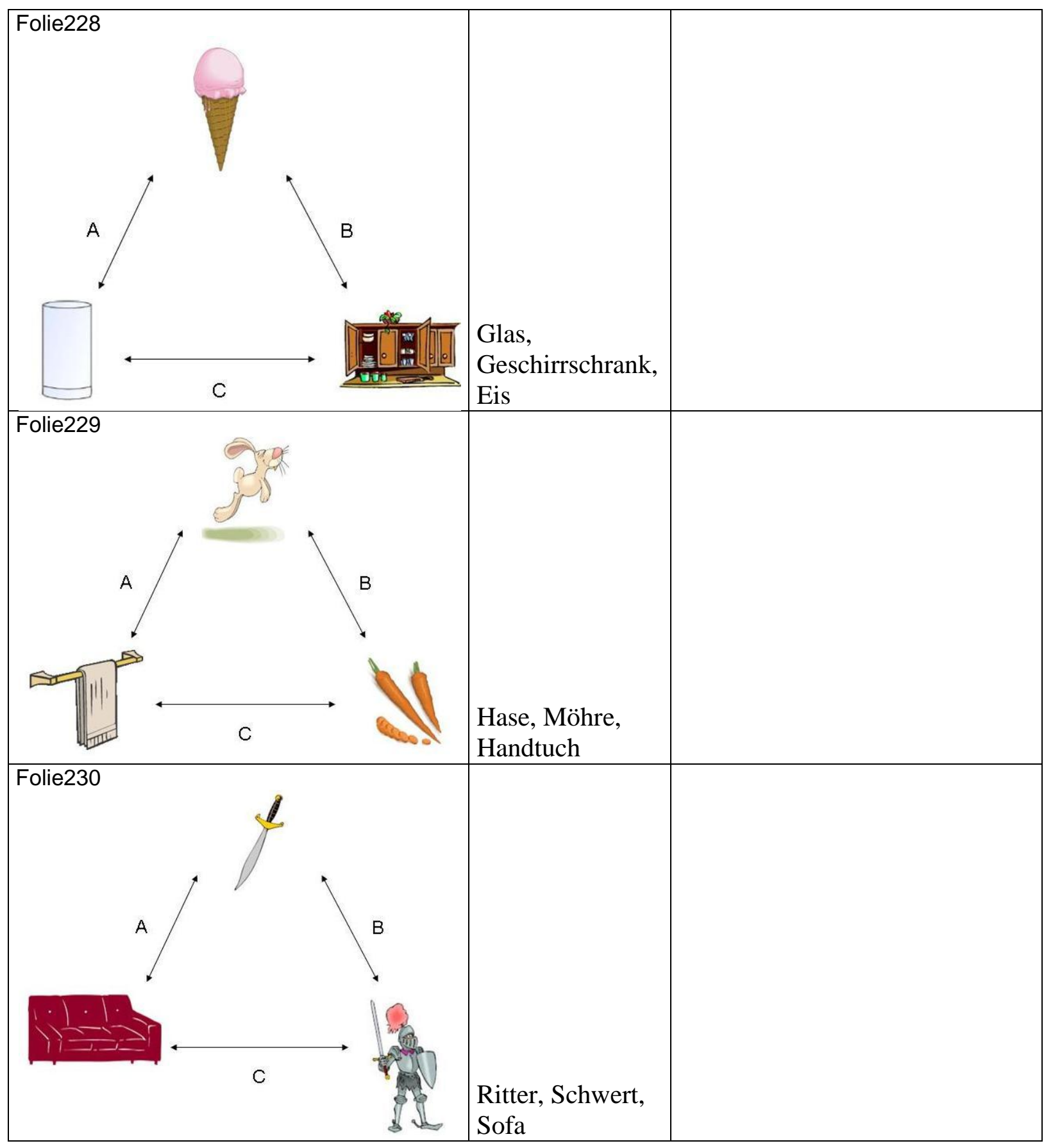




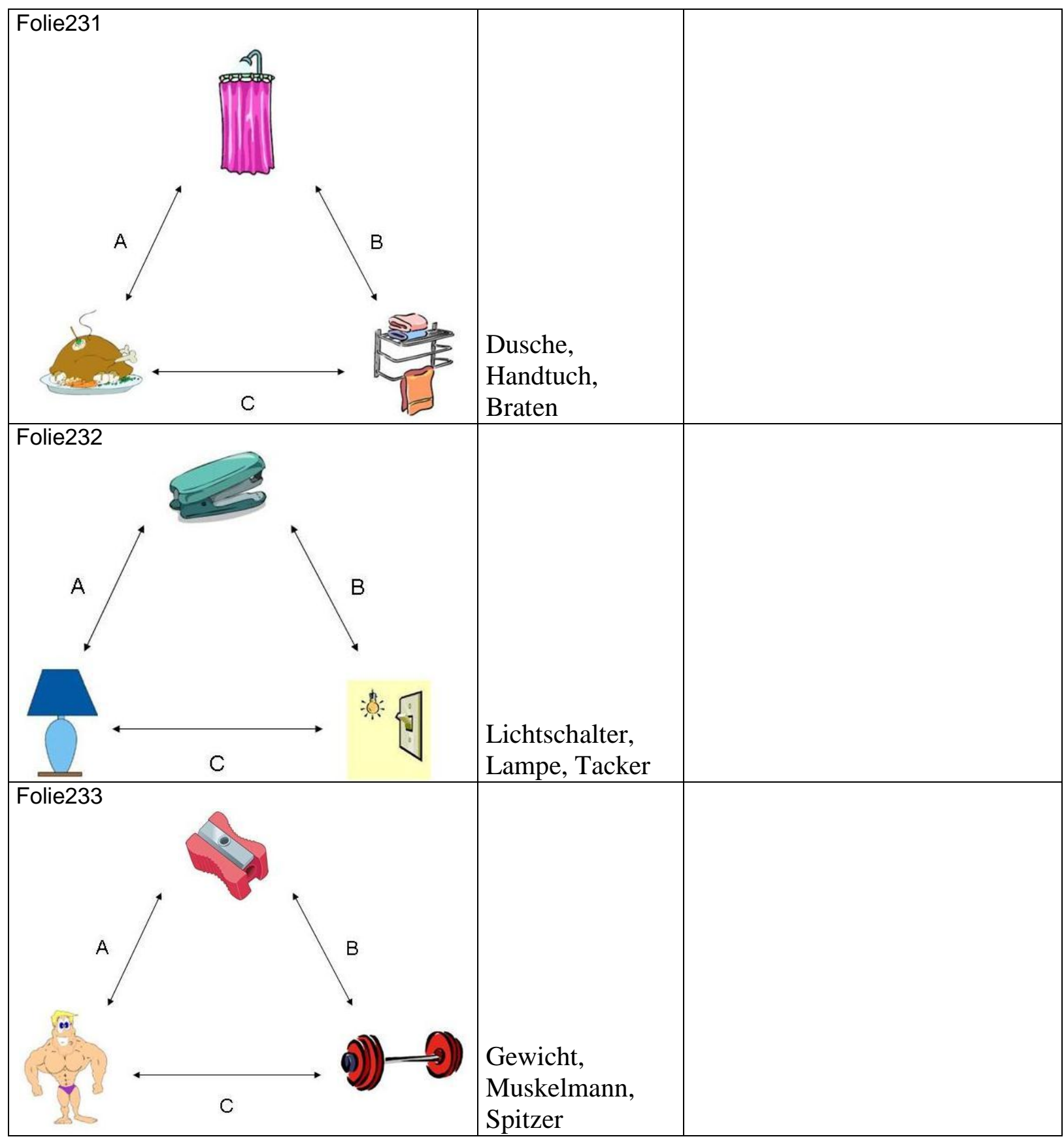




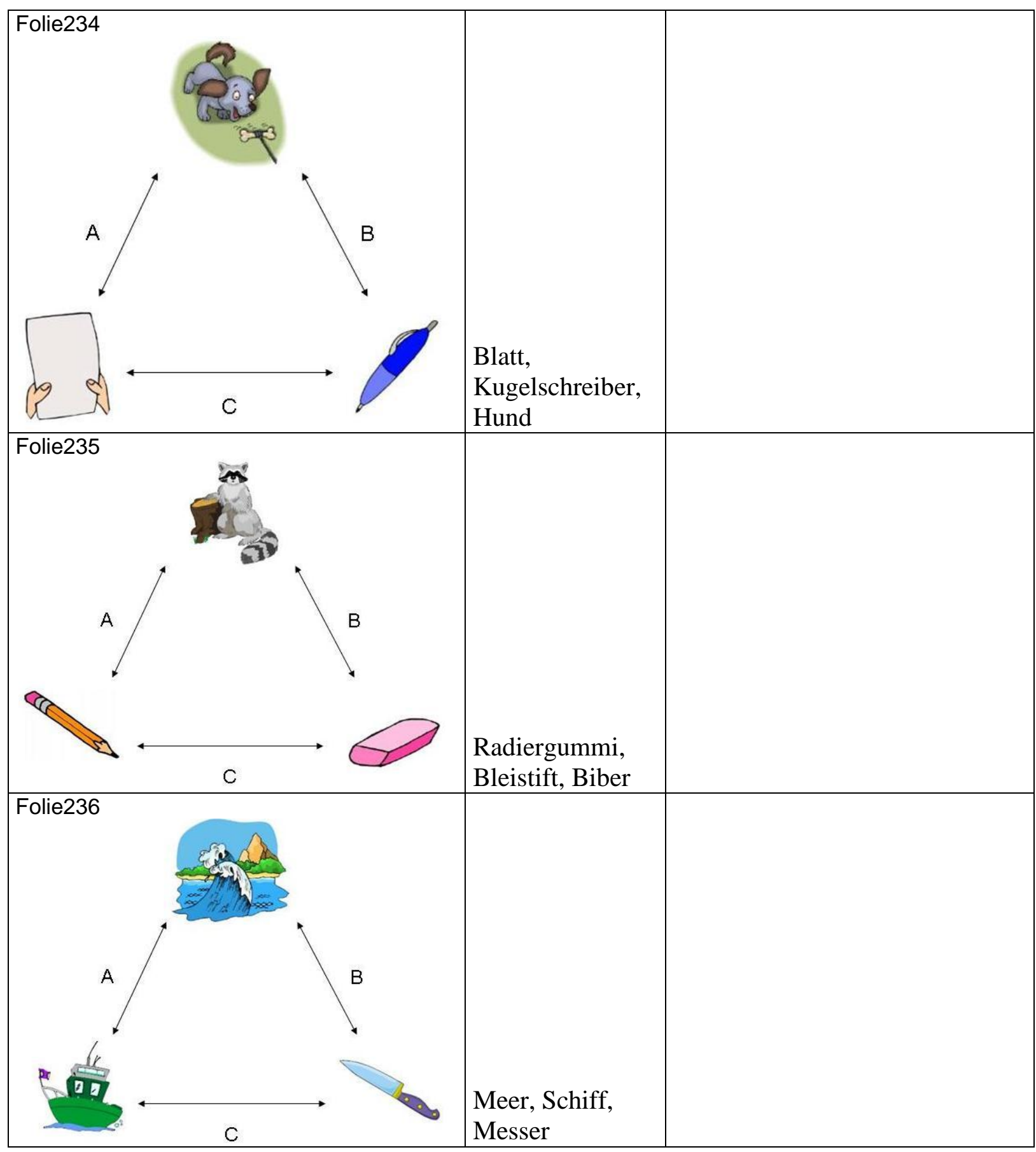




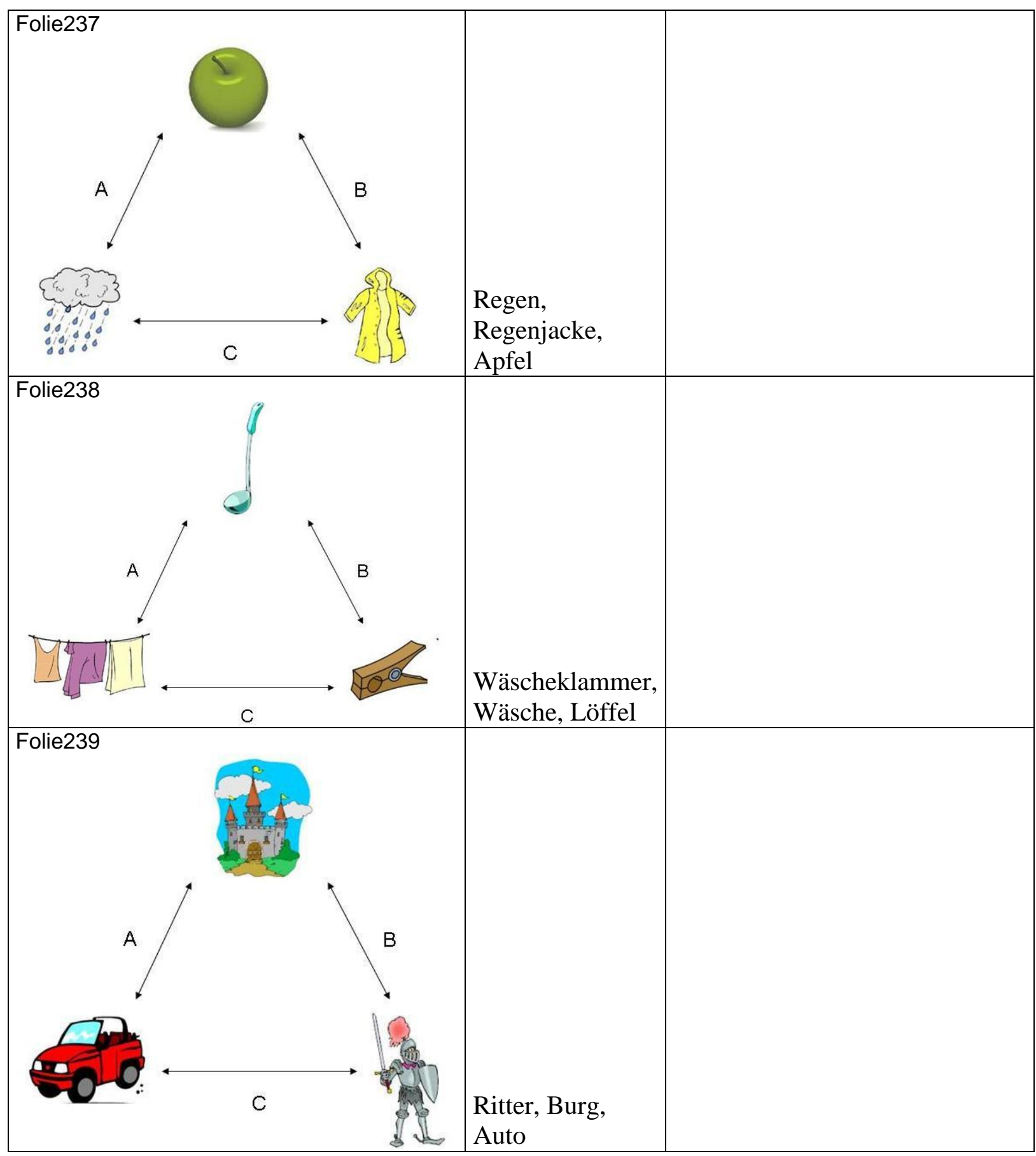




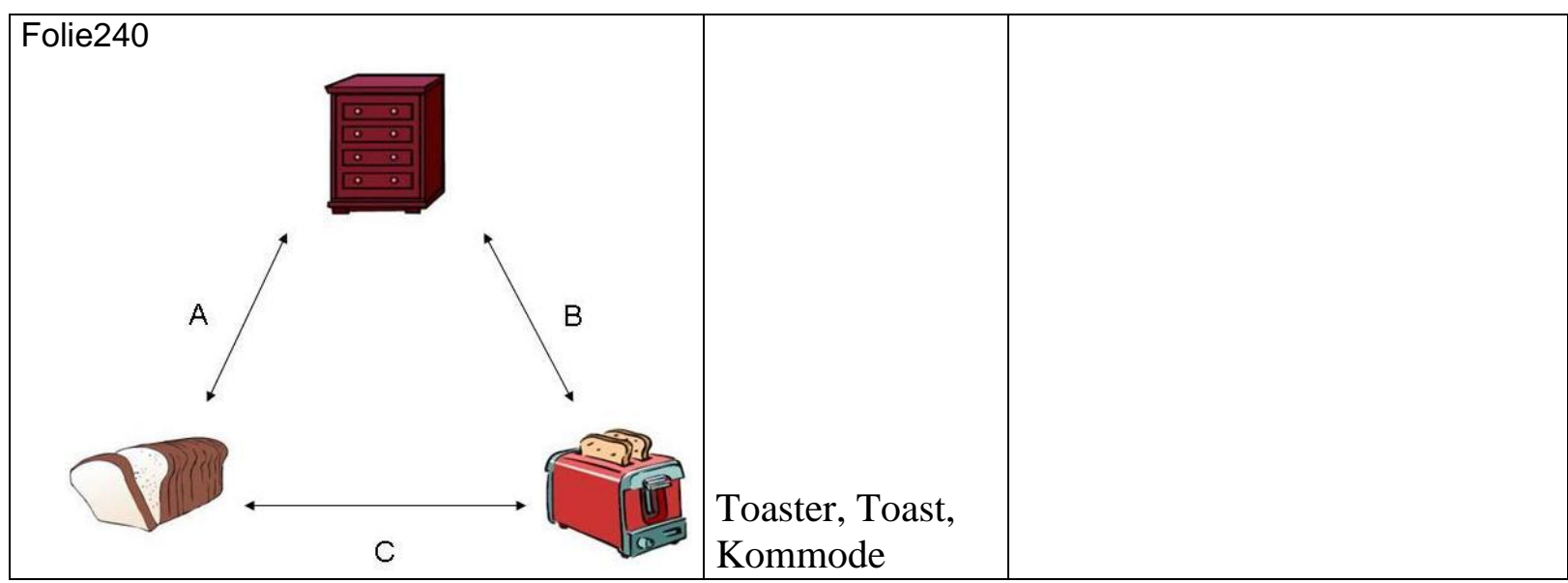


Anhang E: Instruktion Experiment 2 „Kategorisierung über Bildtripel“6

$\underline{\text { Deutsche Instruktion Seite } 1}$

Liebe/r Versuchsteilnehmer/in,

vielen Dank für deine Bereitschaft an diesem Versuch teilzunehmen!

Bitte lies dir die folgende Instruktion aufmerksam durch.

Im Verlauf des Versuchs werden dir auf dem Bildschirm Dreierpaare von Bildern präsentiert. Bitte beurteile, welche zwei Bilder am ehesten zusammen passen. Die beiden Bilder können z.B. einer gemeinsamen Kategorie angehören oder sie haben eine funktionale oder logische Verbindung zueinander. Versuche so schnell wie möglich zu antworten.

Der genaue Versuchsablauf wird im Folgenden anhand eines Beispiels verdeutlicht.

Bitte Leertaste drücken! 
Deutsche Instruktion Seite 2

Richte deinen Blick zunächst auf das Kreuz, welches auf dem Bildschirm erscheint! $+$

Fixiere dieses Kreuz solange, bis die Bilder präsentiert werden.

Beispiel:

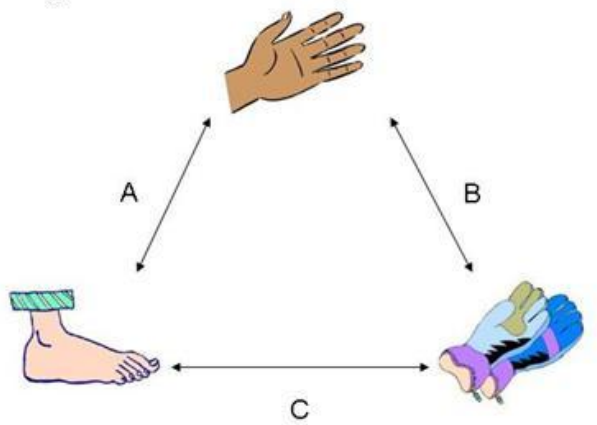

Wähle nun aus, welches Paar am ehesten zusammengehört und gebe deine Antwort über die markierten Tasten. Sollte für dich Verbindung A stimmig sein, so drücke die Taste A Sollte hingegen Verbindung B für dich am ehesten zutreffen, antworte mit Taste B. Es gibt oft keine richtigen oder falschen Antworten. Gib deine Antwort einfach danach ab, welche Verbindung für dich am ehesten zutrifft. Versuche so schnell wie möglich zu antworten.

Hast Du deine Antwort abgegeben, erscheint als nächstes wieder das Kreuz auf dem Bildschirm und der nächste Durchgang beginnt.

Das Experiment besteht aus mehreren Blöcken, wobei dir nach Vollendung jeden Blocks die Möglichkeit gegeben wird, solange Pause zu machen, wie du möchtest. Du kannst den nächsten Block selbständig starten, indem Du die Leertaste drückst.

Bei Fragen wende Dich bitte jetzt an die Versuchsleiter/in! 


\section{Chinesische Instruktion Seite 1}

亲爱的参试者:

非常感谢你参与这个实验!

请你仔细阅读以下说明。

在实验过程中你将会每次在屏幕上看到三对同时呈现的图片。

请你直觉地判断, 哪两幅图最为相互匹配。

这两幅图可能是属于同一类, 或者它们之间存在功能上的或者逻辑上的联系。 请你尽可能地快速回答。

下面将通过一个具体例子, 以使你了解实验的具体过程。

请按空格键！ 


\section{Chinesische Instruktion Seite 2}

请你先注意屏幕上出现的“十"字！

$+$

请注视这个“十"字, 直到图片出现。

例子:

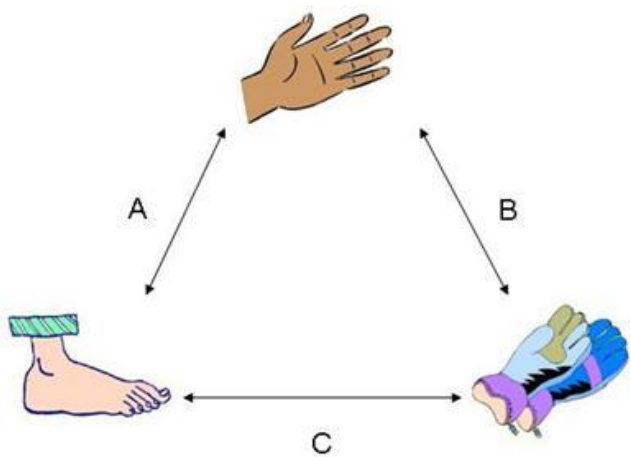

请选出, 哪一对最为匹配, 并通过指定的按键给出你的回答。 如果你同意 $A$ 组合, 请按 $A$ 键。如果你认为 $B$ 组合更为合适, 就请按 $B$ 键。 没有正确或错误的回答, 你只需要按直觉给出你认为最为合适的组合。 请尽可能地快速回答。

在你给出答案之后, 屏幕上又会出现“十“字, 下一轮的测试回答又开始了。 这个实验分为几个部分。每一个部分完成后会有间歇休息, 其时间由自己控制。 通过按空格键你可以启动下一部分实验, 继续进行回答。 若有问题, 请现在向主试者提出来! 
Anhang F: Itemmaterial Experiment 3 ,Sprachproduktion“

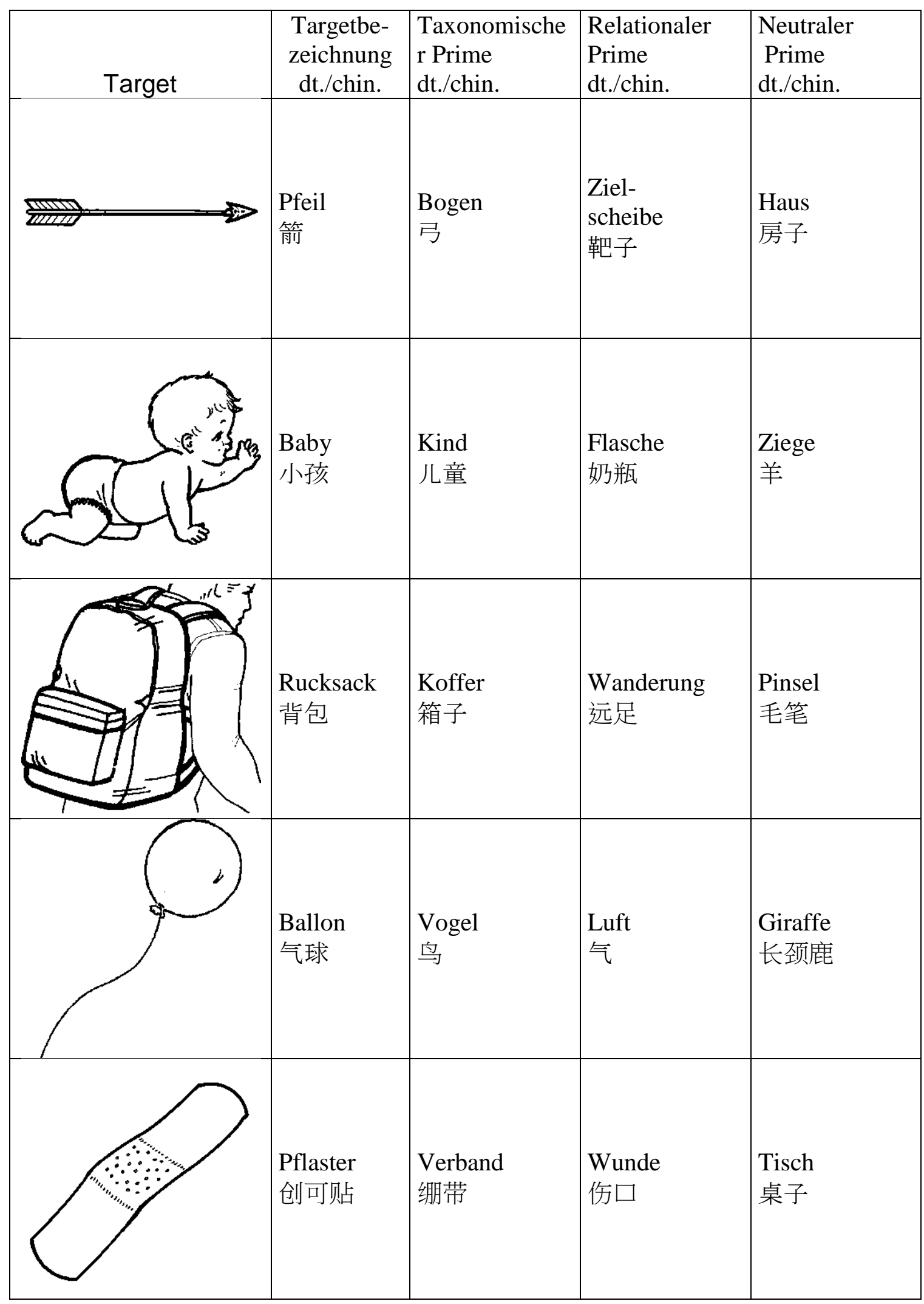




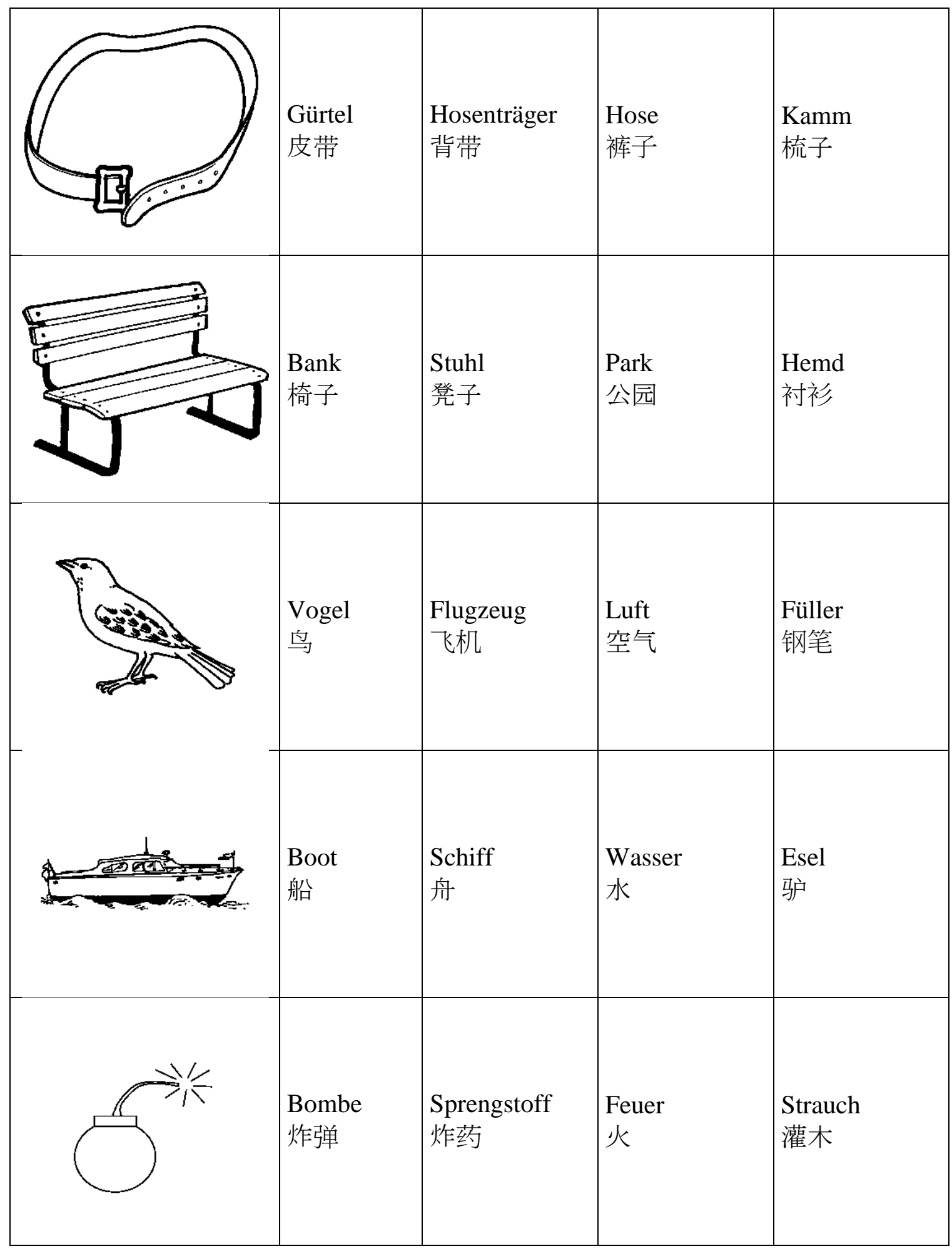




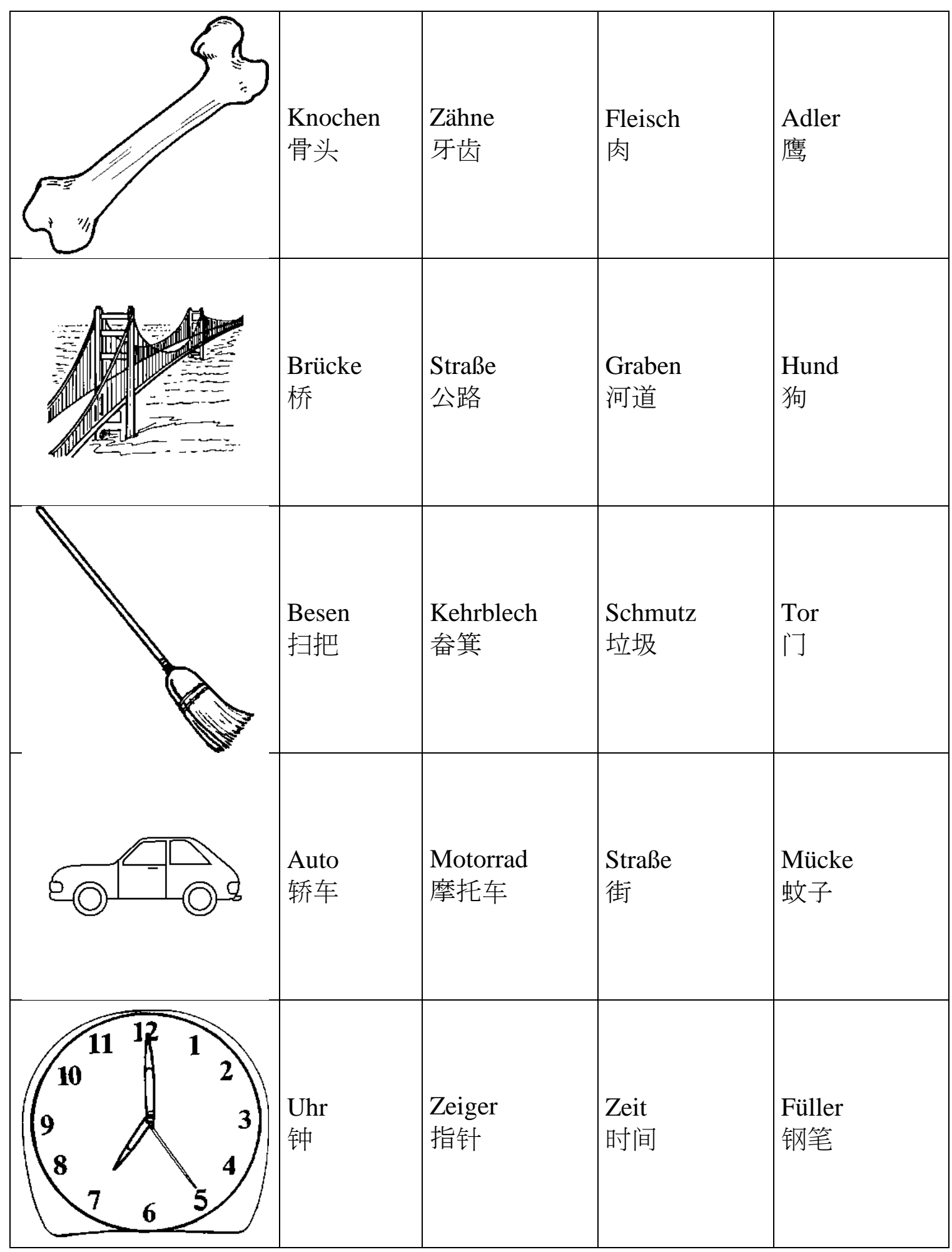




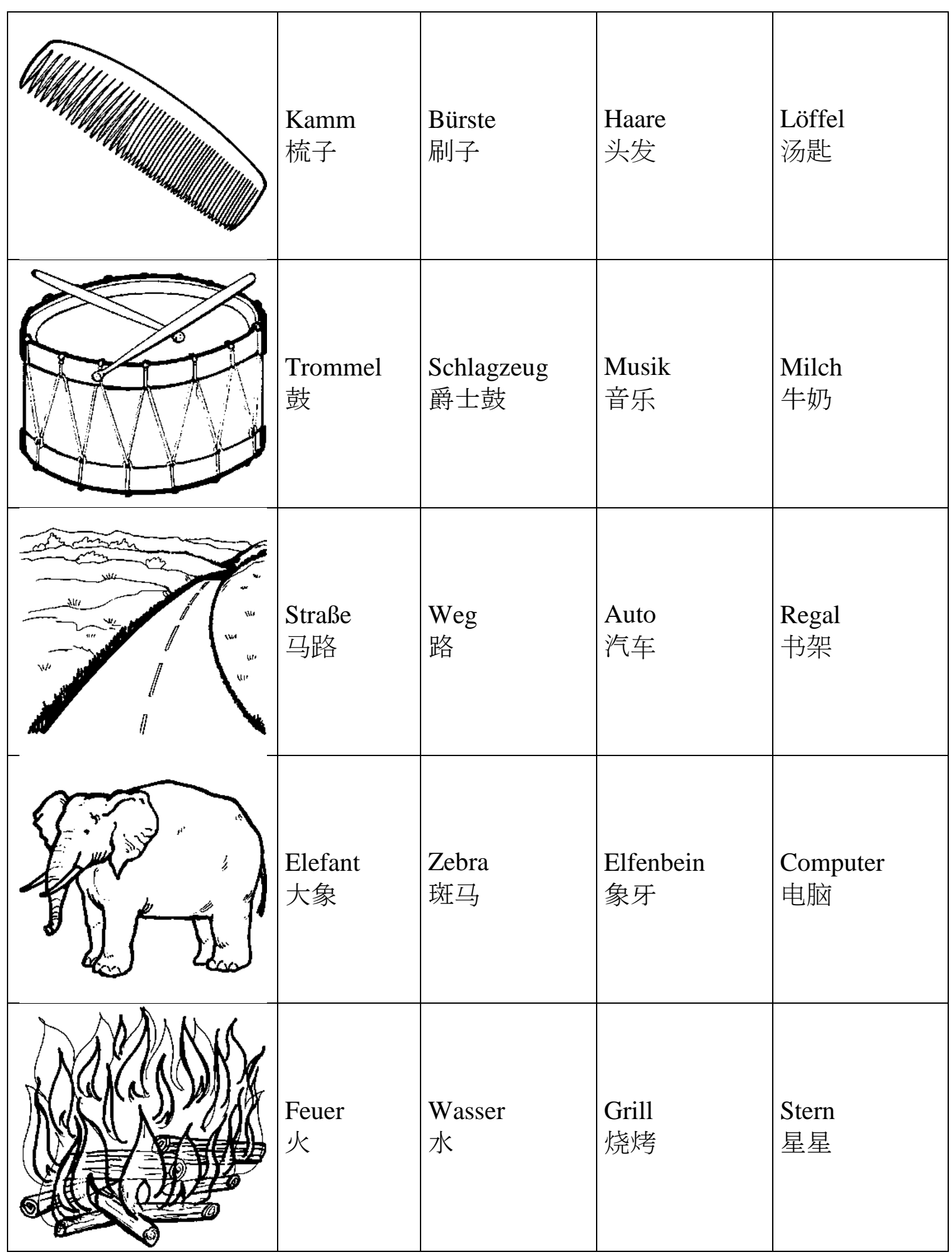




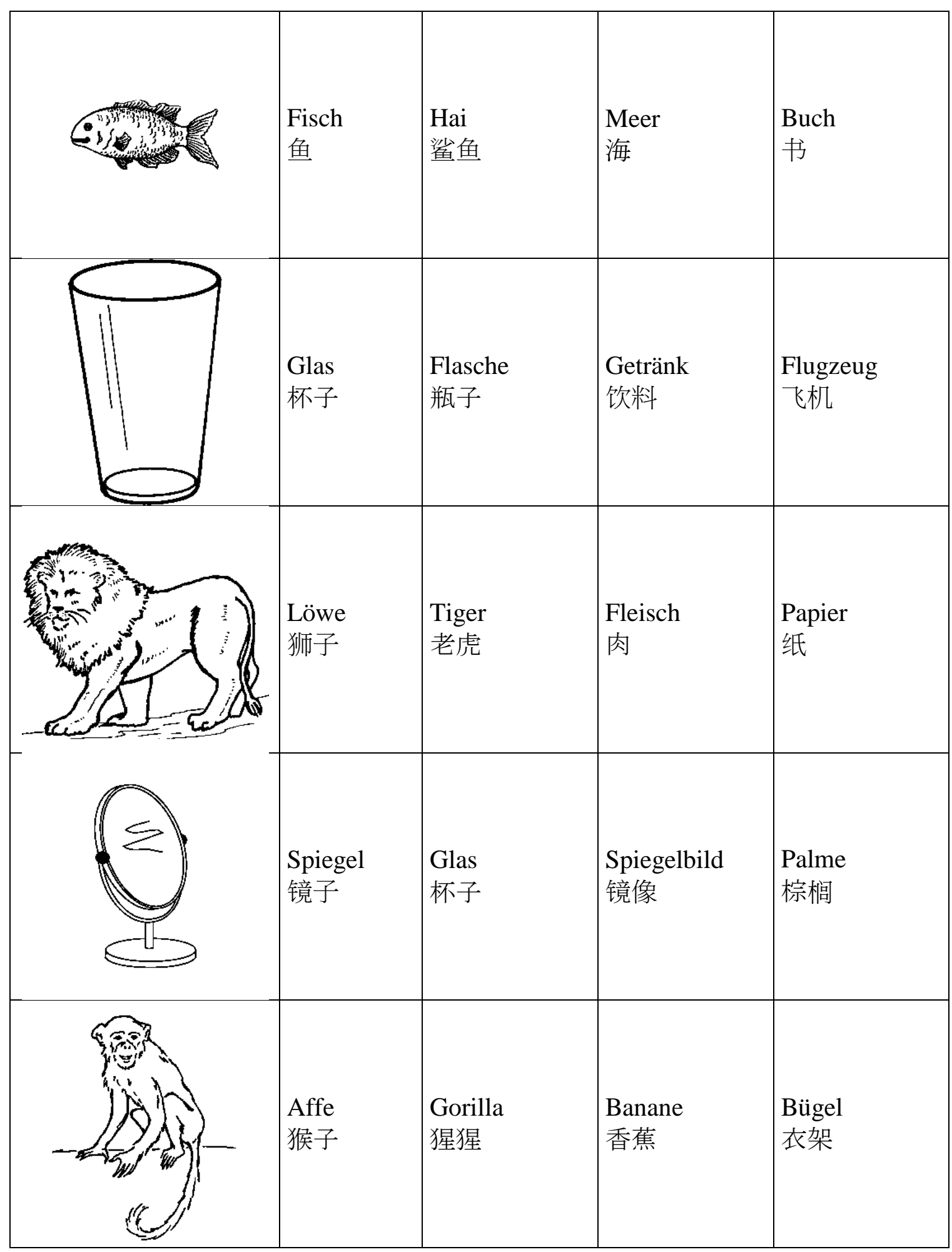




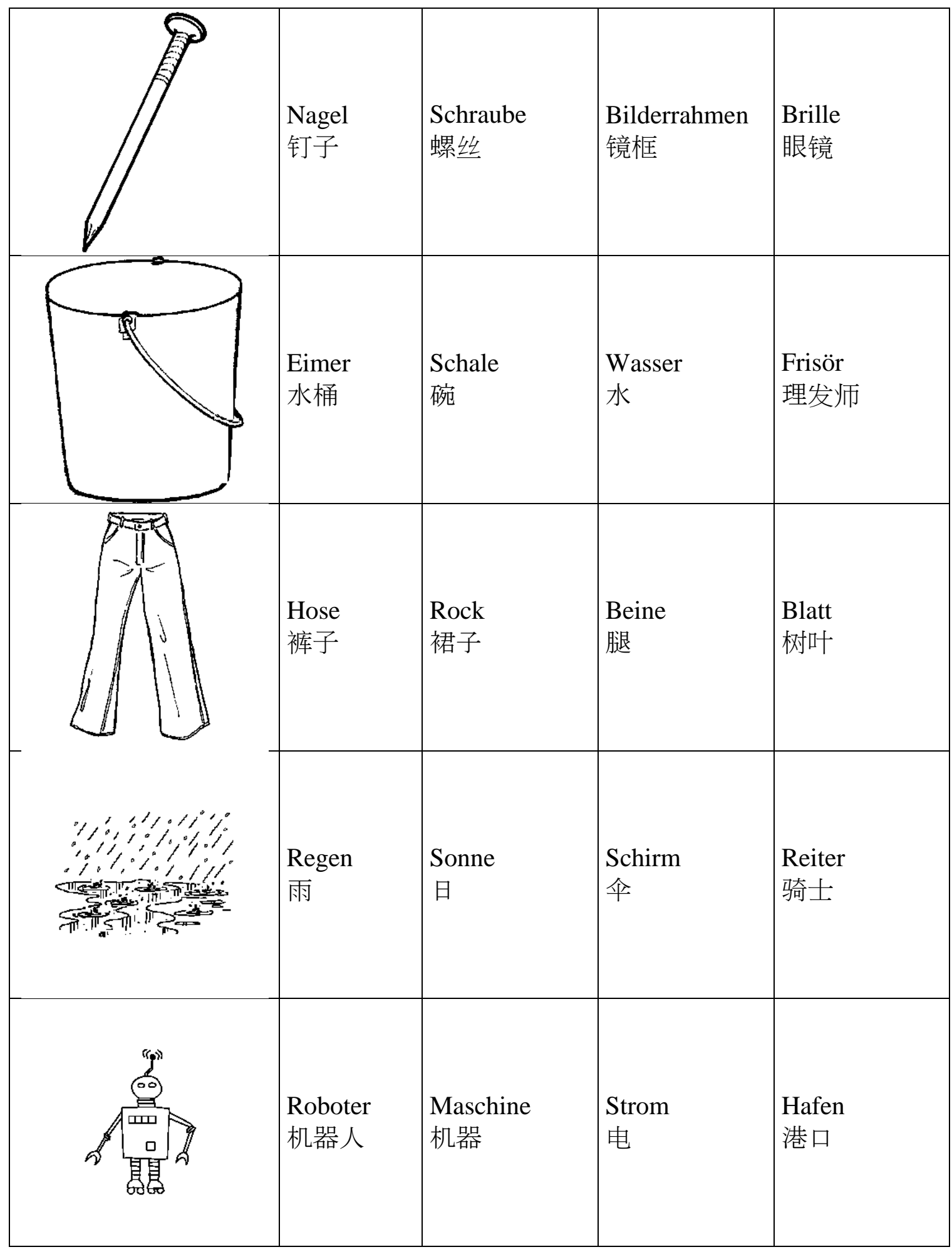




\section{Anhang G: Instruktion Experiment 3 ,Sprachproduktion“}

\section{Instruktion}

Vielen Dank für dein Interesse, an unserem Experiment teilzunehmen.

Das Experiment gliedert sich in mehrere Teile:

Im ersten Teil des Experiments wirst du die Bezeichnung einiger Bilder lernen.

Im zweiten Teil sollst du dann die Bilder so schnell wie möglich benennen und dabei die zuvor gelernten Bezeichnungen verwenden.

\section{Teil}

Zuerst werden dir auf dem Bildschirm nacheinander eine Anzahl von Bildern gezeigt, und zwar wird jedes Bild zusammen mit der passenden sprachlichen Bezeichnung gezeigt.

Es ist dabei wichtig, dass du dir für jedes Bild die gezeigte Bezeichnung einprägst. Um zu kontrollieren, ob du dir die richtige Bezeichnung gemerkt hast, werden dir die Bilder anschließend ohne Bezeichnung gezeigt, und du benennst jedes Bild mit der zuvor gelernten Bezeichnung.

\section{Teil}

In dem nächsten Teil des Experimentes, der auch am Computer stattfindet, sollst du versuchen, die gezeigten Bilder so schnell wie möglich mit den gelernten Bezeichnungen zu benennen. Fixiere zunächst das Kreuz in der Mitte des Bildschirms, bis das Bild erscheint, und benenne dieses dann. Sprich die Bezeichnung bitte laut und deutlich aus. Deine Antwort wird per Mirkofon aufgezeichnet. Auf dem Bildschirm wird kurz vor oder nach dem Bild oder auch gleichzeitig zusätzlich noch ein Wort erscheinen. Dieses Wort ist jedoch für die von dir erwartete Benennung irrelevant. Wichtig ist allein, dass du das Bild mit der zuvor gelernten Bezeichnung benennst.

Bitte antworte bei jedem Bild so schnell wie möglich, denn es werden immer mehrere Bilder hintereinander kommen. Vor jedem Bild wird das Fixationskreuz zu sehen sein. Es sind aber auch Pausen vorgesehen! Diese Pausen kannst du selbständig beenden.

Solltest du einen Fehler machen, unterbrich das Experiment bitte nicht, sondern mache einfach normal weiter. 


\section{Instruction}

We thank you very much for your interest in participating in our experiment. The experiment comprises different parts:

In the first part of the experiment you will learn how to name a set of pictures.

In the second part, your task is to name these pictures as fast as possible using the picture names, that you have learned before.

Part 1

We will start by showing you one by one a set of pictures on the srceen. Each picture will be accompanied by the appropriate name. It is important that you try to remember these names. As a test, the pictures will then be presented without their names and you will have to name them.

\section{Part 2}

In the following part of the experiment your task is to name the pictures as fast as possible by the names that you have learned before. First a cross will appear in the middle of the screen, then the picture. Please look at the cross until the picture is presented. As soon as the picture appears please name it by pronouncing the appropriate name that you have learned. Please try to speak loud and clearly because your answers are registered via a microphone. In addition to the picture there will appear a word on the screen either just before or after the presentation of the picture or simultaniously. This word is of no importance for your answer. You just have to name the picture by the name that you have learned before.

Please try to answer as fast as possible when a picture is presented because several pictures will be presented in rapid succession. But don't worry there will be breaks and you are free to choose the length of these breaks.

If you feel that you have made an error in naming please do not interrupt the experiment but try to continue the naming task .

If you still have questions please ask the experimenter. 
Anhang H: Nachtestfragebögen Experiment 3 „Sprachproduktion“

Deutscher Nachfragebogen

Vp.-Nr.:

Bitte ankreuzen:

1. Bist du

$\mathbf{O}$ männlich oder $\mathbf{O}$ weiblich ?

2. Wie alt bist du?

Jahre

3. Was studierst du?

4. Hast du eine bestimmte Strategie verwendet?

5. Hast du irgendwelche Kommentare?

Vielen Dank für deine Teilnahme! 


\section{Chinesisches Nachfragebogen}

Vp.-Nr.:

Please make a cross:

1. Are you

$\mathbf{O}$ male or $\mathbf{O}$ female ?

2. How old are you? years

3. What are you studying?

4. Did you use any specific strategy?

5. Do you have any comments?

Thank you very much for your participation! 
Hiermit versichere ich, dass ich die Dissertation mit dem Titel „Untersuchung kulturbedingter Kategorisierungspräferenzen bei chinesischen und deutschen Probandinnen und Probanden“ selbständig und ohne Benutzung anderer als der angegeben Hilfsmittel angefertigt habe. Alle Stellen, die wörtlich oder sinngemäß aus Veröffentlichungen oder anderen Quellen entnommen sind, sind als solche kenntlich gemacht.

Göttingen, den 20.12.2012

Markus Reitt 